\title{
PISA 2003 Data Analysis Manual
}

\section{SPSS ${ }^{\circledR}$ USERS}

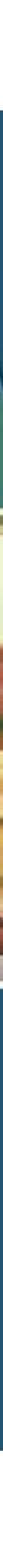


Programme for International Student Assessment

\section{PISA 2003 \\ Data Analysis Manual \\ SPSS ${ }^{\circledR}$ Users}





\section{Foreword}

The OECD's Programme for International Student Assessment (PISA) surveys, which take place every three years, have been designed to collect information about 15-year-old students in participating countries. PISA examines how well students are prepared to meet the challenges of the future, rather than how well they master particular curricula. The data collected during each PISA cycle are an extremely valuable source of information for researchers, policy makers, educators, parents and students. It is now recognised that the future economic and social well-being of countries is closely linked to the knowledge and skills of their populations. The internationally comparable information provided by PISA allows countries to assess how well their 15-year-old students are prepared for life in a larger context and to compare their relative strengths and weaknesses.

The PISA 2003 database, on which this manual is focused, contains information on over a quarter of a million students from 41 countries. It includes not only information on their performance in the four main areas of assessment - reading, mathematics, science and problem solving - but also their responses to the Student Questionnaire that they complete as part of the assessment. Data from the school principals are also included.

The PISA 2003 Data Analysis Manual has evolved from the analytical workshops held in Sydney, Vienna, Paris and Bratislava, which exposed participants to the various techniques needed to correctly analyse the complex databases. It allows analysts to confidently replicate procedures used for the production of the PISA 2003 initial reports, Learning for Tomorrow's World - First Results from PISA 2003 (OECD, 2004a) and Problem Solving for Tomorrow's World - First Measures of Cross-Curricular Competencies from PISA 2003 (OECD, 2004b), and to accurately undertake new analyses in areas of special interest. In addition to the inclusion of the necessary techniques, the manual also includes a detailed account of the variables constructed from the student and school questionnaires. This information was previously published in the Manual for the PISA 2000 Database (OECD, 2002a).

The PISA 2003 Data Analysis Manual is in four parts - the first two sections give a detailed theoretical background and instructions for analysing the data; the third section lists the program codes (syntaxes and the macros), which are needed to carry out the analyses; and the fourth section contains a detailed description of the database.

PISA is a collaborative effort by the participating countries, and guided by their governments on the basis of shared policy-driven interests. Representatives of each country form the PISA Governing Board which decides on the assessment and reporting of results in PISA.

There are two versions of this manual - one for SPSS ${ }^{\circledR}$ users and one for SAS ${ }^{\circledR}$ users. The OECD recognises the creative work of Christian Monseur in preparing the text for both versions of the manual in collaboration with Sheila Krawchuk and Keith Rust, as well as his preparation of the program coding for the SAS ${ }^{\circledR}$ users' manual. The coding for the SPSS ${ }^{\circledR}$ users' manual was prepared by Wolfram Schulz and Eveline Gebhardt. The main editorial work was completed at the OECD Secretariat by Miyako Ikeda, Sophie Vayssettes, John Cresswell, Claire Shewbridge and Kate Lancaster. The PISA assessments and the data underlying the manuals were prepared by the PISA Consortium under the direction of Raymond Adams. 



\section{Table of Contents \\ .}

\section{CHAPTER 1}

The OECD's Programme for International Student Assessment ................................................... 11

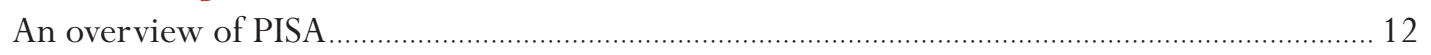

What makes PISA unique? ........................................................................................................ 13

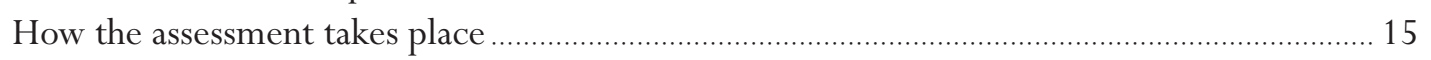

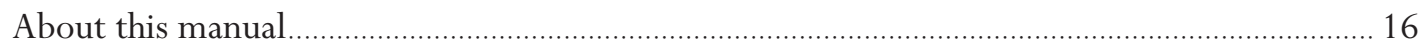

\section{CHAPTER 2}

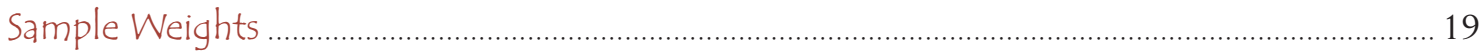

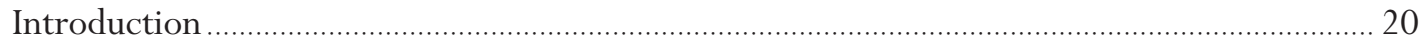

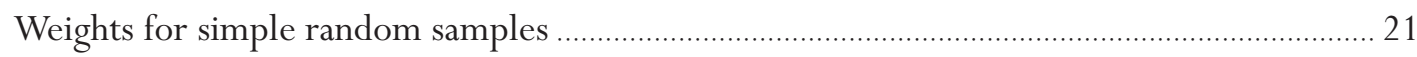

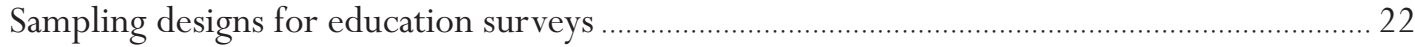

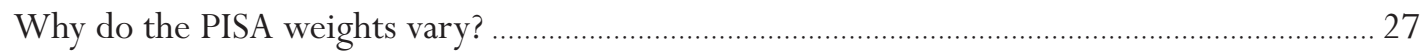

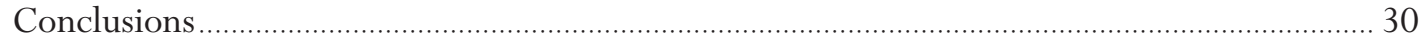

\section{CHAPTER 3}

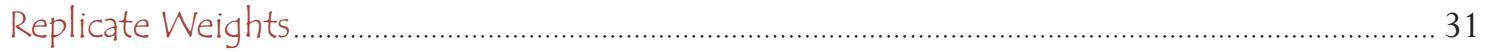

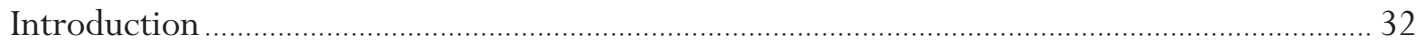

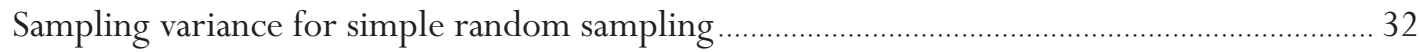

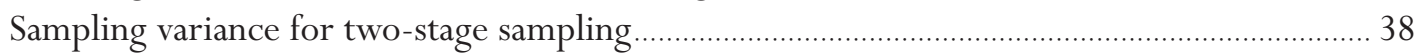

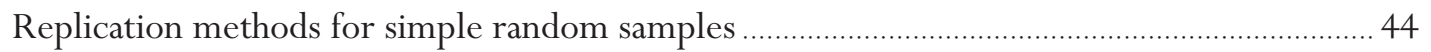

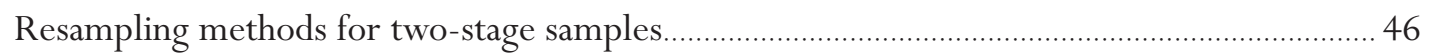

The Jackknife for unstratified two-stage sample designs ......................................................... 47

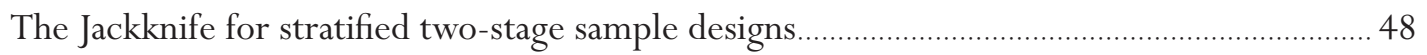

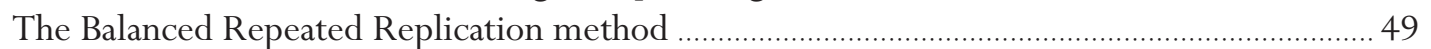

Other procedures for accounting for clustered samples ....................................................... 51

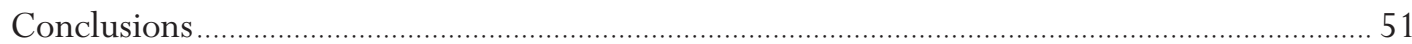

\section{CHAPTER 4}

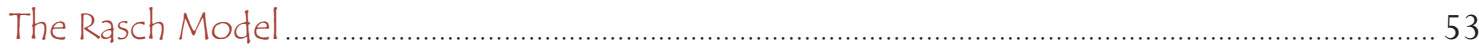

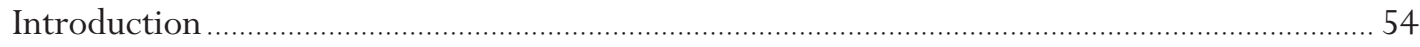

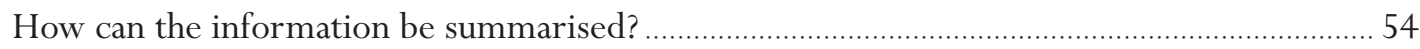

The Rasch model for dichotomous items ......................................................................................... 56

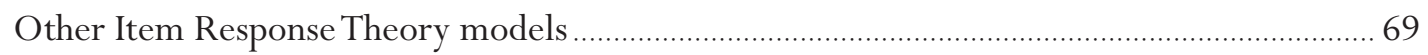

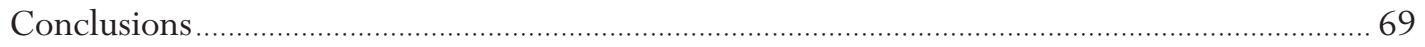


CHAPTER 5

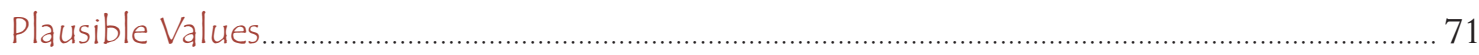

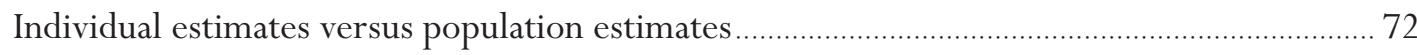

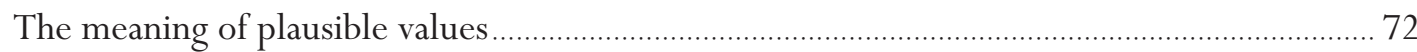

Comparison of the efficiency of Warm Likelihood Estimates, Expected A Posteriori estimates and plausible values for the estimation of some population statistics ....................... 76

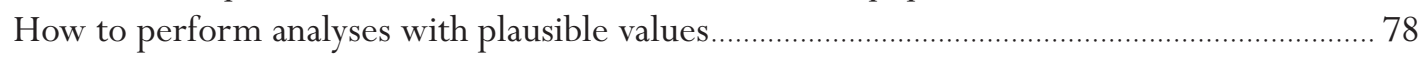

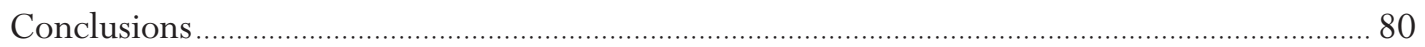

CHAPTER 6

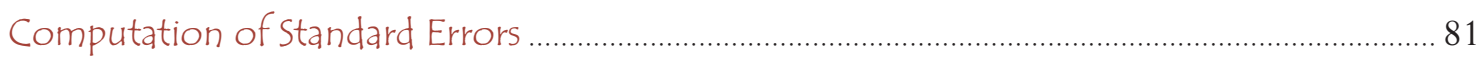

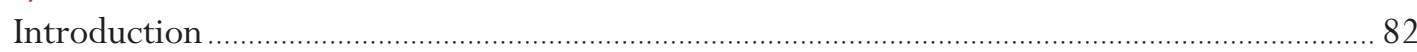

The standard error on univariate statistics for numerical variables .......................................... 82

The SPSS ${ }^{\circledR}$ macro for computing the standard error on a mean ….......................................... 85

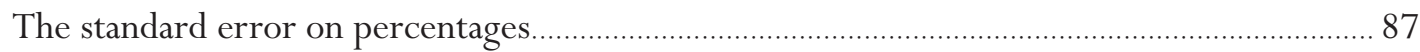

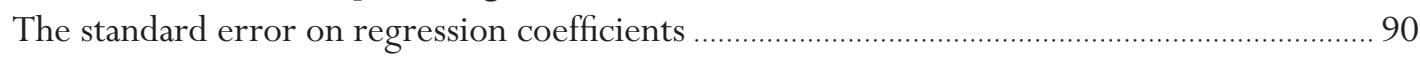

The standard error on correlation coefficients ............................................................................... 92

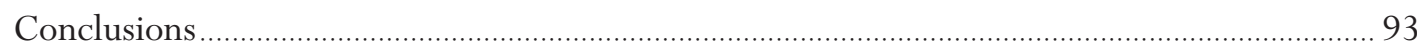

CHAPTER 7

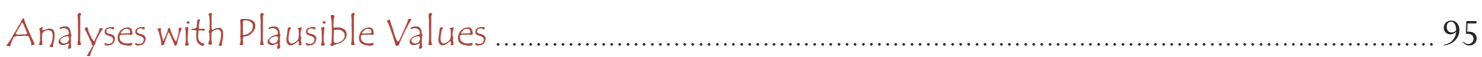

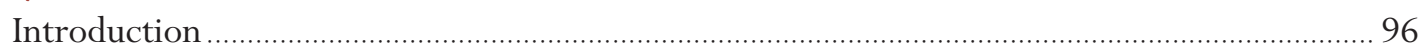

Univariate statistics on plausible values …………......................................................................... 96

The standard error on percentages with plausible values ..................................................... 101

The standard error on regression coefficients with plausible values ....................................... 101

The standard error on correlation coefficients with plausible values ........................................... 104

Correlation between two sets of plausible values ....................................................................... 104

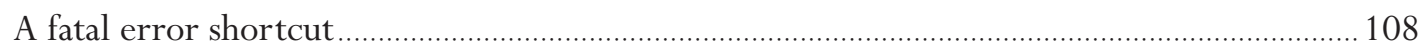

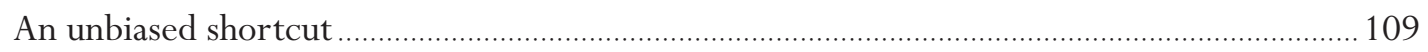

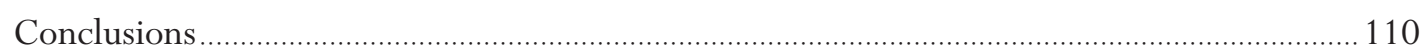

CHAPTER 8

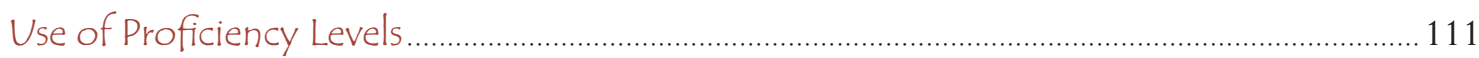

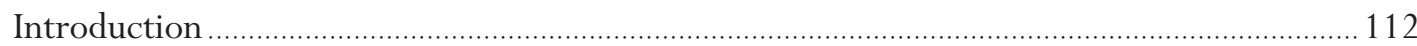

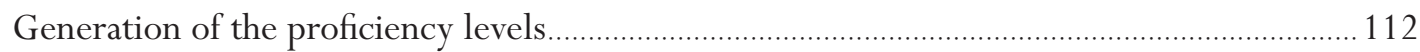

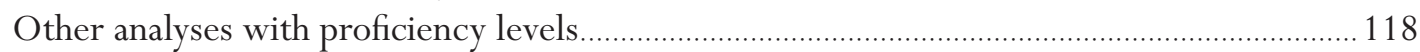

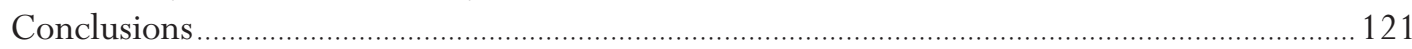

CHAPTER 9

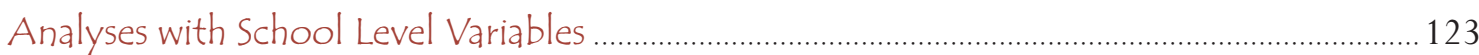

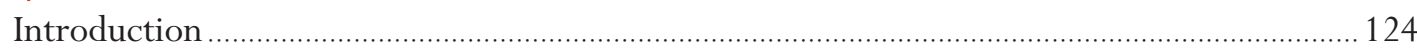

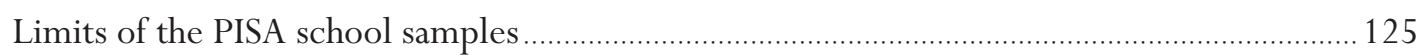

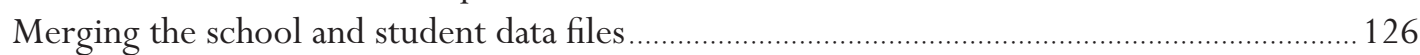

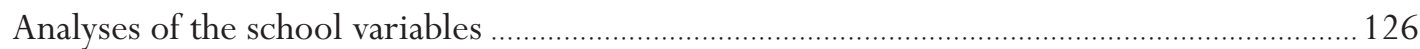

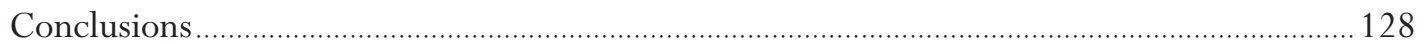


CHAPTER 10

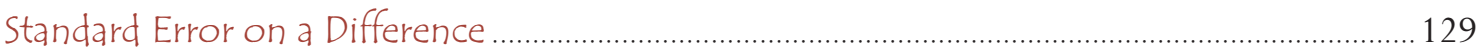

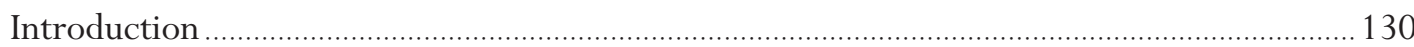

The standard error of a difference without plausible values ................................................... 132

The standard error of a difference with plausible values............................................................ 137

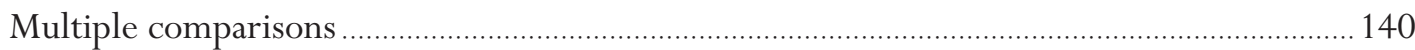

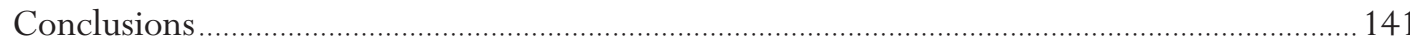

CHAPTER 11

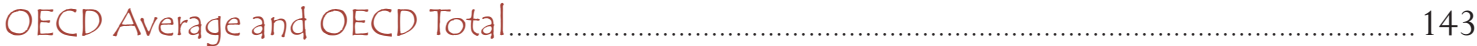

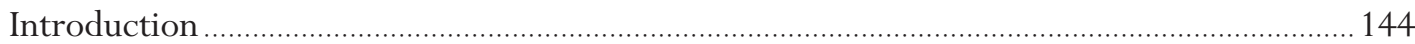

Recoding of the database for the estimation of the OECD total and OECD average ............. 144

Duplication of the data for avoiding three runs of the procedure .......................................... 147

Comparisons between OECD average or OECD total estimates and a country estimate ......... 147

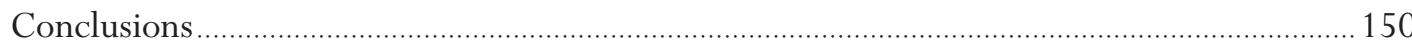

CHAPTER 12

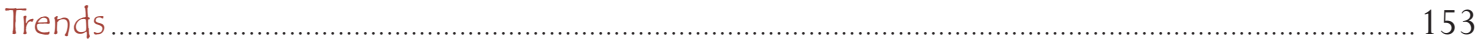

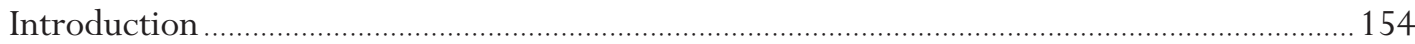

The computation of the standard error for trend indicators on variables other

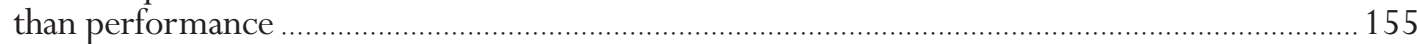

The computation of the standard error for trend indicators on performance variables ........ 158

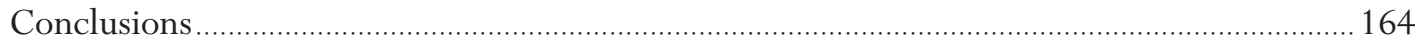

CHAPTER 13

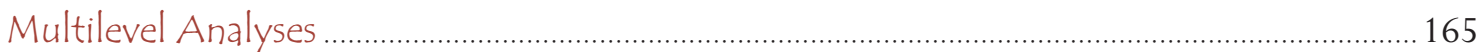

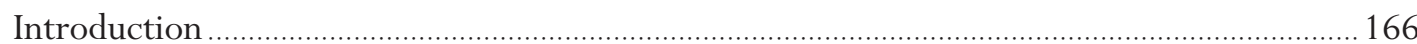

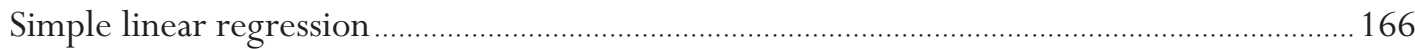

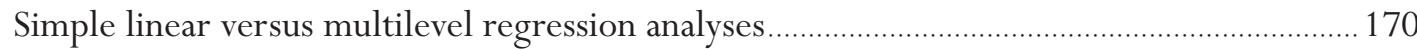

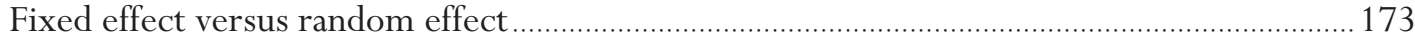

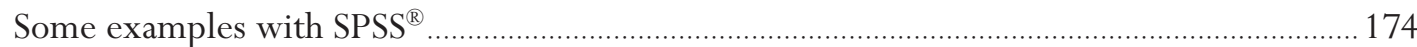

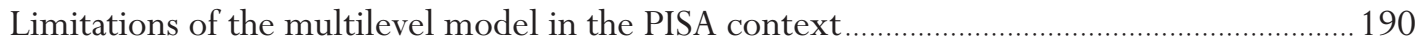

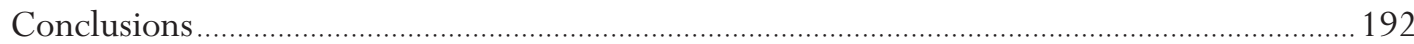

CHAPTER 14

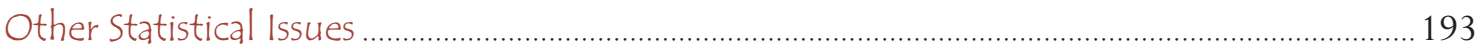

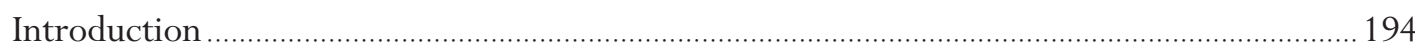

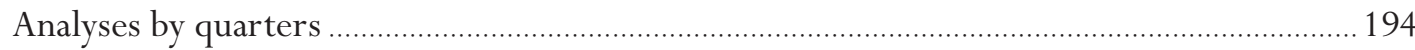

The concepts of relative risk and attributable risk ................................................................. 198

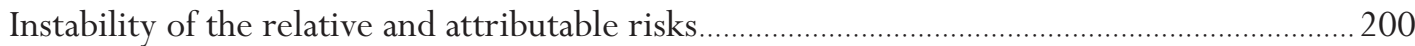

Computation of the relative risk and attributable risk ................................................................2201

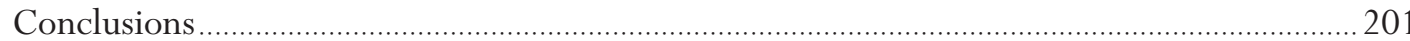

CHAPTER 15

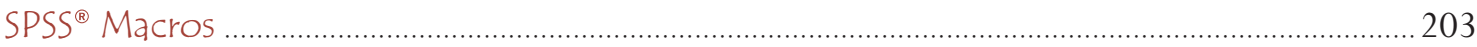

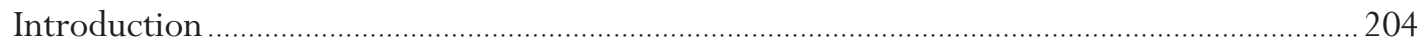

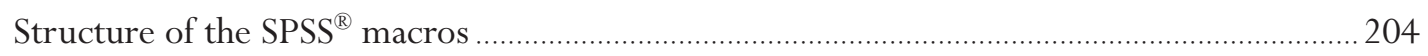




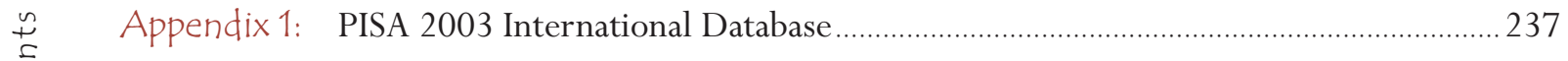

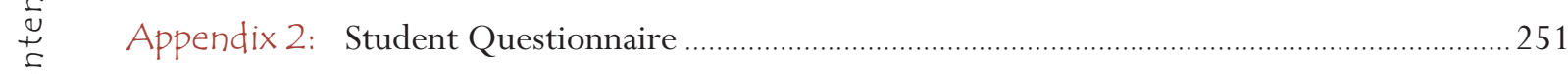

$\cup \quad$ Appendix 3: Educational Career Questionnaire ……................................................................. 265

ᄂ Appendix 4: Information Communication Technology (ICT) Questionnaire ..............................267

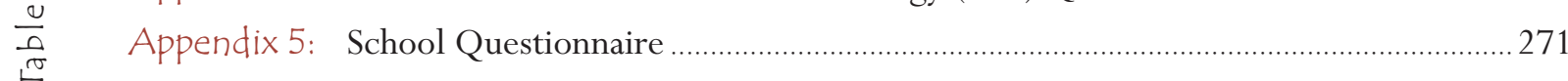

Appendix 6: Student Questionnaire Data File Codebook ..............................................................2 285

Appendix 7: School Questionnaire Data File Codebook ……........................................................ 331

Appendix 8: Student Cognitive Test Data File Codebook............................................................... 345

Appendix 9: Student and School Questionnaire Indices ............................................................... 375

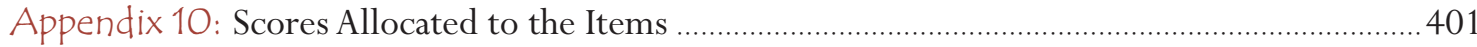

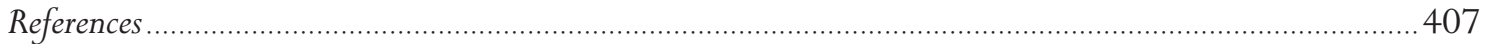




\section{USERS'GUIDE}

\section{Preparation of data files}

All data files (in text format) and the SPSS ${ }^{\circledR}$ control files are available on the PISA Web site (www.pisa.oecd.org).

\section{SPSS ${ }^{\circledR}$ users}

The PISA 2003 student data file and the PISA 2003 school data file need to be saved under "c: \PISA \Data2003" before running the syntax in the following chapters.

Student data file: c: \PISA \Data2003\INT_stui_2003.sav

School data file: c: \PISA \Data2003\INT_schi_2003.sav

The SPSS ${ }^{\circledR}$ macros presented in Chapter 15 need to be saved under “c: $\backslash$ PISA $\backslash$ macros".

\section{SPSS ${ }^{\circledR}$ syntax and macros}

All syntaxes and macros used in this manual can be copied from the PISA Web site (www.pisa. oecd.org). Each chapter of the manual contains a complete set of syntaxes, which must be done sequentially, for all of them to run correctly, within the chapter.

\section{Rounding of figures}

In the tables and formulas, figures were rounded to a convenient number of decimal places, although calculations were always made with the full number of decimal places.

\section{Country abbreviations used in this manual}

\begin{tabular}{l|l|l|l|l|l|l|l}
\hline AUS & Australia & FRA & France & KOR & Korea & PRT & Portugal \\
\hline AUT & Austria & GBR & United Kingdom & LIE & Liechtenstein & RUS & Russian Federation \\
\hline BEL & Belgium & GRC & Greece & LUX & Luxembourg & SVK & Slovakia \\
\hline BRA & Brazil & HKG & Hong Kong-China & LVA & Latvia & SWE & Sweden \\
\hline CAN & Canada & HUN & Hungary & MAC & Macao-China & THA & Thailand \\
\hline CHE & Switzerland & IDN & Indonesia & MEX & Mexico & TUN & Tunisia \\
\hline CZE & Czech Republic & IRL & Ireland & NLD & Netherlands & TUR & Turkey \\
\hline DEU & Germany & ISL & Iceland & NOR & Norway & URY & Uruguay \\
\hline DNK & Denmark & ITA & Italy & NZL & New Zealand & USA & United States \\
\hline ESP & Spain & JPN & Japan & POL & Poland & YUG & Serbia \\
\hline FIN & Finland & & & & & &
\end{tabular}

\section{Socio-economic status}

The highest occupational status of parents (HISEI) is referred to as the socio-economic status of the students throughout this manual. It should be noted that occupational status is only one aspect of socio-economic status, which can also include education and wealth. The PISA 2003 database also includes a broader socio-economic measure called the index of Economic, Social and Cultural Status (ESCS), which is derived from the highest occupational status of parents, the highest educational level and an estimate related to household possessions. 


\section{Further documentation}

For further information on the PISA 2003 results, see the PISA 2003 initial reports: Learning for Tomorrow's World - First Results from PISA 2003 (OECD, 2004a) and Problem Solving for Tomorrow's World - First Measures of Cross-Curricular Competencies from PISA 2003 (OECD, 2004b). For further information on the PISA assessment instruments and the method used in PISA, see the PISA 2003 Technical Report (OECD, forthcoming) and the PISA Web site (www.pisa.oecd.org). 


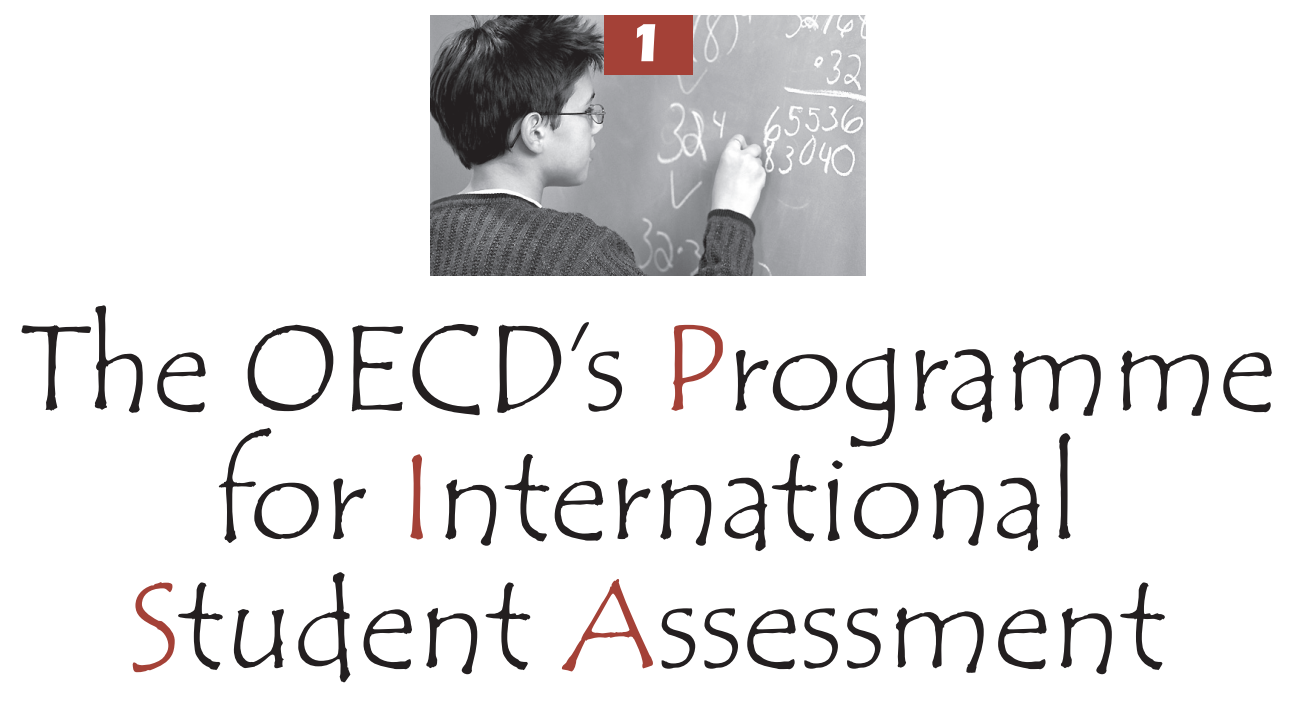

An overview of PISA

What makes PISA unique?

How the assessment takes place

About this manual 


\section{AN OVERVIEW OF PISA}

The OECD's Programme for International Student Assessment (PISA) is a collaborative effort, involving all OECD countries and a significant number of partner countries, to measure how well 15-year-old students are prepared to meet the challenges of today's knowledge societies. The assessment looks to the future, focusing on young people's ability to use their knowledge and skills to meet real-life challenges, rather than on the mastery of specific school curricula. This orientation reflects a change in the goals and objectives of curricula themselves, which are increasingly concerned with knowledge application rather than merely knowledge acquisition. The age of 15 is used because in most OECD countries it is the age at which students are approaching the end of compulsory schooling.

PISA is the most comprehensive and rigorous international effort to date to assess student performance and to collect data about the student, as well as about the family and institutional factors potentially affecting performance. Decisions about the scope and nature of the assessment and the background information to be collected were made by leading experts in participating countries and steered jointly by their governments on the basis of shared, policy-driven interests. Substantial efforts and resources were devoted to achieving wide cultural and linguistic coverage in the assessment materials. Stringent quality assurance mechanisms were applied in translation, sampling and data collection. As a consequence, the results of PISA have a high degree of validity and reliability, and they can significantly improve understanding of the outcomes of education in a large number of the world's countries.

PISA is based on a dynamic model of lifelong learning in which new knowledge and skills necessary for successful adaptation to a changing world are continuously acquired throughout life. PISA focuses on skills that 15-year-olds will need in the future and seeks to assess their ability to perform them. PISA does assess students' knowledge, but it also examines their potential to reflect on their knowledge and experiences, and to apply that knowledge and those experiences to real-world issues. For example,

Table 1.1 Participating countries in PISA 2000 and in PISA 2003

\begin{tabular}{|c|c|c|}
\hline & PISA 2000 & PISA 2003 \\
\hline $\begin{array}{l}\text { OECD } \\
\text { countries }\end{array}$ & $\begin{array}{l}\text { Australia, Austria, Belgium, Canada, } \\
\text { Czech Republic, Denmark, Finland, France, } \\
\text { Germany, Greece, Hungary, Iceland, } \\
\text { Ireland, Italy, Japan, Korea, Luxembourg, } \\
\text { Mexico, Netherlands, }{ }^{a} \text { New Zealand, } \\
\text { Norway, Poland, Portugal, Spain, Sweden, } \\
\text { Switzerland, United Kingdom, } \\
\text { United States. }\end{array}$ & $\begin{array}{l}\text { Australia, Austria, Belgium, Canada, } \\
\text { Czech Republic, Denmark, Finland, France, } \\
\text { Germany, Greece, Hungary, Iceland, } \\
\text { Ireland, Italy, Japan, Korea, Luxembourg, } \\
\text { Mexico, Netherlands, New Zealand, } \\
\text { Norway, Poland, Portugal, Slovak Republic, } \\
\text { Spain, Sweden, Switzerland, Turkey, } \\
\text { United Kingdom, United States. }\end{array}$ \\
\hline $\begin{array}{l}\text { Partner } \\
\text { countries }\end{array}$ & $\begin{array}{l}\text { Albania, Argentina, Brazil, Bulgaria, Chile, } \\
\text { Hong Kong-China, Indonesia, Israel, Latvia, } \\
\text { Liechtenstein, Macedonia, Peru, Romania, } \\
\text { Russian Federation, Thailand }\end{array}$ & $\begin{array}{l}\text { Brazil, Hong Kong-China, Indonesia, } \\
\text { Liechtenstein, Latvia, Macao-China, } \\
\text { Russian Federation, Thailand, Tunisia, } \\
\text { Uruguay, Serbia. }^{c}\end{array}$ \\
\hline
\end{tabular}

a. Response rate is too low to ensure comparability. See Annex 3 in Literacy Skills for the World of Tomorrow - Further Results From PISA 2000 (OECD, 2003a).

b. Response rate is too low to ensure comparability. See Annex 3 in Learning for Tomorrow's World - First Results from PISA 2003 (OECD, 2004a).

c. For the country Serbia and Montenegro, data for Montenegro are not available in PISA 2003. The latter accounts for 7.9 per cent of the national population. The name "Serbia" is used as a shorthand for the Serbian part of Serbia and Montenegro. 
in order to understand and evaluate scientific advice on food safety, an adult would not only need to know some basic facts about the composition of nutrients, but should also be able to apply that information. The term "literacy" is used to encapsulate this broader concept of knowledge and skills.

PISA is an ongoing survey with a data collection every three years. The first PISA survey was conducted in 2000 in 32 countries, using written tasks answered in schools under independently supervised test conditions following consistently applied standards. Another 11 countries participated in the same survey in late 2001 or early 2002. The second survey was conducted in 2003 in 41 countries. Table 1.1 gives the list of participating countries for PISA 2000 and PISA 2003.

PISA mainly assesses reading, mathematical and scientific literacy. For each data collection, one of these three domains is chosen as the major domain, while the others are considered as minor domains. PISA 2000 focused on reading, while the major domain for PISA 2003 was mathematical literacy. About 70 per cent of the testing time is devoted to the major domain and the remainder is shared by the minor domains.

Table 1.2 - Assessment domains covered per data collection

\begin{tabular}{l|l|l}
\hline & Major domain & Minor domains \\
\hline PISA 2000 & Reading literacy & Mathematical literacy, scientific literacy \\
\hline PISA 2003 & Mathematical literacy & $\begin{array}{l}\text { Reading literacy } \\
\text { Scientific literacy } \\
\text { Problem solving }\end{array}$ \\
\hline PISA 2006 & Scientific literacy & $\begin{array}{l}\text { Mathematical literacy } \\
\text { Reading literacy }\end{array}$ \\
\hline
\end{tabular}

In 2009, the major domain will again be reading literacy.

\section{WHAT MAKES PISA UNIQUE?}

PISA is not the first international comparative survey of student achievement. Others have been conducted over the past 40 years, primarily developed by the International Association for the Evaluation of Educational Achievement (IEA) and by the Education Testing Service's International Assessment of Educational Progress (IAEP).

These surveys have concentrated on outcomes linked directly to those parts of the curriculum that are essentially common across the participating countries. Aspects of the curriculum unique to one country or a smaller number of countries have usually not been taken into account in the assessments, regardless of how significant those parts of the curriculum are for the countries involved.

Key features associated with PISA include:

- Its policy orientation, with design and reporting methods determined by the need of governments to draw policy lessons;

- Its innovative "literacy" concept, which is concerned with the capacity of students to apply knowledge and skills in key subject areas and to analyse, reason and communicate effectively as they pose, solve and interpret problems in a variety of situations;

- Its relevance to lifelong learning, which does not limit PISA to assessing students' curricular and cross-curricular competencies but also asks them to report on their own motivation to learn, beliefs about themselves and learning strategies; 
- Its regularity, which will enable countries to monitor their progress in meeting key learning objectives;

- Its breadth of geographical coverage and collaborative nature, with the 47 countries that have participated in a PISA assessment so far and the 13 additional countries that join the PISA 2006 assessment representing a total of one-third of the world population and almost nine-tenths of the world's gross domestic product (GDP) $;^{1}$ and

- Its aged-based coverage of young people near the end of their compulsory schooling, which will enable countries to assess the performance of education systems. While most young people in OECD countries continue their initial education beyond the age of 15 , this is normally close to the end of the initial period of basic schooling in which all young people follow a broadly common curriculum. It is useful to determine, at that stage, the extent to which they have acquired knowledge and skills that will help them in the future, including the individualized paths of further learning they may follow.

This emphasis on testing in terms of mastery and broad concepts is particularly significant in light of the concern among nations to develop human capital, which the OECD defines as the knowledge, skills, competencies and other attributes embodied in individuals that are relevant to personal, social and economic well-being.

Estimates of human capital have tended, at best, to be derived using proxies, such as level of education completed. When the interest in human capital is extended to include attributes that permit full social and democratic participation in adult life and that equip people to become lifelong learners, the inadequacy of these proxies becomes even clearer.

By directly testing for knowledge and skills close to the end of basic schooling, PISA examines the degree of preparedness of young people for adult life and, to some extent, the effectiveness of education systems. Its aim is to assess achievement in relation to the underlying objectives (as defined by society) of education systems, not in relation to the teaching and learning of a body of knowledge. This view of educational outcomes is needed if schools and education systems are encouraged to focus on modern challenges.

PISA defines the assessment domains as follows:

- Mathematical literacy An individual's capacity to identify and understand the role that mathematics plays in the world, to make well-founded judgements and to use and engage with mathematics in ways that meet the needs of that individual's life as a constructive, concerned and reflective citizen.

- Reading literacy An individual's capacity to understand, use and reflect on written texts, in order to achieve one's goals, to develop one's knowledge and potential and to participate in society.

- Scientific literacy The capacity to use scientific knowledge, to identify questions and to draw evidence-based conclusions in order to understand and help make decisions about the natural world and the changes made to it through human activity.

- Problem-solving skills An individual's capacity to use cognitive processes to confront and resolve real, cross-disciplinary situations where the solution path is not immediately obvious and where the literacy domains or curricular areas that might be applicable are not within a single domain of mathematics, science or reading. 
More information on the assessment domains can be found in these PISA publications:

- Measuring Student Knowledge and Skills - A New Framework for Assessment (OECD, 1999a);

- Sample Tasks from the PISA 2000 Assessment - Reading, Mathematical and Scientific Literacy (OECD, 2002b);

- Literacy Skills for the World of Tomorrow - Further Results from PISA 2000 (OECD, 2003a);

- The PISA 2003 Assessment Framework - Mathematics, Reading, Science and Problem Solving Knowledge and Skills (OECD, 2003b);

- Learning for Tomorrow's World - First Results from PISA 2003 (OECD, 2004a); and

- Problem Solving for Tomorrow's World - First Measures of Cross-Curricular Competencies (OECD, 2004b).

\section{HOW THE ASSESSMENT TAKES PLACE}

\section{The assessment of student performance}

The PISA 2000 and the PISA 2003 assessments consisted of paper-and-pencil tests. The question format in the assessment is varied. Some questions require students to select or produce simple responses that can be directly compared with a single correct answer, such as multiple choice or closed constructed response items. Others are more constructive, requiring students to develop their own responses designed to measure broader constructs than those captured by more traditional surveys, allowing for a wider range of acceptable responses and more complex marking that can include partially correct responses.

Literacy in PISA is assessed through units consisting of a stimulus (e.g. text, table, chart, figure, etc.), followed by a number of tasks associated with this common stimulus. This is an important feature, allowing questions to go into greater depth than they could if each question introduced an entirely new context. It allows time for the student to assimilate material that can then be used to assess multiple aspects of performance.

Examples of items of the PISA 2000 assessment are available in Sample Tasks from the PISA 2000 Assessment - Reading, Mathematical and Scientific Literacy (OECD, 2002b).

Examples of items of the PISA 2003 assessment are available in The PISA 2003 Assessment Framework - Mathematics, Reading, Science and Problem Solving Knowledge and Skills (OECD, 2003b).

\section{The context questionnaires and their use}

To gather contextual information, PISA asks students and the principals of the participating schools to respond to background questionnaires of around 20 to 30 minutes in length. These questionnaires are central to the analysis of the results because they provide information about a range of student and school characteristics.

The questionnaires seek information about:

- The students and their family backgrounds, including the economic, social, and cultural capital of the students and their families;

- Aspects of students' lives, such as their attitudes to learning, their habits and life inside school and their family environment; 
- Aspects of schools, such as the quality of the school's human and material resources, public and private funding, decision-making processes and staffing practices;

- The context of instruction, including instructional structures and types, class size and the level of parental involvement;

- Strategies of self-regulated learning, motivational preferences and goal orientations, self-regulated cognition mechanisms, action control strategies, preferences for different types of learning situations, learning styles and social skills required for cooperative learning (these aspects were part of an international option in the PISA 2000 assessment, but were included in the compulsory student questionnaire in PISA 2003); and

- Aspects of learning and instruction, including students' motivation, engagement and confidence in relation to the major domain of assessment, and the impact of learning strategies on achievement in this domain.

In PISA 2003 as well as in PISA 2000, an information and communication technology (ICT) questionnaire was offered as an international option. It focused on: i) availability and use of information technologies (IT), including the location where IT is mostly used as well as the type of use; ii) IT confidence and attitudes, including self-efficacy and attitudes towards computers; and iii) learning background of IT, focusing on where students learned to use computers and the Internet.

In PISA 2003, an educational career questionnaire was also offered as an international option. It collected data on aspects of the students' educational career in three areas: i) students' past education including grade repetition, interruptions of schooling, changes of schools and changes of study programme; ii) students' current education on aspects involving mathematics, focusing on the type of mathematics classes and their current level of achievement; and iii) students' future and occupation, focusing on expected education level and expected occupation at the age of 30 .

The PISA 2003 questionnaires are available in Appendices 2 to 5 of this volume, as well as on the PISA Web site (www.pisa.oecd.org).

Several indices at the student level and at the school level were derived from the questionnaire data. These indices combine several answers provided by students or principals to build a broader concept that is not directly observable. For instance, one cannot directly observe the student's reading engagement, but it is possible to ask several questions like "I like talking about books with other people" that reflect the student's level of reading engagement.

More information on how these indices were constructed and their psychometric properties can be found in Appendix 9 as well as in PISA 2003 Technical Report (OECD, forthcoming).

\section{ABOUT THIS MANUAL}

PISA implemented complex methodological procedures to ensure reliable population estimates and their respective standard errors. More precisely, PISA 2000 and PISA 2003 used plausible values for reporting population achievement estimates and replicate weights for the computation of their respective standard errors.

In addition to these two methodological complexities, PISA collects data on a regular basis, in a particular context, and with standardised procedures. 
This manual is designed to explain these complex methodologies through examples using the PISA data. The manual does not detail every aspect of the methodologies, but nevertheless, they are described to ensure that all potential PISA database users can understand them and use the PISA data in an appropriate way.

Analysing the PISA data is a process that has been simplified by using programming procedures within statistical software packages, such as $\mathrm{SAS}^{\mathbb{R}}$ and $\mathrm{SPSS}^{\circledR}$. Consequently, this manual also contains examples of these procedures. There are, in fact, two versions of the manual - one for users of SAS ${ }^{\circledR}$ and one for users of SPSS ${ }^{\circledR}$. Each version of the manual consists of four parts.

The first part, Chapter 1 to Chapter 5, is identical in both versions of the manual. It presents concepts and theories which are used in PISA. These chapters are:

1. The OECD's Programme for International Student Assessment

2. Sample Weights

3. Replicate Weights

4. The Rasch Model

5. Plausible Values

The second part, Chapter 6 to Chapter 14, is different in each manual. In each, they describe how to correctly analyse the PISA data and contain the necessary coding - either SAS ${ }^{\circledR}$ or SPSS ${ }^{\circledR}$. These chapters are:

6. Computation of Standard Errors

7. Analyses with Plausible Values

8. Use of Proficiency Levels

9. Analyses with School-Level Variables

10. Standard Error on a Difference

11. OECD Average and OECD Total

12. Trends

13. Multilevel Analyses

14. Other Statistical Issues

The third part is also different in each manual: it consists of Chapter 15, which presents either the SAS ${ }^{\circledR}$ or the SPSS ${ }^{\circledR}$ macros that facilitate the computation of the estimates and standard errors.

The fourth part is identical in both versions of the manual. It consists of appendices that describe the details of the PISA 2003 data files. ${ }^{2}$

While chapters are organized by type of analyses, the manual progressively builds upon the statistical knowledge and the SAS ${ }^{\mathbb{R}}$ or $\mathrm{SPSS}^{\mathbb{R}}$ syntax knowledge previously presented. It is therefore advised to read the chapters in order, starting with Chapter 1.

There also exist specialised software packages that are configured to deal with complex samples and plausible values. These include WesVar ${ }^{\circledR}$, from Westat Inc. (www.westat.com/wesvar); AM, from the American Institutes for Research (www.am.air.org); and SUDAAN, from the Research Triangle Institute (www.rti.org/sudaan). 
In addition, the OECD has developed an interactive website that automatically performs simple statistical analyses - mainly computation of means and percentages - using the plausible value methodologies and the replicate weights (http://pisaweb.acer.edu.au/oecd_2003/oecd_pisa_data.html). This site also contains the complete PISA 2003 databases in ASCII format.

1. The combined population of all countries (excluding Chinese Taipei) that have or will have participated in the PISA 2000, 2003 and 2006 assessments amounts to 32 per cent of the 2002 world population. The GDP of these countries amounts to 87 per cent of the 2002 world GDP. The data on GDP and population sizes were derived from the U.N. World Development Indicators database.

2. The description of the PISA 2000 data files is covered in Manual for the PISA 2000 Database (OECD, 2002a). 


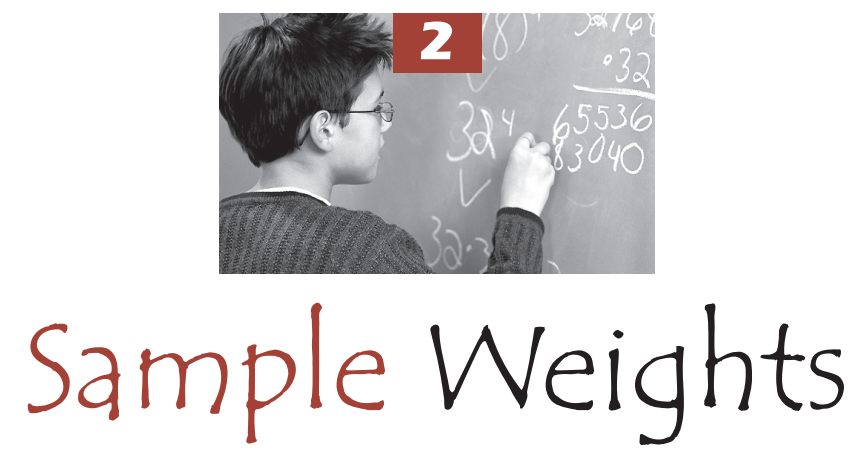

Introduction

Weights for simple random samples

Sampling designs for education surveys

Why do the PISA weights vary?

Conclusions

30 


\section{INTRODUCTION}

National or international surveys usually collect data from a sample. Dealing with a sample rather than the whole population is preferable for several reasons.

First, for a census, all members of the population need to be identified. This identification process presents no major difficulty for human populations in some countries, where national databases with the name and address of all or nearly all citizens may be available. However, in other countries, it is not possible for the researcher to identify all members or sampling units of the target population, mainly because it would be too time consuming or because of the nature of the target population.

Second, even if all members of a population are easily identifiable, researchers may still draw a sample, because dealing with the whole population:

- Might require unreasonable budgets;

- Is time consuming and thus incompatible with publication deadlines; and

- Does not necessarily help with obtaining additional and/or required information.

Drawing a sample can be done in several ways depending on the population characteristics and the survey research questions. All sample designs aim to avoid bias in the selection procedure and achieve the maximum precision in view of the available resources. Nevertheless, biases in the selection can arise:

- If the sampling is done by a non-random method, which generally means that the selection is consciously or unconsciously influenced by human choices. The importance of randomness in the selection procedure should not be underestimated; and

- If the sampling frame (list, index or other population record) that serves as the basis for selection does not cover the population adequately, completely or accurately.

Biases can also arise if some sections of the population are impossible to find or refuse to co-operate. In educational surveys, schools might refuse to participate and within participating schools, some students might refuse to participate or simply be absent on the day of the assessment. The size of the bias introduced by the school or student non-response is proportional to the correlation between the school, or the student, propensity to participate and the survey measures. For instance, it may be that low achievers are more likely to be absent on the day of the assessment than high achievers. This is the reason why international education surveys require a minimal student participation rate. For PISA, this minimum is 80 per cent.

Finally, if the sampling units do not have the same chances to be selected and if the population parameters are estimated without taking into account these varying probabilities, then results might also be biased. To compensate for these varying probabilities, data need to be weighted. Weighting consists of acknowledging that some units in the sample are more important than others and have to contribute more than others for any population estimates. A sampling unit with a very small probability of selection will be considered as more important than a sampling unit with a high probability of selection. Weights are therefore inversely proportional to the probability of selection.

Nevertheless, a sample is only useful to the extent that it allows the estimation of some characteristics of the whole population. This means that the statistical indices computed on the sample, like a mean, a standard deviation, a correlation, a regression coefficient, and so on, can be generalized to the population. This generalization is more reliable if the sampling requirements have been met.

Depending on the sampling design, selection probabilities and procedures to compute the weights will vary. These variations are discussed in the next sections. 


\section{WEIGHTS FOR SIMPLE RANDOM SAMPLES}

Selecting members of a population by simple random sampling is the most straightforward procedure. There are several ways to draw such a sample, for example:

- The $N$ members ${ }^{1}$ of a population are numbered and $n$ of them are selected by random numbers without replacement;

- $N$ numbered discs are placed in a container, mixed well, and $n$ of them are selected at random;

- The $N$ population members are arranged in a random order, and every $\frac{N}{n}^{\text {th }}$ member is then selected; or

- The $N$ population members are each assigned a random number. The random numbers are sorted from lowest to highest or highest to lowest. The first $n$ members make up one random sample.

The simple random sample gives an equal probability of selection to each member of the population. If $n$ members are selected from a population of $N$ members according to a simple random procedure, then the probability of each member, $i$, to be part of the sample is equal to:

$$
p_{i}=\frac{n}{N}
$$

For example, if 40 students are randomly selected from a population of 400 students, the probability of each student, $i$, to be part of the sample is equal to:

$$
p_{i}=\frac{n}{N}=\frac{40}{400}=0.1
$$

In other words, each student has one chance out of ten to be selected.

As mentioned previously, weights are usually defined as the inverse of the probability of selection. In the case of a simple random sample, the weight will be equal to:

$$
w_{i}=\frac{1}{p_{i}}=\frac{N}{n}
$$

The weight of each of the 40 students selected from a population of 400 students will therefore be equal to:

$$
w_{i}=\frac{1}{p_{i}}=\frac{N}{n}=\frac{400}{40}=10
$$

This means that each student in the sample represents himself or herself, as well as nine other students. Since each unit has the same selection probability in a simple random sample, the weight attached to each selected unit will also be identical. Therefore, the sum of the weights of the selected units will be equal to the population size, i.e. $N$ :

$$
\sum_{i=1}^{n} w_{i}=\sum_{i=1}^{n} \frac{N}{n}=N
$$

In the example,

$$
\sum_{i=1}^{40} 10=400
$$


Furthermore, since all sampled units have the same weight, the estimation of any population parameter will not be affected by the weights. For instance, consider the mean of some characteristic, $X$. The weighted mean is equivalent to the sum of the product of the weight and $X$ divided by the sum of the weights.

$$
\hat{\mu}_{(X)}=\frac{\sum_{i=1}^{n} w_{i} x_{i}}{\sum_{i=1}^{n} w_{i}}
$$

Since $w_{i}$ is a constant, the weighted mean and the unweighted mean will be equal.

$$
\hat{\boldsymbol{\mu}}_{(X)}=\frac{\sum_{i=1}^{n} w_{i} x_{i}}{\sum_{i=1}^{n} w_{i}}=\frac{w_{i} \sum_{i=1}^{n} x_{i}}{w_{i} \sum_{i=1}^{n} 1}=\frac{\sum_{i=1}^{n} x_{i}}{n}
$$

\section{SAMPLING DESIGNS FOR EDUCATION SURVEYS}

Simple random sampling is very rarely used in education surveys because:

- It is too expensive. Indeed, depending on the school population size, it is quite possible that selected students would attend many different schools. This would require the training of a large number of test administrators, the reimbursement of a large amount of travel expenses and so on;

- It is not practical. One would have to contact too many schools; and

- It would be impossible to link, from a statistical point of view, student variables and school, class, or teacher variables. Educational surveys usually try to understand the statistical variability of the student's outcome measure by school or class level variables. With just one or only a few students per school, this statistical relationship would have no stability.

Therefore, surveys in education usually draw a student sample in two steps. First, a sample of schools is selected from a complete list of schools containing the student population of interest. Then, a simple random sample of students or classes is drawn from within the selected schools. In PISA, usually 35 students from the population of 15 -year-olds are randomly selected within the selected schools. If less than 3515 -year-olds attend a selected school, then all of the students will be invited to participate.

This two-stage sampling procedure will have an impact on the calculation of the weights and, similarly, the school selection procedure will affect the characteristics and properties of the student sample.

Suppose that the population of 400 students is distributed in ten schools, each school containing 40 students. Four schools are selected randomly and within schools, ten students are selected according to a similar procedure. Each school, denoted $i$, has a selection probability equal to:

$$
p_{1 \_i}=\frac{n_{s c}}{N_{s c}}=\frac{4}{10}=0.4
$$


Within the four selected schools, each student, denoted $j$, has a selection probability equal to:

$$
p_{2_{-i j}}=\frac{n_{i}}{N_{i}}=\frac{10}{40}=0.25
$$

with $N_{i}$ being the number of students in school $i$ and $n_{i}$ the number of students sampled in school $i$. It means that within each selected school, each student has a chance of one in four to be sampled.

The final selection probability for student $j$ attending school $i$ is equal to the product of the school selection probability by the student selection probability within the school, i.e.:

$$
p_{i j}=p_{1_{-} i} p_{2_{-} i j}=\frac{n_{s c} n_{i}}{N_{s c} N_{i}}
$$

In the example, the final student probability is equal to:

$$
p_{i j}=p_{1_{-} i} p_{2_{-} i j}=\frac{n_{s c} n_{i}}{N_{s c} N_{i}}=\frac{4 * 10}{10 * 40}=0.4 * 0.25=0.10
$$

The school weight, denoted $w_{1 \_i}$, the within-school weight, denoted $w_{2 \_i j}$, and the final school weight, denoted $w_{i j}$, are respectively equal to:

$$
\begin{aligned}
& w_{1_{-} i}=\frac{1}{p_{1_{-} i}}=\frac{1}{0.4}=2.5 \\
& w_{2_{-} i j}=\frac{1}{p_{2_{-} i j}}=\frac{1}{0.25}=4 \\
& w_{i j}=\frac{1}{p_{i j}}=\frac{1}{0.1}=10
\end{aligned}
$$

Table 2.1 presents the selection probability at the school level, at the within-school level, and the

\begin{tabular}{|c|c|c|c|c|c|c|c|c|}
\hline $\begin{array}{c}\text { School } \\
\text { label }\end{array}$ & $\begin{array}{c}\begin{array}{c}\text { School } \\
\text { size }\end{array} \\
N_{i}\end{array}$ & $\begin{array}{c}\text { School } \\
\text { prob. } \\
p_{1 \_i}\end{array}$ & $\begin{array}{c}\text { School } \\
\text { weight } \\
w_{1 \_i}\end{array}$ & $\begin{array}{c}\text { Within- } \\
\text { school } \\
\text { prob. } \\
p_{2 \_i j}\end{array}$ & $\begin{array}{c}\text { Within- } \\
\text { school } \\
\text { weight } \\
w_{2 \_i j}\end{array}$ & $\begin{array}{c}\text { Final } \\
\text { student } \\
\text { prob. } \\
p_{i j}\end{array}$ & $\begin{array}{c}\text { Final } \\
\text { student } \\
\text { weight } \\
w_{i j}\end{array}$ & $\begin{array}{c}\text { Sum } \\
\text { of final } \\
\text { weights } \\
n_{i} w_{i j}\end{array}$ \\
\hline 1 & 40 & & & & & & & \\
\hline 2 & 40 & 0.4 & 2.5 & 0.25 & 4 & 0.1 & 10 & 100 \\
\hline 3 & 40 & & & & & & & \\
\hline 4 & 40 & & & & & & & \\
\hline 5 & 40 & 0.4 & 2.5 & 0.25 & 4 & 0.1 & 10 & 100 \\
\hline 6 & 40 & & & & & & & \\
\hline 7 & 40 & 0.4 & 2.5 & 0.25 & 4 & 0.1 & 10 & 100 \\
\hline 8 & 40 & & & & & & & \\
\hline 9 & 40 & & & & & & & \\
\hline 10 & 40 & 0.4 & 2.5 & 0.25 & 4 & 0.1 & 10 & 100 \\
\hline Total & & & 10 & & & & & 400 \\
\hline
\end{tabular}
final probability of selection for the selected students as well as the weight for these different levels where schools 2, 5, 7 and 10 have been selected.

\section{Table 2.1 - School, within-school, and final probability of selection and corresponding weights for a two-stage simple random sample with the first stage units being schools of equal size}


As shown by Table 2.1, the sum of the school weights corresponds to the number of schools in the population, i.e. 10 , and the sum of the final weights corresponds to the number of students in the population, i.e. 400 .

In practice, of course, schools differ in size. School enrolment numbers tend to be larger in urban areas as compared to rural areas. If schools are selected by simple random sampling, the school probability will not change, but within the selected schools, the student selection probability will vary according to the school size. In a small school, this probability will be large, while in a very large school, this probability will be small. Table 2.2 shows an example of the results obtained from schools of different sizes.

\section{Table 2.2 - School, within-school, and final probability of selection and corresponding weights for a two-stage simple random sample with the first stage units being schools of unequal size}

\begin{tabular}{|c|c|c|c|c|c|c|c|c|}
\hline $\begin{array}{c}\text { School } \\
\text { label }\end{array}$ & $\begin{array}{l}\text { School } \\
\text { size }\end{array}$ & $\begin{array}{c}\text { School } \\
\text { prob. }\end{array}$ & $\begin{array}{l}\text { School } \\
\text { weight }\end{array}$ & $\begin{array}{l}\text { Within- } \\
\text { school } \\
\text { prob. }\end{array}$ & $\begin{array}{l}\text { Within- } \\
\text { school } \\
\text { weight }\end{array}$ & $\begin{array}{l}\text { Final } \\
\text { student } \\
\text { prob. }\end{array}$ & $\begin{array}{l}\text { Final } \\
\text { student } \\
\text { weight }\end{array}$ & $\begin{array}{l}\text { Sum } \\
\text { of final } \\
\text { weights }\end{array}$ \\
\hline 1 & 10 & & & & & & & \\
\hline 2 & 15 & 0.4 & 2.5 & 0.66 & 1.5 & 0.27 & 3.75 & 37.5 \\
\hline 3 & 20 & & & & & & & \\
\hline 4 & 25 & & & & & & & \\
\hline 5 & 30 & 0.4 & 2.5 & 0.33 & 3 & 0.13 & 7.5 & 75 \\
\hline 6 & 35 & & & & & & & \\
\hline 7 & 40 & 0.4 & 2.5 & 0.25 & 4 & 0.1 & 10 & 100 \\
\hline 8 & 45 & & & & & & & \\
\hline 9 & 80 & & & & & & & \\
\hline 10 & 100 & 0.4 & 2.5 & 0.1 & 10 & 0.04 & 25 & 250 \\
\hline Total & 400 & & 10 & & & & & 462.5 \\
\hline
\end{tabular}

With a simple random sample of schools of unequal size, all schools will have the same selection probability and, as before, the sum of school weights will be equal to the number of schools in the population. Unfortunately, the sum of final student weights will not necessarily be equal to the number of students in the population. Further, the student final weight will differ among schools depending on the size of each school. This variability will reduce the reliability of all population parameter estimates.

Table 2.3 and Table 2.4 present the different probabilities and weights if the four smallest schools or the four largest schools are selected. As shown in these two tables, the sums of final student weights vary substantially from the expected value of 400 . The sum of school weights, however, will always be equal to the number of schools in the population. 
Table 2.3 - School, within-school, and final probability of selection and corresponding weights for a simple and random sample of schools of unequal size (smaller schools)

\begin{tabular}{c|c|c|c|c|c|c|c|c}
\hline $\begin{array}{c}\text { School } \\
\text { label }\end{array}$ & $\begin{array}{c}\text { School } \\
\text { size }\end{array}$ & $\begin{array}{c}\text { School } \\
\text { prob. }\end{array}$ & $\begin{array}{c}\text { School } \\
\text { weight }\end{array}$ & $\begin{array}{c}\text { Within- } \\
\text { school } \\
\text { prob. }\end{array}$ & $\begin{array}{c}\text { Within- } \\
\text { school } \\
\text { weight }\end{array}$ & $\begin{array}{c}\text { Final } \\
\text { student } \\
\text { prob. }\end{array}$ & $\begin{array}{c}\text { Final } \\
\text { student } \\
\text { weight }\end{array}$ & $\begin{array}{c}\text { Sum } \\
\text { of final } \\
\text { weight }\end{array}$ \\
\hline 1 & 10 & 0.4 & 2.5 & 1 & 1 & 0.4 & 4 & 40 \\
2 & 15 & 0.4 & 2.5 & 0.66 & 1.5 & 0.27 & 3.75 & 37.5 \\
3 & 20 & 0.4 & 2.5 & 0.5 & 2 & 0.2 & 5 & 50 \\
4 & 25 & 0.4 & 2.5 & 0.4 & 2.5 & 0.16 & 6.25 & 62.5 \\
\hline Total & & & $\mathbf{1 0}$ & & & & & 190 \\
\hline
\end{tabular}

Table 2.4 - School, within-school, and final probability of selection and corresponding weights for a simple and random sample of schools of unequal size (larger schools)

\begin{tabular}{c|c|c|c|c|c|c|c|c}
\hline $\begin{array}{c}\text { School } \\
\text { label }\end{array}$ & $\begin{array}{c}\text { School } \\
\text { size }\end{array}$ & $\begin{array}{c}\text { School } \\
\text { prob. }\end{array}$ & $\begin{array}{c}\text { School } \\
\text { weight }\end{array}$ & $\begin{array}{c}\text { Within- } \\
\text { school } \\
\text { prob. }\end{array}$ & $\begin{array}{c}\text { Within- } \\
\text { school } \\
\text { weight }\end{array}$ & $\begin{array}{c}\text { Final } \\
\text { student } \\
\text { prob. }\end{array}$ & $\begin{array}{c}\text { Final } \\
\text { student } \\
\text { weight }\end{array}$ & $\begin{array}{c}\text { Sum } \\
\text { of final } \\
\text { weight }\end{array}$ \\
\hline 7 & 40 & 0.4 & 2.5 & 0.250 & 4 & 0.10 & 10.00 & 100.0 \\
8 & 45 & 0.4 & 2.5 & 0.222 & 4.5 & 0.88 & 11.25 & 112.5 \\
9 & 80 & 0.4 & 2.5 & 0.125 & 8 & 0.05 & 20.00 & 200.0 \\
10 & 100 & 0.4 & 2.5 & 0.100 & 10 & 0.04 & 25.00 & 250.0 \\
\hline Total & & & $\mathbf{1 0}$ & & & & & $\mathbf{6 6 2 . 5}$ \\
\hline
\end{tabular}

The focus of international education surveys such as PISA is more on the student sample than on the school sample. Many authors even consider that such studies do not draw a school sample per se. They just consider the school sample as an operational stage to draw the student sample. Therefore, a sampling design that consists of a simple random sample of schools is inappropriate as it would underestimate or overestimate the student population size. It would also result in an important variability of final weights and consequently increase the sampling variance.

In order to avoid these disadvantages, schools are selected with probabilities proportional to their size (PPS). Larger schools will therefore have a higher probability of selection than smaller schools, but students in larger schools have a smaller within-school probability of being selected than students in small schools. With such procedures, the probability of a school to be selected is equal to the ratio of the school size multiplied by the number of schools to be sampled and divided by the total number of students in the population:

$$
p_{1_{-} i}=\frac{N_{i}^{*} n_{s c}}{N}
$$

The formulae for computing the within-school probabilities and weights remain unchanged. The final probability and weight are still the product of the school and within-school probabilities or weights. For instance, the school probability for school 9 is equal to:

$$
p_{1_{-} 9}=\frac{N_{9} * n_{s c}}{N}=\frac{80 * 4}{400}=\frac{4}{5}=0.8
$$


The student within-school probability for school 9 is equal to:

$$
p_{2-9 j}=\frac{n_{9}}{N_{9}}=\frac{10}{80}=0.125
$$

The final probability is equal to:

$$
p_{9 j}=0.8 * 0.125=0.1
$$

Table 2.5 - School, within-school, and final probability of selection and corresponding weights for a PPS sample of schools of unequal size

\begin{tabular}{r|c|c|c|c|c|c|c|c}
\hline $\begin{array}{c}\text { School } \\
\text { label }\end{array}$ & $\begin{array}{c}\text { School } \\
\text { size }\end{array}$ & $\begin{array}{c}\text { School } \\
\text { prob. }\end{array}$ & $\begin{array}{c}\text { School } \\
\text { weight }\end{array}$ & $\begin{array}{c}\text { Within- } \\
\text { school } \\
\text { prob. }\end{array}$ & $\begin{array}{c}\text { Within- } \\
\text { school } \\
\text { weight }\end{array}$ & $\begin{array}{c}\text { Final } \\
\text { student } \\
\text { prob. }\end{array}$ & $\begin{array}{c}\text { Final } \\
\text { student } \\
\text { weight }\end{array}$ & $\begin{array}{c}\text { Sum } \\
\text { of final } \\
\text { weight }\end{array}$ \\
\hline 1 & 10 & & & & & & & \\
2 & 15 & & & & & & & \\
3 & 20 & 0.2 & 5.00 & 0.500 & 2.0 & 0.1 & 10 & \\
4 & 25 & & & & & & & \\
5 & 30 & & & & & & & \\
6 & 35 & & & & & & & \\
7 & 40 & 0.4 & 2.50 & 0.250 & 4.0 & 0.1 & 100 \\
8 & 45 & & & & & & & \\
9 & 80 & 0.8 & 1.25 & 0.125 & 8.0 & 0.1 & 10 & 100 \\
10 & 100 & 1 & 1.00 & 0.100 & 10.0 & 0.1 & 10 & 100 \\
\hline Total & 400 & & 9.75 & & & & & 400 \\
\hline
\end{tabular}

As shown in Table 2.5, the school and within-school weights differ among schools, but final student weights do not vary. The weights will therefore not increase sampling variability. Further, the sum of final weights corresponds to the total number of students in the population. However, the sum of school weight differs from the expected value of 10 , but this does not present a major problem as such educational surveys are mainly interested in the student sample.

With a PPS sample of schools, and an equal number of students selected in each selected school, the sum of the final student weights will always be equal to the total number of students in the population (non-response being ignored at this stage). This will be the case even if the smallest or the largest schools get selected. The sum of the school weights however will not be equal to the number of schools in the population. If the four smallest schools get selected, the sum of school weights will be equal to 25.666. If the four largest schools get selected, the sum of school weights will be equal to 6.97 .

In order to keep the difference between the number of schools in the population and the sum of the school weights in the sample minimal, schools are selected according to a systematic procedure. The procedure consists of first sorting the schools according to their size. A sampling interval is computed as the ratio between the total number of students in the population and the number of schools in the sample, i.e.:

$$
\text { Int }=\frac{N}{n_{s c}}=\frac{400}{4}=100
$$


Table 2.6 - Selection of schools according to a PPS and systematic procedure

\begin{tabular}{c|cccc}
\hline $\begin{array}{c}\text { School } \\
\text { label }\end{array}$ & $\begin{array}{c}\text { School } \\
\text { size }\end{array}$ & $\begin{array}{c}\text { From student } \\
\text { number }\end{array}$ & $\begin{array}{c}\text { To student } \\
\text { number }\end{array}$ & $\begin{array}{c}\text { Part of } \\
\text { the sample }\end{array}$ \\
\hline 1 & 10 & 1 & 10 & No \\
2 & 15 & 11 & 25 & No \\
3 & 20 & 26 & 45 & No \\
4 & 25 & 46 & 70 & No \\
5 & 30 & 71 & 100 & Yes \\
6 & 35 & 101 & 135 & No \\
7 & 40 & 136 & 175 & No \\
8 & 45 & 176 & 220 & Yes \\
9 & 80 & 221 & 300 & Yes \\
10 & 100 & 301 & 400 & Yes \\
\hline
\end{tabular}

A random number from a uniform distribution [0.1] is drawn. Let us say 0.752. This random number is then multiplied by the sampling interval, i.e. 0.752 by $100=75.2$. The school which contains the first student number greater than 75.2 is selected. Then the sampling interval is added to the value 75.2. The school which contains the student having the first student number greater than 175.2 will be selected. This systematic procedure is applied until the number of schools needed in the sample has been reached. In the example, the four selection numbers will be the following: 75.2, 175.2, 275.2 and 375.2 .

Sorting the school sampling frame by the measure of size and then using a systematic selection procedure prevents obtaining a sample of only small schools or (more likely) a sample with only large schools. This therefore reduces the sampling variance on the sum of the school weights which is an estimate of the school population size.

\section{WHY DO THE PISA WEIGHTS VARY?}

As demonstrated in the previous section, a two-stage sample design with a PPS sample of schools should guarantee that all students will have the same probability of selection and therefore the same weight. However, the PISA data still needs to be weighted.

Table 2.7 clearly shows that PISA 2003 final weights present some variability. This variability is quite small for countries, such as Iceland, Luxembourg and Tunisia, but appears to be greater in countries such as Canada, Italy and the United Kingdom.

Table 2.8 presents the weighted and unweighted means per country on the mathematics scale for PISA 2003. The differences between the weighted and unweighted means are small for countries with small weight variability, such as Iceland, Luxembourg and Tunisia. On the contrary, the effect of the weights on the mean might be substantial for countries that present a large variability in weight. For instance, not using the weights would overestimate the mathematics performance of the Italian students by about 30 points on the PISA mathematics scale and underestimate the average performance of the Canadian students by nearly 11 score points. 
Table 2.7 The $10^{\text {th }}, \mathbf{2 5 ^ { \text { th } }} \mathbf{\mathbf { 5 }} \mathbf{5 0}^{\text {th }}, \mathbf{7 5 ^ { \text { th } }}$ and $\mathbf{9 0}{ }^{\text {th }}$ percentiles of PISA 2003 final weights

\begin{tabular}{|c|c|c|c|c|c|}
\hline & Percentile 10 & Percentile 25 & Percentile 50 & Percentile 75 & Percentile 90 \\
\hline AUS & 4.70 & 11.86 & 19.44 & 25.06 & 29.55 \\
\hline AUT & 13.00 & 14.92 & 17.24 & 20.33 & 25.53 \\
\hline BEL & 4.09 & 10.48 & 12.96 & 15.32 & 19.22 \\
\hline BRA & 222.44 & 309.68 & 407.59 & 502.14 & 627.49 \\
\hline CAN & 1.16 & 2.18 & 5.09 & 13.17 & 36.28 \\
\hline CHE & 1.35 & 2.88 & 6.70 & 15.55 & 21.76 \\
\hline CZE & 5.19 & 12.55 & 17.77 & 23.77 & 27.33 \\
\hline DEU & 140.10 & 160.05 & 180.05 & 208.72 & 243.21 \\
\hline DNK & 8.86 & 10.07 & 11.73 & 13.29 & 16.22 \\
\hline ESP & 3.97 & 4.38 & 15.50 & 48.73 & 83.84 \\
\hline FIN & 2.80 & 9.94 & 11.60 & 12.24 & 13.29 \\
\hline FRA & 142.51 & 148.21 & 159.98 & 177.56 & 213.43 \\
\hline GBR & 7.73 & 10.71 & 23.12 & 136.69 & 180.64 \\
\hline GRC & 15.07 & 17.18 & 21.71 & 27.56 & 30.90 \\
\hline HKG & 13.31 & 14.26 & 15.15 & 16.60 & 19.36 \\
\hline HUN & 16.13 & 19.27 & 22.25 & 25.37 & 29.41 \\
\hline IDN & 21.82 & 42.47 & 106.18 & 272.23 & 435.96 \\
\hline IRL & 11.33 & 12.01 & 13.51 & 15.31 & 17.99 \\
\hline ISL & 1.06 & 1.12 & 1.16 & 1.20 & 1.36 \\
\hline ITA & 2.56 & 14.93 & 20.65 & 66.11 & 108.66 \\
\hline JPN & 217.14 & 248.47 & 258.13 & 281.97 & 314.52 \\
\hline KOR & 80.82 & 89.60 & 96.72 & 107.86 & 117.81 \\
\hline LIE & 1.00 & 1.00 & 1.01 & 1.03 & 1.06 \\
\hline LUX & 1.00 & 1.01 & 1.03 & 1.06 & 1.09 \\
\hline LVA & 4.26 & 5.17 & 6.47 & 7.40 & 8.92 \\
\hline MAC & 1.14 & 3.12 & 4.80 & 6.60 & 8.09 \\
\hline MEX & 3.09 & 6.36 & 13.00 & 27.49 & 67.09 \\
\hline NLD & 24.84 & 35.41 & 43.80 & 52.42 & 65.60 \\
\hline NOR & 11.11 & 11.59 & 12.47 & 13.53 & 14.76 \\
\hline NZL & 7.41 & 8.99 & 10.77 & 12.34 & 13.98 \\
\hline POL & 103.73 & 110.45 & 118.72 & 130.28 & 144.73 \\
\hline PRT & 13.90 & 16.33 & 18.70 & 22.66 & 28.82 \\
\hline RUS & 172.98 & 245.92 & 326.11 & 426.26 & 596.07 \\
\hline SVK & 4.39 & 6.98 & 8.64 & 11.02 & 16.79 \\
\hline SWE & 17.95 & 19.54 & 22.03 & 24.47 & 28.81 \\
\hline THA & 74.96 & 101.57 & 119.35 & 130.48 & 154.26 \\
\hline TUN & 31.27 & 31.41 & 32.19 & 33.32 & 34.62 \\
\hline TUR & 22.06 & 50.49 & 109.69 & 135.98 & 152.65 \\
\hline URY & 1.81 & 2.79 & 4.43 & 8.06 & 11.66 \\
\hline USA & 296.10 & 418.79 & 554.25 & 704.78 & 885.84 \\
\hline YUG & 8.68 & 12.83 & 16.62 & 18.20 & 19.73 \\
\hline
\end{tabular}


Table 2.8 Weighted and unweighted country means on the PISA 2003 mathematics scale

\begin{tabular}{|c|c|c|c|}
\hline & Weighted mean & Unweighted mean & Difference \\
\hline AUS & 524.27 & 522.33 & 1.94 \\
\hline AUT & 505.61 & 511.86 & -6.25 \\
\hline BEL & 529.29 & 533.19 & -3.90 \\
\hline BRA & 356.02 & 360.41 & -4.40 \\
\hline CAN & 532.49 & 521.40 & 11.09 \\
\hline $\mathrm{CHE}$ & 526.55 & 518.24 & 8.31 \\
\hline CZE & 516.46 & 534.95 & -18.50 \\
\hline DEU & 502.99 & 508.41 & -5.43 \\
\hline DNK & 514.29 & 513.69 & 0.60 \\
\hline ESP & 485.11 & 494.78 & -9.67 \\
\hline FIN & 544.29 & 542.81 & 1.48 \\
\hline FRA & 510.80 & 514.73 & -3.93 \\
\hline GBR & 508.26 & 514.44 & -6.18 \\
\hline GRC & 444.91 & 440.88 & 4.04 \\
\hline HKG & 550.38 & 555.86 & -5.48 \\
\hline HUN & 490.01 & 488.59 & 1.42 \\
\hline IDN & 360.16 & 361.51 & -1.35 \\
\hline IRL & 502.84 & 504.68 & -1.84 \\
\hline ISL & 515.11 & 515.05 & 0.05 \\
\hline ITA & 465.66 & 496.00 & -30.34 \\
\hline JPN & 534.14 & 533.51 & 0.62 \\
\hline KOR & 542.23 & 540.60 & 1.62 \\
\hline LIE & 535.80 & 536.46 & -0.67 \\
\hline LUX & 493.21 & 493.48 & -0.27 \\
\hline LVA & 483.37 & 486.17 & -2.80 \\
\hline MAC & 527.27 & 522.79 & 4.48 \\
\hline MEX & 385.22 & 405.40 & -20.18 \\
\hline NLD & 537.82 & 542.12 & -4.29 \\
\hline NOR & 495.19 & 495.64 & -0.46 \\
\hline NZL & 523.49 & 525.62 & -2.13 \\
\hline POL & 490.24 & 489.00 & 1.24 \\
\hline PRT & 466.02 & 465.23 & 0.79 \\
\hline RUS & 468.41 & 472.44 & -4.03 \\
\hline SVK & 498.18 & 504.12 & -5.94 \\
\hline SWE & 509.05 & 507.95 & 1.09 \\
\hline THA & 416.98 & 422.73 & -5.75 \\
\hline TUN & 358.73 & 359.34 & -0.61 \\
\hline TUR & 423.42 & 426.72 & -3.30 \\
\hline URY & 422.20 & 412.99 & 9.21 \\
\hline USA & 482.88 & 481.47 & 1.41 \\
\hline YUG & 436.87 & 436.36 & 0.51 \\
\hline
\end{tabular}


Different factors contribute to the variability of weights:

- Oversampling or undersampling of some strata of the population: usually, the school population is divided into different subgroups, called strata. For instance, a country might decide for convenience to separate the urban schools from the rural schools in the list of schools. In most cases, the number of students selected in the rural stratum and in the urban stratum will be proportional to what these two strata represent in the whole population. This stratification process guarantees for instance that a predefined number of schools within each stratum will be selected. Without the stratification, this number might vary. Nevertheless, for national reporting purposes, a country might decide to sample more students than what would have been sampled based on a proportional allocation in some part of the student population. Suppose that 90 per cent of the student population in a country attends academic tracks and 10 per cent of the students attend vocational tracks. If the national centre staff wants to compare the performance of the students by track, then it will be necessary to sample more vocational students than what would be sampled based on a proportional allocation.

- Lack of accuracy or no updated size measure for schools on the school sampling frame: when schools are selected with a probability proportional to their size, a measure of size needs to be included in the school list. In PISA, this measure of size is the number of 15 -year-olds in each school in the population, but national statistics per school and per date of birth are not always available. Therefore, the measure of size can be the number of students in the modal grade for 15-year-olds, or the total number of students in the school divided by the number of grades. Further, even if national statistics per school and per date of birth are available, these data might be one or two years old. Therefore, inconsistencies between the number of 15-year-olds at the testing time and the measure of size used in the school sample frame generate some variability in the final weights. Let us suppose that school 9 in Table 5 has 100 15-year-old students at the time of testing. When schools were selected from the list of schools, the measure of size was set at 80. The school weight was set at 1.25 . The within-school weight will be equal to 100 divided by 10 , i.e. 10 rather than 8 . Therefore, the final weight will be equal to 12.5 instead of the expected 10 .

- School and within-school weight adjustment for school and student non-response: some schools, and within the selected and participating schools, some students, might refuse to participate. To compensate for this non-response, a weight adjustment is applied at each level where non-response occurs. For instance, if only 25 students out of the 35 selected students from a participating school are present on the day of the assessment, then the weight of the participating students will be multiplied by a ratio of 35 by $25 .^{2}$ The student participation rate will vary from one school to another, and therefore the final weights will vary. A similar procedure is also applied to compensate for the school non-response. More information about these adjustment factors is available in the PISA 2003 Technical report (OECD, forthcoming).

\section{CONCLUSIONS}

This chapter has briefly described: i) what a weight is and how to compute it; ii) what the PISA sampling design is and why such a design is considered as the most appropriate; iii) why the PISA weights show some variability; and iv) the impact of the weights on population estimates.

All statistical analyses or procedures on the PISA data should be weighted. Unweighted analyses will provide biased population parameter estimates.

\section{Notes}

1. $N$ usually represents the size of the population and $n$ the size of the sample.

2. In PISA 2003, the student weight adjustment for student non response might also differ in a particular school. 


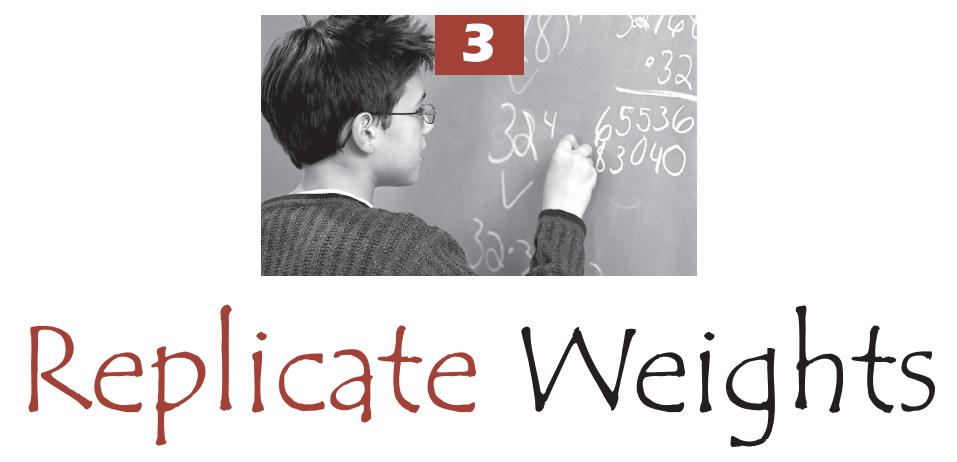

Introduction

Sampling variance for simple random sampling ……………......... 32

Sampling variance for two-stage sampling ………………………..... 38

Replication methods for simple random samples ........................... 44

Resampling methods for two-stage samples ………………………...... 46

The Jackknife for unstratified two-stage sample designs ................ 47

The Jackknife for stratified two-stage sample designs...................... 48

The Balanced Repeated Replication method ..................................... 49

Other procedures for accounting for clustered samples ………...... 51

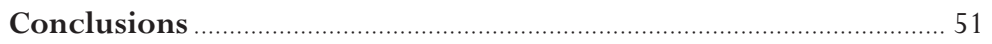




\section{INTRODUCTION}

In most cases, as mentioned in Chapter 2, national or international surveys collect data from a sample instead of conducting a full a census. However, for a particular population, there are thousands, even millions of possible samples, and each of them does not necessarily yield the same estimates of population statistics. Every generalisation made from a sample, i.e. every estimate of a population statistic, has an associated uncertainty or risk of error. The sampling variance corresponds to the measure of this uncertainty due to sampling.

This chapter explains the statistical procedures used for computing the sampling variance and its square root, the standard error. More specifically, this chapter discusses how to estimate sampling variances for population estimates derived from a complex sample design using replicate weights. First, the concept of sampling variance will be examined through a fictitious example for simple random sampling. Second, the computation of the standard error will be investigated for two-stage sampling. Third, replication methods for estimating sampling variances will be introduced for simple random samples and for two-stage samples respectively.

\section{SAMPLING VARIANCE FOR SIMPLE RANDOM SAMPLING}

Suppose that a teacher decides to implement the mastery learning approach in his or her classroom. This methodology requires that each lesson be followed by a student assessment. In the example given, the teacher's class has 36 students. The teacher quickly realises that it would be too time consuming to grade all assessments and therefore decides to select a sample of quizzes to find out whether the material taught has been assimilated (Bloom, 1979).

However, the random sampling of a few quizzes can result in the selection of high achievers or low achievers only, which would introduce an important error in the class mean performance estimate. These situations are extreme examples, but drawing a random sample will always generate some uncertainty.

In the same example, before selecting some quizzes, the teacher grades all of them and analyzes the results for the first lesson. Figure 3.1 presents the distribution of the 36 students' results. One student gets a grade 5 , two students get a grade 6 , and so on.

Figure 3.1 Distribution of the results of the 36 students

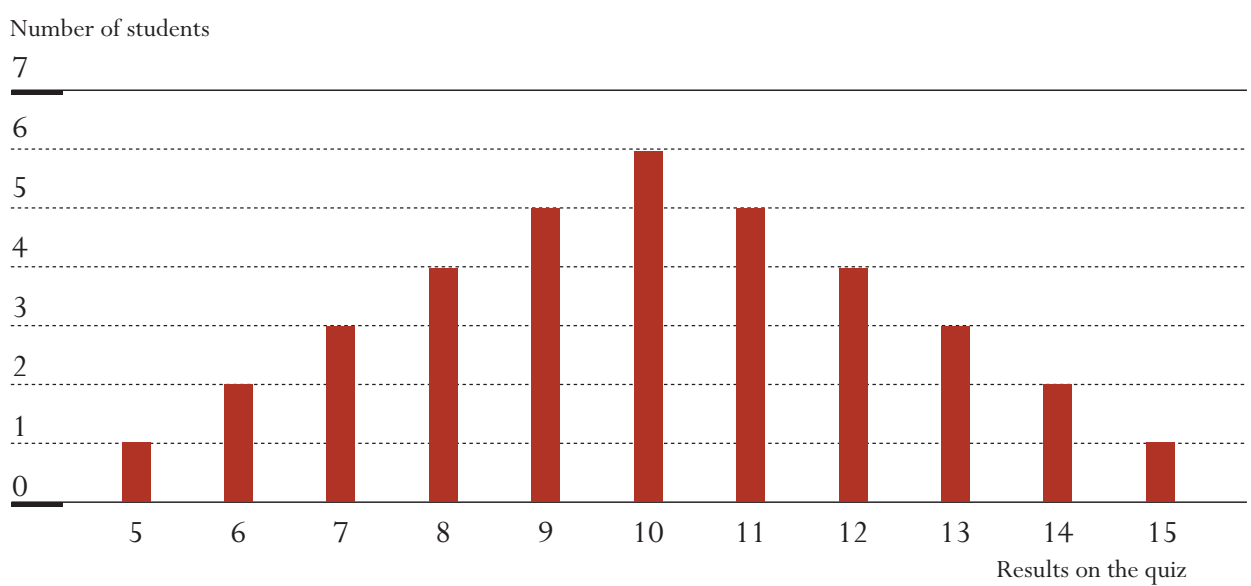


The distribution of the student grades corresponds to a normal distribution. The population mean and the population variance are respectively equal to:

$$
\begin{aligned}
& \mu=\frac{1}{N} \sum_{i=1}^{N} x_{i}=\frac{(5+6+6+7+\ldots+14+14+15)}{36}=\frac{360}{36}=10 \\
& \sigma^{2}=\frac{1}{N} \sum_{i=1}^{N}\left(x_{i}-\mu\right)^{2}=\frac{\left[(5-10)^{2}+(6-10)^{2}+\ldots+(14-10)^{2}+(15-10)^{2}\right]}{36}=\frac{240}{36}=5.8333
\end{aligned}
$$

The standard deviation is therefore equal to:

$$
\sigma=\sqrt{\sigma^{2}}=\sqrt{5.833}=2.415
$$

The teacher then decides to randomly select a sample of two students after the next lesson to save on grading time. The number of possible samples of 2 students out of a population of 36 students is equal to:

$$
C_{36}^{2}=\frac{36 !}{(36-2) ! 2 !}=630
$$

There are 630 possible samples of 2 students out of a population of 36 students. Table 3.1 describes these 630 possible samples. For instance, there are two possible samples which provide a mean estimate of 5.5 for student performance. These two samples are: i) the student with a grade 5 and the first student with a grade 6; and ii) the student with a 5 and the second student with a 6 . Similarly, there are two ways of selecting a sample that would produce a mean grade of $6:$ i) the two sampled students both receive a grade 6 ; or ii) one student receives a 5 and the second student receives a 7 . As only two students obtained a grade 6 (Figure 3.1), there is only one possible sample with two grades 6 . Since Figure 3.1 shows that there is only one student who received a grade 5 and three students who received

\begin{tabular}{|c|c|c|c|}
\hline Sample mean & $\begin{array}{l}\text { Results of } \\
\text { the two sampled students }\end{array}$ & $\begin{array}{c}\text { Number of combinations } \\
\text { of the two results }\end{array}$ & Number of samples \\
\hline 5.5 & 5 and 6 & 2 & 2 \\
\hline 6 & $\begin{array}{l}6 \text { and } 6 \\
5 \text { and } 7\end{array}$ & $\begin{array}{l}1 \\
3\end{array}$ & 4 \\
\hline 6.5 & $\begin{array}{l}5 \text { and } 8 \\
6 \text { and } 7\end{array}$ & $\begin{array}{l}4 \\
6\end{array}$ & 10 \\
\hline 7 & $\begin{array}{l}7 \text { and } 7 \\
5 \text { and } 9 \\
6 \text { and } 8\end{array}$ & $\begin{array}{l}3 \\
5 \\
8\end{array}$ & 16 \\
\hline 7.5 & $\begin{array}{r}5 \text { and } 10 \\
6 \text { and } 9 \\
7 \text { and } 8\end{array}$ & $\begin{array}{r}6 \\
10 \\
12\end{array}$ & 28 \\
\hline 8 & $\begin{array}{r}8 \text { and } 8 \\
5 \text { and } 11 \\
6 \text { and } 10 \\
7 \text { and } 9\end{array}$ & $\begin{array}{r}6 \\
5 \\
12 \\
15 \\
\end{array}$ & 38 \\
\hline
\end{tabular}
a grade 7 , there are three possible samples of two students with a grade 5 and a grade 7 .

\section{Table 3.1 - Description of the 630 possible samples of 2 students selected from 36 according to their mean}


Table 3.1 (continued) - Description of the $\mathbf{6 3 0}$ possible samples of $\mathbf{2}$ students selected from 36 according to their mean

\begin{tabular}{|c|c|c|c|}
\hline Sample mean & $\begin{array}{l}\text { Results of } \\
\text { the two sampled students }\end{array}$ & $\begin{array}{l}\text { Number of combinations } \\
\text { of the two results }\end{array}$ & Number of samples \\
\hline 8.5 & $\begin{array}{r}5 \text { and } 12 \\
6 \text { and } 11 \\
7 \text { and } 10 \\
8 \text { and } 9\end{array}$ & $\begin{array}{r}4 \\
10 \\
18 \\
20\end{array}$ & 52 \\
\hline 9 & $\begin{array}{l}9 \text { and } 9 \\
5 \text { and } 13 \\
6 \text { and } 12 \\
7 \text { and } 11 \\
8 \text { and } 10\end{array}$ & $\begin{array}{r}10 \\
3 \\
8 \\
15 \\
24 \\
\end{array}$ & 60 \\
\hline 9.5 & $\begin{array}{l}5 \text { and } 14 \\
6 \text { and } 13 \\
7 \text { and } 12 \\
8 \text { and } 11 \\
9 \text { and } 10\end{array}$ & $\begin{array}{r}2 \\
6 \\
12 \\
20 \\
30 \\
\end{array}$ & 70 \\
\hline 10 & $\begin{array}{r}10 \text { and } 10 \\
5 \text { and } 15 \\
6 \text { and } 14 \\
7 \text { and } 13 \\
8 \text { and } 12 \\
9 \text { and } 11\end{array}$ & $\begin{array}{r}15 \\
1 \\
4 \\
9 \\
16 \\
25\end{array}$ & 70 \\
\hline 10.5 & $\begin{array}{r}6 \text { and } 15 \\
7 \text { and } 14 \\
8 \text { and } 13 \\
9 \text { and } 12 \\
10 \text { and } 11 \\
\end{array}$ & $\begin{array}{r}2 \\
6 \\
12 \\
20 \\
30 \\
\end{array}$ & 70 \\
\hline 11 & $\begin{array}{r}7 \text { and } 15 \\
8 \text { and } 14 \\
9 \text { and } 13 \\
10 \text { and } 12 \\
11 \text { and } 11 \\
\end{array}$ & $\begin{array}{r}3 \\
8 \\
15 \\
24 \\
10 \\
\end{array}$ & 60 \\
\hline 11.5 & $\begin{array}{r}8 \text { and } 15 \\
9 \text { and } 14 \\
10 \text { and } 13 \\
11 \text { and } 12 \\
\end{array}$ & $\begin{array}{r}4 \\
10 \\
18 \\
20 \\
\end{array}$ & 52 \\
\hline 12 & $\begin{array}{r}9 \text { and } 15 \\
10 \text { and } 14 \\
11 \text { and } 13 \\
12 \text { and } 12 \\
\end{array}$ & $\begin{array}{r}5 \\
12 \\
15 \\
6 \\
\end{array}$ & 38 \\
\hline 12.5 & $\begin{array}{l}10 \text { and } 15 \\
11 \text { and } 14 \\
12 \text { and } 13\end{array}$ & $\begin{array}{r}6 \\
10 \\
12 \\
\end{array}$ & 28 \\
\hline 13 & $\begin{array}{l}11 \text { and } 15 \\
12 \text { and } 14 \\
13 \text { and } 13\end{array}$ & $\begin{array}{l}5 \\
8 \\
2\end{array}$ & 16 \\
\hline 13.5 & $\begin{array}{l}12 \text { and } 15 \\
13 \text { and } 14\end{array}$ & $\begin{array}{l}4 \\
6\end{array}$ & 10 \\
\hline 14 & $\begin{array}{l}13 \text { and } 15 \\
14 \text { and } 14 \\
\end{array}$ & $\begin{array}{l}3 \\
1 \\
\end{array}$ & 4 \\
\hline 14.5 & 14 and 15 & 2 & 2 \\
\hline
\end{tabular}


As shown in Table 3.1, there are two possible samples with a mean of 5.5, four possible samples with a mean of 6 , ten possible samples with a mean of 6.5 , sixteen possible samples with a mean of 7 , and so on.

Figure 3.2 is a chart of the frequency of samples by their mean estimates for all possible samples of 2 students from 36.

Figure 3.2 Sampling variance distribution of the mean

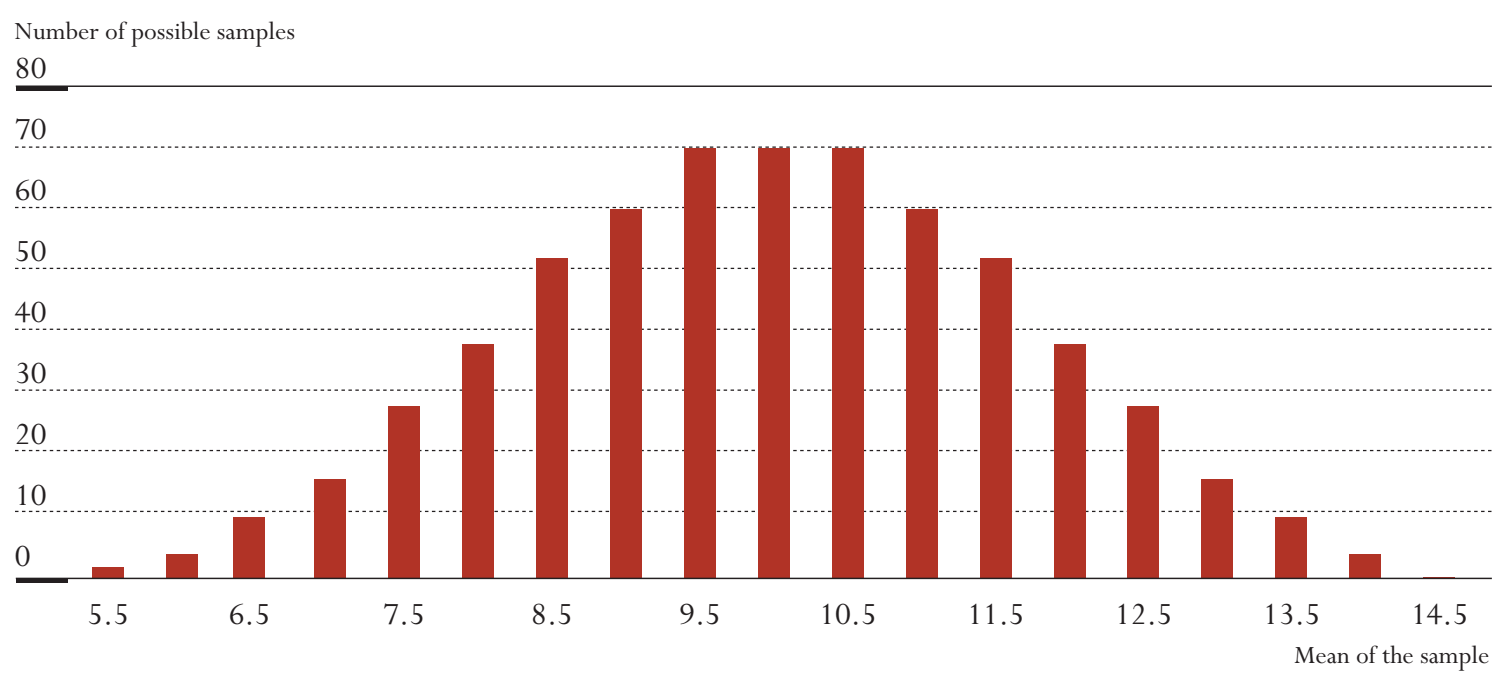

As for all distributions, this distribution of the means of all possible samples can be summarized by central tendency indices and dispersion indices, such as the mean and the variance (or its square root, i.e. the standard deviation).

$$
\mu_{(\hat{\mu})}=[(2 \times 5.5)+(4 \times 6)+(10 \times 6.5)+(16 \times 7)+(28 \times 7.5)+(38 \times 8)+\ldots . .+(2 \times 14.5)] / 630=10
$$

The mean of all possible sample means is equal to the student population mean, i.e. 10. This result is not a coincidence, but a fundamental property of the mean of a simple random sample, i.e. the mean of the means of all possible samples is equal to the population mean. In more formal language, the sample mean is an unbiased estimate of the population mean. Stated differently, the expected value of the sample mean is equal to the population mean.

However, it should be noted that there is an important variation around this expectation. In the example considered, sample means range from 5.5 to 14.5. The variance of this distribution, usually denoted as the sampling variance of the mean, can be computed as:

$$
\sigma_{(\hat{\mu})}^{2}=\left[(5.5-10)^{2}+(5.5-10)^{2}+(6-10)^{2}+\ldots+(14.5-10)^{2}+(14.5-10)^{2}\right] / 630=2.833
$$

Its square root, denoted as the standard error, is equal to:

$$
\sigma_{(\hat{\mu})}=\sqrt{\sigma_{(\hat{\mu})}^{2}}=\sqrt{2.833}=1.68
$$


However, what information does the standard error of the mean give, or more specifically, what does the value 1.68 tell us? The distribution of the means of all possible samples follows approximately a normal distribution. Therefore, based on the mathematical properties of the normal distribution, it can be said that:

- $68.2 \%$ of all possible sample means fall between -1 standard error and +1 standard error around the mean; and

- 95.4\% of all possible sample means fall between -2 standard errors and +2 standard errors.

Let us check the mathematical properties of the normal distribution on the sampling variance distribution of the mean. Remember that, the mean of the sampling variance distribution is equal to 10 and its standard deviation, denoted by the term "standard error", is equal to 1.68.

How many samples have a mean between $\mu_{(\hat{\mu})}-\sigma_{(\hat{\mu})}$ and $\mu_{(\hat{\mu})}+\sigma_{(\hat{\mu})}$, i.e. between $(10-1.68)$ and $(10+1.68)$, or between 8.32 and 11.68 ?

Table 3.2 Distribution of all possible samples with a mean between 8.32 and $\mathbf{1 1 . 6 8}$

\begin{tabular}{c|c|c|c}
\hline Sample mean & Number of samples & Percentage of samples & Cumulative \% of sample \\
\hline 8.5 & 52 & 0.0825 & 0.0825 \\
\hline 9 & 60 & 0.0952 & 0.1777 \\
\hline 9.5 & 70 & 0.1111 & 0.2888 \\
\hline 10 & 70 & 0.1111 & 0.4 \\
\hline 10.5 & 70 & 0.1111 & 0.5111 \\
\hline 11 & 60 & 0.0952 & 0.6063 \\
\hline 11.5 & 52 & 0.0825 & 0.6888 \\
\hline
\end{tabular}

Table 3.2 shows that there are 434 samples out of 630 with a mean comprised between 8.32 and 11.68 ; these represent $68.8 \%$ of all samples. It can also be demonstrated that the percentage of samples with means between $\mu_{(\hat{\mu})}-2 \sigma_{(\hat{\mu})}$ and $\mu_{(\hat{\mu})}+2 \sigma_{(\hat{\mu})}$, i.e. between 6.64 and 13.36 is equal to 94.9 .

To estimate the standard error of the mean, the mean of all possible samples has been computed. In reality though, only the mean of one sample is known. This, as will be shown, is enough to calculate an estimate of the sampling variance. It is therefore important to identify the factors responsible for the sampling variance from the one sample chosen.

The first determining factor is the size of the sample. If the teacher, in our example, decides to select four quizzes instead of two, then the sampling distribution of the mean will range from 6 (the four lowest results being 5, 6, 6 and 7) to 14 (the four highest results being 13, 14, 14 and 15). Remember that the sampling distribution ranged from 5.5 to 14.5 with samples of two units. Increasing the sample size reduces the variance of the distribution.

There are 58905 possible samples of 4 students out of a population of 36 students. Table 3.3 gives the distribution of all possible samples of four students for a population of 36 students. 
Table 3.3 - Distribution of the mean of all possible samples of four students out of a population of 36 students

\begin{tabular}{|c|c|}
\hline Sample mean & Number of possible samples \\
\hline 6.00 & 3 \\
\hline 6.25 & 10 \\
\hline 6.50 & 33 \\
\hline 6.75 & 74 \\
\hline 7 & 159 \\
\hline 7.25 & 292 \\
\hline 7.50 & 510 \\
\hline 7.75 & 804 \\
\hline 8 & 1213 \\
\hline 8.25 & 1700 \\
\hline 8.50 & 2288 \\
\hline 8.75 & 2896 \\
\hline 9 & 3531 \\
\hline 9.25 & 4082 \\
\hline 9.50 & 4553 \\
\hline 9.75 & 4830 \\
\hline 10 & 4949 \\
\hline 10.25 & 4830 \\
\hline 10.50 & 4553 \\
\hline 10.75 & 4082 \\
\hline 11 & 3531 \\
\hline 11.25 & 2896 \\
\hline 11.50 & 2288 \\
\hline 11.75 & 1700 \\
\hline 12 & 1213 \\
\hline 12.25 & 804 \\
\hline 12.50 & 510 \\
\hline 12.75 & 292 \\
\hline 13 & 159 \\
\hline 13.25 & 74 \\
\hline 13.50 & 33 \\
\hline 13.75 & 10 \\
\hline 14 & 3 \\
\hline
\end{tabular}

It can be easily shown that this distribution has a mean of 10 and a standard deviation, denoted standard error, of 1.155 .

This proves that the size of the sample does not affect the expected value of the sample mean, but it does reduce the variance of the distribution of the sample means: the bigger the sample size, the lower the sampling variance of the mean.

The second factor that contributes to the sampling variance is the variance of the population itself. For example, if the results are reported out of a total score of 40 instead of 20, (i.e. the student results are all multiplied by two), then the mean of the student results will be 20, the variance will be 23.333 (i.e. four times 5.8333 ) and the standard deviation will be equal to 4.83 (i.e. two times 2.415).

It can be shown that the sampling variance from a sample of two students will be equal to 11.333 and that the standard error of the mean will be equal to 3.3665 (i.e. two times 1.68). 
The standard error of the mean is therefore proportional to the population variance. Based on these examples, it can be established that the sampling variance of the mean is equal to:

$$
\sigma_{(\hat{\boldsymbol{u}})}^{2}=\frac{\sigma^{2}}{n}\left(\frac{N-n}{N-1}\right)
$$

and the standard error of the sample mean is equal to:

$$
\sigma_{(\hat{\mu})}=\sqrt{\sigma_{(\hat{\mu})}^{2}}=\frac{\sigma}{\sqrt{n}} \sqrt{\frac{N-n}{N-1}}
$$

Where:

$$
\begin{aligned}
& \sigma^{2}=\text { variance of the population; } \\
& \sigma=\text { standard deviation of the population; } \\
& n=\text { sample size; and } \\
& N=\text { population size. }
\end{aligned}
$$

This formula can be checked with the example:

$$
\sigma_{(\hat{\mu})}^{2}=\frac{\sigma^{2}}{n}\left(\frac{N-n}{N-1}\right)=\frac{5.833}{2}\left(\frac{36-2}{36-1}\right)=2.8333
$$

As the size of the population increases, the ratio $\left(\frac{N-n}{N-1}\right)$ tends toward 1. In such cases, a close approximation of the sampling variance of the mean is given by:

$$
\sigma_{(\hat{\mu})}^{2}=\frac{\sigma^{2}}{n}
$$

However, in practice, the population variance is unknown and is estimated from a sample. The sampling variance estimate on the mean, just as a mean estimate, can vary depending on the sample. Therefore, being based on a sample, only an estimate of the sampling variance on the mean (or any other estimate) can be computed.

In the remainder of this manual, the concepts of sampling variance and estimations of the sampling variance will be confounded to simplify the text and the mathematical notations. That is, symbols depicting the estimates of sampling variance will not have a hat $\left({ }^{\wedge}\right)$ to differentiate them from true values, but the fact that they are estimates is to be understood.

\section{SAMPLING VARIANCE FOR TWO-STAGE SAMPLING}

Education surveys and more particularly international surveys rarely sample students by simply selecting a random sample of students. Schools get selected first and, within each selected school, classes or students are randomly sampled.

One of the differences between simple random sampling and two-stage sampling is that for the latter, selected students attending the same school cannot be considered as independent observations. This is because students within a school will usually have more common characteristics than students 
from different educational institutions. For instance, they are offered the same school resources, may have the same teachers, and therefore are taught a common curriculum, and so on. Differences between students from different schools are also greater if different educational programs are not available in all schools. For instance, one would expect to observe more differences between students from a vocational school and students from an academic school, than those that would be observed between students from two vocational schools.

Further, it is well known that within a country, within sub-national entities, and within cities, people tend to live in areas according to their financial resources. As children usually attend schools close to their homes, it is likely that students attending the same school come from similar social and economic backgrounds.

A simple random sample of 4000 students is thus likely to cover the diversity of the population better than a sample of 100 schools with 40 students observed within each school. It follows that the uncertainty associated with any population parameter estimate (i.e. standard error) will be greater for a two-stage sample than for a simple random sample of the same size.

The increase of the uncertainty due to the two-stage sample is directly proportional to the differences between the first stage units, known as primary sampling units (PSUs), i.e. schools for education surveys. The consequences of this uncertainty for two extreme and fictitious situations are given below:

- All students in the population are randomly assigned to schools. Therefore, there should not be any differences between schools. Randomly selecting 100 schools and then within the selected schools randomly drawing 40 students would be similar from a statistical point of view to directly selecting randomly 4000 students as there are no differences between schools. The uncertainty associated with any population parameter estimate would be equal to the uncertainty obtained from a simple random sample of 4000 students.

- All schools are different but within schools, all students are perfectly identical. Since within a particular school, all students are identical: observing only one student, or 40, would provide the same amount of information. Therefore, if 100 schools are selected and 40 students are observed per selected school, the effective sample size of this sample would be equal to 100 . Therefore, the uncertainty associated with any population parameter estimate would be equal to the uncertainty obtained from a simple random sample of 100 students.

Of course, there is no educational system in the world that can be identified with either of these extreme and fictitious situations. Nevertheless, in some educational systems, school differences, at least regarding the survey's measure, for example, the academic performance, appear to be very small, while in some other educational systems, school differences can be quite substantial.

The academic performance of each student can be represented by a test score, or by the difference between his or her score and the country average score. In education research, it is common to split the difference between the student's score and the country average score into three parts: i) the distance between the student's performance and the corresponding class mean; ii) the distance between this class mean and the corresponding school mean; iii) the distance between this school mean and the country mean. The first difference relates to the within-class variance 
(or the residual variance in terms of variance analysis). It indicates how much student scores can vary within a particular class. The second difference - the distance between the class mean and the school mean - is related to the between-classes-within-school variance. This difference reflects the range of differences between classes within schools. This between-classes-withinschool variance might be substantial in educational institutions that offer both academic and vocational education. The third distance - the difference between the school average and the country average - is called the between-school variance. This difference indicates how much student performance varies among schools.

To obtain an estimate of these three components of the variance, it would be necessary to sample several schools, at least two classes per school and several students per class. PISA randomly selects 15 -year-olds directly from student lists within the participating schools. Therefore, generally speaking, it is impossible to distinguish the between- and within-classes variances. PISA can only provide estimates of the between- and the within-school variances.

Table 3.4 provides the between-school and within-school variances on the mathematics scale for PISA 2003. In northern European countries, the between-school variances are very small compared to their within-school variance estimates. In these countries, the student variance mainly lies at the within-school level. In terms of student achievement then, schools in such countries do not vary greatly. However, in Austria, Belgium, Germany, Hungary and Turkey, for instance, more than 50 per cent of the student differences in performance are accounted for at the school level. This means that the student performance differs substantially among schools. Therefore, the uncertainty associated with any population parameters will be larger for these countries when compared to the uncertainty for northern European countries, given a comparable sample size of schools and students.

As Kish (1987) noted:

Standard methods for statistical analysis have been developed on assumptions of simple random sampling. Assuming independence for individual elements (or observations) greatly facilitates the mathematics used for distribution theories of formulas for complex statistics. ... However, independent selection of elements is seldom realised in practice, because much research is actually and necessarily accomplished with complex sample designs. It is economical to select clusters that are natural grouping of elements, and these tend to be somewhat homogeneous for most characteristics. The assumptions may fail mildly or badly; hence standard statistical analysis tends to result in mild or bad underestimates in length of reported probability intervals. Overestimates are possible, but rare and mild.

Kish established a state of the art knowledge of the sampling variance according to the type of estimator and the sampling design. The sampling variance distributions are well known for univariate and multivariate estimators for simple random samples. The use of stratification variables with a simple random sample still allows the mathematical computation of the sampling variances, but with a substantial increase of complexity. As shown in Table 3.5, the computation of sampling variances for two-stage samples is available for some designs, but it becomes quite difficult to compute for multivariate indices. 
Table 3.4 Between-school and within-school variances on the mathematics scale in PISA 2003

\begin{tabular}{|c|c|c|}
\hline & Between-school variance & Within-school variance \\
\hline AUS & 1919.11 & 7169.09 \\
\hline AUT & 5296.65 & 4299.71 \\
\hline BEL & 7328.47 & 5738.33 \\
\hline BRA & 4128.49 & 5173.60 \\
\hline CAN & 1261.58 & 6250.12 \\
\hline $\mathrm{CHE}$ & 3092.60 & 6198.65 \\
\hline CZE & 4972.45 & 4557.50 \\
\hline DEU & 6206.92 & 4498.70 \\
\hline DNK & 1109.45 & 7357.14 \\
\hline ESP & 1476.85 & 6081.74 \\
\hline FIN & 336.24 & 6664.98 \\
\hline FRA & 3822.62 & 4536.22 \\
\hline GBR & 1881.09 & 6338.25 \\
\hline GRC & 3387.52 & 5991.75 \\
\hline HKG & 4675.30 & 5298.26 \\
\hline HUN & 5688.56 & 4034.66 \\
\hline IDN & 2769.48 & 3343.87 \\
\hline IRL & 1246.70 & 6110.71 \\
\hline ISL & 337.56 & 7849.99 \\
\hline ITA & 4922.84 & 4426.67 \\
\hline JPN & 5387.17 & 4668.82 \\
\hline KOR & 3531.75 & 5011.56 \\
\hline LIE & 3385.41 & 5154.08 \\
\hline LUX & 2596.36 & 5806.97 \\
\hline LVA & 1750.22 & 6156.52 \\
\hline MAC & 1416.99 & 6449.96 \\
\hline MEX & 2476.01 & 3916.46 \\
\hline NLD & 5528.99 & 3326.09 \\
\hline NOR & 599.49 & 7986.58 \\
\hline NZL & 1740.61 & 7969.97 \\
\hline POL & 1033.90 & 7151.46 \\
\hline PRT & 2647.70 & 5151.93 \\
\hline RUS & 2656.62 & 6021.44 \\
\hline SVK & 3734.56 & 4873.69 \\
\hline SWE & 986.03 & 8199.46 \\
\hline THA & 2609.38 & 4387.08 \\
\hline TUN & 2821.00 & 3825.36 \\
\hline TUR & 6188.40 & 4891.13 \\
\hline URY & 4457.08 & 5858.42 \\
\hline USA & 2395.38 & 6731.45 \\
\hline YUG & 2646.00 & 4661.59 \\
\hline
\end{tabular}

a. The results are based on the first plausible value for the mathematics scale, denoted PV1MATH in the PISA 2003 database (www.pisa.oecd.org). 
Table 3.5 - Current status of sampling errors

\begin{tabular}{l|lll}
\hline $\begin{array}{l}\text { Selection } \\
\text { methods }\end{array}$ & $\begin{array}{l}\text { Means and total of } \\
\text { entire samples }\end{array}$ & $\begin{array}{l}\text { Subclass means } \\
\text { and differences }\end{array}$ & $\begin{array}{l}\text { Complex analytical statistics, } \\
\text { e.g. coefficients in regression }\end{array}$ \\
\hline $\begin{array}{l}\text { Simple random selection } \\
\text { of elements }\end{array}$ & Known & Known & Known \\
\hline Stratified selection of elements & Known & Available & Conjectured \\
\hline Complex cluster sampling & $\begin{array}{l}\text { Known for some } \\
\text { sampling design }\end{array}$ & Available & Difficult \\
\hline
\end{tabular}

Note: Row 1 refers to standard statistical theory (Kish and Frankel, 1974).

Authors of sampling manuals usually distinguish two types of two-stage sampling:

- Two-stage sampling with first-stage units of equal sizes; and

- Two-stage sampling with first-stage units of unequal sizes.

Beyond this distinction, different characteristics of the population and of the sampling design need to be taken into account in the computation of the sampling variance, because they affect the sampling variance. Some of the factors to be considered are:

- Is the population finite or infinite?

- Was size a determining criterion in the selection of the first-stage units?

- Was a systematic procedure used for selecting first-stage or second-stage units?

- Does the sampling design include stratification variables?

The simplest two-stage sample design occurs with infinite populations of stage one and stage two units. As both stage units are infinite populations, PSUs are considered to be of equal sizes. If a simple random sample of PSUs is selected and if, within each selected PSU, a simple random sample of stage two units is selected then the sampling variance of the mean will be equal to:

$$
\sigma_{(\hat{\mu})}^{2}=\frac{\sigma_{\text {between_PSU }}^{2}}{n_{P S U}}+\frac{\sigma_{\text {within_PSU }}^{2}}{n_{P S U} n_{\text {within }}}
$$

Let us apply this formula to an education survey and let us consider the population of schools as infinite and the population of students within each school as infinite. The computation of the sampling variance of the mean is therefore equal to:

$$
\sigma_{(\hat{\boldsymbol{u}})}^{2}=\frac{\sigma_{\text {between_school }}^{2}}{n_{\text {school }}}+\frac{\sigma_{\text {within_school }}^{2}}{n_{\text {students }}}
$$

Table 3.6 - Between-school and within-school variances, number of participating students and schools in Denmark and Germany in PISA 2003

\begin{tabular}{l|cc}
\hline & Denmark & Germany \\
\hline Between-school variance & 1109.45 & 6206.92 \\
Within-school variance & 7357.14 & 4498.70 \\
Number of participating schools & 206 & 216 \\
Number of participating students & 4218 & 4660 \\
\hline
\end{tabular}


Under these assumptions, the sampling variance of the mean and its square root, i.e. the standard error, in Denmark are equal to:

$$
\begin{aligned}
& \sigma_{(\hat{\mu})}^{2}=\frac{1109.45}{206}+\frac{7357.14}{4218}=5.39+1.74=7.13 \\
& \sigma_{(\hat{\mu})}=\sqrt{7.13}=2.67
\end{aligned}
$$

The sampling variance of the mean and its square root, i.e. the standard error, in Germany are equal to:

$$
\begin{aligned}
& \sigma_{(\hat{\mu})}^{2}=\frac{6206.92}{216}+\frac{4498.70}{4660}=28.74+0.97=29.71 \\
& \sigma_{(\hat{\mu})}=\sqrt{29.71}=5.45
\end{aligned}
$$

If both samples were considered as simple random samples, then the standard error of the mean for Denmark and Germany would be respectively equal to 1.42 and 1.51 .

Based on these results, we can make the following observations:

- The standard error of the mean is larger for two-stage sampling than for simple random sampling. For example, in the case of Germany, the standard errors for simple random sampling and for twostage sampling are 1.51 and 5.45 respectively. Considering a two-stage sample as a simple random sample will therefore substantially underestimate standard errors and consequently confidence intervals will be too narrow. The confidence interval on the mathematic scale average, i.e. 503, would be equal to: $[503-(1.96 * 1.51) ; 503+(1.96 * 1.51)]=[500.05 ; 505.96]$ in the case of a simple random sample, but equal to $[484-(1.96 * 5.45) ; 484+(1.96 * 5.45)]=[492.32 ; 513.68]$ in the case of a two-stage sample. This indicates that any estimated mean value between 492.32 and 500.05 and between 505.96 and 513.68 may or may not be considered as statistically different from the German average, depending on the standard error used.

- The sampling variance of the mean for two-stage samples is mainly dependent on the betweenschool variance and the number of participating schools. Indeed, the between-school variance accounts for 76 percent of the total sampling variance in Denmark, i.e. 5.39/7.13=0.76. For Germany, the between-school variance accounts for 97 per cent of the total sampling variance $(28.74 / 29.71=0.97)$. Therefore, one should expect larger sampling variance in countries with larger between-school variance, such as Germany and Austria for example.

However, the PISA population cannot be considered as an infinite population of schools with an infinite population of students. Further,

- Schools have unequal sizes;

- The PISA sample is a sample without replacement, i.e. a school cannot be selected twice;

- Schools are selected proportionally to their sizes and according to a systematic procedure; and

- Stratification variables are included in the sample design.

These characteristics of the sampling design will influence the sampling variance, so that the formula used above is also inappropriate. Indeed, Learning for Tomorrow's World - First Results from PISA 2003 (OECD, 2004a) indicates that the standard errors on the mathematics scale mean for Denmark and Germany are 2.7 and 3.3, respectively. 
This shows that the PISA sample design is quite efficient in reducing the sampling variance. However, the design becomes so complex that there is no easy formula for computing the sampling variance, or even estimators, such as means.

Since the IEA 1990 reading literacy study, replication or resampling methods have been used to compute estimates of the sampling variance for international education surveys. Even though these methods were known since the late 50s, they were not often used as they require numerous computations. With the availability of powerful personal computers in the 1990s and the increased use of international databases by non-mathematicians, international coordinating centres were encouraged to use resampling methods for estimating sampling variances from complex sample designs.

According to Rust and Rao (1996):

The common principle that these methods have is to use computational intensity to overcome difficulties and inconveniences in utilizing an analytic solution to the problem at hand. Briefly, the replication approach consists of estimating the variance of a population parameter of interest by using a large number of somewhat different subsamples (or somewhat different sampling weights) to calculate the parameter of interest. The variability among the resulting estimates is used to estimate the true sampling error of the initial or full-sample estimate.

These methods will first be described for simple random samples and for two-stage samples. The PISA replication method will be presented subsequently.

\section{REPLICATION METHODS FOR SIMPLE RANDOM SAMPLES}

There are two main types of replication methods for simple random samples. These are known as the Jackknife and the Bootstrap. One of the most important differences between the Jackknife and the Bootstrap is related to the procedure used to produce the repeated subsamples or replicate samples. From a sample of $n$ units, the Jackknife generates in a systematic way $n$ replicate samples of $n-1$ units. The Bootstrap randomly generates a large number of repetitions of $n$ units selected with replacement, with each unit having more than one chance of selection.

Since PISA does not use a Bootstrap replication method adapted to multi-stage sample designs, this section will only present the Jackknife method.

Suppose that a sample of ten students has been selected by simple random sampling. The Jackknife method will then generate ten subsamples, or replicate samples, each of nine students, as follows:

Table 3.7 - Jackknife replicate samples and their means

\begin{tabular}{l|c|c|c|c|c|c|c|c|c|c|c}
\hline Student & 1 & 2 & 3 & 4 & 5 & 6 & 7 & 8 & 9 & 10 & Mean \\
\hline Value & 10 & 11 & 12 & 13 & 14 & 15 & 16 & 17 & 18 & 19 & 14.50 \\
\hline Replication 1 & 0 & 1 & 1 & 1 & 1 & 1 & 1 & 1 & 1 & 1 & 15.00 \\
\hline Replication 2 & 1 & 0 & 1 & 1 & 1 & 1 & 1 & 1 & 1 & 1 & 14.88 \\
\hline Replication 3 & 1 & 1 & 0 & 1 & 1 & 1 & 1 & 1 & 1 & 1 & 14.77 \\
\hline Replication 4 & 1 & 1 & 1 & 0 & 1 & 1 & 1 & 1 & 1 & 1 & 14.66 \\
\hline Replication 5 & 1 & 1 & 1 & 1 & 0 & 1 & 1 & 1 & 1 & 1 & 14.55 \\
\hline Replication 6 & 1 & 1 & 1 & 1 & 1 & 0 & 1 & 1 & 1 & 1 & 14.44 \\
\hline Replication 7 & 1 & 1 & 1 & 1 & 1 & 1 & 0 & 1 & 1 & 1 & 14.33 \\
\hline Replication 8 & 1 & 1 & 1 & 1 & 1 & 1 & 1 & 0 & 1 & 1 & 14.22 \\
\hline Replication 9 & 1 & 1 & 1 & 1 & 1 & 1 & 1 & 1 & 0 & 1 & 14.11 \\
\hline Replication 10 & 1 & 1 & 1 & 1 & 1 & 1 & 1 & 1 & 1 & 0 & 14.00 \\
\hline
\end{tabular}


As shown in Table 3.7, the Jackknife generates ten replicate samples of nine students. The sample mean based on all ten students is equal to 14.5. For the first replicate sample, student 1 is not included in the calculation of the mean, and the mean of the nine students included in replicate sample 1 is 15.00 . For the second replicate sample, the second student is not included and the mean of the other 9 students is equal to 14.88 , and so on.

The Jackknife estimate of sampling variance of the mean is equal to:

$$
\sigma_{\text {jack }}^{2}=\frac{n-1}{n} \sum_{i=1}^{n}\left(\hat{\theta}_{(i)}-\hat{\theta}\right)^{2} \text { with }
$$

$\hat{\boldsymbol{\theta}}_{(i)}$ representing the statistic estimate for replicate sample $i$, and $\hat{\boldsymbol{\theta}}$ representing the statistic estimate based on the whole sample.

Based on the data from Table 3.7, the Jackknife sampling variance of the mean is equal to:

$$
\begin{aligned}
& \sigma_{(\hat{\mu})}^{2}=\frac{9}{10}\left[(15.00-14.50)^{2}+(14.88-14.50)^{2}+\ldots .+(15.11-14.50)^{2}+(14.00-14.50)^{2}\right] \\
& \sigma_{(\hat{\mu})}^{2}=\frac{9}{10}(1.018519)=0.9167
\end{aligned}
$$

The usual population variance estimator is equal to:

$$
\sigma^{2}=\frac{1}{n-1} \sum_{i=1}^{n}\left(x_{i}-\hat{\mu}\right)^{2}=\frac{1}{9}\left[(10-14.5)^{2}+(11-14.5)^{2}+\ldots+(18-14.5)^{2}+(19-14.5)^{2}\right]=9.17
$$

Therefore, the sampling variance of the mean, estimated by the mathematical formula, is equal to:

$$
\sigma_{(\hat{\mu})}^{2}=\frac{\sigma^{2}}{n}=\frac{9.17}{10}=0.917
$$

As shown in this example, the Jackknife method and the mathematical formula provide identical estimation of the sampling variance. Rust (1996) mathematically demonstrates this equality.

$$
\begin{aligned}
& \hat{\mu}_{(i)}-\hat{\mu}=\frac{\left[\left(\sum_{i=1}^{n} x_{i}\right)-x_{i}\right]}{n-1}-\frac{\left[\sum_{i=1}^{n} x_{i}\right]}{n}=-\frac{x_{i}}{n-1}+\left[\sum_{i=1}^{n} x_{i}\right]\left[\frac{1}{n-1}-\frac{1}{n}\right] \\
& =-\frac{1}{(n-1)}\left[x_{i}-\left(\sum_{i=1}^{n} x_{i}\right)\left(1-\frac{(n-1)}{n}\right)\right]=-\frac{1}{(n-1)}\left[x_{i}-\hat{\mu}(n-(n-1)]=-\frac{1}{(n-1)}\left(x_{i}-\hat{\mu}\right)\right.
\end{aligned}
$$

Therefore,

$$
\begin{aligned}
& \left(\hat{\mu}_{(i)}-\hat{\mu}\right)^{2}=\frac{1}{(n-1)^{2}}\left(x_{i}-\hat{\mu}\right)^{2} \\
& \Rightarrow \sum_{i=1}^{n}\left(\hat{\mu}_{(i)}-\hat{\mu}\right)^{2}=\frac{1}{(n-1)^{2}} \sum_{i=1}^{n}\left(x_{i}-\hat{\mu}\right)^{2}=\frac{1}{(n-1)} \frac{\sum_{i=1}^{n}\left(x_{i}-\hat{\mu}\right)^{2}}{(n-1)}=\frac{1}{(n-1)} \hat{\sigma}^{2} \\
& \Rightarrow \sigma_{\text {jack }}^{2}=\frac{n-1}{n} \sum_{i=1}^{n}\left(\hat{\mu}_{(i)}-\hat{\mu}\right)^{2}=\frac{(n-1)}{n} \frac{1}{(n-1)} \hat{\sigma}^{2}=\frac{\hat{\sigma}^{2}}{n}
\end{aligned}
$$


The Jackknife method can also be applied to compute the sampling variance for other statistics, such as regression coefficients. In this particular example, the procedure will consist of the computation of 11 regression coefficients: one based on the whole sample and ten others with each being based on one replicate sample. The comparison between the whole sample regression coefficient and each of the ten replicate regression coefficients will provide an estimate of the sampling variance of that statistic.

Table 3.8 Values on variables $\mathbf{X}$ and $\mathbf{Y}$ for a sample of 10 students

\begin{tabular}{|c|c|c|c|c|c|c|c|c|c|c|}
\hline Student & 1 & 2 & 3 & 4 & 5 & 6 & 7 & 8 & 9 & 10 \\
\hline ValueY & 10 & 11 & 12 & 13 & 14 & 15 & 16 & 17 & 18 & 19 \\
\hline Value X & 10 & 13 & 14 & 19 & 11 & 12 & 16 & 17 & 18 & 15 \\
\hline
\end{tabular}

The regression coefficient for the whole sample is equal to 0.53 .

Table 3.9 - Regression coefficients for each replicate sample

\begin{tabular}{l|c} 
& Regression coefficient \\
\hline Replicate 1 & 0.35 \\
Replicate 2 & 0.55 \\
Replicate 3 & 0.56 \\
Replicate 4 & 0.64 \\
Replicate 5 & 0.51 \\
Replicate 6 & 0.55 \\
Replicate 7 & 0.51 \\
Replicate 8 & 0.48 \\
Replicate 9 & 0.43 \\
Replicate 10 & 0.68 \\
\hline
\end{tabular}

The Jackknife formula, i.e. $\sigma_{\text {jack }}^{2}=\frac{n-1}{n} \sum_{i=1}^{n}\left(\hat{\theta}_{(i)}-\hat{\theta}\right)^{2}$, can be applied to compute the sampling variance of the regression coefficient.

$$
\sigma_{\text {jack }}^{2}=\frac{n-1}{n} \sum_{i=1}^{n}\left(\hat{\theta}_{(i)}-\hat{\theta}\right)^{2}=\frac{9}{10}\left[(0.35-0.53)^{2}+(0.55-0.53)^{2}+\ldots(0.68-0.53)^{2}\right]=0.07
$$

This result is identical to the result that the usual sampling variance formula for a regression coefficient would render.

\section{RESAMPLING METHODS FOR TWO-STAGE SAMPLES}

There are three types of replication methods for two-stage samples:

- The Jackknife, with two variants: one for unstratified samples and another one for stratified samples;

- The Balanced Repeated Replication (BRR) and its variant, Fay's modification;

- The Bootstrap.

PISA uses BRR with Fay's modification. 
If a simple random sample of PSUs is drawn without the use of any stratification variables, then it can be shown that the sampling variance of the mean obtained using the Jackknife method is mathematically equal to the formula provided in section 2 of this chapter, i.e.:

$$
\sigma_{(\hat{\mu})}^{2}=\frac{\sigma_{\text {between_PSU }}^{2}}{n_{P S U}}+\frac{\sigma_{\text {within_PSU }}^{2}}{n_{P S U} n_{\text {within }}}
$$

Consider a sample of ten schools and within selected schools, a simple random sample of students. The Jackknife method for an unstratified two-stage sample consists of generating ten replicates of nine schools. Each school is removed only once, in a systematic way.

Table 3.10 - The Jackknife replicate samples for unstratified two-stage sample

\begin{tabular}{l|c|c|c|c|c|c|c|c|c|c}
\hline Replicate & $\mathbf{R} 1$ & $\mathbf{R} 2$ & $\mathbf{R} 3$ & $\mathbf{R} 4$ & $\mathbf{R 5}$ & $\mathbf{R 6}$ & $\mathbf{R 7}$ & $\mathbf{R 8}$ & $\mathbf{R 9}$ & $\mathbf{R} 10$ \\
\hline School 1 & 0.00 & 1.11 & 1.11 & 1.11 & 1.11 & 1.11 & 1.11 & 1.11 & 1.11 & 1.11 \\
\hline School 2 & 1.11 & 0.00 & 1.11 & 1.11 & 1.11 & 1.11 & 1.11 & 1.11 & 1.11 & 1.11 \\
\hline School 3 & 1.11 & 1.11 & 0.00 & 1.11 & 1.11 & 1.11 & 1.11 & 1.11 & 1.11 & 1.11 \\
\hline School 4 & 1.11 & 1.11 & 1.11 & 0.00 & 1.11 & 1.11 & 1.11 & 1.11 & 1.11 & 1.11 \\
\hline School 5 & 1.11 & 1.11 & 1.11 & 1.11 & 0.00 & 1.11 & 1.11 & 1.11 & 1.11 & 1.11 \\
\hline School 6 & 1.11 & 1.11 & 1.11 & 1.11 & 1.11 & 0.00 & 1.11 & 1.11 & 1.11 & 1.11 \\
\hline School 7 & 1.11 & 1.11 & 1.11 & 1.11 & 1.11 & 1.11 & 0.00 & 1.11 & 1.11 & 1.11 \\
\hline School 8 & 1.11 & 1.11 & 1.11 & 1.11 & 1.11 & 1.11 & 1.11 & 0.00 & 1.11 & 1.11 \\
\hline School 9 & 1.11 & 1.11 & 1.11 & 1.11 & 1.11 & 1.11 & 1.11 & 1.11 & 0.00 & 1.11 \\
\hline School 10 & 1.11 & 1.11 & 1.11 & 1.11 & 1.11 & 1.11 & 1.11 & 1.11 & 1.11 & 0.00 \\
\hline
\end{tabular}

For the first replicate, denoted R1, school 1 has been removed. The weights of the other schools in the first replicate are adjusted by a factor of 1.11 , i.e. $\frac{10}{9}$ or, as a general rule, by a factor of $\frac{G}{G-1}$, with $G$ being the number of PSUs in the sample. This adjustment factor is then applied when school replicate weights and within school replicate weights are combined to give the student replicate weights. For the second replicate, school 2 is removed and the weights in the remaining schools are adjusted by the same factor, and so on.

The statistic of interest is computed for the whole sample, and then again for each replicate. The replicate estimates are then compared to the whole sample estimate to obtain the sampling variance, as follows:

$$
\sigma_{(\hat{\theta})}^{2}=\frac{(G-1)}{G} \sum_{i=1}^{G}\left(\hat{\theta}_{(i)}-\hat{\theta}\right)^{2}
$$

This formula is identical to the one used for a simple random sample, except that instead of using $n$ replicates, $n$ being the number of units in the sample, this formula uses $G$ replicates, with $G$ being the number of PSUs. 


\section{THE JACKKNIFE FOR STRATIFIED TWO-STAGE SAMPLE DESIGNS}

As mentioned at the beginning of Chapter 2, two major principles underlie all sample designs. The first is the concern to avoid bias in the selection procedure, the second to achieve the maximum precision in view of the available financial resources.

To reduce the uncertainty, or to minimize the sampling variance without modifying the sample size, international and national education surveys usually implement the following procedures in the sampling design:

- PSUs are selected proportionally to their size and according to a systematic procedure. This procedure leads to an efficient student sampling procedure. Equal-sized samples of students can be selected from each school. At the same time, the overall selection probabilities (combining the school and student sampling components) do not vary much.

- National centres are encouraged to identify stratification variables that are statistically associated with the student performance. Characteristics, such as rural versus urban, academic versus vocational, private versus public, are associated with the student performance. The sampling variance reduction will be proportional to the explanatory power of these stratification variables on student performance.

The Jackknife for stratified two-stage samples allows the reduction of the sampling variance by taking both of these aspects into consideration. Failing to do so, would lead to a systematic overestimation of sampling variances.

Suppose that the list of schools in the population is divided into two parts called strata: rural schools and urban schools. Further, within these two strata, schools are sorted by size. Within each stratum, ten schools are selected systematically and proportionally to their size.

The Jackknife method for stratified two-stage sample designs consists of systematically pairing sampled schools within each stratum in the order in which they were selected. Therefore, schools will be paired with other similar schools.

Table 3.11 - The Jackknife replicates for stratified two-stage sample designs

\begin{tabular}{c|c|c|c|c|c|c|c|c|c|c|c}
\hline Pseudo-stratum & School & R1 & R2 & R3 & R4 & R5 & R6 & R7 & R8 & R9 & R10 \\
\hline 1 & 1 & 2 & 1 & 1 & 1 & 1 & 1 & 1 & 1 & 1 & 1 \\
\hline 1 & 2 & 0 & 1 & 1 & 1 & 1 & 1 & 1 & 1 & 1 & 1 \\
\hline 2 & 3 & 1 & 0 & 1 & 1 & 1 & 1 & 1 & 1 & 1 & 1 \\
\hline 2 & 4 & 1 & 2 & 1 & 1 & 1 & 1 & 1 & 1 & 1 & 1 \\
\hline 3 & 5 & 1 & 1 & 2 & 1 & 1 & 1 & 1 & 1 & 1 & 1 \\
\hline 3 & 6 & 1 & 1 & 0 & 1 & 1 & 1 & 1 & 1 & 1 & 1 \\
\hline 4 & 7 & 1 & 1 & 1 & 0 & 1 & 1 & 1 & 1 & 1 & 1 \\
\hline 4 & 8 & 1 & 1 & 1 & 2 & 1 & 1 & 1 & 1 & 1 & 1 \\
\hline 5 & 9 & 1 & 1 & 1 & 1 & 2 & 1 & 1 & 1 & 1 & 1 \\
\hline 5 & 10 & 1 & 1 & 1 & 1 & 0 & 1 & 1 & 1 & 1 & 1 \\
\hline 6 & 11 & 1 & 1 & 1 & 1 & 1 & 2 & 1 & 1 & 1 & 1 \\
\hline 6 & 12 & 1 & 1 & 1 & 1 & 1 & 0 & 1 & 1 & 1 & 1 \\
\hline 7 & 14 & 1 & 1 & 1 & 1 & 1 & 1 & 0 & 1 & 1 & 1 \\
\hline 7 & 15 & 1 & 1 & 1 & 1 & 1 & 1 & 2 & 1 & 1 & 1 \\
\hline 8 & 16 & 1 & 1 & 1 & 1 & 1 & 1 & 1 & 2 & 1 & 1 \\
\hline 8 & 17 & 1 & 1 & 1 & 1 & 1 & 1 & 1 & 1 & 0 & 1 \\
\hline 9 & 18 & 1 & 1 & 1 & 1 & 1 & 1 & 1 & 1 & 2 & 1 \\
\hline 10 & 19 & 1 & 1 & 1 & 1 & 1 & 1 & 1 & 1 & 1 & 2 \\
\hline 10 & 20 & 1 & 1 & 1 & 1 & 1 & 1 & 1 & 1 & 1 & 0 \\
\hline
\end{tabular}


Table 3.11 describes how replicates are generated for this method. Schools 1 to 10 are rural, and schools 11 to 20 are urban. Within each stratum, there are therefore five school pairs, or pseudostrata (also called variance strata).

The Jackknife for stratified two-stage samples will generate as many replicates as there are pairs or pseudo strata. In this example, ten replicates will therefore be generated. For each replicate sample, one school is randomly removed within a particular pseudo-stratum and the weight of the remaining school in the pseudo-stratum is doubled. For replicate 1, denoted R1, school 2 is removed and the weight of school 1 is doubled in pseudo-stratum 1. For replicate 2, school 3 is removed and the weight of school 4 is doubled in pseudo-stratum 2, and so on.

As previously mentioned, the statistic of interest is computed based on the whole sample and then again based on each replicate sample. The replicate estimates are then compared to the whole sample estimate to obtain the sampling variance, as follows:

$$
\sigma_{(\hat{\theta})}^{2}=\sum_{i=1}^{G}\left(\hat{\theta}_{(i)}-\hat{\theta}\right)^{2}
$$

This replication method is now generally used in IEA studies.

\section{THE BALANCED REPEATED REPLICATION METHOD}

While the Jackknife method consists of removing only one school for each replicate sample, the Balanced Repeated Replication (BRR) method proceeds by selecting at random one school within each pseudo-stratum to have its weight set to 0 , and by doubling the weights of the remaining schools.

As this method results in a large set of possible replicates, a balanced set of replicate samples is generated according to Hadamard matrices in order to avoid lengthy computations. The number of replicates is the smallest multiple of four, greater than or equal to the number of pseudo-strata. In this example, as there are ten pseudo-strata, 12 replicates will be generated.

Table $3.12 \backsim$ The BRR replicates

\begin{tabular}{c|c|c|c|c|c|c|c|c|c|c|c|c|c}
\hline Pseudo-stratum & School & $\mathrm{R} 1$ & $\mathrm{R} 2$ & $\mathrm{R} 3$ & $\mathrm{R} 4$ & $\mathrm{R} 5$ & $\mathrm{R} 6$ & $\mathrm{R} 7$ & $\mathrm{R} 8$ & $\mathrm{R} 9$ & $\mathrm{R} 10$ & $\mathrm{R} 11$ & $\mathrm{R} 12$ \\
\hline 1 & 1 & 2 & 0 & 0 & 2 & 0 & 0 & 0 & 2 & 2 & 2 & 0 & 2 \\
1 & 2 & 0 & 2 & 2 & 0 & 2 & 2 & 2 & 0 & 0 & 0 & 2 & 0 \\
\hline 2 & 3 & 2 & 2 & 0 & 0 & 2 & 0 & 0 & 0 & 2 & 2 & 2 & 0 \\
2 & 4 & 0 & 0 & 2 & 2 & 0 & 2 & 2 & 2 & 0 & 0 & 0 & 2 \\
\hline 3 & 5 & 2 & 0 & 2 & 0 & 0 & 2 & 0 & 0 & 0 & 2 & 2 & 2 \\
3 & 6 & 0 & 2 & 0 & 2 & 2 & 0 & 2 & 2 & 2 & 0 & 0 & 0 \\
\hline 4 & 7 & 2 & 2 & 0 & 2 & 0 & 0 & 2 & 0 & 0 & 0 & 2 & 2 \\
4 & 8 & 0 & 0 & 2 & 0 & 2 & 2 & 0 & 2 & 2 & 2 & 0 & 0 \\
\hline 5 & 9 & 2 & 2 & 2 & 0 & 2 & 0 & 0 & 2 & 0 & 0 & 0 & 2 \\
5 & 10 & 0 & 0 & 0 & 2 & 0 & 2 & 2 & 0 & 2 & 2 & 2 & 0 \\
\hline 6 & 11 & 2 & 2 & 2 & 2 & 0 & 2 & 0 & 0 & 2 & 0 & 0 & 0 \\
6 & 12 & 0 & 0 & 0 & 0 & 2 & 0 & 2 & 2 & 0 & 2 & 2 & 2 \\
\hline 7 & 13 & 2 & 0 & 2 & 2 & 2 & 0 & 2 & 0 & 0 & 2 & 0 & 0 \\
7 & 14 & 0 & 2 & 0 & 0 & 0 & 2 & 0 & 2 & 2 & 0 & 2 & 2 \\
\hline 8 & 15 & 2 & 0 & 0 & 2 & 2 & 2 & 0 & 2 & 0 & 0 & 2 & 0 \\
8 & 16 & 0 & 2 & 2 & 0 & 0 & 0 & 2 & 0 & 2 & 2 & 0 & 2 \\
\hline 9 & 17 & 2 & 0 & 0 & 0 & 2 & 2 & 2 & 0 & 2 & 0 & 0 & 2 \\
9 & 18 & 0 & 2 & 2 & 2 & 0 & 0 & 0 & 2 & 0 & 2 & 2 & 0 \\
\hline 10 & 19 & 2 & 2 & 0 & 0 & 0 & 2 & 2 & 2 & 0 & 2 & 0 & 0 \\
10 & 20 & 0 & 0 & 2 & 2 & 2 & 0 & 0 & 0 & 2 & 0 & 2 & 2 \\
\hline
\end{tabular}


The statistic of interest is again computed based for the whole sample and then again for each replicate. The replicate estimates are then compared with the whole sample estimate to estimate the sampling variance, as follows:

$$
\sigma_{(\hat{\theta})}^{2}=\frac{1}{G} \sum_{i=1}^{G}\left(\hat{\theta}_{(i)}-\hat{\theta}\right)^{2}
$$

With this replication method, each replicate sample only uses half of the available observations. This large reduction in sample might therefore become problematic for the estimation of a statistic on a rare subpopulation. Indeed, the number of remaining observations might be so small, even equal to 0 , that the estimation of the population parameter for a particular replicate sample is impossible. To overcome this disadvantage, Fay developed a variant to the BRR method. Instead of multiplying the school weights by a factor of 0 or 2, Fay suggested multiplying the weights by a deflating factor $k$ between 0 and 1 , with the second inflating factor being equal to 2 minus $k$. For instance, if the deflating weight factor, denoted $k$, is equal to 0.6 , then the inflating weight factor will be equal to 2- k, i.e. $1-0.6=1.4$ (Judkins, 1990).

PISA uses the Fay method with a factor of 0.5. Table 3.13 describes how the replicate samples and weights are generated for this method.

Table 3.13 - The Fay replicates

\begin{tabular}{c|c|c|c|c|c|c|c|c|c|c|c|c|c}
\hline $\begin{array}{c}\text { Pseudo- } \\
\text { stratum }\end{array}$ & School & R1 & R2 & R3 & R4 & R5 & R6 & R7 & R8 & R9 & R 10 & R 11 & R 12 \\
\hline 1 & 1 & 1.5 & 0.5 & 0.5 & 1.5 & 0.5 & 0.5 & 0.5 & 1.5 & 1.5 & 1.5 & 0.5 & 1.5 \\
1 & 2 & 0.5 & 1.5 & 1.5 & 0.5 & 1.5 & 1.5 & 1.5 & 0.5 & 0.5 & 0.5 & 1.5 & 0.5 \\
\hline 2 & 3 & 1.5 & 1.5 & 0.5 & 0.5 & 1.5 & 0.5 & 0.5 & 0.5 & 1.5 & 1.5 & 1.5 & 0.5 \\
2 & 4 & 0.5 & 0.5 & 1.5 & 1.5 & 0.5 & 1.5 & 1.5 & 1.5 & 0.5 & 0.5 & 0.5 & 1.5 \\
\hline 3 & 5 & 1.5 & 0.5 & 1.5 & 0.5 & 0.5 & 1.5 & 0.5 & 0.5 & 0.5 & 1.5 & 1.5 & 1.5 \\
3 & 6 & 0.5 & 1.5 & 0.5 & 1.5 & 1.5 & 0.5 & 1.5 & 1.5 & 1.5 & 0.5 & 0.5 & 0.5 \\
\hline 4 & 7 & 1.5 & 1.5 & 0.5 & 1.5 & 0.5 & 0.5 & 1.5 & 0.5 & 0.5 & 0.5 & 1.5 & 1.5 \\
4 & 8 & 0.5 & 0.5 & 1.5 & 0.5 & 1.5 & 1.5 & 0.5 & 1.5 & 1.5 & 1.5 & 0.5 & 0.5 \\
\hline 5 & 9 & 1.5 & 1.5 & 1.5 & 0.5 & 1.5 & 0.5 & 0.5 & 1.5 & 0.5 & 0.5 & 0.5 & 1.5 \\
5 & 10 & 0.5 & 0.5 & 0.5 & 1.5 & 0.5 & 1.5 & 1.5 & 0.5 & 1.5 & 1.5 & 1.5 & 0.5 \\
\hline 6 & 11 & 1.5 & 1.5 & 1.5 & 1.5 & 0.5 & 1.5 & 0.5 & 0.5 & 1.5 & 0.5 & 0.5 & 0.5 \\
6 & 12 & 0.5 & 0.5 & 0.5 & 0.5 & 1.5 & 0.5 & 1.5 & 1.5 & 0.5 & 1.5 & 1.5 & 1.5 \\
\hline 7 & 13 & 1.5 & 0.5 & 1.5 & 1.5 & 1.5 & 0.5 & 1.5 & 0.5 & 0.5 & 1.5 & 0.5 & 0.5 \\
7 & 14 & 0.5 & 1.5 & 0.5 & 0.5 & 0.5 & 1.5 & 0.5 & 1.5 & 1.5 & 0.5 & 1.5 & 1.5 \\
\hline 8 & 15 & 1.5 & 0.5 & 0.5 & 1.5 & 1.5 & 1.5 & 0.5 & 1.5 & 0.5 & 0.5 & 1.5 & 0.5 \\
8 & 16 & 0.5 & 1.5 & 1.5 & 0.5 & 0.5 & 0.5 & 1.5 & 0.5 & 1.5 & 1.5 & 0.5 & 1.5 \\
\hline 9 & 17 & 1.5 & 0.5 & 0.5 & 0.5 & 1.5 & 1.5 & 1.5 & 0.5 & 1.5 & 0.5 & 0.5 & 1.5 \\
9 & 18 & 0.5 & 1.5 & 1.5 & 1.5 & 0.5 & 0.5 & 0.5 & 1.5 & 0.5 & 1.5 & 1.5 & 0.5 \\
\hline 10 & 19 & 1.5 & 1.5 & 0.5 & 0.5 & 0.5 & 1.5 & 1.5 & 1.5 & 0.5 & 1.5 & 0.5 & 0.5 \\
10 & 20 & 0.5 & 0.5 & 1.5 & 1.5 & 1.5 & 0.5 & 0.5 & 0.5 & 1.5 & 0.5 & 1.5 & 1.5 \\
\hline
\end{tabular}

As with all replication methods, the statistic of interest is computed on the whole sample and then again on each replicate. The replicate estimates are then compared to the whole sample estimate to get the sampling variance, as follows:

$$
\sigma_{(\hat{\theta})}^{2}=\frac{1}{G(1-k)^{2}} \sum_{i=1}^{G}\left(\hat{\theta}_{(i)}-\hat{\theta}\right)^{2}
$$


In PISA, it was decided to generate 80 replicate samples and therefore 80 replicate weights. Therefore, the formula becomes:

$$
\sigma_{(\hat{\theta})}^{2}=\frac{1}{G(1-k)^{2}} \sum_{i=1}^{G}\left(\hat{\theta}_{(i)}-\hat{\theta}\right)^{2}=\frac{1}{80(1-0.5)^{2}} \sum_{i=1}^{80}\left(\hat{\theta}_{(i)}-\hat{\theta}\right)^{2}=\frac{1}{20} \sum_{i=1}^{80}\left(\hat{\theta}_{(i)}-\hat{\theta}\right)^{2}
$$

\section{OTHER PROCEDURES FOR ACCOUNTING FOR CLUSTERED SAMPLES}

For the past two decades, multi-level models and software packages have been introduced in the education research field. There is no doubt that these models allowed a break through in the unraveling of education phenomena. Indeed, multi-level regression models offer the possibility of taking into account the fact that students are nested within classes and schools: each contributing factor can be evaluated when establishing the outcome measure.

Multi-level regression software packages, such as MLWin or HLM, just like any professional statistical package, provide an estimate of the standard error for each of the estimated population parameters. While SAS ${ }^{\circledR}$ and SPSS ${ }^{\circledR}$ consider the sample as a simple random sample of population elements, MLWin and HLM recognize the hierarchical structure of the data, but consider that the school sample is a simple random one. They therefore do not take into account the complementary sample design information used in PISA to reduce the sampling variance. Consequently, in PISA, the sampling variances estimated with multi-level models will always be greater than the sampling variances estimated with Fay replicate samples.

As these multi-level model packages do not incorporate the additional sample design information, their standard error estimates are similar to the Jackknife method for unstratified samples. For instance, the German PISA 2003 data were analyzed using the multi-level model proposed by SAS ${ }^{\circledR}$ and called PROC MIXED. The standard errors of the mean of the five plausible values ${ }^{2}$ for the combined reading literacy scale were respectively 5.4565, 5.3900, 5.3911, 5.4692, and 5.3461. The average of these five standard errors is equal to 5.41. Recall that the use of the formula in section 2 of this chapter produces an estimate of the sampling variance equal to 5.45 .

With multi-level software packages, using replicates cannot be avoided if unbiased estimates of the standard errors for the estimates want to be obtained.

\section{CONCLUSIONS}

Since international education surveys use a two-stage sample design most of the time, it would be inappropriate to apply the sampling distribution formulas developed for simple random sampling. Doing so would lead to an underestimation of the sampling variances.

Sampling designs in education surveys can be very intricate. As a result, sampling distributions might not be available or too complex even for simple estimators, such as means. Since the 1990 IEA reading literacy study, sampling variances have been estimated through replication methods. These methods function by generating several subsamples, or replicate samples, from the whole sample. The statistic of interest is then estimated for each of these replicate samples and then compared to the whole sample estimate to provide an estimate of the sampling variance.

A replicate sample is formed simply through a transformation of the full sample weights according to an algorithm specific to the replication method. These methods therefore can be applied to any 
estimators ${ }^{3}$ - means, medians, percentiles, correlations, regression coefficients, etc. - which can be easily computed thanks to advanced computing resources. Further, using these replicate weights does not require an extensive knowledge in statistics, since these procedures can be applied regardless of the statistic of interest.

1. See reasons for this decision in the PISA 2000 Technical Report (OECD, 2002c).

2. See Chapter 4 for a description of plausible values.

3. Several empirical or theoretical studies have compared the different resampling methods for complex sampling design. As Rust and Krawchuk noted: "A benefit of both BRR and modified BRR over the Jackknife is that they have a sound theoretical basis for use with nonsmooth statistics, such as quantiles like the median. It has long been known that the Jackknife is inconsistent for estimating the variances of quantiles. That is, as the sample size increases for a given sample design, the estimation of the variances of quantiles does not necessarily become more precise when using the Jackknife." (Rust and Krawchuk, 2002). 


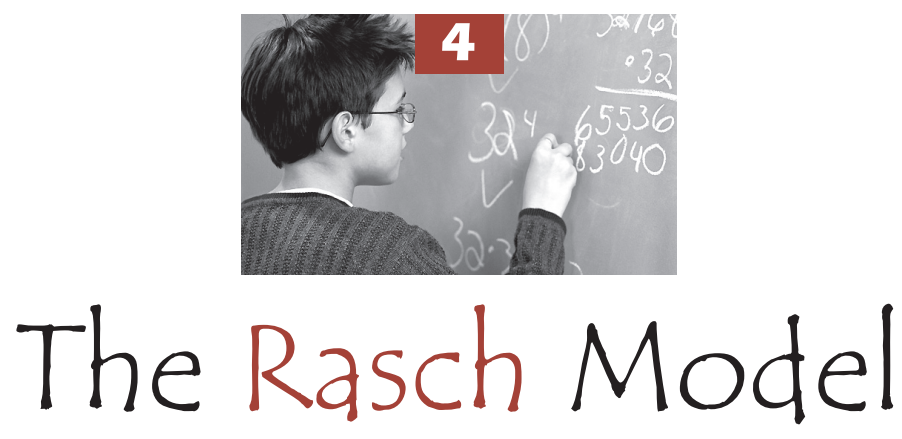

Introduction

How can the information be summarised? …………………........ 54

The Rasch model for dichotomous items ....................................... 56

Other Item Response Theory models .......................................... 69

Conclusions 69 


\section{INTRODUCTION}

International surveys in education such as PISA are designed to estimate the performance in particular subject areas of various subgroups of students, at specific age or grade levels.

For the surveys to be considered valid, many items need to be developed and included in the final tests. The OECD publications related to the assessment frameworks indicate the breadth and depth of the PISA domains, showing that many items are needed to assess a domain as broadly defined as, for example, mathematical literacy.

At the same time, it is unreasonable and perhaps undesirable to assess each sampled student with the whole item battery because:

- After extended testing time, students' results start to be affected by fatigue and this would therefore bias the outcomes of the surveys; and

- School principals would refuse to free their students for the very long testing period that would be required. This would reduce the school participation rate, which in turn might substantially bias the outcomes of the results.

To overcome the conflicting demands of limited student-level testing time and broad coverage of the assessment domain, students are assigned a subset of the item pool. The result of this is that only certain sub-samples of students respond to each item.

If the survey purpose is to estimate performance by reporting the percentage of correct answers for each item, it would not be necessary to report the performance of individual students. However, typically there is a need to summarise detailed item level information for communicating the outcomes of the survey to the research community, to the public and also to policy makers. In addition, educational surveys aim to explain the difference in results between countries, between schools and between students. For instance, a researcher might be interested in the difference in performance between boys and girls.

\section{HOW CAN THE INFORMATION BE SUMMARISED?}

At the country level, the most straightforward procedure for summarizing the item-level information would be to compute the average percentage of correct answers. This has been largely used in previous national or international surveys and is still used in some current international surveys, even when more complex models are implemented. These surveys may report the overall percentage of correct answers in mathematics and in science, as well as by content areas (for example, biology, physics, chemistry, earth sciences and so on). For instance, in mathematics, the overall percentage of correct answers for one country might be 54 per cent and for another, 65 per cent.

The great advantage of this type of reporting is that it can be understood by everyone. Everybody can imagine a mathematics test and can envision what is represented by 54 per cent and 65 per cent of correct answers. These two numbers also give a sense of the difference between the two countries.

Nevertheless, there are some weaknesses in this approach because the percentage of correct answers depends on the difficulty of the test. The actual size of the difference in results between two countries depends on the difficulty of the test and this may lead to misinterpretation. 
International surveys do not aim to just report an overall level of performance. Over the past few decades, policy makers have also largely been interested in equity indicators. They may also be interested in the amount of dispersion of results in their country. In some countries the results may be clustered around the mean and in other countries there may be large numbers of students scoring very high results and very low results.

It would be impossible to compute dispersion indices with only the difficulty indices, based on percentage of correct answers of all the items. To do so, the information collected through the test needs also to be summarised at the student level.

To compare the results of two students assessed by two different tests, the tests must have exactly the same average difficulty. For PISA, as all items included in the main study are usually field trialled, test developers have some idea of the item difficulties and therefore can allocate the items to the different tests in such a way that the items in each test have more or less the same average difficulty. However, the two tests will never have exactly the same difficulty.

The distribution of the item difficulties will affect the distribution of the students' performance expressed as a raw score. For instance, a test with only items of medium difficulty will generate a different student score distribution to a test that consists of a large range of item difficulties.

This is also complicated to a further degree in PISA as it assesses three or even four domains per cycle. This multiple assessment reduces the number of items available for each domain per test and it is easier to guarantee the comparability of two tests of 60 items than it is with, for example, 15 items.

If the different tests are randomly assigned to students, then the equality of the sub-populations in terms of mean score and variance of the student's performance can be assumed. In other words,

- The mean of the raw score should be identical for the different tests; and

- The variance of the student raw scores should be identical for the different tests.

If this is not the case, then it would mean that the different tests do not have exactly the same psychometric properties. To overcome this problem of comparability of the student performance between tests, the student's raw scores can be standardised per test. As the equality of the subpopulations can be assumed, differences in the results are due to differences in the test characteristics. The standardisation would then neutralise the effect of test differences on student's performance.

However, usually, only a sample of students from the different sub-populations is tested. As explained in the two previous chapters, this sampling process generates an uncertainty around any population estimates. Therefore, even if different tests present exactly the same psychometric properties and are randomly assigned, the mean and standard deviation of the students' performance between the different tests can slightly differ. As the test characteristics and the sampling variability are confounded, the assumption cannot be made that the student raw scores obtained with different tests are fully comparable.

Other psychometric arguments can also be invoked against the use of raw scores based on the percentage of correct answers to assess student performance. Raw scores are on a ratio scale in so far as the interpretation of the results is limited to the number of correct answers. A student who 
gets a 0 on this scale did not provide any correct answers, but could not be considered as having no competencies, while a student who gets 10 has twice the number of correct answers as a student who gets 5, but does not necessarily, have twice the competencies. Similarly, a student with a perfect score could not be considered as having all competencies (Wright and Stone, 1979).

\section{THE RASCH MODEL FOR DICHOTOMOUS ITEMS}

\section{Introduction}

Let us suppose that someone wants to estimate the competence of a high jumper. It might be measured or expressed as his or her:

- Individual record;

- Individual record during an official and international event;

- Mean performance during a particular period of time; or

- Most frequent performance during a particular period of time.

Figure 4.1 presents the proportion of success of two high jumpers per height for the last year of competition.

Figure 4.1 Proportion of success per height of the jump

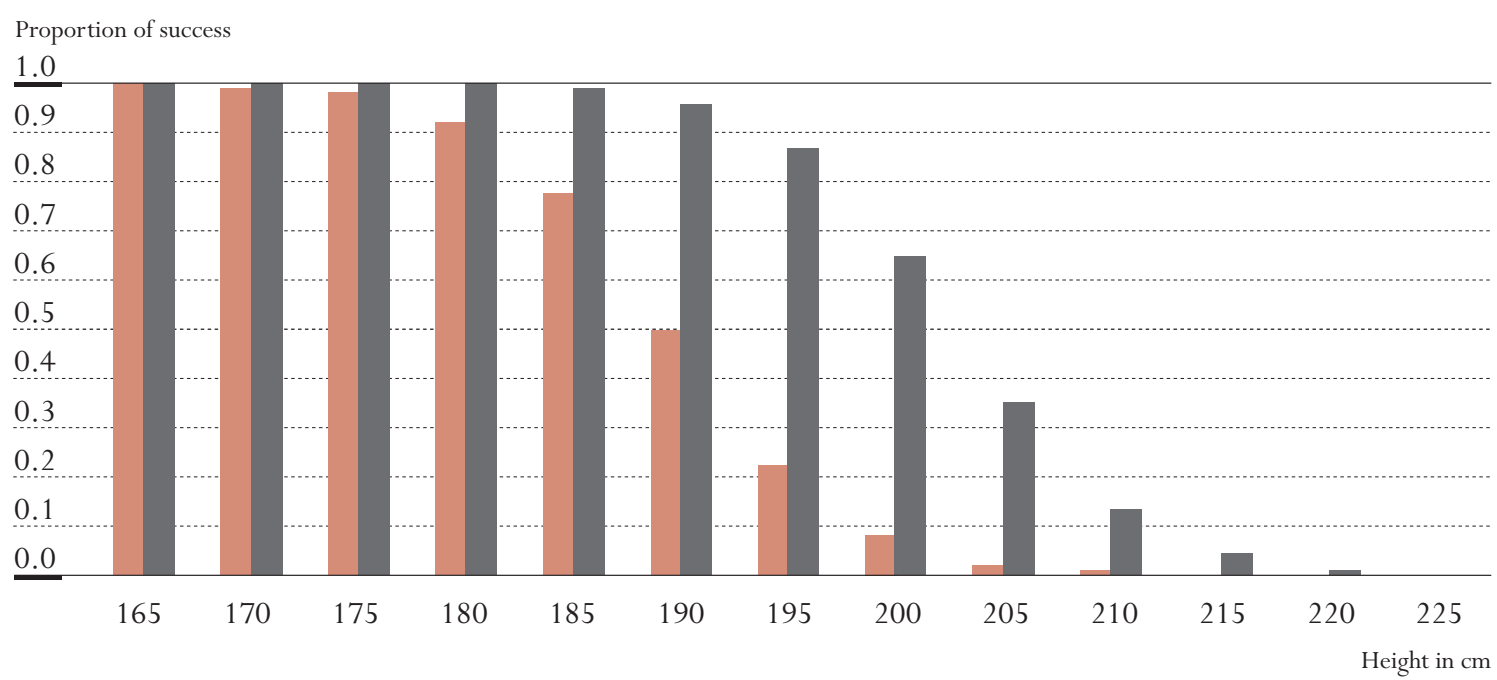

The two high jumpers always succeeded at 165 centimetres. Then the proportion of success progressively decreases to reach 0 for both at 225 centimetres. While it starts to decrease at 170 for the first high jumper, however, it starts to decrease at 185 for the second.

These data can be depicted by a logistic regression model. This statistical analysis consists of explaining a dichotomous variable by a continuous variable. In this example, the continuous variable will explain the success or the failure of a particular jumper by the height of the jump. The outcome of this analysis will allow the estimation of the probability of success, given any height. Figure 4.2 presents the probability of success for the two high jumpers. 


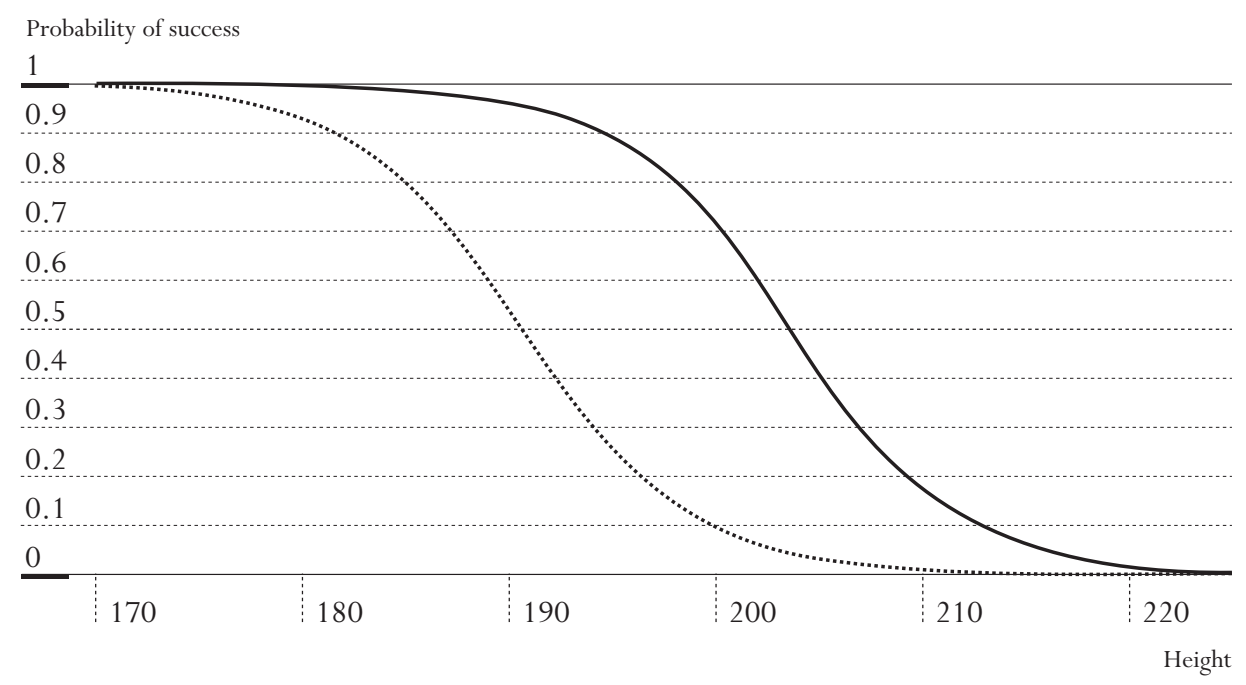

These two functions model the probability of success for the two high jumpers. The dotted curve represents the probability of success for the first high jumper and the solid curve, the probability of success for the second high jumper.

By convention, ${ }^{2}$ the performance level would be defined as the height where the probability of success is equal to 0.50 . This makes sense as below that level, the probability of success is lower than the probability of failure and beyond that level, this is the inverse.

In this particular example, the performance of the two high jumpers is respectively 190 and 202.5. Note that from Figure 4.1, the performance of the first jumper is directly observable whereas for jumper 2, it is not and needs to be estimated from the model. A key property of this kind of approach is that the level (i.e. the height) of the crossbar and the performance of the high jumpers are expressed on the same metric or scale.

Scaling cognitive data according to the Rasch model follows the same principle. The difficulty of the items is analogous to the difficulty of the jump based on the height of the crossbar. Further, just as a particular jump has two possible outcomes, i.e. success or failure, the answer of a student to a particular question is either correct or incorrect. Finally, just as each jumper's performance was defined at the point where the probability of success was 0.5 , the student's performance/ability is likewise measured where the probability of success on an item equals 0.5 .

A feature of the Rasch model is that it will create a continuum on which both student performance and item difficulty will be located and a probabilistic function links these two components. Low ability students and easy items will be located on the left side of the continuum or scale while high ability students and difficult items will be located on the right side of the continuum. Figure 4.3 represents the probability of success (dotted curve) and the probability of failure (solid curve) for an item of difficulty zero. 


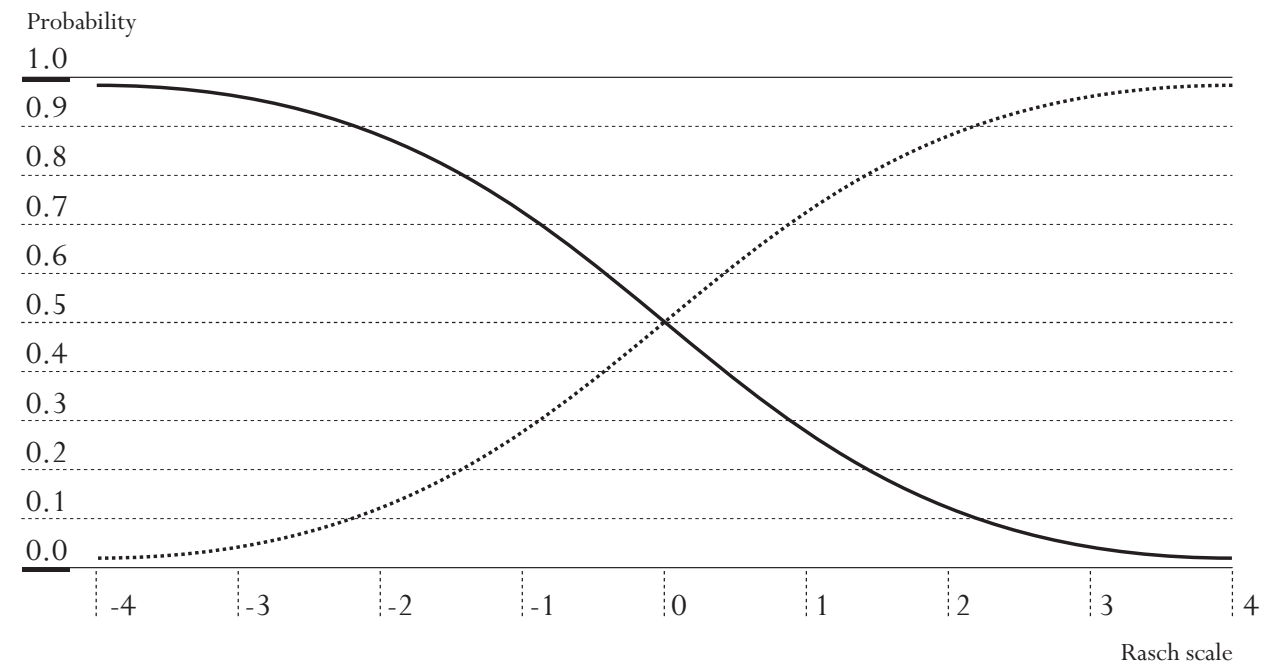

As shown by Figure 4.3, a student with an ability of zero has a probability of 0.5 of success on an item of difficulty zero and a probability of 0.5 of failure. A student with an ability of -2 has a probability of a bit more than 0.10 of success and a probability of a bit less than 0.90 of failure on the same item of difficulty zero. But this student will have a probability of 0.5 of succeeding on an item of difficulty -2 .

From a mathematical point of view, the probability that a student $i$, with an ability denoted $\beta_{i}$, provides a correct answer to item $j$ of difficulty $\delta_{j}$ is equal to:

$$
P\left(X_{i j}=1 \mid \beta_{i}, \delta_{j}\right)=\frac{\exp \left(\beta_{i}-\delta_{j}\right)}{1+\exp \left(\boldsymbol{\beta}_{i}-\delta_{j}\right)}
$$

Similarly, the probability of failure is equal to:

$$
P\left(X_{i j}=0 \mid \boldsymbol{\beta}_{\mathrm{i}}, \boldsymbol{\delta}_{j}\right)=\frac{1}{1+\exp \left(\beta_{i}-\delta_{j}\right)}
$$

It can be easily shown that:

$$
P\left(X_{i j}=1 \mid \beta_{\mathrm{i}}, \delta_{j}\right)+P\left(X_{i j}=0 \mid \boldsymbol{\beta}_{i}, \delta_{j}\right)=1
$$

In other words, the probability of success and the probability of failure always sum to one. Table 4.1 to Table 4.5 present the probability of success for different student abilities and different item difficulties.

Table 4.1 — Probability of success when student ability equals item difficulty

\begin{tabular}{c|c|c}
\hline Student ability & Item difficulty & Probability of success \\
\hline-2 & -2 & 0.50 \\
-1 & -1 & 0.50 \\
0 & 0 & 0.50 \\
1 & 1 & 0.50 \\
2 & 2 & 0.50 \\
\hline
\end{tabular}


Table 4.2 - Probability of success when student ability is less than the item difficulty by 1 unit

\begin{tabular}{c|c|c}
\hline Student ability & Item difficulty & Probability of success \\
\hline-2 & -1 & 0.27 \\
-1 & 0 & 0.27 \\
0 & 1 & 0.27 \\
1 & 2 & 0.27 \\
2 & 3 & 0.27 \\
\hline
\end{tabular}

Table 4.3 - Probability of success when student ability is greater than the item difficulty by 1 unit

\begin{tabular}{c|c|c}
\hline Student ability & Item difficulty & Probability of success \\
\hline-2 & -3 & 0.73 \\
-1 & -2 & 0.73 \\
0 & -1 & 0.73 \\
1 & 0 & 0.73 \\
2 & 3 & 0.73 \\
\hline
\end{tabular}

Table 4.4 - Probability of success when student ability is less than the item difficulty by 2 units

\begin{tabular}{c|c|c}
\hline Student ability & Item difficulty & Probability of success \\
\hline-2 & 0 & 0.12 \\
-1 & 1 & 0.12 \\
0 & 2 & 0.12 \\
1 & 3 & 0.12 \\
2 & 4 & 0.12 \\
\hline
\end{tabular}

Table 4.5 Probability of success when student ability is greater than the item difficulty by 2 units

\begin{tabular}{c|c|c}
\hline Student ability & Item difficulty & Probability of success \\
\hline-2 & -4 & 0.88 \\
-1 & -3 & 0.88 \\
0 & -2 & 0.88 \\
1 & -1 & 0.88 \\
2 & 0 & 0.88 \\
\hline
\end{tabular}

It should be noted that:

- When the student ability is equal to the item difficulty, the probability of success will always be equal to 0.50 , regardless of the student ability and item difficulty locations on the continuum.

- If the item difficulty exceeds the student ability by one Rasch unit, denoted as a logit, then the probability of success will always be equal to 0.27 , regardless of the location of the student ability on the continuum. 
- If the student ability exceeds the item difficulty by one logit, the probability of success will always be equal to 0.73 , regardless of the location of the student ability on the continuum.

- If two units separate the student ability and the item difficulty, the probabilities of success will be 0.12 and 0.88 respectively.

From these observations, it is evident that the only factor that influences the probability of success is the distance on the Rasch continuum between the student ability and the item difficulty.

These examples also illustrate the symmetry of the scale. If the student ability is lower than the item difficulty by one logit, then the probability of success will be 0.27 which is 0.23 lower than the probability of success when ability and difficulty are equal. If the student ability is higher than the item difficulty by one logit, the probability of success will be 0.73 , which is 0.23 higher than the probability of success when ability and difficulty are equal. Similarly, a difference of two logits generates a change of 0.38 .

\section{Item calibration}

Of course, in real settings a student's answer will either be correct or incorrect, so what then is the meaning of a probability of 0.5 of success in terms of correct or incorrect answers? In simple terms the following interpretations can be made:

- If 100 students each having an ability of 0 have to answer a item of difficulty 0 , then the model will predict 50 correct answers and 50 incorrect answers;

- If a student with an ability of 0 has to answer 100 items, all of difficulty 0 , then the model will predict 50 correct answers and 50 incorrect answers.

As described, the Rasch model, through a probabilistic function, builds a relative continuum on which the item's difficulty and the student's ability are located. With the example of high jumpers, the continuum already exists, i.e. this is the physical continuum of the meter height. With cognitive data, the continuum has to be built. By analogy, this consists of building a continuum on which the unknown height of the crossbars, i.e. the difficulty of the items, will be located. Three major principles underlie the construction of the Rasch continuum.

- The relative difficulty of an item results from the comparison of that item with all other items. Let us suppose that a test consists of only two items. Intuitively, the response pattern $(0,0)$ and $(1,1)$ ( 1 denotes a success and 0 denotes a failure), where the ordered pairs refer to the responses to items 1 and 2, respectively, is uninformative for comparing the two items. The responses in these patterns are identical. On the other hand, responses $(1,0)$ and $(0,1)$ are different and are informative on just that comparison. If 50 students have the $(0,1)$ response pattern and only 10 students have the $(1,0)$ response pattern, then the second item is substantially easier than the first item. Indeed, 50 students succeeded on the second item while failing the first one and only 10 students succeeded on the first item while failing the second. This means that if one person succeeds on one of these two items, the probability of succeeding on the second item is five times higher than the probability of succeeding on first item. It is, therefore, easier to succeed on the second than it is to succeed on the first. Note that the relative difficulty of the two items is independent of the student abilities. 
- As difficulties are determined through comparison of items, this creates a relative scale, and therefore there is an infinite number of scale points. Broadly speaking, the process of overcoming this issue is comparable to the need to create anchor points on the temperature scales. For example, Celsius fixed two reference points: the temperature at which the water freezes and the temperature at which water boils. He labelled the first reference point as 0 and the second reference point at 100 and consequently defined the measurement unit as one-hundredth of the distance between the two reference points. In the case of the Rasch model, the measurement unit is defined by the probabilistic function involving the item difficulty and student ability parameters. Therefore, only one reference point has to be defined. The most common reference point consists of centring the item difficulties on zero. However, other arbitrary reference points can be used, like centring the student's abilities on zero.

- This continuum allows the computation of the relative difficulty of items partly submitted to different sub-populations. Let us suppose that the first, item was administered to all students and the second item was only administered to the low ability students. The comparison of items will only be performed on the subpopulation who was administered both items, i.e. the low ability student population. The relative difficulty of the two items will be based on this common subset of students.

Once the item difficulties have been placed on the Rasch continuum, the student scores can be computed. The line in Figure 4.4 represents a Rasch continuum. The item difficulties are located above that line and the item numbers are located below the line. For instance, item 7 represents a difficult item and item 17, an easy item. This test includes a few easy items, a large number of medium difficulty items and a few difficult items. The $x$ symbols above the line represent the distribution of the student scores.

\section{Figure 4.4 - Student score and item difficulty distributions on a Rasch continuum}

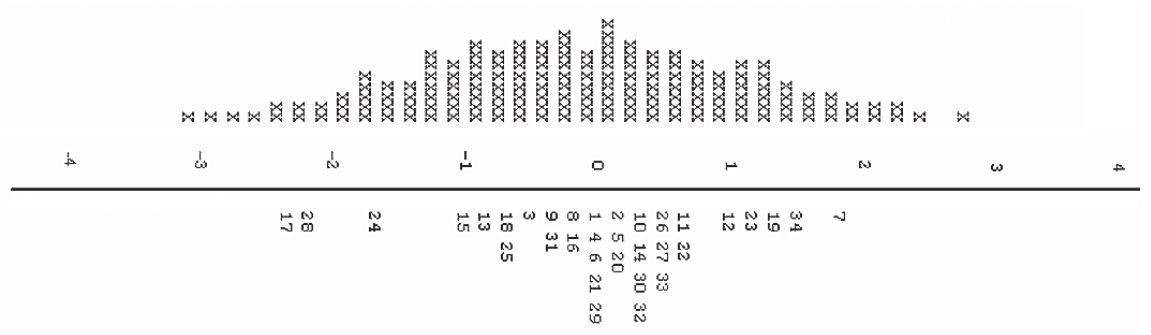

\section{Computation of a student's score}

Once the item difficulties have been located on the Rasch scale, student scores can be computed. In a previous section, it was mentioned that the probability that a student $i$, with an ability denoted $\beta_{i}$, provides a correct answer to item $j$ of difficulty $\delta_{j}$ is equal to:

$$
P\left(X_{i j}=1 \mid \beta_{i}, \delta_{j}\right)=\frac{\exp \left(\beta_{i}-\delta_{j}\right)}{1+\exp \left(\boldsymbol{\beta}_{i}-\delta_{j}\right)}
$$

Similarly, the probability of failure is equal to:

$$
P\left(X_{i j}=0 \mid \boldsymbol{\beta}_{i}, \boldsymbol{\delta}_{j}\right)=\frac{1}{1+\exp \left(\beta_{i}-\delta_{j}\right)}
$$


The Rasch model assumes the independence of the items, i.e. the probability of a correct answer does not depend on the responses given to the other items. Consequently, the probability of succeeding on two items is equal to the product of the two individual probabilities of success.

Let us consider a test of four items with the following items difficulties: $-1,-0.5,0.5$ and 1 . There are 16 possible responses patterns. These 16 patterns are presented in Table 4.6.

\section{Table 4.6 - Possible response patterns for a test of four items}

\begin{tabular}{c|l}
\hline Raw score & Response patterns \\
\hline 0 & $(0,0,0,0)$ \\
1 & $(1,0,0,0),(0,1,0,0),(0,0,1,0),(0,0,0,1)$ \\
2 & $(1,1,0,0),(1,0,1,0),(1,0,0,1),(0,1,1,0),(0,1,0,1),(0,0,1,1)$ \\
3 & $(1,1,1,0),(1,1,0,1),(1,0,1,1),(0,1,1,1)$ \\
4 & $(1,1,1,1)$ \\
\hline
\end{tabular}

For any student ability denoted $\beta_{i}$, it is possible to compute the probability of any response pattern. Let us compute the probability of the response pattern $(1,1,0,0)$ for three students with an ability of $-1,0$, and 1 .

Table 4.7 Probability for the response pattern $(\mathbf{1 , 1 , 0 , 0 )}$ for three student abilities

\begin{tabular}{l|l|l|c|c|c}
\hline & & & $\beta_{i}=-1$ & $\beta_{i}=0$ & $\beta_{i}=1$ \\
\hline Item 1 & $\delta_{1}=-1$ & Response $=1$ & 0.50 & 0.73 & 0.88 \\
\hline Item 2 & $\delta_{2}=-0.5$ & Response $=1$ & 0.38 & 0.62 & 0.82 \\
\hline Item 3 & $\delta_{3}=0.5$ & Response $=0$ & 0.82 & 0.62 & 0.38 \\
\hline Item 4 & $\delta_{4}=1$ & Response $=0$ & 0.88 & 0.73 & 0.50 \\
\hline
\end{tabular}

The probability of success for the first student on the first item is equal to:

$$
P\left(X_{i j}=1 \mid \beta_{i}, \delta_{j}\right)=P\left(X_{1,1}=1 \mid-1,-1\right) \frac{\exp (-1-(-1))}{1+\exp (-1-(-1))}=0.5
$$

The probability of success for the first student on the second item is equal to:

$$
P\left(X_{i j}=1 \mid \beta_{i}, \delta_{j}\right)=P\left(X_{1,2}=1 \mid-1,-0.5\right) \frac{\exp (-1-(0.5))}{1+\exp (-1-(-0.5))}=0.38
$$


The probability of failure for the first student on the third item is equal to:

$$
P\left(X_{i j}=0 \mid \beta_{i}, \delta_{j}\right)=P\left(X_{1,3}=0 \mid-1,0.5\right) \frac{1}{1+\exp (-1-0.5)}=0.82
$$

The probability of failure for the first student on the fourth item is equal to:

$$
P\left(X_{i j}=0 \mid \beta_{i}, \delta_{j}\right)=P\left(X_{1,4}=0 \mid-1,1\right) \frac{1}{1+\exp (-1-1)}=0.88
$$

As these four items are considered as independent, the probability of the response pattern $(1,1,0,0)$ for a student with an ability $\beta_{i}=-1$ is equal to:

$0.50 \times 0.38 \times 0.82 \times 0.88=0.14$

Given the item difficulties, a student with an ability $\beta_{i}=-1$ has 14 chances out of 100 to provide a correct answer to items 1 and 2 and to provide an incorrect answer to items 3 and 4. Similarly, a student with an ability of $\beta_{i}=0$ has a probability of 0.21 to provide the same response pattern and a student with an ability of $\beta_{i}=1$ has a probability of 0.14 .

Figure 4.5 Response pattern probabilities for the response pattern $(\mathbf{1 , 1 , 0 , 0 )}$

Response pattern probability

0.25

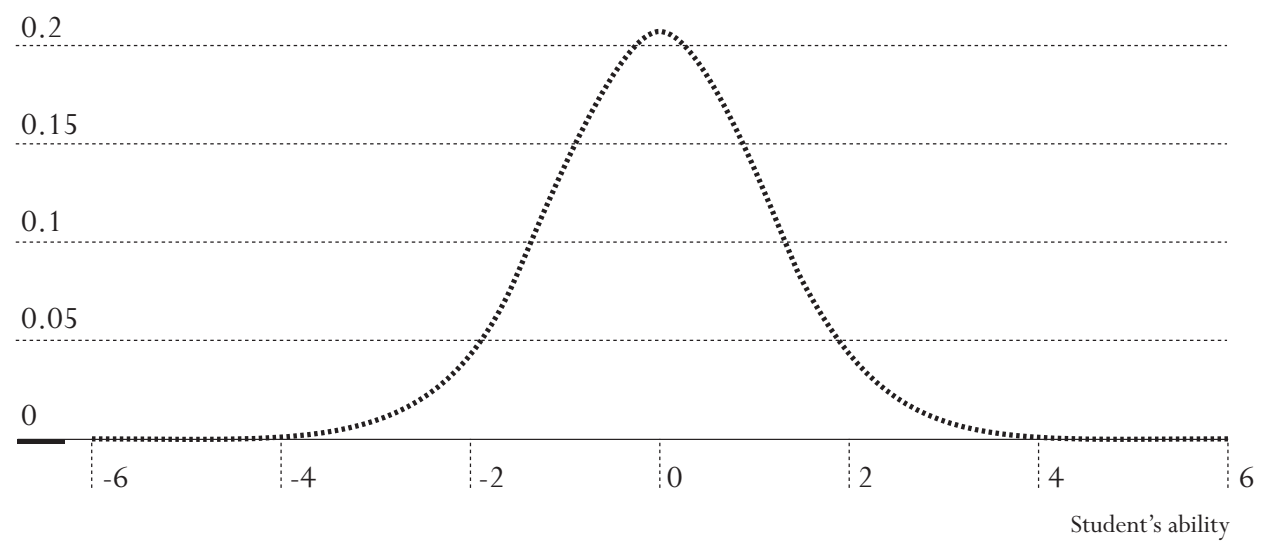

This process can be applied for a large range of student abilities and for all possible response patterns. Figure 4.5 presents the probability of observing the response pattern $(1,1,0,0)$ for all students' abilities between -6 and +6 . As shown, the most likely value corresponds to a student ability of 0 . Therefore, the Rasch model will estimate the ability of any students with a response pattern $(1,1,0,0)$ to 0 .

Figure 4.6 presents the distribution of the probabilities for all response patterns with only one correct item. As shown in Table 4.6, there are four responses patterns with only one correct item, i.e. $(1,0,0,0),(0,1,0,0),(0,0,1,0),(0,0,0,1)$. 


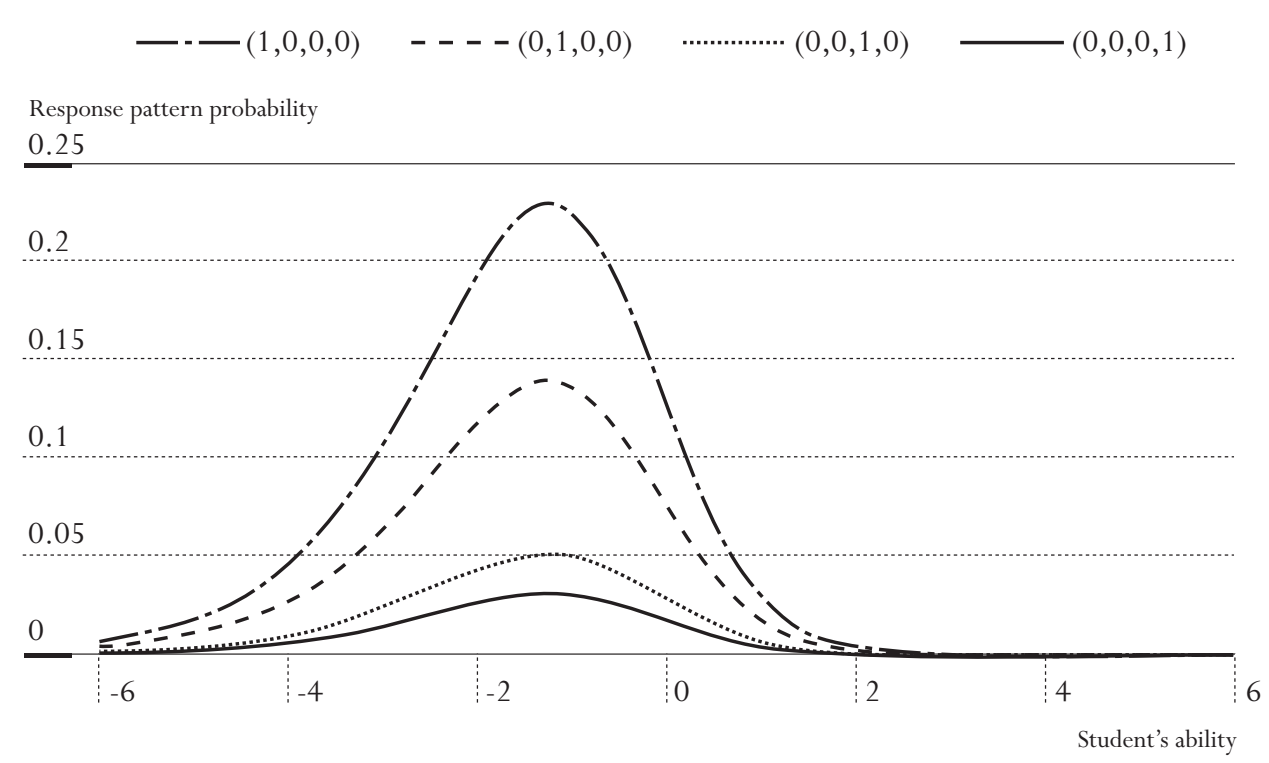

Figure 4.6 clearly shows that:

- The most likely response pattern for any students who succeed on only 1 item is $(1,0,0,0)$ and the most unlikely response pattern is $(0,0,0,1)$. When a student only provides one correct answer, it is expected that the correct answer was provided for the easiest item, i.e. item 1. It is also unexpected that this correct answer was provided for the most difficult item, i.e. item 4.

- Whatever the response pattern, the most likely value always corresponds to the same value for student ability. For instance, the most likely student ability for the response pattern $(1,0,0,0)$ is around -1.25 . This is also the most likely student's ability for the other response patterns.

The Rasch model will therefore return the value -1.25 for any students who get only one correct answer, whichever item was answered correctly.

Similarly, as shown by Figure 4.7 and by Figure 4.8:

- The most likely response pattern with two correct items is $(1,1,0,0)$;

- The most likely student's ability is always the same for any response pattern that includes two correct answers (0 in this case);

- The most likely response pattern with three correct items is $(1,1,1,0)$;

- The most likely student's ability is always the same for any response pattern that includes three correct answers $(+1.25$ in this case).

This type of Rasch ability estimate is usually denoted the Maximum Likelihood Estimate. As shown by these figures, per raw score, i.e. 0 correct answer, one correct answers, two correct answers, and so on, the Rasch model will return only one Maximum Likelihood Estimate. 


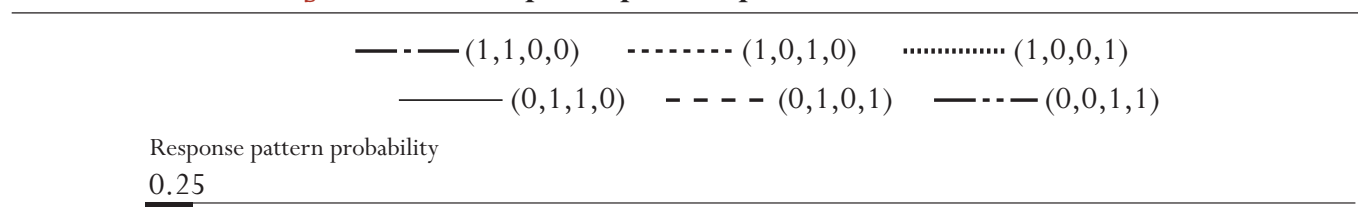

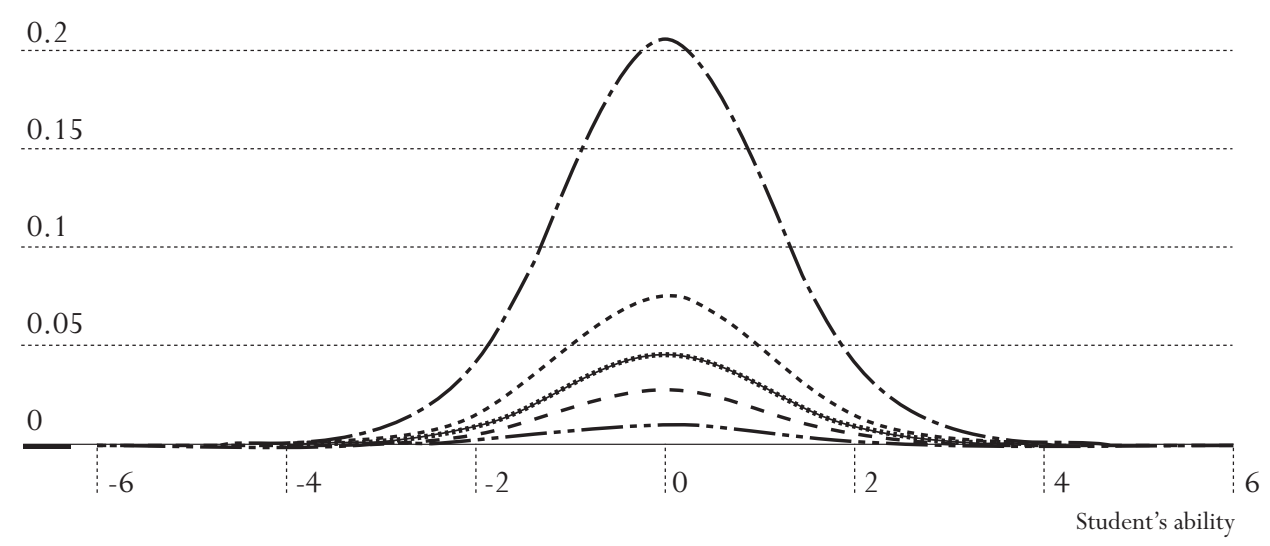

a. In this example, since the likelihood function for the response pattern $(1,0,0,1)$ is perfectly similar to that for response pattern $(0,1,1,0)$, these two lines are overlapped in the figure.

Figure 4.8 - Response pattern probabilities for a raw score of 3

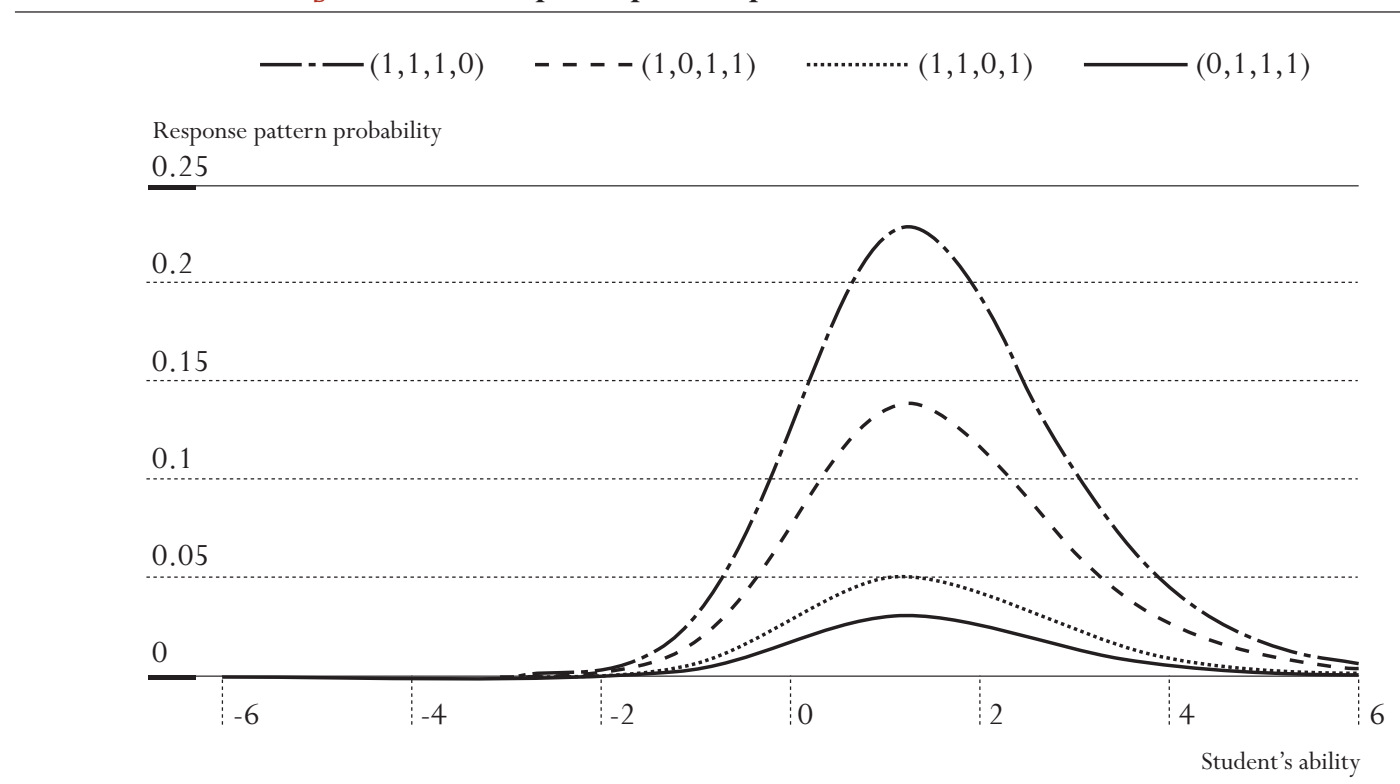

It has been shown that this Maximum Likelihood Estimate (or MLE) is biased and proposed to weight the contribution of each item by the information this item can provide (Warm, 1989). For instance, a difficult item does not provide much information for a low ability student. On the other hand, this item can provide more information for a high ability student. Therefore, for a low ability student, easy items will contribute more than difficult items and similarly, for a high ability student, difficult item will contribute more than easy items. So Warm estimates and MLEs are similar types of student individual ability estimates. 
As the Warm estimate corrects the small bias in the MLE, it is usually preferred as the estimate of an individual's ability. Therefore, in the PISA, Weighted Likelihood Estimates (WLEs) are calculated by applying weights to MLE in order to account for the bias inherent in MLE as Warm proposed.

\section{Computation of a student's score for incomplete designs}

As stated previously, PISA uses a rotated booklet design for overcoming the conflicting demands of limited student level testing time and the broad coverage of the assessment domain. A testing design where students are assigned a subset of items is denoted as an incomplete design. The principles for computing the student's individual ability estimate described in the previous section are still applicable for incomplete designs.

Let us suppose that two students with abilities of -1 and 1 have to answer two out of the four items presented in Table 4.8. The student with $\beta_{i}=-1$ has to answer the first two items, i.e. the two easiest items and the student with $\beta_{i}=1$ has to answer the last two items, i.e. the two most difficult items. Both students succeed on their first item and fail on their second item.

Table 4.8 Probability for the response pattern $(\mathbf{1 , 0 )}$ for two students of different ability in an incomplete test design

\begin{tabular}{l|l|l|c|c}
\hline \multicolumn{3}{l|}{} & $\beta_{i}=-1$ & $\beta_{i}=1$ \\
\hline Item 1 & $\delta_{1}=-1$ & Response $=1$ & 0.50 & \\
\hline Item 2 & $\delta_{2}=-0.5$ & Response $=0$ & 0.62 & \\
\hline Item 3 & $\delta_{3}=0.5$ & Response $=1$ & & 0.62 \\
\hline Item 4 & $\delta_{4}=1$ & Response $=0$ & & 0.50 \\
\hline
\end{tabular}

Both patterns have a probability of 0.31 respectively for an ability of -1 and 1 . As previously, these probabilities can be computed for a large range of student's abilities. Figure 4.9 presents the $(1,0)$ response pattern probabilities for the easy test (dotted line) and for the difficult test (solid line).

Figure 4.9 - Response pattern likelihood for an easy test and a difficult test

Response pattern probability

0.35

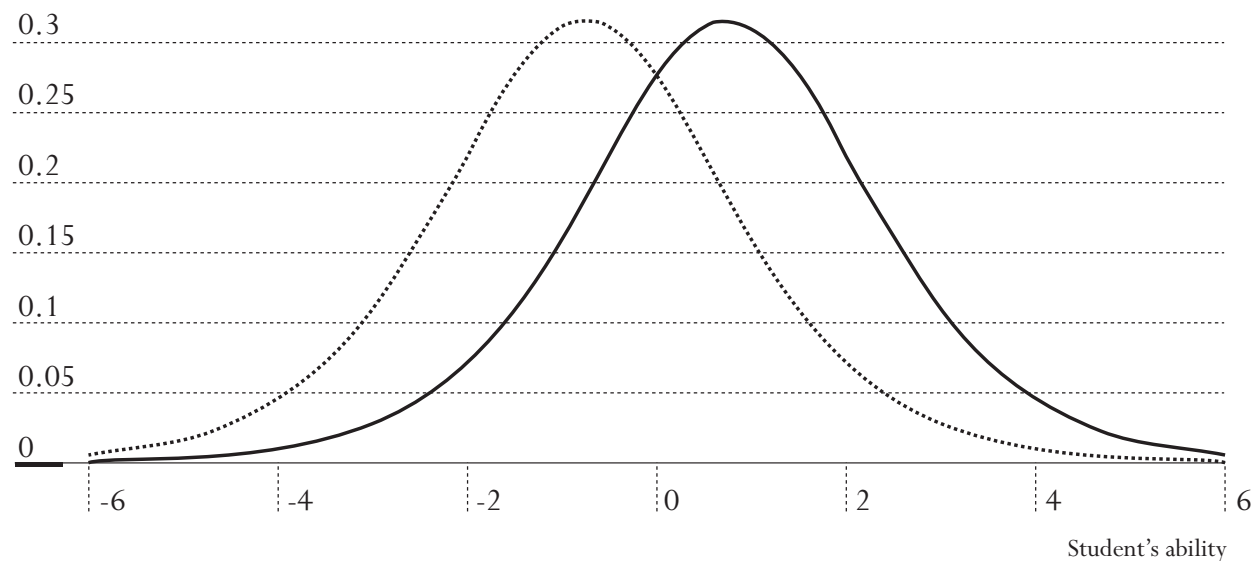


Based on Figure 4.9, we can state that for any student that succeeded on one item of the easy test, the model will estimate the student ability at -0.75 and that for any student that succeed one item of the difficult test, the model will estimate the student ability at 0.75 . If raw scores were used as estimates of student ability, in both cases, we would get 1 out of 2 , or 0.5 .

In summary, the raw score does not take into account the difficulty of the item for the estimation of the raw score and therefore, the interpretation of the raw score depends on the item difficulties. On the other hand, the Rasch model uses the number of correct answers and the difficulties of the items administered to a particular student for his or her ability estimate. Therefore, a Rasch score can be interpreted independently of the item difficulties. As far as all items can be located on the same continuum, the Rasch model can return fully comparable student's ability estimates, even if students were assessed with different subset of items. Note, however, that valid ascertainment of the student's Rasch score depends upon having an accurate knowledge of the item difficulties.

\section{Optimal conditions for linking items}

Some conditions have to be satisfied when different tests are used. First of all, the data collected through these tests must be linked. Without any links, the data collected through two different tests cannot be reported on a single scale. Usually, tests are linked by having different students doing common items or having the same students assessed with the different tests.

Let us suppose that a researcher wants to estimate the growth in reading performance between a population of grade 2 students and a population of grade 4 students. Two tests will be developed and both will be targeted at the expected proficiency level of both populations. To ensure that both tests can be scaled on the same continuum, a few difficult items from the grade 2 test will be included in the grade 4 test, let us say items 7, 34, 19, 23 and 12 .

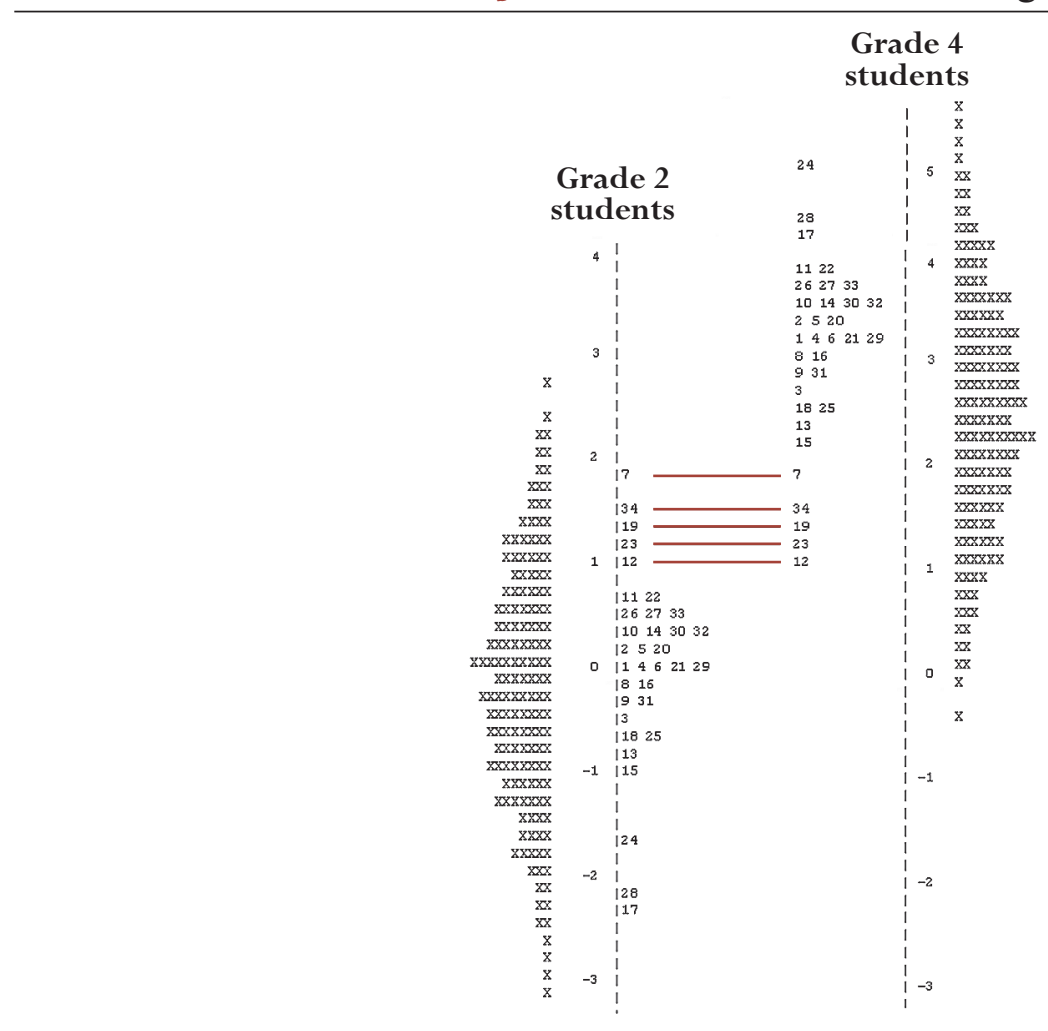


Figure 4.10 represents this item anchoring process. The left part of Figure 4.10 presents the outputs of the scaling of the grade 2 test with items centred on zero. For the scaling of grade 4 data, the reference point will be the grade 2 difficulty of the anchoring items. Then the difficulty of the other grade 4 items will be fixed according to this reference point, as shown on the right side of Figure 4.10.

With this anchoring process grade 2 and grade 4 item difficulties will be located on a single continuum. Therefore, the grade 2 and grade 4 students' ability estimates will also be located on the same continuum.

To accurately estimate the increase between grades 2 and 4, the researcher will ensure that the location of the anchor items is similar in both tests.

From a theoretical point of view, only one item is needed to link two different tests. However, this situation is far from being optimal. A balanced incomplete design presents the best guarantee for reporting the data of different tests on a single scale. This was adopted by PISA 2003 where the item pool was divided into 13 clusters of items. The item allocation to clusters takes into account the expected difficulty of the items and the expected time needed to answer the items. Table 4.9 presents the PISA 2003 test design. Thirteen clusters of items were denoted as C1 to C13 respectively. Thirteen booklets were developed and each of them has four parts, denoted as block 1 to block 4. Each booklet consists of four clusters. For instance, booklet 1 consists of cluster 1, cluster 2, cluster 4 and cluster 10 .

Table 4.9 - PISA 2003 test design

\begin{tabular}{l|c|c|c|c} 
& Block 1 & Block 2 & Block 3 & Block 4 \\
\hline Booklet 1 & C1 & C2 & C4 & C10 \\
\hline Booklet 2 & C2 & C3 & C5 & C11 \\
\hline Booklet 3 & C3 & C4 & C6 & C12 \\
\hline Booklet 4 & C4 & C5 & C7 & C13 \\
\hline Booklet 5 & C5 & C6 & C8 & C1 \\
\hline Booklet 6 & C6 & C7 & C9 & C2 \\
\hline Booklet 7 & C7 & C8 & C10 & C3 \\
\hline Booklet 8 & C8 & C9 4 & C5 \\
\hline Booklet 9 & C9 11 & C12 \\
\hline Booklet 10 & C10 & C10 & C13 & C7 \\
\hline Booklet 11 & C11 & C12 & C1 & C8 \\
\hline Booklet 12 & C12 & C13 & C2 & C9 \\
\hline Booklet 13 & C13 & C1 & C3 & \\
\hline
\end{tabular}

With such design, each cluster appears four times, once in each position. Further, each pair of clusters appears once and only once.

This design should ensure that the link process will not be influenced by the respective location of the link items in the different booklets. 


\section{Extension of the Rasch model}

Wright and Masters have generalised the original Rasch model to polytomous items, usually denoted as the partial credit model (Wright and Masters, 1982). With this model, items can be scored as incorrect, partially correct and correct. The PISA cognitive items were calibrated according to this model.

This polytomous items model can also be applied on Likert scale data. There is of course no correct or incorrect answer for such scales but the basic principles are the same: the possible answers can be ordered. PISA questionnaire data are scaled with the one-parameter logistic model for polytomous items.

\section{OTHER ITEM RESPONSE THEORY MODELS}

A classical distinction between Item Response Theory models concerns the number of parameters used to describe items. The Rasch model is designated as a one-parameter model because item characteristic curves only depend on the item difficulty. In the three-parameter logistic model, the item characteristic curves depend on: i) the item difficulty parameter; ii) the item discrimination parameter; and iii) what can be termed the "guessing" parameter. This last parameter accounts for the fact that, on a multiple choice test, all students have some chance of answering the item correctly, no matter how difficult the item is.

\section{CONCLUSIONS}

The Rasch model was designed to build a symmetric continuum on which both item difficulty and student ability are located. The item difficulty and the student ability are linked by a logistic function. With this function, it is possible to compute the probability that a student succeeds on an item.

Further, due to this probabilistic link, it is not a requirement to administer the whole item battery to every student. If some link items are guaranteed, the Rasch model will be able to create a scale on which every item and every student will be located. This last feature of the Rasch model constitutes one of the major reasons why this model has become fundamental in educational surveys.

1. See Measuring Student Knowledge and Skills - A New Framework for Assessment (OECD, 1999a) and The PISA 2003 Assessment Framework - Mathematics, Reading, Science and Problem Solving Knowledge and Skills (OECD, 2003b).

2. The probability of 0.5 was firstly used by psychophysics theories (Guilford, 1954). 



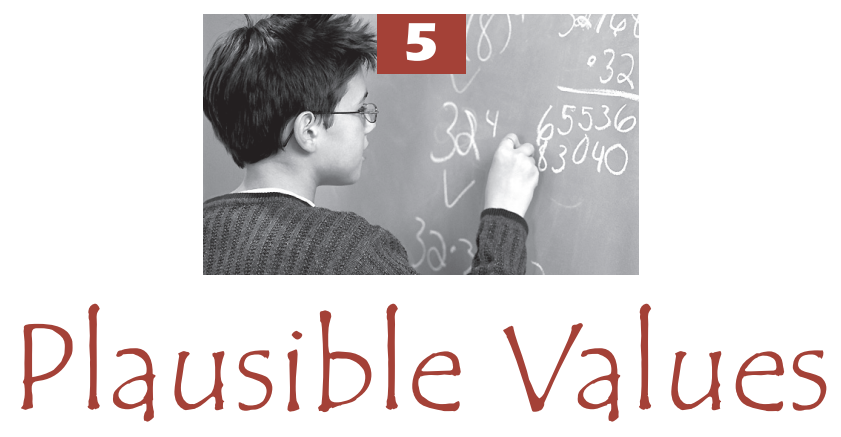

Individual estimates versus population estimates ………………..... 72

The meaning of plausible values …........................................... 72

Comparison of the efficiency of Warm Likelihood Estimates, Expected A Posteriori estimates and plausible values for the estimation of some population statistics.......................................... 76

How to perform analyses with plausible values …………………..... 78

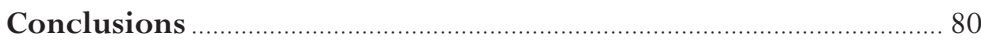




\section{INDIVIDUAL ESTIMATES VERSUS POPULATION ESTIMATES}

Education tests can have two major purposes:

- To measure the knowledge and skills of particular students. The performance of each student usually will have an impact on his or her future (school career, admission to post-secondary education, and so on). It is therefore particularly important to minimize the measurement error associated with each individual's estimate.

- To assess the knowledge or skills of a population. The performance of individuals will have no impact on their school career or professional life. In such a case, the goal of reducing error in making inferences about the target population is more important than the goal of reducing error at the individual level.

National or international education surveys belong to the second category.

International surveys such as PISA report student performance through plausible values (PVs). ${ }^{1}$ The remainder of this chapter will explain the conceptual meaning of plausible values and the advantage of reporting with them. Individual estimators (such as the WLE defined in Chapter 4) will be compared with PVs for the purposes of estimating a range of population statistics.

\section{THE MEANING OF PLAUSIBLE VALUES}

An example taken from the physical sciences, measurement area can help illustrate this complex concept. Suppose that a city board decides to levy a new building tax to increase the city's revenue. This new tax will be proportional to the length of the family house living room. Inspectors visit all city houses to measure the length of the living rooms. They are given a measuring tape and are instructed to record the length in term of integers only, i.e. 1 metre, 2 metres, 3 metres, 4 metres and so on.

The results of this measure are shown in Figure 5.1. About 3 per cent of the living rooms have a reported length of 4 metres; slightly over 16 per cent of the living rooms have a reported length of 9 metres and so on.

Figure 5.1 - Living room length expressed in integers

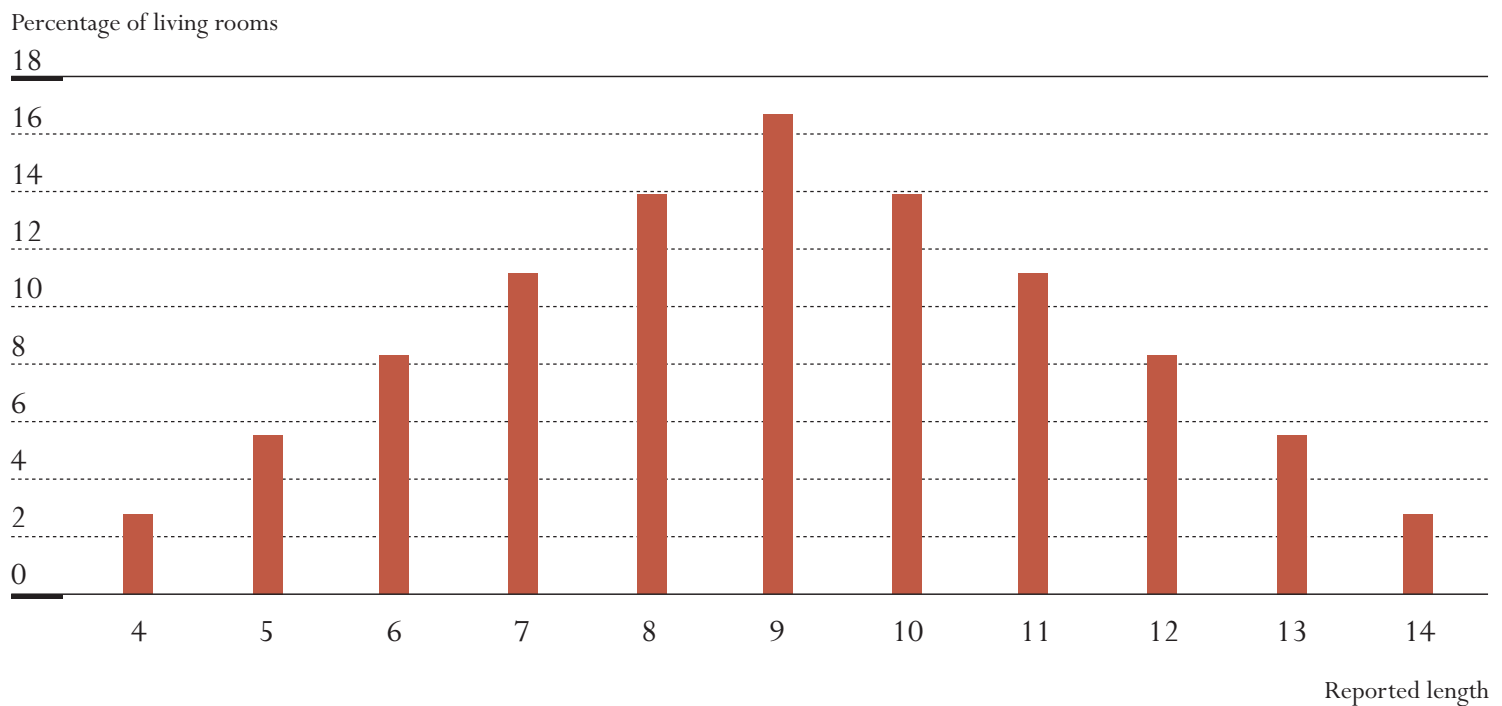


Of course, the reality is quite different as length is a continuous variable. With a continuous variable, observations can take any value between the minimum and the maximum. On the other hand, with a discontinuous variable, observations can only take a predefined number of values. Figure 5.2 gives the length distribution of the living rooms per reported length.

Figure 5.2 - Real length per reported length

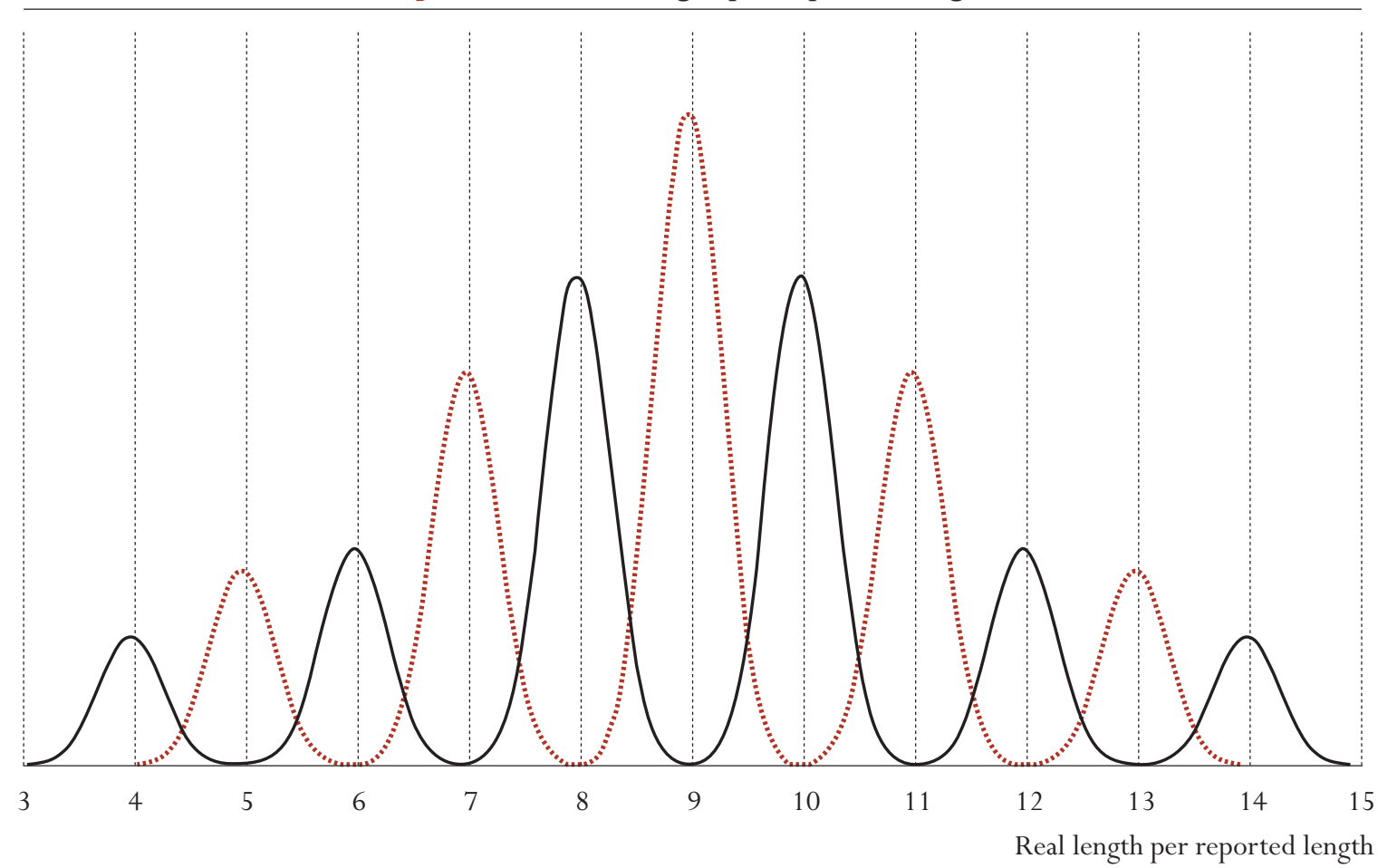

All living rooms with a reported length of 5 metres are not exactly 5 metres long. On average, they are 5 metres long, but their length varies around the mean. The difference between reported length and real length is due to the rounding process and measurement error. An inspector might incorrectly report 5 metres for a particular living room, when it really measures 4.15 metres. If the rounding process were the only source of error, then the reported length should be 4 metres. The second source of error, the error in measuring, explains the overlapping of the distribution.

In this particular example, the lengths of the living rooms are normally distributed around the mean, which is also the reported length. If the difference between the length and the closest integer is small, then the probability of not reporting this length with the closest integer is very small. For instance, it is unlikely that a length of 4.15 will be be reported as 5 metres or 3 metres. However, as the distance between the real length and the closest integer increases, the probability of not reporting this length with the closest integer will also increase. For instance, it is likely that a length of 4.95 will be reported as 5 metres, whereas a length of 4.50 will be reported equally as many times as 4 metres as it is 5 metres.

The methodology of PVs consists of:

- Mathematically computing distributions (denoted as posterior distributions) around the reported values and the reported length in the example; and

- Assigning to each observation a set of random values drawn from the posterior distributions. 
PVs can therefore be defined as random values from the posterior distributions. In the example, a living room of 7.154 metres that was reported as 7 metres might be assigned any value from the normal distribution around the reported length of 7. It might be 7.45 as well as 6.55 or 6.95 . Therefore, plausible values should not be used for individual estimation.

This fictitious example from the physical sciences can be translated successfully to the social sciences. For example, with a test of 6 dichotomous items, a continuous variable (i.e. mental ability) can be transformed into a discontinuous variable. The discontinuous variable will be the student raw score or the number of correct answers. The only possible scores are: 0, 1, 2, 3, 4, 5 and 6.

Contrary to most measures in the physical sciences, psychological or education measures encompass substantial measurement errors because:

- The concept to be measured is broader;

- They might be affected by the mental and physical dispositions of the students on the day of the assessment; and

- The conditions in which students are tested might also affect the results.

This means that there are large overlaps in the posterior distributions, as shown in Figure 5.3.

Further, with the example of the living room, the measurement error of the posterior distributions can be considered as independent of the living room. ${ }^{2}$ In education, the measurement error is not always independent of the proficiency level of the students. It may be smaller for average students, and larger for low and high achievers.

Further, in this particular example, the posterior distributions for score 0 and score 6 are substantially skewed, as the posterior distributions of the living rooms with a reported length of 4 and 14 metres would be, if all living rooms smaller than 4 metres were reported as 4 and if all living rooms longer than 14 metres were reported as 14 . This means that the posterior distributions are not normally distributed, as shown in Figure 5.3.

Figure 5.3 A posterior distribution on a test of 6 items

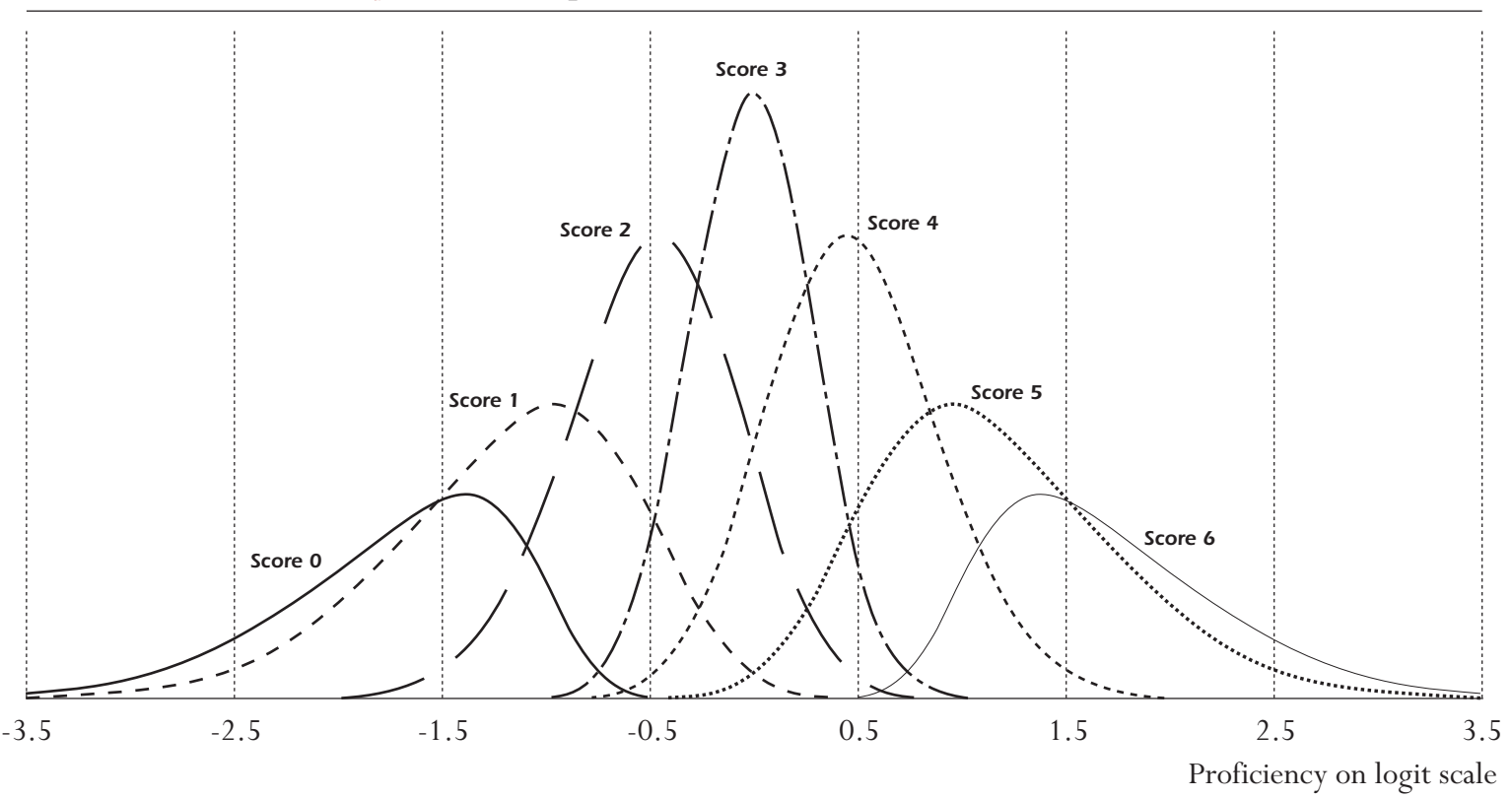


Generating PVs on an education test consists of drawing random numbers from the posterior distributions. This example clearly shows that plausible values should not be used as individual performance. Indeed, a student who scores 0 might get -3, but also -1. A student who scores 6 might get 3 , but also 1 .

It has been noted that

The simplest way to describe plausible values is to say that plausible values are a representation of the range of abilities that a student might reasonably have. ... Instead of directly estimating a student's ability $\theta$, a probability distribution for a student's $\theta$, is estimated. That is, instead of obtaining a point estimate for $\theta$, (like a WLE), a range of possible values for a student's $\theta$, with an associated probability for each of these values is estimated. Plausible values are random draws from this (estimated) distribution for a student's $\theta$ (Wu and Adams, 2002). ${ }^{3}$

All this methodology aims at building a continuum from a collection of discontinuous variables (i.e. the test score). It is meant to prevent biased inferences occurring as a result of measuring an unobservable underlying ability through a test using a relatively small number of items.

Finally, an individual estimate of student ability can also be derived from the posterior distributions. This derived individual estimate is called the Expected A Posteriori estimator (EAP). Instead of assigning a set of random values from the posterior distributions, the mean of the posterior distributions is assigned. Therefore, the EAP can be considered as the mean of an infinite set of plausible values for a particular student.

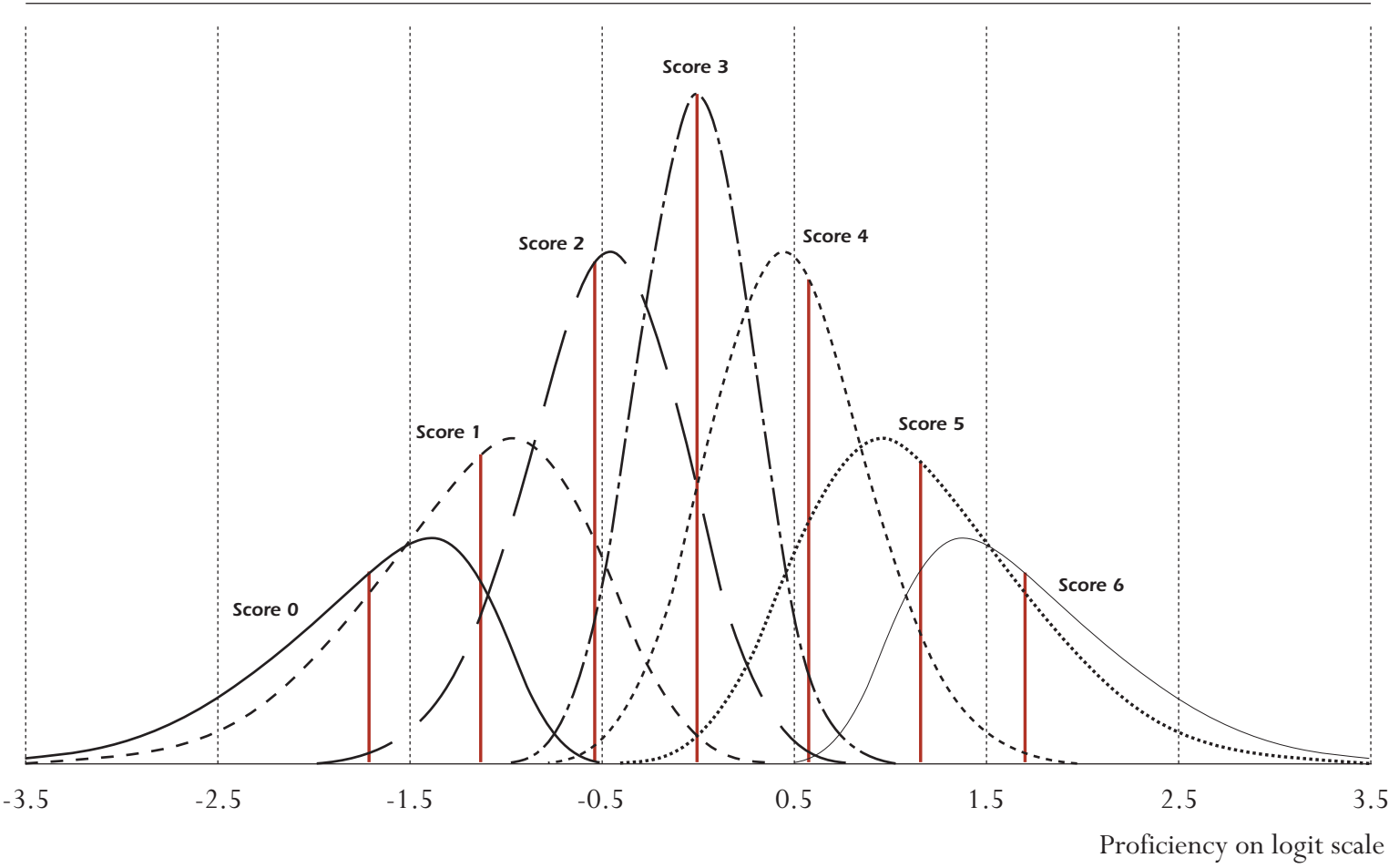


As only one value is assigned per posterior distribution, the EAP estimator is also a discontinuous variable. ${ }^{4}$ However, EAP estimates and WLEs differ as the former requires a population distribution assumption, which is not the case for the latter. Further, while any raw score for a particular test will always be associated with one and only one WLE, different EAP values can be associated with a particular raw score, depending on the regressors used as conditioning variables.

Researchers not used to working with plausible values might consider this apparent randomization as a source of imprecision. The comparison of the different types of Rasch ability estimators (WLE, EAP and PVs) through the estimation of population statistics will overcome this perception. Although the PISA 2003 database only includes PVs , ${ }^{5}$ the comparison will incorporate EAP estimates to show biases that occur when data analysts average the plausible values at the student levels to obtain one score value per student.

\section{COMPARISON OF THE EFFICIENCY OF WARM LIKELIHOOD ESTIMATES, EXPECTED A POSTERIORI ESTIMATES AND PVS FOR THE ESTIMATION OF SOME POPULATION STATISTICS ${ }^{6}$}

A comparison between different student ability estimators can be performed on real data. Such a comparison will outline differences, but it will not identify the best estimators for a particular population statistic. A simulation can be used to illustrate this:

The simulation consists of three major steps:

- The generation of a data set including a continuous variable that represents the student abilities (i.e. denoted as the latent variable), some background variables (including the gender and an index of social background), denoted HISEI, and a pattern of item responses coded 0 for incorrect answer and 1 for a correct answer. The results presented hereafter are based on a fictitious test of 15 items. $^{7}$

- The computation of the student ability estimator, in particular the WLEs, EAP estimates and PVs. ${ }^{8}$

- The estimation of some population parametres using the student ability (i.e. latent variable) and the different student ability estimators. A comparison will be made for:

- Mean, variance and percentiles;

- Correlation; and

- Between- and within-school variance.

The data set contains 5250 students distributed in 150 schools with 35 students per school. Table 5.1 presents the structure of the simulated data set before the importation of the Rasch student abilities estimators.

Table 5.1 - Structure of the simulated data

\begin{tabular}{c|c|c|c|c|c|c|c|c}
\hline School ID & Student ID & Sex & HISEI & Item 1 & Item 2 & $\ldots$ & Item 14 & Item 15 \\
\hline 001 & 01 & 1 & 32 & 1 & 1 & & 0 & 0 \\
\hline 001 & 02 & 0 & 45 & 1 & 0 & & 1 & 0 \\
\hline$\ldots$ & $\ldots$ & & & & & & & \\
\hline 150 & 34 & 0 & 62 & 0 & 0 & & 1 & 1 \\
\hline 150 & 35 & 1 & 50 & 0 & 1 & & 1 & 1 \\
\hline
\end{tabular}


Table 5.2 presents the mean and the variance of the latent variable, the WLEs, the five PVs and the EAP estimates. The average of the five PVs mean is also included.

Table 5.2 Means and variances for the latent variable and the different student ability estimators

\begin{tabular}{c|c|c}
\hline & Mean & Variance \\
\hline Latent variable & 0.00 & 1.00 \\
\hline WLE & 0.00 & 1.40 \\
\hline EAP & 0.00 & 0.75 \\
\hline PV1 & 0.01 & 0.99 \\
\hline PV2 & 0.00 & 0.99 \\
\hline PV3 & 0.00 & 1.01 \\
\hline PV4 & 0.00 & 1.01 \\
\hline PV5 & -0.01 & 0.00 \\
\hline Average of the 5 PV statistics & 0.00 & 1.00 \\
\hline
\end{tabular}

Table 5.2 shows that a good estimate of the population's mean (i.e. the latent variable estimate) is obtained regardless of the type of the latent variable used (WLEs, EAP estimates or PVs). It can be empirically demonstrated that none of the estimates significantly differ from the expected mean, i.e. 0.00 in this particular case (Wu and Adams, 2002). Additionally, it can also be shown that the mean of the WLEs will not be biased if the test is well targeted, i.e. if the average of the item difficulties is around 0 on the Rasch scale (Wu and Adams, 2002). That is, on a well targeted test, students will obtain a raw score of about 50 per cent correct answers. If the test is too easy then the mean of the WLEs will be underestimated (this is called the ceiling effect), while if it is too difficult then the mean of the WLEs will be overestimated (this is called the floor effect).

These last results explain why the mean of the WLEs provided in the PISA 2000 data base differs from the mean of the plausible values, especially for non OECD countries. For the reading reflecting scale, the means obtained for Canada usingWLEs and PVs are respectively 538.4 and 542.5 (i.e. very close). In contrast, the means obtained for Peru, using WLEs and PVs are respectively 352.2 and 322.7, which is a difference of about 0.3 standard deviations. There is bias whenWLEs are used to estimate the mean, if the test is not well targeted. This comparison cannot be performed on the PISA 2003 database as it only reports student performance with plausible values.

For the population variance, Table 5.2 shows that PVs give estimates closest to the expected value, while WLEs overestimate it and the EAP underestimates it. These results are consistent with other simulation studies.

Table 5.3 presents some percentiles computed on the different ability estimators. For example, because the variance computed using plausible values is not biased, the percentiles based on PVs are also unbiased. However, because the EAP estimates and WLEs variances are biased, the percentiles and in particular extreme percentiles will also be biased. These results are consistent with other simulation studies previously cited.

Table 5.4 presents the correlation between the social background index (HISEI), gender and the latent variables and the different estimators of students' abilities. The correlation coefficients with 
the WLEs are both underestimated, while the correlation coefficients with the EAP estimates are overestimated. Only the correlation coefficients with the plausible values are unbiased. ${ }^{?}$

Table 5.3 - Percentiles for the latent variable and the different student ability estimators

\begin{tabular}{c|c|c|c|c|c|c|c}
\hline & P5 & P10 & P25 & P50 & P75 & P90 & P95 \\
\hline Latent variable & -1.61 & -1.26 & -0.66 & 0.01 & 0.65 & 1.26 & 1.59 \\
\hline WLE & -2.15 & -1.65 & -0.82 & -0.1 & 0.61 & 1.38 & 1.81 \\
\hline EAP & -1.48 & -1.14 & -0.62 & -0.02 & 0.55 & 1.08 & 1.37 \\
\hline PV1 & -1.68 & -1.29 & -0.71 & -0.03 & 0.64 & 1.22 & 1.59 \\
\hline PV2 & -1.67 & -1.31 & -0.69 & -0.03 & 0.62 & 1.22 & 1.58 \\
\hline PV3 & -1.67 & -1.32 & -0.70 & -0.02 & 0.64 & 1.21 & 1.56 \\
\hline PV4 & -1.69 & -1.32 & -0.69 & -0.03 & 0.63 & 1.23 & 1.55 \\
\hline PV5 & -1.65 & -1.3 & -0.71 & -0.02 & 0.62 & 1.2 & 1.55 \\
\hline $\begin{array}{c}\text { Average of the } \\
\text { 5 PV statistics }\end{array}$ & -1.67 & -1.31 & -0.70 & -0.03 & 0.63 & 1.22 & 1.57 \\
\hline
\end{tabular}

Table 5.4 - Correlation between HISEI, GENDER and the latent variable, the different student ability estimators

\begin{tabular}{c|c|c}
\hline & HISEI & GENDER \\
\hline Latent variable & 0.40 & 0.16 \\
\hline WLE & 0.33 & 0.13 \\
\hline EAP & 0.46 & 0.17 \\
\hline PV1 & 0.41 & 0.15 \\
\hline PV2 & 0.42 & 0.15 \\
\hline PV3 & 0.42 & 0.13 \\
\hline PV4 & 0.40 & 0.15 \\
\hline PV5 & 0.40 & 0.14 \\
\hline Average of the 5 PV statistics & 0.41 & 0.14 \\
\hline
\end{tabular}

It should be noted that the regression coefficients are all unbiased for the different types of estimators. Nevertheless, as variances are biased for some estimators, residual variances will also be biased. Therefore, the standard error on the regression coefficients will be biased in the case of the WLEs and the EAP estimates.

Finally, Table 5.5 presents the between- and within-school variances. Between-school variances for the different estimators do not differ from the expected value of 0.33 . However, WLEs overestimate the within school variance, while the EAP estimates underestimate it. These results are consistent with other simulation studies (Monseur and Adams, 2002).

As this example shows, PVs provide unbiased estimates.

\section{HOW TO PERFORM ANALYSES WITH PLAUSIBLE VALUES}

As stated in the previous section, a set of PVs, usually five, are drawn for each student for each scale or subscale. Population statistics should be estimated using each plausible value separately. The reported population statistic is then the average of each plausible value statistic. For instance, if 
Table 5.5 - Between- and within-school variances

\begin{tabular}{c|c|c} 
& $\begin{array}{c}\text { Between-school } \\
\text { variance }\end{array}$ & $\begin{array}{c}\text { Within-school } \\
\text { variance }\end{array}$ \\
\hline Latent variable & 0.33 & 0.62 \\
\hline WLE & 0.34 & 1.02 \\
\hline EAP & 0.35 & 0.38 \\
\hline PV1 & 0.35 & 0.61 \\
\hline PV2 & 0.36 & 0.60 \\
\hline PV3 & 0.36 & 0.61 \\
\hline PV4 & 0.35 & 0.61 \\
\hline PV5 & 0.35 & 0.61 \\
\hline Average of the 5 PV statistics & 0.35 & 0.61 \\
\hline
\end{tabular}

one is interested in the correlation coefficient between the social index and the reading performance in PISA, then five correlation coefficients should be computed and then averaged.

Data analysts should never average the plausible values at the student level, i.e. computing in the data set the mean of the five plausible values at the student level and then computing the statistic of interest once using that average PV value. Doing so would be equivalent to an EAP estimate, with a bias as described in the previous section.

Mathematically, secondary analyses with plausible values can be described as follows. If $\theta$ is the population statistic and $\theta_{i}$ is the statistic of interest computed on one plausible value, then:

$\theta=\frac{1}{M} \sum_{i=1}^{M} \theta_{i}$, with $M$ being the number of plausible values.

The plausible values also allow computing the uncertainty in the estimate of $\theta$ due to the lack of precision of the measurement test. If a perfect test could be developed, then the measurement error would be equal to zero and the five statistics from the plausible values would be exactly identical. Unfortunately, perfect tests do not exist and never will. This measurement variance, usually denoted imputation variance, is equal to:

$$
B_{M}=\frac{1}{M-1} \sum_{i=1}^{M}\left(\theta_{i}-\theta\right)^{2}
$$

It corresponds to the variance of the five plausible value statistics of interest. The final stage is to combine the sampling variance and the imputation variance as follows:

$$
V=U+\left(1+\frac{1}{M}\right) B_{M} \text {, with } U \text { being the sampling variance. }
$$

In the following chapters, we will show how to compute sampling variances and imputation variances and how to combine them, using the PISA 2003 database. 


\section{CONCLUSIONS}

This chapter was devoted to the meaning of the plausible values and the steps that are required when analysing data with PVs. A comparison between PVs and alternate individual ability estimates was presented to convince PISA data users of the superiority of this methodology for reporting population estimates.

\section{Notes}

1. The methodology of PVs was first implemented in NAEP studies (see Beaton, 1987).

2. The measurement error will be independent of the length of the living rooms if the inspectors are using a measuring instrument that is at least 15 metres long (such as a measuring tape). If they are using a standard metre, then the overall measurement error will be proportional to the length of the living room.

3. The probability distribution for a student's $\theta$ can be based on the cognitive data only, i.e. the item response pattern, but can also include additional information, such as student gender, social background, and so on. The probability distribution becomes therefore conditioned by this additional information. A mathematical explanation of the model used for the scaling of the PISA 2000 scaling can be found in the PISA 2000 Technical Report (OECD, 2002c).

4. If several regressors are used as conditioning variables, then the EAP estimator tends to a continuous variable.

5. PISA 2000 data files include both WLEs and PVs.

6. PVs and EAP estimators can be computed with or without regressors. As the PISA 2000 PVs were generated based on all variables collected through the student questionnaires, this comparison will only include PVs and EAP estimators with the use of regressors.

7. The data generation starts with a factorial analysis on a 3 by 3 squared correlation matrix. The correlation between the latent variable and gender was set at 0.20 , the correlation between the latent variable and the social background indicator was set at 0.40 and the correlation between gender and the social background indicator was set at 0.00 . Three random variables are drawn from normal distributions and combined according to the factorial regression coefficients to create the three variables of interest, i.e. reading, gender and social background. Based on the student score on the latent variable and a predefined set of 20 item difficulties; probabilities of success are computed according to the Rasch model. These probabilities are then compared to uniform distribution and recoded into 0 and 1. Finally, gender is recoded into a dichotomous variable.

8. The estimators were computed with the Conquest Software developed by M.L.Wu, R.J Adams and M.R. Wilson.

9. The results on the EAP and PV correlation coefficients are observed when the probability distributions are generated with conditioning variables. Without the conditioning, the correlation with the plausible values would be underestimated. 


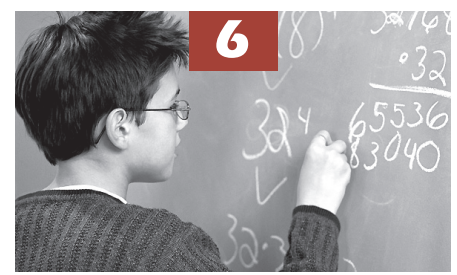

\section{Computation
of Standard Errors}

Introduction

The standard error on univariate statistics for numerical variables ... 82

The SPSS ${ }^{\circledR}$ macro for computing the standard error on a mean $\ldots . .85$

The standard error on percentages …………………..................... 87

The standard error on regression coefficients …………………....... 90

The standard error on correlation coefficients ................................. 92

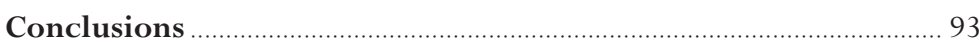




\section{INTRODUCTION}

As shown in Chapter 3, replicates have to be used for the computation of the standard error for any population estimate. This chapter will give examples of such computations.

For PISA 2000 and PISA 2003, the Fay's variant of the Balanced Repeated Replication is used. The general formula for computing the sampling variance with this method is:

$$
\sigma_{(\hat{\theta})}^{2}=\frac{1}{G(1-k)^{2}} \sum_{i=1}^{G}\left(\hat{\theta}_{(i)}-\hat{\theta}\right)^{2}
$$

Since the PISA databases include 80 replicates and since the Fay coefficient was set to 0.5 for both data collections, the above formula can be simplified as follows:

$$
\sigma_{(\hat{\theta})}^{2}=\frac{1}{G(1-k)^{2}} \sum_{i=1}^{G}\left(\hat{\theta}_{(i)}-\hat{\theta}\right)^{2}=\frac{1}{80(1-0.5)^{2}} \sum_{i=1}^{80}\left(\hat{\theta}_{(i)}-\hat{\theta}\right)^{2}=\frac{1}{20} \sum_{i=1}^{80}\left(\hat{\theta}_{(i)}-\hat{\theta}\right)^{2}
$$

\section{THE STANDARD ERROR ON UNIVARIATE STATISTICS FOR NUMERICAL VARIABLES}

To compute the mean and its respective standard error, it is necessary to first compute this statistic by weighting the data with the student final weight, i.e. W_FSTUWT, and then to compute 80 other means, each of them by weighting the data with one of the 80 replicates, i.e. W_FSTR1 to W_FSTR80.

Box 6.1 presents the SPSS ${ }^{\circledR}$ syntax for computing these 81 means based on the social background index (denoted HISEI for the PISA 2003 data for Germany) and Table 6.1 presents the HISEI final estimates as well as the 80 replicate estimates.

\section{Box 6.1 - SPSS $^{\circledR}$ syntax for the computation of 81 means}

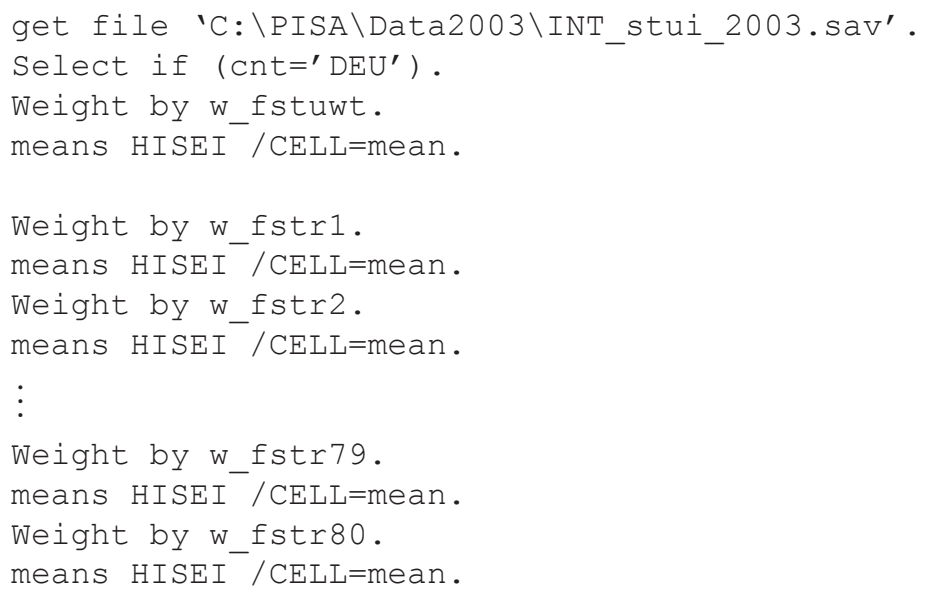

The mean that will be reported is equal to 49.33 , i.e. the estimate obtained with the student final weight W_FSTUWT. The 80 replicate estimates are just used to compute the standard error on the mean of 49.33 . 
Table 6.1 - HISEI mean estimates

\begin{tabular}{|c|c|c|c|}
\hline Weight & Mean estimate & Weight & Mean estimate \\
\hline Final weight & 49.33 & & \\
\hline Replicate 1 & 49.44 & Replicate 41 & 49.17 \\
\hline Replicate 2 & 49.18 & Replicate 42 & 49.66 \\
\hline Replicate 3 & 49.12 & Replicate 43 & 49.18 \\
\hline Replicate 4 & 49.46 & Replicate 44 & 49.04 \\
\hline Replicate 5 & 49.24 & Replicate 45 & 49.42 \\
\hline Replicate 6 & 49.34 & Replicate 46 & 49.72 \\
\hline Replicate 7 & 49.13 & Replicate 47 & 49.48 \\
\hline Replicate 8 & 49.08 & Replicate 48 & 49.14 \\
\hline Replicate 9 & 49.54 & Replicate 49 & 49.57 \\
\hline Replicate 10 & 49.20 & Replicate 50 & 49.36 \\
\hline Replicate 11 & 49.22 & Replicate 51 & 48.78 \\
\hline Replicate 12 & 49.12 & Replicate 52 & 49.53 \\
\hline Replicate 13 & 49.33 & Replicate 53 & 49.27 \\
\hline Replicate 14 & 49.47 & Replicate 54 & 49.23 \\
\hline Replicate 15 & 49.40 & Replicate 55 & 49.62 \\
\hline Replicate 16 & 49.30 & Replicate 56 & 48.96 \\
\hline Replicate 17 & 49.24 & Replicate 57 & 49.54 \\
\hline Replicate 18 & 48.85 & Replicate 58 & 49.14 \\
\hline Replicate 19 & 49.41 & Replicate 59 & 49.27 \\
\hline Replicate 20 & 48.82 & Replicate 60 & 49.42 \\
\hline Replicate 21 & 49.46 & Replicate 61 & 49.56 \\
\hline Replicate 22 & 49.37 & Replicate 62 & 49.75 \\
\hline Replicate 23 & 49.39 & Replicate 63 & 48.98 \\
\hline Replicate 24 & 49.23 & Replicate 64 & 49.00 \\
\hline Replicate 25 & 49.47 & Replicate 65 & 49.35 \\
\hline Replicate 26 & 49.51 & Replicate 66 & 49.27 \\
\hline Replicate 27 & 49.35 & Replicate 67 & 49.44 \\
\hline Replicate 28 & 48.89 & Replicate 68 & 49.08 \\
\hline Replicate 29 & 49.44 & Replicate 69 & 49.09 \\
\hline Replicate 30 & 49.34 & Replicate 70 & 49.15 \\
\hline Replicate 31 & 49.41 & Replicate 71 & 49.29 \\
\hline Replicate 32 & 49.18 & Replicate 72 & 49.29 \\
\hline Replicate 33 & 49.50 & Replicate 73 & 49.08 \\
\hline Replicate 34 & 49.12 & Replicate 74 & 49.25 \\
\hline Replicate 35 & 49.05 & Replicate 75 & 48.93 \\
\hline Replicate 36 & 49.40 & Replicate 76 & 49.45 \\
\hline Replicate 37 & 49.20 & Replicate 77 & 49.13 \\
\hline Replicate 38 & 49.54 & Replicate 78 & 49.45 \\
\hline Replicate 39 & 49.32 & Replicate 79 & 49.14 \\
\hline Replicate 40 & 49.35 & Replicate 80 & 49.27 \\
\hline
\end{tabular}

There are three major steps for the computation of the standard error:

1. Each replicate estimate will be compared with the final estimate 49.33 and the difference will be squared. Mathematically, it corresponds to $\left(\hat{\theta}_{(i)}-\hat{\theta}\right)^{2}$ or in this particular case, $\left(\hat{\mu}_{i}-\hat{\mu}\right)^{2}$. For the first replicate, it will be equal to: $(49.44-49.33)^{2}=0.0140$. For the second replicate, it corresponds to: $(49.18-49.33)^{2}=0.0228$. Table 6.2 presents the squared differences.

2. The sum of the squared differences is computed, and then divided by 20. Mathematically, it corresponds to $1 / 20 . \sum_{i=1}^{80}\left(\hat{\mu}_{(i)}-\hat{\mu}\right)^{2}$. In the example, the sum is equal to $(0.0140+0.0228+\ldots+0.0354+0.0031)=3.5195$

The sum divided by 20 is therefore equal to $3.5159 / 20=0.1760$. This value represents the sampling variance on the mean estimate for HISEI. 
3. The standard error is equal to the square root of the sampling variance, i.e.:

$$
\sigma_{(\hat{\mu})}=\sqrt{\sigma_{(\hat{\mu})}^{2}}=\sqrt{0.1760}=0.4195
$$

This means that the sampling distribution on the HISEI mean for Germany has a standard deviation of 0.4195 . This value also allows building a confidence interval around this mean. With a risk of type I error equal to 0.05 , usually denoted $\alpha$, the confidence interval will be equal to:

$$
[49.33-(1.96 * 0.4195) ; 49.33+(1.96 * 0.4195)]
$$

[48.51;50.15]

In other words, there are 5 chances out of 100 that an interval formed in this way will fail to capture the population mean. It also means that the German population mean for HISEI is significantly different from a value of 51 , for example, as this number is not included in the confidence interval.

Chapter 9 will show how this standard error can be used for comparisons either between two or several countries, or between sub-populations within a particular country.

\begin{tabular}{|c|c|c|c|}
\hline Weight & Squared difference & Weight & Squared difference \\
\hline $\begin{array}{l}\text { Replicate } 1 \\
\text { Replicate } 2 \\
\text { Replicate } 3 \\
\text { Replicate } 4 \\
\text { Replicate } 5 \\
\text { Replicate } 6 \\
\text { Replicate } 7 \\
\text { Replicate } 8 \\
\text { Replicate } 9 \\
\text { Replicate } 10 \\
\text { Replicate } 11 \\
\text { Replicate } 12 \\
\text { Replicate } 13 \\
\text { Replicate } 14 \\
\text { Replicate } 15 \\
\text { Replicate } 16 \\
\text { Replicate } 17 \\
\text { Replicate } 18 \\
\text { Replicate } 19 \\
\text { Replicate } 20 \\
\text { Replicate } 21 \\
\text { Replicate } 22 \\
\text { Replicate } 23 \\
\text { Replicate } 24 \\
\text { Replicate } 25 \\
\text { Replicate } 26 \\
\text { Replicate } 27 \\
\text { Replicate } 28 \\
\text { Replicate } 29 \\
\text { Replicate } 30 \\
\text { Replicate } 31 \\
\text { Replicate } 32 \\
\text { Replicate } 33 \\
\text { Replicate } 34 \\
\text { Replicate } 35 \\
\text { Replicate } 36 \\
\text { Replicate } 37 \\
\text { Replicate } 38 \\
\text { Replicate } 39 \\
\text { Replicate } 40\end{array}$ & $\begin{array}{l}0.0140 \\
0.0228 \\
0.0421 \\
0.0189 \\
0.0075 \\
0.0002 \\
0.0387 \\
0.0583 \\
0.0472 \\
0.0167 \\
0.0124 \\
0.0441 \\
0.0000 \\
0.0205 \\
0.0048 \\
0.0009 \\
0.0074 \\
0.2264 \\
0.0077 \\
0.2604 \\
0.0182 \\
0.0016 \\
0.0041 \\
0.0093 \\
0.0199 \\
0.0344 \\
0.0007 \\
0.1919 \\
0.0139 \\
0.0001 \\
0.0071 \\
0.0215 \\
0.0302 \\
0.0411 \\
0.0778 \\
0.0052 \\
0.0150 \\
0.0445 \\
0.0000 \\
0.0004\end{array}$ & $\begin{array}{l}\text { Replicate } 41 \\
\text { Replicate } 42 \\
\text { Replicate } 43 \\
\text { Replicate } 44 \\
\text { Replicate } 45 \\
\text { Replicate } 46 \\
\text { Replicate } 47 \\
\text { Replicate } 48 \\
\text { Replicate } 49 \\
\text { Replicate } 50 \\
\text { Replicate } 51 \\
\text { Replicate } 52 \\
\text { Replicate } 53 \\
\text { Replicate } 54 \\
\text { Replicate } 55 \\
\text { Replicate } 56 \\
\text { Replicate } 57 \\
\text { Replicate } 58 \\
\text { Replicate } 59 \\
\text { Replicate } 60 \\
\text { Replicate } 61 \\
\text { Replicate } 62 \\
\text { Replicate } 63 \\
\text { Replicate } 64 \\
\text { Replicate } 65 \\
\text { Replicate } 66 \\
\text { Replicate } 67 \\
\text { Replicate } 68 \\
\text { Replicate } 69 \\
\text { Replicate } 70 \\
\text { Replicate } 71 \\
\text { Replicate } 72 \\
\text { Replicate } 73 \\
\text { Replicate } 74 \\
\text { Replicate } 75 \\
\text { Replicate } 76 \\
\text { Replicate } 77 \\
\text { Replicate } 78 \\
\text { Replicate } 79 \\
\text { Replicate } 80\end{array}$ & $\begin{array}{l}0.0239 \\
0.1090 \\
0.0203 \\
0.0818 \\
0.0082 \\
0.1514 \\
0.0231 \\
0.0349 \\
0.0590 \\
0.0014 \\
0.3003 \\
0.0431 \\
0.0032 \\
0.0086 \\
0.0868 \\
0.1317 \\
0.0438 \\
0.0354 \\
0.0034 \\
0.0081 \\
0.0563 \\
0.1761 \\
0.1173 \\
0.1035 \\
0.0008 \\
0.0030 \\
0.0139 \\
0.0618 \\
0.0557 \\
0.0324 \\
0.0016 \\
0.0011 \\
0.0603 \\
0.0052 \\
0.1575 \\
0.0157 \\
0.0378 \\
0.0155 \\
0.0354 \\
0.0031\end{array}$ \\
\hline & & Sum of squared differences & 3.5195 \\
\hline
\end{tabular}

Table 6.2 - Squared differences between replicate estimates and the final estimate 


\section{THE SPSS ${ }^{\circledR}$ MACRO FOR COMPUTING THE STANDARD ERROR ON A MEAN}

Writing all the SPSS ${ }^{\circledR}$ syntax to compute these 81 means and then transferring them into an Microsoft ${ }^{\circledR}$ Excel $^{\circledR}$ spreadsheet to finally obtain the standard error would be very time consuming. Fortunately, SPSS ${ }^{\circledR}$ macros simplify iterative computations. The software package will execute $\mathrm{N}$ times the commands included between the beginning command (!DO !I=1 !TO N) and the ending command (!DOEND). Further, it also saves the results in a temporary file that can be used subsequently for the computation of the standard error.

About 12 SPSS $^{\circledR}$ macros have been written to simplify the main PISA computations. These macros have been saved in different files (with the extension .sps). Box 6.2 shows a SPSS $^{\circledR}$ syntax where a macro is called for computing the mean and standard error of the variable HISEI.

\section{Box 6.2 - SPSS $^{\circledR}$ syntax for the computation of the mean of HISEI and its respective standard error}

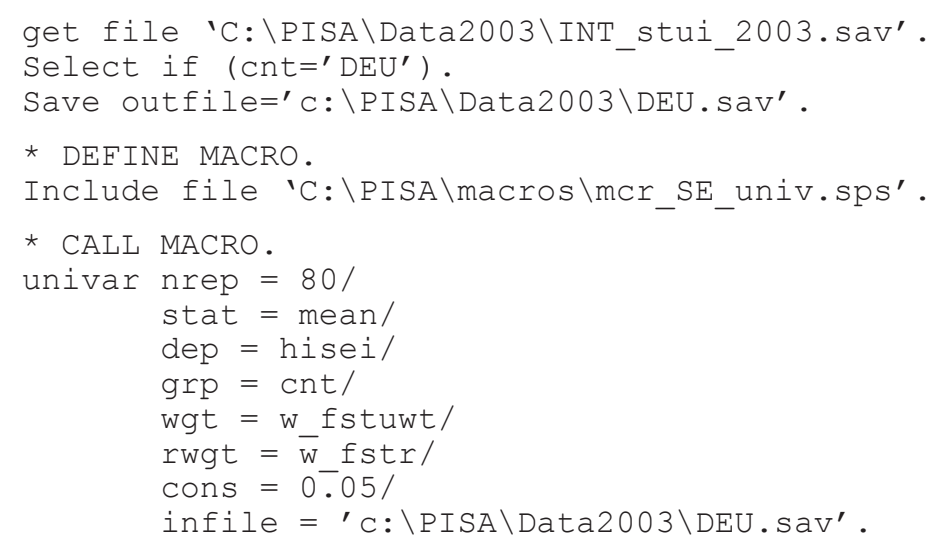

After selecting the German data from the PISA 2003 student database and saving a temporary data file, the command "Include file 'C: \PISA \macros\mcr_SE_univ.sps'." will create and save a new procedure to calculate a univariate statistic and its standard error for later use. This procedure is named 'UNIVAR' and will run when the macro is called.

When calling the macro, the arguments have to be defined by the user. NREP is the number of replicates. STAT is the statistic that is being computed. The statistic is computed with the aggregate command, which means that the statistics displayed in Table 6.4 are available for this macro. DEP is the variable the statistic is computed for and GRP is the group or break variable. In this example, the group variable country (CNT) is a constant (as the data file only contains German data, only one statistic and one standard error will be computed). WGT is the full student weight and RWGT is the root of the replicate weights (the macro will concatenate this root with the numbers 1 to 80: W_FSTR1 to W_FSTR80 in this case). CONS is the constant that is used when calculating the sampling variance. This constant is :

$\frac{1}{G(1-k)^{2}}$

where $\mathrm{G}$ is the number of replicates and $k$ is Fay's factor (PISA uses 0.5 , see Chapter 3 and the beginning of this chapter). INFILE is the data file used for the procedure. 
As these iterative computations might be CPU consuming, the procedure will only read in the variables that are needed.

From the temporary input data file, this macro will compute per country the mean of HISEI and its standard error by using the final full student weight (W_FSTUWT) and the 80 replicate weights (W_FSTR1 to W_FSTR80). This macro will return exactly the same values for the mean estimate and its respective standard error as the ones obtained through Table 6.1 and Table 6.2.

The structure of the output data file is presented in Table 6.3.

Table 6.3 - Structure of the output data file from Box $\mathbf{6 . 2}$

\begin{tabular}{c|c|c}
\hline CNT & STAT & SE \\
\hline DEU & 49.33 & 0.42 \\
\hline
\end{tabular}

If the data set had not been reduced to the data for Germany, then the number of rows in the output data file would be equal to the number of countries in the database.

There are a few restrictions as well as a few options with this macro:

- Only one input data file can be specified;

- Several group or breakdown variables can be specified; for instance, if results per gender are needed, then the group variables will be CNT and ST03Q01 (grp = cnt st03q01);

- Only one numerical variable can be specified in the VAR statement;

- Only one statistic can be specified. The available statistics are presented in Table 6.4; and

- The output file will not be saved by the macro.

Table 6.4 Available statistics with the UNIV macro ${ }^{2}$

\begin{tabular}{l|l}
\hline Statistics available & Meaning \\
\hline SUM & (Sum $)$ \\
\hline MEAN & (Mean) \\
\hline SD & (Std.deviation) \\
\hline PGT & $(\%$ cases gt value $)$ \\
\hline PLT & $(\%$ cases lt value) \\
\hline PIN & $(\%$ between values $)$ \\
\hline POUT & $(\%$ not in range) \\
\hline FGT & (Fraction gt value $)$ \\
\hline FLT & (Fraction lt value) \\
\hline FIN & (Fraction in range $)$ \\
\hline FOUT & (Frac.not in range) \\
\hline
\end{tabular}

a. Some other statistics are also available through the aggregate function in SPSS ${ }^{\circledR}$, such as the minimum, the maximum, the first, the last, the number of observations, and so on. Nevertheless, they are not included in the table, either because it does not make sense to apply these statistics on the PISA data, or else because the Fay's method cannot be applied on these statistics. For instance, as no weights are set to 0 in any replicates, the minimum or maximum value for a particular variable will always be the same. Therefore, the macro will return the value of 0 , which is meaningless.

Box 6.3 presents the syntax for the computation of the standard deviation per gender and Table 6.5 the structure of the output data base. 


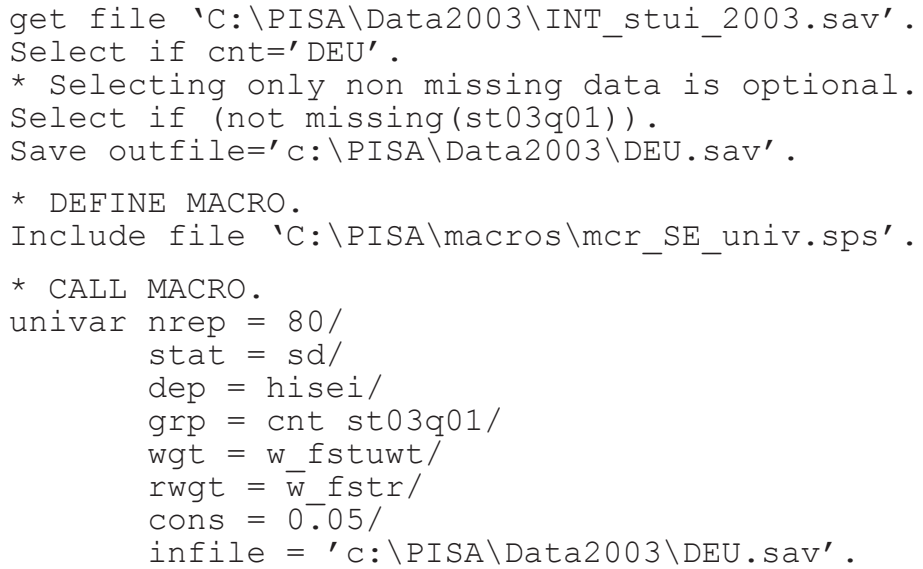

Table 6.5 - Structure of the output data file from Box $\mathbf{6 . 3}$

\begin{tabular}{c|c|c|c}
\hline CNT & ST03Q01 & STAT & SE \\
\hline DEU & 1 & 16.12 & 0.29 \\
\hline DEU & 2 & 16.34 & 0.23 \\
\hline
\end{tabular}

\section{THE STANDARD ERROR ON PERCENTAGES}

For variables such as gender, the statistic of interest is usually the percentage per category. The procedure for estimating the standard error is identical to the procedure used for the estimation of the standard error on a mean or a standard deviation, i.e. per category of the variable, 81 percentages have to be computed.

Box 6.4 presents the SPSS ${ }^{\circledR}$ syntax for running the macro that will compute the percentages and their respective standard errors for each category of the gender variable. The structure of the output data file is presented in Table 6.6.

\section{Box 6.4 $\square$ SPSS $^{\circledR}$ syntax for the computation of percentages and their respective standard error for gender}

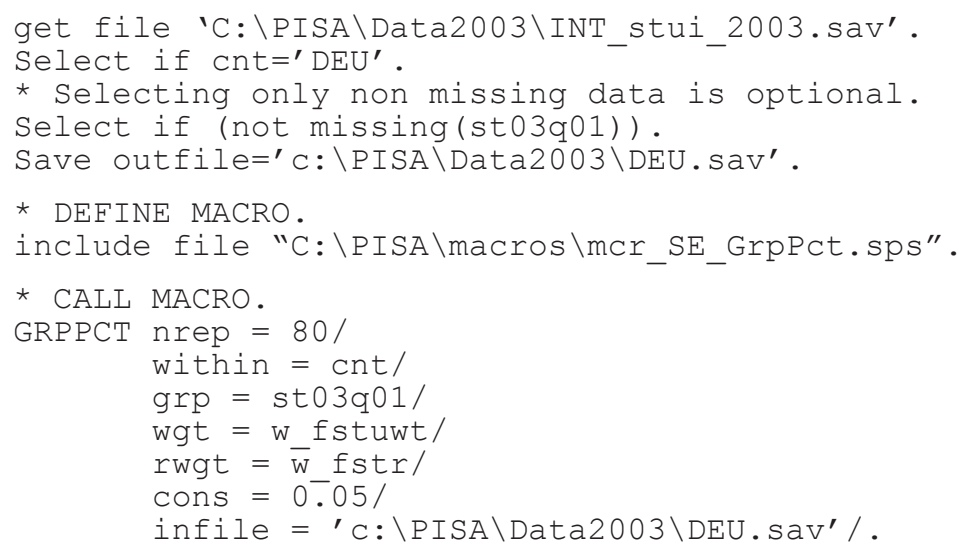


Table 6.6 Structure of the output data file from Box $\mathbf{6 . 4}$

\begin{tabular}{c|c|c|c}
\hline CNT & ST03Q01 & STAT & SE \\
\hline DEU & 1 & 49.66 & 1.04 \\
\hline DEU & 2 & 50.34 & 1.04 \\
\hline
\end{tabular}

Table 6.7 presents the estimates of the percentage of girls for the 81 weights and the squared differences. The percentage of girls that will be reported is equal to 49.66 , i.e. the percentage obtained with the final student weight.

As previously, there are three major steps for the computation of the standard error:

1. Each replicate estimate will be compared with the final estimate 49.66 and the difference will be squared. Mathematically, it corresponds to $\left(\hat{\pi}_{(i)}-\hat{\pi}\right)^{2}$. For the first replicate, it will be equal to: $(49.82-49.66)^{2}=0.0256$.

2. The sum of the squared differences is computed, and then divided by 20. Mathematically, it corresponds to $1 / 20 . \sum_{i=1}^{80}\left(\hat{\pi}_{(i)}-\hat{\pi}\right)^{2}$. In the example, the sum is equal to:

$(0.0252+0.1044+\ldots+0.3610+0.1313)=21.4412$

The sum divided by 20 is therefore equal to $\frac{21.4412}{20}=1.07206$. This value represents the sampling variance on the percentage estimate of girls.

3. The standard error is equal to the square root of the sampling variance, i.e.

$$
\sigma_{(\hat{\pi})}=\sqrt{\sigma_{(\hat{\pi})}^{2}}=\sqrt{1.07206}=1.035
$$

The same process can be used for the percentage of boys. It should be noted that the standard error for boys is equal to the one for girls. Indeed, it can be mathematically shown that the standard error on $\pi$ is equal to the standard error on $1-\pi$, i.e. $\sigma_{(p)}=\sigma_{(1-p)}$. Nevertheless, if missing data for gender are kept in the data file, the standard error on the percentage of boys can slightly differ from the standard error on the percentage of girls.

Just as for the macro for numerical variables, more than one breakdown variable can be used. In PISA 2003, the first question in the student questionnaire (ST01Q01) provides the students' grade. German 15-year-olds are distributed from grade 7 to grade 11.

Box 6.5 presents the $\operatorname{SPSS}^{\circledR}$ syntax and Table 6.8 presents the distribution of students per grade per gender. The percentages within the 'WITHIN' group variable add up to 100 per cent. In this example, the percentages of pupils in grades 7 to 11 within gender and country add up to 100 per cent. If 'WITHIN $=\mathrm{CNT}$ ' and 'GRP=ST03Q01 ST01Q01' the sum of the percentages of all ten groups within the country will be 100 per cent. 
Table 6.7 Percentage of girls for the final and replicate weights and squared differences

\begin{tabular}{|c|c|c|c|c|c|}
\hline Weight & $\begin{array}{c}\% \\
\text { Estimate }\end{array}$ & $\begin{array}{c}\text { Squared } \\
\text { difference }\end{array}$ & Weight & $\begin{array}{c}\% \\
\text { Estimate }\end{array}$ & $\begin{array}{c}\text { Squared } \\
\text { difference }\end{array}$ \\
\hline Final weight & 49.66 & & & & \\
\hline Replicate 1 & 49.82 & 0.03 & Replicate 41 & 50.00 & 0.11 \\
\hline Replicate 2 & 49.98 & 0.10 & Replicate 42 & 49.95 & 0.09 \\
\hline Replicate 3 & 49.44 & 0.05 & Replicate 43 & 49.70 & 0.00 \\
\hline Replicate 4 & 49.32 & 0.11 & Replicate 44 & 50.59 & 0.87 \\
\hline Replicate 5 & 49.39 & 0.07 & Replicate 45 & 49.07 & 0.35 \\
\hline Replicate 6 & 49.06 & 0.36 & Replicate 46 & 48.82 & 0.71 \\
\hline Replicate 7 & 48.59 & 1.14 & Replicate 47 & 49.88 & 0.05 \\
\hline Replicate 8 & 48.85 & 0.66 & Replicate 48 & 49.14 & 0.27 \\
\hline Replicate 9 & 49.06 & 0.36 & Replicate 49 & 49.53 & 0.02 \\
\hline Replicate 10 & 49.72 & 0.00 & Replicate 50 & 49.81 & 0.02 \\
\hline Replicate 11 & 50.05 & 0.15 & Replicate 51 & 49.87 & 0.04 \\
\hline Replicate 12 & 49.31 & 0.13 & Replicate 52 & 49.82 & 0.02 \\
\hline Replicate 13 & 49.29 & 0.13 & Replicate 53 & 49.42 & 0.06 \\
\hline Replicate 14 & 49.47 & 0.04 & Replicate 54 & 48.99 & 0.45 \\
\hline Replicate 15 & 49.90 & 0.06 & Replicate 55 & 50.07 & 0.17 \\
\hline Replicate 16 & 50.82 & 1.35 & Replicate 56 & 50.68 & 1.04 \\
\hline Replicate 17 & 49.11 & 0.30 & Replicate 57 & 50.34 & 0.46 \\
\hline Replicate 18 & 49.51 & 0.02 & Replicate 58 & 49.54 & 0.02 \\
\hline Replicate 19 & 49.79 & 0.02 & Replicate 59 & 48.75 & 0.83 \\
\hline Replicate 20 & 50.75 & 1.18 & Replicate 60 & 50.14 & 0.23 \\
\hline Replicate 21 & 50.24 & 0.33 & Replicate 61 & 49.45 & 0.05 \\
\hline Replicate 22 & 49.79 & 0.02 & Replicate 62 & 49.46 & 0.04 \\
\hline Replicate 23 & 49.87 & 0.04 & Replicate 63 & 50.11 & 0.20 \\
\hline Replicate 24 & 49.37 & 0.08 & Replicate 64 & 49.64 & 0.00 \\
\hline Replicate 25 & 49.50 & 0.02 & Replicate 65 & 49.72 & 0.00 \\
\hline Replicate 26 & 49.82 & 0.02 & Replicate 66 & 50.79 & 1.27 \\
\hline Replicate 27 & 49.92 & 0.07 & Replicate 67 & 49.73 & 0.00 \\
\hline Replicate 28 & 49.55 & 0.01 & Replicate 68 & 49.96 & 0.09 \\
\hline Replicate 29 & 50.22 & 0.31 & Replicate 69 & 50.31 & 0.42 \\
\hline Replicate 30 & 49.16 & 0.25 & Replicate 70 & 49.17 & 0.24 \\
\hline Replicate 31 & 50.51 & 0.73 & Replicate 71 & 50.10 & 0.19 \\
\hline Replicate 32 & 49.98 & 0.10 & Replicate 72 & 49.93 & 0.07 \\
\hline Replicate 33 & 50.67 & 1.02 & Replicate 73 & 49.55 & 0.01 \\
\hline Replicate 34 & 49.29 & 0.13 & Replicate 74 & 49.42 & 0.06 \\
\hline Replicate 35 & 48.96 & 0.49 & Replicate 75 & 49.60 & 0.00 \\
\hline Replicate 36 & 49.98 & 0.10 & Replicate 76 & 49.45 & 0.05 \\
\hline Replicate 37 & 50.23 & 0.33 & Replicate 77 & 49.80 & 0.02 \\
\hline Replicate 38 & 48.25 & 1.99 & Replicate 78 & 49.91 & 0.07 \\
\hline Replicate 39 & 49.56 & 0.01 & Replicate 79 & 49.06 & 0.36 \\
\hline Replicate 40 & 49.66 & 0.00 & Replicate 80 & 50.02 & 0.13 \\
\hline
\end{tabular}

\section{Box 6.5 - SPSS $^{\circledR}$ syntax for the computation of percentages of grade per gender}

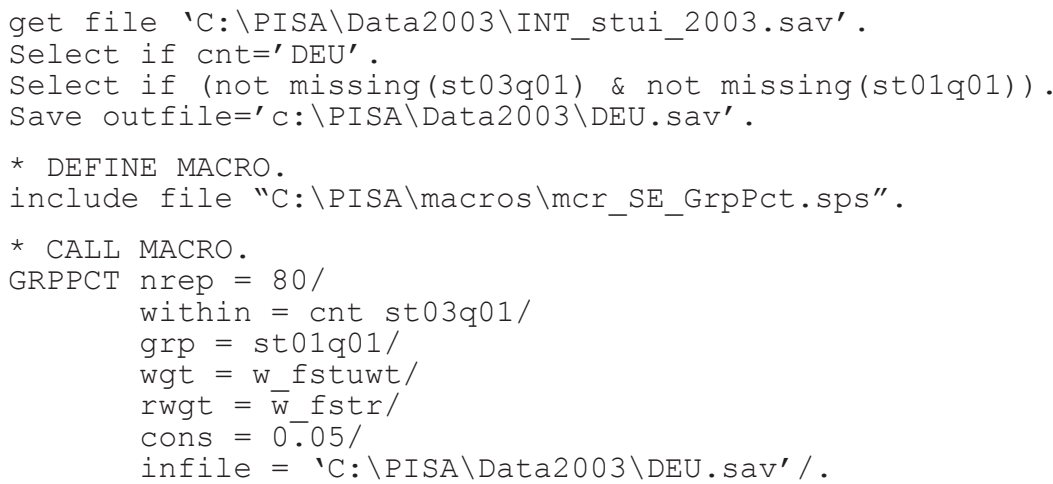


As shown in Table 6.8, more boys tend to be in lower grades than girls and more girls tend to be in upper grades in Germany.

Table 6.8 - Structure of the output data file from Box $\mathbf{6 . 5}$

\begin{tabular}{c|r|r|r|c}
\hline CNT & ST03Q01 & ST01Q01 & STAT & SE \\
\hline DEU & 1 & 7 & 1.15 & 0.26 \\
\hline DEU & 1 & 8 & 13.09 & 0.83 \\
\hline DEU & 1 & 9 & 59.33 & 1.00 \\
\hline DEU & 1 & 10 & 26.28 & 1.08 \\
\hline DEU & 1 & 11 & 0.17 & 0.08 \\
\hline DEU & 2 & 7 & 2.28 & 0.45 \\
\hline DEU & 2 & 8 & 16.92 & 1.04 \\
\hline DEU & 2 & 9 & 60.32 & 1.06 \\
\hline DEU & 2 & 10 & 20.41 & 0.79 \\
\hline DEU & 2 & 11 & 0.08 & 0.05 \\
\hline
\end{tabular}

\section{THE STANDARD ERROR ON REGRESSION COEFFICIENTS}

For any requested statistic, the computation of the estimate and its standard error will follow exactly the same procedure as the ones described for the mean of HISEI and for the percentage of girls. The remainder of this chapter will explain the use of two other SPSS ${ }^{\circledR}$ macros developed for analyzing PISA data.

The first macro is for simple linear regression analyses. Besides the arguments common to all SPSS ${ }^{\circledR}$ macros described in this manual, i.e. (i) $\mathrm{NREP}=$, (ii) $\mathrm{GRP}=$, (iii) W_FSTUWT=, (iv) W_FSTR=, (v) CONS $=$, and (vi) INFILE $=$, two arguments need to be specified: the dependent variable and the independent variables. Only one dependent variable can be specified, whereas several independent variables can be specified.

Box 6.6 provides the syntax for running the simple linear regression macro. In this example, the dependent variable is the expected student job at the age of 30 (BSMJ) and the independent variables are the family socio-economic index (HISEI) and the students' gender after recoding (GENDER). After a recoding of the gender variable into a dichotomous 0-1 variable, the macro is defined by the “include" statement.

\section{Box 6.6 $\operatorname{SPSS}^{\circledR}$ syntax for the regression analyses (1)}

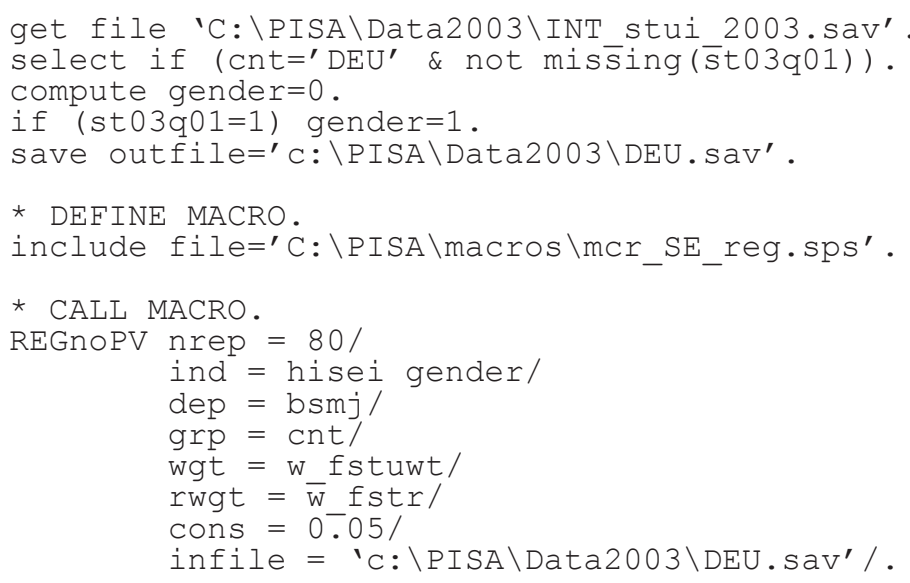


Table 6.9 presents the structure of the output data file of the regression analysis. ${ }^{1}$

Table 6.9 - Structure of the output data file from Box 6.6

\begin{tabular}{c|c|c|c}
\hline CNT & IND & STAT & SE \\
\hline DEU & $\mathrm{b}_{0}$ & 32.90 & 1.29 \\
\hline DEU & hisei & 0.37 & 0.03 \\
\hline DEU & gender & 2.07 & 0.62 \\
\hline
\end{tabular}

Where $b_{0}$ is the intercept and HISEI and GENDER are the slopes of the corresponding variables. To restructure the data so that every country (group) has one record in the data file, the commands from Box 6.7 can be applied (this command is not available in $\mathrm{SPSS}^{\circledR}$ versions older than SPSS ${ }^{\circledR} 11$ ). The structure of the data output file is displayed in Table 6.10.

Box 6.7 - SPSS $^{\circledR}$ syntax restructuring data after regression analyses

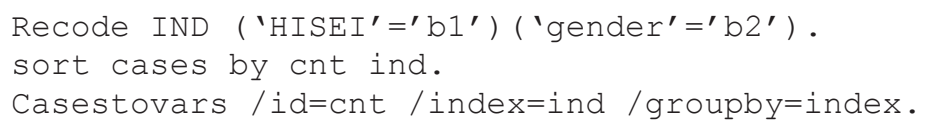

Table 6.10 - Restructure of the output data file

\begin{tabular}{c|c|c|c|c|c|c}
\hline CNT & STAT.B0 & SE.B0 & STAT.B1 & SE.B1 & STAT.B2 & SE.B2 \\
\hline DEU & 32.90 & 1.29 & 0.37 & 0.03 & 2.07 & 0.62 \\
\hline
\end{tabular}

More complex macros like this one for calculating SEs for regression coefficients sometimes cause errors that are not always visible. These errors occur especially when the same macro is run more than once within the same SPSS ${ }^{\circledR}$ session. It is worthwhile to always test the results from the macro by running the analysis outside the macro using the full student weight and check if the regression coefficients (or any other statistics) are correct. If they are not the same, the best step is to turn off $\mathrm{SPSS}^{\circledR}$, and often even to log off and on again. Deleting all files in 'C: $\backslash$ Temp $\backslash$ ' will also sometimes solve the problem.

There are two ways to determine whether the regression coefficients are significantly different from 0 . The first method consists of building a confidence interval around the estimated regression coefficient. The confidence interval for the GENDER regression coefficient on BSMJ can be computed for a value of $\alpha$ equal to 0.05 as:

$[2.07-(1.96 * 0.62) ; 2.07+(1.96 * 0.62)]=[0.85 ; 3.29]$

As the value 0 is not included in this confidence interval, the regression coefficient is significantly different from 0 . As the value 0 was assigned to the boys and the value 1 to the girls, it means that on average, girls have significantly higher job expectations.

Another way to test the null hypothesis of the regression coefficient consists of dividing the regression coefficient by its standard error. This procedure will standardize the regression coefficient. It also means that the sampling distribution of the standardized regression coefficient, under the null hypothesis, has an expected mean of 0 and a standard deviation of 1 . Therefore, if the ratio of 
the regression coefficient to its standard error is lower than -1.96 or higher than 1.96 , it will be considered as significantly different from 0 .

It should be mentioned that the syntax in Box 6.8 will provide different results from Box 6.6. In Box 6.6, GENDER is considered as an explanatory variable, whereas in Box 6.8, GENDER is used as a breakdown variable. In the second model, there is only one explanatory variable, i.e. HISEI.

\section{Box 6.8 - SPSS $^{\circledR}$ syntax for the regression analyses (2)}

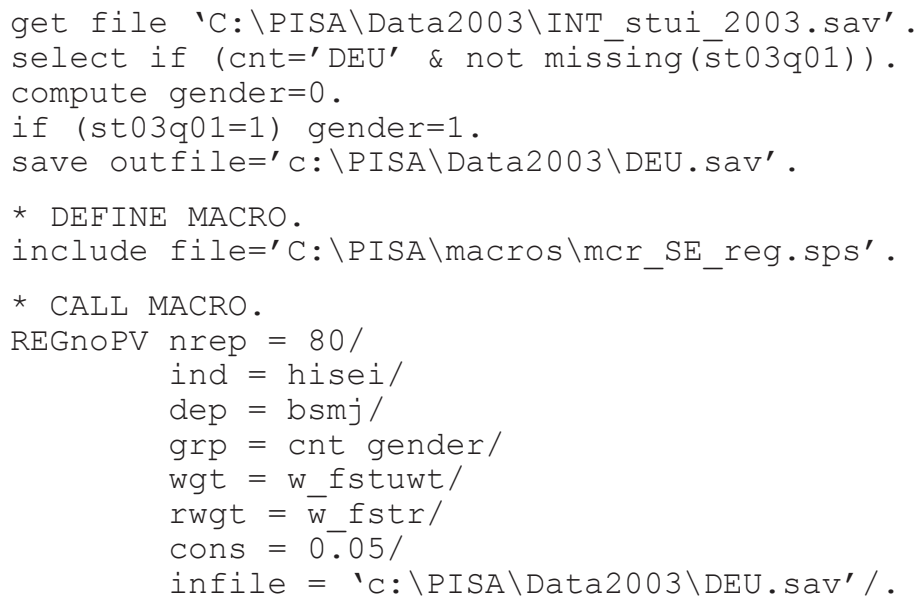

Table 6.11 presents the structure of the output data file for the second model.

Table 6.11 - Structure of the output data file from Box 6.8

\begin{tabular}{c|c|c|c|c}
\hline CNT & GENDER & IND & STAT & SE \\
\hline DEU & 0 & $\mathrm{~b}_{0}$ & 32.54 & 1.44 \\
\hline DEU & 0 & HISEI & 0.37 & 0.03 \\
\hline DEU & 1 & $\mathrm{~b}_{0}$ & 35.33 & 1.66 \\
\hline DEU & 1 & HISEI & 0.36 & 0.03 \\
\hline
\end{tabular}

\section{THE STANDARD ERROR ON CORRELATION COEFFICIENTS}

Table 6.12 and Box 6.9 present, respectively, the SPSS ${ }^{\circledR}$ syntax and the structure of the output data file for the macro devoted to the computation of a correlation between two variables. Note that cases with missing values for either of the variables are deleted by the macro from the data file to apply listwise deletion of missing values.

Table 6.12 - Structure of the output data file from Box 6.9

\begin{tabular}{c|c|c} 
CNT & STAT & SE \\
\hline DEU & 0.34 & 0.02 \\
\hline
\end{tabular}




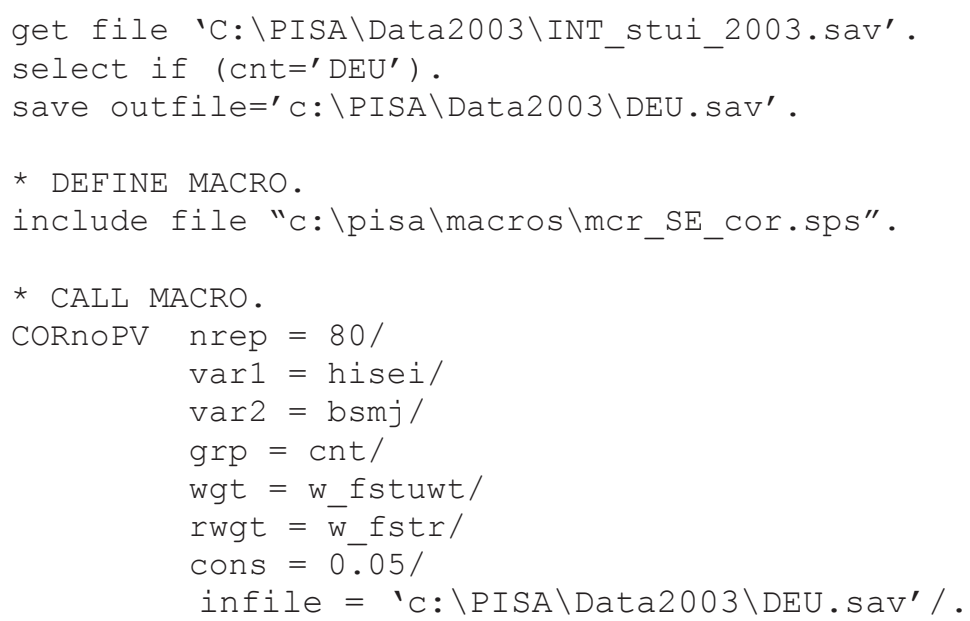

\section{CONCLUSIONS}

This chapter described the computation of the standard error by using the 80 replicates. For any given statistic, the procedure is the same.

Further, by using examples, the SPSS ${ }^{\circledR}$ syntax for running the SPSS $^{\circledR}$ macros, developed to facilitate the computation of the standard errors, has been provided.

However, none of the macros described in this chapter can be used if plausible values are included in the analyses. Chapter 7 will describe how to proceed with such variables.

1. SPSS $^{\circledR}$ produces many tables in the output viewer when replicating the regression 80 times. This slows down the computer and makes the computer unavailable for other tasks as long as the macro is running. SPSS ${ }^{\circledR} 12$ has introduced an Output Management System (OMS), which gives the user, among other output control, the opportunity to choose what is printed in the output. Adding the command OMS / select tables warnings headings /destination viewer=no. before calling the macro will prevent SPSS ${ }^{\circledR}$ printing headings, tables, logs and warnings into the output viewer. This will be active until the OMS is turned off by the command OMSEND. 



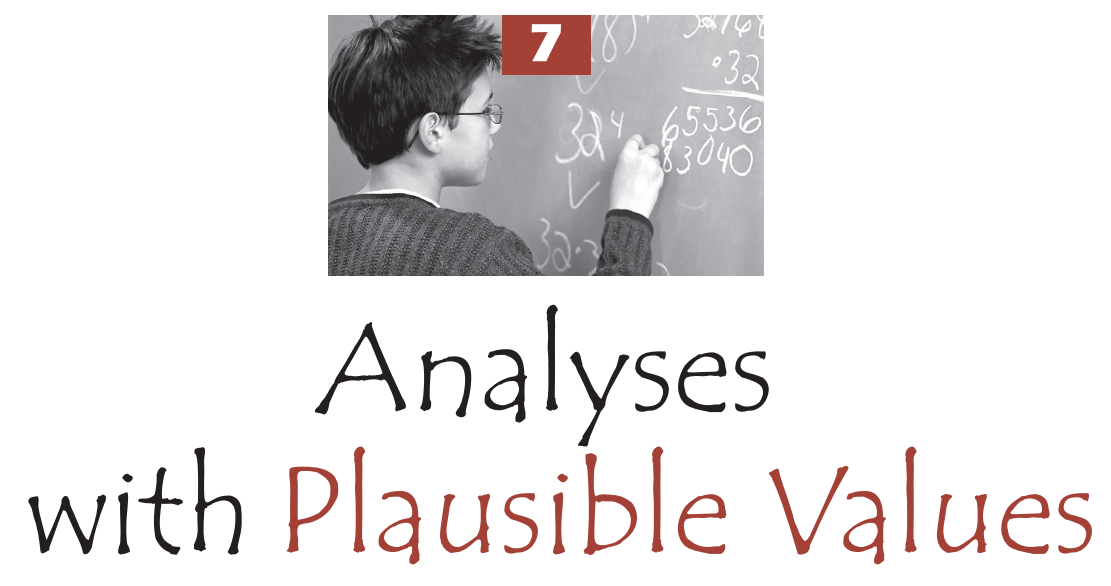

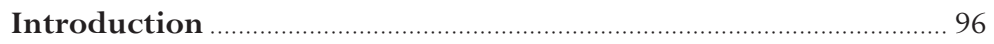

Univariate statistics on plausible values …………………………....... 96

The standard error on percentages with plausible values ……...... 101

The standard error on regression coefficients

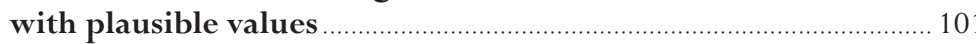

The standard error on correlation coefficients

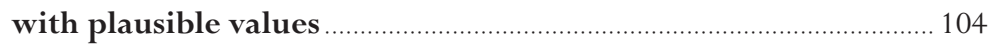

Correlation between two sets of plausible values........................... 104

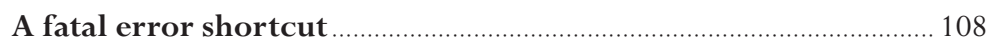

An unbiased shortcut .................................................................. 109

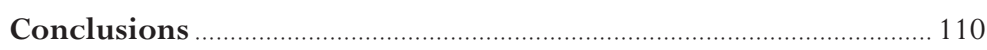




\section{INTRODUCTION}

Mathematics literacy was the major domain in PISA 2003, while reading, science, and problem solving were minor domains. One scale was created for each minor domain, while five scales were generated for the mathematics assessment: a mathematics scale and four subscales (space and shape, change and relationships, quantity, and uncertainty).

As described in Chapter 5 , these cognitive data were scaled with the Rasch model and the performance of students was denoted with plausible values. For each scale and subscale, five plausible values per student were included in the international databases. This chapter describes how to perform analyses with plausible values (PVs).

Since PVs were mainly used for reporting student performance on the cognitive test, this chapter is only useful when conducting analyses on achievement data and their relationships with student or school characteristics.

\section{UNIVARIATE STATISTICS ON PLAUSIBLE VALUES}

The computation of a statistic on plausible values will always consist of six steps, regardless of the required statistic.

1. The required statistic and its respective standard error have to be computed for each plausible value. In Chapter 6, it was mentioned that 81 estimates were necessary to get the final estimate and its standard error. Therefore, any analysis that involves five plausible values will require 405 estimates. If a mean needs to be estimated, then 405 means will be calculated. The means estimated with the final weight are denoted $\hat{\mu}_{1}, \hat{\mu}_{2}, \hat{\mu}_{3}, \hat{\mu}_{4}$ and $\hat{\mu}_{5}$. From the 80 replicates applied on each of the five plausible values, five sampling variances are estimated, denoted respectively $\sigma_{\left(\hat{\mu}_{1}\right)}^{2}, \sigma_{\left(\hat{\mu}_{2}\right)}^{2}, \sigma_{\left(\hat{\mu}_{3}\right)}^{2}, \sigma_{\left(\hat{\mu}_{4}\right)}^{2}$ and $\sigma_{\left(\hat{\mu}_{5}\right)}^{2}$. These five mean estimates and their respective sampling variances are given in Table 7.1.

2. The final mean estimate is equal to the average of the five mean estimates, i.e.

$$
\hat{\mu}=\frac{1}{5}\left(\hat{\mu}_{1}+\hat{\mu}_{2}+\hat{\mu}_{3}+\hat{\mu}_{4}+\hat{\mu}_{5}\right)
$$

3. The final sampling variance is equal to the average of the five sampling variances, i.e.

$$
\sigma_{(\hat{\mu})}^{2}=\frac{1}{5}\left(\sigma_{\left(\hat{\mu}_{1}\right)}^{2}+\sigma_{\left(\hat{\mu}_{2}\right)}^{2}+\sigma_{\left(\hat{\mu}_{3}\right)}^{2}+\sigma_{\left(\hat{\mu}_{4}\right)}^{2}+\sigma_{\left(\hat{\mu}_{5}\right.}^{2}\right.
$$

4. The imputation variance, also denoted measurement error variance, is computed as $\sigma_{(\text {test })}^{2}=\frac{1}{4} \sum_{i=1}^{5}\left(\hat{\mu}_{i}-\hat{\mu}\right)^{2}$. Indeed, as PISA returns five plausible values per scale, then $\sigma_{(\text {test })}^{2}=\frac{1}{M-1} \sum_{i=1}^{M}\left(\hat{\mu}_{i}-\hat{\mu}\right)^{2}=\frac{1}{4} \sum_{i=1}^{3}\left(\hat{\mu}_{i}-\hat{\mu}\right)$. This formula is similar to the one used for the estimation of a population variance, except that in this particular case, observations are not compared with the population mean, but each PV mean is compared with the final mean estimate.

5. The sampling variance and the imputation variance are combined to obtain the final error variance as $\sigma_{(\text {error })}^{2}=\sigma_{(\hat{\mu})}^{2}+\left(1.2 \sigma_{(\text {test })}^{2}\right)$.

Indeed, $\sigma_{(\text {error })}^{2}=\sigma_{(\hat{\mu})}^{2}+\left(\left(1+\frac{1}{M}\right) \sigma_{(\text {test })}^{2}\right)=\sigma_{(\hat{\mu})}^{2}+\left(\left(1+\frac{1}{5}\right) \sigma_{(\text {test })}^{2}\right)=\sigma_{(\hat{\mu})}^{2}+\left((1.2) \sigma_{(\text {test })}^{2}\right)$

6. The standard error is equal to the square root of the error variance. 
Table 7.1 - The 405 mean estimates

\begin{tabular}{|c|c|c|c|c|c|}
\hline Weight & PV1 & PV2 & PV3 & PV4 & PV5 \\
\hline Final & $\hat{\mu}_{1}$ & $\hat{\boldsymbol{\mu}}_{2}$ & $\hat{\mu}_{3}$ & $\hat{\boldsymbol{\mu}}_{4}$ & $\hat{\mu}_{5}$ \\
\hline Replicate 1 & $\hat{\mu}_{1 \_1}$ & $\hat{\boldsymbol{\mu}}_{2 \_1}$ & $\hat{\boldsymbol{\mu}}_{3-1}$ & $\hat{\boldsymbol{\mu}}_{4-1}$ & $\hat{\boldsymbol{\mu}}_{5 \_1}$ \\
\hline Replicate 2 & $\hat{\mu}_{1 \_2}$ & $\hat{\boldsymbol{\mu}}_{2{ }_{2} 2}$ & $\hat{\boldsymbol{\mu}}_{3 \_2}$ & $\hat{\boldsymbol{\mu}}_{4 \_2}$ & $\hat{\boldsymbol{\mu}}_{5-2}$ \\
\hline Replicate 3 & $\hat{\mu}_{1-3}$ & $\hat{\boldsymbol{\mu}}_{2 \_3}$ & $\hat{\boldsymbol{\mu}}_{3 \_3}$ & $\hat{\boldsymbol{\mu}}_{4-3}$ & $\hat{\boldsymbol{\mu}}_{5-3}$ \\
\hline ........... & n........ & ............ & ............ & ........... & ............ \\
\hline ............ & ............ & ............ & ............ & ............ & ............ \\
\hline Replicate 80 & $\hat{\mu}_{1 \_80}$ & $\hat{\mu}_{2 \_80}$ & $\hat{\mu}_{3 \_80}$ & $\hat{\boldsymbol{\mu}}_{4 \_80}$ & $\hat{\boldsymbol{\mu}}_{5 \_80}$ \\
\hline $\begin{array}{l}\text { Sampling } \\
\text { variance }\end{array}$ & $\sigma_{\left(\hat{\mu}_{1}\right)}^{2}$ & $\sigma_{\left(\hat{\mu}_{2}\right)}^{2}$ & $\sigma_{\left(\hat{\mu}_{3}\right)}^{2}$ & $\sigma_{\left(\hat{\mu}_{4}\right)}^{2}$ & $\sigma_{\left(\hat{\mu}_{5}\right)}^{2}$ \\
\hline
\end{tabular}

The mean estimate of the mathematics scale and its respective standard error for the PISA 2003 German data can be computed. The macro described in Chapter 6 and labeled MCR_SE_UNIV.SPS can be sequentially used five times and the results can be combined in an Microsoft ${ }^{\circledR}$ Excel $^{\circledR}$ spreadsheet. Table 7.2 presents the different PV means and their respective sampling variances, as well as the mean estimates on the first and last replicates.

Table 7.2 - Mean estimates and their respective sampling variances on the mathematics scale for Germany

\begin{tabular}{|c|c|c|c|c|c|}
\hline Weight & PV1 & PV2 & PV3 & PV4 & PV5 \\
\hline Final & 503.08 & 503.10 & 502.72 & 503.03 & 503.00 \\
\hline Replicate 1 & 503.58 & 504.16 & 503.43 & 503.96 & 503.94 \\
\hline ........... & ........... & ........... & ........... & ........... & ........... \\
\hline Replicate 80 & 503.18 & 503.62 & 503.46 & 503.30 & 503.83 \\
\hline $\begin{array}{l}\text { Sampling } \\
\text { variance }\end{array}$ & $(3.34)^{2}$ & $(3.27)^{2}$ & $(3.36)^{2}$ & $(3.28)^{2}$ & $(3.32)^{2}$ \\
\hline
\end{tabular}

Box 7.1 presents the SPSS ${ }^{\circledR}$ syntax for running sequentially the MCR_SE_UNIV.SPS macro described in Chapter 6.

The final mean estimate for Germany on the mathematics scale is equal to ( $\mu_{1}=$ stat1, etc. in the data file):

$$
\begin{aligned}
& \hat{\mu}=\frac{1}{5}\left(\hat{\mu}_{1}+\hat{\mu}_{2}+\hat{\mu}_{3}+\hat{\mu}_{4}+\hat{\mu}_{5}\right), \text { i.e. } \\
& \hat{\mu}=\frac{(503.08+503.10+502.72+503.03+503.00)}{5}=502.99
\end{aligned}
$$


The final sampling variance on the mean estimate for the mathematics literacy scale is equal to $\left(\sigma_{(\mu 1)}=\right.$ se1, etc. in the data file $)$ :

$$
\begin{aligned}
& \sigma_{(\hat{\mu})}^{2}=\frac{1}{5}\left(\sigma_{\left(\hat{\mu}_{1}\right)}^{2}+\sigma_{\left(\hat{\mu}_{2}\right)}^{2}+\sigma_{\left(\hat{\mu}_{3}\right)}^{2}+\sigma_{\left(\hat{\mu}_{4}\right)}^{2}+\sigma_{\left(\hat{\mu}_{5}\right)}^{2}\right), \text { i.e. } \\
& \sigma_{(\hat{\mu})}^{2}=\frac{(3.34)^{2}+(3.27)^{2}+(3.36)^{2}+(3.28)^{2}+(3.32)^{2}}{5}=10.98
\end{aligned}
$$

The imputation variance is equal to:

$$
\begin{aligned}
& \sigma_{(\text {test })}^{2}=\frac{1}{4} \sum_{i=1}^{5}\left(\hat{\mu}_{i}-\hat{\mu}\right)^{2}, \text { i.e. } \\
& \sigma_{\text {(test) }}^{2}=\frac{\left\lfloor(503.08-502.99)^{2}+(503.10-502.99)^{2}+\ldots+(503.00-502.99)^{2}\right\rfloor}{4}=\frac{0.09}{4}=0.02
\end{aligned}
$$

The final error variance is equal to:

$$
\begin{aligned}
& \sigma_{(\text {error })}^{2}=\sigma_{(\hat{\mu})}^{2}+\left(1.2 \sigma_{(\text {test })}^{2}\right)_{\text {,i.e. }} \\
& \sigma_{(\text {error })}^{2}=10.98+(1.2 * 0.02)=11.00
\end{aligned}
$$

The final standard error is therefore equal to:

$$
S E=\sqrt{\sigma_{(\text {error })}^{2}}=\sqrt{11.00}=3.32
$$

\section{Box 7.1 - SPSS ${ }^{\circledR}$ syntax for computing the mean on the mathematics scale}

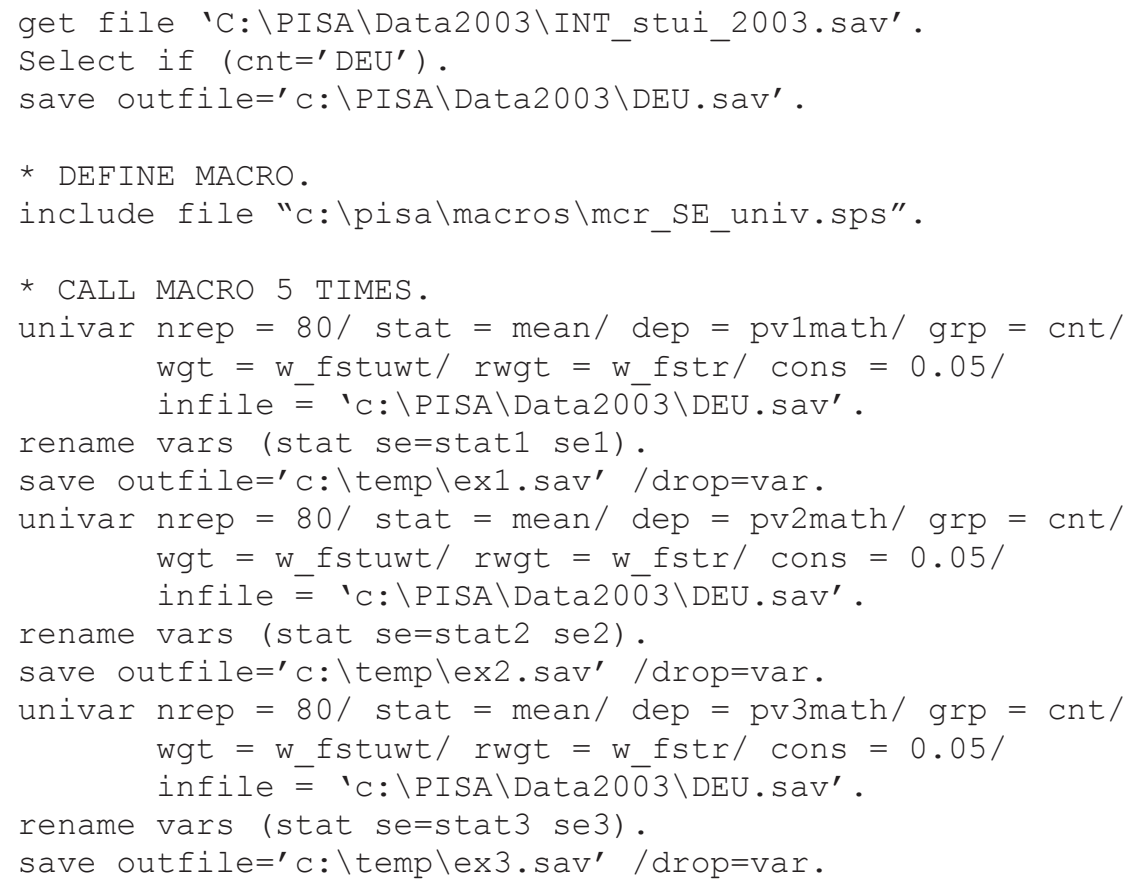




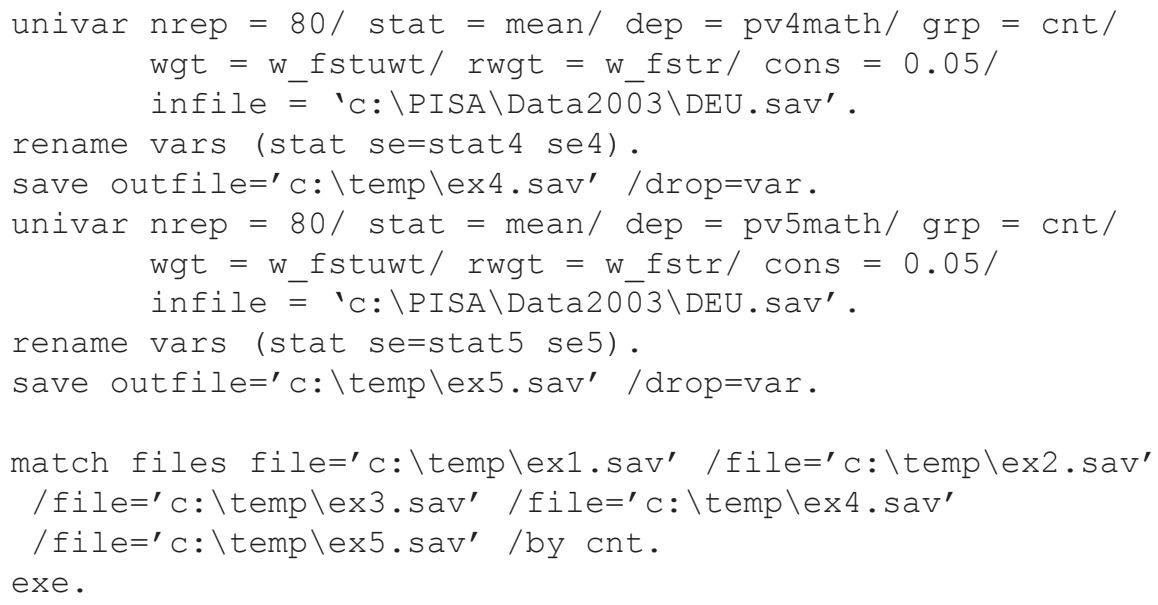

Running sequentially the UNIVAR macro five times and combining the results can be avoided: an $\mathrm{SPSS}^{\circledR}$ macro has been developed for dealing with PVs. This macro also computes:

- The five mean estimates (STAT1 to STAT5).

- The final estimate (STAT).

- The five sampling variances (VAR1 to VAR5).

- The mean of the five sampling variances (PV_VAR).

- The imputation variance (PVMERR).

- The final standard error by combining the final sampling variance and the imputation variance (SE).

\section{Box 7.2 - SPSS $^{\circledR}$ syntax for computing the mean and its standard error on plausible values}

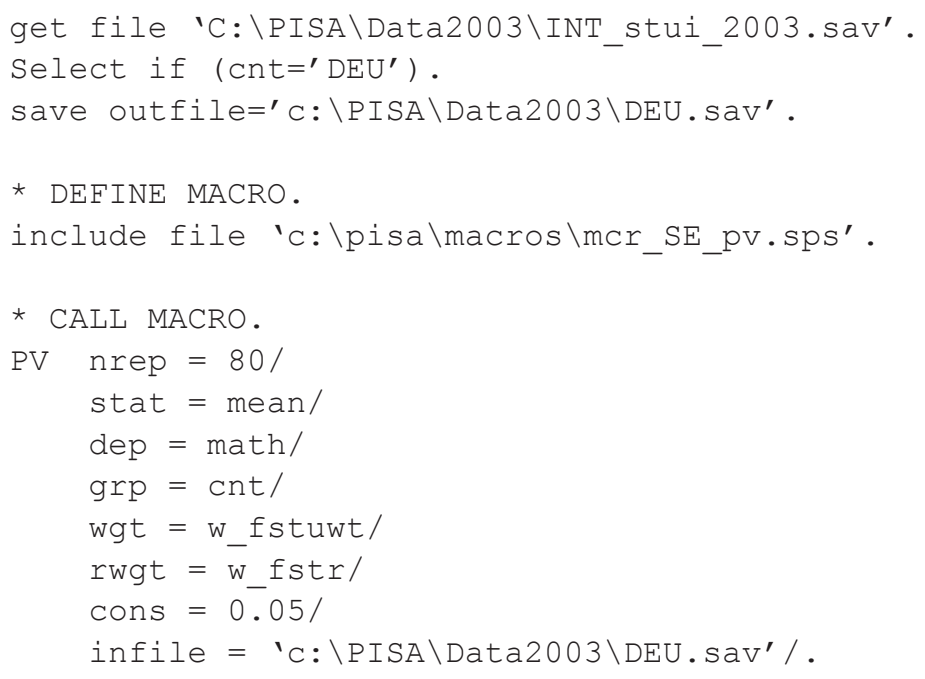


The arguments are identical to the arguments in the macro for univariate statistics without PV's described in Chapter 6. The difference is the description of the 'DEP' argument. Only the root of the variable is needed (the dimension), the macro will concatinate the root with 'pv1' to 'pv5'. That is, when 'DEP $=$ READ' the macro will read in the variables pv1read to pv5read. When 'DEP=MATH1', the macro will read in pv1math1 to pv5math1 and therefore compute the statistics for the first subscale of mathematics.

The structure of the output data file is presented in Table 7.3.

Table 7.3 - Structure of the output data file from Box $\mathbf{7 . 2}$

\begin{tabular}{c|c|c}
\hline CNT & STAT & SE \\
\hline DEU & 502.99 & 3.32 \\
\hline
\end{tabular}

Similar to the $\operatorname{SPSS}^{\circledR}$ macros described in the previous chapter, more than one breakdown variable can be used. For instance, if one wants to determine whether the dispersion of mathematics performance is larger for girls than for the boys, the macro PV can be used as follows:

Box 7.3 - SSPS syntax for computing the standard deviation and its standard error on plausible values per gender

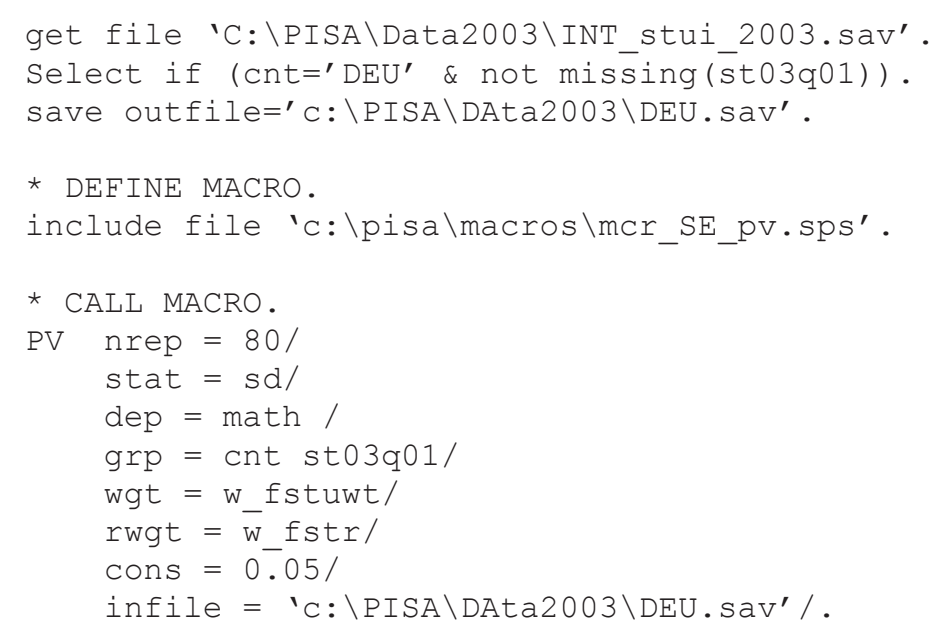

The structure of the output data file is presented in Table 7.4.

Table 7.4 @ Structure of the output data file from Box $\mathbf{7 . 3}$

\begin{tabular}{c|c|c|c}
\hline CNT & ST03Q01 & STAT & SE \\
\hline DEU & 1 & 99.29 & 2.05 \\
\hline DEU & 2 & 105.05 & 2.54 \\
\hline
\end{tabular}

According to Table 7.4, the standard deviation ('STAT') is larger for boys than for girls. Unfortunately, as it will be explained in Chapter 10, these two standard errors ('SE') cannot be used to test the equality of the two standard deviation coefficients, since the standard deviation estimates for boys and girls may be correlated. 


\section{THE STANDARD ERROR ON PERCENTAGES WITH PLAUSIBLE VALUES}

The second macro, first presented in Chapter 6, was developed for the computation of percentages and their respective standard errors. Chapter 8 will deal with applying this macro to plausible values: an entire chapter needs to be devoted to this type of analyses because of the issues involved.

\section{THE STANDARD ERROR ON REGRESSION COEFFICIENTS WITH PLAUSIBLE VALUES}

Suppose that the statistical effect of gender and student socio-economic background on the performance in mathematics needs to be estimated. Just like estimating a mean, this question can be solved by sequentially applying five times the macro REGNOPV described in Chapter 6 .

Box 7.4 presents a SPSS ${ }^{\circledR}$ syntax for such an approach.

\section{Box 7.4 - SPSS $^{\circledR}$ syntax for computing regression coefficients and their standard} errors on plausible values

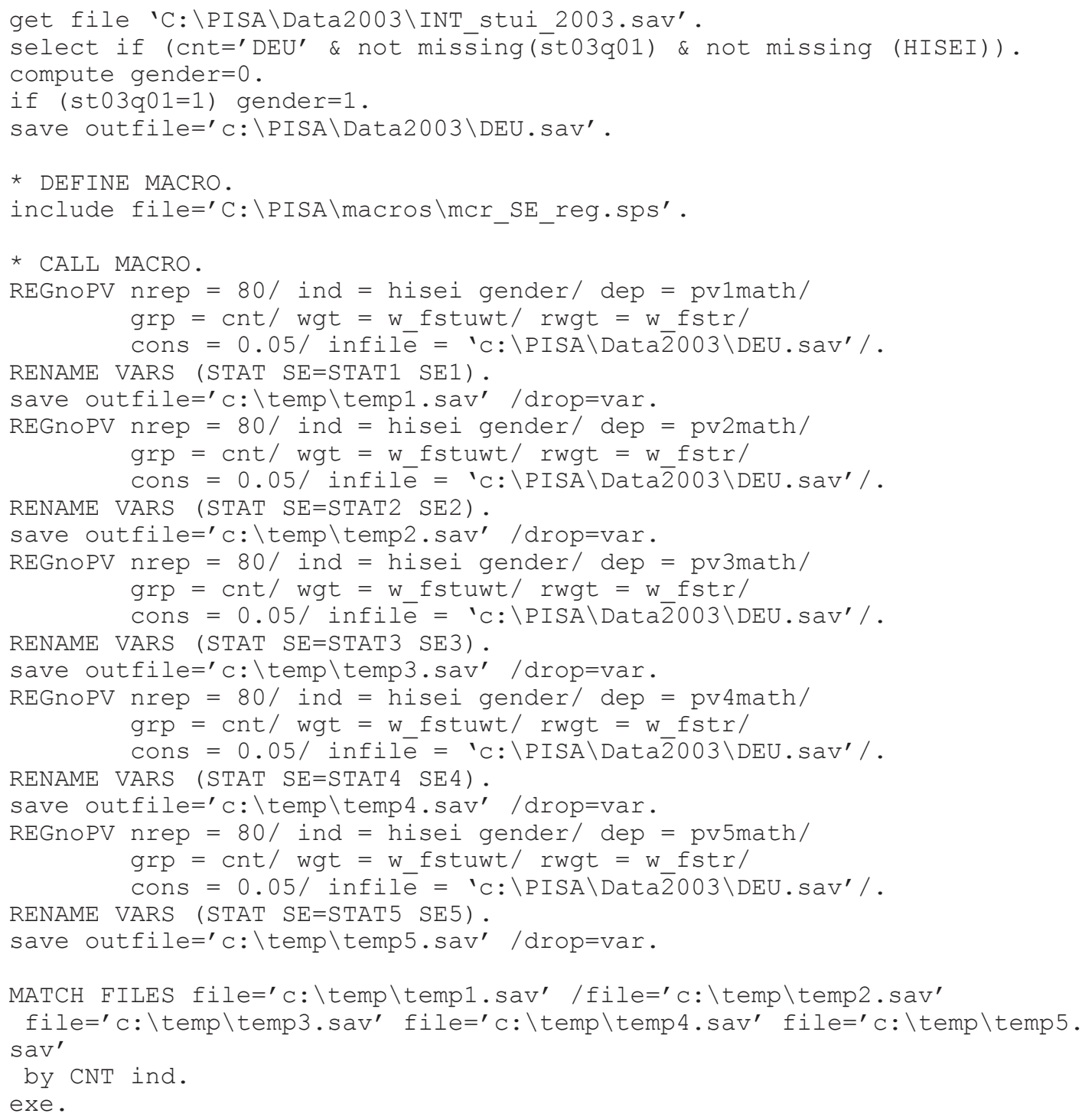


Just like the computation of a mean and its standard error, the computation of regression coefficients and their respective standard errors will consist of six steps:

1. For each plausible value and for each explanatory variable, computation of regression coefficients with the final and the 80 replicate weights. 405 regression coefficients per explanatory variable will be computed. The SPSS ${ }^{\circledR}$ macro REGNOPV applied sequentially five times will return, per explanatory variable, five estimates, denoted $\hat{\beta}_{1}, \ldots, \hat{\beta}_{5}$ and five standard errors, denoted $\sigma_{\left(\hat{\beta}_{1}\right)}, \ldots$, $\sigma_{\left(\hat{\beta}_{5}\right)}$. Table 7.5 gives the mathematical expression for these 405 estimates and Table 7.6 gives some of the values for the 405 regression coefficients obtained on the German data for the HISEI variable.

2. The final regression coefficient estimate is equal to $\hat{\beta}=\frac{\hat{\boldsymbol{\beta}}_{1}+\hat{\boldsymbol{\beta}}_{2}+\hat{\boldsymbol{\beta}}_{3}+\hat{\boldsymbol{\beta}}_{4}+\hat{\boldsymbol{\beta}}_{5}}{5}$, i.e. for HISEI $\hat{\beta}=\frac{2.30+2.27+2.26+2.31+2.34}{5}=2.30$

3. The final sampling variance estimate is equal to:

$$
\begin{aligned}
& \sigma_{(\hat{\beta})}^{2}=\frac{1}{5}\left(\sigma_{\left(\hat{\beta}_{1}\right)}^{2}+\sigma_{\left(\hat{\beta}_{2}\right)}^{2}+\sigma_{\left(\hat{\beta}_{3}\right)}^{2}+\sigma_{\left(\hat{\beta}_{4}\right)}^{2}+\sigma_{\left(\hat{\beta}_{5}\right)}^{2}\right) \text {, i.e. for HISEI } \\
& \sigma_{(\hat{\beta})}^{2}=\frac{(0.11)^{2}+(0.11)^{2}+(0.11)^{2}+(0.11)^{2}+(0.11)^{2}}{5}=0.012
\end{aligned}
$$

4. The imputation variance is equal to $\sigma_{(\text {test })}^{2}=\frac{1}{4} \sum_{i=1}^{5}\left(\hat{\boldsymbol{\beta}}_{i}-\hat{\boldsymbol{\beta}}\right)^{2}$, i.e. for HISEI

$$
\sigma_{(\text {test })}^{2}=\frac{(2.30-2.30)^{2}+(2.27-2.30)^{2}+\ldots+(2.34-2.30)^{2}}{4}=\frac{0.0041}{4}=0.001
$$

5. The error variance is equal to $\sigma_{(\text {error })}^{2}=\sigma_{(\hat{\beta})}^{2}+\left(1.2 \sigma_{(\text {test })}^{2}\right)$, i.e. for HISEI

$$
\sigma_{(\text {error })}^{2}=0.01248+(1.2 * 0.001)=0.01368
$$

\begin{tabular}{|c|c|c|c|c|c|}
\hline Weight & PV1 & PV2 & PV3 & PV4 & PV5 \\
\hline Final & $\hat{\beta}_{1}$ & $\hat{\beta}_{2}$ & $\hat{\beta}_{3}$ & $\hat{\beta}_{4}$ & $\hat{\beta}_{5}$ \\
\hline Replicate 1 & $\hat{\beta}_{1_{-1}}$ & $\hat{\beta}_{2-1}$ & $\hat{\beta}_{3 \_1}$ & $\hat{\beta}_{4-1}$ & $\hat{\beta}_{5-1}$ \\
\hline Replicate 2 & $\hat{\beta}_{1 \_2}$ & $\hat{\beta}_{2 \_2}$ & $\hat{\beta}_{3 \_2}$ & $\hat{\beta}_{4 \_2}$ & $\hat{\beta}_{5-2}$ \\
\hline Replicate 3 & $\hat{\beta}_{1 \_3}$ & $\hat{\beta}_{2-3}$ & $\hat{\beta}_{3 \_3}$ & $\hat{\beta}_{4-3}$ & $\hat{\beta}_{5 \_3}$ \\
\hline ........... & ........... & ........... & ........... & ........... & ........... \\
\hline .......... & ........... & .......... & .......... & .......... & ........... \\
\hline Replicate 80 & $\hat{\boldsymbol{\beta}}_{1 \_80}$ & $\hat{\boldsymbol{\beta}}_{2 \_80}$ & $\hat{\beta}_{3 \_80}$ & $\hat{\boldsymbol{\beta}}_{4 \_80}$ & $\hat{\boldsymbol{\beta}}_{5 \_80}$ \\
\hline $\begin{array}{l}\text { Sampling } \\
\text { variance }\end{array}$ & $\sigma_{\left(\hat{\beta}_{1}\right)}^{2}$ & $\sigma_{\left(\hat{\beta}_{2}\right)}^{2}$ & $\sigma_{\left(\hat{\beta}_{3}\right)}^{2}$ & $\sigma_{\left(\hat{\beta}_{4}\right)}^{2}$ & $\sigma_{\left(\hat{\beta}_{5}\right)}^{2}$ \\
\hline
\end{tabular}

6. The standard error is equal to $S E=\sqrt{\sigma_{(\text {error })}^{2}}=\sqrt{0.01368}=0.117$

As 2.30 divided by 0.117 is 19.66 , the regression coefficient for HISEI is significantly different from 0 .

\section{Table 7.5 - The $\mathbf{4 0 5}$ regression coefficient estimates}


Table 7.6 - HISEI regression coefficient estimates and their respective sampling variance on the mathematics literacy scale for Germany after controlling for gender

\begin{tabular}{|c|c|c|c|c|c|}
\hline Weight & PV1 & PV2 & PV3 & PV4 & PV5 \\
\hline Final & 2.30 & 2.27 & 2.26 & 2.31 & 2.34 \\
\hline Replicate 1 & 2.31 & 2.30 & 2.31 & 2.33 & 2.35 \\
\hline ............ & n.......... & ............ & n.......... & n.......... & ............. \\
\hline Replicate 80 & 2.24 & 2.21 & 2.21 & 2.23 & 2.27 \\
\hline $\begin{array}{l}\text { Sampling } \\
\text { variance }\end{array}$ & $(0.11)^{2}$ & $(0.11)^{2}$ & $(0.11)^{2}$ & $(0.11)^{2}$ & $(0.11)^{2}$ \\
\hline
\end{tabular}

An SPSS $^{\circledR}$ macro has also been developed for regression analyses with plausible values as dependent variables. The $\operatorname{SPSS}^{\circledR}$ syntax is presented in Box 7.5.

\section{Box 7.5 \&PSS ${ }^{\circledR}$ syntax for running the simple linear regression macro with PVs}

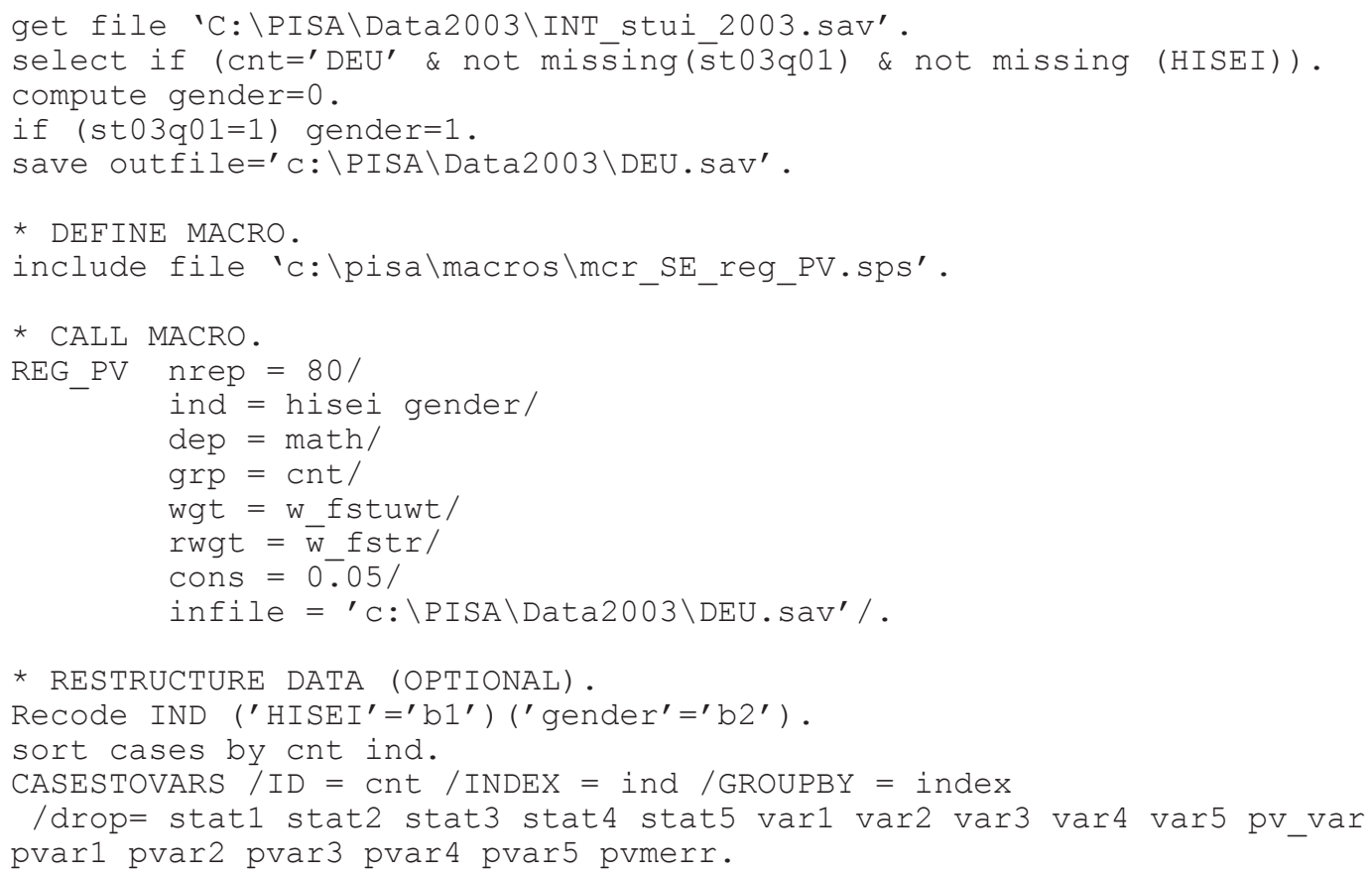

Besides the arguments common to all macros, the root of the plausible value variable names has to be specified as well as the list of independent variables. The structure of the output data file is presented in Table 7.7 .

Table 7.7 - Structure of the output data file from Box 7.5

\begin{tabular}{c|c|c|c}
\hline CNT & CLASS & STAT & SE \\
\hline DEU & $\mathrm{b}_{0}$ & 409.20 & 7.22 \\
\hline DEU & hisei & 2.30 & 0.117 \\
\hline DEU & gender & -13.83 & 3.56 \\
\hline
\end{tabular}


A quick overview of these results shows that all regression parameters are significantly different from 0 .

\section{THE STANDARD ERROR ON CORRELATION COEFFICIENTS WITH PLAUSIBLE VALUES}

An SPSS ${ }^{\circledR}$ macro has also been developed for computing the correlation between a set of plausible values and another variable. The SPSS ${ }^{\circledR}$ syntax for running this macro is presented in Box 7.6 and the structure of the output data file is presented in Table 7.8.

Box 7.6 - SPSS $^{\circledR}$ syntax for running the correlation macro with PVs

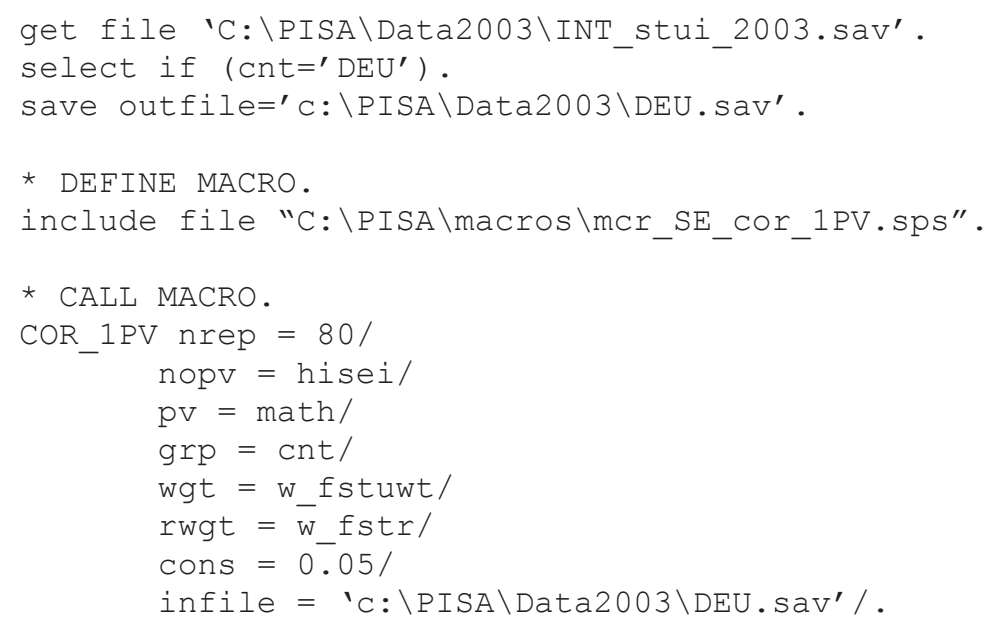

Table 7.8 \& Structure of the output data file from Box $\mathbf{7 . 6}$

\begin{tabular}{c|c|c}
\hline CNT & STAT & SE \\
\hline DEU & 0.39 & 0.02 \\
\hline
\end{tabular}

\section{CORRELATION BETWEEN TWO SETS OF PLAUSIBLE VALUES}

Some researchers might be interested in the correlation between the different domains and subdomains. For instance, some might want to compute the correlation between the reading sub-domains or between the mathematics sub-domains, or between reading and mathematics using the PISA 2000 and PISA 2003 databases.

As described in the PISA 2003 Technical report (OECD, forthcoming), the PISA assessment used incomplete assessment designs, i.e. students have to answer a subset of the item battery. Further, while all students were assessed in the major domain, only a subset of students were assessed in minor domains.

PISA 2000 only included PVs for students for a minor domain if they answered questions for that minor domain. Therefore, computing the correlation between reading and mathematics for example, using the PISA 2000 database, would require working on a subset of students. ${ }^{1}$ 
To facilitate secondary analyses, PISA 2003 returned PVs for all domains and for all students, regardless of whether they were actually assessed or not. Ignoring the assessment status is possible, because the cognitive data in PISA are scaled according to multi dimensional models.

Since this is easier to illustrate graphically, suppose that only two domains were assessed, more specifically mathematics/space and shape and mathematics/quantity. If the mathematics/space and shape and mathematics/quantity materials were scaled independently, the correlation between the two sub-domains would be largely underestimated. In order to avoid this problem, both materials are scaled together. The model will build a two dimensional posterior distribution, instead of two one dimensional posterior distributions as described in Chapter 5. Figure 7.1 graphically presents a two dimensional normal distribution.

\section{Figure 7.1 a two-dimensional distribution}

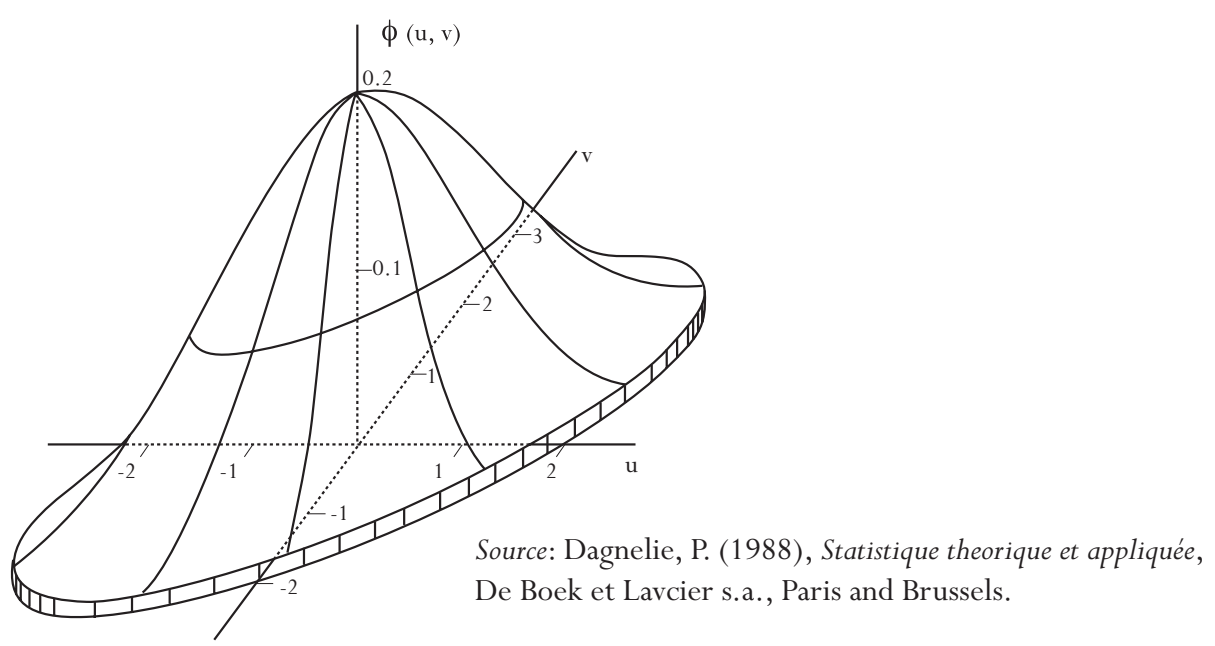

To correctly describe such distributions, two means, two variances, and one correlation are needed. If the correlation is equal to 0 , then the two axes will be orthogonal. As the absolute value of the correlation starts to increase, the angle formed by the two axes becomes less than 90 degrees. ${ }^{2}$ Two axes perfectly overlapping would represent a correlation of 1.0 (or -1.0). These different cases are illustrated in Figure 7.2.

Figure 7.2 - Axes for two-dimensional normal distributions

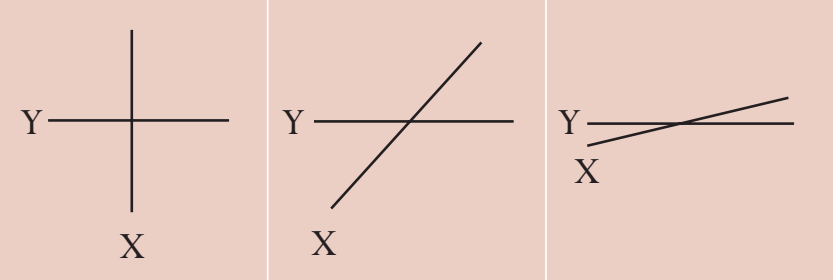

With a two-dimensional model, the first plausible value for mathematics/quantity will be drawn at the same time as the first plausible value for mathematics/space and shape. Per student, this will consist of randomly drawing one dot in the scatter plot. The values of the two plausible values will be the coordinates of the dot on the two axes. The same procedure is applied for the second, third, fourth and fifth PVs. 
As the PISA domains and sub-domains highly correlate, as shown by the graph on the far right most graph in Figure 7.2, it is very unlikely for a student to get a high score for the first plausible value in mathematics / quantity (PV1MATH4) and a low score for the first plausible value in mathematics/space and shape (PV1MATH1). If plausible values were drawn independently for these two mathematics subdomains, such a case would be possible and therefore the correlation would be underestimated.

Since each draw is independent, to calculate the correlation between the two domains, the correlation between each set of plausible values below needs to be computed:

- PV1MATH1 and PV1MATH4;

- PV2MATH1 and PV2MATH4;

- PV3MATH1 and PV3MATH4;

- PV4MATH1 and PV4MATH4; and

- PV5MATH1 and PV5MATH4.

Table 7.9 presents the 25 correlation coefficients between the five plausible values in mathematics / quantity and mathematics/ space and shape respectively for Germany for PISA 2003.

Table 7.9 - Correlation between the five plausible values for each domain, mathematics/quantity and mathematics/space and shape

\begin{tabular}{l|c|c|c|c|c}
\hline & PV1MATH1 & PV2MATH1 & PV3MATH1 & PV4MATH1 & PV5MATH1 \\
\hline PV1MATH4 & 0.90 & 0.83 & 0.84 & 0.84 & 0.83 \\
\hline PV2MATH4 & 0.83 & 0.90 & 0.84 & 0.84 & 0.83 \\
\hline PV3MATH4 & 0.84 & 0.83 & 0.90 & 0.84 & 0.83 \\
\hline PV4MATH4 & 0.83 & 0.83 & 0.84 & 0.90 & 0.83 \\
\hline PV5MATH4 & 0.83 & 0.83 & 0.84 & 0.84 & 0.90 \\
\hline
\end{tabular}

As shown in Table 7.9, the correlation coefficients on the diagonal of the square matrix are substantially higher than the other correlation coefficients. Therefore, the final correlation estimate between these two mathematics sub-domains will be the average of the five correlation coefficients on the diagonal.

The $\operatorname{SPSS}^{\circledR}$ syntax is given in Box 7.7.

\section{Box 7.7 - SPSS ${ }^{\circledR}$ syntax for the computation of the correlation between mathematics/quantity and mathematics/space and shape}

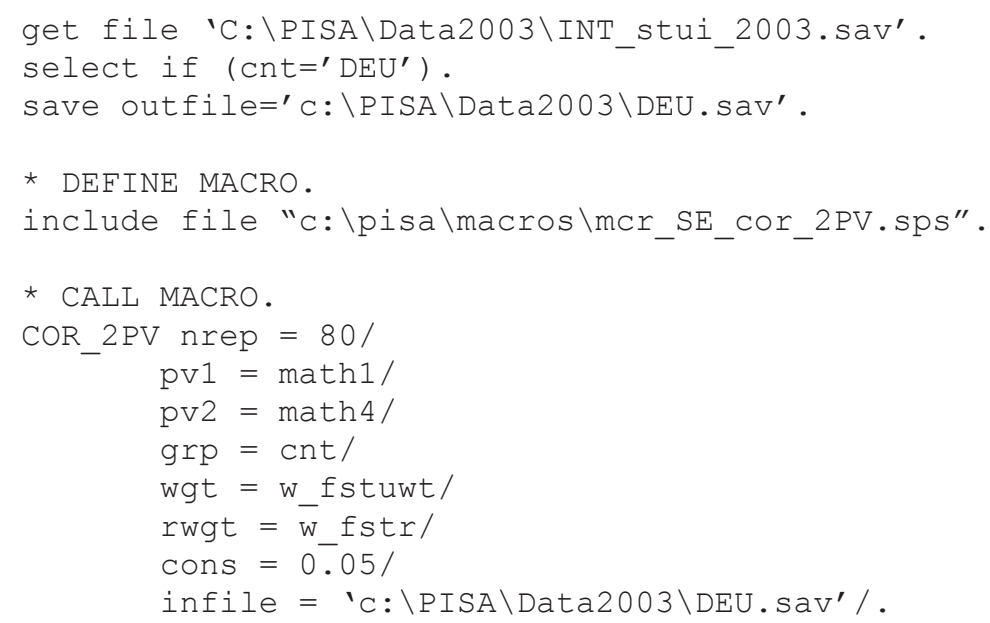


The five correlation estimates and their respective standard errors are given in Table 7.10.

Table 7.10 The five correlation estimates between mathematics/quantity and mathematics/space and shape and their respective sampling variance

\begin{tabular}{l|c|c|c|c|c}
\hline & PV1 & PV2 & PV3 & PV4 & PV5 \\
\hline Correlation & 0.8953 & 0.8964 & 0.8996 & 0.8978 & 0.8958 \\
\hline $\begin{array}{l}\text { Sampling } \\
\text { variance }\end{array}$ & $(0.0040)^{2}$ & $(0.0033)^{2}$ & $(0.0034)^{2}$ & $(0.0037)^{2}$ & $(0.0038)^{2}$ \\
\hline
\end{tabular}

The final correlation estimate is equal to:

$$
\begin{aligned}
& \hat{\rho}=\frac{\hat{\rho}_{1}+\hat{\rho}_{2}+\hat{\rho}_{3}+\hat{\rho}_{4}+\hat{\rho}_{5}}{5}, \text { i.e. } \\
& \hat{\rho}=\frac{0.8953+0.8964+\ldots+0.8958}{5}=0.8970
\end{aligned}
$$

The final sampling variance is equal to:

$$
\begin{aligned}
& \sigma_{(\hat{\rho})}^{2}=\frac{\sum_{i=1}^{5} \sigma_{\left(\hat{\rho}_{i}\right)}^{2}, \text { i.e. }}{5} \\
& \sigma_{(\hat{\rho})}^{2}=\frac{(0.0040)^{2}+(0.0033)^{2}+\ldots+(0.0038)^{2}}{5}=0.000013
\end{aligned}
$$

The measurement variance can be estimated as:

$$
\sigma_{(\text {test })}^{2}=\frac{1}{4} \sum_{i=1}^{5}\left(\hat{\rho}_{i}-\hat{\rho}\right)^{2}=0.000003
$$

The error variance is equal to:

$$
\sigma_{(\text {error })}^{2}=\sigma_{(\hat{\rho})}^{2}+\left(1.2 \sigma_{(\text {test }}^{2}\right)=0.000017
$$

The standard error is equal to:

$$
S E=\sqrt{\sigma_{(\text {error })}^{2}}=\sqrt{0.000017}=0.0041
$$

The computation of the correlation between two domains or between a sub-domain and a domain might be problematic in some cases in the PISA databases. PISA 2000 used two scaling models:

- A three-dimensional model with mathematics, reading and science;

- A five-dimensional model with mathematics, reading (retrieving information, interpreting and reflecting) and science. 
PISA 2003 used also two scaling models:

- A four-dimensional model with mathematics, problem solving, reading and science; and

- A seven-dimensional model with mathematics/space and shape, mathematics/change and relationships, mathematics/uncertainty, mathematics/quantity, problem solving, reading and science.

The PISA databases should contain two sets of plausible values for each of the minor domains. As this would be too confusing, only one set was provided. Therefore the correlation coefficients are underestimated.

This can be confirmed by examining the data. In the case of a minor domain and a subscale of the major domain, the correlation coefficients on the diagonal do not differ from the other correlations, since these two sets of plausible values were generated by two different models.

In PISA 2003 as well as in PISA 2000, the plausible values for the minor domains included in the databases were generated with the major domain as a combined scale. This means that:

- The correlation between a minor domain and the combined scale of the major domain can be computed;

- The correlation between two minor domains can be computed;

- The correlation between the sub-domains can be computed; and

- It is not possible to compute the correlation between minor domains and one of the sub-scales of the major domain.

\section{A FATAL ERROR SHORTCUT}

A common fatal error when analysing with plausible values involves computing the the mean of the five plausible values, before further analysis.

In Chapter 5, the EAP student performance estimator was described. As a reminder, the EAP estimator is equal to the mean of the posterior distribution. Therefore, computing at the student level the mean of the five PVs is more or less equal to the EAP estimate.

In Chapter 5, the efficiency of the EAP estimator was also compared with the WLE and the PVs for some statistics estimations. It was indicated that the EAP estimator:

- Underestimates the standard deviation;

- Overestimates the correlation between the student performance and some background variables; and

- Underestimates the within school variance.

Therefore, computing the mean of the five PVs and then computing statistics on this new score would bias the results just as the EAP does. Table 7.11 provides, per country, the standard deviation of the mathematics scale using the correct method as described in this chapter and also the incorrect method of averaging the five PVs at the student level and then computing the standard deviation on this new score. The result of the latter is denoted as pseudo-EAP. 
As shown by Table 7.11, the pseudo-EAP underestimates the standard deviation.

Table 7.11 - Standard deviations for mathematics scale using the correct method (plausible values) and by averaging the plausible values at the student level (pseudo-EAP)

\begin{tabular}{|c|c|c|c|c|c|}
\hline & $\begin{array}{c}\text { Plausible } \\
\text { values }\end{array}$ & Pseudo EAP & & $\begin{array}{c}\text { Plausible } \\
\text { values }\end{array}$ & Pseudo EAP \\
\hline AUS & 95.42 & 91.90 & KOR & 92.38 & 89.07 \\
\hline AUT & 93.09 & 89.91 & LIE & 99.06 & 95.42 \\
\hline BEL & 109.88 & 106.65 & LUX & 91.86 & 88.28 \\
\hline BRA & 99.72 & 94.79 & LVA & 87.90 & 83.92 \\
\hline CAN & 87.11 & 83.37 & MAC & 86.95 & 82.72 \\
\hline CHE & 98.38 & 94.97 & MEX & 85.44 & 80.52 \\
\hline $\mathrm{CZE}$ & 95.94 & 92.50 & NLD & 92.52 & 89.89 \\
\hline DEU & 102.59 & 99.54 & NOR & 92.04 & 88.31 \\
\hline DNK & 91.32 & 87.52 & NZL & 98.29 & 95.07 \\
\hline ESP & 88.47 & 84.52 & POL & 90.24 & 86.49 \\
\hline FIN & 83.68 & 79.77 & PRT & 87.63 & 83.91 \\
\hline FRA & 91.70 & 88.07 & RUS & 92.25 & 87.81 \\
\hline GBR & 92.26 & 89.18 & SVK & 93.31 & 89.86 \\
\hline GRC & 93.83 & 89.49 & SWE & 94.75 & 91.07 \\
\hline HKG & 100.19 & 96.99 & THA & 81.95 & 77.15 \\
\hline HUN & 93.51 & 89.71 & TUN & 81.97 & 76.86 \\
\hline IDN & 80.51 & 74.86 & TUR & 104.74 & 100.79 \\
\hline IRL & 85.26 & 82.03 & URY & 99.68 & 95.21 \\
\hline ISL & 90.36 & 86.55 & USA & 95.25 & 92.12 \\
\hline ITA & 95.69 & 92.00 & YUG & 84.65 & 80.43 \\
\hline JPN & 100.54 & 96.96 & & & \\
\hline
\end{tabular}

\section{AN UNBIASED SHORTCUT}

Table 7.1 and Table 7.5 respectively give the 405 mean and regression estimates needed for the computation of a mean or regression coefficient final estimate and the respective standard errors.

On average, analysing one PV instead of five PVs provides unbiased population estimates as well as unbiased sampling variances on these estimates. It will not be possible to estimate the imputation variance using this method, however.

Therefore, an unbiased shortcut could consist of:

- Computing, using one of the five PVs, the statistical estimate and its sampling variance by using the final student weight as well as the 80 replicate weights;

- Computing the statistical estimate by using the final student weight on the four other PVs;

- Computing the final statistical estimate by averaging the plausible value statistical estimates;

- Computing the imputation variance, as previously described; and

- Combining the imputation variance and the sampling variance, as previously described. 
This unbiased shortcut is presented in Table 7.12 for the estimation of a mean and its standard error. This shortcut only requires the computation of 85 estimates instead of 405 . The final estimate of this shortcut will be equal to the one obtained with the long procedure, but the standard error might differ slightly.

Table 7.12 - Unbiased shortcut for a population estimate and its standard error

\begin{tabular}{|c|c|c|c|c|c|}
\hline Weight & PV1 & PV2 & PV3 & PV4 & PV5 \\
\hline Final & $\hat{\mu}_{1}$ & $\hat{\boldsymbol{\mu}}_{2}$ & $\hat{\mu}_{3}$ & $\hat{\boldsymbol{\mu}}_{4}$ & $\hat{\boldsymbol{\mu}}_{5}$ \\
\hline Replicate 1 & $\hat{\mu}_{1 \_1}$ & & & & \\
\hline Replicate 2 & $\hat{\mu}_{1 \_2}$ & & & & \\
\hline Replicate 3 & $\hat{\mu}_{1 \_3}$ & & & & \\
\hline .......... & .......... & & & & \\
\hline ........... & ........... & & & & \\
\hline Replicate 80 & $\hat{\mu}_{1 \_80}$ & & & & \\
\hline $\begin{array}{l}\text { Sampling } \\
\text { variance }\end{array}$ & $\sigma_{\left(\hat{\mu}_{1}\right)}^{2}$ & & & & \\
\hline
\end{tabular}

\section{CONCLUSIONS}

This chapter describes the different steps for analysing data with plausible values. It also provides some SPSS ${ }^{\circledR}$ macros to facilitate the computations.

Attention was also drawn to a common error that consists of computing the average of the plausible values at the student level and adding this value to the database to be used as the student score in analyses. Unlike that method, the correct method involves the averaging process always occurring at the latest stage, that is on the statistic that will be reported.

The particular issue of analysing two sets of plausible values was also presented in the case of a correlation. The procedure that was applied can also be extended to a linear regression analysis.

Finally, an unbiased shortcut was described which is useful for time consuming procedures, such as multilevel procedures.

1. For more information, see the Manual for the PISA 2000 Database (OECD, 2002b).

2. A correlation coefficient can be expressed by the cosines of the angle formed by the two variables. 


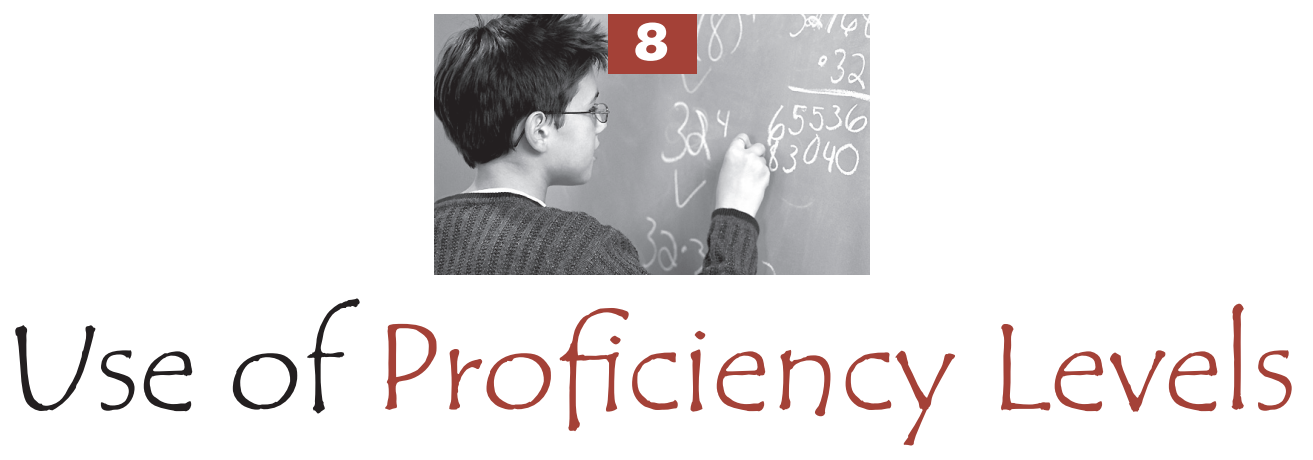

Introduction

Generation of the proficiency levels

Other analyses with proficiency levels

Conclusions 


\section{INTRODUCTION}

The values for student performance in reading, mathematics and science literacy are usually considered as continuous latent variables. In order to facilitate the interpretation of the scores assigned to students, the combined reading literacy scale and the mathematics and science scales were designed to have an average score of 500 points and a standard deviation of 100 across OECD countries in PISA 2000. This means that about two thirds of the OECD students perform between 400 and 600 points.

In PISA 2003, five mathematics scales, namely, the mathematics scale, the mathematics/space and shape scale, the mathematics/change and relationships scale, the mathematics quantity scale and the mathematics/uncertainty scale, were constructed for the first time, in order to have an average score among OECD countries of 500 points. However, unlike the mathematics scale, the PISA 2003 reading and science scales were anchored to the results from PISA 2000.

In order to improve the accessibility of the results to policy makers and educators, described proficiency scales were developed for the assessment domains. Since these scales are divided according to levels of difficulty and performance, a ranking of the student performance can be obtained, as well as a description of the skill associated with that proficiency level. Each successive level is associated with tasks of increased difficulty.

In PISA 2000, five levels of reading proficiency were defined and reported in the PISA 2000 initial report Knowledge and Skills for Life: First Results from PISA 2000 (OECD, 2001). In PISA 2003, six levels of mathematics proficiency levels were also defined and reported in the PISA 2003 initial report Learning for Tomorrow's World - First Results from PISA 2003 (OECD, 2004a).

This chapter will show how to derive the proficiency levels from the PISA databases and how to use them.

\section{GENERATION OF THE PROFICIENCY LEVELS}

Proficiency levels are not included in the PISA databases, but they can be derived from the plausible values.

In PISA 2003, the cutpoints that frame the proficiency levels in mathematics are precisely: 357.77 , 420.07, 482.38, 544.68, 606.99 and 669.3. ${ }^{1}$ While some researchers might understand that different possible scores can be assigned to each student, understanding that different levels can be assigned to a single student is more difficult. Therefore, they might be tempted to compute the average of the five plausible values and then assign to each student a proficiency level based on this average.

As discussed in Chapter 5 and Chapter 7, such procedure is similar to assigning to each student an EAP score, and the biases of such estimators are now well known. Since using EAP scores underestimates the standard deviation, estimation of percentages of students at each level of proficiency will consequently underestimate the percentages at the lowest and highest levels, and overestimate the percentages at the central levels.

As already stated, international education surveys do not intend to precisely estimate the performance of particular students; they aim to describe population characteristics. Therefore, particular students 
can be allocated different proficiency levels for different plausible values. Thus, five plausible proficiency levels will be assigned to each student respectively according to their five plausible values. The $\operatorname{SPSS}^{\circledR}$ syntax for the generation of the plausible proficiency levels in mathematics is provided in Box 8.1.

PISA 2000 provided cutpoints for proficiency levels in reading only. Therefore, proficiency levels can only be generated on the combined reading literacy scale and on the three subscales.

PISA 2003 provided cutpoints for proficiency levels in mathematics. Therefore, proficiency levels can be generated on the mathematics scale and on the four mathematics subscales as well as on the combined reading literacy scale.

The iterative process will recode each of 25 plausible value variables into a new variable with seven categories labelled 0 to 6 .

Box 8.1 - $\operatorname{SPSS}^{\circledR}$ syntax for the generation of the proficiency levels

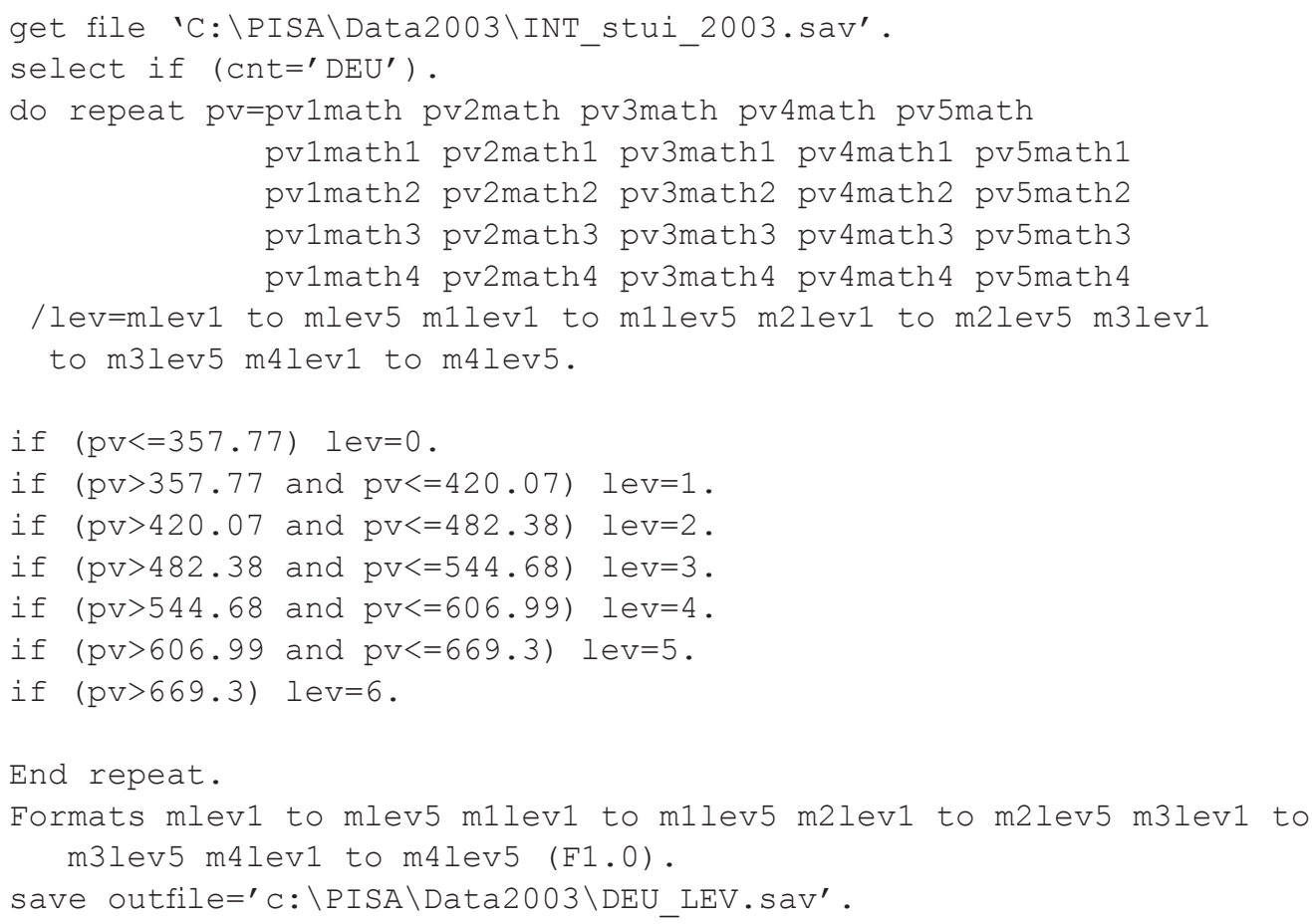

The computation of the percentage of students at each proficiency level and its respective standard error is exactly similar to the computation of a mean estimate and its standard error as described in Chapter 7, i.e.:

- For each plausible value, the percentage of students at each proficiency level and its respective standard error have to be computed. Per proficiency level, 5 percentage estimates denoted $\hat{\pi}_{1}$, $\hat{\boldsymbol{\pi}}_{7}, \hat{\boldsymbol{\pi}}_{2}, \hat{\boldsymbol{\pi}}_{4}$ and $\hat{\boldsymbol{\pi}}_{5}$ will be obtained. Out of the 80 replicates applied on each of the 5 proficiency level variables, per level of proficiency, 5 sampling variances will be estimated, denoted 
$\stackrel{n}{\stackrel{n}{v}}$ respectively $\sigma_{\left(\hat{\pi}_{1}\right)}^{2}, \sigma_{\left(\hat{\pi}_{2}\right)}^{2}, \sigma_{\left(\hat{\pi}_{3}\right)}^{2}, \sigma_{\left(\hat{\pi}_{4}\right) \text { and }}^{2} \sigma_{\left(\hat{\pi}_{5}\right)}^{2}$. These five percentage estimates and their respective sampling variances are given in Table 8.1 .

- The final mean estimate is equal to the average of the 5 mean estimates, i.e.:

$$
\hat{\pi}=\frac{1}{5}\left(\hat{\boldsymbol{\pi}}_{1}+\hat{\boldsymbol{\pi}}_{2}+\hat{\pi}_{3}+\hat{\boldsymbol{\pi}}_{4}+\hat{\pi}_{5}\right)
$$

- The final sampling variance is equal to the average of the 5 sampling variances, i.e.:

$$
\sigma_{(\hat{\pi})}^{2}=\frac{1}{5}\left(\sigma_{\left(\hat{\pi}_{1}\right)}^{2}+\sigma_{\left(\hat{\pi}_{2}\right)}^{2}+\sigma_{\left(\hat{\pi}_{3}\right)}^{2}+\sigma_{\left(\hat{\pi}_{4}\right)}^{2}+\sigma_{\left(\hat{\pi}_{5}\right)}^{2}\right)
$$

- The imputation variance, also denoted measurement error variance is computed as: ${ }^{2}$

$$
\sigma_{(t e s t)}^{2}=\frac{1}{4} \sum_{i=1}^{5}\left(\hat{\pi}_{i}-\hat{\pi}\right)^{2}
$$

- The sampling variance and the imputation variance are combined to obtain the final error variance as

$$
\sigma_{(\text {error })}^{2}=\sigma_{(\hat{\pi})}^{2}+\left(1.2 \sigma_{(\text {test })}^{2}\right)
$$

\begin{tabular}{|c|c|c|c|c|c|}
\hline Weight & $\mathrm{MLEV}_{\mathrm{i}}$ PV1 & MLEV $_{\mathrm{i}}$ PV2 & $\mathrm{MLEV}_{\mathrm{i}}$ PV3 & $\mathrm{MLEV}_{\mathrm{i}} \mathrm{PV} 4$ & $M{ }^{2} V_{\mathrm{i}} \mathrm{PV} 5$ \\
\hline Final & $\hat{\pi}_{1}$ & $\hat{\boldsymbol{\pi}}_{2}$ & $\hat{\boldsymbol{\pi}}_{3}$ & $\hat{\pi}_{4}$ & $\hat{\boldsymbol{\pi}}_{5}$ \\
\hline Replicate 1 & $\hat{\boldsymbol{\pi}}_{1-1}$ & $\hat{\boldsymbol{\pi}}_{2 \_1}$ & $\hat{\boldsymbol{\pi}}_{3 \_1}$ & $\hat{\boldsymbol{\pi}}_{4-1}$ & $\hat{\boldsymbol{\pi}}_{5_{-} 1}$ \\
\hline Replicate 2 & $\hat{\boldsymbol{\pi}}_{1_{12}}$ & $\hat{\boldsymbol{\pi}}_{2 \_2}$ & $\hat{\boldsymbol{\pi}}_{3 \_2}$ & $\hat{\boldsymbol{\pi}}_{4 \_2}$ & $\hat{\boldsymbol{\pi}}_{5 \_2}$ \\
\hline Replicate 3 & $\hat{\boldsymbol{\pi}}_{1 \_3}$ & $\hat{\boldsymbol{\pi}}_{2-3}$ & $\hat{\boldsymbol{\pi}}_{3 \_3}$ & $\hat{\boldsymbol{\pi}}_{4-3}$ & $\hat{\boldsymbol{\pi}}_{5-3}$ \\
\hline ........... & ........... & ........... & ........... & ........... & ........... \\
\hline ............ & ............ & ............ & ........... & ............ & ............ \\
\hline Replicate 80 & $\hat{\boldsymbol{\pi}}_{1 \_80}$ & $\hat{\boldsymbol{\pi}}_{2 \_80}$ & $\hat{\boldsymbol{\pi}}_{3 \_80}$ & $\hat{\boldsymbol{\pi}}_{4 \_80}$ & $\hat{\boldsymbol{\pi}}_{5 \_80}$ \\
\hline $\begin{array}{l}\text { Sampling } \\
\text { variance }\end{array}$ & $\sigma_{\left(\hat{\pi}_{1}\right)}^{2}$ & $\sigma_{\left(\hat{\pi}_{2}\right)}^{2}$ & $\sigma_{\left(\hat{\pi}_{3}\right)}^{2}$ & $\sigma_{\left(\hat{\pi}_{4}\right)}^{2}$ & $\sigma_{\left(\hat{\pi}_{5}\right)}^{2}$ \\
\hline
\end{tabular}

- The standard error is equal to the square root of the error variance.

This process is repeated for each proficiency level.

Table 8.1 - 405 percentage estimates for a particular proficiency level $\boldsymbol{i}$

In this way 405 percentages will be estimated per proficiency level. As there are seven levels in mathematics, it means that 2835 percentages will be estimated. 
The seven proficiency levels in mathematics are:

1. Below Level 1;

2. Level 1;

3. Level 2;

4. Level 3;

5. Level 4;

6. Level 5; and

7. Level 6.

Applying sequentially five times the GRPPCT macro described in Chapter 5 will return, per proficiency level, five percentage estimates and five standard error estimates that can be combined to get the final estimate and its standard error.

Box 8.2 presents the SPSS ${ }^{\circledR}$ syntax for running sequentially five times the GRPPCT macro. Table 8.2 presents per proficiency level, the five estimates and their respective sampling variances.

\section{Box 8.2 - SPSS $^{\circledR}$ syntax for computing the percentages of students} per proficiency level in mathematics

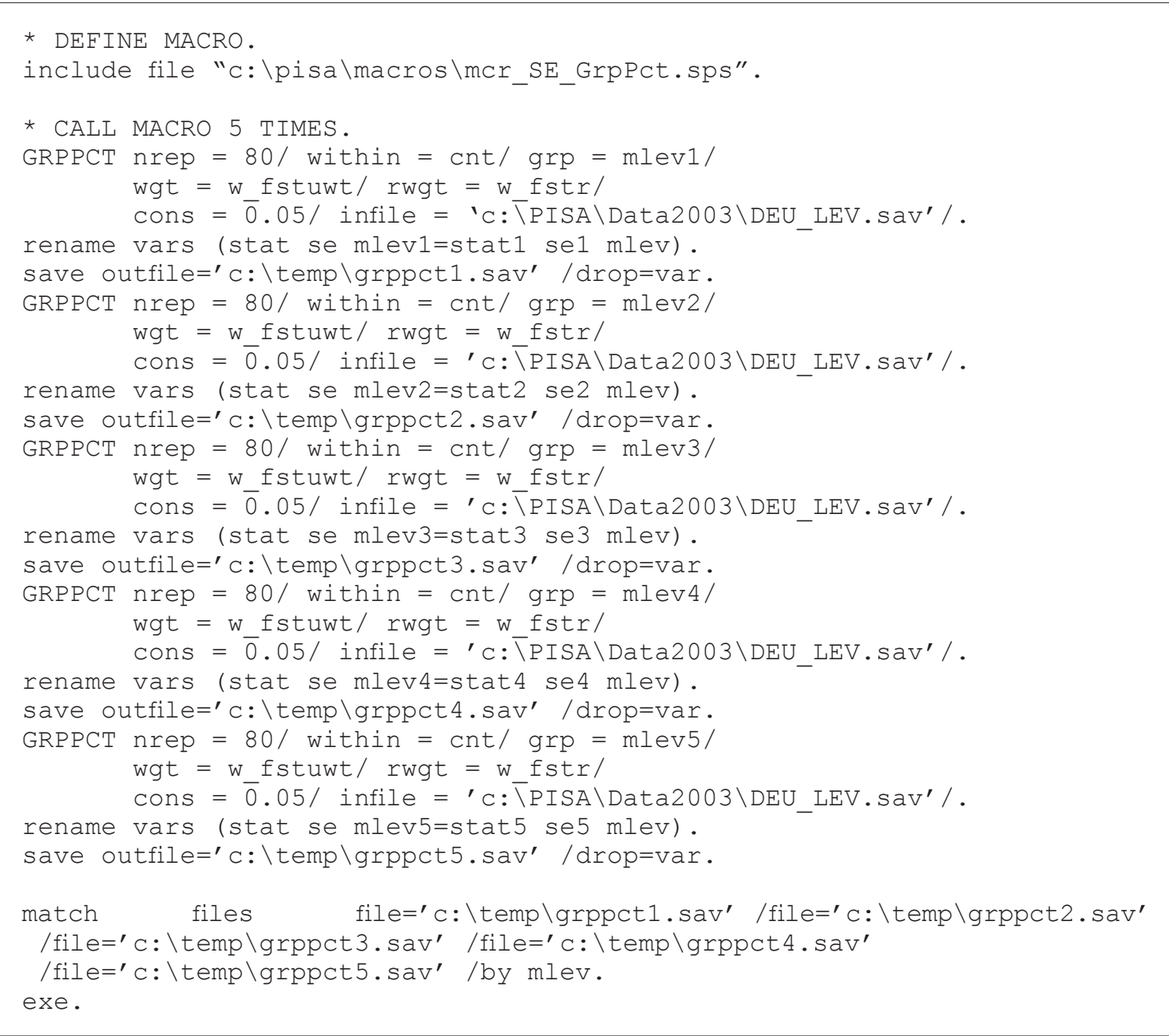


To combine the results:

- Per proficiency level the five percentage estimates are averaged;

- Per proficiency level, the five sampling variances are averaged;

- By comparing the final estimate and the five PV estimates, the imputation variance is computed;

- The final sampling variance and the imputation variance are combined as usual to get the final error variance; and

- The standard error is obtained by taking the square root of the error variance.

Table 8.2 Estimates and sampling variances per proficiency level in mathematics for Germany

\begin{tabular}{|c|c|c|c|c|c|c|}
\hline Level & & PV1 & PV2 & PV3 & PV4 & PV5 \\
\hline \multirow{2}{*}{$\begin{array}{l}\text { Below } \\
\text { Level } 1\end{array}$} & $\hat{\boldsymbol{\pi}}_{i}$ & 9.69 & 9.02 & 9.12 & 9.36 & 8.75 \\
\hline & $\sigma_{\left(\hat{\pi}_{i}\right)}^{2}$ & $(0.79)^{2}$ & $(0.73)^{2}$ & $(0.75)^{2}$ & $(0.74)^{2}$ & $(0.71)^{2}$ \\
\hline \multirow[b]{2}{*}{ Level 1} & $\hat{\boldsymbol{\pi}}_{i}$ & 11.87 & 12.68 & 12.67 & 12.33 & 12.52 \\
\hline & $\sigma_{\left(\hat{\pi}_{i}\right)}^{2}$ & $(0.74)^{2}$ & $(0.74)^{2}$ & $(0.72)^{2}$ & $(0.71)^{2}$ & $(0.72)^{2}$ \\
\hline \multirow{2}{*}{ Level 2} & $\hat{\boldsymbol{\pi}}_{i}$ & 18.20 & 18.83 & 19.53 & 18.87 & 19.56 \\
\hline & $\sigma_{\left(\hat{\pi}_{i}\right)}^{2}$ & $(0.80)^{2}$ & $(0.80)^{2}$ & $(0.86)^{2}$ & $(0.89)^{2}$ & $(0.88)^{2}$ \\
\hline \multirow{2}{*}{ Level 3} & $\hat{\boldsymbol{\pi}}_{i}$ & 23.11 & 22.69 & 22.14 & 22.23 & 22.66 \\
\hline & $\sigma_{\left(\hat{\pi}_{i}\right)}^{2}$ & $(0.72)^{2}$ & $(0.67)^{2}$ & $(0.68)^{2}$ & $(0.62)^{2}$ & $(0.81)^{2}$ \\
\hline \multirow{2}{*}{ Level 4} & $\hat{\pi}_{i}$ & 21.05 & 20.95 & 20.30 & 20.85 & 19.91 \\
\hline & $\sigma_{\left(\hat{\pi}_{i}\right)}^{2}$ & $(0.89)^{2}$ & $(0.93)^{2}$ & $(0.85)^{2}$ & $(0.82)^{2}$ & $(0.85)^{2}$ \\
\hline \multirow{2}{*}{ Level 5} & $\hat{\boldsymbol{\pi}}_{i}$ & 11.65 & 11.74 & 12.50 & 12.13 & 12.82 \\
\hline & $\sigma_{\left(\hat{\pi}_{i}\right)}^{2}$ & $(0.65)^{2}$ & $(0.66)^{2}$ & $(0.70)^{2}$ & $(0.65)^{2}$ & $(0.73)^{2}$ \\
\hline \multirow{2}{*}{ Level 6} & $\hat{\pi}_{i}$ & 4.42 & 4.09 & 3.74 & 4.23 & 3.78 \\
\hline & $\sigma_{\left(\hat{\pi}_{i}\right)}^{2}$ & $(0.35)^{2}$ & $(0.38)^{2}$ & $(0.33)^{2}$ & $(0.37)^{2}$ & $(0.36)^{2}$ \\
\hline
\end{tabular}

The final results are presented in Table 8.3.

Table 8.3 - Final estimates of the percentage of students per proficiency level in mathematics and their respective standard errors for Germany

\begin{tabular}{c|c|c}
\hline Proficiency level & $\%$ & SE \\
\hline Below Level 1 & 9.19 & 0.84 \\
\hline Level 1 & 12.42 & 0.81 \\
\hline Level 2 & 19.00 & 1.05 \\
\hline Level 3 & 22.57 & 0.82 \\
\hline Level 4 & 20.61 & 1.02 \\
\hline Level 5 & 12.17 & 0.87 \\
\hline Level 6 & 4.05 & 0.48 \\
\hline
\end{tabular}


An SPSS ${ }^{\circledR}$ macro has been developed for computing the percentage of students at each proficiency level as well as its respective standard error in one run. Box 8.3 presents the SPSS ${ }^{\circledR}$ syntax for running the macro and Table 8.4 presents the structure of the output data file. For the mathematics scale the GRP argument will be set as MLEV.

\section{Box 8.3 - $\operatorname{SPSS}^{\circledR}$ syntax for computing the percentage of students per proficiency}

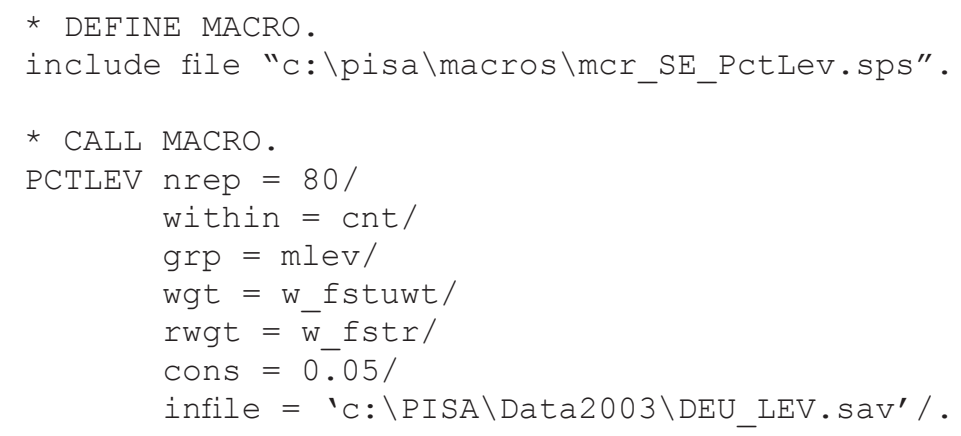

Table 8.4 - Structure of the output data file from Box 8.3

\begin{tabular}{c|c|c|c}
\hline CNT & MLEV & STAT & SE \\
\hline DEU & 0 & 9.19 & 0.84 \\
\hline DEU & 1 & 12.42 & 0.81 \\
\hline DEU & 2 & 19.00 & 1.05 \\
\hline DEU & 3 & 22.57 & 0.82 \\
\hline DEU & 4 & 20.61 & 1.02 \\
\hline DEU & 5 & 12.17 & 0.87 \\
\hline DEU & 6 & 4.05 & 0.48 \\
\hline
\end{tabular}

As before, several breakdown variables can be used. For instance, the distribution of students across proficiency levels per gender can be obtained as in Box 8.4.

Box 8.4 - SAS syntax for computing the percentage of students per proficiency level and per gender

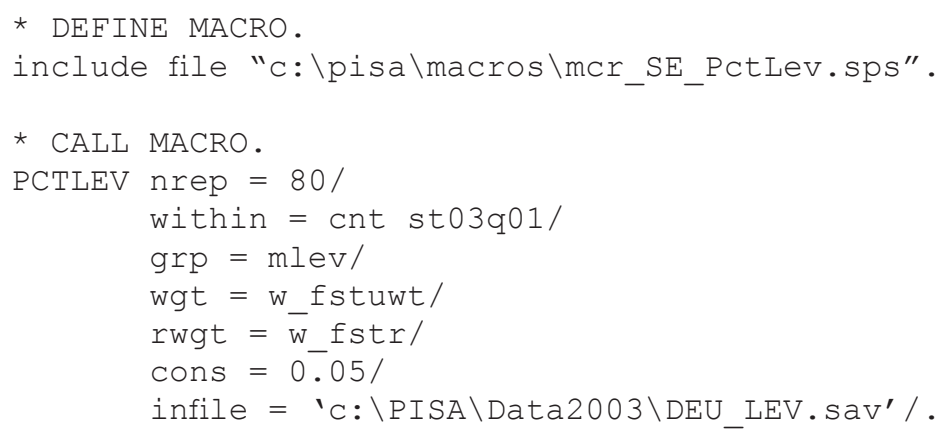


In this case, the sum of the percentages will be equal to 100 per country and per gender, as shown by Table 8.5 .

Table 8.5 - Structure of the output data file from Box $\mathbf{8 . 4}$

\begin{tabular}{ccc|rc}
\hline CNT & ST03Q01 & MLEV & STAT & SE \\
\hline DEU & 1 & 0 & 9.24 & 1.05 \\
DEU & 1 & 1 & 12.15 & 1.02 \\
DEU & 1 & 2 & 19.92 & 1.42 \\
DEU & 1 & 3 & 23.92 & 1.37 \\
DEU & 1 & 4 & 20.65 & 1.18 \\
DEU & 1 & 5 & 11.25 & 0.97 \\
DEU & 1 & 6 & 2.87 & 0.57 \\
DEU & 2 & 0 & 8.88 & 1.04 \\
DEU & 2 & 1 & 12.53 & 0.99 \\
DEU & 2 & 2 & 18.14 & 1.21 \\
DEU & 2 & 3 & 21.43 & 0.98 \\
DEU & 2 & 4 & 20.72 & 1.32 \\
DEU & 2 & 5 & 13.03 & 1.14 \\
DEU & 2 & 6 & 5.27 & 0.65 \\
\hline
\end{tabular}

As shown by Table 8.5 , the percentage of girls at Level 6 is higher than the percentage of boys at Level 6 .

The statistical significance of these differences cannot be evaluated with this procedure. More details on this issue will be provided in Chapter 10.

\section{OTHER ANALYSES WITH PROFICIENCY LEVELS}

One of the indices constructed in PISA 2003 is an index of mathematics self-efficacy, denoted MATHEFF.

For PISA 2003, analysing the relationship between proficiency levels and mathematics self-efficacy is relevant, as there is probably a reciprocal relationship between these two concepts. A better selfperception in mathematics is thought to increase the student's proficiency in mathematics, but an increase in the latter might in return affect the former.

Suppose that the statistic of interest is the average self-efficacy per proficiency level. In statistical terms, mathematics self-efficacy is considered as the dependent variable and the level of proficiency, the independent variable. There is no macro that can directly compute the mean of a continuous variable per proficiency level. On the other hand, the UNIV macro described in Chapter 6 can be applied sequentially five times and the results could be combined in an Microsoft ${ }^{\circledR}$ Excel $^{\circledR}$ spreadsheet for instance. This will be the case whenever proficiency levels are used as independent or as classification variables.

Box 8.5 presents the SPSS ${ }^{\circledR}$ syntax for computing the mean of student self-efficacy per proficiency level. The mean estimates and their respective standard errors are presented in Table 8.6. 


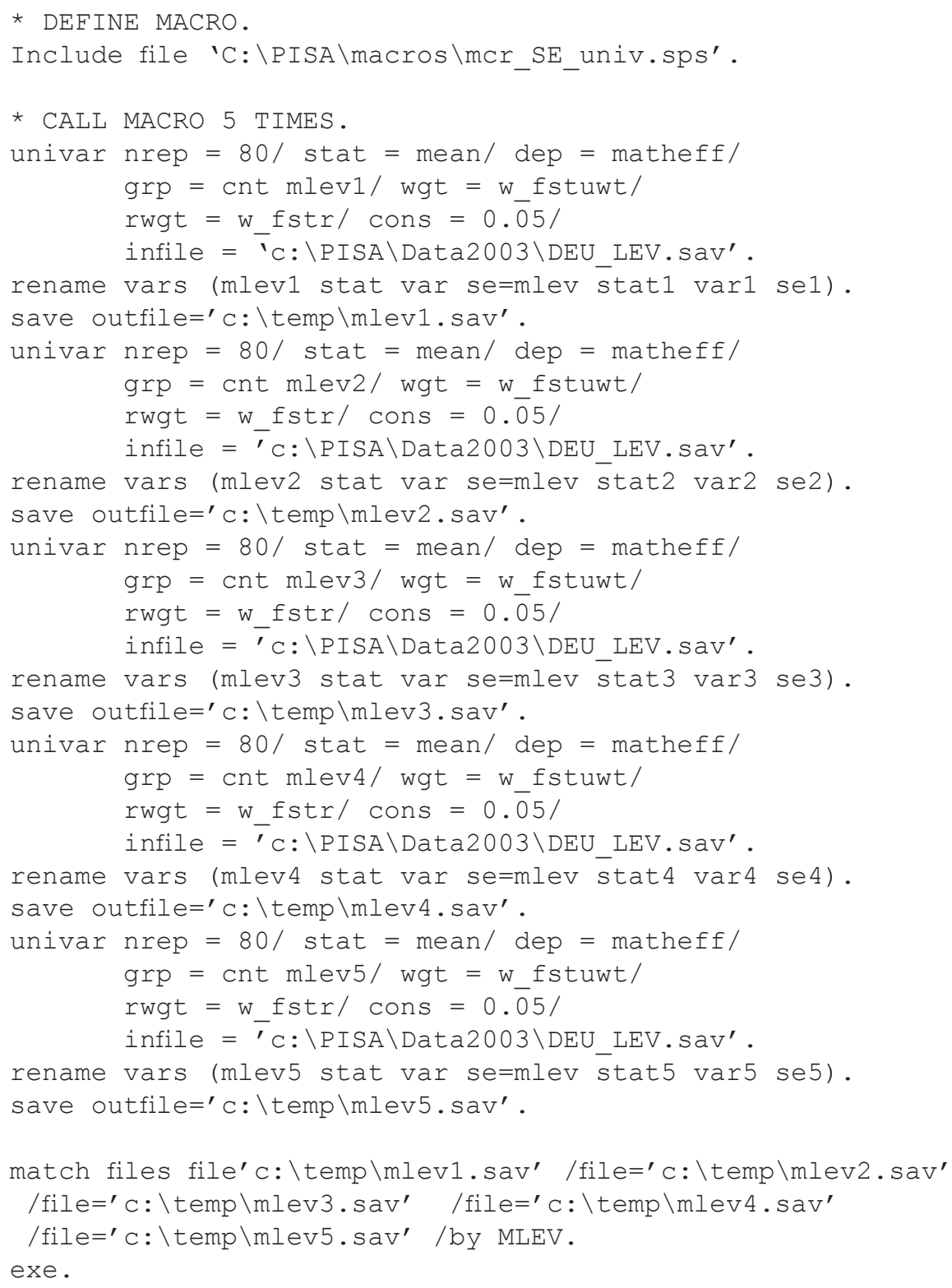

To combine the results:

- Per proficiency level, the five mean estimates are averaged;

- Per proficiency level, the five sampling variances are averaged;

- By comparing the final estimate and the five PV estimates, the imputation variance is computed;

- The final sampling variance and the imputation variance are combined as usual to get the final error variance; and

- The standard error is obtained by taking the square root of the error variance. 
Table 8.6 Mean estimates and standard errors for self-efficacy in mathematics per proficiency level

\begin{tabular}{l|c|c|c|c|c|c}
\hline \multirow{2}{*}{$\begin{array}{l}\text { Level } \\
\text { Lelow }\end{array}$} & $\hat{\mu}_{i}$ & -0.68 & -0.70 & -0.74 & -0.72 & PV1 \\
\cline { 2 - 7 } & $\sigma_{\left(\hat{\mu}_{i}\right)}^{2}$ & $(0.06)^{2}$ & $(0.06)^{2}$ & $(0.06)^{2}$ & $(0.05)^{2}$ & $(0.06)^{2}$ \\
\hline \multirow{3}{*}{ Level 1 } & $\hat{\mu}_{i}$ & -0.44 & -0.45 & -0.42 & -0.43 & -0.40 \\
\cline { 2 - 7 } & $\sigma_{\left(\hat{\mu}_{i}\right)}^{2}$ & $(0.06)^{2}$ & $(0.05)^{2}$ & $(0.06)^{2}$ & $(0.04)^{2}$ & $(0.05)^{2}$ \\
\hline \multirow{2}{*}{ Level 2 } & $\hat{\mu}_{i}$ & -0.18 & -0.16 & -0.17 & -0.18 & -0.18 \\
\cline { 2 - 7 } & $\sigma_{\left(\hat{\mu}_{i}\right)}^{2}$ & $(0.03)^{2}$ & $(0.03)^{2}$ & $(0.03)^{2}$ & $(0.03)^{2}$ & $(0.03)^{2}$ \\
\hline \multirow{3}{*}{ Level 3 } & $\hat{\mu}_{i}$ & 0.09 & 0.09 & 0.12 & 0.11 & 0.10 \\
\cline { 2 - 7 } & $\sigma_{\left(\hat{\mu}_{i}\right)}^{2}$ & $(0.03)^{2}$ & $(0.03)^{2}$ & $(0.03)^{2}$ & $(0.03)^{2}$ & $(0.03)^{2}$ \\
\hline \multirow{3}{*}{ Level 4 } & $\hat{\mu}_{i}$ & 0.43 & 0.45 & 0.41 & 0.45 & 0.44 \\
\cline { 2 - 7 } & $\sigma_{\left(\hat{\mu}_{i}\right)}^{2}$ & $(0.03)^{2}$ & $(0.03)^{2}$ & $(0.03)^{2}$ & $(0.03)^{2}$ & $(0.03)^{2}$ \\
\hline \multirow{2}{*}{ Level 5 } & $\hat{\mu}_{i}$ & 0.85 & 0.84 & 0.86 & 0.79 & 0.82 \\
\cline { 2 - 7 } & $\sigma_{\left(\hat{\mu}_{i}\right)}^{2}$ & $(0.04)^{2}$ & $(0.04)^{2}$ & $(0.03)^{2}$ & $(0.04)^{2}$ & $(0.04)^{2}$ \\
\hline \multirow{2}{*}{ Level 6 } & $\hat{\mu}_{i}$ & 1.22 & 1.23 & 1.27 & 1.28 & 1.29 \\
\cline { 2 - 7 } & $\sigma_{\left(\hat{\mu}_{i}\right)}^{2}$ & $(0.05)^{2}$ & $(0.05)^{2}$ & $(0.06)^{2}$ & $(0.05)^{2}$ & $(0.07)^{2}$ \\
\hline
\end{tabular}

Final results are presented in Table 8.7.

The syntax in Box 8.5 can be improved by adding commands that will compute the final estimate and its standard error, as in Box 8.6. As the results have been stored in the five output data files with exactly the same variable names, it is necessary to relabel them first.

\section{Box 8.6 - SPSS $^{\circledR}$ syntax for computing the mean of self-efficacy in mathematics} per proficiency level

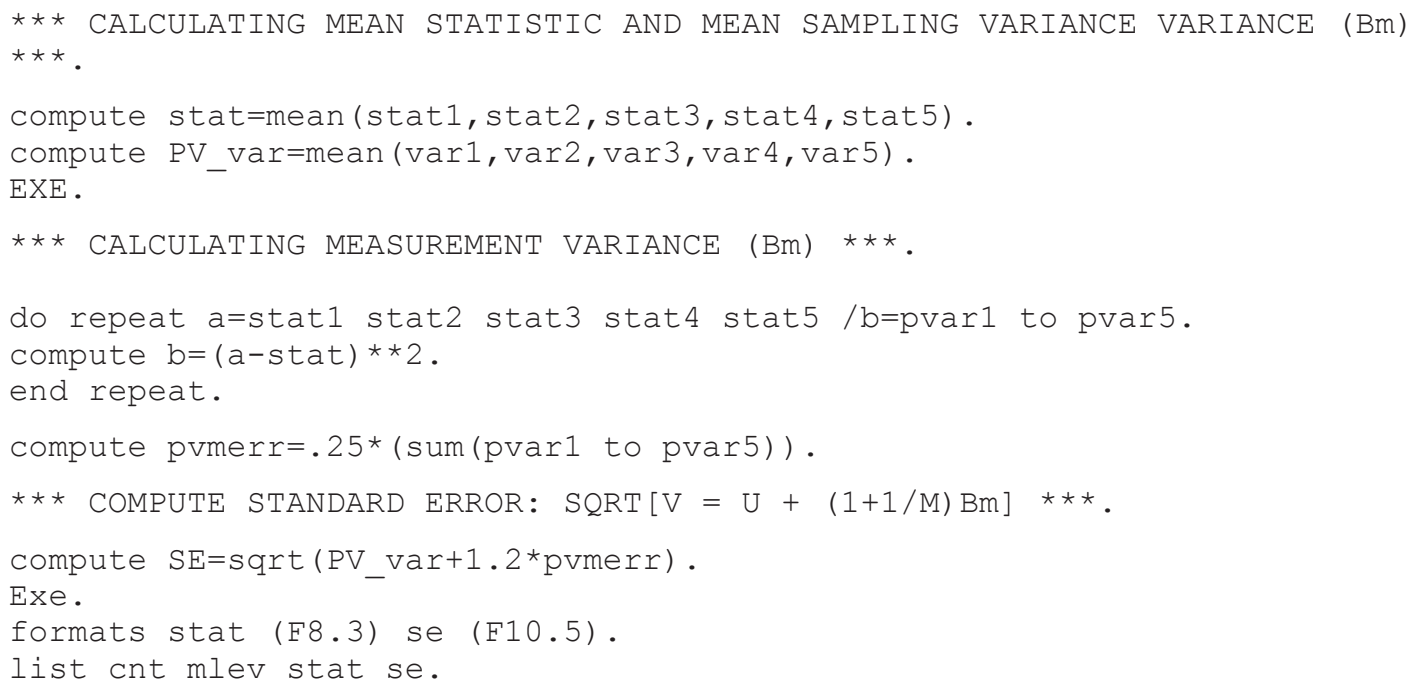


Once the output data files are merged according to the breakdown variables (in this particular case, by CNT) and by proficiency levels (MLEV):

- The final estimate is computed by averaging the five estimates;

- The final sampling variance is computed by averaging the five sampling variances;

- The imputation variance is computed; and

- The standard error is computed by combining the sampling variance and the imputation variance and taking the square root.

The structure of the output data file is presented in Table 8.7.

Table 8.7 - Structure of the output data file from Box $\mathbf{8 . 6}$

\begin{tabular}{c|c|c|c} 
CNT & MLEV & STAT & SE \\
\hline DEU & 0 & -0.72 & 0.07 \\
\hline DEU & 1 & -0.43 & 0.06 \\
\hline DEU & 2 & -0.17 & 0.03 \\
\hline DEU & 3 & 0.10 & 0.03 \\
\hline DEU & 4 & 0.44 & 0.03 \\
\hline DEU & 5 & 0.83 & 0.05 \\
\hline DEU & 6 & 1.26 & 0.07 \\
\hline
\end{tabular}

Table 8.7 shows that high mathematics self-efficacy (STAT) is associated with higher proficiency level (MLEV).

\section{CONCLUSIONS}

This chapter shows how to compute the percentage of students per proficiency level. As shown, the algorithm is similar to the one used for other statistics.

The difficulty of conducting analyses using proficiency levels as the explanatory (independent) variables was also discussed.

\section{Notes}

1. In PISA 2000, the cutpoints that frame the proficiency levels in reading are precisely: $334.7526,407.4667$, $480.1807,552.8948$ and 625.6088 .

2. This formula is a simplification of the general formula provided in Chapter 4. M, denoting the number of plausible values, has been replaced by 5 . 



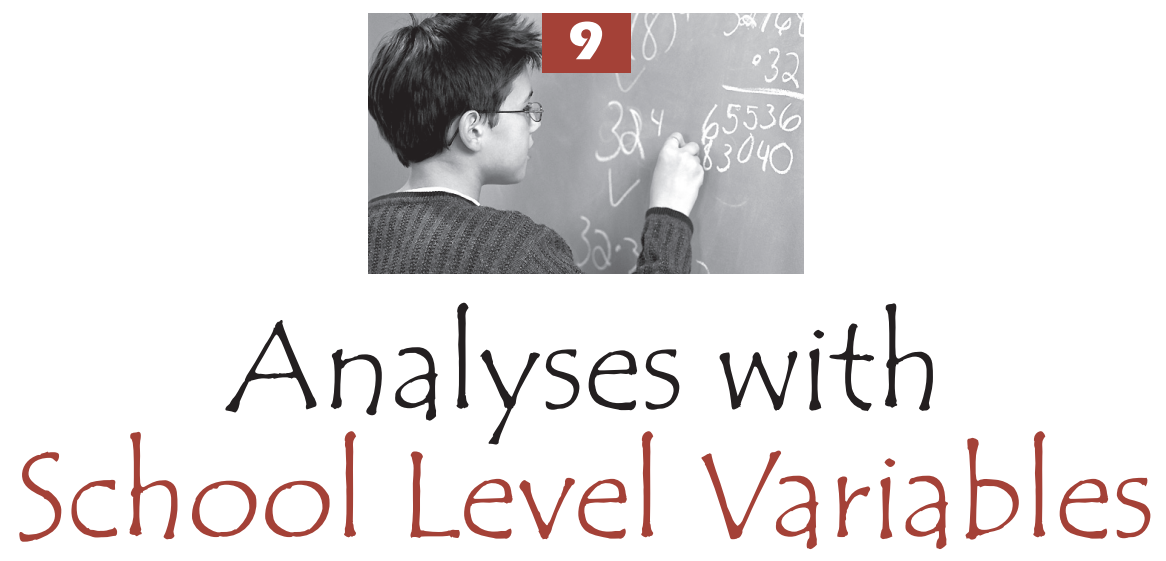

Introduction

Limits of the PISA school samples

Merging the school and student data files

Analyses of the school variables 


\section{INTRODUCTION}

The target population in PISA is 15-year-old students. This population was chosen because, at this age in most OECD countries, students are nearing the end of their compulsory schooling, thus PISA should be able to give an indication of the cumulative effect of the education for the student over the years. There is a two-stage sampling procedure used in PISA. After the population is defined, school samples are selected with a probability proportional to size. Subsequently, 35 students are randomly selected from each school. As the target population is based on age, it is therefore possible that the students will come from a variety of grades.

Table 9.1 presents the distribution of 15-year-olds per country and per grade in PISA 2003.

Table 9.1 Estimates of student percentage per grade and per country in PISA 2003

\begin{tabular}{|c|c|c|c|c|c|c|}
\hline & 7 & 8 & 9 & 10 & 11 & 12 \\
\hline AUS & 0.01 & 0.14 & 8.34 & 72.26 & 19.21 & 0.05 \\
\hline AUT & 0.30 & 5.07 & 43.18 & 51.45 & & \\
\hline BEL & 0.33 & 3.69 & 29.64 & 65.49 & 0.85 & \\
\hline BRA & 13.70 & 24.82 & 42.89 & 18.08 & 0.51 & \\
\hline CAN & 0.57 & 2.47 & 13.74 & 82.04 & 1.17 & 0.00 \\
\hline $\mathrm{CHE}$ & 0.75 & 16.90 & 62.77 & 19.40 & 0.18 & \\
\hline CZE & 0.15 & 2.82 & 44.67 & 52.36 & & \\
\hline DEU & 1.70 & 14.99 & 59.94 & 23.25 & 0.12 & \\
\hline DNK & 0.07 & 9.10 & 86.96 & 3.83 & 0.05 & \\
\hline ESP & 0.03 & 3.18 & 27.03 & 69.73 & 0.02 & \\
\hline FIN & 0.26 & 12.43 & 87.31 & & & \\
\hline FRA & 0.20 & 5.37 & 34.86 & 57.29 & 2.23 & 0.05 \\
\hline GBR & & & 0.02 & 33.81 & 63.56 & 2.61 \\
\hline GRC & 0.22 & 2.09 & 6.55 & 76.13 & 15.01 & \\
\hline HKG & 5.12 & 10.75 & 25.70 & 58.36 & 0.08 & \\
\hline HUN & 1.08 & 5.00 & 65.13 & 28.76 & 0.02 & \\
\hline IDN & 2.40 & 12.68 & 48.78 & 34.51 & 1.57 & 0.07 \\
\hline IRL & 0.02 & 2.78 & 60.87 & 16.68 & 19.65 & \\
\hline ISL & & & & 100.00 & & \\
\hline ITA & 0.18 & 1.38 & 14.20 & 79.95 & 4.28 & \\
\hline JPN & & & & 100.00 & & \\
\hline KOR & & & 1.57 & 98.33 & 0.10 & \\
\hline LIE & 0.61 & 20.37 & 71.26 & 7.75 & & \\
\hline LUX & & 14.85 & 55.79 & 29.25 & 0.10 & \\
\hline LVA & 1.09 & 16.76 & 75.96 & 6.08 & 0.13 & \\
\hline MAC & 12.30 & 25.88 & 36.82 & 24.66 & 0.34 & \\
\hline MEX & 3.62 & 10.95 & 40.76 & 43.69 & 0.93 & 0.04 \\
\hline NLD & 0.14 & 4.44 & 45.61 & 49.32 & 0.47 & 0.02 \\
\hline NOR & & & 0.62 & 98.68 & 0.69 & \\
\hline NZL & & 0.06 & 6.79 & 89.38 & 3.74 & 0.02 \\
\hline POL & 0.72 & 3.07 & 95.70 & 0.51 & & \\
\hline PRT & 4.25 & 10.58 & 20.26 & 64.32 & 0.58 & \\
\hline RUS & 0.35 & 2.58 & 28.74 & 67.23 & 1.10 & \\
\hline SVK & 0.58 & 0.92 & 37.10 & 60.93 & 0.46 & \\
\hline SWE & 0.03 & 2.36 & 93.00 & 4.61 & & \\
\hline THA & 0.18 & 1.09 & 44.06 & 53.26 & 1.41 & \\
\hline TUN & 15.39 & 21.99 & 25.15 & 34.52 & 2.94 & \\
\hline TUR & 0.84 & 4.39 & 3.20 & 52.12 & 39.19 & 0.25 \\
\hline URY & 5.67 & 9.67 & 18.22 & 59.36 & 7.09 & \\
\hline USA & 0.28 & 2.40 & 29.71 & 60.63 & 6.98 & \\
\hline YUG & & & 97.60 & 2.40 & & \\
\hline
\end{tabular}

a. The results are based on the information provided in the student tracking forms. These results are therefore not biased due to a differential grade participation rate. 
In a few countries, especially, most of the 15-year-old population tend to be in a modal grade, whereas in others, the 15 -year-old population is spread across several grades.

The PISA target population can spread over several grades for different reasons:

- If the student does not pass a particular grade examination, he or she has to repeat the grade. For example in some countries there may be up to about 35 per cent of students who have already repeated at least one grade.

- Even if grade retention is not used, the 15-year-old population might be separated at the testing time into two grades. For logistical reasons, PISA testing takes place in a single calendar year. As the recommended testing window is around April (in the northern hemisphere), the PISA target population is defined as all students between 15 years and 3 months old and 16 years and 2 months old at the beginning of the testing period. If the entrance rules for compulsory education are defined in terms of complete calendar years, then the PISA target population will attend just one grade.

As the 15-year-old population attends different grades in most OECD countries, the within-school samples can only consist of a random sample of students. Consequently, the PISA participating students are attending several grades and within a particular grade, are in several classes, depending on the school size. Largely because the PISA sample is not class based, PISA 2000 and PISA 2003 did not collect data at the teacher level. However, PISA collects data at the school level. This chapter describes how and why school level data should be analysed.

Since the PISA target population attends several grades in most countries, it would be interesting to compute the average performance growth between two adjacent grades, so that performance differences between countries could be translated into school year effect. However, this would certainly lead to an overestimation of the performance growth: 15-year-olds attending lower grades are either lower achievers or younger students, and 15-year-olds attending higher grades are either high achievers or older students. Therefore, comparisons of different grade sub-populations cannot be made with confidence. Equalizing these sub-populations by controlling the student performance by a set of background characteristics can be attempted, but things are really never equal.

\section{LIMITS OF THE PISA SCHOOL SAMPLES}

As mentioned earlier, the following statement is valid for both PISA and IEA studies:

Although the student's samples were drawn from within a sample of schools, the school sample was designed to optimize the resulting sample of students, rather than to give an optimal sample of schools. For this reason, it is always preferable to analyse the school-level variables as attributes of students, rather than as elements in their own right (Gonzalez and Kennedy, 2003).

This advice is particularly important in PISA as the target population is not defined as a grade, but as all students of a particular age.

In some countries, lower secondary and upper secondary education are provided by the same school, whereas in others, this is not the case because -i.e.lower and upper secondary education are provided different schools. In these countries, usually, the transition between lower and upper secondary education occurs around the age of 15, i.e. in most cases, at the end of compulsory education. As PISA focuses on the 15-year-old population, it means that one part of the target population 
is attending upper secondary education, while the other is attending lower secondary education. Consequently, in some countries, 15-year-olds can be in different educational institutions.

As discussed in Chapter 2, schools are selected from the school sample frame by the PPS sampling method, i.e. proportionally to the number of 15 -year-olds attending the school. This might mean, for example that upper secondary schools only attended by students over the PISA age of 15 should not be included in the school sample frame. Similarly, lower secondary schools without any 15-yearolds should not be included in the school sample frame.

Thus, neither the lower secondary school population, nor the upper secondary school population represents the 15-year-old school population. In other words, the PISA school target population does not necessarily match the school population(s) within a particular country.

This lack of a perfect match between the usual school population(s) and the PISA school population affects the way school data should be analysed. To avoid biases for the population estimates, school data have to be imported into the student data files and have to be analysed with the student final weight. This means, for example, that one will not estimate the percentage of public schools versus private schools, but will estimate the percentage of 15 -year-olds attending private schools versus public schools. From a pedagogical and a policy point of views, what is really important is not the percentage of schools that present such characteristics, but the percentage of students who are affected by these characteristics, i.e. the percentage of students attending a school with such characteristics.

\section{MERGING THE SCHOOL AND STUDENT DATA FILES}

Box 9.1 provides the SPSS ${ }^{\circledR}$ syntax for merging the student data file and the school data file. Both files need first to be sorted by the identification variables, i.e. CNT, SCHOOLID and STIDSTD in the student data file and CNT and SCHOOLID in the school data file. Afterwards, the two sorted data files can be merged according to the common identification variables, i.e. CNT and SCHOOLID.

Box 9.1 - SPSS syntax for merging the student data file and the school data file

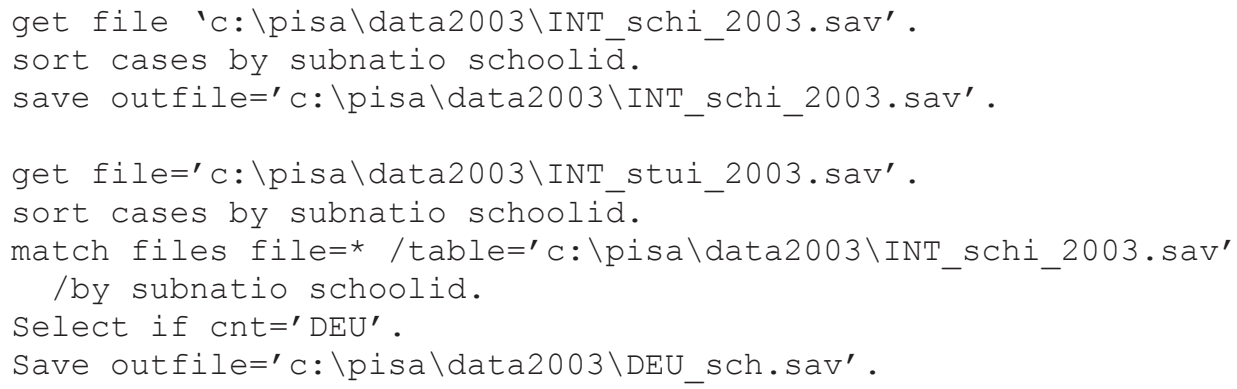

\section{ANALYSES OF THE SCHOOL VARIABLES}

After merging the student data file and the school data file, school data can be analysed like any student level variables as school variables are now considered as attributes of students. However, in this case, it is even more critical to use the replicate weights to compute sampling errors. Failure to do so would give a completely misleading inference. 
The remainder of this chapter explains the methods for computing the percentages of students by school location and their respective standard errors as well as the student average performance on the mathematics scale per school location.

Box 9.2 presents the question about school location in the school questionnaire.

\section{Box 9.2 - School question on school location in PISA 2003}

\section{Q1 Which of the following best describes the community in which} your school is located?

(Please $<$ tick $>$ only one box.)

A < village, hamlet or rural area $>$ (fewer than 3000 people)

A $<$ small town $>$ (3 000 to about 15000 people $)$

A $<$ town $>(15000$ to about 100000 people $)$

A $<_{\text {city }}>$ (100 000 to about 1000000 people)

A large $<$ city $>$ with over 1000000 people

Box 9.3 provides the SPSS ${ }^{\circledR}$ syntax. As previously indicated, the SPSS ${ }^{\circledR}$ macro might be CPUconsuming and thus it is advised to keep only the variables indispensable for the analyses.

\section{Box 9.3 - SPSS ${ }^{\circledR}$ syntax for computing the percentage of students and} the average mathematics performance per school location

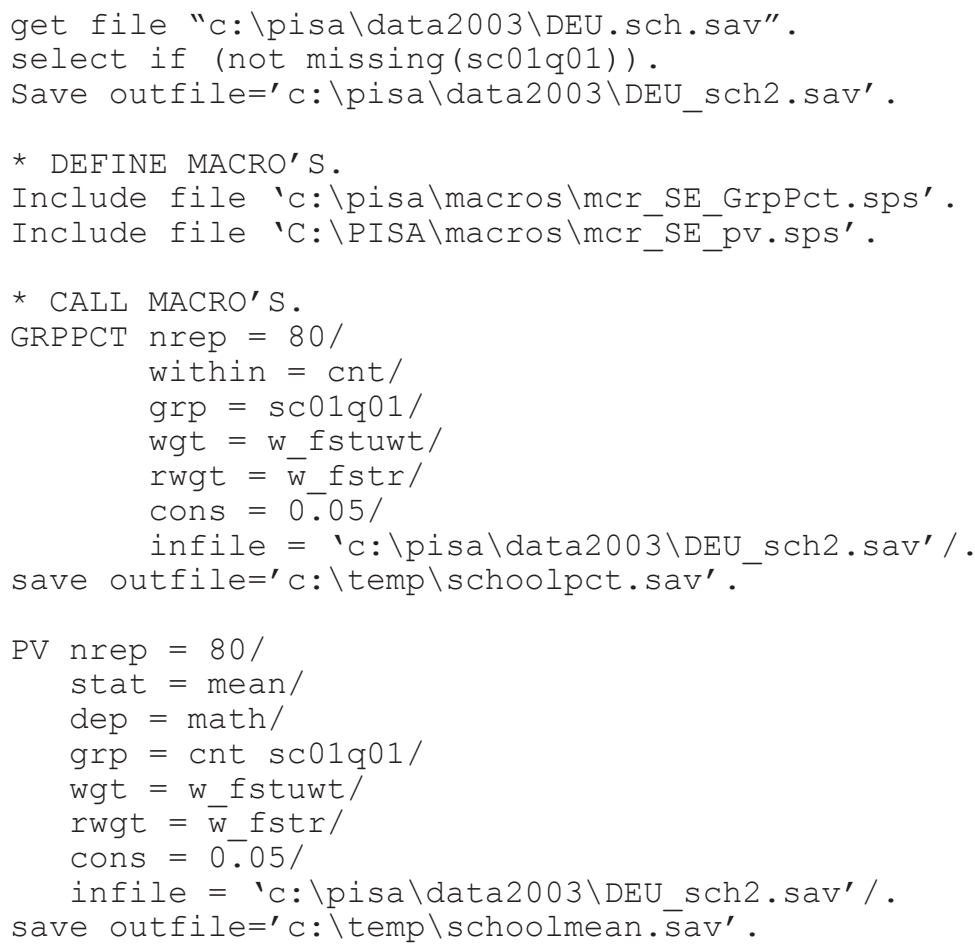


Table 9.2 and Table 9.3 present the structure of the output data files.

Table 9.2 - Structure of the output data file schoolpct.sav

\begin{tabular}{c|c|c|c}
\hline CNT & SC01Q01 & STAT & SE \\
\hline DEU & 1 & 5.04 & 1.37 \\
\hline DEU & 2 & 24.61 & 2.70 \\
\hline DEU & 3 & 38.76 & 3.75 \\
\hline DEU & 4 & 19.53 & 2.77 \\
\hline DEU & 5 & 12.06 & 1.98 \\
\hline
\end{tabular}

Table 9.3 - Structure of the output data file schoolmean.sav

\begin{tabular}{c|c|c|c}
\hline CNT & SC01Q01 & STAT & SE \\
\hline DEU & 1 & 489.65 & 14.81 \\
\hline DEU & 2 & 507.46 & 6.19 \\
\hline DEU & 3 & 496.74 & 8.92 \\
\hline DEU & 4 & 510.24 & 13.19 \\
\hline DEU & 5 & 507.07 & 14.13 \\
\hline
\end{tabular}

As a reminder, the school data was analysed at the student level and weighted by the student final weight. Therefore, results should be interpreted as: 5.04 per cent of the 15 -year-olds are attending a school located in a village with less than 3000 people. Twenty-five per cent of the students are attending a school located in a small town (from 3000 to 15000 people) and so on. The students attending a school located in a small village on average perform at 489.65 and so on.

As the percentages for some categories might be small, the standard error will be large for the mean estimates.

All the SPSS ${ }^{\circledR}$ macros described in the previous chapters can be used on the school variables once they have been imported in the student data file.

\section{CONCLUSIONS}

For statistical and pedagogical reasons, the data collected through the school questionnaire, as well as the variables derived from that instrument, have to be analysed at the student level.

All the SPSS ${ }^{\circledR}$ macros developed can be used without any modifications. The interpretation of the results should clearly state the analysis level, i.e. for instance the percentage of students attending a school located in a small village and not the percentage of schools located in a small village. 


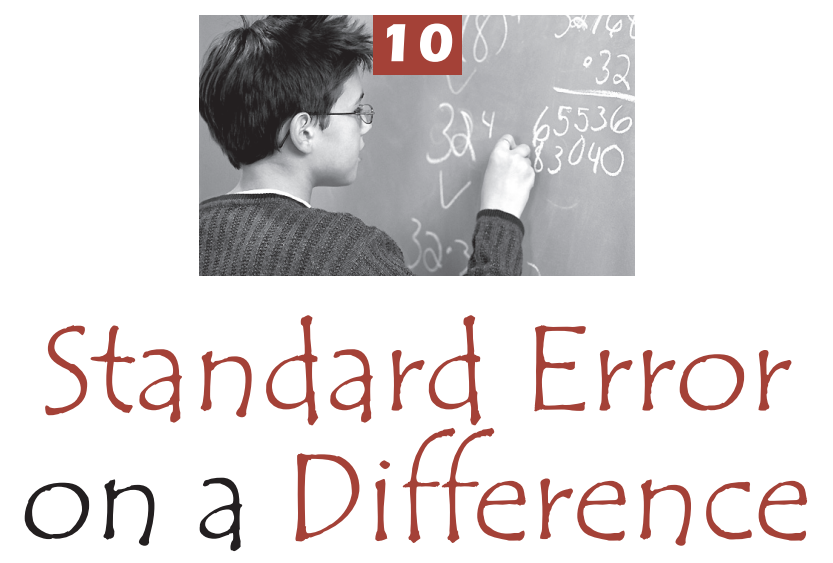

Introduction

The standard error of a difference without plausible values......... 132

The standard error of a difference with plausible values............... 137

Multiple comparisons ................................................................ 140

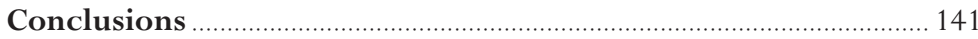




\section{INTRODUCTION}

Suppose that X represents the student score for a mathematics test and $\mathrm{Y}$ the student score for a science test for the same sample of students. To summarize the score distribution for both tests, one can compute:

- $\boldsymbol{\mu}_{(X)}, \boldsymbol{\mu}_{(Y)}$, representing respectively the mean of X and the mean of $\mathrm{Y}$,

- $\sigma_{(X)}^{2}, \sigma_{(Y)}^{2}$, representing respectively the variance of $\mathrm{X}$ and the variance of $\mathrm{Y}$

It can be shown that:

$$
\begin{aligned}
& \mu_{(X+Y)}=\mu_{(X)}+\mu_{(Y)} \text { and } \\
& \sigma_{(X+Y)}^{2}=\sigma_{(X)}^{2}+\sigma_{(Y)}^{2}+2 \operatorname{cov}(X, Y)
\end{aligned}
$$

If a total score is computed by just adding the mathematics and science scores, then according to these two formulae, the mean of this total score will be the sum of the two initial means and the variance of the total score will be equal to the sum of the variance of the two initial variables $\mathrm{X}$ and $\mathrm{Y}$ plus 2 times the covariance between $\mathrm{X}$ and $\mathrm{Y}$. This covariance represents the relationship between $\mathrm{X}$ and Y. Usually, high achievers in mathematics are also high achievers in science and thus, one should expect in this particular example a positive and high covariance.

Similarly,

$$
\begin{aligned}
& \mu_{(X-Y)}=\mu_{(X)}-\mu_{(Y)} \text { and } \\
& \sigma_{(X-Y)}^{2}=\sigma_{(X)}^{2}+\sigma_{(Y)}^{2}-2 \operatorname{cov}(X, Y)
\end{aligned}
$$

In other words, the variance of a difference is equal to the sum of the variances of the two initial variables minus two times the covariance between the two initial variables.

As described in Chapter 3, a sampling distribution has the same characteristics as any distribution, except that units consist of sample estimates and not observations. Therefore,

$$
\sigma_{\left(\hat{\mu}_{X}-\hat{\mu}_{Y}\right)}^{2}=\sigma_{\left(\hat{\mu}_{X}\right)}^{2}+\sigma_{\left(\hat{\mu}_{Y}\right)}^{2}-2 \operatorname{cov}\left(\hat{\mu}_{X}, \hat{\mu}_{Y}\right)
$$

The sampling variance of a difference is equal to the sum of the two initial sampling variances minus two times the covariance between the two sampling distributions on the estimates.

Suppose that one wants to determine whether the girls' performance is on average higher than the boys' performance. As for all statistical analyses, the null hypothesis has to be tested. In this particular example, it will consist of computing the difference between the boys' performance mean and the girls' performance mean or the inverse. The null hypothesis will be:

$$
H_{0}: \hat{\mu}_{(b o y s)}-\hat{\mu}_{(g i r l s)}=0
$$

To test this null hypothesis, the standard error on this difference has to be computed and then compared to the observed difference. The respective standard errors on the mean estimate for boys and for girls $\left(\sigma_{\left(\hat{\mu}_{\text {boys }}\right)}, \sigma_{\left(\hat{\mu}_{\text {girls }}\right)}\right)$ can be easily computed. 
What does the covariance between the two variables, i.e. $\hat{\boldsymbol{\mu}}_{(\text {boys })}, \hat{\boldsymbol{\mu}}_{(\text {girls })}$, tell us? A positive covariance means that if $\hat{\boldsymbol{\mu}}_{\text {(boys) }}$ increases, then $\hat{\boldsymbol{\mu}}_{\text {(girls) }}$ will also increase. A covariance equal or close to 0 means that $\hat{\boldsymbol{\mu}}_{(\text {boys })}$ can increase or decrease with $\hat{\boldsymbol{\mu}}_{(\mathrm{girls})}$ remaining unchanged. Finally, a negative covariance means that if $\hat{\mu}_{(\text {boys })}$ increases, then $\hat{\mu}_{(\text {girls })}$ will decrease, and inversely.

How are $\hat{\boldsymbol{\mu}}_{(\text {boys })}$ and $\hat{\boldsymbol{\mu}}_{\text {(girls) }}$ correlated? Suppose that in the school sample, a coeducational school attended by low achievers is replaced by a coeducational school attended by high achievers. The country mean will increase slightly, as well as the boys and the girls means. If the replacement process is continued, $\hat{\boldsymbol{\mu}}_{(\text {boys })}$ and $\hat{\boldsymbol{\mu}}_{(\text {girls })}$ will likely increase in a similar pattern. Indeed, a coeducational school attended by high achieving boys is usually also attended by high achieving girls. Therefore, the covariance between $\hat{\boldsymbol{\mu}}_{(\text {boys })}$ and $\hat{\boldsymbol{\mu}}_{(\text {girls })}$ will be positive.

Let us now suppose that all schools are single gender. A boys' school can replace a girls school in the sample and therefore $\hat{\boldsymbol{\mu}}_{(b o y s)}$ and $\hat{\boldsymbol{\mu}}_{(\text {girls) }}$ will change. If gender is used as a stratification variable, i.e. all girls schools are allocated to an explicit stratum and all boys schools are allocated to another explicit stratum, then a girls school can only be replaced by another girls school. In this case, only $\hat{\boldsymbol{\mu}}_{(g i r l s)}$ will change. As might change without affecting $\hat{\boldsymbol{\mu}}_{(\text {boys })}$, the expected value of the covariance between $\hat{\boldsymbol{\mu}}_{(\text {boys })}$ and $\hat{\boldsymbol{\mu}}_{(\mathrm{girls})}$ is 0 .

Finally, a negative covariance means that if a school is attended by high achieving boys, then that school is also attended by low achieving girls or the inverse. This situation in not so likely.

In summary, the expected value of the covariance will be equal to 0 if the two sub-samples are independent. If the two sub-samples are not independent, then the expected value of the covariance might differ from 0 .

In PISA, as well as in IEA studies, country samples are independent. Therefore, for any comparison between two countries, the expected value of the covariance will be equal to 0 , and thus the standard error on the estimate is:

$$
\sigma_{\left(\hat{\theta}_{i}-\hat{\theta}_{j}\right)}=\sqrt{\sigma_{\left(\hat{\theta}_{i}\right)}^{2}+\sigma_{\left(\hat{\theta}_{j}\right)}^{2}} \text {, with } \theta \text { being any statistic. }
$$

For instance, on the mathematics literacy scale in PISA 2003, the German mean is equal to 503 with a standard error of 3.3, and the Belgian mean is equal to 529 with a standard error of 2.3.Therefore, the difference between Belgium and Germany will be 529-503=26 and the standard error on this difference is:

$$
\sigma_{\left(\hat{\theta}_{i}-\hat{\theta}_{j}\right)}=\sqrt{\sigma_{\left(\hat{\theta}_{i}\right)}^{2}+\sigma_{\left(\hat{\theta}_{j}\right)}^{2}}=\sqrt{(3.3)^{2}+(2.3)^{2}}=\sqrt{10.89+5.29}=\sqrt{16.18}=4.02
$$

The difference divided by its standard error, i.e. $26 / 4.02=6.46$, is greater than 1.96 , which is significant. This means that Belgian's performance is greater than Germany's.

Similarly, the percentage of students below Level 1 is equal to 9.2 in Germany (with a standard error of 0.8) and to 7.2 in Belgium (with a standard error of 0.6). The difference is equal to 9.2 $-7.2=2.0$ and the standard error on this difference is equal to:

$$
\sigma_{\left(\hat{\theta}_{i}-\hat{\theta}_{j}\right)}=\sqrt{\sigma_{\left(\hat{\theta}_{i}\right)}^{2}+\sigma_{\left(\hat{\theta}_{j}\right)}^{2}}=\sqrt{(0.6)^{2}+(0.8)^{2}}=\sqrt{0.36+0.64}=\sqrt{1}=1 .
$$


The standardised difference is equal to 2 (i.e. 2/1), which is significant. Thus the percentage of students below Level 1 is greater in Germany than in Belgium.

Within a particular country, any sub-samples will be considered as independent if the categorical variable used to define the sub-samples was used as an explicit stratification variable. For instance, since Canada used the provinces as an explicit stratification variable, then these sub-samples are independent and any comparison between two provinces does not require the estimation of the covariance between the sampling distributions.

As a general rule, any comparison between countries does not require the estimation of the covariance, but it is strongly advised to estimate the covariance between the sampling distributions for any within-country comparisons.

As described earlier in this section, the estimation of the covariance between, for instance, $\hat{\boldsymbol{\mu}}_{\text {(boys) }}$ and $\hat{\mu}_{\text {(girls) }}$ would require the selection of several samples and then the analysis of the variation of

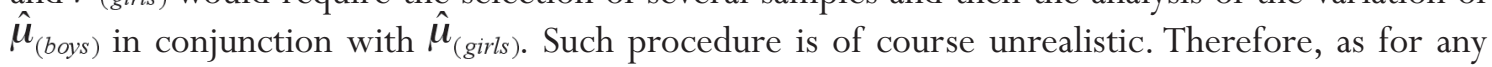
computation of a standard error in PISA, replication methods using the supplied replicate weights will be used to estimate the standard error on a difference.

\section{THE STANDARD ERROR OF A DIFFERENCE WITHOUT PLAUSIBLE VALUES}

Let us suppose that a researcher wants to test whether girls in Germany have higher job expectations than boys.

As described in Chapter 6, the $\operatorname{SPSS}^{\circledR}$ macro UNIVAR can be used to estimate the average job expectation for boys and girls respectively.

Box 10.1 presents the $\operatorname{SPSS}^{\circledR}$ syntax for the computation of the mean for the job expectations at the age of 30 (BSMJ) per gender. Table 10.1 presents the structure of the output data file as well as the results per gender.

Box 10.1 = SPSS $^{\circledR}$ syntax for computing the mean of job expectations per gender

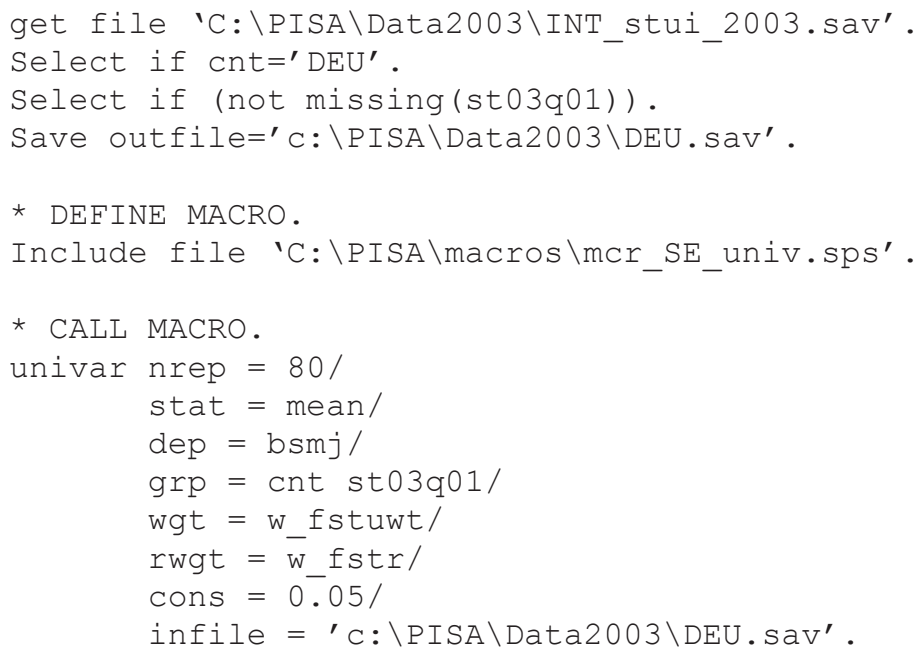


Table 10.1 - Structure of the output data file from Box $\mathbf{1 0 . 1}$

\begin{tabular}{c|c|c|c}
\hline CNT & ST03Q01 & STAT & SE \\
\hline DEU & 1 & 53.05 & 0.57 \\
\hline DEU & 2 & 50.58 & 0.69 \\
\hline
\end{tabular}

On average, the job expectation is 53.05 for girls and 50.58 for boys. As German schools are usually coeducational and as gender is not used as an explicit stratification variable, the expected value of the covariance might differ from 0 .

To compute the standard error per gender, it is necessary to compute the mean estimate for each of the 80 replicate weights. Table 10.2 presents the mean estimate per weight and per gender.

The final difference estimate will be the difference between the two final estimates, i.e. $53.05-50.58=2.47$.

The procedure to estimate the final standard error is quite straightforward. It is exactly similar to the procedure described in Chapter 6 , except that $\theta$ is now a difference, and not a mean or a regression coefficient. The different steps are:

- The difference between the girls and the boys means is computed per replicate;

- each of the 80 difference estimates is compared with the final difference estimate, then squared;

- the sum of the square is computed then divided by 20 to obtain the sampling variance on the difference; and

- The standard error is the square root of the sampling variance.

These different steps can be summarized as:

$\sigma_{(\hat{\theta})}=\sqrt{\frac{1}{20} \sum_{i=1}^{80}\left(\hat{\theta}_{(i)}-\hat{\theta}\right)^{2}}$ with $\theta$ being a difference.

Concretely:

- For the first replicate, the difference between the girls mean estimate and the boys mean estimate is equal to $(53.29-50.69)=2.60$. For the second replicate, the difference estimate will be equal to $(53.16-50.53)=2.63$ and so on for the 80 replicates. All these difference estimates are presented in Table 10.3.

- Each of the 80 replicate difference estimates is compared with the final difference estimate and this difference is squared. For the first replicate, it will be $(2.60-2.47)^{2}=0.0164$. For the second replicates, it will be $(2.63-2.47)^{2}=0.0258$. These squared differences are also presented in Table 10.3

- These squared differences are summed. This sum is equal to:

$(0.0164+0.0258+\ldots . .+0.0641)=9.7360$. The sampling variance on the difference is therefore equal to:

$$
\frac{9.7360}{20}=0.4868
$$

- The standard error is equal to the square root of 0.4868 , i.e. 0.6977 . 
As $\frac{2.47}{0.6977}$ is greater than 1.96 , job expectations for girls are statistically greater than job expectations for boys in Germany.

If the researcher had considered the two German sub-samples as independent, then he/she would have obtained for the standard error on this difference:

$$
\sigma_{\left(\hat{\theta}_{i}-\hat{\theta}_{j}\right)}=\sqrt{\sigma_{\left(\hat{\theta}_{i}\right)}^{2}+\sigma_{\left(\hat{\theta}_{j}\right)}^{2}}=\sqrt{(0.57)^{2}+(0.69)^{2}}=0.895
$$

In this particular case, the difference between the unbiased estimate of the standard error (i.e. 0.698) and the biased estimate of the standard error (i.e. 0.895) is quite small. As it will be shown later in this chapter, the difference between the biased and unbiased estimates of the standard error can be substantial.

Table 10.2 Mean estimates for the final and 80 replicate weights per gender

\begin{tabular}{|c|c|c|c|c|c|}
\hline Weight & $\begin{array}{l}\text { Mean estimate } \\
\text { for girls }\end{array}$ & $\begin{array}{l}\text { Mean estimate } \\
\text { for boys }\end{array}$ & Weight & $\begin{array}{l}\text { Mean estimate } \\
\text { for girls }\end{array}$ & $\begin{array}{c}\text { Mean estimate } \\
\text { for boys }\end{array}$ \\
\hline Final weight & 53.05 & 50.58 & & & \\
\hline Replicate 1 & 53.29 & 50.69 & Replicate 41 & 52.69 & 50.55 \\
\hline Replicate 2 & 53.16 & 50.53 & Replicate 42 & 53.28 & 51.23 \\
\hline Replicate 3 & 53.16 & 50.45 & Replicate 43 & 53.07 & 50.39 \\
\hline Replicate 4 & 53.30 & 50.70 & Replicate 44 & 52.95 & 49.72 \\
\hline Replicate 5 & 52.79 & 50.28 & Replicate 45 & 53.31 & 51.04 \\
\hline Replicate 6 & 53.14 & 50.76 & Replicate 46 & 53.72 & 50.80 \\
\hline Replicate 7 & 53.04 & 50.36 & Replicate 47 & 52.91 & 51.03 \\
\hline Replicate 8 & 52.97 & 50.11 & Replicate 48 & 53.10 & 50.53 \\
\hline Replicate 9 & 53.28 & 51.37 & Replicate 49 & 53.05 & 50.81 \\
\hline Replicate 10 & 53.01 & 50.55 & Replicate 50 & 53.79 & 50.90 \\
\hline Replicate 11 & 53.26 & 50.70 & Replicate 51 & 52.65 & 50.15 \\
\hline Replicate 12 & 53.16 & 49.86 & Replicate 52 & 53.30 & 50.45 \\
\hline Replicate 13 & 52.81 & 50.94 & Replicate 53 & 52.68 & 50.12 \\
\hline Replicate 14 & 53.21 & 50.71 & Replicate 54 & 52.74 & 50.01 \\
\hline Replicate 15 & 53.39 & 50.23 & Replicate 55 & 53.50 & 50.11 \\
\hline Replicate 16 & 53.06 & 50.46 & Replicate 56 & 52.54 & 50.58 \\
\hline Replicate 17 & 53.34 & 50.48 & Replicate 57 & 53.31 & 51.03 \\
\hline Replicate 18 & 52.71 & 50.42 & Replicate 58 & 53.13 & 50.34 \\
\hline Replicate 19 & 53.18 & 50.87 & Replicate 59 & 52.72 & 50.37 \\
\hline Replicate 20 & 52.82 & 50.44 & Replicate 60 & 53.49 & 51.43 \\
\hline Replicate 21 & 53.36 & 50.74 & Replicate 61 & 53.13 & 50.71 \\
\hline Replicate 22 & 53.15 & 50.72 & Replicate 62 & 53.61 & 51.27 \\
\hline Replicate 23 & 53.24 & 50.65 & Replicate 63 & 52.74 & 50.15 \\
\hline Replicate 24 & 52.68 & 50.51 & Replicate 64 & 53.19 & 50.25 \\
\hline Replicate 25 & 52.76 & 50.44 & Replicate 65 & 53.28 & 51.04 \\
\hline Replicate 26 & 52.79 & 50.43 & Replicate 66 & 52.91 & 50.94 \\
\hline Replicate 27 & 53.01 & 50.58 & Replicate 67 & 53.25 & 50.85 \\
\hline Replicate 28 & 53.24 & 50.12 & Replicate 68 & 53.12 & 50.74 \\
\hline Replicate 29 & 52.86 & 50.68 & Replicate 69 & 53.08 & 50.31 \\
\hline Replicate 30 & 52.85 & 50.02 & Replicate 70 & 52.92 & 50.44 \\
\hline Replicate 31 & 52.90 & 50.85 & Replicate 71 & 53.35 & 50.63 \\
\hline Replicate 32 & 53.25 & 50.60 & Replicate 72 & 53.25 & 50.75 \\
\hline Replicate 33 & 53.32 & 50.54 & Replicate 73 & 52.54 & 50.42 \\
\hline Replicate 34 & 52.42 & 50.55 & Replicate 74 & 52.58 & 50.20 \\
\hline Replicate 35 & 52.91 & 50.72 & Replicate 75 & 52.49 & 49.75 \\
\hline Replicate 36 & 53.06 & 50.36 & Replicate 76 & 52.98 & 50.96 \\
\hline Replicate 37 & 52.67 & 50.73 & Replicate 77 & 53.04 & 50.24 \\
\hline Replicate 38 & 53.36 & 50.16 & Replicate 78 & 53.30 & 50.44 \\
\hline Replicate 39 & 52.57 & 50.36 & Replicate 79 & 52.93 & 50.36 \\
\hline Replicate 40 & 53.07 & 50.58 & Replicate 80 & 52.98 & 50.76 \\
\hline
\end{tabular}


Table 10.3 - Difference estimates for the final and 80 replicate weights

\begin{tabular}{|c|c|c|c|c|c|}
\hline Weight & $\begin{array}{c}\text { Difference } \\
\text { between } \\
\text { boys and girls } \\
(\mathrm{G}-\mathrm{B})\end{array}$ & $\begin{array}{l}\text { Squared } \\
\text { difference } \\
\text { between the } \\
\text { replicate and the } \\
\text { final estimates }\end{array}$ & Weight & $\begin{array}{l}\text { Difference } \\
\text { between } \\
\text { boys and } \\
\text { girls }(\mathrm{G}-\mathrm{B})\end{array}$ & $\begin{array}{c}\text { Squared } \\
\text { difference } \\
\text { between the } \\
\text { replicate and the } \\
\text { final estimates }\end{array}$ \\
\hline Final weight & 2.47 & & & & \\
\hline Replicate 1 & 2.60 & 0.0164 & Replicate 41 & 2.14 & 0.1079 \\
\hline Replicate 2 & 2.63 & 0.0258 & Replicate 42 & 2.05 & 0.1789 \\
\hline Replicate 3 & 2.72 & 0.0599 & Replicate 43 & 2.68 & 0.0440 \\
\hline Replicate 4 & 2.61 & 0.0180 & Replicate 44 & 3.23 & 0.5727 \\
\hline Replicate 5 & 2.51 & 0.0011 & Replicate 45 & 2.28 & 0.0373 \\
\hline Replicate 6 & 2.39 & 0.0067 & Replicate 46 & 2.92 & 0.2038 \\
\hline Replicate 7 & 2.68 & 0.0450 & Replicate 47 & 1.88 & 0.3488 \\
\hline Replicate 8 & 2.86 & 0.1483 & Replicate 48 & 2.56 & 0.0084 \\
\hline Replicate 9 & 1.92 & 0.3085 & Replicate 49 & 2.23 & 0.0567 \\
\hline Replicate 10 & 2.46 & 0.0002 & Replicate 50 & 2.89 & 0.1768 \\
\hline Replicate 11 & 2.57 & 0.0089 & Replicate 51 & 2.49 & 0.0004 \\
\hline Replicate 12 & 3.30 & 0.6832 & Replicate 52 & 2.85 & 0.1440 \\
\hline Replicate 13 & 1.87 & 0.3620 & Replicate 53 & 2.56 & 0.0072 \\
\hline Replicate 14 & 2.50 & 0.0009 & Replicate 54 & 2.73 & 0.0667 \\
\hline Replicate 15 & 3.16 & 0.4756 & Replicate 55 & 3.39 & 0.8520 \\
\hline Replicate 16 & 2.60 & 0.0173 & Replicate 56 & 1.96 & 0.2631 \\
\hline Replicate 17 & 2.87 & 0.1577 & Replicate 57 & 2.28 & 0.0351 \\
\hline Replicate 18 & 2.29 & 0.0327 & Replicate 58 & 2.79 & 0.1017 \\
\hline Replicate 19 & 2.31 & 0.0269 & Replicate 59 & 2.35 & 0.0158 \\
\hline Replicate 20 & 2.38 & 0.0078 & Replicate 60 & 2.05 & 0.1749 \\
\hline Replicate 21 & 2.62 & 0.0221 & Replicate 61 & 2.42 & 0.0027 \\
\hline Replicate 22 & 2.43 & 0.0014 & Replicate 62 & 2.34 & 0.0164 \\
\hline Replicate 23 & 2.59 & 0.0142 & Replicate 63 & 2.59 & 0.0137 \\
\hline Replicate 24 & 2.17 & 0.0901 & Replicate 64 & 2.94 & 0.2230 \\
\hline Replicate 25 & 2.32 & 0.0227 & Replicate 65 & 2.24 & 0.0539 \\
\hline Replicate 26 & 2.36 & 0.0132 & Replicate 66 & 1.97 & 0.2524 \\
\hline Replicate 27 & 2.43 & 0.0015 & Replicate 67 & 2.40 & 0.0050 \\
\hline Replicate 28 & 3.12 & 0.4225 & Replicate 68 & 2.38 & 0.0089 \\
\hline Replicate 29 & 2.18 & 0.0844 & Replicate 69 & 2.76 & 0.0848 \\
\hline Replicate 30 & 2.84 & 0.1333 & Replicate 70 & 2.48 & 0.0002 \\
\hline Replicate 31 & 2.06 & 0.1709 & Replicate 71 & 2.72 & 0.0609 \\
\hline Replicate 32 & 2.65 & 0.0312 & Replicate 72 & 2.50 & 0.0006 \\
\hline Replicate 33 & 2.78 & 0.0970 & Replicate 73 & 2.12 & 0.1217 \\
\hline Replicate 34 & 1.87 & 0.3611 & Replicate 74 & 2.39 & 0.0073 \\
\hline Replicate 35 & 2.19 & 0.0809 & Replicate 75 & 2.73 & 0.0693 \\
\hline Replicate 36 & 2.69 & 0.0490 & Replicate 76 & 2.02 & 0.2031 \\
\hline Replicate 37 & 1.94 & 0.2825 & Replicate 77 & 2.80 & 0.1058 \\
\hline Replicate 38 & 3.20 & 0.5355 & Replicate 78 & 2.86 & 0.1519 \\
\hline Replicate 39 & 2.21 & 0.0683 & Replicate 79 & 2.57 & 0.0091 \\
\hline Replicate 40 & 2.48 & 0.0001 & Replicate 80 & 2.22 & 0.0641 \\
\hline & & & \multicolumn{2}{|c|}{ Sum of squared differences } & 9.7360 \\
\hline
\end{tabular}


An SPSS ${ }^{\circledR}$ macro has been developed for the computation of standard errors on differences. Box 10.2 presents the SPSS ${ }^{\circledR}$ syntax for running this macro.

\section{Box 10.2 - SPSS $^{\circledR}$ syntax for the computation of standard errors on differences}

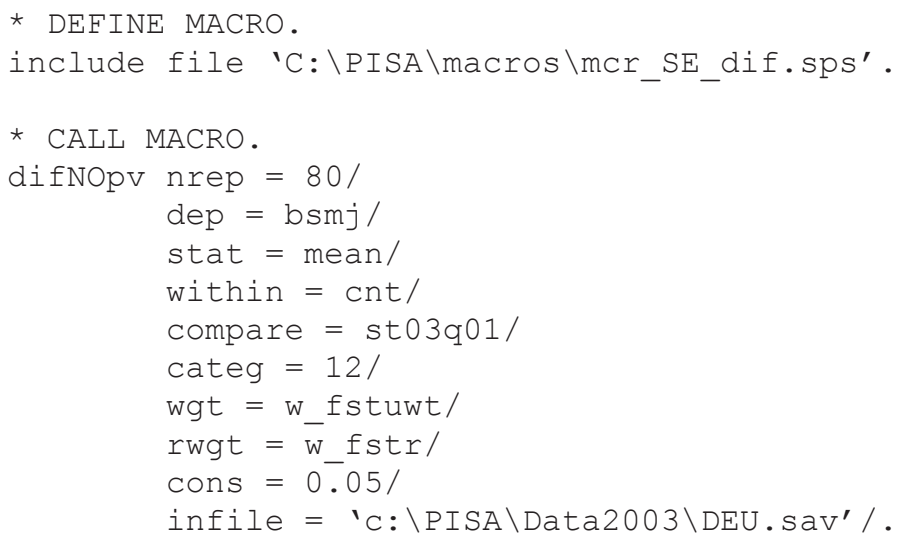

Beside the arguments common to all SPSS ${ }^{\circledR}$ macros, four other arguments have to be specified:

- The DEP argument informs the macro of the numerical variable on which a mean or a standard deviation will be computed per value of a categorical variable. In the example, DEP equals BSMJ.

- The COMPARE argument specifies the categorical variables on which the contrasts will be based.

- The CATEG argument specifies the values of the categorical variables for which contrasts are required. As gender has only two categories, denoted 1 and 2, the CATEG statement is set as "12/". There should be no spaces or other characters between the categories in the CATEG argument and the '/' should follow directly after the last category. If a categorical variable has four categories and if these four categories are specified in the CATEGORY statement (like CATEG = 1234/), then the macro will compute the standard error on the difference between:

- Category 1 and category 2;

- Category 1 and category 3;

- Category 1 and category 4;

- Category 2 and category 3;

- Category 2 and category 4; and

- Category 3 and category 4.

This macro has a few limitations:

- A maximum of 9 categories can be specified of the compare variable;

- The categorical compare variable should be defined as numeric, so string variables should be converted into numeric variables; and

- The values of the categories should be one digit only. Double digit values have to be recoded first.

- The STAT argument specifies the required statistic. See Chapter 5 for available statistics. 
Table 10.4 - Structure of the output data file from Box $\mathbf{1 0 . 2}$

\begin{tabular}{c|c|c}
\hline CNT & STAT & SE \\
\hline DEU & 2.47 & 0.6977 \\
\hline
\end{tabular}

It is worth noting that for dichotomous variables, the standard error on the difference can also be computed by a regression model.

Box 10.3 - An alternative SPSS syntax for computing the standard error on a difference for dichotomous variable

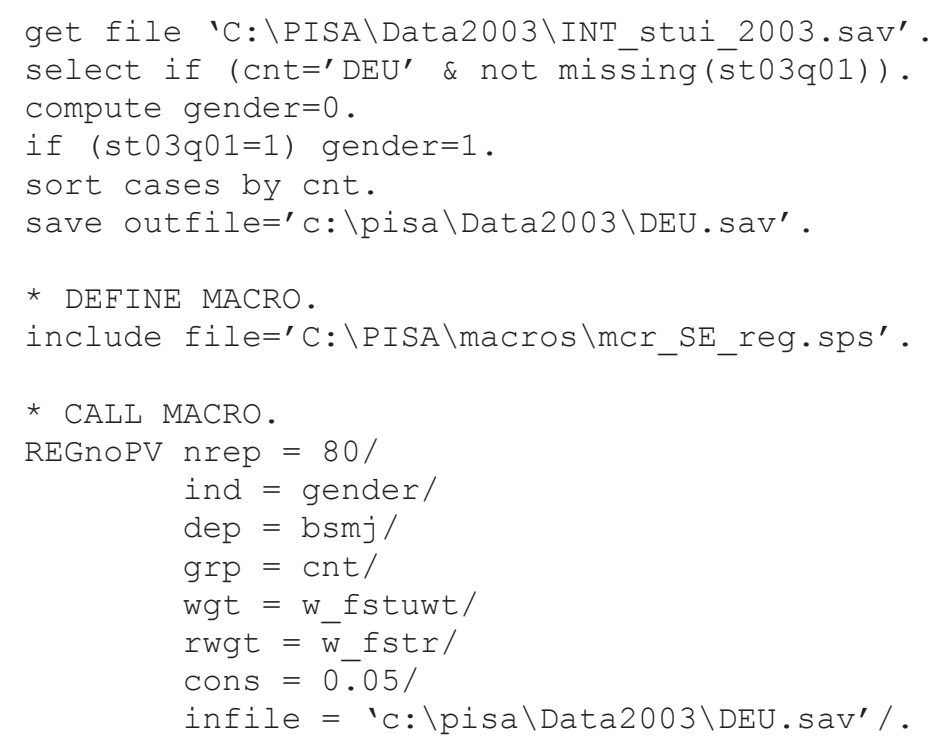

Table 10.5 - Structure of the output data file from Box $\mathbf{1 0 . 3}$

\begin{tabular}{c|c|c|c}
\hline CNT & CLASS & STAT & SE \\
\hline DEU & $\mathrm{b}_{0}$ & 50.58 & 0.686 \\
\hline DEU & gender & 2.47 & 0.698 \\
\hline
\end{tabular}

The difference estimate and its respective standard error are equal to the regression coefficient estimate and its standard error. For polytomous categorical variables, the use of the regression macro would require the recoding of the categorical variables into $h-1$ dichotomous variables, with $h$ being equal to the number of categories. Further, the regression macro will compare each category with the reference category (in the table above, the reference group is the boys), while the macro DIFNOPV will provide all contrasts.

\section{THE STANDARD ERROR OF A DIFFERENCE WITH PLAUSIBLE VALUES}

The procedure for computing the standard error on a difference that involves plausible values consists of: 
- Using each plausible value and for the final and 80 replicate weights, the requested statistic, a mean for example, has to be computed per value of the categorical variable;

- Computing per contrast, per plausible value and per replicate weight, the difference between the two categories. There will be 405 difference estimates: Table 10.6 presents the structure of these 405 differences;

- A final difference estimate equal to the average of the five difference estimates;

- Computing, per plausible value, the sampling variance by comparing the final difference estimate with the 80 replicate estimates;

- A final sampling variance equal to the average of the five sampling variances;

- Computing the imputation variance, also denoted as measurement error variance;

- Combining the sampling variance and the imputation variance to obtain the final error variance; and

- A standard error equal to the square root of the error variance.

Table 10.6 - Gender difference estimates and their respective sampling variances on the mathematics scale

\begin{tabular}{l|c|c|c|c|c}
\hline Weight & PV1 & PV2 & PV3 & PV4 & PV5 \\
\hline Final & -8.94 & -9.40 & -8.96 & -7.46 & -10.12 \\
\hline Replicate 1 & -9.64 & -10.05 & -10.29 & -8.74 & -11.45 \\
\hline$\ldots \ldots \ldots$. & & & & & \\
\hline Replicate 80 & -8.56 & -8.52 & -8.85 & -7.70 & -9.84 \\
\hline $\begin{array}{l}\text { Sampling } \\
\text { variance }\end{array}$ & $(4.11)^{2}$ & $(4.36)^{2}$ & $(4.10)^{2}$ & $(4.31)^{2}$ & $(4.28)^{2}$ \\
\hline
\end{tabular}

An SPSS ${ }^{\circledR}$ macro has been developed to compute standard errors on differences that involve plausible values. Box 10.4 provides the SPSS ${ }^{\circledR}$ syntax. In this example, the standard error on the difference between the boys' and the girls' performance on the combined reading literacy scale is computed.

\section{Box 10.4 . SPSS $^{\circledR}$ syntax for computing standard errors on differences that involve plausible values}

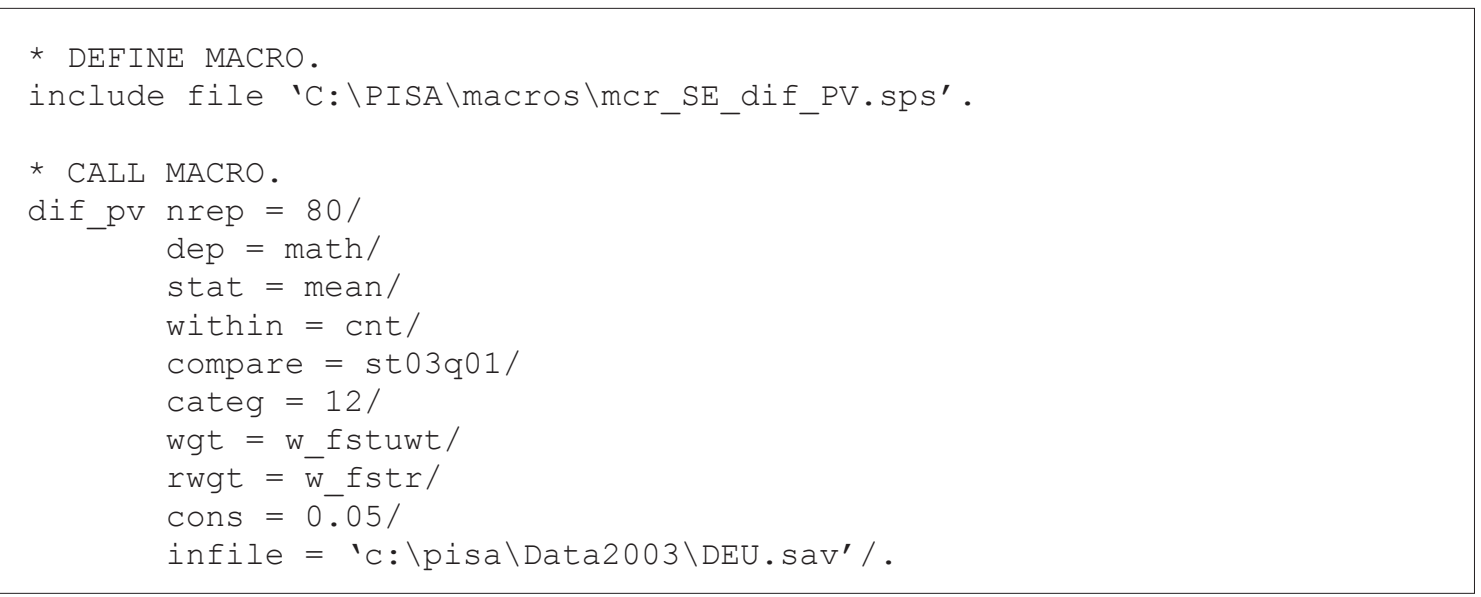


Table 10.7 presents the structure of the output data file.

Table 10.7 - Structure of the output data file from Box 10.4

\begin{tabular}{c|c|c}
\hline CNT & STAT & SE \\
\hline DEU & -8.98 & 4.37 \\
\hline
\end{tabular}

As the ratio between the difference estimate and its respective standard error is greater than 1.96, the null hypothesis is rejected. Thus girls perform on average lower than boys in Germany. It is also worth noting that these results might also be obtained through the regression macro for plausible values.

Table 10.8 provides for all PISA 2003 countries the gender difference estimates on the mathematics scale, as well as the unbiased standard errors and the biased standard errors.

\section{Table 10.8 - Gender differences on the mathematics scale, unbiased standard errors} and biased standard errors

\begin{tabular}{|c|c|c|c|c|c|c|c|}
\hline Country & $\begin{array}{c}\text { Mean } \\
\text { difference }\end{array}$ & $\begin{array}{l}\text { Unbiased } \\
\text { standard } \\
\text { error }\end{array}$ & $\begin{array}{c}\text { Biased } \\
\text { standard } \\
\text { error }\end{array}$ & Country & $\begin{array}{c}\text { Mean } \\
\text { difference }\end{array}$ & $\begin{array}{l}\text { Unbiased } \\
\text { standard } \\
\text { error }\end{array}$ & $\begin{array}{c}\text { Biased } \\
\text { standard } \\
\text { error }\end{array}$ \\
\hline AUS & -5.34 & 3.75 & 4.04 & KOR & -23.41 & 6.77 & 6.90 \\
\hline AUT & -7.57 & 4.40 & 5.59 & LIE & -28.84 & 10.92 & 9.58 \\
\hline BEL & -7.51 & 4.81 & 4.69 & LUX & -17.17 & 2.81 & 2.40 \\
\hline BRA & -16.26 & 4.06 & 7.49 & LVA & -2.81 & 3.97 & 5.97 \\
\hline $\mathrm{CAN}$ & -11.17 & 2.13 & 2.78 & MAC & -21.26 & 5.83 & 5.83 \\
\hline CHE & -16.63 & 4.87 & 5.98 & MEX & -10.90 & 3.94 & 5.91 \\
\hline $\mathrm{CZE}$ & -14.97 & 5.08 & 6.11 & NLD & -5.12 & 4.29 & 5.36 \\
\hline DEU & -8.98 & 4.37 & 5.59 & NOR & -6.22 & 3.21 & 4.04 \\
\hline DNK & -16.58 & 3.20 & 4.50 & NZL & -14.48 & 3.90 & 4.23 \\
\hline ESP & -8.86 & 2.98 & 4.02 & POL & -5.59 & 3.14 & 4.18 \\
\hline FIN & -7.41 & 2.67 & 3.24 & PRT & -12.25 & 3.31 & 5.41 \\
\hline FRA & -8.51 & 4.15 & 4.60 & RUS & -10.12 & 4.36 & 6.75 \\
\hline GBR & -6.66 & 4.90 & 4.84 & SVK & -18.66 & 3.65 & 5.30 \\
\hline GRC & -19.40 & 3.63 & 6.11 & SWE & -6.53 & 3.27 & 4.30 \\
\hline HKG & -4.06 & 6.64 & 7.96 & THA & 4.02 & 4.24 & 5.22 \\
\hline HUN & -7.79 & 3.54 & 4.69 & TUN & -12.17 & 2.51 & 4.01 \\
\hline IDN & -3.34 & 3.39 & 6.02 & TUR & -15.13 & 6.16 & 10.33 \\
\hline IRL & -14.81 & 4.19 & 4.54 & URY & -12.09 & 4.15 & 5.51 \\
\hline ISL & 15.41 & 3.46 & 3.15 & USA & -6.25 & 2.89 & 4.65 \\
\hline ITA & -17.83 & 5.89 & 5.96 & YUG & -1.21 & 4.36 & 6.14 \\
\hline JPN & -8.42 & 5.89 & 7.04 & & & & \\
\hline
\end{tabular}

In nearly all countries, the unbiased standard error is smaller than the biased standard error, reflecting a positive covariance between the two sampling distributions. In a few countries, the difference between the two standard errors is small, but it is substantial for some other countries, such as Brazil, Greece, Indonesia and Turkey. 


\section{MULTIPLE COMPARISONS}

In Chapter 3, it was noted that every statistical inference is associated with what is usually called a type I error. This error represents the risk of rejecting a null hypothesis that is true.

Let us suppose that at the population level, there is no difference in the reading performance between boys and girls. A sample is drawn and the gender difference in reading performance is computed. As this difference is based on a sample, a standard error on the difference has to be computed. If the standardised difference, i.e. the gender difference divided by its standard error, is less than -1.96 or greater than 1.96, that difference would be reported as significant. In fact, there are 5 chances out of 100 to observe a standardised difference lower than -1.96 or higher than 1.96 and still have the null hypothesis true. In other words, there are 5 chances out of 100 to reject the null hypothesis, when there is no true gender difference in the population.

If 100 countries are participating in the international survey and if the gender difference is computed for each of them, then it is statistically expected to report 5 of the 100 gender differences as significant, when there are no true differences at the population level.

For every country, the type I error is set at 0.05 . For two countries, as countries are independent samples, the probability of not making a type I error, i.e. accepting both null hypotheses, is now equal to 0.9025 (0.95 times 0.95 ). Table 10.9 presents the cross tabulation of the different probabilities.

Table 10.9 - The cross tabulation of the different probabilities

\begin{tabular}{|c|c|c|c|}
\hline & & \multicolumn{2}{|c|}{ Country A } \\
\hline & & 0.05 & 0.95 \\
\hline \multirow{2}{*}{ Country B } & 0.05 & 0.0025 & 0.0475 \\
\hline & 0.95 & 0.0475 & 0.9025 \\
\hline
\end{tabular}

This statistical issue is even more amplified for tables of multiple comparisons of achievement. Suppose that the means of three countries need to be compared. This will involve three tests: country A versus country B, country A versus country C, and country B versus country C. The probability of not making a type I error is therefore equal to:

$$
(1-\alpha)(1-\alpha)(1-\alpha)=(1-\alpha)^{3} .
$$

Broadly speaking, if X comparisons are tested, then the probability of not making a type I error is equal to:

$$
(1-\alpha)^{\mathrm{x}}
$$

Dunn (1961) developed a general procedure that is appropriate for testing a set of a priori hypotheses, while controlling the probability of making a type I error. It consists of adjusting the value $\alpha$. Precisely, the value $\alpha$ is divided by the number of comparisons and then its respective critical value is used.

In the case of three comparisons, the critical value for an $\alpha=0.05$ will therefore be equal to 2.24 instead of 1.96. Indeed,

$$
\frac{0.05}{3}=0.01666
$$


As the risk is shared by both tails of the sampling distribution, one has to find the $\mathrm{z}$ score that corresponds to the cumulative proportion of 0.008333. Consulting the cumulative function of the standardised normal distribution will return the value -2.24 .

Nevertheless, the researcher still has to decide how many comparisons are involved. In PISA, it was decided that no correction of the critical value will be applied, except on multiple comparison tables. Indeed, in many cases, readers are primarily interested in finding out whether a given value in a particular country is different from a second value in the same or another country, e.g. whether females in a country perform better than males in the same country. Therefore, as only one test is performed at a time, then no adjustment is required.

On the other hand, with multiple comparison tables, the reader is interested in comparing the performance of one country with all other countries. For example, if one wants to compare the performance of country 1 with all other countries, we will have the following comparisons: country 1 versus country 2, country 1 versus country 3 , and country 1 versus country L. Therefore, the adjustment will be based on L-1 comparisons.

In PISA 2003, as the results of 40 countries were published in the initial reports, the critical value will be based on 39 comparisons and will be equal to 3.2272. As more countries participated in PISA 2003, this critical value is slightly higher than the critical value for PISA $2000 .{ }^{1}$

\section{CONCLUSIONS}

This chapter was devoted to the computation of standard errors on differences. After a description of the statistical issues for such estimates, the different steps for computing such standard errors were presented. The SPSS ${ }^{\circledR}$ macros to facilitate such computations were also described.

It was clearly stated that any comparison between countries does not require the estimation of the covariance, so macros from previous chapters can be used and the standard errors can be pooled. However, it is strongly advised that the covariance between the sampling distributions for any within country comparisons should be estimated. New macros are introduced for these estimations.

Finally, the correction of the critical value for multiple comparisons was discussed.

1. The critical value in the multiple comparisons for PISA 2000 was 3.144. 



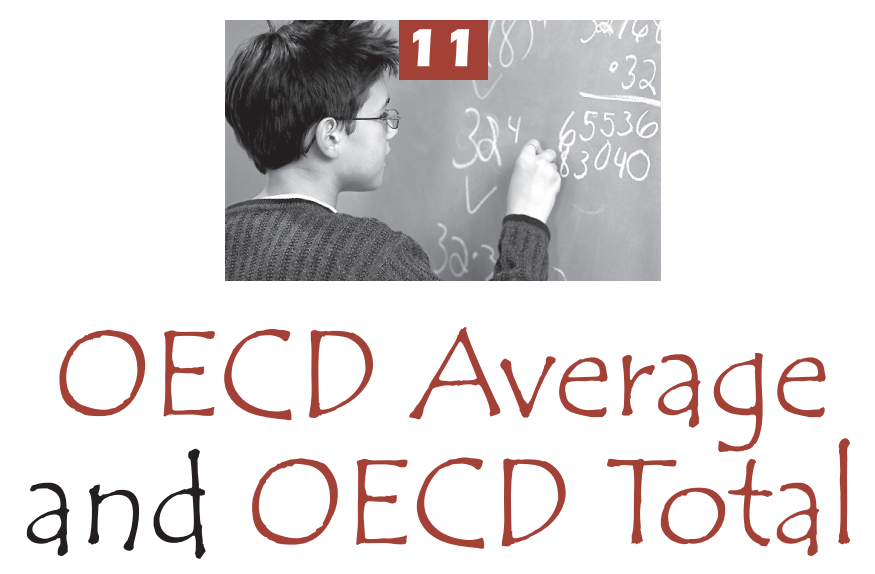

Introduction

Recoding of the database for the estimation of the OECD total and OECD average.

Duplication of the data for avoiding three runs of the procedure... 147 Comparisons between OECD average or OECD total estimates and a country estimate

Conclusions 


\section{INTRODUCTION}

In all PISA initial and thematic reports, the OECD gives the results for each country, but also two additional aggregated estimates: the OECD average and the OECD total.

The OECD average, sometimes also referred to as the country average, is the mean of the data values for all OECD countries for which data are available or can be estimated. The OECD average can be used to see how a country compares on a given indicator with a typical OECD country. The OECD average does not take into account the absolute size of the population in each country, i.e. each country contributes equally to the average. The contribution of the smallest OECD country, i.e. Luxembourg, is equivalent to the one of the largest country, i.e. the United States.

The OECD total considers all the OECD countries as a single entity, to which each country contributes proportionally to the number of 15 -year-olds enrolled in its schools. It illustrates how a country compares with the OECD as a whole.

In PISA 2003, all OECD countries as well as several partner countries participated. It is possible, however, that for a particular cycle, data for one or several OECD countries may not be available for specific indicators. Researchers should, therefore, keep in mind that the terms OECD average and OECD total refer to the OECD countries included in the respective comparisons for each cycle and for a particular comparison.

For simple statistics such as a mean or a percentage, the OECD average and the OECD total statistics and their respective standard errors can be mathematically computed. If $C$ OECD countries participated, then the OECD average mean and its respective sampling variance are equal to:

$$
\hat{\boldsymbol{\mu}}=\frac{\sum_{i=1}^{C} \hat{\mu}_{i}}{C} \text { and } \quad \sigma_{(\hat{\mu})}^{2}=\frac{\sum_{i=1}^{C} \sigma_{\left(\hat{\mu}_{i}\right)}^{2}}{C^{2}}
$$

The OECD total mean and its respective sampling variance are equal to:

$$
\hat{\boldsymbol{\mu}}=\frac{\sum_{i=1}^{C} w_{i} \hat{\boldsymbol{\mu}}_{i}}{\sum_{i=1}^{C} w_{i}} \text { and } \sigma_{(\hat{\mu})}^{2}=\frac{\sum_{i=1}^{C} w_{i}^{2} \sigma_{\left(\hat{\mu}_{i}\right)}^{2}}{\left[\sum_{i=1}^{C} w_{i}\right]^{2}} \text {, }
$$

with $w_{i}$ being the sum of the student final weights for a particular country.

While these formulae can be used for the computation of a mean or a percentage, they cannot be used for most other statistics. Such statistics can only be obtained directly from the data set.

\section{RECODING OF THE DATABASE FOR THE ESTIMATION OF THE OECD TOTAL AND OECD AVERAGE}

As stated in Chapter 3, the sum of the student final weights per country is an estimate of the 15 yearold population in that country. Therefore, the OECD total statistic can easily be obtained by deleting the partner country data. Then the statistic is computed using the OECD variable as breakdown 
variable (the OECD variable is a constant since the partner countries have been deleted from the file) instead of country (CNT). The standard error is obtained as usual by using the 80 replicates. Box 11.1 provides the SPSS ${ }^{\circledR}$ syntax for the computation of the mathematics performance per gender for the OECD total and Table 11.1 provides the results of the procedure.

\section{Box 11.1 - SPSS ${ }^{\circledR}$ syntax for the OECD total for the mathematics performance per gender}

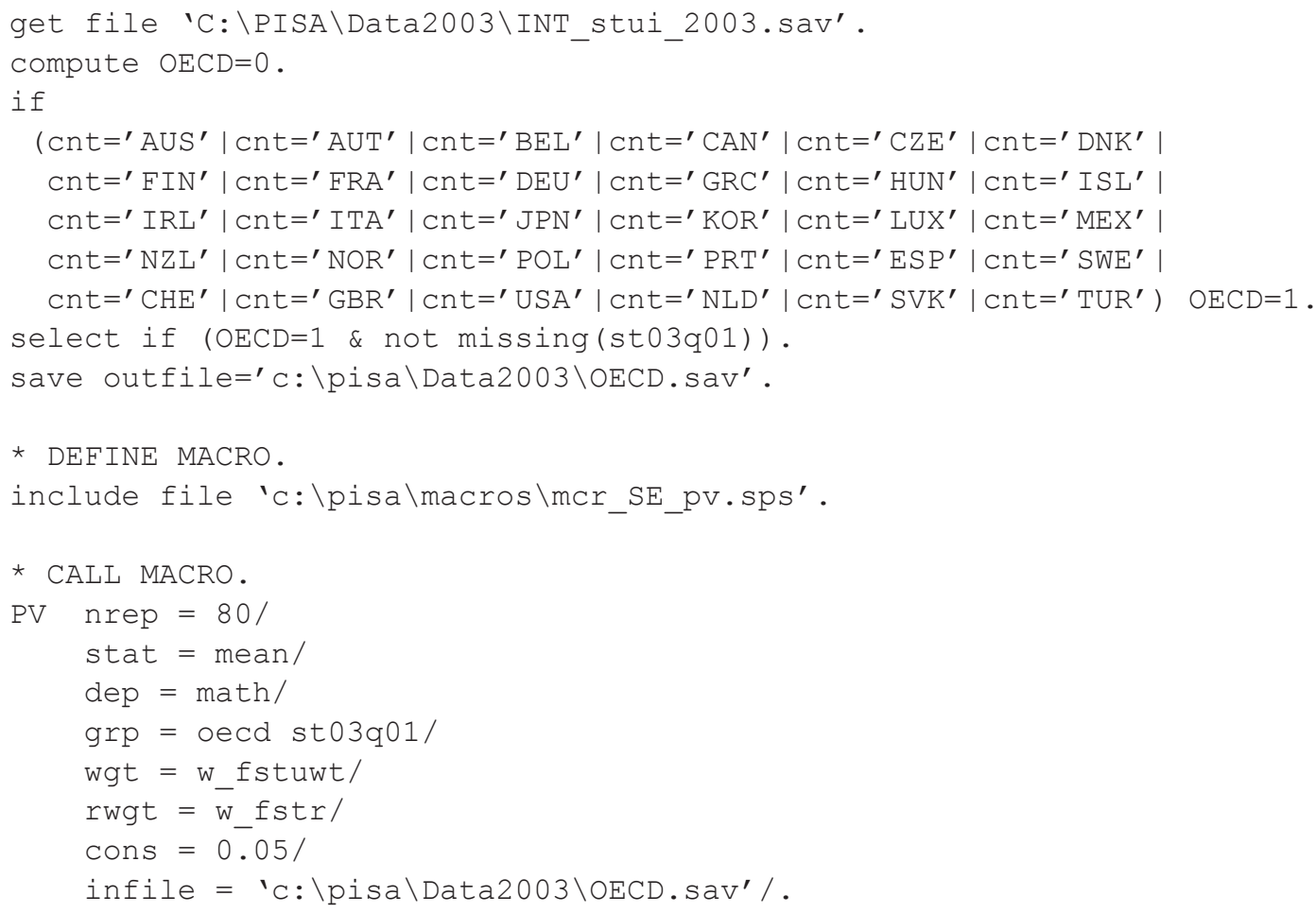

Table 11.1 \& Structure of the output data file from Box $\mathbf{1 1 . 1}$

\begin{tabular}{c|c|c}
\hline ST03Q01 & STAT & SE \\
\hline 1 & 483.93 & 1.25 \\
\hline 2 & 494.04 & 1.32 \\
\hline
\end{tabular}

The OECD average requires an additional step. The student final weights need to be recoded, so that the sum of the student final weights per country is equal to a constant, e.g. 1000. This can easily be implemented with the procedure described in Box 11.2. ${ }^{1}$ Table 11.2 presents the results of the procedure. The breakdown variables are OECD and ST03Q01 as in the example above for calculating OECD total and its standard error. The two weight arguments are different. The full student weight (W_FSTUWT) is replaced by the senate weight (SENATE). The replicate weights have been transformed by the same linear transformation as the full student weight and are now called S_FSTR1 to S_FSTR80. 


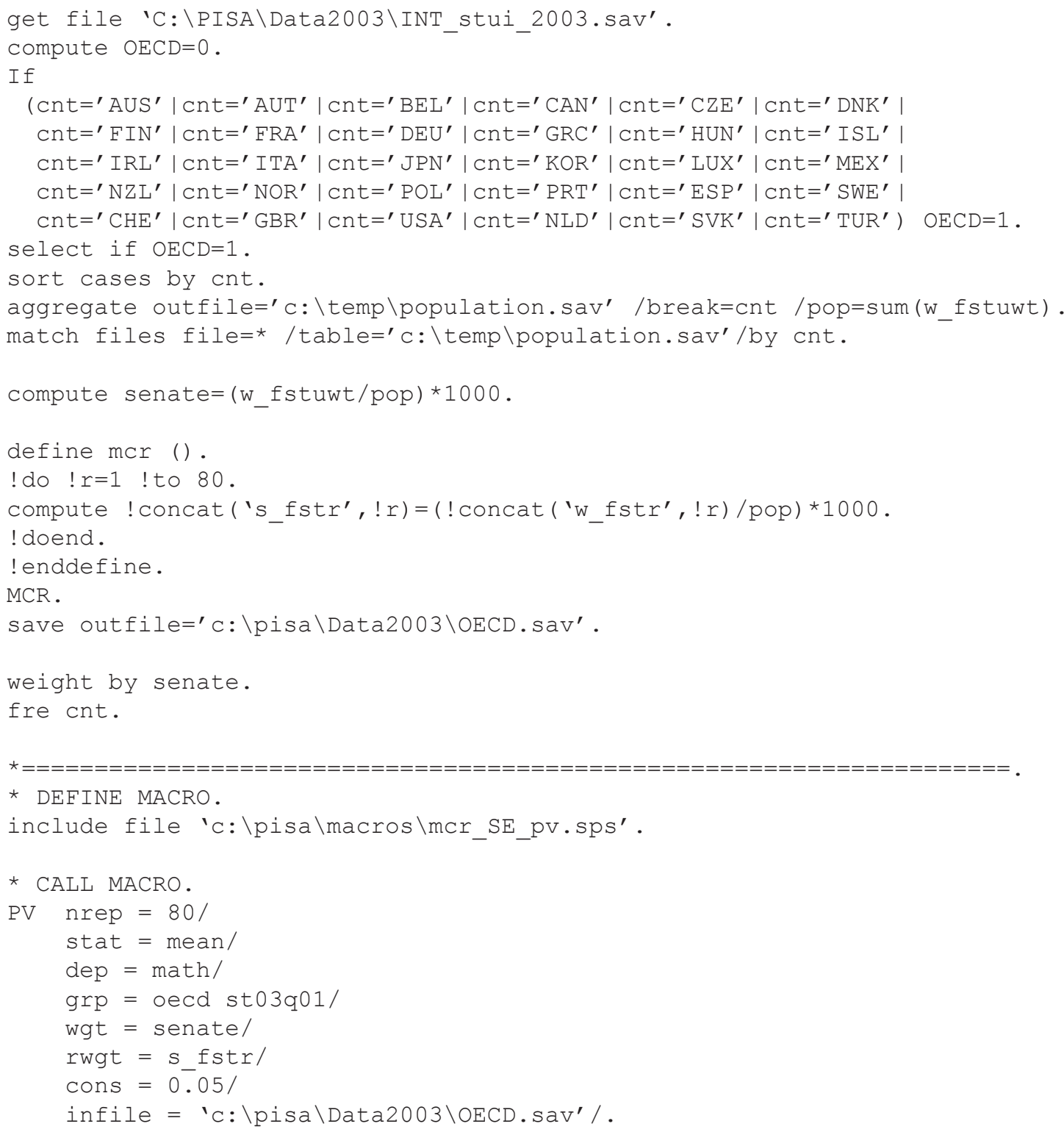

Table 11.2 — Structure of the output data file from Box 11.2

\begin{tabular}{c|c|c}
\hline ST03Q01 & STAT & SE \\
\hline 1 & 494.41 & 0.76 \\
\hline 2 & 505.53 & 0.75 \\
\hline
\end{tabular}

It is worth noting that the standard error is higher for the OECD total than it is for the OECD average. In the case of the OECD total, 40 per cent of the data come from just two countries (the United States and Japan), and these two countries do not have large sample sizes compared to the other OECD countries. 


\section{DUPLICATION OF THE DATA FOR AVOIDING THREE RUNS OF THE PROCEDURE}

If a researcher is interested in the country estimates as well as the OECD total and the OECD average, then three runs of the procedure are needed: one for the country estimates, one for the OECD total estimate and one for the OECD average estimate.

In order to avoid such repetitions, it is possible to duplicate three times the data for the OECD countries in such a way that the procedure directly provides the estimates for each country, as well as the OECD total and the OECD average estimates.

Box 11.3 presents the SPSS $^{\circledR}$ syntax for the generation of such data sets. It consists of the following steps:

- A new categorical variable, denoted OECD and separating OECD and partner countries, is computed. A value of 1 for this variable designates OECD countries, whereas a value of 4 designates partner countries. A second alphanumerical variable, denoted COUNTRY and set as CNT, is created.

- OECD countries are selected and saved in TEMP2. The variable OECD is set as 2 and the COUNTRY variable is set as TOT.

- On the TEMP2 file, the sum of the student final weights per country is computed through the procedure described in Box 11.2. The final weights are transformed in such a way that the sum per country is equal to 1000 . The same linear transformation is applied to the 80 replicates. The CNT variable is set as AVG and the OECD variable is set as 3. The new weight variables overwrite the old weight variables. These new data are saved into TEMP3.

- After sorting TEMP2 andTEMP3, the three temporary files are merged and saved in a final SPSS ${ }^{\circledR}$ data file.

SPSS macros presented in the previous chapters can be applied on this new data file. The breakdown variables are now OECD and COUNTRY instead of CNT. The output data file will consist of 43 rows. The first 30 rows will be the results of OECD countries. The next two rows will present the OECD total and the OECD average estimates. Finally, the 11 last rows will present the estimates for the partner countries.

\section{COMPARISONS BETWEEN OECD AVERAGE OR OECD TOTAL ESTIMATES AND A COUNTRY ESTIMATE}

Only OECD countries that are fully adjudicated contribute to the OECD average and the OECD total estimates and their respective standard errors. Therefore, the expected value of the covariance between a country sampling variance and the OECD aggregate sampling variance will differ from 0 if the country's values are included in the OECD aggregate values, because the two are not independent. Indeed, if the sampling variance of one country increases, then the OECD aggregate sampling variance will also increase.

If a researcher wants to test the null hypothesis between an OECD country and the OECD aggregate estimate, then the covariance should be estimated, as explained in Chapter 10. Since the covariance is expected to be positive, then the correct standard error estimate should be smaller than the standard error obtained from the formulae. 

computation of the OECD total and the OECD average

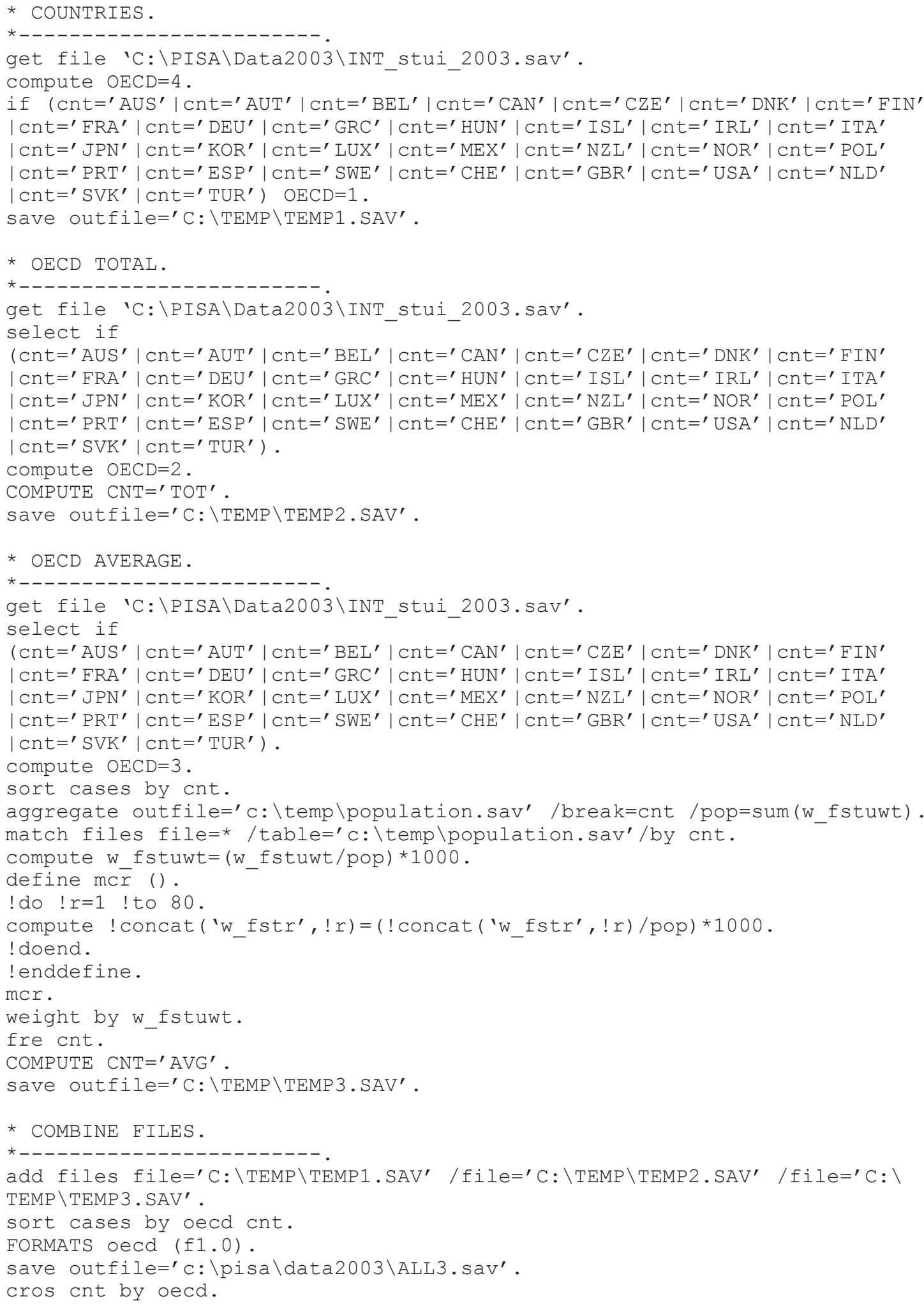




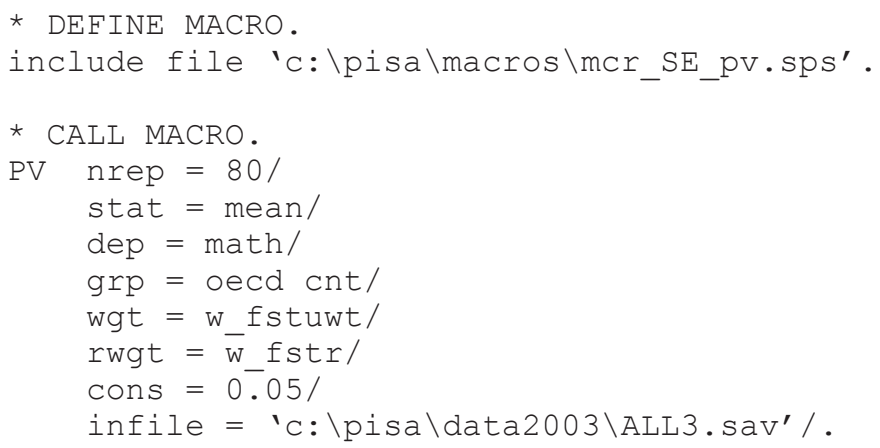

Since partner countries do not contribute at all to the OECD aggregate estimates, estimating the covariance is not necessary. The standard error on the difference can be directly obtained from the country standard error and the aggregate standard error.

Table 11.3 provides:

- The country mean performance in mathematics as well as the OECD average and the OECD total;

- The standard error on these mean estimates;

- The difference between the country and the OECD total;

- The standard error on this difference, using the formula provided in Chapter 10, i.e. without an estimation of the covariance;

- The standard error on this difference, using the replicate, i.e. with an estimation of the covariance;

- The difference between the country and the OECD average;

- The standard error on this difference, using the formula provided in Chapter 10, i.e. without an estimation of the covariance; and

- The standard error on this difference, using the replicate, i.e. with an estimation of the covariance.

The correct standard error estimates are in bold. The differences between the biased and unbiased estimates for OECD countries are not very large, except in the case of the United States and Germany for the OECD total.

The differences for partner countries are not very large either. As the expected covariance for partner countries are 0 , both standard errors are on average unbiased. However, it is recommended to use the standard error directly obtained with the formula.

Table 11.3 - Country mathematics performance means and their respective standard errors, country difference with OECD total and OECD average, their respective standard errors with or without estimation of the covariance

\begin{tabular}{l|c|c|c|c|c|c|c|c}
\hline & \multicolumn{2}{|c|}{ Country } & \multicolumn{3}{c|}{ OECD total } & \multicolumn{3}{c}{ OECD average } \\
\hline & Mean & SE & DIF & SE without & SE with & DIF & SE without & SE with \\
\hline AUS & 524.27 & 2.15 & 35.27 & 2.40 & $\mathbf{2 . 1 2}$ & 24.27 & 2.24 & $\mathbf{2 . 0 3}$ \\
\hline AUT & 505.61 & 3.27 & 16.61 & 3.44 & $\mathbf{3 . 4 9}$ & 5.61 & 3.33 & $\mathbf{3 . 2 7}$ \\
\hline BEL & 529.29 & 2.29 & 40.29 & 2.52 & $\mathbf{2 . 4 2}$ & 29.29 & 2.37 & $\mathbf{2 . 2 3}$ \\
\hline CAN & 532.49 & 1.82 & 43.49 & 2.11 & $\mathbf{2 . 0 8}$ & 32.49 & 1.92 & $\mathbf{1 . 9 6}$ \\
\hline
\end{tabular}


Table 11.3 (continued) - Country mathematics performance means and their respective standard errors, country difference with OECD total and OECD average, their respective standard errors with or without estimation of the covariance

\begin{tabular}{|c|c|c|c|c|c|c|c|c|}
\hline & \multicolumn{2}{|c|}{ Country } & \multicolumn{3}{|c|}{ OECD total } & \multicolumn{3}{|c|}{ OECD average } \\
\hline & Mean & SE & $D I F$ & SE without & SE with & $D I F$ & SE without & SE with \\
\hline $\mathrm{CHE}$ & 526.55 & 3.38 & 37.56 & 3.55 & 3.48 & 26.55 & 3.44 & 3.38 \\
\hline CZE & 516.46 & 3.55 & 27.46 & 3.70 & 3.90 & 16.46 & 3.60 & 3.51 \\
\hline DEU & 502.99 & 3.32 & 13.99 & 3.49 & 3.42 & 2.99 & 3.38 & 3.30 \\
\hline DNK & 514.29 & 2.74 & 25.29 & 2.95 & 2.99 & 14.29 & 2.82 & 2.67 \\
\hline ESP & 485.11 & 2.41 & -3.89 & 2.64 & 2.60 & -14.89 & 2.49 & 2.47 \\
\hline FIN & 544.29 & 1.87 & 55.29 & 2.15 & 2.07 & 44.29 & 1.97 & 1.91 \\
\hline FRA & 510.80 & 2.50 & 21.80 & 2.72 & 2.45 & 10.80 & 2.58 & 2.46 \\
\hline GBR & 508.26 & 2.43 & 19.26 & 2.65 & 2.41 & 8.26 & 2.51 & 2.39 \\
\hline GRC & 444.91 & 3.90 & -44.09 & 4.05 & 3.94 & -55.09 & 3.95 & 3.81 \\
\hline HUN & 490.01 & 2.84 & 1.02 & 3.03 & 3.20 & -9.99 & 2.91 & 2.95 \\
\hline IRL & 502.84 & 2.45 & 13.84 & 2.67 & 2.56 & 2.84 & 2.53 & 2.41 \\
\hline ISL & 515.11 & 1.42 & 26.11 & 1.78 & 1.78 & 15.11 & 1.56 & 1.48 \\
\hline ITA & 465.66 & 3.08 & -23.33 & 3.26 & 3.11 & -34.34 & 3.14 & 2.98 \\
\hline JPN & 534.14 & 4.02 & 45.14 & 4.16 & 3.88 & 34.14 & 4.06 & 3.94 \\
\hline KOR & 542.23 & 3.24 & 53.23 & 3.41 & 3.34 & 42.23 & 3.30 & 3.16 \\
\hline LUX & 493.21 & 0.97 & 4.21 & 1.45 & 1.48 & -6.79 & 1.16 & 1.20 \\
\hline MEX & 385.22 & 3.64 & -103.78 & 3.80 & 3.55 & -114.78 & 3.70 & 3.64 \\
\hline NLD & 537.82 & 3.13 & 48.83 & 3.31 & 3.19 & 37.82 & 3.19 & 3.10 \\
\hline NOR & 495.19 & 2.38 & 6.19 & 2.61 & 2.69 & -4.81 & 2.46 & 2.41 \\
\hline NZL & 523.49 & 2.26 & 34.49 & 2.50 & 2.41 & 23.49 & 2.34 & 2.31 \\
\hline POL & 490.24 & 2.50 & 1.24 & 2.72 & 2.82 & -9.76 & 2.58 & 2.54 \\
\hline PRT & 466.02 & 3.40 & -22.98 & 3.57 & 3.30 & -33.98 & 3.46 & 3.23 \\
\hline SVK & 498.18 & 3.35 & 9.19 & 3.51 & 3.46 & -1.82 & 3.41 & 3.31 \\
\hline SWE & 509.05 & 2.56 & 20.05 & 2.77 & 2.48 & 9.05 & 2.64 & 2.40 \\
\hline TUR & 423.42 & 6.74 & -65.58 & 6.82 & 6.48 & -76.58 & 6.77 & 6.46 \\
\hline USA & 482.88 & 2.95 & -6.11 & 3.14 & 2.38 & -17.12 & 3.02 & 2.90 \\
\hline TOT & 489.00 & 1.07 & & & & & & \\
\hline AVE & 500.00 & 0.63 & & & & & & \\
\hline BRA & 356.02 & 4.83 & -132.98 & 4.95 & 4.89 & -143.98 & 4.87 & 4.77 \\
\hline HKG & 550.38 & 4.54 & 61.39 & 4.66 & 4.80 & 50.38 & 4.58 & 4.68 \\
\hline IDN & 360.16 & 3.91 & -128.84 & 4.05 & 4.03 & -139.84 & 3.96 & 3.88 \\
\hline LIE & 535.80 & 4.12 & 46.80 & 4.26 & 4.16 & 35.80 & 4.17 & 4.13 \\
\hline LVA & 483.37 & 3.69 & -5.62 & 3.84 & 3.88 & -16.62 & 3.74 & 3.76 \\
\hline MAC & 527.27 & 2.89 & 38.27 & 3.08 & 3.13 & 27.27 & 2.95 & 2.85 \\
\hline RUS & 468.41 & 4.20 & -20.59 & 4.33 & 4.47 & -31.59 & 4.24 & 4.33 \\
\hline THA & 416.98 & 3.00 & -72.02 & 3.18 & 3.38 & -83.02 & 3.06 & 3.20 \\
\hline TUN & 358.73 & 2.54 & -130.26 & 2.75 & 2.55 & -141.27 & 2.61 & 2.57 \\
\hline URY & 422.20 & 3.29 & -66.80 & 3.46 & 3.41 & -77.80 & 3.35 & 3.30 \\
\hline YUG & 436.87 & 3.75 & -52.13 & 3.90 & 3.85 & -63.13 & 3.81 & 3.78 \\
\hline
\end{tabular}

\section{CONCLUSIONS}

This chapter was devoted to the concept of the OECD total and the OECD average. For simple statistics such as a mean or a percentage, these aggregated estimates and their respective standard errors can directly be obtained from the country individual estimates. 
In most cases, nevertheless, the estimate and its standard error can only be computed from the data base. SPSS ${ }^{\circledR}$ syntax was provided.

In order to avoid three runs for obtaining individual country estimates as well as OECD aggregate estimates, the SPSS ${ }^{\circledR}$ syntax for creating a larger data set was also provided.

Finally, following the issues raised in the previous chapter devoted to comparisons, any comparison that involves a particular country and an OECD aggregate estimate was discussed

1. As an alternative, a country weight, CNTFAC1, can be also used. 



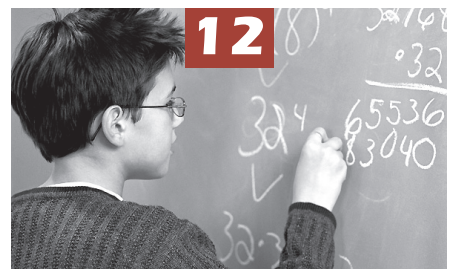

\section{Trends}

Introduction

The computation of the standard error for trend indicators

on variables other than performance

The computation of the standard error for trend indicators on performance variables

Conclusions 


\section{INTRODUCTION}

Policy makers and researchers demand information on how indicators change over time. The longer the time period the more reliable the trend indicator. One example would be an analysis of the impact of reforms to the education system, where policy makers would seek to measure changes in the targeted area to gauge how effective their policies have been. In the early 1960s, for example, most of the OECD countries implemented education reforms to facilitate access to tertiary education, mainly through financial help. One indicator of the impact of these reforms would be to calculate the percentage of the population with a tertiary qualification for several different years to show how this has evolved. Computing this trend indicator is a straightforward statistical manipulation, since the measure (i.e. whether or not an individual has completed tertiary education) is quite objective and the information is in most cases available at the population level. Nevertheless, such measures can be slightly biased by, for example, differing levels of immigration over the time period, student exchange programmes, and so on.

Of course trends over time on one particular indicator need careful interpretation. Policy makers would also need to take into account changes to the economic context of the country, such as rising unemployment rates. For example, an increase in the percentage of tertiary graduates does not necessarily prove that the reform to the education system was effective. Further, when comparing trend indicators across countries it is import to consider how comparable the definition of the indicator is from country to country, for example tertiary education might mean something different in each country.

The PISA project offers a unique opportunity to extend the computation of trend indicators on educational outcomes by looking at student performance in reading, mathematical and scientific literacy.

For the trend measures to be reliable, the comparability of the target population, the data collection procedures, and the assessment framework need to be consistent over time. Being able to use the results from PISA as trend indicators is one of the major aims of the project.

PISA 2000 and PISA 2003 used the same assessment frameworks and the data collection procedures were essentially unchanged. In PISA 2000, the target population was defined as all 15-year-olds in grade 5 or higher grades. In PISA 2003, it was defined as all 15-year-olds in grade 7 or higher grades. In PISA 2000, only a very small percentage of 15 -year-olds were attending grade 5 or grade 6 (Austria $=0.03 \%$, Canada $=0.03 \%$, Czech Republic $=0.06 \%$, Germany $=0.02 \%$, Hungary $=0.59 \%$, Latvia $=0.27 \%$, Portugal $=1.25 \%$ and Russia $=0.04 \%$ ). Therefore, except for Portugal, the change in the target population should not significantly affect trend indicators.

Other issues that need to be included in the interpretation of trend indicators are student and school participation rates and coverage indices. A higher or a lower school participation rate might explain partly observed differences.

Behind these preliminary precautions, the computation of trend indicators in PISA raises two statistical issues:

- PISA collects data on a sample and therefore any statistic has to be associated with a sampling error. The next section will discuss how to compute such sampling error on a trend indicator.

- Between 20 and 30 items per domain from the 2000 assessments were included in the 2003 assessment to ensure a psychometric link. These anchor items were used to scale the PISA 2000 and the PISA 2003 assessments on a common scale. As one can easily imagine, selecting other 
anchor items would have returned slightly different results on the trend performance indicators. It follows that any comparison between two PISA cycles in the student performance will require the addition of another error component, i.e. the item sampling error.

\section{THE COMPUTATION OF THE STANDARD ERROR FOR TREND INDICATORS ON VARIABLES OTHER THAN PERFORMANCE}

For any country, the PISA 2000 and the PISA 2003 samples are independent. Therefore, the standard error on any trend indicators not involving achievement variables can be computed as follows:

$$
\sigma_{\left(\hat{\theta}_{2003}-\hat{\theta}_{2000}\right)}=\sqrt{\sigma_{\left(\hat{\theta}_{2003}\right)}^{2}+\sigma_{\left(\hat{\theta}_{2000}\right)}^{2}} \text {, with } \theta \text { representing any statistic. }
$$

However, the computation of a difference between PISA 2000 and PISA 2003 and its standard error are relevant only if the two measures are identical. For instance, in the PISA 2000 and PISA 2003 databases, there are several indices derived from the student questionnaires with exactly the same variable names (for instance, HEDRES for home educational resources, BELONG for the student's sense of belonging to the school, and so on). The questions that were used to derive these indices have not changed, but as the scaling was done independently in 2000 and in 2003, there is no guarantee that the 2000 and the 2003 metrics are comparable. Further, these indices were standardised at the OECD level to get a mean of 0 and a standard deviation of 1 . The 2000 standardisation differs from the 2003 one. It is therefore not recommended to compute trend indicators on questionnaire indices.

In the case of the PISA questionnaire indices, as the questions have not been modified, the underlying concepts are similar. Therefore, the correlation coefficients between these indices and the student performance can directly be compared. However, as the item parameters were estimated in 2003 without any link with the PISA 2000 data, the metric of the scales might be slightly different and an absolute increase in, for example, the sense of belonging might be simply a result of the scaling, or the standardisation, without any attitudinal change in the student. For the same reasons, regression coefficients for indices derived from student questionnaire data cannot be compared between 2000 and 2003.

The Highest International Social and Economic Index (denoted HISEI in the databases) satisfies all the conditions for the computation of trend indicators. Indeed, the questions were not changed and the transformation used on the ISCO categories in 2000 was implemented in 2003 without any modification.

Table 12.1 presents, per country, the mean estimate of HISEI and its standard error for PISA 2000 and PISA 2003, as well as the difference between the two estimates, the standard error of this difference and the standardised difference, i.e. the difference divided by its standard error.

For Germany (DEU), the means for HISEI in 2000 and in 2003 are respectively equal to 48.85 and 49.33. The difference between these two data collection is therefore equal to:

$49.33-48.55=0.48$.

The standard errors on these mean estimates are equal to 0.32 and 0.42 . The standard error on the difference estimate is equal to:

$$
\sigma_{\left(\hat{\theta}_{2003}-\hat{\theta}_{2000}\right)}=\sqrt{\sigma_{\left(\hat{\theta}_{2003}\right)}^{2}+\sigma_{\left(\hat{\theta}_{2000}\right)}^{2}}=\sqrt{(0.32)^{2}+(0.42)^{2}}=0.53
$$


The standardised difference, i.e. the difference estimate divided by its standard error, is equal to:

$$
\frac{0.48}{0.53}=0.91
$$

As the standardised difference is included in the interval [-1.96;1.96], the difference on the mean estimate for HISEI between 2000 and 2003 is not statistically different from 0 with a type I error of 0.05 .

Table 12.1 shows that the difference is statistically different from 0 in 13 countries: Austria, Belgium, Brazil, the Czech Republic, Indonesia, Iceland, Korea, Liechtenstein, Luxembourg, Mexico, Thailand, the United Kingdom and the United States.

It would be unrealistic to consider these differences as simply a reflection of social and economic changes in these 13 countries. In a period of three years, some changes can occur, but these cannot explain by themselves the size of the observed increases or decreases.

Table 12.1 - Trend indicators between PISA 2000 and PISA 2003 for HISEI per country

\begin{tabular}{|c|c|c|c|c|c|c|c|}
\hline & \multicolumn{2}{|c|}{ PISA 2000} & \multicolumn{2}{|c|}{ PISA 2003} & \multicolumn{3}{|c|}{ Difference } \\
\hline & Mean & SE & Mean & SE & Estimate & SE & $\begin{array}{c}\text { STD } \\
\text { difference }\end{array}$ \\
\hline AUS & 52.25 & $(0.50)$ & 52.59 & $(0.30)$ & 0.34 & $(0.58)$ & 0.59 \\
\hline AUT & 49.72 & $(0.29)$ & 47.06 & $(0.52)$ & -2.66 & $(0.59)$ & -4.49 \\
\hline BEL & 48.95 & $(0.39)$ & 50.59 & $(0.38)$ & 1.65 & $(0.54)$ & 3.05 \\
\hline BRA & 43.93 & $(0.59)$ & 40.12 & $(0.64)$ & -3.81 & $(0.87)$ & -4.39 \\
\hline CAN & 52.83 & $(0.22)$ & 52.58 & $(0.27)$ & -0.25 & $(0.35)$ & -0.73 \\
\hline CHE & 49.21 & $(0.53)$ & 49.30 & $(0.43)$ & 0.09 & $(0.68)$ & 0.13 \\
\hline CZE & 48.31 & $(0.27)$ & 50.05 & $(0.34)$ & 1.74 & $(0.44)$ & 3.98 \\
\hline DEU & 48.85 & $(0.32)$ & 49.33 & $(0.42)$ & 0.48 & $(0.53)$ & 0.91 \\
\hline DNK & 49.73 & $(0.43)$ & 49.26 & $(0.45)$ & -0.47 & $(0.63)$ & -0.75 \\
\hline ESP & 44.99 & $(0.62)$ & 44.29 & $(0.58)$ & -0.70 & $(0.85)$ & -0.83 \\
\hline FIN & 50.00 & $(0.40)$ & 50.23 & $(0.36)$ & 0.23 & $(0.54)$ & 0.42 \\
\hline FRA & 48.27 & $(0.44)$ & 48.66 & $(0.47)$ & 0.39 & $(0.64)$ & 0.61 \\
\hline GBR & 51.26 & $(0.35)$ & 49.65 & $(0.39)$ & -1.61 & $(0.52)$ & -3.07 \\
\hline GRC & 47.76 & $(0.60)$ & 46.94 & $(0.72)$ & -0.83 & $(0.93)$ & -0.88 \\
\hline HUN & 49.53 & $(0.47)$ & 48.58 & $(0.33)$ & -0.95 & $(0.57)$ & -1.65 \\
\hline IDN & 36.38 & $(0.77)$ & 33.65 & $(0.61)$ & -2.73 & $(0.98)$ & -2.77 \\
\hline IRL & 48.43 & $(0.48)$ & 48.34 & $(0.49)$ & -0.09 & $(0.69)$ & -0.13 \\
\hline ISL & 52.73 & $(0.28)$ & 53.72 & $(0.26)$ & 0.99 & $(0.38)$ & 2.62 \\
\hline ITA & 47.08 & $(0.31)$ & 46.83 & $(0.38)$ & -0.24 & $(0.49)$ & -0.50 \\
\hline JPN & 50.54 & $(0.62)$ & 49.98 & $(0.31)$ & -0.56 & $(0.69)$ & -0.80 \\
\hline KOR & 42.80 & $(0.42)$ & 46.32 & $(0.36)$ & 3.52 & $(0.55)$ & 6.36 \\
\hline LIE & 47.46 & $(0.94)$ & 50.73 & $(0.75)$ & 3.27 & $(1.21)$ & 2.71 \\
\hline LUX & 44.79 & $(0.27)$ & 48.17 & $(0.22)$ & 3.38 & $(0.35)$ & 9.76 \\
\hline LVA & 50.15 & $(0.54)$ & 50.28 & $(0.52)$ & 0.13 & $(0.75)$ & 0.18 \\
\hline MEX & 42.48 & $(0.68)$ & 40.12 & $(0.68)$ & -2.37 & $(0.96)$ & -2.46 \\
\hline NLD & 50.85 & $(0.47)$ & 51.26 & $(0.38)$ & 0.42 & $(0.61)$ & 0.68 \\
\hline NOR & 53.91 & $(0.38)$ & 54.63 & $(0.39)$ & 0.72 & $(0.54)$ & 1.33 \\
\hline NZL & 52.20 & $(0.37)$ & 51.46 & $(0.36)$ & -0.74 & $(0.51)$ & -1.45 \\
\hline POL & 46.03 & $(0.47)$ & 44.96 & $(0.34)$ & -1.07 & $(0.58)$ & -1.85 \\
\hline PRT & 43.85 & $(0.60)$ & 43.10 & $(0.54)$ & -0.75 & $(0.81)$ & -0.92 \\
\hline RUS & 49.38 & $(0.45)$ & 49.86 & $(0.38)$ & 0.49 & $(0.59)$ & 0.82 \\
\hline SWE & 50.57 & $(0.39)$ & 50.64 & $(0.38)$ & 0.07 & $(0.55)$ & 0.12 \\
\hline THA & 33.02 & $(0.57)$ & 36.01 & $(0.43)$ & 2.99 & $(0.72)$ & 4.18 \\
\hline USA & 52.40 & $(0.79)$ & 54.55 & $(0.37)$ & 2.15 & $(0.87)$ & 2.47 \\
\hline
\end{tabular}


It is also possible that the quality of the samples might explain some of the differences. As a student's propensity to participate positively correlate with his or her academic records and as on average low achievers come from lower social background variables than high achievers, an increase or a decrease in the student participation rates might affect the HISEI mean.

A change in the percentage of missing data for the HISEI variable would be another explanation that can be easily verified. On average, students who do not provide their parents' jobs are lower achievers. Therefore, one should expect low social background characteristics, so that an increase of missing data could be associated with an increase of the HISEI mean and inversely.

Table 12.2 provides the percentages of missing data for the HISEI variables in PISA 2000 and PISA 2003 databases. These results do not really confirm the hypothesis. For instance, in the United States, the percentages of missing data were respectively about 14 per cent in 2000 and about 6 per cent in 2003 and the means of HISEI were respectively 52.40 and 54.55. In 9 out of the 13 countries where the HISEI means significantly differ, either an increase of the HISEI mean is associated with a decrease of the percentage of missing data or the inverse. In the three other countries, i.e. Belgium, the Czech Republic and Mexico, the relationship is consistent with the hypothesis.

Table 12.2 - Percentages of missing data for HISEI

\begin{tabular}{|c|c|c|c|c|c|c|c|}
\hline & \multicolumn{2}{|c|}{ PISA 2000} & \multicolumn{2}{|c|}{ PISA 2003} & \multicolumn{3}{|c|}{ Difference } \\
\hline & $\%$ & SE & $\%$ & SE & Estimate & SE & $\begin{array}{c}\text { STD } \\
\text { difference }\end{array}$ \\
\hline AUS & 4.15 & $(0.38)$ & 7.91 & $(1.56)$ & 3.76 & (1.61) & 2.33 \\
\hline AUT & 2.06 & $(0.20)$ & 3.62 & $(0.32)$ & 1.56 & $(0.38)$ & 4.13 \\
\hline BEL & 5.02 & $(0.45)$ & 6.11 & $(0.48)$ & 1.09 & $(0.66)$ & 1.66 \\
\hline BRA & 7.90 & $(0.62)$ & 8.75 & (1.03) & 0.86 & $(1.20)$ & 0.71 \\
\hline CAN & 3.00 & $(0.18)$ & 12.34 & $(0.76)$ & 9.34 & $(0.78)$ & 11.93 \\
\hline $\mathrm{CHE}$ & 3.36 & $(0.32)$ & 3.06 & $(0.26)$ & -0.30 & $(0.41)$ & -0.72 \\
\hline $\mathrm{CZE}$ & 1.90 & $(0.42)$ & 5.65 & (1.19) & 3.75 & $(1.26)$ & 2.97 \\
\hline DEU & 3.05 & $(0.34)$ & 9.92 & $(0.63)$ & 6.87 & $(0.72)$ & 9.55 \\
\hline DNK & 7.12 & $(0.85)$ & 2.73 & $(0.37)$ & -4.40 & $(0.92)$ & -4.76 \\
\hline ESP & 4.48 & $(0.49)$ & 3.70 & $(0.37)$ & -0.78 & $(0.62)$ & -1.27 \\
\hline FIN & 1.96 & $(0.22)$ & 1.44 & $(0.16)$ & -0.52 & $(0.27)$ & -1.92 \\
\hline FRA & 6.23 & $(0.51)$ & 4.61 & $(0.45)$ & -1.61 & $(0.68)$ & -2.37 \\
\hline GBR & 5.15 & $(0.44)$ & 7.23 & (1.17) & 2.07 & $(1.25)$ & 1.66 \\
\hline GRC & 4.04 & $(0.57)$ & 5.81 & $(0.41)$ & 1.78 & $(0.70)$ & 2.53 \\
\hline HUN & 3.02 & $(0.36)$ & 5.39 & $(0.42)$ & 2.37 & $(0.55)$ & 4.31 \\
\hline IDN & 6.99 & $(0.64)$ & 8.67 & $(0.53)$ & 1.67 & $(0.83)$ & 2.03 \\
\hline IRL & 3.23 & $(0.34)$ & 4.32 & $(0.57)$ & 1.09 & $(0.66)$ & 1.65 \\
\hline ISL & 2.19 & $(0.24)$ & 2.30 & $(0.25)$ & 0.11 & $(0.35)$ & 0.31 \\
\hline ITA & 2.73 & $(0.46)$ & 2.47 & $(0.28)$ & -0.26 & $(0.54)$ & -0.48 \\
\hline JPN & 62.52 & $(3.47)$ & 11.25 & $(0.81)$ & -51.27 & $(3.56)$ & -14.41 \\
\hline KOR & 7.34 & $(0.49)$ & 2.36 & $(0.21)$ & -4.97 & $(0.54)$ & -9.29 \\
\hline LIE & 5.49 & $(1.41)$ & 3.02 & $(0.85)$ & -2.47 & $(1.64)$ & -1.50 \\
\hline LUX & 9.55 & $(0.50)$ & 3.62 & $(0.29)$ & -5.92 & $(0.58)$ & -10.27 \\
\hline LVA & 5.02 & $(0.52)$ & 3.34 & $(0.39)$ & -1.68 & $(0.66)$ & -2.56 \\
\hline MEX & 8.51 & $(0.59)$ & 5.07 & $(0.44)$ & -3.43 & $(0.74)$ & -4.65 \\
\hline
\end{tabular}




\begin{tabular}{|c|c|c|c|c|c|c|c|}
\hline & \multicolumn{2}{|c|}{ PISA 2000} & \multicolumn{2}{|c|}{ PISA 2003} & \multicolumn{3}{|c|}{ Difference } \\
\hline & $\%$ & SE & $\%$ & SE & Estimate & SE & $\begin{array}{c}\text { STD } \\
\text { difference }\end{array}$ \\
\hline NLD & 3.07 & $(0.65)$ & 7.64 & $(1.34)$ & 4.57 & $(1.49)$ & 3.07 \\
\hline NOR & 2.44 & $(0.31)$ & 3.18 & $(0.39)$ & 0.74 & $(0.50)$ & 1.49 \\
\hline NZL & 3.92 & $(0.39)$ & 14.13 & $(0.43)$ & 10.22 & $(0.58)$ & 17.60 \\
\hline POL & 6.90 & $(0.79)$ & 2.33 & $(0.30)$ & -4.57 & $(0.85)$ & -5.39 \\
\hline PRT & 3.72 & $(0.42)$ & 2.76 & $(0.28)$ & -0.96 & $(0.50)$ & -1.90 \\
\hline RUS & 3.16 & $(0.33)$ & 2.14 & $(0.30)$ & -1.02 & $(0.45)$ & -2.27 \\
\hline SWE & 2.48 & $(0.30)$ & 2.63 & $(0.31)$ & 0.15 & $(0.43)$ & 0.35 \\
\hline THA & 10.95 & $(1.38)$ & 5.85 & $(0.64)$ & -5.09 & $(1.52)$ & -3.35 \\
\hline USA & 14.58 & $(1.95)$ & 5.88 & $(0.38)$ & -8.70 & $(1.99)$ & -4.38 \\
\hline
\end{tabular}

This simple example shows that the interpretation of trend indicators is quite complex. The social and economic structure of a country should remain unchanged over a period of three years, so that no differences occur between two cycles. However, as shown, this difference appears significant in all 13 countries.

Changes in the school or student participation rates and in the distribution of missing data might sometimes explain these significant differences. It is therefore recommended to implement some verification before trying to interpret calculated differences as a real change in the population characteristics.

\section{THE COMPUTATION OF THE STANDARD ERROR FOR TREND INDICATORS ON PERFORMANCE VARIABLES}

\section{Anchoring of the PISA 2000 and PISA 2003 performance scales}

The PISA 2000 database contains five plausible values for each of the following domains or subdomains:

- Mathematics

- Reading

- Reading/retrieving information

- Reading/interpreting

-Reading/reflecting

- Science

The PISA 2003 database also contains five plausible values for each of the following domains or subdomains:

- Mathematics

- Mathematics/space and shape

- Mathematics/change and relationship

- Mathematics/uncertainty

- Mathematics/quantity 
- Problem solving

- Reading

- Science

The psychometric procedures used to link the PISA 2000 and PISA 2003 performance scales are different for mathematics than they are for reading and science.

Reading was the major domain in 2000 and 28 of the 140 items developed for the 2000 assessment were used for the 2003 assessment. The 2003 data were therefore reported on the 2000 reading scale. The science assessment data of 2003 are also reported on the 2000 science scale as 25 of the 30 items developed for the 2000 assessment were used for the 2003 assessment.

Mathematics, as the major domain, was the subject of major development work for PISA 2003. Further, the mathematics assessment in 2000 only covered two of the four content areas (space and shape and change and relationships). Twenty items out of the 85 items used in 2003 assessment come from the 2000 assessment. Because of this broadening in the assessment, it was deemed inappropriate to report the PISA 2003 mathematics scores base on the scale for the PISA 2000 mathematics scores.

However, to provide countries with some trend indicators, the mathematics subscales space and shape, and change and relationship of the PISA 2000 were reported on the PISA 2003 scales.

The steps for anchoring the PISA 2003 reading and science data on the 2000 scales are:

1. Calibration of the 2003 reading and science data to get the PISA 2003 item parameters, i.e. the relative difficulty of the item on the Rasch scale.

2. Based on these item parameters, generation of the plausible values for reading and science on the PISA 2003 data.

3. Based on the item parameters of step 1, but only on the link items, generation of plausible values for Reading and Science on the PISA 2000 data. By this time, two sets for plausible values are available for PISA 2000: the original set of plausible values included in the PISA 2000 database and the set of plausible values based on the PISA 2003 item parameters. Unfortunately, the mean and the standard deviation of the new set of plausible values will slightly differ from the PISA 2000 original plausible values. These differences reflect the changes in the difficulty of the link items between 2000 and 2003. As a reminder, the mean and the standard deviation for the OECD average were set respectively at 500 and 100 in 2000. Let us suppose that the new set of plausible values return a mean of 505 and a standard deviation of 110 . The new set of plausible values for the PISA 2000 data has to be transformed so that their mean and standard deviation is respectively equal to 500 and 100 .

4. This step consists of the computation of the linear transformation that will guarantee that the mean and the standard deviation of the new set of plausible values on the PISA 2000 data has a mean of 500 and a standard deviation of 100 . This linear transformation can be written as $P V_{c a l_{-} 2000}=\alpha+\beta * P V_{c a l_{-} 2003}$ with $\beta=\frac{\sigma_{c a l_{-} 2000}}{\sigma_{c a l_{-} 2003}}$ and $\alpha=\left(\mu_{c a l_{-} 2000}-\beta *\left(\mu_{c a l \_2003}\right)\right)$.

In the example, $\beta=100 / 110=0.909$ and $\alpha=(500-(0.909 * 505))=40.955 ;^{2}$ and 
5. This linear transformation is applied on the PISA 2003 plausible values. This linear transformation applied on the Reading or Science PISA 2003 plausible values guarantees that the student performance in 2003 is comparable to the student performance in 2000.

As stated earlier, with another set of link items, the linear transformation would have been different. As a consequence, there is an uncertainty in the transformation due to sampling of the link items, referred as the linking error.

The steps for anchoring the two mathematics PISA 2000 subscales on the PISA 2003 subscales are:

1. Calibration of the 2003 mathematics data to get the PISA 2003 item parameter;

2. Based on these item parameters, generation of the PISA 2003 plausible values; and

3. Based on the 2003 item parameters, generation of plausible values for the mathematics PISA 2000 data.

Similarly, the estimation of the trend would have been slightly different with another set of anchor items in reading and in science. It is therefore important to integrate this error component in the standard error of the trend indicator.

\section{Inclusion of the linking error in the computation of the standard error}

For each link item, we have two item parameter estimates that are now on the same metric: the 2000 item parameter and the 2003 item parameter. Some of these link items show an increase of the relative difficulty, some show a decrease, but on average, the difference is equal to 0 . This means that some items seem more difficult in 2003 than they were in 2000 or the inverse.

As the subset of link items can be considered as a simple random sample of an infinite population of link items, the linking error can be computed as:

$$
\sigma_{(\text {Linking_error })}=\sqrt{\frac{\sigma^{2}}{n}} \text { where: }
$$

$\sigma^{2}$ represents the variance of the item parameter differences, and $n$ denotes the number of link items used.

If the item parameters from the 2003 calibration perfectly match the item parameters from the 2000 calibration, then the relative difficulty of the link items would not have changed. All the differences between the relative difficulty in 2000 and in 2003 would be equal to 0 and therefore, the linking error would be equal to 0 .

As the differences in the item parameters increase, the variance of these differences will increase and consequently the linking error will increase. It makes sense for the uncertainty around the trend to be proportional to the changes in the item parameters.

Also, the uncertainty around the trend indicators is inversely proportional to the number of link items. From a theoretical point of view, only one item is needed to measure a trend, but with only one item, the uncertainty will be very large. If the number of link items increases, the uncertainty will decrease. 
Table 12.3 provides the centred item parameters (i.e. item difficulty differences) for the reading link items for PISA 2000 and PISA 2003, as well as the difference between the two sets of estimates.

Table 12.3 - Item parameter estimates in $\mathbf{2 0 0 0}$ and 2003 for the reading link items

\begin{tabular}{l|c|c|c}
\hline Item Name & Centered Delta in 2003 & Centered Delta in 2000 & Difference \\
\hline R055Q01 & -1.28 & -1.347 & -0.072 \\
\hline R055Q02 & 0.63 & 0.526 & -0.101 \\
\hline R055Q03 & 0.27 & 0.097 & -0.175 \\
\hline R055Q05 & -0.69 & -0.847 & -0.154 \\
\hline R067Q01 & -2.08 & -1.696 & 0.388 \\
\hline R067Q04 & 0.25 & 0.546 & 0.292 \\
\hline R067Q05 & -0.18 & 0.212 & 0.394 \\
\hline R102Q04A & 1.53 & 1.236 & -0.290 \\
\hline R102Q05 & 0.87 & 0.935 & 0.067 \\
\hline R102Q07 & -1.42 & -1.536 & -0.116 \\
\hline R104Q01 & -1.47 & -1.205 & 0.268 \\
\hline R104Q02 & 1.44 & 1.135 & -0.306 \\
\hline R111Q01 & 2.17 & 1.905 & -0.267 \\
\hline R111Q02B & -0.19 & -0.023 & 0.164 \\
\hline R111Q06B & 1.54 & 1.395 & -0.147 \\
\hline R219Q01T & 0.89 & 0.838 & -0.051 \\
\hline 2219Q01E & -0.59 & -0.520 & 0.069 \\
\hline R219Q02 & 0.10 & 0.308 & 0.210 \\
\hline R220Q01 & -1.13 & -0.887 & 0.243 \\
\hline R220Q02B & 0.86 & 0.815 & -0.041 \\
\hline R220Q04 & -0.14 & -0.114 & 0.027 \\
\hline R220Q05 & -0.10 & 0.193 & 0.297 \\
\hline R220Q06 & -1.39 & -1.569 & -0.184 \\
\hline R227Q01 & -0.34 & -0.142 & 0.196 \\
\hline R227Q02T & 0.40 & 0.226 & -0.170 \\
\hline R227Q03 & 0.16 & 0.075 & -0.086 \\
\hline R227Q06 & 0.46 & 0.325 & -0.132 \\
\hline & -0.56 & -0.886 & \\
\hline & & & \\
\hline
\end{tabular}

The variance of the difference is equal to 0.047486 . The link error is therefore equal to:

$$
\sigma_{(\text {Linking_error })}=\sqrt{\frac{\sigma^{2}}{n}}=\sqrt{\frac{0.047486}{28}}=0.041 \text {. }
$$

On the PISA reading scale with a mean of 500 and a standard deviation of 100, it corresponds to 3.75.

The linking errors between PISA 2000 and PISA 2003 are:

- Reading 3.75

- Science

- Mathematics/space and shape. 6.01

- Mathematics/change and relationship...... 4.84

A common transformation has been estimated from the link items, and this transformation is applied to all participating countries. It follows that any uncertainty that is introduced through the linking 
is common to all students and all countries. Thus, for example, suppose that the unknown linking error between PISA 2000 and PISA 2003 in reading resulted in an overestimation of student scores by two points on the PISA 2000 scale. It follows that every student's score would be overestimated by two score points. This overestimation would have effects on certain, but not all, summary statistics computed from the PISA 2003 data. For example, consider the following:

- Each country's mean would be overestimated by an amount equal to the link error, in our example this is two score points;

- The mean performance of any subgroup would be overestimated by an amount equal to the linking error, in our example this is two score points;

- The standard deviation of student scores would not be affected because the overestimation of each student by a common error does not change the standard deviation;

- The difference between the mean scores of two countries in PISA 2003 would not be influenced because the overestimation of each student by a common error would have distorted each country's mean by the same amount;

- The difference between the mean scores of two groups (e.g. males and females) in PISA 2003 would not be influenced, because the overestimation of each student by a common error would have distorted each group's mean by the same amount;

- The difference between the performance of a group of students (e.g. a country) between PISA 2000 and PISA 2003 would be influenced because each student's score in PISA 2003 would be influenced by the error; and

- A change in the difference between two groups from PISA 2000 to PISA 2003 would not be influenced. This is because neither of the components of this comparison, which are differences in scores in 2000 and 2003 respectively, is influenced by a common error that is added to all student scores in PISA 2003.

In general terms, the linking error need only be considered when comparisons are being made between PISA 2000 and PISA 2003 results, and then usually when group means are being compared.

The most obvious example of a situation where there is a need to use the linking error is in the comparison of the mean performance for a country between PISA 2000 and PISA 2003.

In PISA 2000, the mean in reading literacy for Germany is equal to 483.99 with a standard error of 2.47. In PISA 2003, the mean for Germany is equal to 491.36 and the standard error is equal to 3.39. The difference between 2000 and 2003 is therefore equal to $491.36-483.99=7.37$. The average performance of the German students has therefore increased by 7.37 scores on the PISA 2000 reading scale.

The standard error on this difference, as mentioned here above, is influenced by the linking error. The standard error is therefore equal to:

$$
\begin{aligned}
& S E=\sqrt{\sigma_{\left(\hat{\mu}_{2000}\right)}^{2}+\sigma_{\left(\hat{\mu}_{2003}\right)}^{2}+\sigma_{(\text {linking_error })}^{2}} \\
& S E=\sqrt{(2.47)^{2}+(3.39)^{2}+(3.75)^{2}}=5.63
\end{aligned}
$$


As the standardised difference between PISA 2000 and PISA 2003, i.e. (7.37/5.63) is included in the interval $[-1.96 ; 1.96]$, the null hypothesis of no difference is not rejected. In other words, Germany's performance in reading has not changed between 2000 and 2003.

Table 12.4 provides the estimates of the reading performance in Germany per gender in 2000 and 2003, with their respective standard errors, as well as the difference estimates and their respective standard errors.

Table 12.4 Mean performance in reading per gender for Germany

\begin{tabular}{l|l|c|c}
\hline & & Performance in reading & Standard error \\
\hline 2003 & Girls & 512.93 & 3.91 \\
\hline & Boys & 470.80 & 4.23 \\
\hline & Difference & 42.13 & 4.62 \\
\hline & Girls & 502.20 & 3.87 \\
\hline & Boys & 467.55 & 3.17 \\
\hline & Difference & 34.65 & 5.21 \\
\hline
\end{tabular}

As the comparison for a particular country between 2000 and 2003 is affected by the linking error, the comparison for a particular subgroup between 2000 and 2003 is also affected by the linking error. Therefore, the standard error has to include the linking error.

The trend indicators for German boys and for German girls are, respectively, equal to:

$$
\begin{aligned}
& \text { Trends }_{\text {Girls }}=512.93-502.20=10.73 \\
& S E_{\text {Girls }}=\sqrt{(3.91)^{2}+(3.87)^{2}+(3.75)^{2}}=6.66 \\
& \text { Trends }_{\text {Boys }}=470.80-467.55=3.25 \\
& S E_{\text {Boys }}=\sqrt{(4.23)^{2}+(3.17)^{2}+(3.75)^{2}}=6.48
\end{aligned}
$$

Both differences are not statistically different from 0 .

On the other hand, the gender difference in 2003 is not affected by the linking error. Indeed, both subgroup estimates will be underestimated or overestimated by the same amount and therefore the computation of the difference will neutralize this difference. Consequently, the trend indicator on the gender difference and its standard error will be equal to:

$$
\begin{aligned}
& \text { Trends }_{\text {Gender_dif }}=42.13-34.65=7.43 \\
& S E_{\text {Gender_dif }}=\sqrt{(4.62)^{2}+(5.21)^{2}}=6.96
\end{aligned}
$$

This means that the change in gender difference in Germany for Reading between 2000 and 2003 was not statistically significant, even though it appears from Table 3 to have widened considerably.

In the PISA 2000 and PISA 2003 initial reports, student performance is also reported by proficiency levels (see Chapter 8). As the linking error affects the country mean estimate, the percentages of 
$\underset{v}{u} \quad$ students at each level will also be affected. However, an overestimation or an underestimation of the PISA 2003 results of $X$ points on the PISA scale will have a different impact on the percentages of students at each proficiency level for each country. If the percentage is small, then the impact will be small. If the percentage is large, then the impact will be larger. It would have been too complex to provide for each country and for each proficiency level a linking error. It was therefore decided not to take into account the linking error for the comparison of percentages of students at each proficiency level between PISA 2000 and PISA 2003. This means that the standard errors on the difference between 2000 and 2003 are underestimated.

\section{CONCLUSIONS}

This chapter was devoted to the computation of the standard error on trend indicators. The comparison of any variable other than performance variables is quite straightforward as the PISA 2000 and the PISA 2003 samples are independent. However, as stated previously, such comparisons are only relevant if the 2000 and the 2003 measures are comparable.

The comparison of performance mean estimates is more complex as it might require the inclusion of the linking error in the standard error depending on the statistic. For instance, Figure 2.6d in Learning for Tomorrow's World - First Results from PISA 2003 (OECD, 2004a), presents the trends in mathematics/space and shape average performance between 2000 and 2003. The trend indicator has integrated the linking error in its standard error. Figure 2.6c in the same report presents the trends between 2000 and 2003 on the $5^{\text {th }}, 10^{\text {th }}, 25^{\text {th }}, 75^{\text {th }}, 90^{\text {th }}$ and $95^{\text {th }}$ percentiles and the linking error was not integrated into the standard error of the trends. Broadly speaking, the PISA 2003 initial report has integrated the linking error only in tables where the country mean performance is compared between 2000 and 2003.

Due to the growing interest in trend indicators and their political impacts, it is essential to interpret significant changes with caution. A significant change might simply be due to a difference in the school or student participation rate or in the pattern of missing data.

1. The PISA 2000 database has been updated to integrate this new set of plausible values.

2. Actually, the linear transformation was applied on the plausible values before their transformation on the PISA scale with a mean of 500 and a standard deviation of 100. Further, different transformations were applied by gender (i.e. girls, boys and missing gender). The linear transformations per gender are: (i) girls: 2000_PVs $=0.0970+$ $\left(0.8739 * 2003 \_P V s\right)$, (ii) boys: $2000 \_P V s=0.0204+\left(0.8823 * 2003 \_P V s\right)$, (iii) missing gender: 2000_PVs $=$ $0.0552+\left(0.8830 * 2003 \_\right.$PVs $)$. In science, the linear transformation is: 2000_PVs $=-0.01552+(1.0063 *$ 2003_PVs). 


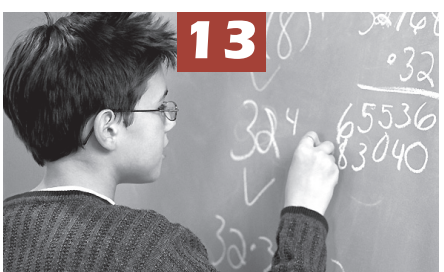

Multilevel Analyses

Introduction

166

Simple linear regression

166

Simple linear versus multilevel regression analyses

Fixed effect versus random effect

Some examples with SAS ${ }^{\circledR}$

Limitations of the multilevel model in the PISA context

Conclusions 


\section{INTRODUCTION}

For the past two decades, education survey data have increasingly been analyzed with multilevel models. Indeed, since linear regression models fail to take into account the potential effects that may arise from the way in which students are assigned to schools or to classes within schools, they may give an incomplete or misleading representation of the efficiency of the education systems. In some countries, for instance, the socio-economic background of a student may partly determine the type of school that he or she attends and there may be little variation therefore in the socioeconomic background of students within each school. In other countries or systems, schools may draw on students from a wide range of socio-economic backgrounds, but within the school, the socio-economic background of the student impacts the type of class he or she is allocated to and, as a result the within-school variance. A linear regression model that does not take into account the hierarchical structure of the data will not differentiate between these two systems.

The use of multilevel models (Goldstein, 1995), also called hierarchical linear models (Bryk and Raudenbush, 1992), acknowledges the fact that students are nested within classes and schools. The relative variation in the outcome measure - between students, within the same school and between schools - can therefore be evaluated.

\section{SIMPLE LINEAR REGRESSION}

A linear equation can always be represented by a straight line. An equation with two variables will be represented in a two dimension space, and an equation with three variables, in a three dimensional space, and so on.

The following equation is graphically represented in Figure 13.1.

$$
Y=5+4 X
$$

Since all linear equations are represented by a straight line, it is only necessary to identify two points that belong to the line to be able to graph it. If $X$ is equal to 1 , then $Y$ will be equal to 9 . If $X$ is equal to 10 , then $Y$ will be equal to 45 . The straight line with the points $(1,9)$ and $(10,45)$ corresponds to the equation.

\section{Figure 13.1 - Graphical representation of a linear equation}

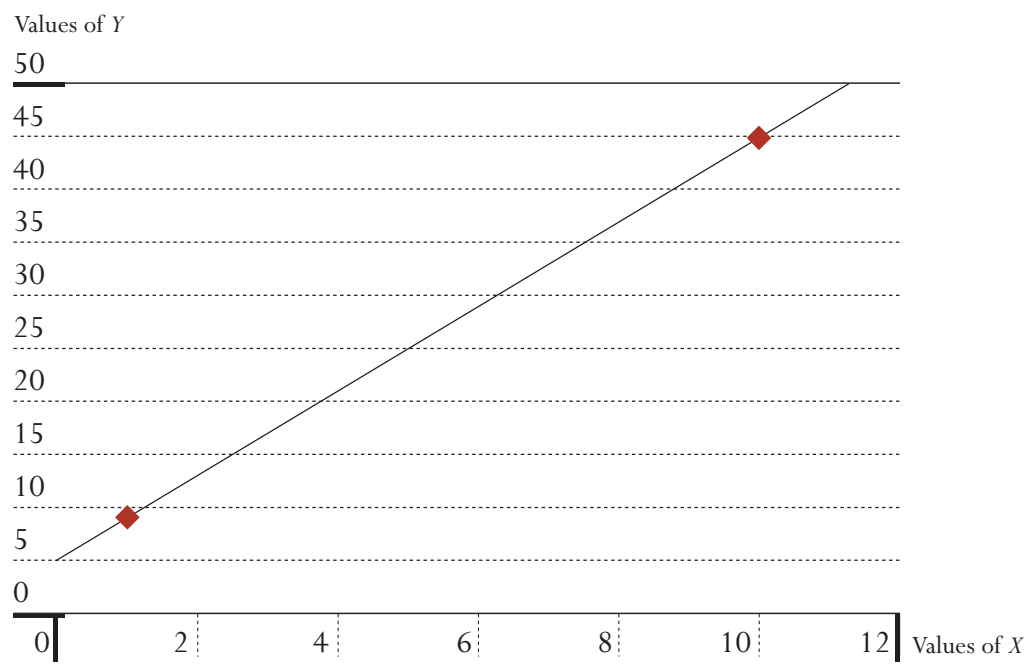


Figure 13.1 shows the graphical representation of the equation $Y=5+4 X$. As the figure shows, the line crosses the $Y$ axis at 5 . The point $(0,5)$ is called the intercept. It gives the value of $Y$ when $X$ is equal to 0 . The $X$ factor, or regression coefficient in statistical terms, gives the slope of the straight line. It tells us about the $Y$ increase for an additional unit on the $X$ axis. In the example considered, if $X$ increases by one unit, then $Y$ increases by four units.

The general expression of a linear equation with two variables is:

$$
Y=a+b X \text {, with } a \text { the intercept and } b \text { the regression coefficient. }
$$

Although human processes can also be described with a similar approach, they are less deterministic. Let us graphically represent the relationship that might exist between the family socio-economic background (SES) of students and their academic performance at school.

\section{Figure 13.2 — Relationship between student socio-economic background} and academic performance

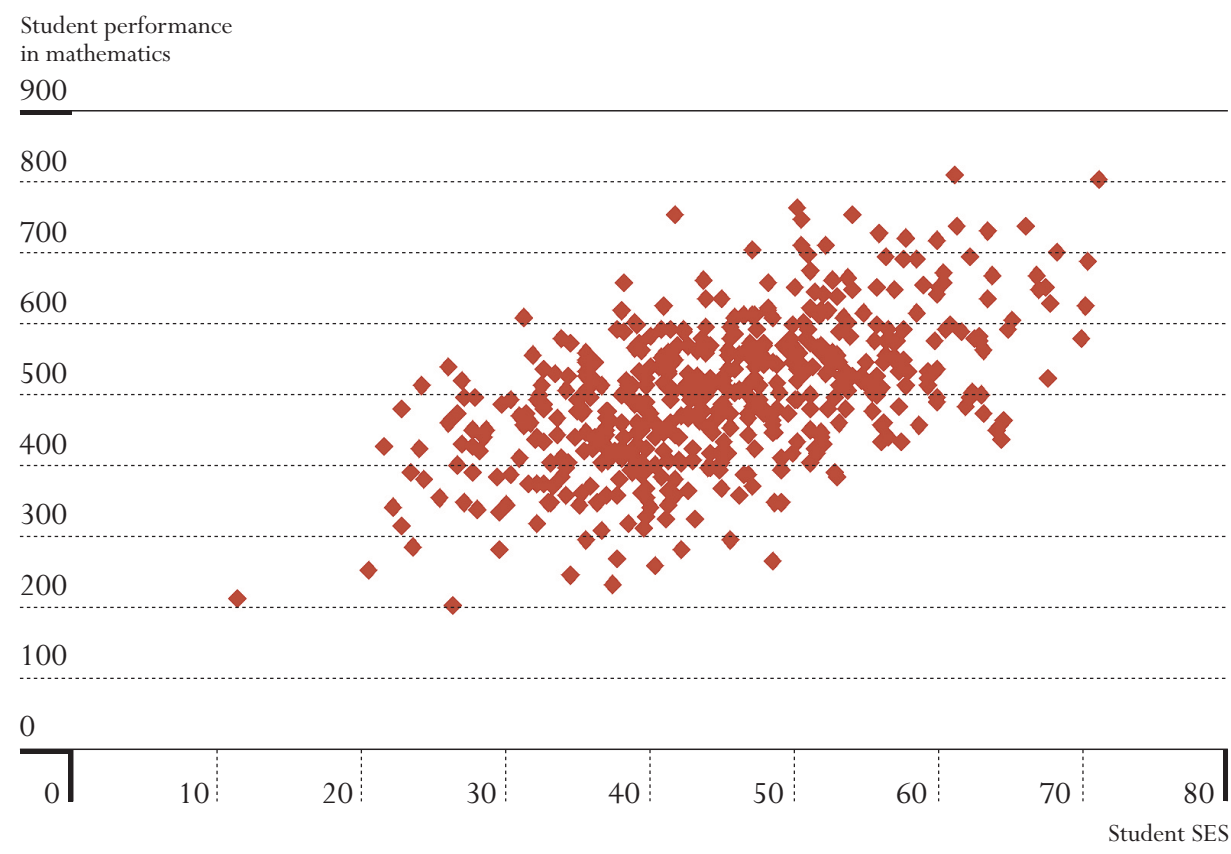

As Figure 13.2 shows, there is a positive relationship between the student's socio-economic background and the academic performance. Students from higher socio-economic backgrounds tend to perform better at school. However, unlike a linear equation, not all points are located on a straight line, meaning that students from a low socio-economic background may perform well academically, and that students from a high socio-economic background may perform poorly.

Statisticians use a linear regression analysis to quantify such relationships. The process in this particular example is similar to a linear equation with two variables. It will consist of computing an equation $Y_{i}=\alpha+\beta X$, with $Y_{i}$ being the academic performance of student $i$, and $X_{i}$ being his or her family socio-economic background. This equation can also be represented by a straight line denoted regression line. 
The regression line in Figure 13.3 corresponds to the regression equation, $Y_{i}=250.5+5.5 X_{i}$. One measure of socio-economic status used for PISA 2000 and for PISA 2003 (Ganzeboom et al., 1992) is the index of highest occupational status of parents called HISEI. This index ranges from 16 to 90 with an average of about 50 and a standard deviation of approximately 15 . The performance in mathematics has an international mean of 500 and a standard deviation of 100 . This equation shows that an increase of one unit on the HISEI scale is associated with an increase of 5.5 points on the PISA mathematics scale, on average.

\section{Figure 13.3 - Regression line of the socio-economic background on} the student performance in mathematics

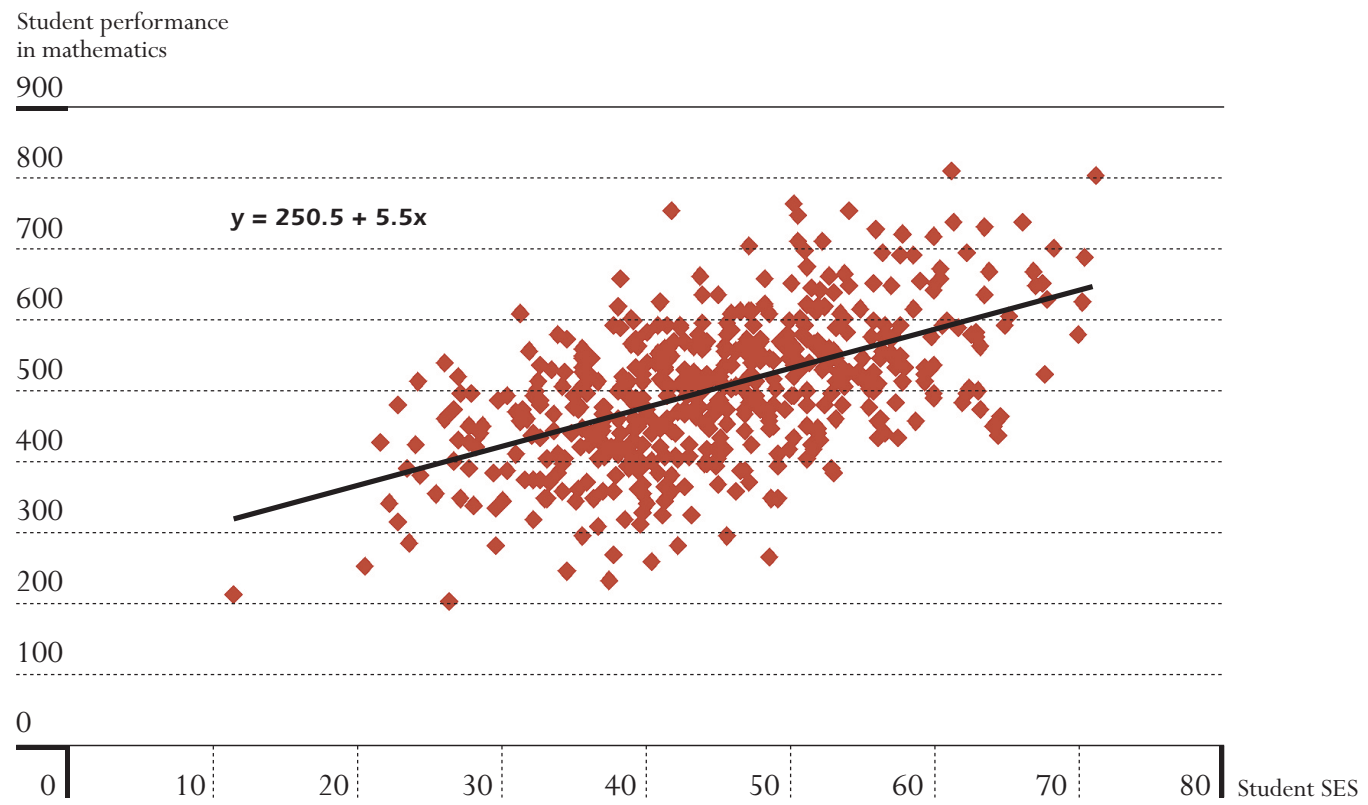

This regression equation can also be used to predict the mathematics performance of a student if the socio-economic background is known. For instance, this regression equation will predict, for each student with a HISEI value of 60 , a score of $250.5+(5.5 \times 60)=580.5$. In other words, any student with a HISEI of 60 will have a predicted score of 580.5. However, as shown by Figure 13.3, some of these students have a performance very close to this predicted score, usually denoted $\hat{Y}_{i}$, but the others either perform better, or at a lower level.

Before the computation of the regression equation, each student in the sample could be characterised by HISEI, $X_{i}$, and by performance in mathematics, $Y_{i}$. Now, each student can also be characterized by his or her predicted score, $\hat{Y}_{1}$, and by the difference between the observed score and predicted score $\left(Y_{i}-\hat{Y}_{i}\right)$, usually denoted as the residual (or $\left.\boldsymbol{\varepsilon}_{i}\right)$.

Table 13.1 - HISEI, mathematics performance, predicted score and residual

\begin{tabular}{c|c|c|c|c}
\hline Students & HISEI & Observed score & Predicted score & Residual \\
\hline $\mathbf{1}$ & 49 & 463 & 520 & -57 \\
\hline $\mathbf{2}$ & 53 & 384 & 542 & -158 \\
\hline $\mathbf{3}$ & 51 & 579 & 531 & +48 \\
\hline $\mathbf{4}$ & 42 & 404 & 481.5 & -77.5 \\
\hline $\mathbf{5}$ & 42 & 282 & 481.5 & -199.5 \\
\hline
\end{tabular}


The first student has an HISEI value of 49 and a mathematics performance of 463 . Based on his or her socio-economic background, one would have predicted a score of 520. This student has, therefore, a lower performance than expected. The residual is equal to -57. On the other hand, the third student has a performance of 579 and an expected score of 531 . This student performs better than expected.

Table 13.1 shows that the observed scores, the predicted scores and the residual scores present some variability on which variance coefficients can be computed. The regression equation and the regression line are constructed in a way that minimise the variance of the residual, denoted residual variance. This means that:

- The regression equation must include the point $\left(\boldsymbol{\mu}_{x}, \boldsymbol{\mu}_{y}\right)$;

- The mean of the predicted score is equal to the mean of the observed score $\left(\boldsymbol{\mu}_{y}=\boldsymbol{\mu}_{\hat{y}}\right)$; and

- The mean of the residual must be equal to 0 .

Finally, a regression analysis can be extended to several explanatory variables. If $k$ predictors are incorporated in the regression, then the equation will be written as:

$$
Y_{i}=\alpha+\beta_{1} X_{1 i}+\beta_{2} X_{2 i}+\ldots \ldots+\beta_{k} X_{k i}
$$

For instance, the mathematics performance on the PISA test can be explained by the student family background, his or her gender, the time spent each week on homework, interest in mathematics and so on.

\section{Box 13.1 - Interpretation of a regression coefficient and an intercept}

A regression coefficient reflects the change of units on the $\mathrm{Y}$ axis (the dependent variable - in this particular case, the increase on the mathematics scale) per unit change of the $\mathrm{X}$ axis. The interpretation of a regression coefficient depends on the measurement unit of an independent variable. Therefore, the statistical effect of different independent variables can not be compared, unless these independent variables have the same measurement units.

To achieve this, independent variables can be standardised so that the measurement units become the standard deviation. If all variables have a standard deviation of 1 , the regression coefficients of different variables can be directly compared. The regression coefficients will reflect the increase on the mathematic scale per standard deviation of the independent variables.

Suppose that two independent variables denoted $\mathrm{X} 1$ and $\mathrm{X} 2$ are used to explain the mathematical performance of students in two countries. The tables below provide the regression coefficients and the standard deviation of X1 and X2 before and after standardising the independent variables.

\begin{tabular}{|l|c|c|c|c|}
\hline \multirow{2}{*}{} & \multicolumn{2}{|c|}{ Xefore standardisation } \\
\cline { 2 - 5 } & $\beta_{1}$ & $\sigma_{\left(x_{1}\right)}$ & $\beta_{1}$ & $\sigma_{\left(x_{2}\right)}$ \\
\hline Country A & 10 & 2 & 15 & 3 \\
\hline Country B & 5 & 1 & 7.5 & 1.5 \\
\hline
\end{tabular}

\begin{tabular}{|l|c|c|c|c|}
\hline \multirow{2}{*}{} & \multicolumn{2}{|c|}{ After standardisation } \\
\cline { 2 - 5 } & $\beta_{1}$ & $\sigma_{\left(x_{1}\right)}$ & $\beta_{1}$ & $\sigma_{\left(x_{2}\right)}$ \\
\hline Country A & 5 & 1 & 5 & 1 \\
\hline Country B & 5 & 1 & 5 & 1 \\
\hline
\end{tabular}


The results are quite different. Based on the regression coefficients after standardisation, it seems that the two independent variables have the same statistical effect on the mathematic performance in both countries. Assume that X1 represents the time spent at home for the homework. In country A, the increase of one hour spent on homework is associated with an increase of 10 points on the mathematic scale while in country B, an additional hour is associated with the increase of 5 points on the mathematic scale. While the standardisation of the variables allows comparisons, the interpretation of a particular regression coefficient becomes more complex as it does not refer anymore to the original scale.

Thus, there is no single algorithm to solve this problem. It depends on the nature of the independent variable and the purpose of the analyses.

The interpretation of the intercept is even more complex as it depends on the standard deviation and the mean of the independent variables. Let us suppose that HISEI is standardised to a mean of zero and a standard deviation of one. The regression coefficient would reflect the increase in mathematics per standard deviation on the socio-economic status scale. The intercept would therefore represent the performance of a student with a transformed HISEI score of 0 . In a model with only standardised variables, it would reflect the performance of a hypothetical student who has average scores for all independent variables.

\section{SIMPLE LINEAR VERSUS MULTILEVEL REGRESSION ANALYSES}

The previous simple linear regression has shown the relationship between socio-economic background and mathematics performance at the population level, i.e. the 15-year-olds attending an educational institution.

A relationship between the socio-economic background of the student and performance in mathematics does not necessarily imply that richer countries will have a higher performance average than developing countries. Further, the relationship observed at the student level across schools does not necessary imply that the same phenomenon will be identified within each school.

Multilevel regression analyses recognize that sampled units are nested within larger units. Instead of computing one regression equation on the whole dataset, the multilevel regression analysis will compute a regression equation per larger unit. In all education surveys, students are nested within schools. Therefore, a multilevel regression analysis will compute a regression equation per school.

Figure 13.4 shows four graphs that highlight the distinction between a linear regression and a multilevel linear regression model. These four graphs represent the relationship between student socio-economic backgrounds and mathematics performance estimates in different countries.

The thick black line represents the regression line when the hierarchical structure of the data is not taken into account. The thin red lines represent the relationship between these two variables within particular schools. For each school, there is a regression line (a red line in this particular example). The larger black point on the linear regression lines (black) represents the point with the mean of 
$X$ and $Y$ as coordinates, $\left(\mu_{x}, \mu_{y}\right)$, and the red point on the multilevel regression lines represents the point with the school mean of $X$ and $Y$ as coordinates, $\left(\mu_{x i}, \mu_{y i}\right)$.

The simple linear regression analysis, graphically represented by the black lines, shows that the expected score of a student from a higher socio-economic background is considerably higher than the expected score of a student from a lower socio-economic background. The comparison between the four graphs shows the similarity of the relationship between the student's socioeconomic background and the student performance at that level between countries. Based on simple linear regression analyses, one would conclude that the relationship between the socio-economic background and the student performance is identical in the different countries.

However, the multilevel regression analyses clearly distinguish the relationship between the two variables in the four countries.

\section{Figure 13.4 — Linear regression analysis versus multilevel regression analysis}

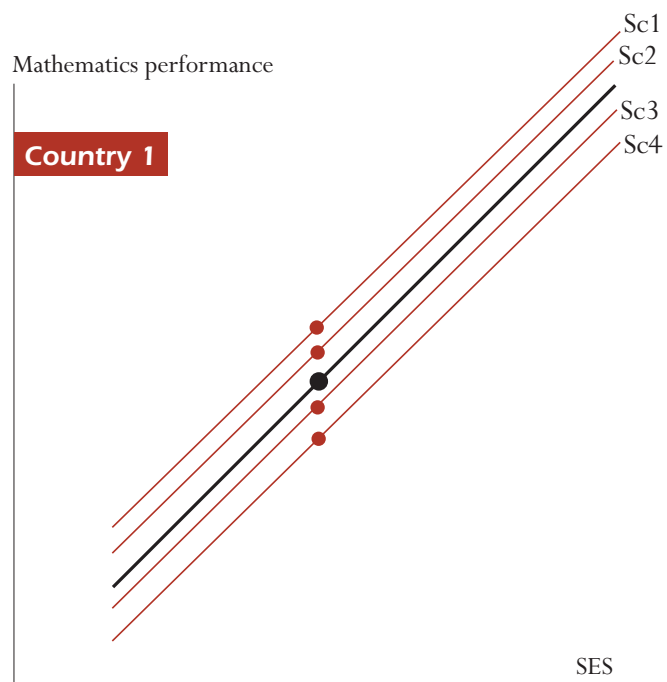

Mathematics performance

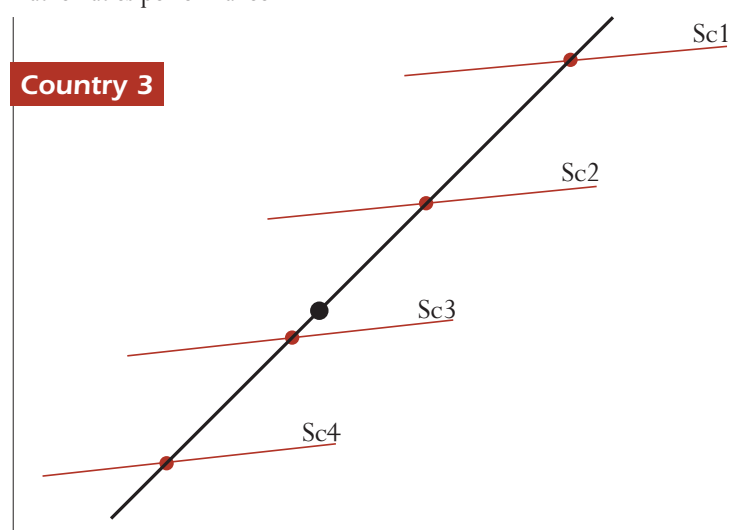

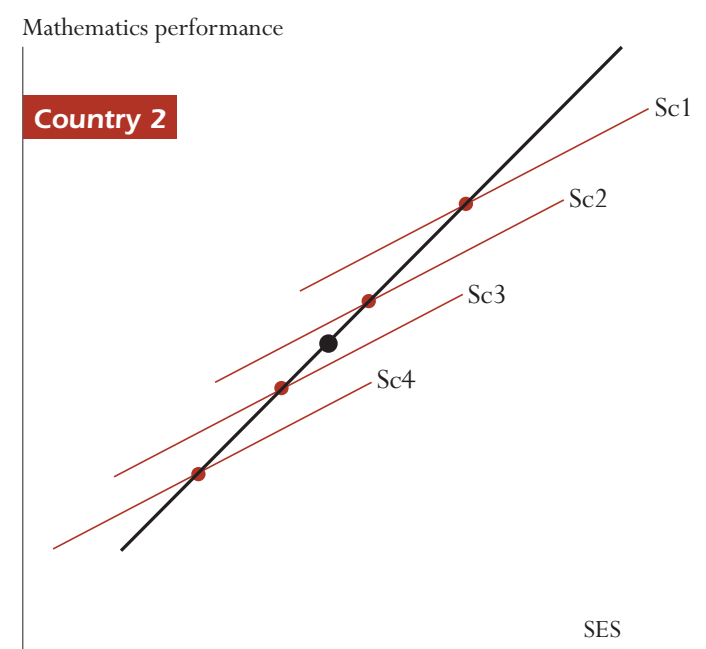

Mathematics performance

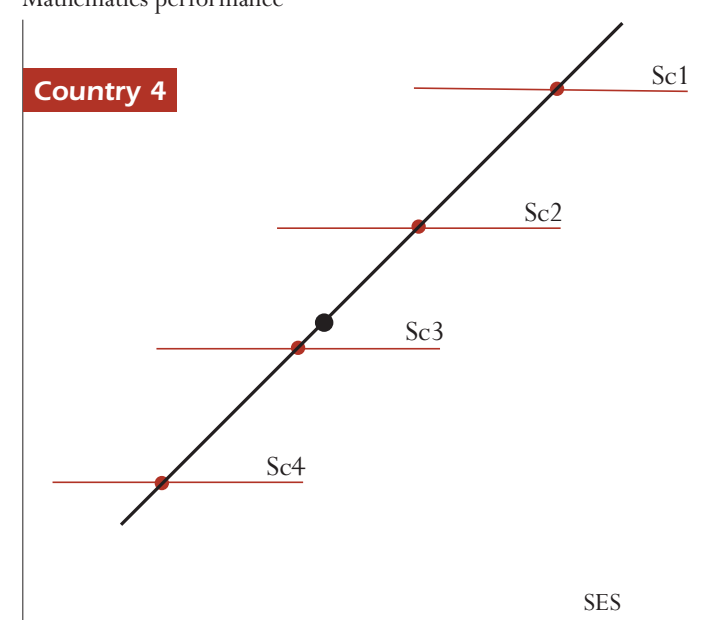


In country 1 , the multilevel regression lines are similar and close to the simple linear regression line. This means that:

- Regarding the socio-economic background of the student ( $X$ axis):

- The different schools are all attended by students coming from a wide range of socio-economic backgrounds. All the within-school regression lines cover the whole range of values on the $X$ axis; and

- The schools have the same socio-economic intake, i.e. the mean of the student socio-economic background. Indeed, the projections of the red dots on the $X$ axis are very close to each other.

- Regarding the student performance in mathematics ( $Y$ axis):

- In each school, there are low, medium and high achievers. All the within-school regression lines cover the $Y$ axis; and

- On average, the schools have a similar level of performance. Indeed, the projections of the red dots on the $Y$ axis are very close to each other. It also means that the between-school variance is quite small.

- Regarding the relationship between the socio-economic background and mathematics performance:

- In each school, there is a strong relationship between socio-economic background and achievement. Within all schools, low socio-economic background students perform well below high socioeconomic background students. The slope of the within-school regression line indicates the strength of the relationship.

Each school in country 1 can therefore be considered as a simple random sample of the population and each school reflects the relationships that exist at the population level.

The opposite of country 1 is graphically represented by country 4 . The multilevel regression lines differ considerably from the simple linear regression line. In that particular case, it means that:

- Regarding the socio-economic background of the student ( $X$ axis):

- The schools do not cover the range of socio-economic backgrounds that exist at the population level. School 1 is mainly attended by high socio-economic background students while school 4 is mainly attended by low socio-economic background students; and

- The schools have therefore different socio-economic intakes as the projections of the red dots on the $X$ axis would show. In other words, there is a significant socio-economic segregation at the school level.

- Regarding the student performance in mathematics ( $Y$ axis):

- The schools do not cover the range of the student performance that exists at the population level. School 1 is mainly attended by high achievers and school 4 is mainly attended by low achievers; and

- Schools largely differ by their average performance level, as the projections of the red dots on the $Y$ axis would show. In country 4, the school performance variance is therefore very important.

- Regarding the relationship between the socio-economic background and mathematics performance:

- In each school, there is no relationship between the socio-economic background and achievement.

- Within a particular school, the socio-economic background of the student does not matter. What does matter is not the socio-economic background of the student but the school he or she will attend. But the socio-economic background of the student will determine the school he or she will attend.

Countries 2 and 3 present intermediate situations between these two extreme examples. 


\section{FIXED EFFECT VERSUS RANDOM EFFECT}

For the cases examined so far, the within-school regression lines were all parallel, but multilevel regression analyses also allow the regression slope to vary. In the former, the effect, i.e. the $X$ effect, will be considered as fixed, while in the latter, the effect will be considered as random. Figure 13.5 represents a case with a random effect.

\section{Figure 13.5 A random multilevel model}

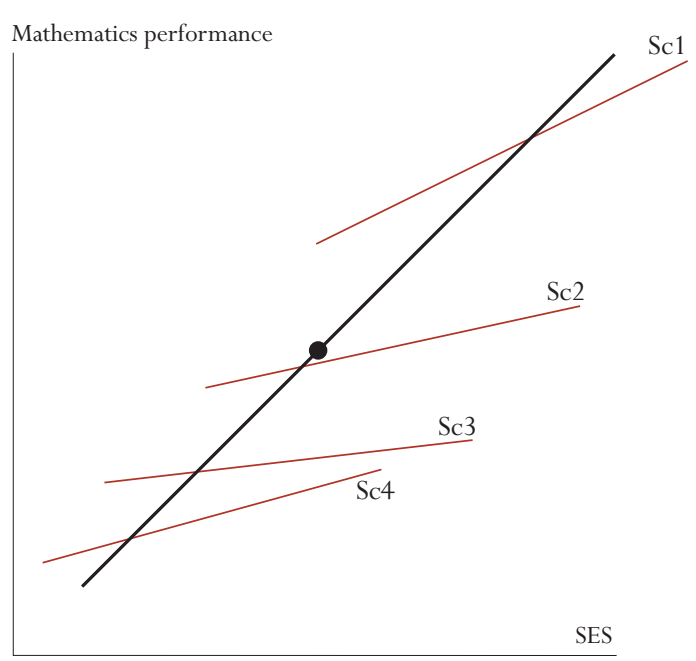

Mathematically, in the case of one explanatory variable, the two models can be differentiated as follows:

$$
\begin{aligned}
& Y_{i j}=\alpha_{j}+\beta X_{i j}+\varepsilon_{i j} \text { for a fixed effect } \\
& \alpha_{j}=\gamma_{00}+U_{0 j}
\end{aligned}
$$

and

$$
\begin{aligned}
& Y_{i j}=\alpha_{j}+\beta_{j} X_{i j}+\varepsilon_{i j} \text { for a random effect } \\
& \alpha_{j}=\gamma_{00}+U_{0 j} \\
& \beta_{j}=\gamma_{10}+U_{1 j}
\end{aligned}
$$

The subscript $i$ in the equations refers to the student ${ }^{1}$ (also denoted level 1 in the multilevel model literature), and the subscript $j$ refers to the school (or level 2). In an equation, the presence of the subscript $j$ for a regression coefficient means that it can vary from one school to another.

The term $\varepsilon_{i j}$ denotes the residual of the equation, i.e. the difference between the observed score $Y_{i j}$ and the predicted score $\hat{Y}_{i j}$. This residual is normally distributed with a mean of 0 and a constant level 1 (i.e. the student level) variance, usually denoted $\sigma^{2}$.

As shown by these two equations, the intercept $\alpha_{j}$ is always considered as a random effect. Considering the intercept as a fixed parameter would reduce the multilevel model to a linear regression analysis. The intercept $\alpha_{j}$ can further be divided into a fixed part, i.e. $\gamma_{00}$ denotes the overall intercept and is equal to the average of the school intercepts $\alpha_{j}$, and secondly into a random part, i.e. $U_{0 j}$, denoting school departure from the overall intercept. This school departure $U_{0 j}$ is assumed to have a mean of 0 and a variance $\boldsymbol{\tau}_{0}^{2}$. 
The $\beta$ coefficient in the first equation has no subscript $j$, meaning that the effect $X$ cannot vary from one school to the other. The regression lines are therefore parallel and thus the $X$ effect is considered as fixed. On the other hand, the $\beta$ coefficient in the second equation has a subscript $j$, meaning that it can vary from one school to another. The regression lines are no longer parallel and thus the $X$ effect is now considered as random. As previously, this regression coefficient $\beta_{j}$ can be divided into a fixed part and a random part. The fixed part $\gamma_{10}$ is called overall regression coefficient and corresponds to the mean of the regression coefficients $\beta_{j}$. The random part $U_{1 j}$ is the school departure from the overall regression coefficient. It has a mean of 0 and a variance denoted $\boldsymbol{\tau}_{1}^{2}$.

Random effects and fixed effects can be combined in a single multilevel regression analysis. For instance, in the following equation, two student explanatory variables are introduced in the model, one is considered as fixed, $X_{1}$, and the other one as random, $X_{2}$.

$$
Y_{i j}=\alpha_{j}+\beta_{1} X_{1 i j}+\beta_{2 j} X_{2 i j}
$$

\section{SOME EXAMPLES WITH SPSS ${ }^{\circledR}$}

Usually, two types of indices are relevant in multilevel analyses: the regression coefficients and the decomposition of the variance into the different levels, i.e. the student level (or level 1) and school level (or level 2).

Multilevel regression analyses always report the residual variance at the different levels - the between-school variance and the within-school variance that are not explained by the predictors included in the model.

However, scientific reports usually show the explained variance. The conversion of the residual variance into percentages of explained variance just requires the comparison of the school and student variance coefficients with their respective residual variance coefficients.

\section{Example 1}

The decomposition of the total variance can be easily obtained with a multilevel regression model. Applying the following model

$$
\begin{aligned}
& Y_{i j}=\alpha_{j}+\varepsilon_{i j} \\
& \alpha_{j}=\gamma_{00}+U_{0 j}
\end{aligned}
$$

will provide unbiased estimates of the between-school variance and the within-school variance. As the regression model has no predictors, the school intercepts, i.e. $\alpha_{j}$ will therefore be equal or close to the school means. The variance of $U_{0_{j}}$ will be equal to the between-school variance. As each student will be assigned his or her school mean as predicted score, the variance of $\varepsilon_{i j}$ will be equal to the within-school variance.

SPSS $^{\circledR}$ offers two procedures for multi-level analyses: VARCOMP enables researchers to do (multilevel) variance decomposition, MIXED is a procedure, which can be used for multi-level modelling. The SPSS ${ }^{\circledR}$ MIXED and VARCOMP procedures allow for modelling multilevel regression. However, both procedures require the standardisation of the weights, i.e. the sum of the weights is equal to the number of students in the dataset. If the BY statement is used, then the standardisation will be done by category of the breakdown variable. 
Box 13.2 provides the SPSS $^{\circledR}$ syntax for this standardisation, as well as a short checking procedure. ${ }^{2}$ Please note that in order to write variance estimates into an output file, the VARCOMP procedures need to be used (see Box 13.3).

\section{Box 13.2 - Standardisation of the PISA 2003 final weights}

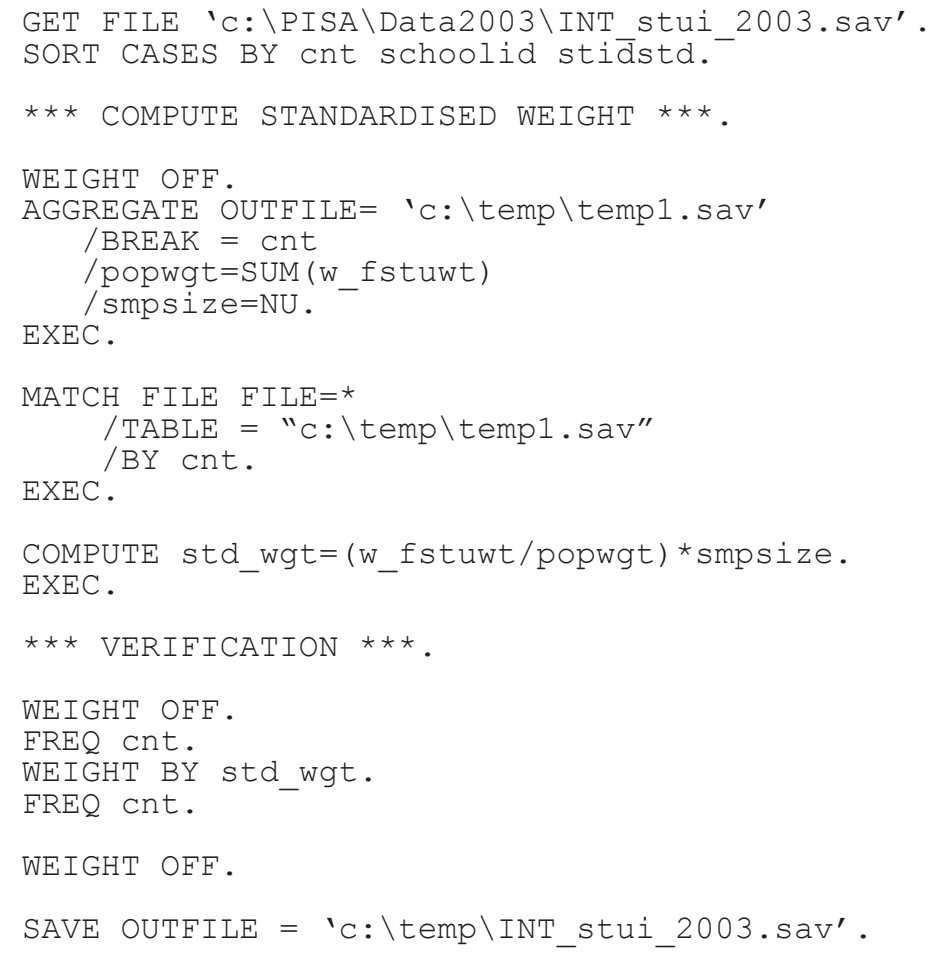

Box 13.3 provides the SPSS $^{\circledR}$ syntax for a multilevel regression model as well as the SPSS $^{\circledR}$ syntax for the computation of the intra-class correlation.

\section{Box 13.3 - SPSS $^{\circledR}$ syntax for a multilevel regression model - Example 1}

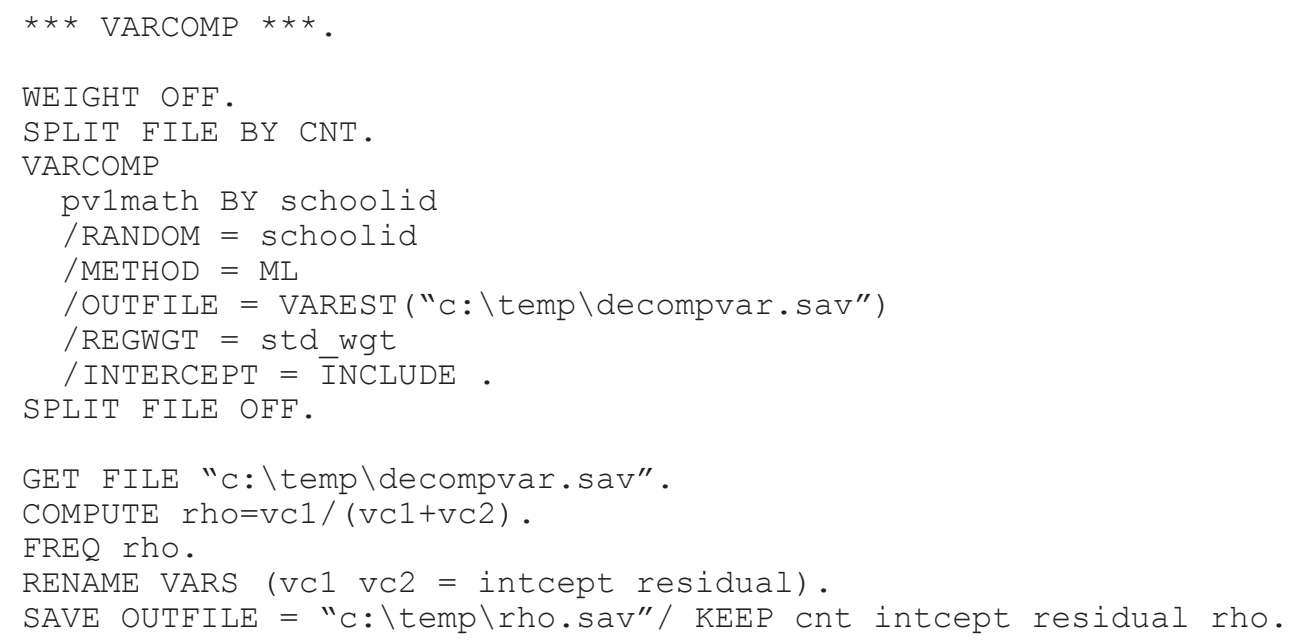


The RANDOM statement defines the second level of the analyses. The first statement following the name of the procedure (either VARCOMP or MIXED) specifies the model, including the dependent and then the predictor variables following the keyword WITH. The VARCOMP procedure also requires to define the second level in the model statement using the BY keyword (for example: pv1math BY schoolid WITH hisei). In this particular example, there is no predictor included in the model statement. Therefore the school and within-school residual variances will be equal to the school and within-school variance estimates. The RANDOM statement distinguishes between fixed and random predictors, as explained in the previous section. It should be noted that when using the MIXED procedure "intercept" always needs to be mentioned. The REGWGT statement should be followed by the normalised student-level weight. In order to get results by country the command should be proceeded by the SPLIT FILE BY (grouping variable) statement and followed by SPLIT FILE OFF. The VARCOMP PROCEDURE allows to include an OUTFILE statement which allows to write the variance estimates into another SPSS $^{\circledR}$ system file. The variance estimates will be saved in the file "decompvar.sav". Further options for OUTFILE statement in VARCOMP are COVB (covariance matrix of variance estimates) and CORB (correlation matrix of variance estimates).

Table 13.2 provides the between-school and within-school variance estimates and the intra-class correlation. These variance estimates were saved in the file "decompvar.sav". As shown in Box 13.3, the intra-class correlation ${ }^{3}$ is equal to:

$$
\rho=\frac{\sigma_{\text {between-school }}^{2}}{\sigma_{\text {between-school }}^{2}+\sigma_{\text {within-school }}^{2}}=\frac{\tau_{0}^{2}}{\tau_{0}^{2}+\sigma^{2}}
$$

with $\sigma_{\text {school }}^{2}$ or $\boldsymbol{\tau}_{0}^{2}$ the between-school variance and $\sigma_{\text {within-school }}^{2}$ or $\sigma^{2}$ the within-school variance. In Australia, the between-school variance is equal to $1919.11^{4}$ and the within-school variance is equal to 7169.09. The intra-class correlation is therefore the percentage of the total variance that is accounted for by the school. It reflects how schools differ in their student average performance. In Australia, the intra-class correlation is therefore equal to $1919.11 /(1919.11+7169.09)=0.21$. The estimate of the intra-class correlation ranges from 0.04 in Iceland to 0.63 in the Netherlands.

\section{Example 2}

The following examples are based on the data of Luxembourg. The school sample size in Luxembourg, i.e. 29, will allow the presentation of the school parameter estimates. In Example 2, the socioeconomic background of the student, denoted HISEI, is introduced as a fixed factor.

\section{Preparation of the data file}

In the PISA databases, there are no missing data for the final weight and for the student performance estimate. However, there are missing values for variables that might be used as predictors in a multilevel regression model. These missing data generate two major issues:

- The sum of the weights will slightly differ from the number of cases that will be used by the regression models. Note that cases with missing values are automatically ${ }^{5}$ dropped from any regression models.

- The school and student variances from different models cannot be compared as missing values are not always random. For instance, low socio-economic background students are usually less likely to provide answers about their mother's and/or father's occupations. 
Table 13.2 a Between- and within-school variance estimates and intra-class correlation

\begin{tabular}{|c|c|c|c|}
\hline Country & Between-school variance & Within-school variance & rho \\
\hline AUS & 1919.11 & 7169.09 & 0.21 \\
\hline AUT & 5296.65 & 4299.71 & 0.55 \\
\hline BEL & 7328.47 & 5738.33 & 0.56 \\
\hline BRA & 4128.49 & 5173.60 & 0.44 \\
\hline CAN & 1261.58 & 6250.12 & 0.17 \\
\hline $\mathrm{CHE}$ & 3092.60 & 6198.65 & 0.33 \\
\hline CZE & 4972.45 & 4557.50 & 0.52 \\
\hline DEU & 6206.92 & 4498.70 & 0.58 \\
\hline DNK & 1109.45 & 7357.14 & 0.13 \\
\hline ESP & 1476.85 & 6081.74 & 0.20 \\
\hline FIN & 336.24 & 6664.98 & 0.05 \\
\hline FRA & 3822.62 & 4536.22 & 0.46 \\
\hline GBR & 1881.09 & 6338.25 & 0.23 \\
\hline GRC & 3387.52 & 5991.75 & 0.36 \\
\hline HKG & 4675.30 & 5298.26 & 0.47 \\
\hline HUN & 5688.56 & 4034.66 & 0.59 \\
\hline IDN & 2769.48 & 3343.87 & 0.45 \\
\hline IRL & 1246.70 & 6110.71 & 0.17 \\
\hline ISL & 337.56 & 7849.99 & 0.04 \\
\hline ITA & 4922.84 & 4426.67 & 0.53 \\
\hline JPN & 5387.17 & 4668.82 & 0.54 \\
\hline KOR & 3531.75 & 5011.56 & 0.41 \\
\hline LIE & 3385.41 & 5154.08 & 0.40 \\
\hline LUX & 2596.36 & 5806.97 & 0.31 \\
\hline LVA & 1750.22 & 6156.52 & 0.22 \\
\hline MAC & 1416.99 & 6449.96 & 0.18 \\
\hline MEX & 2476.01 & 3916.46 & 0.39 \\
\hline NLD & 5528.99 & 3326.09 & 0.62 \\
\hline NOR & 599.49 & 7986.58 & 0.07 \\
\hline NZL & 1740.61 & 7969.97 & 0.18 \\
\hline POL & 1033.90 & 7151.46 & 0.13 \\
\hline PRT & 2647.70 & 5151.93 & 0.34 \\
\hline RUS & 2656.62 & 6021.44 & 0.31 \\
\hline SVK & 3734.56 & 4873.69 & 0.43 \\
\hline SWE & 986.03 & 8199.46 & 0.11 \\
\hline THA & 2609.38 & 4387.08 & 0.37 \\
\hline TUN & 2821.00 & 3825.36 & 0.42 \\
\hline TUR & 6188.40 & 4891.13 & 0.56 \\
\hline URY & 4457.08 & 5858.42 & 0.43 \\
\hline USA & 2395.38 & 6731.45 & 0.26 \\
\hline YUG & 2646.00 & 4661.59 & 0.36 \\
\hline
\end{tabular}

To avoid these two problems, it is recommended to delete any cases with missing data for the different predictors that will be used in the regression models before the weight standardisation. As the next multilevel regression model examples will use two student level variables, i.e. HISEI for the student socio-economic background, and ST03Q01 for the student gender and two school level variables, i.e. the percentage of girls in the school, PCGIRLS, and the type of schools, SCHLTYPE, cases with missing data for at least one of these four variables will be deleted before the weight standardisation. 
Box 13.4 presents the $\operatorname{SPSS}^{\circledR}$ syntax. It consists of:

- Merging the student data file and the school data file with the variables of interest;

- Deleting the cases with at least one missing data for the predictor; and

- Standardising the weight.

Before deletion of cases with missing values, there are 3923 records in the Luxembourg database. After deletion, 3782 are left. About 3.5 per cent of the cases are deleted. If too many cases are deleted, for instance, more than 10 per cent, then either the variables with too many missing values should be dropped from the analyses, or imputation methods should be used.

Rerunning the empty multilevel model

After deletion of cases with missing values with the syntax in Box 13.4, the empty multilevel model, i.e. a multilevel regression model without any predictor in Box 13.3, is run to obtain the betweenschool and within-school variance estimates. The between-school and the within-school variance estimates, saved in the "decompvar.sav" file, are now respectively equal to 2563.30 and 5734.35 instead of 2596.36 and 5806.97.

Box 13.4 SPSS $^{\circledR}$ syntax for standardising PISA 2003 final weights with deletion of cases with missing values in Luxembourg

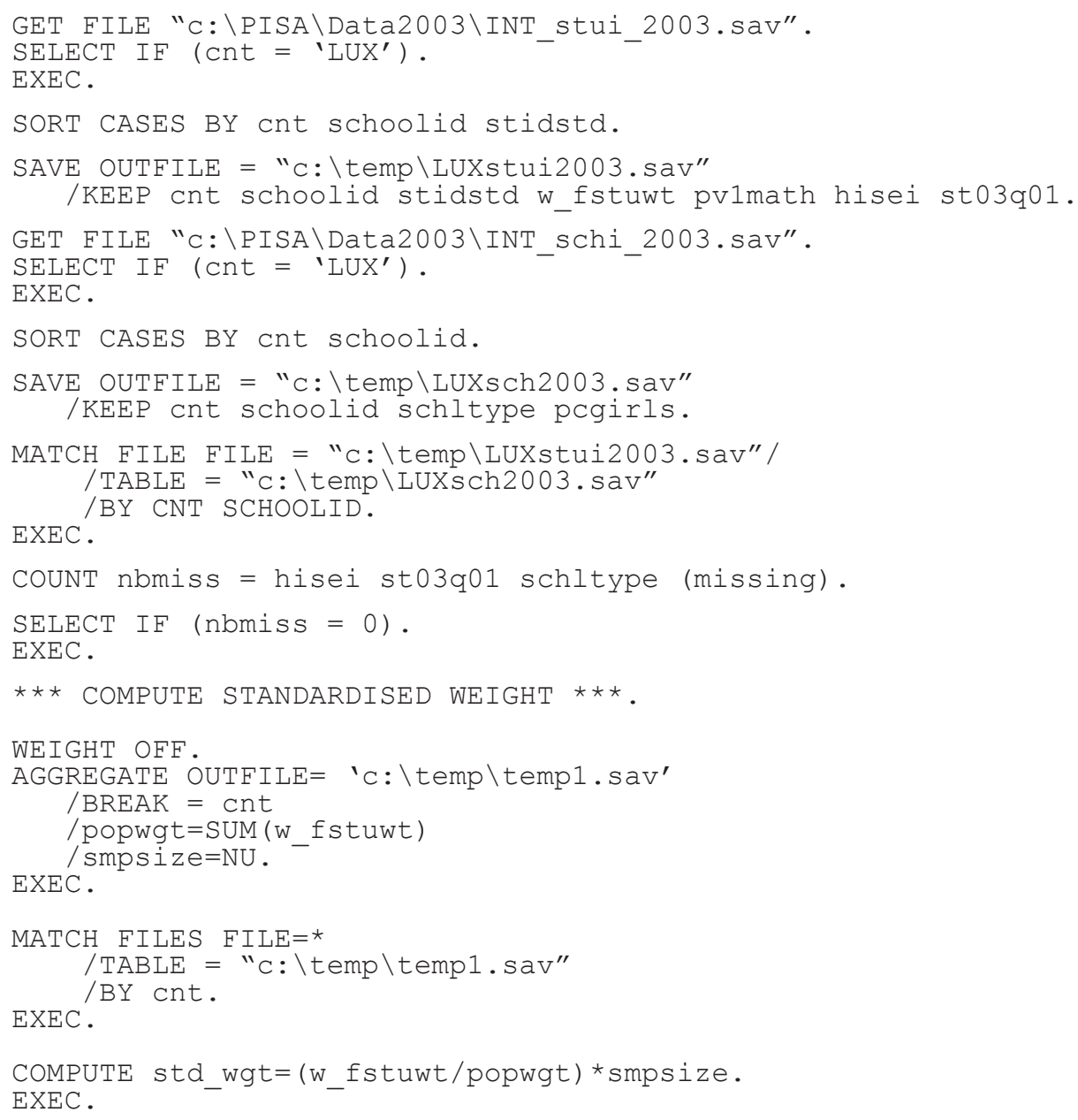




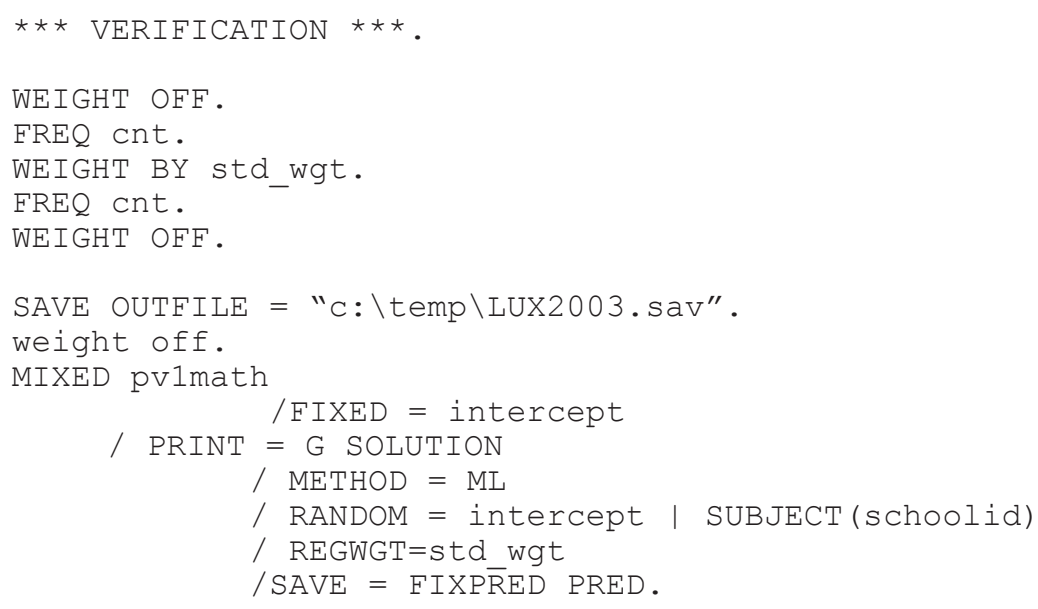

Estimating the "empty" model with the MIXED will have only one fixed parameter $\gamma_{00}$, which is 492.36 for the Luxembourg data.

SPSS $^{\circledR}$ unfortunately does not have output files with the random parameters at the second level units. With an empty model, these random parameters would only include the school departure $U_{0 j}$. Table 13.3 is a printout of a random parameter file which was computed using the SAS program. It contains:

- The breakdown variables used in the model, i.e. CNT;

- The effect, i.e. the intercept or as it will be shown later, the random predictor, the estimate;

- The class variable, i.e. the SCHOOLID;

- The estimate;

- The standard error on the estimate;

- The number of degrees of freedom (the number of students minus the number of schools);

- The $t$ statistic; and

- The probability that the estimates differ from 0 .

For instance, the departure of the school 00001 from the overall intercept 492.36 is only 0.71 . This departure does not differ from 0 , as shown by the $t$ statistic and its associated probability value. In other words, the intercept of school 00001 is not significantly different from the overall intercept. On the other hand, the intercept of school 00002 is significantly higher than the overall intercept.

\section{Shrinkage factor}

In the case of an empty model, one might consider that the sum of the overall intercept $\gamma_{00}$ and a particular school departure $U_{0 j}$ should be perfectly equal to the school performance mean.

Multilevel models shrink the school departures. To illustrate this shrinkage process, suppose we have an educational system with 100 schools. Assume that the school performance means are perfectly identical. In other words, the between-school variance is equal to 0 . If 20 students are tested within each school, it is expected that school mean estimates will slightly differ from the school means. 
Table 13.3 - Printout of the random parameter file as computed with $\mathbf{S A S}^{\circledR}$

\begin{tabular}{l|l|c|c|c|c|c|c}
\hline CNT & Effect & SCHOOLid & Estimate & StdErrPred & DF & tValue & Probt \\
\hline LUX & Intercept & 00001 & 0.71 & 13.00 & 3753 & 0.05 & 0.96 \\
LUX & Intercept & 00002 & 66.39 & 11.63 & 3753 & 5.71 & 0.00 \\
LUX & Intercept & 00003 & -23.71 & 11.03 & 3753 & -2.15 & 0.03 \\
LUX & Intercept & 00004 & -44.68 & 12.18 & 3753 & -3.67 & 0.00 \\
LUX & Intercept & 00005 & -8.56 & 10.68 & 3753 & -0.80 & 0.42 \\
LUX & Intercept & 00006 & 61.90 & 11.34 & 3753 & 5.46 & 0.00 \\
LUX & Intercept & 00007 & -68.69 & 12.39 & 3753 & -5.54 & 0.00 \\
LUX & Intercept & 00008 & 61.14 & 11.62 & 3753 & 5.26 & 0.00 \\
LUX & Intercept & 00009 & 81.64 & 11.10 & 3753 & 7.36 & 0.00 \\
LUX & Intercept & 00010 & -62.00 & 11.37 & 3753 & -5.45 & 0.00 \\
LUX & Intercept & 00011 & 33.19 & 25.14 & 3753 & 1.32 & 0.19 \\
LUX & Intercept & 00012 & -11.35 & 12.54 & 3753 & -0.91 & 0.37 \\
LUX & Intercept & 00013 & 15.56 & 10.47 & 3753 & 1.49 & 0.14 \\
LUX & Intercept & 00014 & 8.01 & 11.25 & 3753 & 0.71 & 0.48 \\
LUX & Intercept & 00015 & 37.55 & 12.36 & 3753 & 3.04 & 0.00 \\
LUX & Intercept & 00016 & -46.59 & 10.95 & 3753 & -4.26 & 0.00 \\
LUX & Intercept & 00017 & -33.61 & 10.98 & 3753 & -3.06 & 0.00 \\
LUX & Intercept & 00018 & -76.02 & 12.54 & 3753 & -6.06 & 0.00 \\
LUX & Intercept & 00019 & -70.43 & 12.96 & 3753 & -5.43 & 0.00 \\
LUX & Intercept & 00020 & 57.54 & 11.17 & 3753 & 5.15 & 0.00 \\
LUX & Intercept & 00021 & 8.04 & 11.01 & 3753 & 0.73 & 0.47 \\
LUX & Intercept & 00022 & -0.67 & 25.14 & 3753 & -0.03 & 0.98 \\
LUX & Intercept & 00023 & 84.27 & 10.90 & 3753 & 7.73 & 0.00 \\
LUX & Intercept & 00024 & 29.88 & 11.12 & 3753 & 2.69 & 0.01 \\
LUX & Intercept & 00025 & 63.74 & 11.69 & 3753 & 5.45 & 0.00 \\
LUX & Intercept & 00026 & -33.65 & 11.15 & 3753 & -3.02 & 0.00 \\
LUX & Intercept & 00027 & -8.29 & 11.53 & 3753 & -0.72 & 0.47 \\
LUX & Intercept & 00028 & -36.89 & 13.84 & 3753 & -2.66 & 0.01 \\
LUX & Intercept & 00029 & -84.43 & 10.96 & 3753 & -7.71 & 0.00 \\
\hline & & & & & & \\
& & & & & \\
\end{tabular}

Indeed, within particular schools, predominantly high achievers or low achievers may be sampled so that the school mean is respectively overestimated or underestimated. As the number of sampled students within schools increases, the difference between the school mean and its estimate is likely to decrease. Therefore, the shrinkage factor is inversely proportional to the number of sampled students within schools.

The shrinkage factor ${ }^{6}$ is equal to:

$$
\frac{n_{j} \sigma_{\text {between-school }}^{2}}{n_{j} \sigma_{\text {between-school }}^{2}+\sigma_{\text {within-school }}^{2}} \text {, }
$$

with $n_{j}$ being the number of students in school $j$ in the sample (Goldstein, 1997). 
Table 13.4 presents, for each school, the average performance in mathematics, the number of students used in the multilevel regression model, the departure from the overall intercept estimated by the empty multilevel regression model, as presented in Table 13.3 and the sum of the overall intercept $\gamma_{00}$ and the school departure $U_{0 j}$. Box 13.5 shows how to compute Table 13.4 using SPSS ${ }^{\circledR}$ (results in output file "schoolmeans.sav").

Box 13.5 — $\operatorname{SPSS}^{\circledR}$ syntax for a multilevel regression model - Example 2 (1)

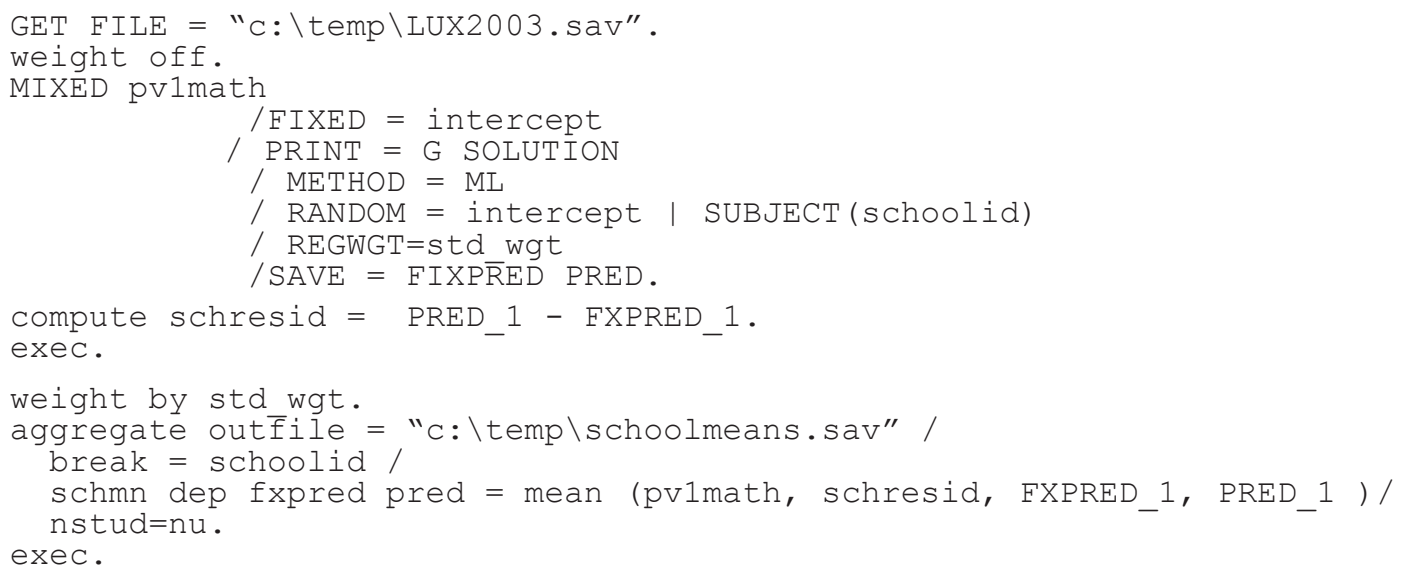

Table 13.4 - School performance in mathematics, number of students per school and corrected mean

\begin{tabular}{|c|c|c|c|c|}
\hline School & School mean & Number of students & Departure $U_{0 j}$ & $\gamma_{00}+U_{0 j}$ \\
\hline 00001 & 493.1 & 67 & 0.7 & 493.1 \\
\hline 00002 & 560.0 & 120 & 66.4 & 558.8 \\
\hline 00003 & 468.3 & 179 & -23.7 & 468.6 \\
\hline 00004 & 446.6 & 94 & -44.7 & 447.7 \\
\hline 00005 & 483.7 & 233 & -8.6 & 483.8 \\
\hline 00006 & 555.2 & 146 & 61.9 & 554.3 \\
\hline 00007 & 421.8 & 83 & -68.7 & 423.7 \\
\hline 00008 & 554.6 & 116 & 61.1 & 553.5 \\
\hline 00009 & 575.1 & 167 & 81.6 & 574.0 \\
\hline 00010 & 429.4 & 131 & -62.0 & 430.4 \\
\hline 00011 & 535.2 & 8 & 33.2 & 525.6 \\
\hline 00012 & 480.7 & 78 & -11.3 & 481.0 \\
\hline 00013 & 508.0 & 289 & 15.6 & 507.9 \\
\hline 00014 & 500.5 & 150 & 8.0 & 500.4 \\
\hline 00015 & 530.9 & 87 & 37.6 & 529.9 \\
\hline 00016 & 445.2 & 184 & -46.6 & 445.8 \\
\hline 00017 & 458.3 & 183 & -33.6 & 458.8 \\
\hline 00018 & 414.2 & 73 & -76.0 & 416.3 \\
\hline 00019 & 419.6 & 66 & -70.4 & 421.9 \\
\hline 00020 & 550.7 & 162 & 57.5 & 549.9 \\
\hline 00021 & 500.5 & 174 & 8.0 & 500.4 \\
\hline 00022 & 491.5 & 8 & -0.7 & 491.7 \\
\hline 00023 & 577.6 & 185 & 84.3 & 576.6 \\
\hline 00024 & 522.7 & 169 & 29.9 & 522.2 \\
\hline 00025 & 557.3 & 117 & 63.7 & 556.1 \\
\hline 00026 & 458.2 & 151 & -33.7 & 458.7 \\
\hline 00027 & 483.9 & 126 & -8.3 & 484.1 \\
\hline 00028 & 453.9 & 53 & -36.9 & 455.5 \\
\hline 00029 & 406.9 & 183 & -84.4 & 407.9 \\
\hline
\end{tabular}


$\triangleq \quad$ As shown, the difference between the school performance mean and the sum $\gamma_{00}+U_{0 j}$ is:

- Proportional to the school departure, i.e. the shrinkage factor mainly affects low and high performing schools; and

- Inversely proportional to the number of observed students in the school.

Introduction of HISEl as a fixed effect

With the introduction of the student level variable HISEI as a fixed effect, the equation can be written as:

$Y_{i j}=\alpha_{j}+\beta_{1}(H I S E I)_{i j}+\varepsilon_{i j}$

$\alpha_{j}=\gamma_{00}+U_{0 j}$

The SPSS ${ }^{\circledR}$ syntax for this model is presented in Box 13.6 and parts of the SPSS $^{\circledR}$ output is presented in Box 13.7.

\section{Box 13.6 - SPSS $^{\circledR}$ syntax for a multilevel regression model - Example 2 (2)}

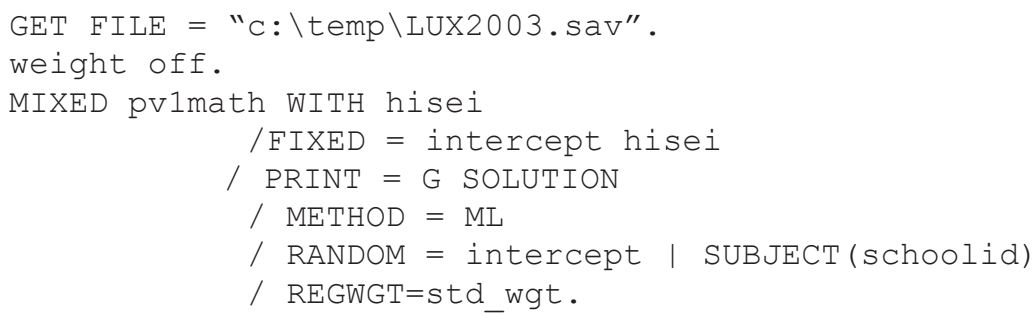

Box 13.7 - SPSS $^{\circledR}$ output - Example 2

\begin{tabular}{|c|c|c|}
\hline Parameter & Estimate & Std. Error \\
\hline Residual & 5551.5060563 & 128.1500411 \\
\hline $\begin{array}{l}\text { Intercept [subject }=\text { SCHOOLID] } \\
\text { Variance }\end{array}$ & 1950.3945680 & 530.9974935 \\
\hline
\end{tabular}

Estimates of Fixed Effects ${ }^{a, b}$

\begin{tabular}{l|r|r|r|r|r|r|r}
\multirow{2}{*}{ Parameter } & \multicolumn{1}{|c|}{ Estimate } & Std. Error & \multicolumn{1}{c|}{$\mathrm{df}$} & $\mathrm{t}$ & \multicolumn{2}{c|}{ Sig. } & \multicolumn{2}{|c}{$95 \%$ Confidence Interval } \\
\cline { 6 - 8 } & & & & & & Lower Bound & Upper Bound \\
\hline Intercept & 446.7649734 & 9.2614577 & 43.341 & 48.239 & .000 & 428.0917086 & 465.4382383 \\
\hline HISEI & .9479007 & .0823676 & 3780.841 & 11.508 & .000 & .7864114 & 1.1093899 \\
\hline
\end{tabular}

a. Dependent variable: plausible value in math.

b. Residual is weighted by std_wgt.

Only one change has been introduced in comparison with the syntax presented in Box 13.5. The name HISEI has been added to the model statement. 
The overall intercept $\gamma_{00}$ is now equal to 446.76 and the within-school regression coefficient $\beta_{1}$ is equal to 0.9479 . This means that, within a particular school, an increase of 1 unit on the HISEI scale will be associated with an increase of 0.9479 on the mathematics scale. By comparison, the linear regression coefficient of HISEI on the mathematics performance is equal to 2.05. The relationship between SES and student achievement in Luxembourg education system seems to be similar to the one in the hypothetical examples for country 2 or as country 3 in Figure 13.4.

The between-school and within-school residual variable estimates, respectively denoted $\tau_{0}^{2}, \sigma^{2}$ are equal to 1949.09 and 5551.53 .

The percentage of variance explained by the HISEI variable can be computed as:

$$
\begin{aligned}
& 1-\frac{1949.09}{2563.07}=0.24 \text { at the school level and } \\
& 1-\frac{5551.53}{5734.39}=0.03 \text { at the student level. }
\end{aligned}
$$

How can a student level variable explain about 24 per cent of the between-school variance and only 3 per cent of the within-school variance? This mainly reflects the school socio-economic background segregation. Some of the Luxembourg schools are mainly attended by students with high socioeconomic backgrounds while some other schools are mainly attended by students with low socioeconomic backgrounds.

Figure 13.6 provides a graphical explanation of this phenomenon. The between-school variance in any case can be graphically represented by the variability of the school intercepts on the $Y$ axis.

Note that the between-school variance can be obtained by an empty multilevel regression model. In that particular case, the intercept is close to the orthogonal projection of the school performance average on the $Y$ axis, as shown by the black line in Figure 13.6. As explained in the previous section, the difference between the school mean and the intercept results from the application of the shrinkage factor.

Figure 13.6 Graphical representation of the between-school variance reduction

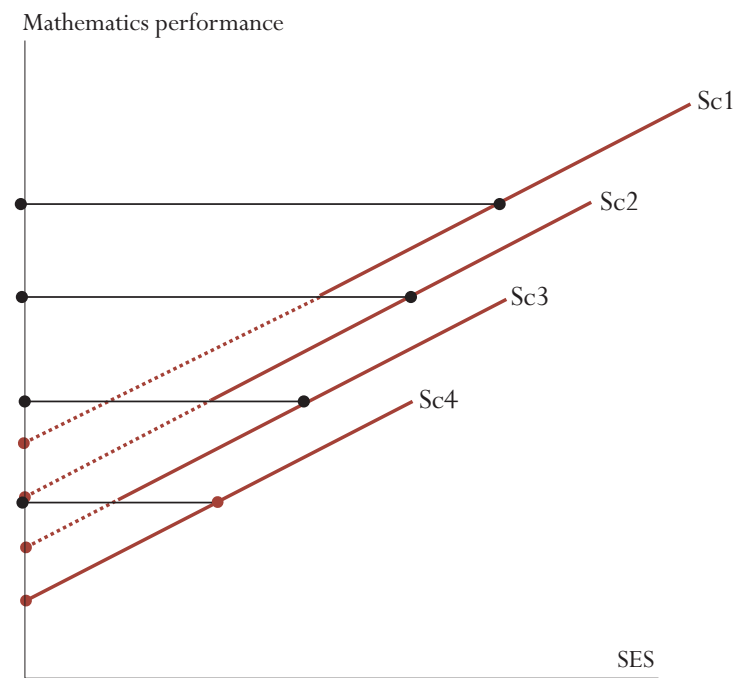


The between-school residual variance can be obtained by the extension of the regression line on the $Y$ axis, as shown by the red discontinuous line in Figure 13.6. As one can see, the range of the black intercepts is larger than the range of the red intercepts.

Broadly speaking, a student level variable will have an impact on the between-school variance if:

- Schools differ in the mean and the range of students in regard with that variable (see countries 2, 3 and 4) in Figure 13.4; and

- The within-school regression coefficient of that variable differs from 0 . The case of country 4 in Figure 13.4 illustrates a case where using the HISEI variable at the student level in the model will not reduce the between-school variance. On the other hand, the introduction of the school socioeconomic intake, i.e. the school HISEI mean, will have in country 4 a substantial impact on the between-school variance.

\section{Example 3}

Example 3 is similar to Example 2, except that HISEI is now considered as a random effect. The SPSS $^{\circledR}$ syntax is presented in Box 13.8. The equation can therefore be written as:

$$
\begin{aligned}
& Y_{i j}=\alpha_{j}+\beta_{1 j}(\text { HISEI })_{i j}+\varepsilon_{i j} \\
& \alpha_{j}=\gamma_{00}+U_{0 j} \\
& \beta_{1 j}=\gamma_{10}+U_{1 j}
\end{aligned}
$$

Box 13.8 - $\operatorname{SPSS}^{\circledR}$ syntax for a multilevel regression model - Example 3

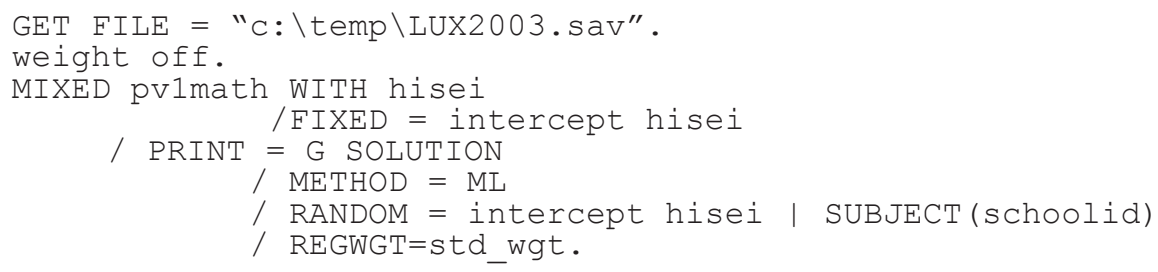

The variable HISEI has been added to the random statement.

The fixed parameter file contains the overall intercept $\gamma_{00}$ and HISEI overall regression coefficient $\gamma_{10}$. Like the school intercepts which are divided into two parts, an overall intercept and a school departure, the within-school regression coefficient is divided into two parts: an overall regression coefficient (the fixed part, denoted $\gamma_{10}$ ) and a school regression coefficient departure (the random part, denoted $U_{1 j}$ ).

The overall intercept and regression coefficient are presented in Table 13.5. The overall intercept is equal to 449.59 and the overall HISEI regression coefficient is equal to 0.89 . As shown by the $t$ statistic and its associated probability, both parameters are significantly different from 0 .

Table 13.5 - Fixed parameter output

\begin{tabular}{c|c|c|c|c|c}
\hline CNT & Effect & Estimate & StdErrPred & tValue & Probt \\
\hline LUX & Intercept & 449.59 & 9.69 & 46.39 & 0.00 \\
\hline LUX & HISEI & 0.89 & 0.11 & 8.17 & 0.00 \\
\hline
\end{tabular}


The random parameter file lists the school departures:

- $U_{0 j}$ from the intercept $\gamma_{00}$, i.e. 449.59 ; and

- $U_{1 j}$ from HISEI regression coefficient $\gamma_{10}$, i.e. 0.89 .

As HISEI is now considered as a random effect, it is meaningless to interpret the school departure from the overall intercept. Table 13.6 presents the school departure from the overall HISEI regression coefficient for the first 13 schools. $^{7}$

Table 13.6 - Random parameter output computed with $\mathbf{S A S}{ }^{\circledR}$

\begin{tabular}{l|l|c|c|c|c|c|c}
\hline CNT & Effect & SCHOOL & Estimate & StdErrPred & DF & tValue & Probt \\
\hline LUX & HISEI & 00001 & 0.22 & 0.31 & 3724 & 0.71 & 0.48 \\
LUX & HISEI & 00002 & 0.04 & 0.26 & 3724 & 0.15 & 0.88 \\
LUX & HISEI & 00003 & 0.29 & 0.26 & 3724 & 1.13 & 0.26 \\
LUX & HISEI & 00004 & -0.51 & 0.29 & 3724 & -1.75 & 0.08 \\
LUX & HISEI & 00005 & -0.08 & 0.25 & 3724 & -0.31 & 0.76 \\
LUX & HISEI & 00006 & 0.07 & 0.28 & 3724 & 0.26 & 0.79 \\
LUX & HISEI & 00007 & -0.04 & 0.29 & 3724 & -0.13 & 0.90 \\
LUX & HISEI & 00008 & -0.13 & 0.27 & 3724 & -0.49 & 0.62 \\
LUX & HISEI & 00009 & -0.29 & 0.25 & 3724 & -1.19 & 0.23 \\
LUX & HISEI & 00010 & -0.17 & 0.26 & 3724 & -0.65 & 0.52 \\
LUX & HISEI & 00011 & 0.07 & 0.34 & 3724 & 0.19 & 0.85 \\
LUX & HISEI & 00012 & -0.04 & 0.28 & 3724 & -0.14 & 0.89 \\
LUX & HISEI & 00013 & 0.82 & 0.22 & 3724 & 3.66 & 0.00 \\
\hline
\end{tabular}

The HISEI regression coefficient for school 00001 is equal to $0.89+0.22=1.11$, but it cannot be considered as significantly different from the overall intercept. Out of the 13 schools presented in Table 13.6, only school 00013 presents a regression coefficient that significantly differs from the overall coefficient, as shown by the $t$ statistics of the probability. The HISEI regression coefficient is equal to $0.89+0.82=1.71$ and as shown by the $t$ statistic or the probability, this within-school regression coefficient is significantly different from the overall regression coefficient.

SPSS now provides three variance estimates:

- The between-school residual variance $\boldsymbol{\tau}_{0}^{2}$, i.e. 2 147.64;

- The within-school residual variance $\sigma^{2}$, i.e. 5 509.34; and

- The variance of HISEI regression coefficients $\boldsymbol{\tau}_{1}^{2}$, i.e. 0.1275 . This is also the variability of the regression coefficient departure.

In comparison with Example 2, the between-school residual variance has slightly increased and the within-school residual variance has slightly decreased. The reduction of the within-school variance is not surprising as the random effect can only better fit the data.

Figure 13.7 helps to understand the increase of the between-school residual variance. The regression coefficient for school 00001 ( $\mathrm{Sc} 1)$ is slightly less steep so that the extension of the regression line will be higher than previously on the $Y$ axis. Further, the regression coefficient is slightly steeper for school 00004 (Sc4), so that the extension of the regression line will be a bit lower on the $Y$ axis. 

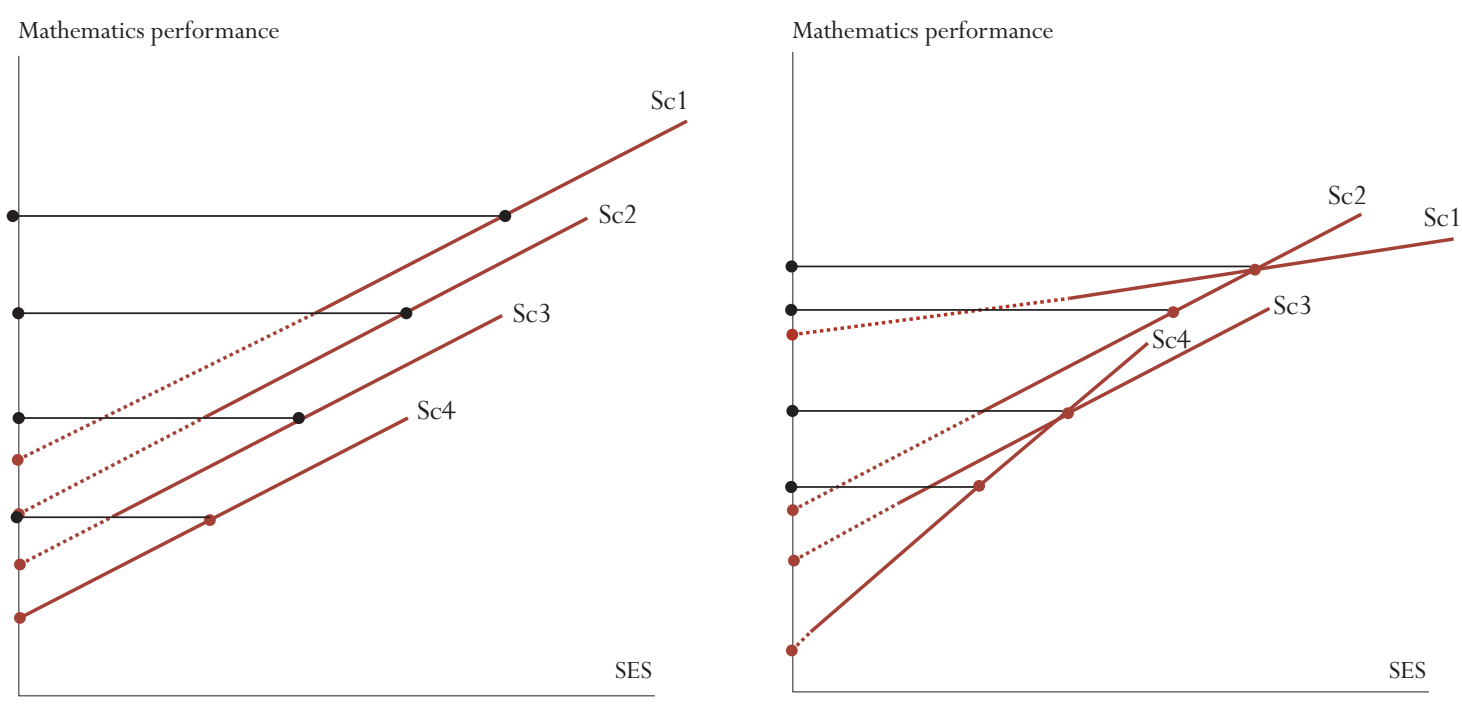

\section{Example 4}

In Example 4, the student gender, denoted ST03Q01 in the PISA database, is added as a fixed factor to the previous model. The equation can be written as:

$$
\begin{aligned}
& Y_{i j}=\alpha_{j}+\beta_{1 j}(\text { HISEI })_{i j}+\beta_{2}(\text { ST03Q01 })_{i j} \\
& \alpha_{j}=\gamma_{00}+U_{0 j} \\
& \beta_{1 j}=\gamma_{10}+U_{1 j}
\end{aligned}
$$

Box 13.9 presents the $\operatorname{SPSS}^{\circledR}$ syntax.

\section{Box 13.9 - SPSS $^{\circledR}$ syntax for a multilevel regression model - Example 4}

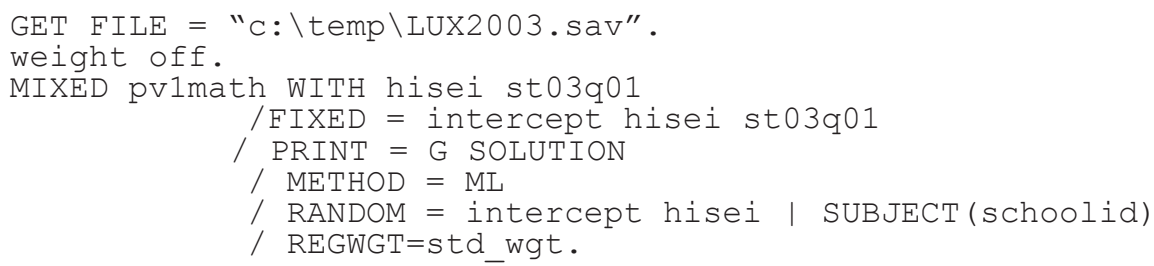

The fixed parameters are respectively equal to 419.68 for the overall intercept, 0.86 for the overall HISEI regression coefficient and 20.7927 for the overall gender coefficient.

The between-school residual variance $\boldsymbol{\tau}_{0}^{2}$ is equal to 2167.41 and the within-school residual variance $\sigma^{2}$ is equal to 5415.34 . Finally, the variance of the school HISEI regression coefficient $\tau_{1}^{2}$ is equal to 0.1313 .

This model explains $1-\frac{2167.41}{2563.07}=15.3$ per cent of the between-school variance and $1-\frac{5415.34}{5734.39}=5.6$ per cent of the within-school variance.

The gender regression coefficient of 20.8 reflects the expected gender difference within any school, after controlling for HISEI. 
The expected within-school gender difference can greatly differ from the overall gender difference, especially in a highly tracked system. It appears that girls are more likely to attend an academic track while boys are more likely to attend a vocational track. The linear regression coefficient of gender on the student performance does not take into account this differential attendance. If the different tracks are organised by different schools, as in Germany for instance, a multilevel regression model will take this differential attendance into account, so that the gender multilevel regression coefficient will substantially be different from the linear regression coefficient. The table below provides the linear and multilevel regression coefficients for gender on the German PISA 2003 data.

At the population level, boys outperform girls by 8.9 in mathematics while girls outperform boys by 42.1 in reading. But within a particular school, the expected differences in mathematics and in reading are respectively equal to 30.7 and -19.3 .

\section{Gender differences in Germany}

\begin{tabular}{l|c|c} 
& Mathematics & Reading \\
\hline Simple linear regression coefficient & 8.9 & -42.1 \\
\hline Multilevel regression coefficient & 30.7 & -19.3 \\
\hline
\end{tabular}

Gender can also be considered as a random factor. The equation can therefore be written as:

$Y_{i j}=\alpha_{j}+\beta_{1 j}(H I S E I)_{i j}+\beta_{2 j}(S T 03 Q 01)_{i j}$

$\alpha_{j}=\gamma_{00}+U_{0 j}$

$\beta_{1 j}=\gamma_{10}+U_{1 j}$

$\boldsymbol{\beta}_{2 j}=\gamma_{20}+U_{2 j}$

Box 13.11 presents the variance estimate of the random parameters as well as the regression coefficient estimates of the fixed parts of the model.

Box 13.11 - SPSS $^{\circledR}$ output - Example 4

Estimates of Fixed Effects ${ }^{a, b}$

\begin{tabular}{l|r|r|r|r|r} 
Parameter & \multicolumn{1}{c|}{ Estimate } & \multicolumn{1}{c|}{ Std. Error } & \multicolumn{1}{c}{$\mathrm{df}$} & \multicolumn{1}{c}{$\mathrm{t}$} & \multicolumn{1}{c}{ Sig. } \\
\hline Intercept & 419.3613485 & 10.0172881 & 43.829 & 41.864 & .000 \\
\hline HISEI & .8606827 & .1097952 & 38.918 & 7.839 & .000 \\
\hline ST03Q01 & 21.0238222 & 3.1530028 & 31.424 & 6.668 & .000 \\
\hline
\end{tabular}

Estimates of Covariance Parameters ${ }^{a, b}$

\begin{tabular}{ll|r|r}
\multicolumn{1}{l|}{ Parameter } & & Estimate & \multicolumn{1}{c}{ Std. Error } \\
\hline Residual & & 5400.8730832 & 125.3934221 \\
\hline Intercept [subject $=$ SCHOOLID] & Variance & 1904.7788816 & 613.6594064 \\
\hline HISEI [subject $=$ SCHOOLID] & Variance & .1348145 & .0714020 \\
\hline ST03Q01 [subject $=$ SCHOOLID] & Variance & 70.5719428 & 56.7028751 \\
\hline
\end{tabular}

a. Dependent variable: plausible value in mathematics.

b. Residual is weighted by std_wgt. 
As shown in Box 13.11, the variability of $U_{2 j}$, i.e. the school departure on the gender regression coefficient is quite large. This indicates that the gender differences vary from one school to another.

\section{Example 5}

The last equation in Example 4 wasY $_{i j}=\alpha_{j}+\beta_{1 j}(H I S E I)_{i j}+\beta_{2 j}(\text { STO3QO1) })_{i j}$. This equation mainly models the student performance variability within schools by introducing student level predictors. However, due to the segregation effect, these student level predictors can explain some of the between-school variance.

It is also possible to introduce a predictor school level variable. Suppose that one is interested in the effect of the school type on the school mean performance. The equation can be written as:

$$
\begin{aligned}
& Y=\alpha_{j}+\beta_{1 j}(\text { HISEI })_{i j}+\beta_{2 j}(\text { STO3QO1 })_{i j}+\varepsilon_{i j} \\
& \alpha_{j}=\gamma_{00}+\gamma_{01}(\text { SCHLTYPE })_{j}+U_{0 j} \\
& \beta_{1 j}=\gamma_{10}+U_{1 j} \\
& \beta_{2 j}=\gamma_{20}+U_{2 j}
\end{aligned}
$$

In other words, as the school type variable is identical for all students within a particular school, this variable will only have an effect on the school intercepts. Given the socio-economic background and the gender composition of the schools, does the school type explain why some schools perform better than expected and why some schools perform at a lower level than expected?

The $\operatorname{SPSS}^{\circledR}$ syntax is presented in Box 13.12 .

Box 13.12 - $\operatorname{SPSS}^{\circledR}$ syntax for a multilevel regression model - Example 5 (1)

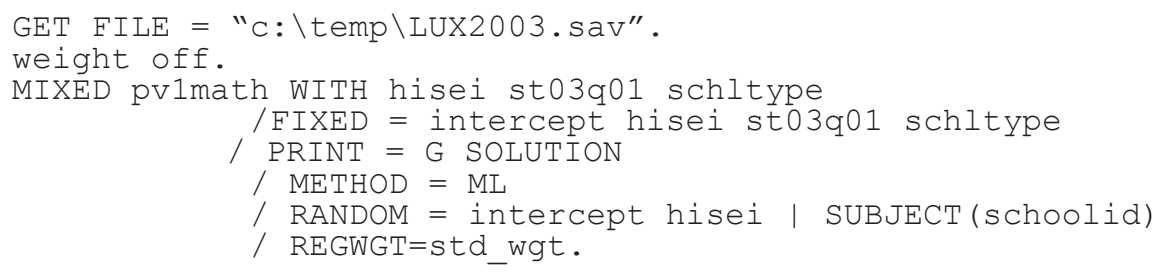

Table 13.7 presents the results for the fixed parameters.

Table 13.7 - Fixed parameters - Example 5

\begin{tabular}{c|c|c|c|c|c|c}
\hline CNT & Effect & Estimate & StdErrPred & DF & tValue & Probt \\
\hline LUX & Intercept & 320.47 & 66.69 & 27 & 4.81 & 0.00 \\
\hline LUX & HISEI & 0.86 & 0.11 & 28 & 7.84 & 0.00 \\
\hline LUX & ST03Q01 & 20.69 & 2.59 & 3723 & 7.98 & 0.00 \\
\hline LUX & SCHLTYPE & 35.14 & 23.36 & 3723 & 1.50 & 0.13 \\
\hline
\end{tabular}

As shown by Table 13.7, the school type variable is not significant. In other words, it cannot be stated that government-dependent private schools differ from public schools once the student socio-economic background and the student gender is controlled. 


\section{Example 6}

The model can finally be extended by trying to understand why the school HISEI and ST03Q01 regression coefficients vary. Two hypotheses to test are:

- The HISEI regression coefficients differ between public school and private government: dependent schools, and

- The ST03Q01 regression coefficients is related to the percentage of boys and girls in the school. The equation can be written as:

$$
\begin{aligned}
& Y_{i j}=\alpha_{j}+\beta_{1 j}(\text { HISEI })_{i j}+\beta_{2 j}(\text { ST03Q01 })_{i j}+\varepsilon_{i j} \\
& \alpha_{j}=\gamma_{00}+\gamma_{01}(\text { SCHLTYPE })_{j}+U_{0 j} \\
& \beta_{1 j}=\gamma_{10}+\gamma_{11}(\text { SCHLTYPE })_{j}+U_{1 j} \\
& \beta_{2 j}=\gamma_{20}+\gamma_{21}(\text { PCGIRLS })_{j}+U_{2 j}
\end{aligned}
$$

Box 13.13 presents the SPSS ${ }^{\circledR}$ syntax for running this model. Testing whether the HISEI regression coefficients differ according to the school type is similar to testing the interaction between the school type and the HISEI regression coefficients. Therefore, in SPSS, the term "hisei * schltype" has to be added in the FIXED statement, as well as "st03q01*pcgirls". Please note that the model statement following the procedure name lists "schltype" and "pcgirls" without the interaction terms.

\section{Box 13.13 — SPSS $^{\circledR}$ syntax for a multilevel regression model - Example 5 (2)}

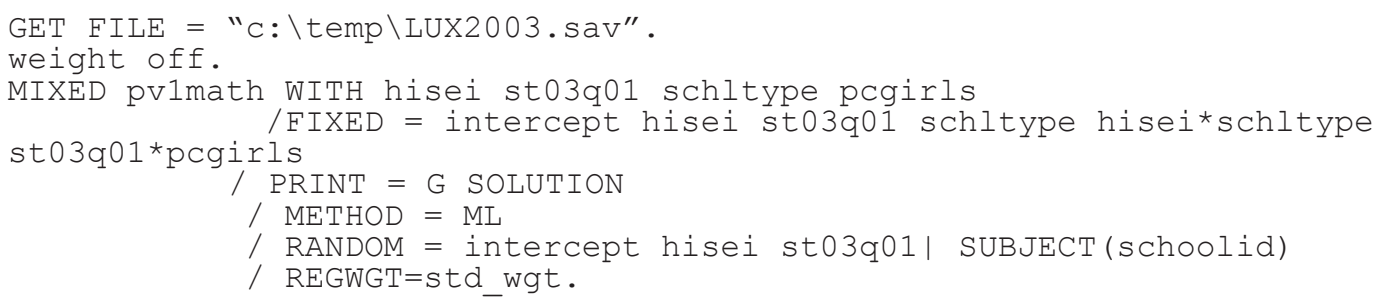

Box 13.14 - SPSS $^{\circledR}$ output - Example 5

\section{Estimates of Fixed Effects $s^{\mathrm{a}, \mathrm{b}}$}

\begin{tabular}{l|r|r|r|r|r} 
Parameter & \multicolumn{1}{|c|}{ Estimate } & \multicolumn{1}{c|}{ Std. Error } & \multicolumn{1}{c}{$\mathrm{df}$} & $\mathrm{t}$ & \multicolumn{1}{c}{ Sig. } \\
\hline Intercept & 291.4022663 & 71.2240565 & 39.885 & 4.091 & .000 \\
\hline HISEI & 1.8607710 & .9019687 & 51.684 & 2.063 & .044 \\
\hline ST03Q01 & 19.8852339 & 11.7315332 & 50.628 & 1.695 & .096 \\
\hline SCHLTYPE & 45.1271348 & 24.8610903 & 39.203 & 1.815 & .077 \\
\hline HISEI * SCHLTYPE & -.3504893 & .3137546 & 50.175 & -1.117 & .269 \\
\hline ST03Q01 * PCGIRLS & 2.6190012 & 24.8681643 & 49.929 & .105 & .917 \\
\hline
\end{tabular}

Estimates of Covariance Parameters ${ }^{a, b}$

\begin{tabular}{ll|r|r} 
Parameter & & \multicolumn{1}{|c}{ Estimate } & \multicolumn{1}{c}{ Std. Error } \\
\hline Residual & & 5397.4444256 & 125.3180631 \\
\hline Intercept [subject = SCHOOLID] & Variance & 1757.3290219 & 578.2565538 \\
\hline HISEI [subject = SCHOOLID] & Variance & .1426724 & .0735291 \\
\hline ST03Q01 [subject = SCHOOLID] & Variance & 71.0821703 & 56.3930823 \\
\hline
\end{tabular}

a. Dependent variable: plausible value in mathematics.

b. Residual is weighted by std_wgt. 
Table 13.8 presents the fixed parameters in relation to the equation. As shown in the SPSS ${ }^{\circledR}$ output in Box 13.14, the random HISEI regression coefficient is not significantly associated with the school type.

Table 13.8 - Fixed parameter estimates - Example 6

\begin{tabular}{l|c|c}
\hline Effect & $\begin{array}{c}\text { Coefficient } \\
\text { estimate }\end{array}$ & Coefficient \\
\hline Intercept & 291.40 & $\gamma_{00}$ \\
\hline HISEI & 1.86 & $\gamma_{10}$ \\
\hline ST03Q01 & 19.88 & $\gamma_{20}$ \\
\hline SCHLTYPE & 45.12 & $\gamma_{01}$ \\
\hline HISEI*SCHLTYPE & -0.35 & $\gamma_{11}$ \\
\hline ST03Q01*PCGIRLS & 2.62 & $\gamma_{21}$ \\
\hline
\end{tabular}

Table 13.9 - Random parameter variance estimates - Example 6

\begin{tabular}{l|c|c} 
Effect & Variance estimate & Coefficient \\
\hline Intercept & 1757.37 & $U_{0 j}$ \\
\hline HISEI & 0.1427 & $U_{1 j}$ \\
\hline ST03Q01 & 71.0154 & $U_{2 j}$ \\
\hline Residual & 5397.46 & $\boldsymbol{\varepsilon}_{i j}$ \\
\hline
\end{tabular}

As shown by the reported probability, both null hypotheses have to be accepted, i.e. the school type is not associated with the HISEI slopes and the within-school gender difference is not associated with the percentage of girls in the school.

\section{LIMITATIONS OF THE MULTILEVEL MODEL IN THE PISA CONTEXT}

This section aims to alert potential PISA data users of the limitations or the dangers of such models in the PISA context.

Such models are designed to decompose the student variance into:

- The between-school variance,

- The within-school variance, and

- The within class variance.

As PISA draws, per participating school, a random sample of an age population across grades and across classes, it allows the decomposition of the variance into two levels: a between-school variance and a within-school variance. Further, the overall variance is expected to be larger with an age sample than with a grade sample, unless the age population is attending a single grade, as in Iceland or Japan.

To allow meaningful international comparisons, these types of indicators require a common definition for a school and for a class. While there are no major issues on what a student is, there are from one country to another important differences between what a school is and what a class is.

International surveys in education are primarily interested in the student sample and therefore one might consider the school sample as a necessary step to draw an efficient sample of students that 
minimizes the cost of testing. In this context, the definition of what a school is or what a class is, does not present any major issues. However, the increasing importance and popularity of multilevel analyses calls for more attention on these definition issues.

PISA 2000 and PISA 2003 do not give a detailed definition of a school. The emphasis in the sampling procedures was on developing a list of units that would guarantee full coverage of the enrolled 15year-old population and that would additionally give acceptable response rates. Once a school was selected, it also had to be practical to sample 35 students or so from that school to assess them. Thus, the school frame was constructed with issues of student coverage and practical implementation of PISA administration in mind, rather than analytic considerations. Therefore, in the PISA databases, it is possible that the school identification represents different educational institutions that may not be comparable without any restriction. For instance, in some PISA countries, schools are defined as administrative units that may consist of several buildings not necessarily located close together. Other countries used the building as the school sampling unit and finally, a few countries defined a school as a track within a particular building. It is likely that the larger these aggregates are, the smaller the differences between these aggregates will be and the larger the differences within these aggregates will be. In this context, one would expect to observe high intra-class correlations in these countries and a nonsignificant withinschool regression coefficient for the student socio-economic background (Kirsch et al., 2002).

Besides this problem of an international definition of a school, data users have to be aware of the following issues:

- The choice of a school definition in a particular country may be dictated by the availability of the data. Indeed, the national centres have to include a measure of size of the 15 -year-old population in the school sample frame (see Chapter 2). This information may be available at the administrative unit level, but not at the building level. In federal countries that count several educational systems, the available data might differ from one system to the other, so that the concept of a school might differ even within a particular country.

- For practical or operational reasons, the concept of schools might differ between two PISA data collections. For instance, some countries used the administrative units in the PISA 2000 school sample frame and the building units in the PISA 2003 school sample frame. Such changes were implemented to increase the school participation rate. These conceptual changes will influence the results of any variance decomposition and might also affect the outcomes of multilevel models. Moving from an administrative definition to a building definition will increase the intraclass correlation and should decrease the slope of the within-school regression coefficient. If such changes occur in a country, it is strongly advised not to compute any trends on variance decomposition or multilevel regressions.

As this example shows, multilevel analyses and variance decomposition analyses need to be interpreted in the light of:

- The structure of the educational systems; and

- The school definition used in the school sample frame.

Under the limitations provided in this section, multilevel regression analyses are certainly suitable and appropriate to describe how students are assigned to schools and what the major criteria are for such assignment. However, 10 or even 20 student and school variables will never be able to 
$\backsim$ model the complexity of an educational system. Further, PISA is measuring a cumulative process of about ten years of schooling. What we are doing today can certainly not explain what we are today. Consequently, the pedagogical practices and the school environment in which 15-year-olds are currently learning is unable to fully explain how these students perform today. In this context, policy recommendations should be made and interpreted with caution.

\section{CONCLUSIONS}

This chapter firstly describes the concept of multilevel analyses and how to perform such models with SPSS ${ }^{\circledR}$. It starts with the simplest model, denoted the empty model, and then progressively adds complexity by adding variables. Finally, in the PISA context, important methodological issues that limit the international comparability of the results have been discussed.

\section{Notes}

1. For consistency with the literature on multilevel regression, subscripts $i$ and $j$ have been inverted in comparison with Chapter 2.

2. Multiplying the full student weight W_FSTUWT with the variable CNTFAC2 produces the same weights (COMPUTE std_wgt $=\mathrm{w}_{-}$fstuwt* ${ }^{*}$ ntfac2) as the syntax in Box 13.2. But the resulting standardised weights should only be used for multi-level models based on variables without any missing values. When estimating multi-level models including variables with missing values different standardised weight should be computed after deleting all cases with missing values.

3. See also Table 4.4 in Chapter 4.

4. Note that the results obtained from the SPSS ${ }^{\circledR}$ syntaxes in this chapter sometimes may differ from the examples in the text of this chapter, which were estimated using the SAS ${ }^{\circledR}$ program. These minor deviations are due to differences in the algorithms used by different statistical software packages.

5. A correlation matrix computed with the pairwise deletion option can however be used as input for a linear regression analysis.

6. This shrinkage factor has to be associated to the expected school mean square in an ANOVA model. Indeed, $E\left(M S_{\text {school }}\right)=n_{j} \sigma_{\text {between-school }}^{2}+\sigma_{\text {within-school. }}^{2}$

7. Note that this output file is not available with $\operatorname{SPSS}^{\circledR}$; the results are presented here for the purpose of illustration and were computed with $\mathrm{SAS}^{\circledR}$. 


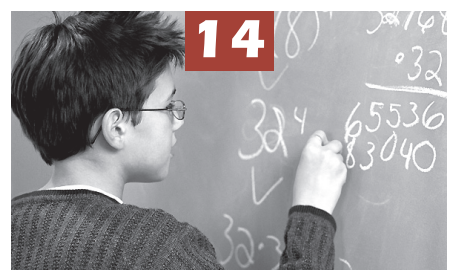

\section{Other Statistical Issues}

Introduction

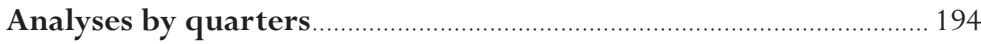

The concepts of relative risk and attributable risk ....................... 198

Instability of the relative and attributable risks .............................. 200

Computation of the relative risk and attributable risk ................. 201

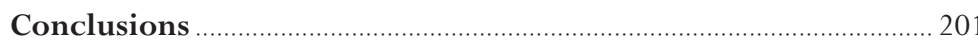




\section{INTRODUCTION}

The PISA 2000 and the PISA 2003 initial reports included descriptions of the relationship between questionnaire indices and student performance by dividing the questionnaire indices into quarters and then reporting the mean achievement per quarter. The PISA reports also include the statistical concepts of relative risk and attributable risk. This chapter is devoted to these two specific issues.

\section{ANALYSES BY QUARTERS}

As described in Chapter 4, the indices derived from the questionnaire data were generated with the Rasch model and students' estimates were reported with the WLEs. As previously mentioned, a WLE individual's estimate is a discontinuous variable.

Table 14.1 presents the distribution of the questionnaire index interest in and enjoyment of mathematics from the German PISA 2003 data set. This table clearly shows the discontinuous character of the variable.

To divide a questionnaire index into quarters, the $25^{\text {th }}, 50^{\text {th }}$, and $75^{\text {th }}$ percentiles have to be computed. These percentiles are respectively $-0.6369,0.029$, and 0.973 for the index of interest in and enjoyment of mathematics for Germany.

There are two possible recoding procedures: lower versus equal or greater and equal or lower versus greater.

The $\mathrm{SPSS}^{\circledR}$ syntax is presented in Box 14.1.

\section{Box 14.1 Two SPSS $^{\circledR}$ syntax procedures for the recoding into quarters}

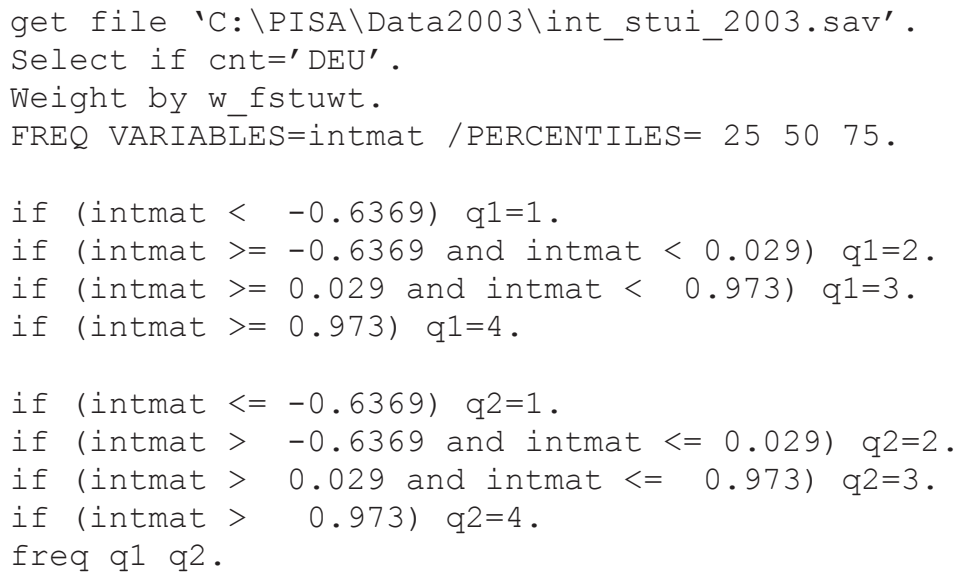

Depending on the procedure adopted, the percentages of students in the bottom quarter, second quarter, third quarter, and top quarter are respectively equal to 24.88, 21.39, 27.80 and 25.93 or $34.53,21.60,25.33$ and 18.54 .

Neither of these two procedures generate quarters that precisely include 25 per cent of the students. Since the percentages of students in each quarter can vary among countries, no international comparisons can be performed. 
Table 14.1 Distribution of the questionnaire index interest in and enjoyment of mathematics for Germany

\begin{tabular}{|c|c|c|c|c|c|}
\hline WLE & Percentage & $\begin{array}{l}\text { Cumulative } \\
\text { percentage }\end{array}$ & WLE & Percentage & $\begin{array}{l}\text { Cumulative } \\
\text { percentage }\end{array}$ \\
\hline-1.783 & 10.20 & 10.20 & 0.477 & 0.10 & 64.30 \\
\hline-1.733 & 0.02 & 10.23 & 0.643 & 0.10 & 64.40 \\
\hline-1.700 & 0.02 & 10.25 & 0.643 & 9.53 & 73.93 \\
\hline-1.469 & 0.02 & 10.27 & 0.869 & 0.03 & 73.96 \\
\hline-1.258 & 7.53 & 17.80 & 0.912 & 0.04 & 74.00 \\
\hline-1.179 & 0.02 & 17.82 & 0.925 & 0.05 & 74.05 \\
\hline-1.147 & 0.02 & 17.85 & 0.940 & 0.02 & 74.07 \\
\hline-1.077 & 0.03 & 17.88 & 0.973 & 7.39 & 81.46 \\
\hline-0.971 & 0.08 & 17.95 & 1.044 & 0.03 & 81.49 \\
\hline-0.929 & 6.77 & 24.73 & 1.146 & 0.03 & 81.52 \\
\hline-0.739 & 0.15 & 24.88 & 1.299 & 5.27 & 86.79 \\
\hline-0.637 & 9.66 & 34.53 & 1.338 & 0.02 & 86.81 \\
\hline-0.619 & 0.13 & 34.66 & 1.346 & 0.04 & 86.85 \\
\hline-0.370 & 0.02 & 34.68 & 1.464 & 0.02 & 86.87 \\
\hline-0.335 & 0.07 & 34.74 & 1.568 & 0.04 & 86.91 \\
\hline-0.319 & 11.37 & 46.11 & 1.587 & 4.58 & 91.49 \\
\hline-0.250 & 0.01 & 46.13 & 1.702 & 0.01 & 91.51 \\
\hline-0.160 & 0.10 & 46.22 & 1.761 & 0.02 & 91.53 \\
\hline-0.045 & 0.05 & 46.27 & 1.792 & 0.04 & 91.57 \\
\hline 0.029 & 9.86 & 56.13 & 1.817 & 0.05 & 91.62 \\
\hline 0.057 & 0.04 & 56.17 & 1.827 & 0.03 & 91.64 \\
\hline 0.068 & 0.08 & 56.25 & 1.891 & 4.72 & 96.37 \\
\hline 0.132 & 0.07 & 56.32 & 2.091 & 0.04 & 96.41 \\
\hline 0.229 & 0.06 & 56.39 & 2.119 & 0.02 & 96.43 \\
\hline 0.300 & 0.02 & 56.41 & 2.161 & 0.07 & 96.50 \\
\hline 0.345 & 7.75 & 64.15 & 2.335 & 0.04 & 96.54 \\
\hline 0.448 & 0.02 & 64.17 & 2.373 & 3.46 & 100.00 \\
\hline 0.462 & 0.02 & 64.20 & & & \\
\hline
\end{tabular}

It was therefore necessary to distribute the students with a WLE equal to one of the 3 percentiles into the two respective adjacent quarters. For instance, 7.39 per cent of the students get a score equal to percentile 75 . As 74.07 per cent of the students get a lower score, it is necessary to sample 0.93 per cent of the students with a score equal to percentile 75 and allocate them to the third quarter. The remaining 6.46 per cent will be allocated to the fourth quarter.

This random subsampling process is implemented by adding a small random variable to the questionnaire index. That random noise will generate more categories and therefore the three new percentiles will be able to divide the index variable into quarters that exactly include 25 per cent of 
the students. Box 14.2 presents the SPSS $^{\circledR}$ syntax for the addition of a random variable, as well as the computation of the percentiles and the recoding into quarters.

Box 14.2 - SPSS $^{\circledR}$ syntax for the questionnaire indices recoding into quarters

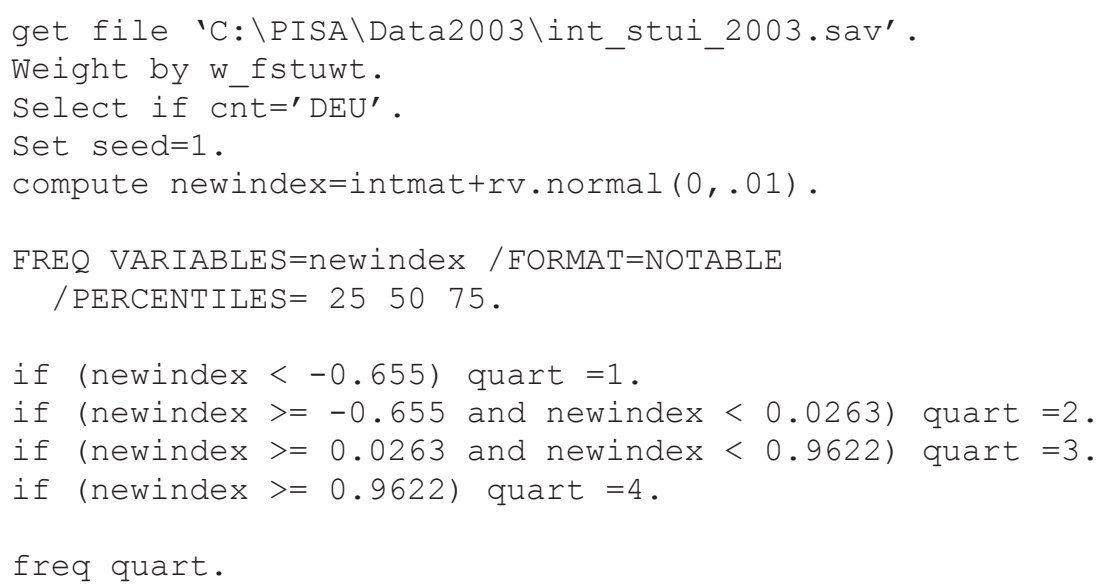

The outcomes of the FREQUENCY procedure will demonstrate that 25 per cent are allocated to each quarter.

This random allocation of some parts of the population to one of the four quarters adds an error component to the standard error. Indeed, in our example, the composition of the 0.93 per cent of the students allocated to the third quarter and the composition of the remaining 6.46 per cent allocated to the fourth quarter might differ between two runs of the procedure (unless the seed is set to a certain integer, like in Box 14.2).

To account for this new error component, the statistical approach adopted for the analyses of plausible values can be implemented. It will therefore consist of:

- Computing for each student a set of five plausible quarters;

- Per plausible quarter, computing the required statistic and its respective sampling variance by using the final and 80 replicate weights;

- Averaging the five estimates and their respective sampling variances;

- Computing the imputation variance; and

- Combining the sampling variance and the imputation variance to obtain the final error variance.

If the dependent variable is a set of plausible values, the procedure described in Chapter 6 will be used, except that each plausible value will be analyzed with a different plausible quarter. Box 14.3 presents the SPSS ${ }^{\circledR}$ syntax for the computation of the average mathematics performance per quarter of any questionnaire derived index.

The different steps of this procedure are:

1. From the initial questionnaire index, five new variables are created by adding a random number;

2. For each new variable, the $25^{\text {th }}, 50^{\text {th }}$ and $75^{\text {th }}$ percentiles are computed and then imported in the temporary data file; 
Box 14.3 - SPSS $^{\circledR}$ syntax for the computation of the average mathematics performance per quarter of a questionnaire derived index

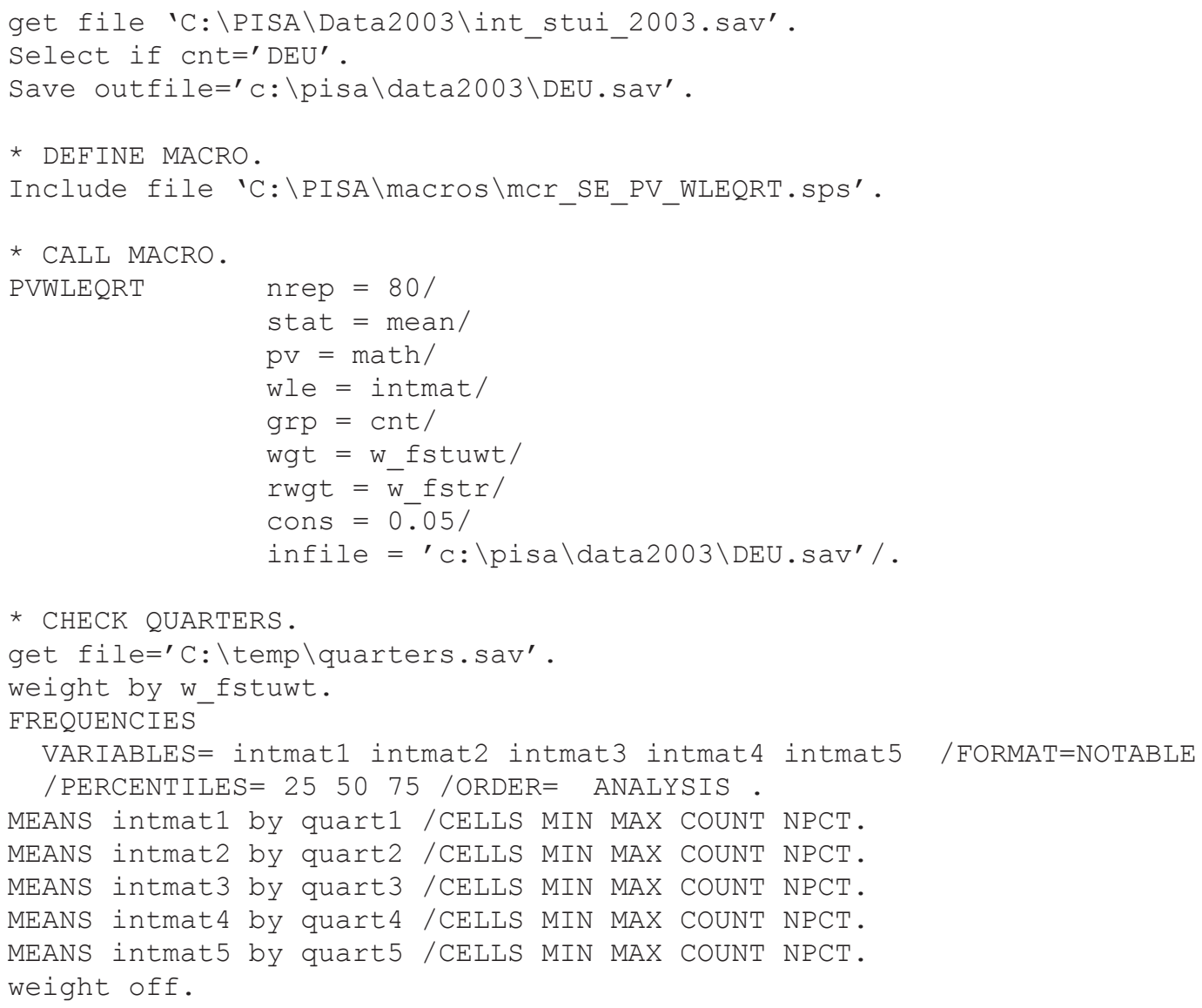

3. The five new variables are compared with their respective percentiles and the quarter allocations are saved in five categorical variables (these 10 new variables are saved in the temporary data file 'C: \temp\quarters.sav');

4. The statistic is computed for each plausible value of mathematics performance by one of the five new categorical variables; and

5. The final estimate and the final standard error are computed.

The results from these steps are presented in Table 14.2.

Table 14.2 Mean scores and standard errors on the mathematics scale for each quarter of index of interest in and enjoyment of mathematics

\begin{tabular}{c|c|c|c}
\hline CNT & quart (INTMAT) & stat (MATH) & SE \\
\hline DEU & 1 & 493 & 4.90 \\
\hline DEU & 2 & 511 & 4.01 \\
\hline DEU & 3 & 520 & 4.67 \\
\hline DEU & 4 & 524 & 4.69 \\
\hline
\end{tabular}




\section{THE CONCEPTS OF RELATIVE RISIK AND ATTRIBUTABLE RISK}

\section{Relative risk}

The notion of relative risk is a measure of association between an antecedent factor and an outcome factor (Cornfield, 1951). The relative risk is simply the ratio of two risks, i.e. the risk of observing the outcome when the antecedent is present, and the risk of observing the outcome when the antecedent is not present. Table 14.3 presents the notation that will be used.

Table 14.3 - Labels used in a two-way table

\begin{tabular}{c|c|c|c|c}
\hline \multicolumn{2}{c|}{} & \multicolumn{3}{|c}{ Outcome measure } \\
\cline { 2 - 5 } \multicolumn{1}{c|}{} & Yes & $P_{11}$ & No & Total \\
\hline \multirow{3}{*}{ Antecedent measure } & No & $P_{21}$ & $P_{12}$ & $P_{1 .}$ \\
\cline { 2 - 5 } & Total & $P_{.1}$ & $P_{22}$ & $P_{2 .}$ \\
\cline { 2 - 5 } & & & $P_{. .}$ \\
\hline
\end{tabular}

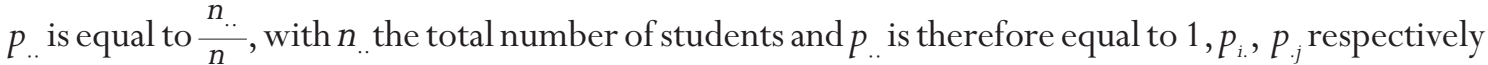
represent the marginal probabilities for each row and for each column. The marginal probabilities are equal to the marginal frequencies divided by the total number of students. Finally, the $p_{i j}$ values represent the probabilities for each cell and are equal to the number of observations in a particular cell divided by the total number of observations.

In this document, the conventions for the two-way table will be the following:

- The rows represent the antecedent factor with:

- The first row for having the antecedent; and

- The second row for "not having the antecedent".

- The columns represent the outcome with:

- The first column for having the outcome; and

- The second column for not having the outcome.

In these conditions, the relative risk is equal to:

$$
R R=\frac{\left(p_{11} / p_{1 .}\right)}{\left(p_{21} / p_{2}\right)}
$$

Let us suppose that a psychologist wants to analyse the risk of a student repeating a grade if the parents recently divorced. The psychologist draws a simple random sample of students of grade 10. In this particular example, the outcome variable is present if the child is repeating grade 10 and the antecedent factor is considered present if the student's parents divorced in the past two years. The results he obtained are presented in Table 14.4 and in Table 14.5. 
Table 14.4 Distribution of $\mathbf{1 0 0}$ students by marital status of the parents and grade repetition

\begin{tabular}{l|c|c|c}
\hline & Repeat the grade & Not repeat the grade & Total \\
\hline Parents divorced & 10 & 10 & 20 \\
\hline Parents not divorced & 5 & 75 & 80 \\
\hline Total & 15 & 85 & 100 \\
\hline
\end{tabular}

Table 14.5 - Probabilities by marital status of the parents and grade repetition

\begin{tabular}{l|c|c|c}
\hline & Repeat the grade & Not repeat the grade & Total \\
\hline Parents divorced & 0.10 & 0.10 & 0.20 \\
\hline Parents not divorced & 0.05 & 0.75 & 0.80 \\
\hline Total & 0.15 & 0.85 & 1.00 \\
\hline
\end{tabular}

The relative risk is therefore equal to:

$$
R R=\frac{\left(p_{11} / p_{1 .}\right)}{\left(p_{21} / p_{2 .}\right)}=\frac{(0.10 / 0.20)}{(0.05 / 0.80)}=\frac{0.5}{0.0625}=8
$$

This means that the probability of repeating grade 10 is eight times larger if the parents recently divorced than if they had not recently divorced.

\section{Attributable risk}

The attributable risk is equal to:

$$
A R=\frac{\left(p_{11} p_{22}\right)-\left(p_{12} p_{21}\right)}{\left(p_{11} p_{2}\right)}
$$

In the previous example, the attributable risk is equal to:

$$
A R=\frac{\left(p_{11} p_{22}\right)-\left(p_{12} p_{21}\right)}{\left(p_{1} p_{2}\right)}=\frac{(0.10 \times 0.75)-(0.10 \times 0.05)}{(0.15 \times 0.80)}=0.583
$$

The attributable risk is interpreted as follows. If the risk factor could be eliminated, then the rate of occurrence of the outcome characteristic in the population would be reduced by this coefficient. With the next version of the formula, the meaning of the attributable risk, i.e. a reduction of the outcome if the risk factor disappears, is more obvious.

$$
A R=\frac{\left(p_{.1}\right)-\left(p_{21} / p_{2 .}\right)}{\left(p_{.1}\right)}
$$

The expression $p_{1}$ represents the proportion of children in the whole sample with the outcome. The expression $\left(p_{21} / p_{2}\right.$. $)$ represents the proportion of children who are not at risk, but nevertheless suffer from the outcome. The difference of these two proportions provides the absolute reduction if the risk was eliminated. Dividing this difference by the first expression transforms this absolute reduction into a relative reduction or a reduction expressed as a percentage. 
These two formulae give the same coefficient:

$$
A R=\frac{\left(p_{.1}\right)-\left(p_{21} / p_{2 .}\right)}{\left(p_{.1}\right)}=\frac{(0.15)-(0.05 / 0.80)}{(0.15)}=0.583
$$

To express this result as a percentage, the coefficient needs to be multiplied by 100 .

\section{INSTABILITY OF THE RELATIVE AND ATTRIBUTABLE RISIKS}

The relative risk and the attributable risk were developed for dichotomous variables. More and more often, these two coefficients are extended and are used with continuous variables. To apply the coefficients to continuous variables, a cutpoint for each variable needs to be set and the continuous variables need to be dichotomised.

It is important to recognise that when applied to dichotomised variables, the computed values of the relative risk and the attributable risk will depend on the value of the chosen cutpoint.

To demonstrate the influence of the cutpoint on the relative and attributable risks, two random variables were generated with a correlation of 0.30 . These two variables were then transformed into dichotomous variables by using respectively the $10^{\text {th }}, 15^{\text {th }}, 20^{\text {th }}, 25^{\text {th }}$ and $30^{\text {th }}$ percentiles as cutpoints. Table 14.6 presents the relative risk and the attributable risk for a range of choices for the cutpoints.

Table 14.6 - Relative risk and attributable risk for different cutpoints

\begin{tabular}{c|c|c} 
Percentile & Relative risk & Attributable risk \\
\hline 10 & 2.64 & 0.13 \\
\hline 15 & 2.32 & 0.16 \\
\hline 20 & 1.90 & 0.15 \\
\hline 25 & 1.73 & 0.15 \\
\hline 30 & 1.63 & 0.15 \\
\hline
\end{tabular}

Table 14.6 shows that the relative risk and, to a lesser extent, the attributable risk coefficients are dependent on the setting of the cutpoints, and therefore the interpretation of the value needs to be made in the light of this observation.

Such a comparison of the relative and attributable risks was computed for the PISA data to identify the changes depending on the cutpoint location. The antecedent factor was the mother's educational level and the outcome variable was reading achievement. Low reading achievement (having the outcome) was successively defined within countries as being below the $10^{\text {th }}, 15^{\text {th }}, 20^{\text {th }}, 25^{\text {th }} 30^{\text {th }}$ and $35^{\text {th }}$ percentiles.

The relative risks for these different cutpoints are respectively on average (across OECD countries) equal to $2.20,1.92,1.75,1.62,1.53$, and 1.46 . The attributable risks are equal to $0.25,0.21,0.19$, $0.17,0.15$, and 0.14 , respectively.

Nevertheless, the correlations between the different relative risks and attributable risks are rather high, as shown in Table 14.7 


\begin{tabular}{c|c|c} 
& RR & AR \\
\hline P15 & 0.96 & 0.98 \\
\hline P20 & 0.93 & 0.97 \\
\hline P25 & 0.92 & 0.96 \\
\hline P30 & 0.90 & 0.94 \\
\hline P35 & 0.87 & 0.92 \\
\hline
\end{tabular}

In PISA, it was decided to use the $25^{\text {th }}$ percentile as the cutpoint for continuous variables when calculating relative and attributable risks.

\section{COMPUTATION OF THE RELATIVE RISK AND ATTRIBUTABLE RISIK}

Depending on the variables involved in the computation of the relative risk and attributable risk, the procedure might differ. Indeed, these two statistical concepts require as input two dichotomous variables, such as gender (ST03Q01).

However, most of the variables in the PISA data bases are not dichotomous; they are categorical or continuous variables.

The recoding of a categorical into a dichotomous variable does not raise special issues. From a theoretical point of view, the purpose of the comparison needs to be decided upon, and the recoding will follow. For instance, in PISA 2000, the education levels of the parents are reported by using the ISCED classification (OECD, 1999b). If the contrast is on the distinction between tertiary versus non tertiary education, then the categorical variable can be recoded into a dichotomous variable. Students whose parents do not have a tertiary qualification will be considered at risk.

Numerical variables also have to be recoded into dichotomous variables. As stated earlier, the OECD has decided to divide numerical variables based on the $25^{\text {th }}$ percentile.

In the PISA 2000 and PISA 2003 databases, all numerical variables, except the performance scales, are discontinuous variables. To ensure that the $25^{\text {th }}$ percentile will divide the variables into two categories that will include, respectively, 25 and 75 per cent, a random variable has to be added to the initial variable, as described in the section devoted to the analyses per quarter. Five relative risk and/or five attributable risk estimates are computed and then combined.

Finally, if plausible values are involved as outcome measures, then five estimates will also be computed and then combined. However, it is not necessary to add a random variable to the initial variable as it constitutes a continuous variable.

\section{CONCLUSIONS}

This chapter was devoted to some statistical issues related to the way the OECD reported the PISA 2000 and PISA 2003 results in the initial reports, in particular questionnaire indices by quarters and the relative and attributable risks. 



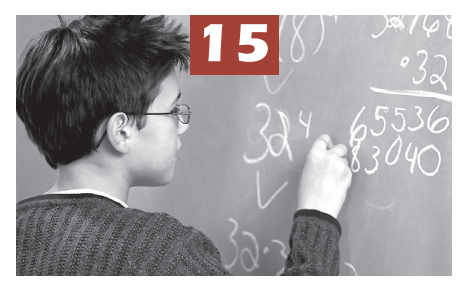

\section{SPSS ${ }^{\oplus}$ Macros}

Introduction

204

Structure of the SPSS ${ }^{\circledR}$ macros.

204 


\section{INTRODUCTION}

This chapter presents the SPSS ${ }^{\circledR}$ syntax of the macros used in the previous chapters. These macros are also included in the CD provided with the manual.

Twelve macros were described. These are summarized in Table 15.1. The file names are in red and the macro names as well as their arguments are in black. All the macros have five common arguments:

- NREP =

- WGT =

- RWGT $=$

- $\mathrm{CONS}=$

- INFILE =

The other arguments are specific to a particular macro. These specific arguments were largely explained in the previous chapters.

\section{STRUCTURE OF THE SPSS ${ }^{\circledR}$ MACROS}

All SPSS ${ }^{\circledR}$ macros have the same structure.

- The first step consists of:

- Reading in the INFILE data file and dropping all variables that are not necessary for the analysis.

- The second step is the iterative part of the macro:

- The SPSS ${ }^{\circledR}$ procedure for computing the estimate is repeated 81 times or 405 times, depending on the procedure; and

- At each run, the results are stored in a temporary file. The number of the replicate and the number of the plausible values if needed are then combined in one file.

- The final step is devoted to the computation of the final statistic and its respective standard error, i.e.:

- The squared differences between the final estimate and the 80 replicates are computed;

- The sum of the squared difference is computed and divided by 20; and

- Final estimates, the sampling variance estimates and, in the case of the plausible values, the measurement variance are computed.

The $\operatorname{SPSS}^{\circledR}$ syntax is presented hereafter. 
Table 15.1 - Synthesis of the twelve SPSS ${ }^{\circledR}$ macros

\begin{tabular}{|c|c|c|}
\hline Requested statistics & Without plausible values & With plausible values \\
\hline $\begin{array}{l}\text { Mean, sd, sum, pgt, plt, } \\
\text { pin, pout, fgt, flt, fin, } \\
\text { fout }\end{array}$ & 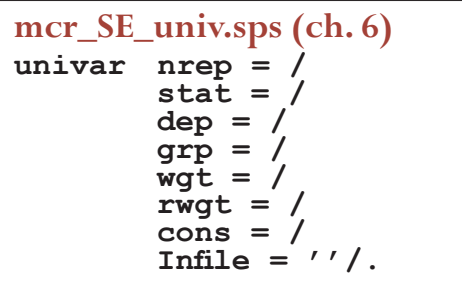 & $\begin{array}{l}\text { mcr_SE_pv.sps (ch. 7) } \\
\text { PV nrep = / } \\
\text { stat = / } \\
\text { dep }=/ \\
\text { grp }=/ \\
\text { wgt }=/ \\
\text { rwgt }=/ \\
\text { cons }=/, 1 / \\
\text { Infile }=1\end{array}$ \\
\hline Percentage & $\begin{array}{ll}\text { mcr_SE_GrpPct.sps (ch.6) } \\
\text { GRPPCT } & \text { nrep = / } \\
& \text { within = / } \\
& \text { grp = / } \\
& \text { wgt = / } \\
& \text { rwgt = / } \\
& \text { cons = / } \\
& \text { infile }=/ /\end{array}$ & $\begin{array}{l}\text { mcr_SE_PctLev.sps (ch. 8) } \\
\text { PCTLEV } \\
\text { nrep = / } \\
\text { within = / } \\
\text { grp = / } \\
\text { wgt = / } \\
\text { rwgt = / } \\
\text { cons = / } \\
\text { infile = '/ }\end{array}$ \\
\hline $\begin{array}{l}\text { Regression } \\
\text { coefficients }\end{array}$ & 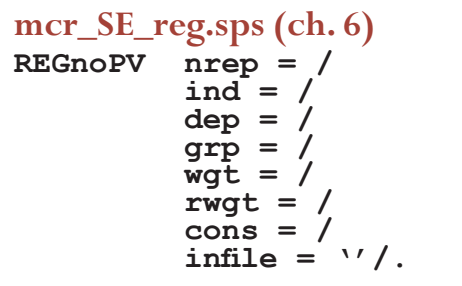 & $\begin{array}{l}\text { mcr_SE_reg_PV.sps (ch. 7) } \\
\text { REG_PV nrep =/ } \\
\text { ind = / } \\
\text { dep }=/ \\
\text { grp = / } \\
\text { wgt }=/ \\
\text { rwgt }=/ \\
\text { cons }=/ \\
\text { infile }=, ' /\end{array}$ \\
\hline $\begin{array}{l}\text { Correlation } \\
\text { coefficients }\end{array}$ & 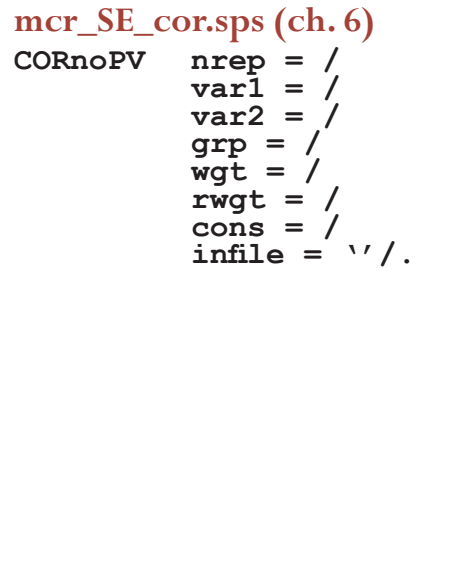 & 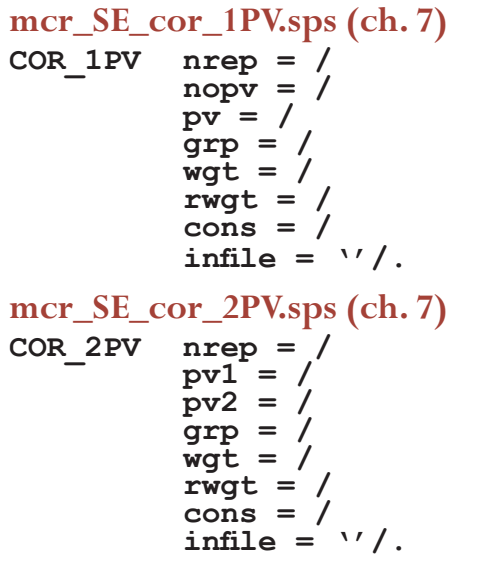 \\
\hline $\begin{array}{l}\text { Differences in mean, sd, } \\
\text { sum, pgt, plt, pin, pout, } \\
\text { fgt, flt, fin, fout }\end{array}$ & 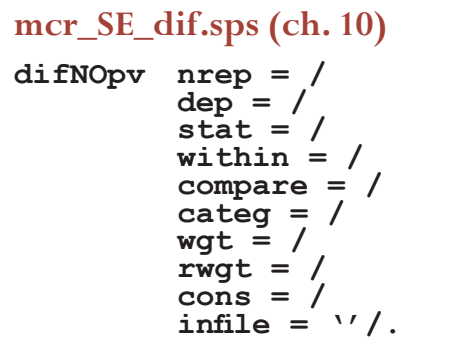 & 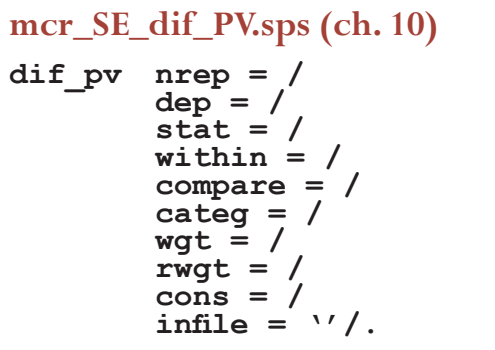 \\
\hline $\begin{array}{l}\text { PV mean, sd, sum, } \\
\text { pgt, plt, pin, pout, } \\
\text { fgt, flt, fin, fout } \\
\text { within quarters of WLE } \\
\text { indices }\end{array}$ & & 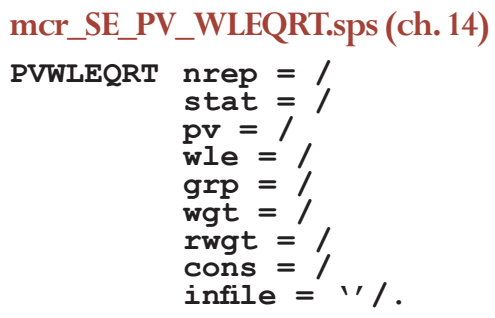 \\
\hline
\end{tabular}




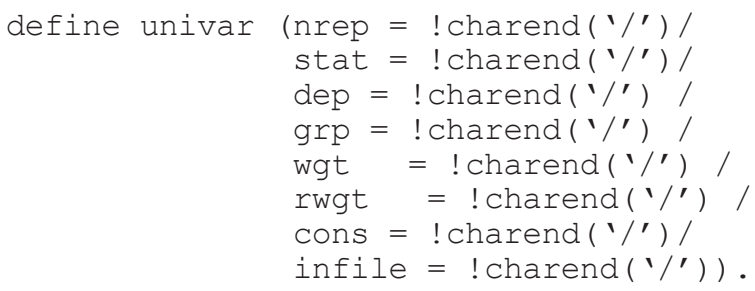

get file ! infile /keep ! grp ! wgt ! concat(!rwgt, 1) to ! concat(!rwgt, !nrep) (dep).

$\star \star *$ COMPUTE ESTIMATE $* \star *$.

weight by !wgt.

aggregate outfile = !quote (!concat ('c: \temp\', !stat,'_all.sav'))

/break=!grp / stat $=$ ! stat $($ ! dep $)$.

* REPLICATES.

! do ! i= 1 ! to ! nrep.

weight by ! concat(!rwgt, ! i) .

aggregate outfile = !quote(!concat ('C: \temp \', !stat,' ', , dep, ! i,'.sav')) /break=! grp / statr $=$ ! stat $($ ! dep $)$.

! doend.

$\star \star *$ COMBINE RESULTS ***.

get file =! quote (! concat ('C: \temp \', !stat,' ', , dep,'1.sav')) .

! Do !e $=2$ ! to !nrep.

add files file=* /file=!quote(!concat ('C: \temp \', !stat,' ', !dep, !e,'. $\left.\mathrm{sav}^{\prime}\right)$ ).

! Doend.

sort cases by !grp.

match files file=* /table=!quote(!concat ('c: \temp\', !stat,' all.sav')) / by ! grp.

exec.

$\star \star \star$ COMPUTE SAMPLING VARIANCE (U) $\star \star \star \star$.

compute $\operatorname{var}=($ statr-stat $) * \star 2$.

save outfile = 'c: \temp\regmod.sav'.

aggregate outfile=*/ break=!grp/ stat= mean(stat) / var=sum(var) .

compute $\operatorname{var}=$ ! cons* ${ }^{*}$ ar

$\star \star \star$ COMPUTE STANDARD ERROR $\star \star \star$.

compute se=sqrt (var) .

exec.

formats stat (f8.3)/ SE (f10.6).

list cases/var= ! grp stat se.

!enddefine. 


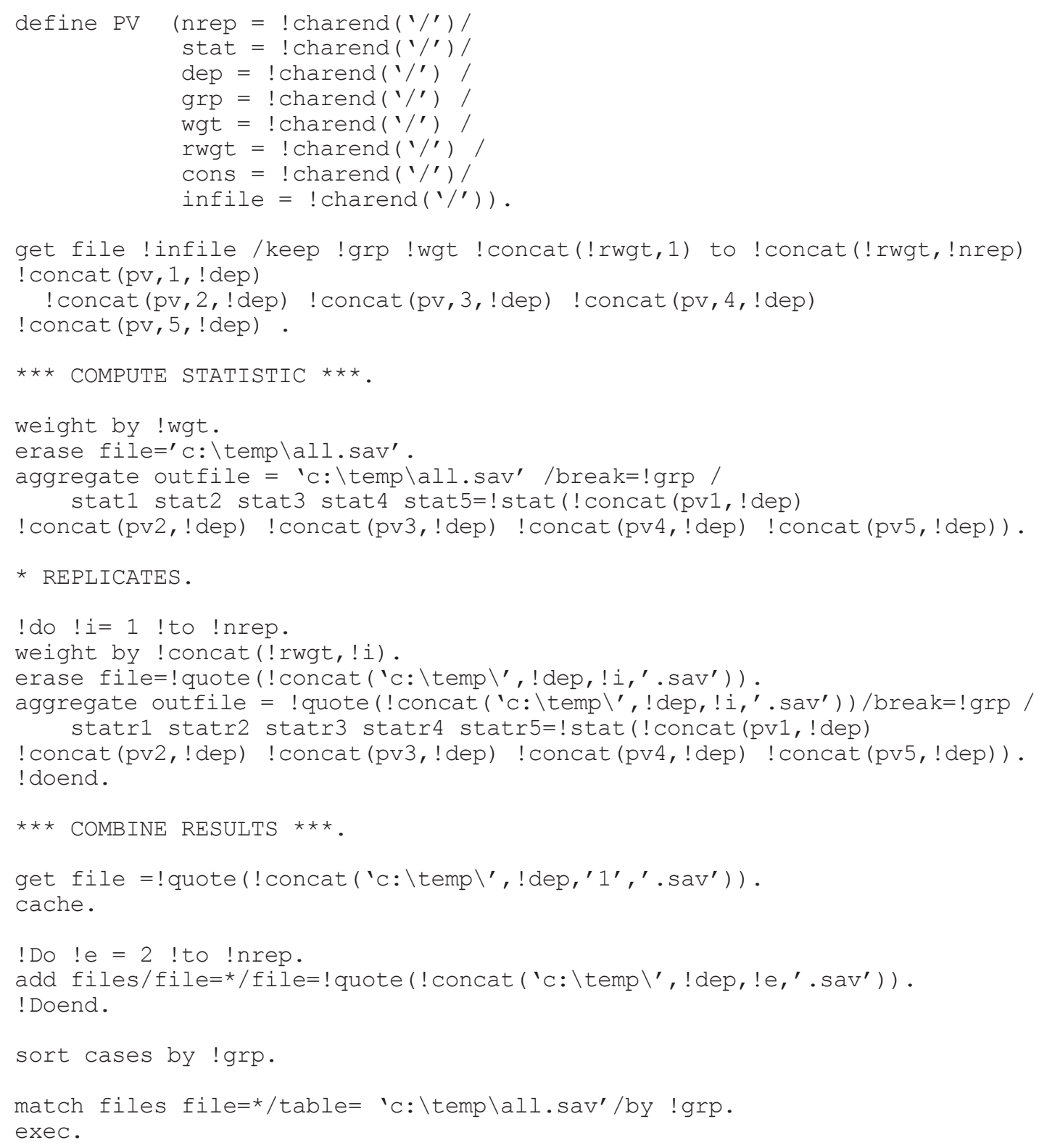




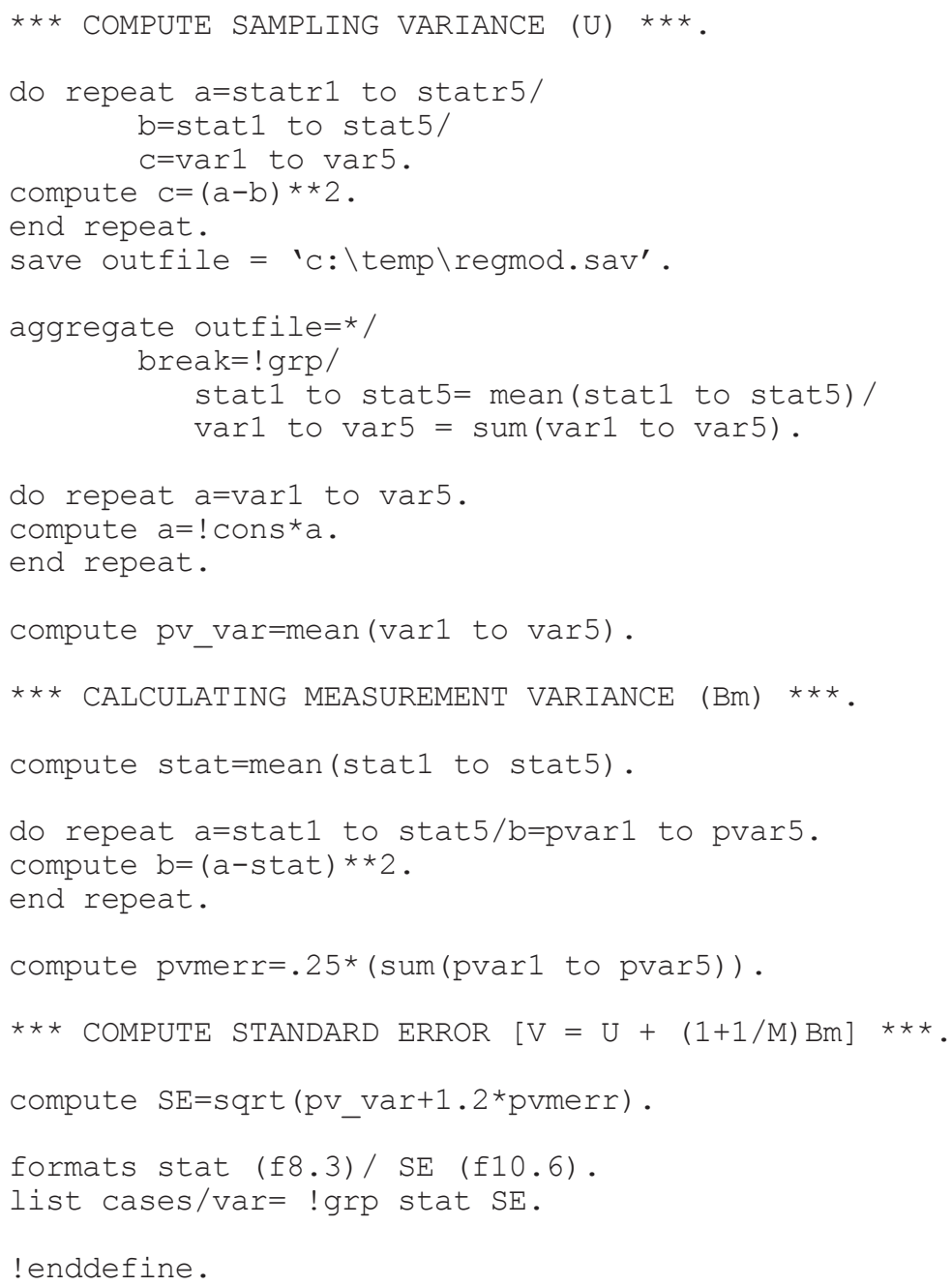


Box 15.3 — SPSS $^{\circledR}$ syntax of mcr_SE_GrpPct.sps.

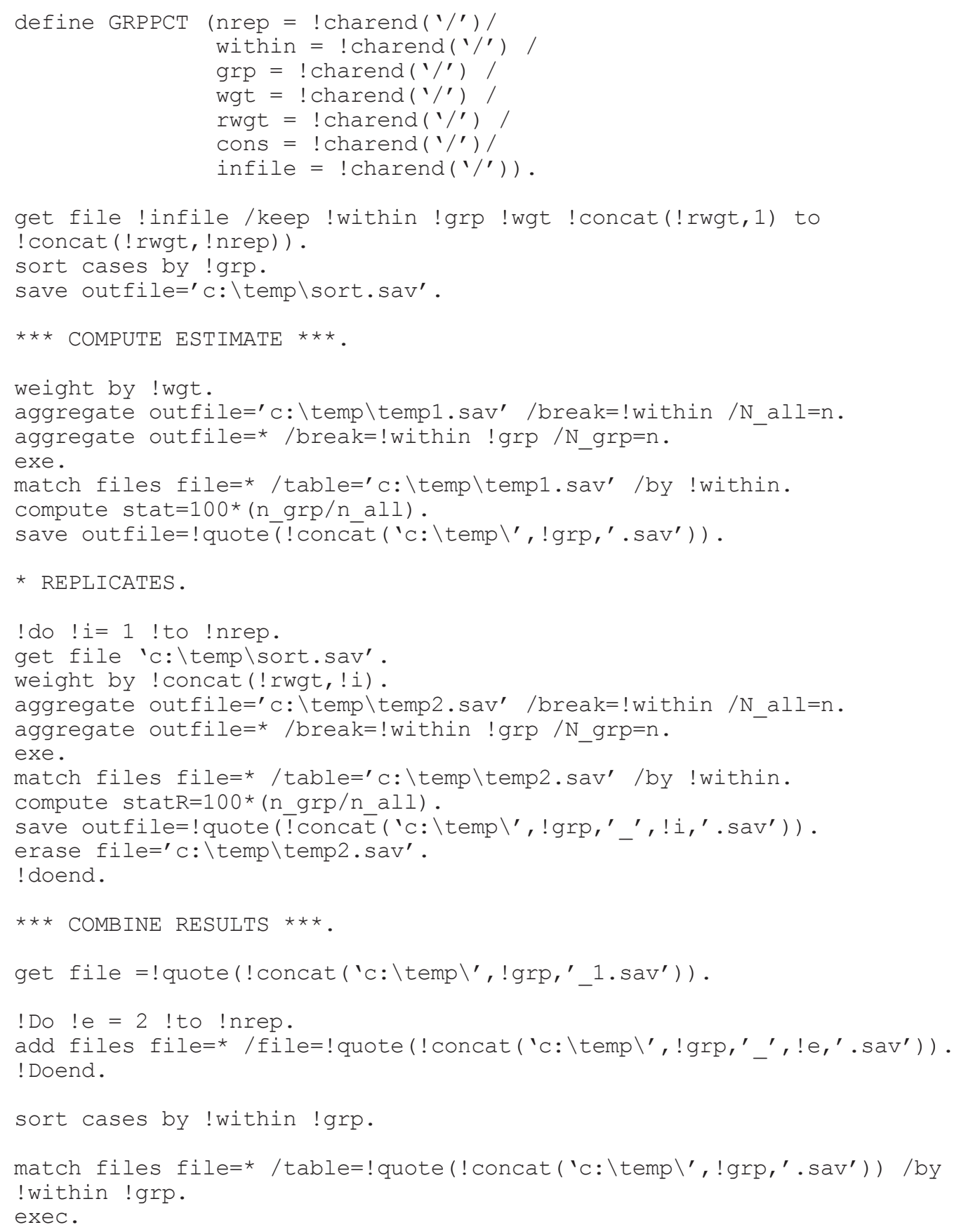




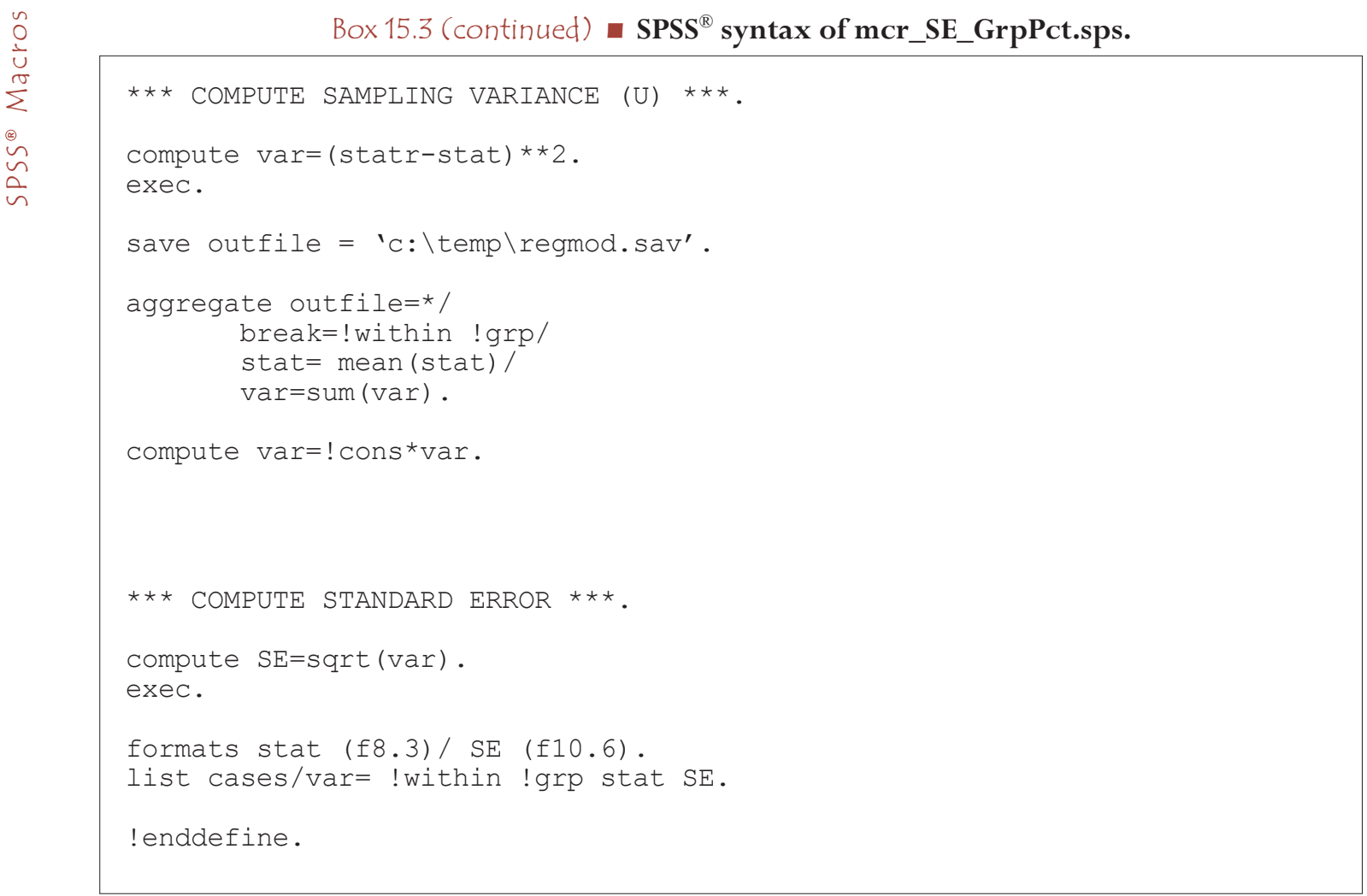




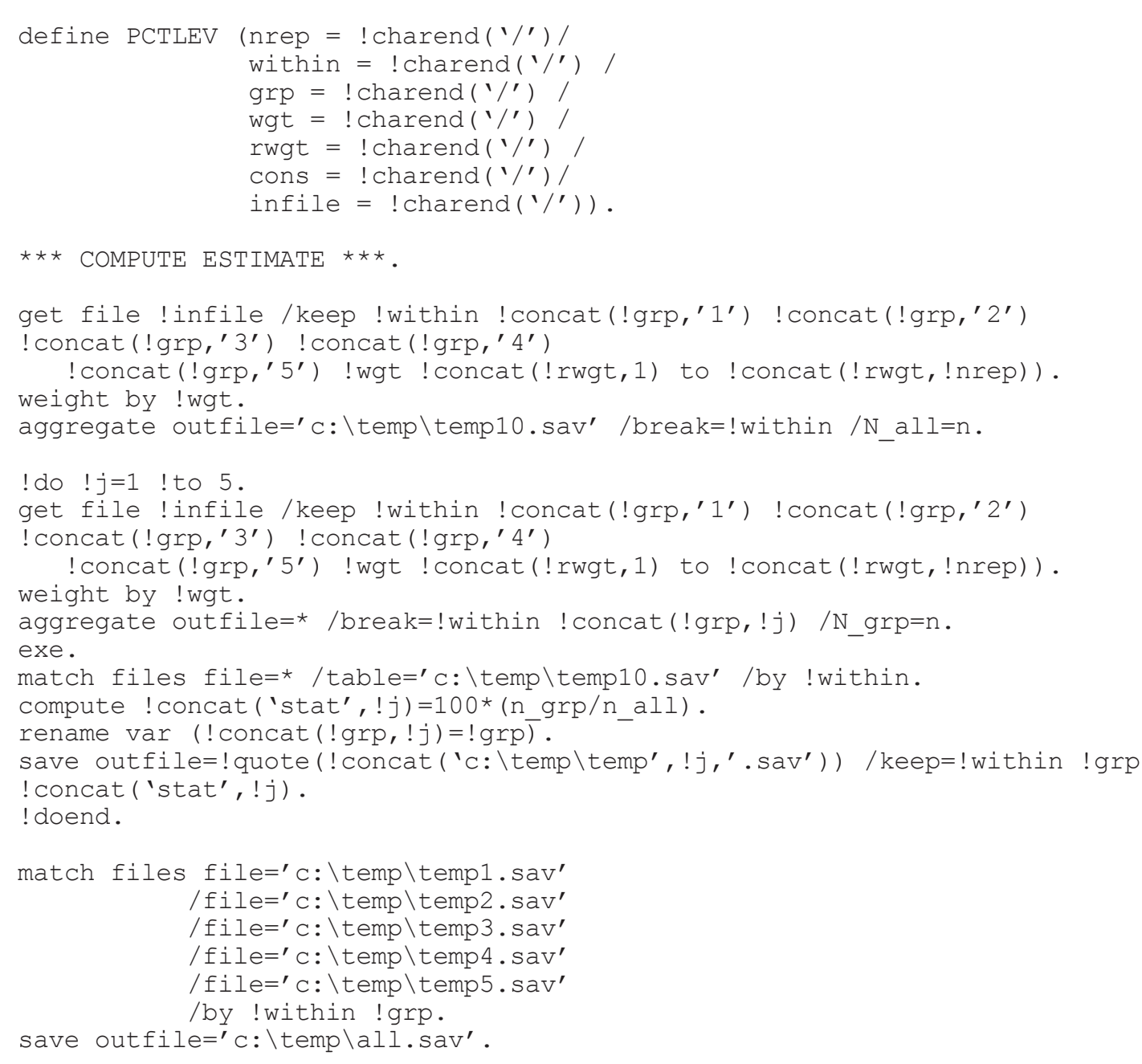




\section{* REPLICATES.}

! do ! i= 1 ! to !nrep.

get file ! infile /keep !within !concat(!grp,'1') !concat(!grp,'2') ! concat (!grp, ' $\left.3^{\prime}\right)$ ! concat (!grp, ' $\left.4^{\prime}\right)$

!concat (!grp,' 5') !wgt !concat(!rwgt, 1) to !concat (!rwgt, !nrep)). weight by ! concat (!rwgt, ! i) .

erase file='c: \temp \temp20.sav'.

aggregate outfile='c: \temp \temp20.sav' /break=!within /N_all=n .

! do $! j=1$ ! to 5 .

get file ! infile /keep !within !concat(!grp,' 1') !concat(!grp,' 2')

! concat (!grp, ' $\left.3^{\prime}\right)$ ! concat (!grp, ' $\left.4^{\prime}\right)$

!concat (!grp, ' $\left.5^{\prime}\right)$ ! wgt ! concat (!rwgt, 1$)$ to !concat (!rwgt, !nrep)) . weight by ! concat (!rwgt, ! i).

aggregate outfile=* /break=!within ! concat(!grp, !j) /N grp=n .

exe.

match files file=* /table='c: \temp \temp20.sav' /by !within.

compute ! concat ('statR', !j)=100* (n grp/n all).

rename $\operatorname{var}($ ! concat $(! g r p, ! j)=! g r p)$.

save outfile=! quote (!concat ('c: \temp\temp',!j,'.sav')) /keep=!within !grp

! concat ('statR', !j).

! doend.

match files file='c: \temp \templ.sav'

/file='c: \temp \temp2.sav'

/file='c: \temp \temp3.sav'

/file='c: \temp \temp4.sav'

/file='c: \temp \temp5.sav'

/by !within ! grp.

save outfile=! quote (! concat ('c: \temp \', !grp,' ', !'i,'.sav')) .

! doend.

$\star \star \star$ COMBINE RESULTS $\star \star \star$.

get file $=$ ! quote (! concat ('c: \temp ', ! grp,'_1.sav')).

! Do $! e=2$ ! to !nrep.

add files file=* /file=!quote(!concat ('c: \temp \',!grp,' ', ,!e,'.sav')).

! Doend.

sort cases by !within ! grp.

match files file=* /table='c: \temp\all.sav' /by !within !grp. exec. 


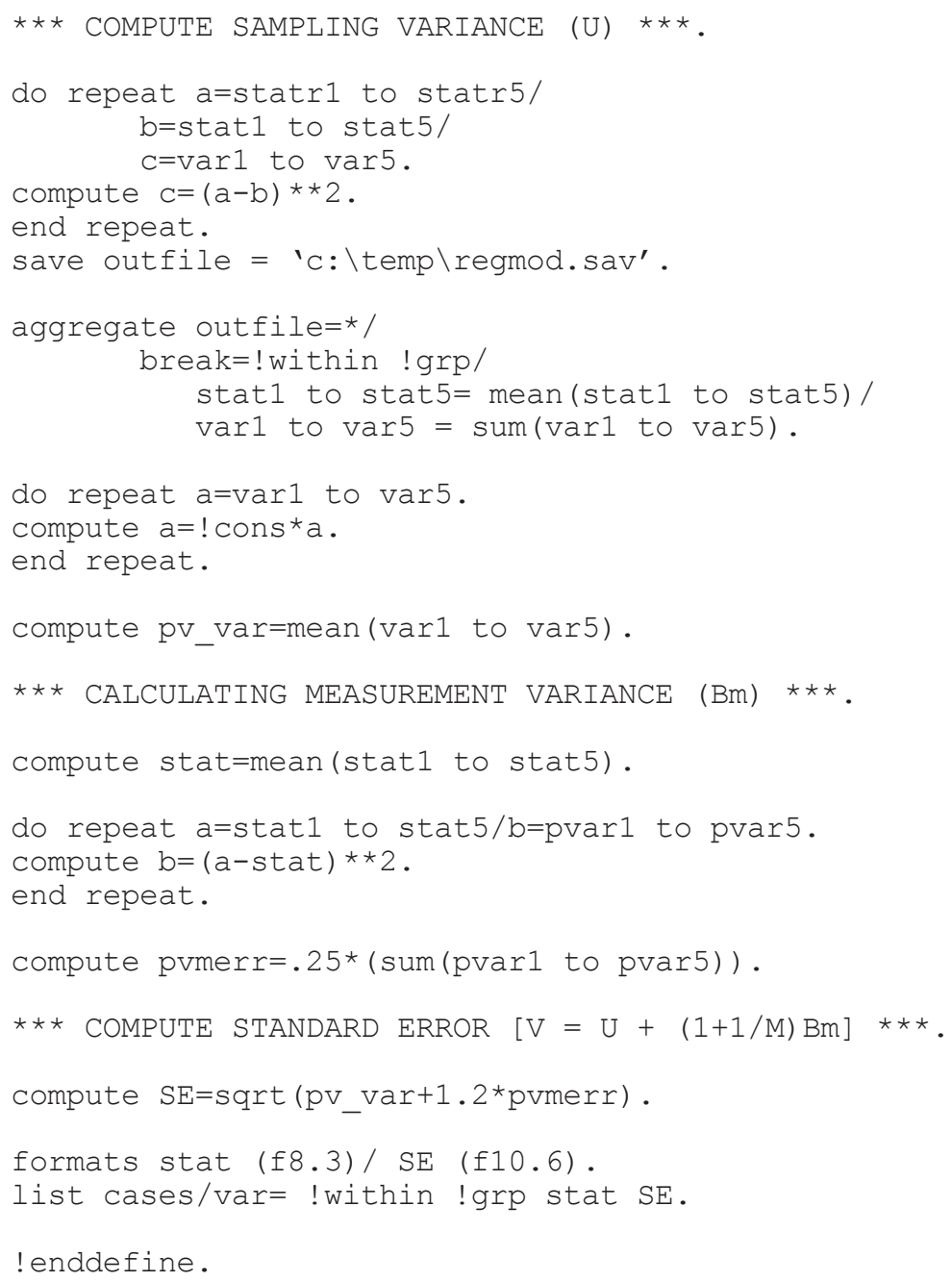




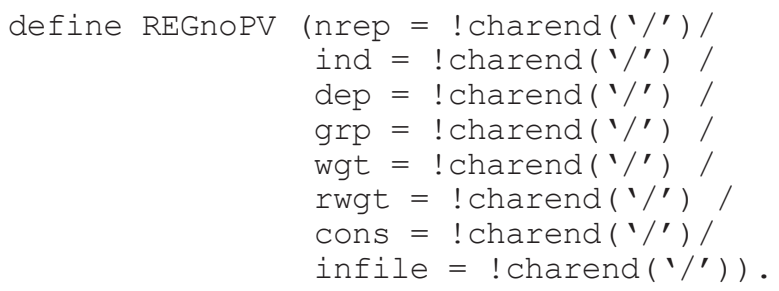




\section{Box 15.5 (continued) $\boldsymbol{\text { aPSS }}{ }^{\circledR}$ syntax of mcr_SE_reg.sps.}

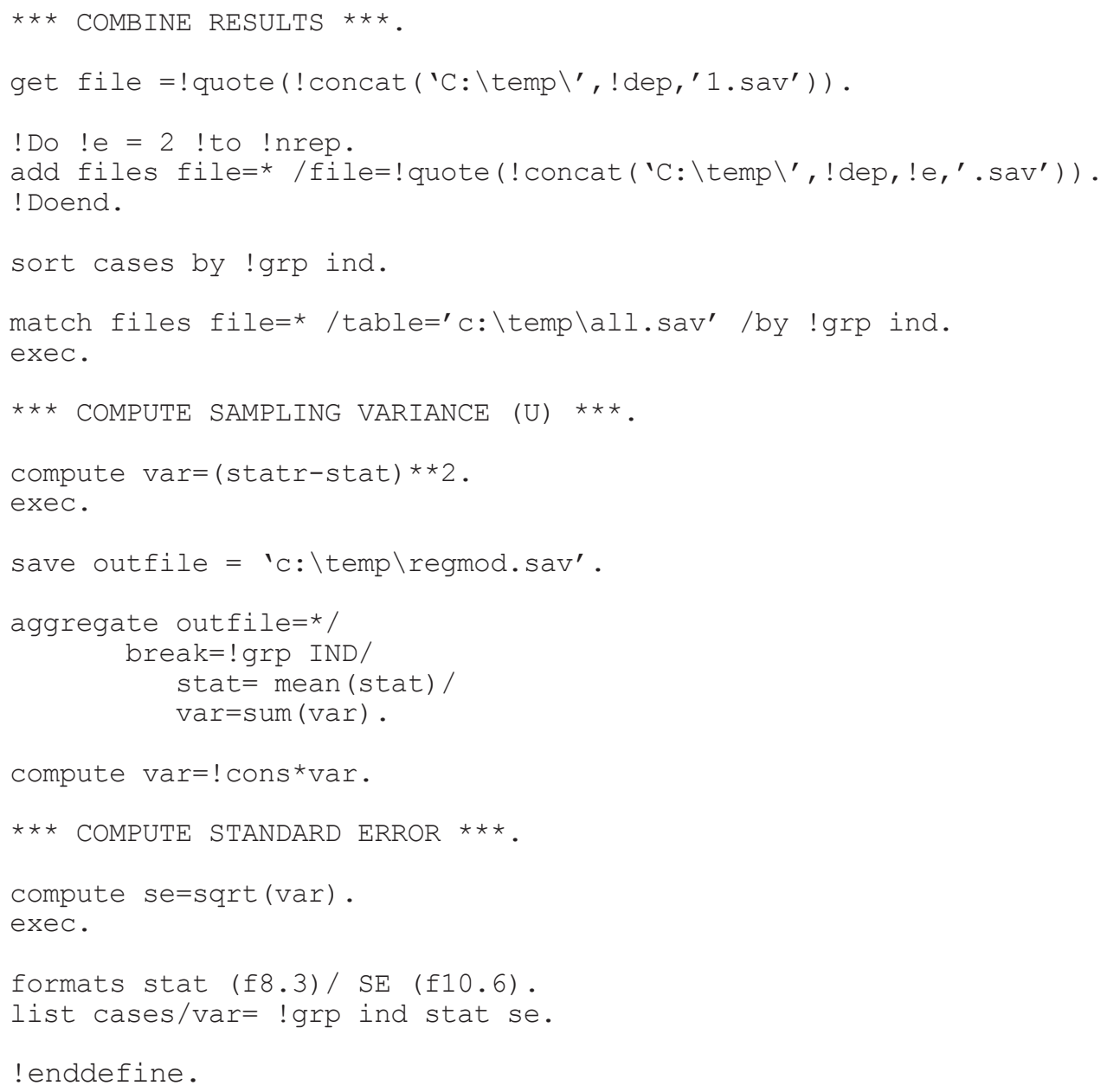




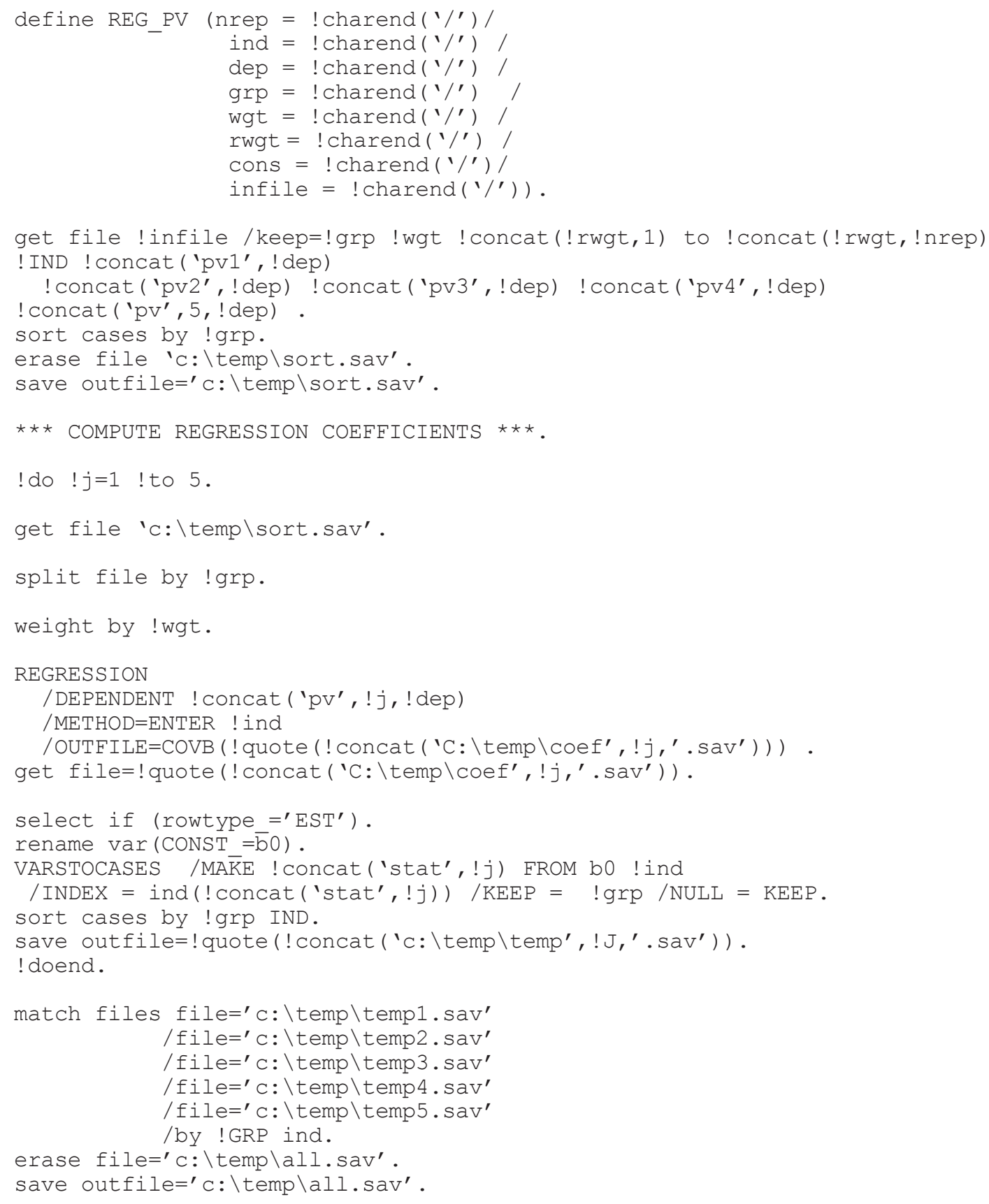




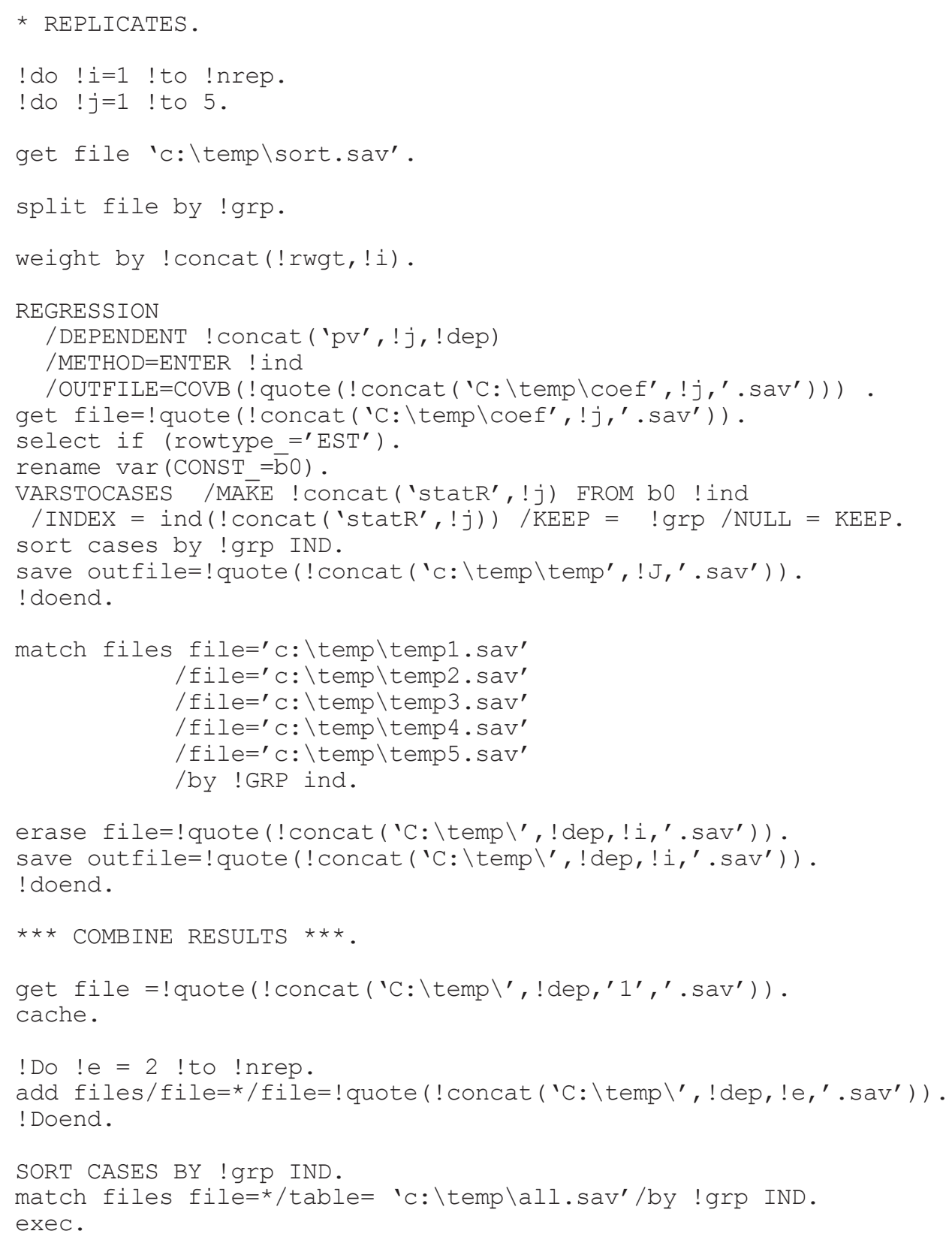




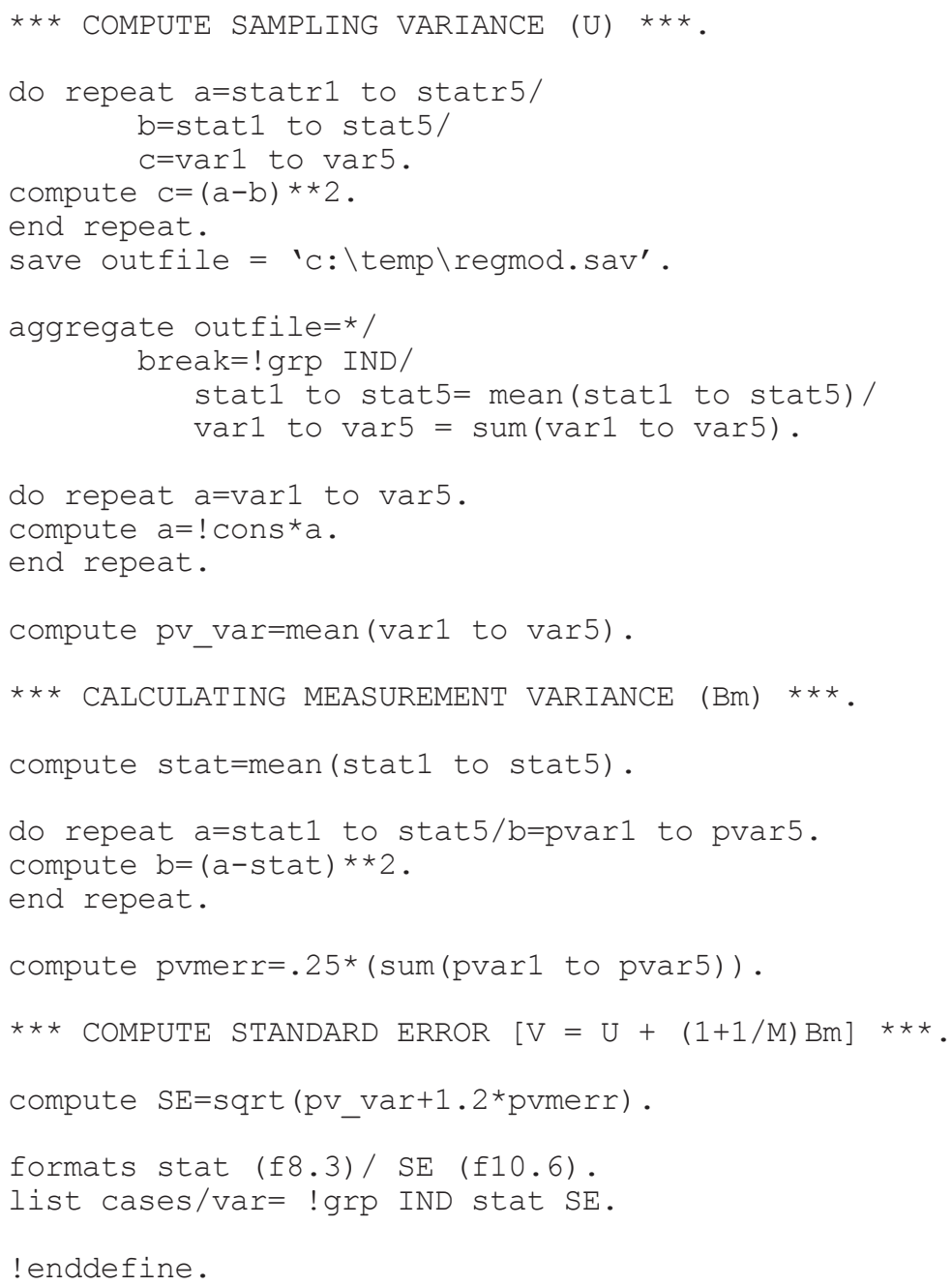




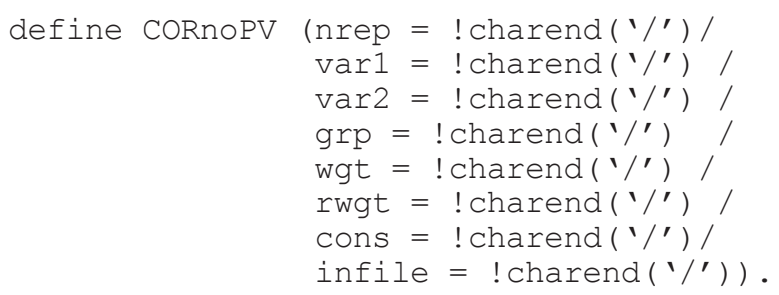




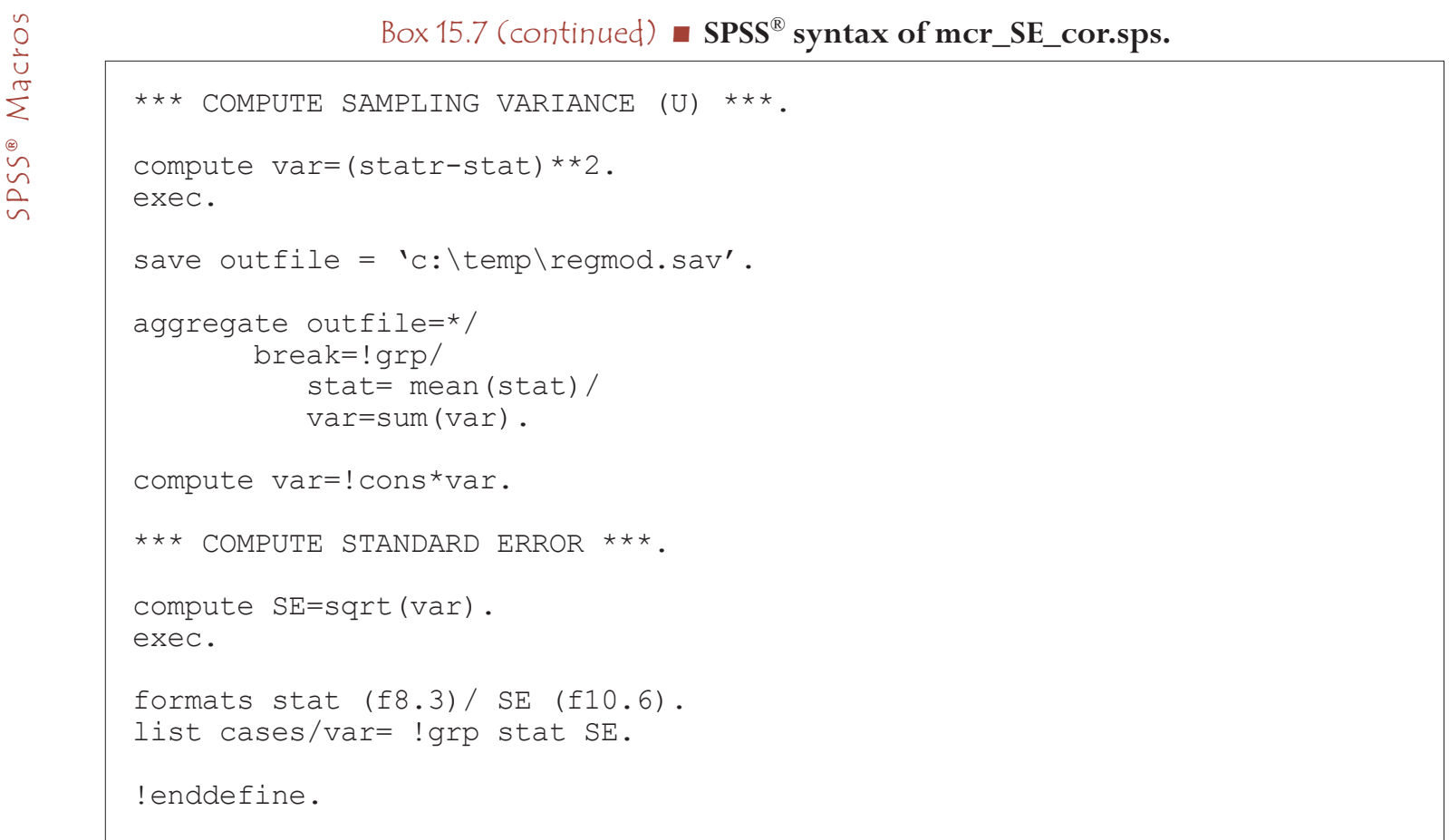




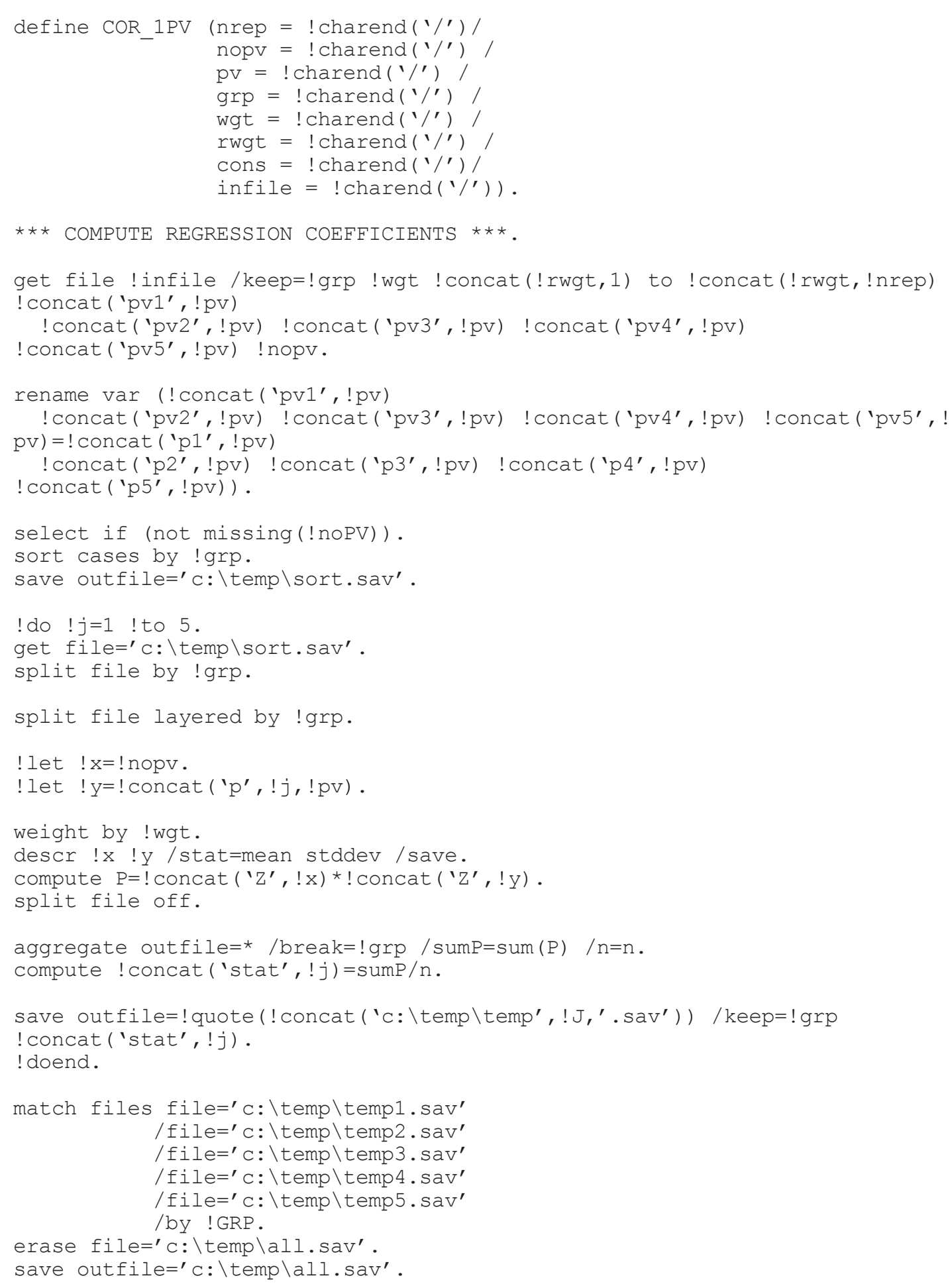




\section{* REPLICATES.}

! do ! i=1 !to !nrep.

! do $! j=1$ ! to 5 .

get file='c: \temp \sort.sav'.

split file by ! grp.

weight by ! concat (!rwgt, !i).

split file layered by ! grp.

! let $! x=!$ nopv

! let $! y=$ ! concat ('p', !j, ! pv).

descr !x !y / stat=mean stddev / save.

compute $\mathrm{P}=$ ! concat (' $\mathrm{Z}$ ',$! \mathrm{x})$ *! concat ( ' $\mathrm{Z}$ ', ! $\mathrm{y}$ ).

split file off.

aggregate outfile=* /break=!grp / sumP $=$ sum (P) /n=n .

compute ! concat ('statR', !j)=sumP/n.

save outfile=! quote (! concat ( 'c: \temp \temp', !J,'.sav')) /keep=!grp

! concat ('statR', !j).

! doend.

match files file='c: \temp \templ.sav'

/file='c: \temp \temp2.sav'

/file='c: \temp \temp3.sav'

/file='c: \temp \temp4.sav'

/file='c: \temp \temp5.sav'

/by ! GRP.

erase file=! quote(! concat ( $C: \backslash$ temp $\backslash$ ', ! pv, ! i,'.sav')) . save outfile=! quote (! concat ('C: \temp \', !pv, ! i,'.sav')).

! doend.

$\star \star \star$ COMBINE RESULTS ***.

get file =! quote (! concat ('C: \temp \', !pv,' 1','.sav')).

cache.

! Do !e $=2$ ! to !nrep.

add files/file=*/file=! quote (! concat ('C: \temp \', !pv, !e,'.sav')) .

! Doend.

sort cases by !grp.

match files file=*/table= 'c: \temp\all.sav'/by !grp.

exec. 


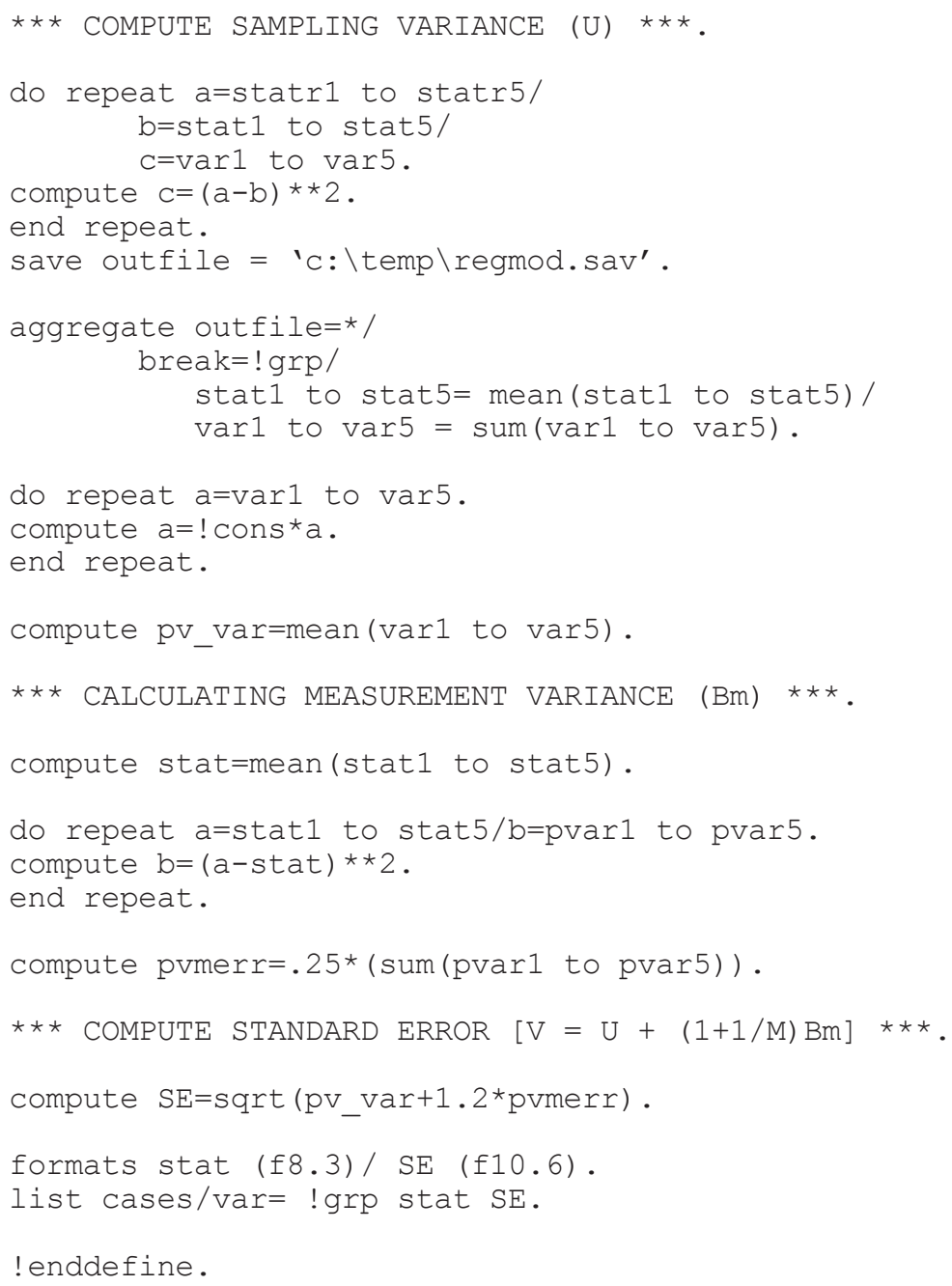




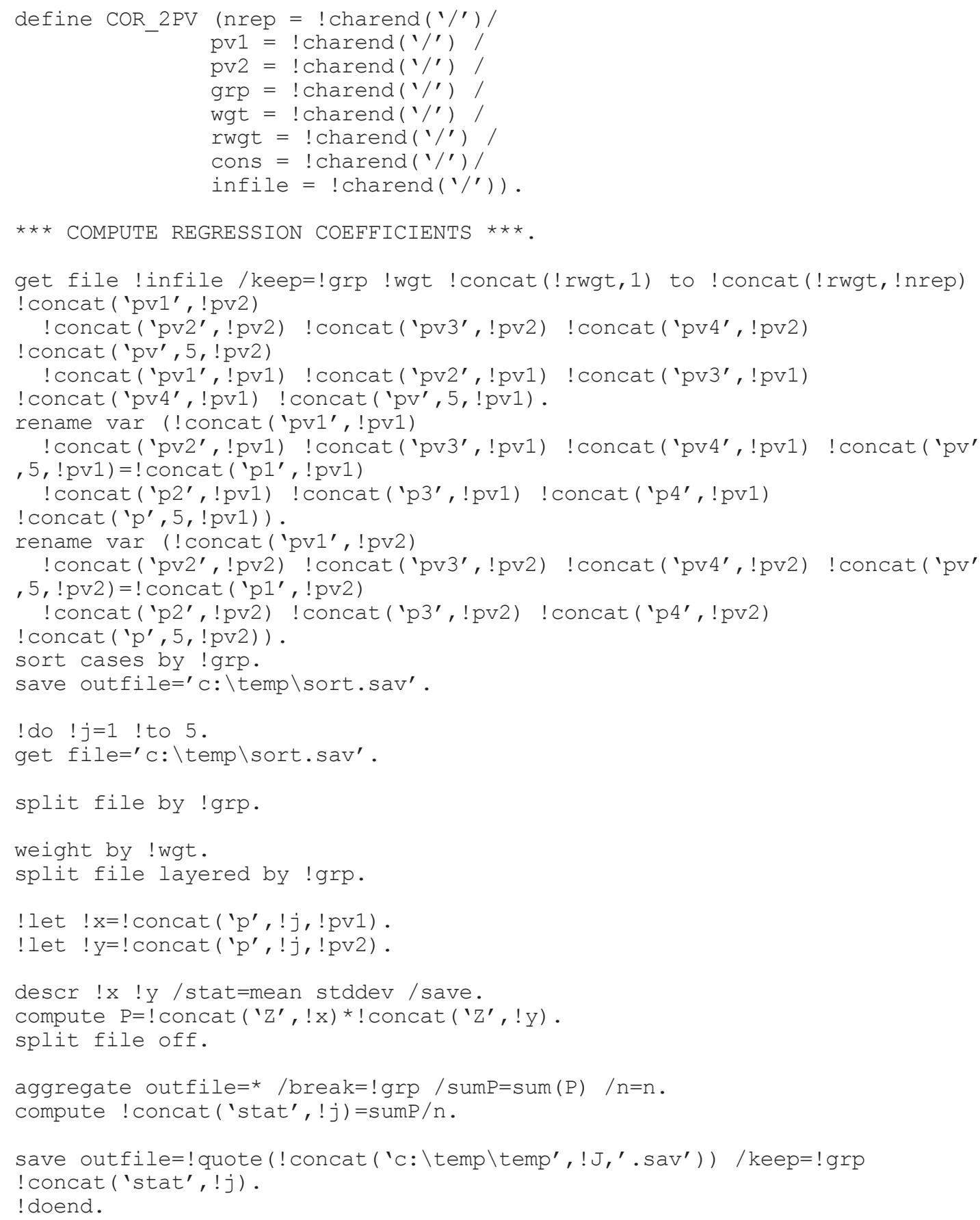


Box 15.9 (continued - 1) ロ SPSS ${ }^{\circledR}$ syntax of mcr_SE_cor_2PV.sps.

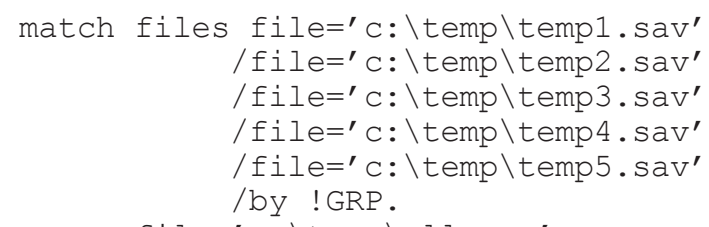

* REPLICATES.

!do ! i=1 !to !nrep.

! do $! j=1$ ! to 5 .

get file='c: \temp\sort.sav'.

split file layered by !grp.

weight by !concat (!rwgt, ! i) .

split file by ! grp.

! let $! x=$ ! concat $(' p ', ! j, ! p v 1)$.

! let $! y=$ ! concat (' $p$ ', ! j, ! pv2).

descr !x !y / stat=mean stddev / save.

compute $\mathrm{P}=$ ! concat ( ' $\mathrm{Z}$ ', ! $\mathrm{x}) *$ ! concat (' $\mathrm{Z}$ ', ! $\mathrm{y})$.

split file off.

aggregate outfile=* /break=!grp / sumP=sum(P) /n=n .

compute ! concat ('statR',$! j$ ) =sumP $/ n$.

save outfile=! quote (!concat ('c: \temp \temp',!J,' .sav')) / keep=! grp

! concat ('statR', !j) .

! doend.

match files file='c: \temp \templ.sav'

/file='c: \temp \temp2.sav'

/file='c: \temp \temp3.sav'

/file='c: \temp \temp4.sav'

/file='c: \temp \temp5.sav'

/by !GRP.

erase file=! quote (! concat ('C: \temp \', !pv2,! i,'.sav')) . save outfile=! quote (! concat ('C: \temp \',!pv2,!i,'.sav') ).

! doend.

$\star \star *$ COMBINE RESULTS $\star * \star$.

get file $=$ ! quote $($ ! concat $(' C: \backslash$ temp $\backslash$ ', ! pv2,' 1','. sav' $)$ ).

cache.

! Do $! e=2$ ! to !nrep.

add files/file=*/file=! quote (! concat ('C: \temp $\backslash$ ', ! pv2, !e,' .sav' ) ).

! Doend.

sort cases by !grp.

match files file=*/table= 'c: \temp\all.sav'/by !grp.

exec. 


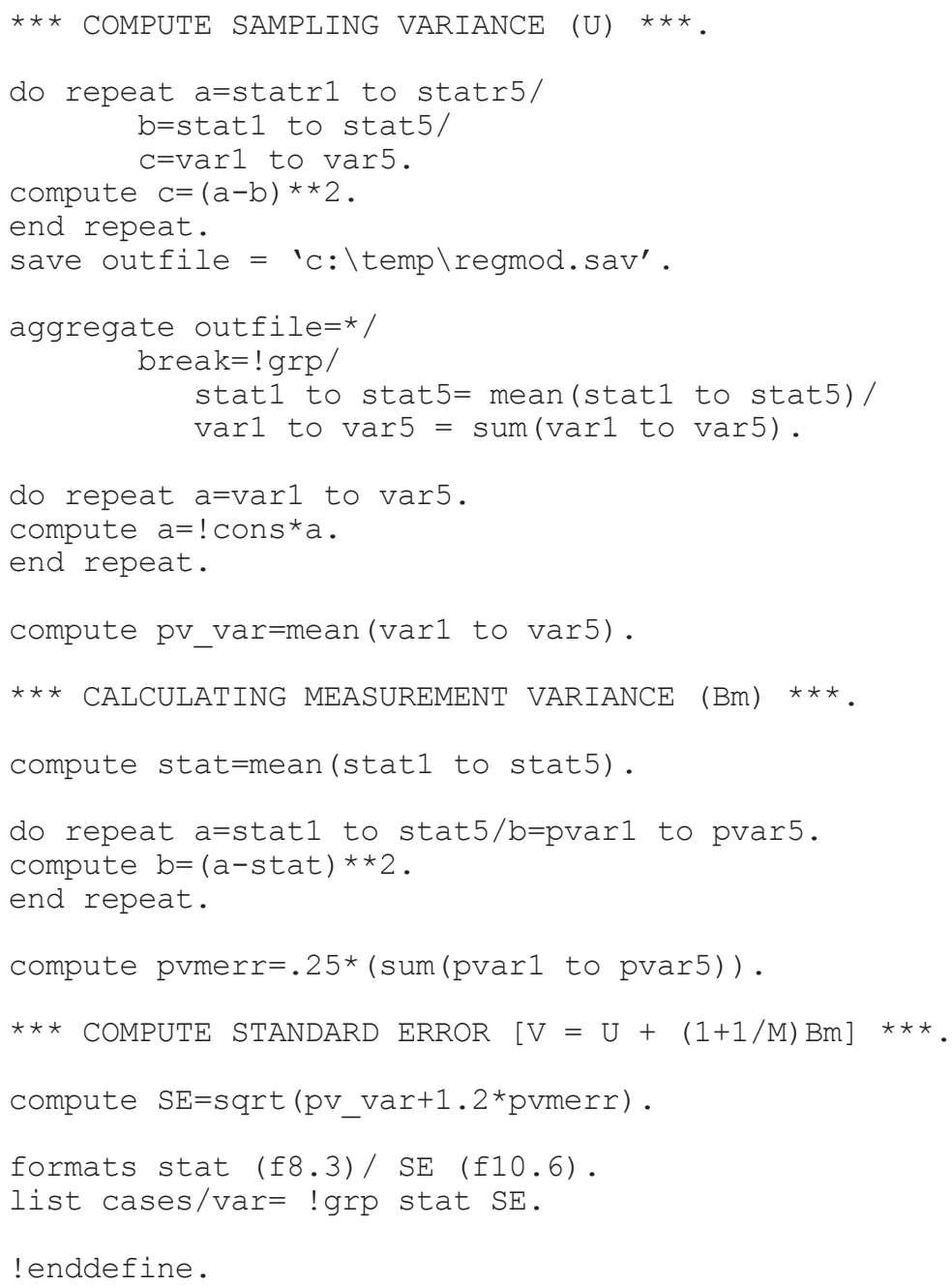




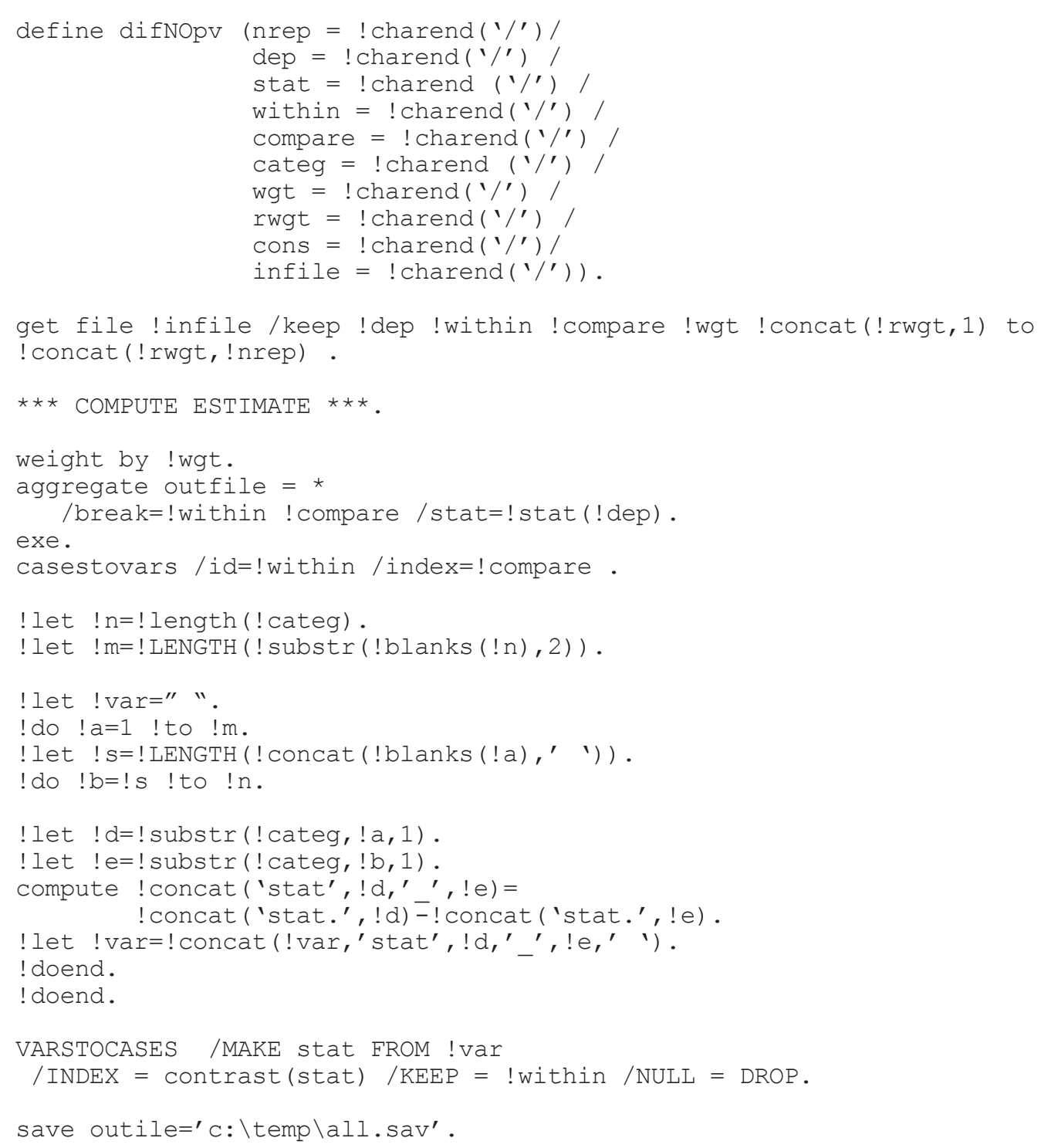




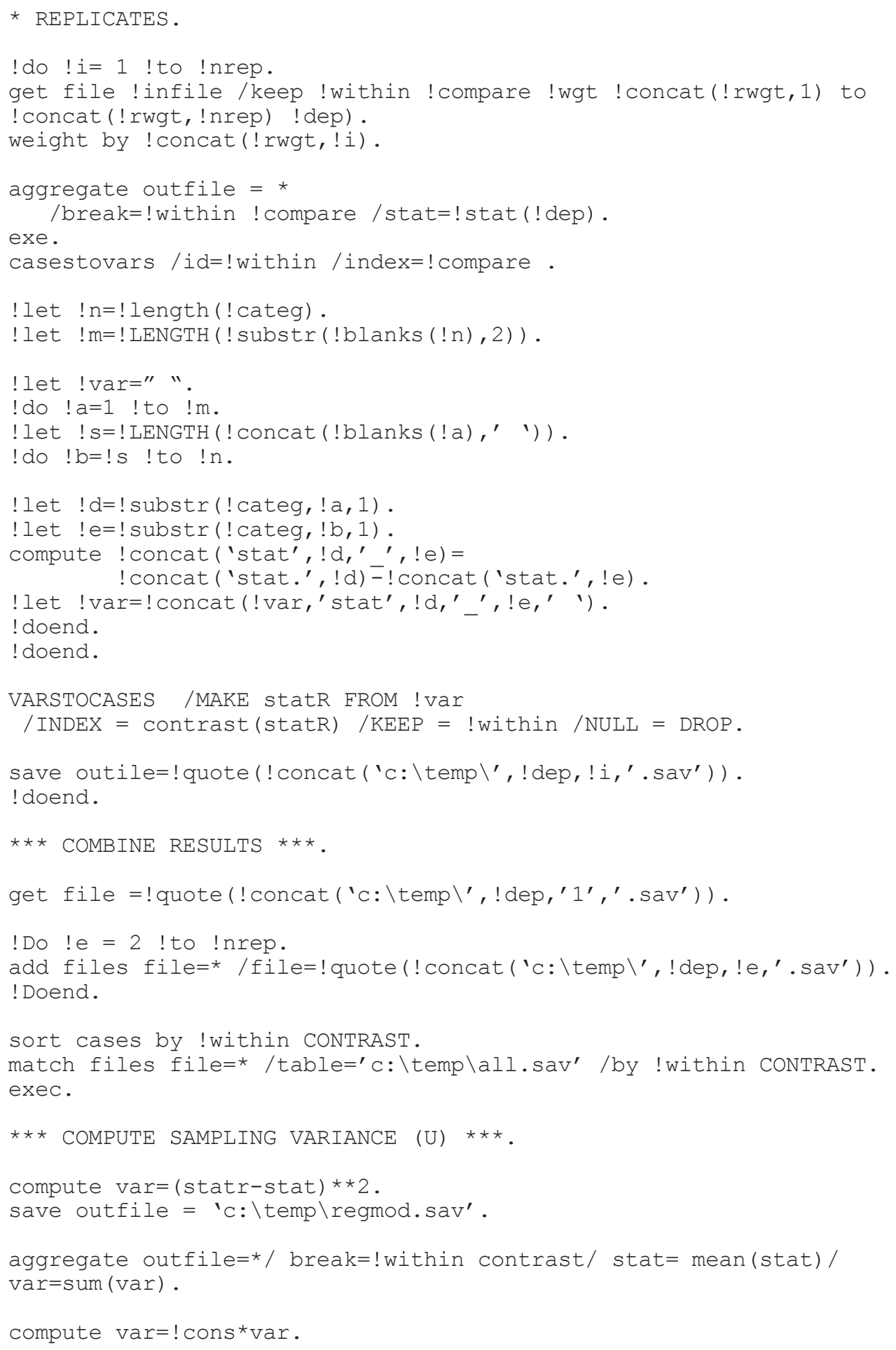


*** COMPUtE StAndARd ERROR ***.

compute se=sqrt ( $\operatorname{var})$.

exec.

formats stat (f8.3)/ SE (f10.6).

list cases/var= !within contrast stat se.

string contr (a3).

compute contr=substr (contrast, 5, 3).

casestovars /id=! within /index=contr /groupby=index /drop=contrast var.

!enddefine. 


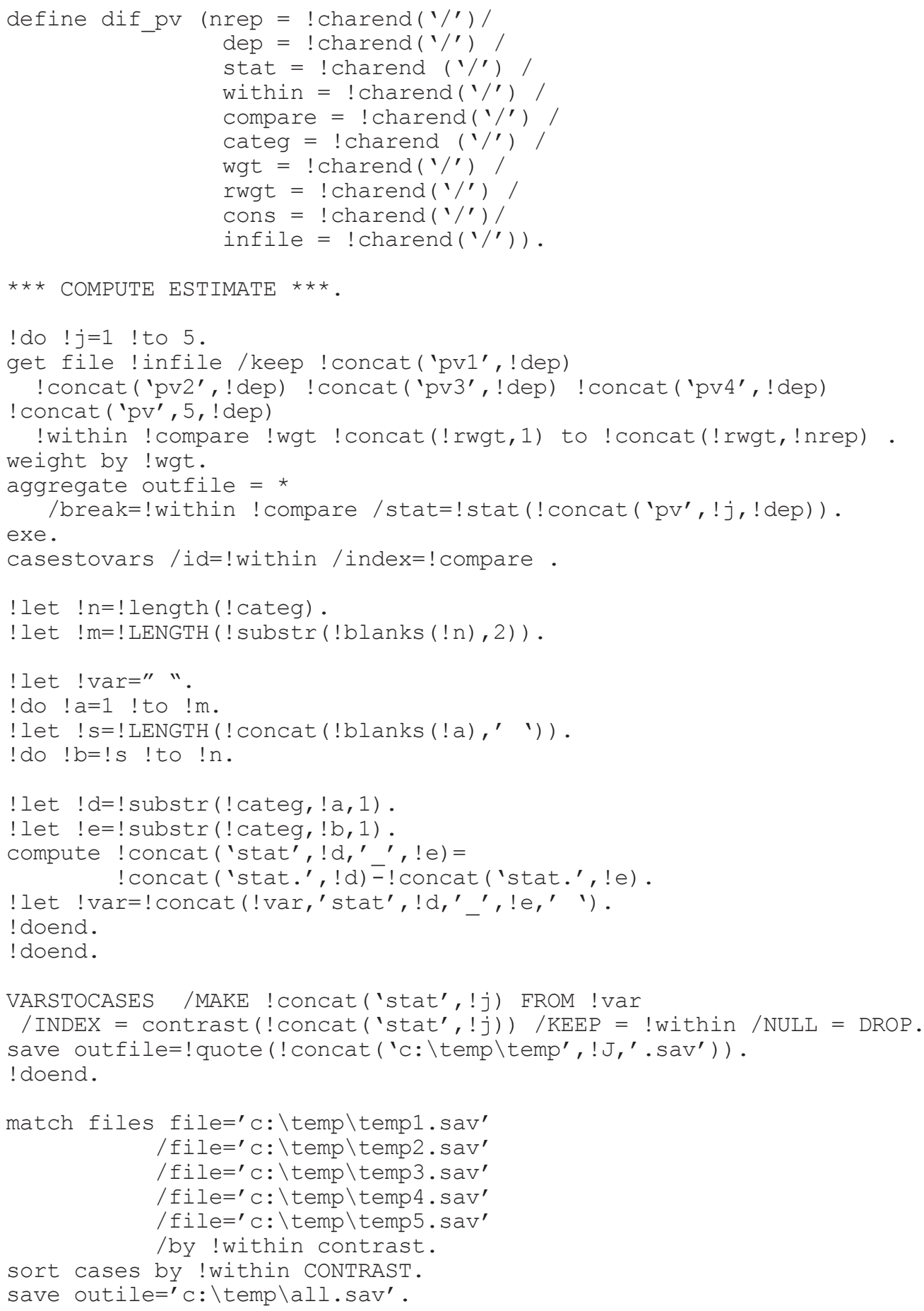




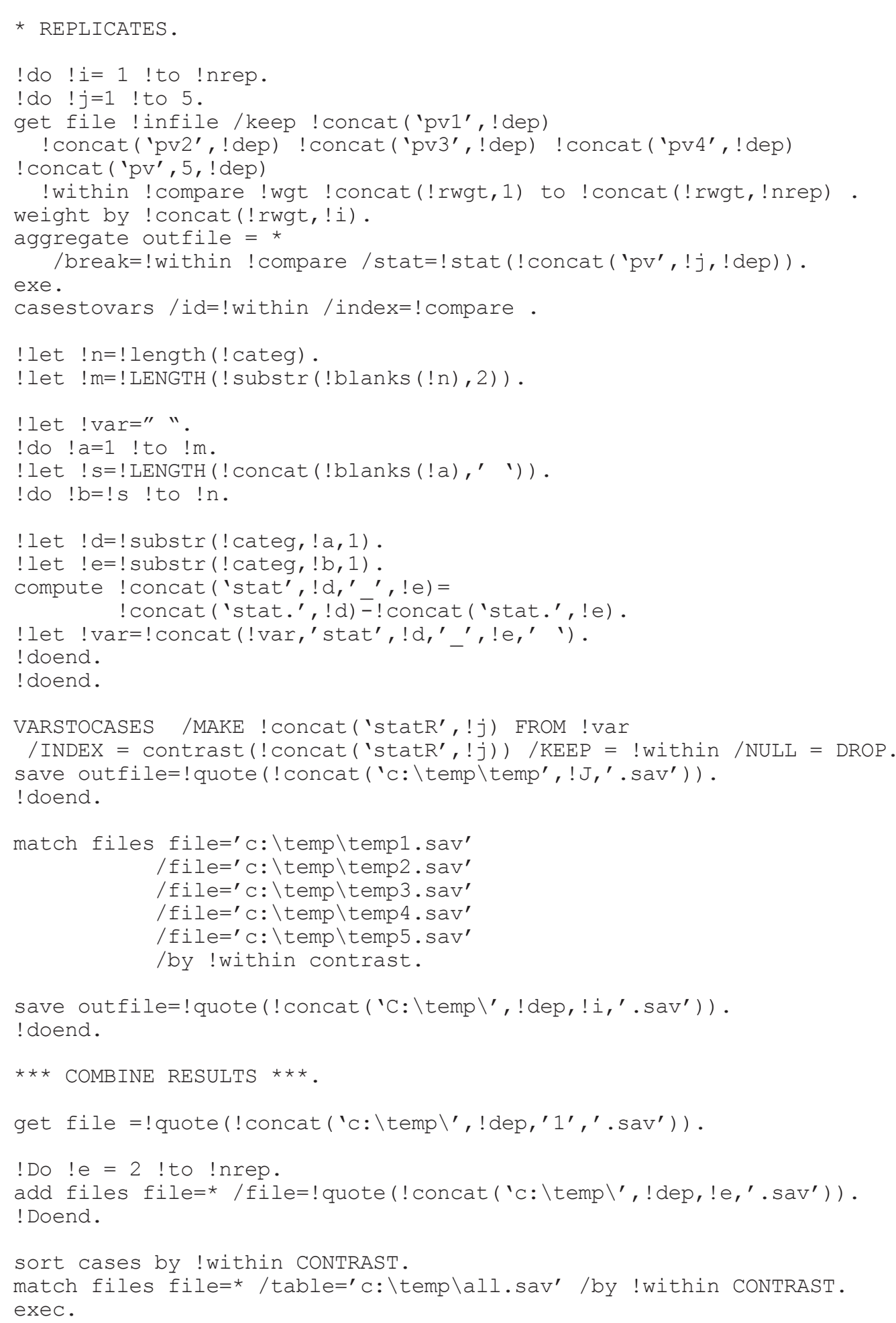




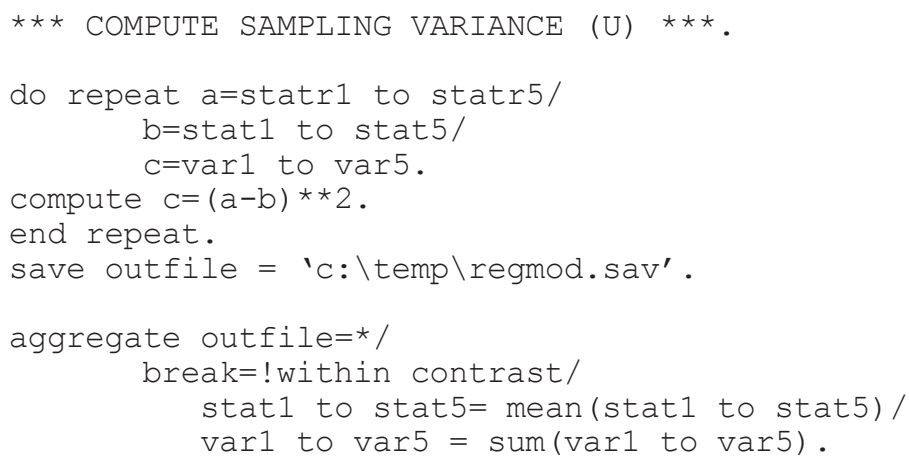




\section{Box 15.12 — SPSS $^{\circledR}$ syntax of mcr_SE_PV_WLEQRT.sps.}

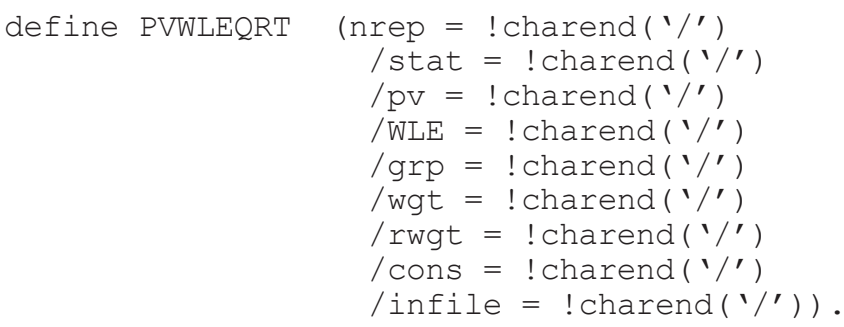

get file=! infile /keep=!grp !wgt !concat(!rwgt,1) to ! concat(!rwgt, !nrep) ! concat (pv, 1, ! pv)

! concat (pv, 2, ! pv) ! concat (pv, 3, !pv) ! concat (pv, 4, ! pv) ! concat (pv, 5, ! pv) ! wle.

select if not missing (!wle).

means ! wle /cell=mean min max.

* COMPUTE CUMULATIVE COUNT WITHin COUNTRIES.

sort cases by cnt.

autorecode cnt/into cnt\#/print.

! do $! s=1$ ! to 5 .

set seed $=$ ! s.

! let ! $\mathrm{v}=$ ! concat $(! \mathrm{wle}, \mathrm{!s})$.

! let ! $c=$ ! concat ( 'cumfreq', !s).

compute ! v=!wle+rv.normal $(0, .01)$.

sort cases by cnt\# !v.

do if (\$casenum=1 or lag (cnt\#) $<>$ cnt\#).

compute ! $\mathrm{C}=\mathrm{w}$ fstuwt.

else if (cnt\#=lag (cnt\#)).

compute $! c=w$ fstuwt $+\operatorname{lag}(! c)$.

end if.

sort cases by cnt.

! doend.

save outfile $=^{\prime} \mathrm{C}: \backslash$ temp \temp.sav' .

* DEFINE CUTSCORES

weight by w fstuwt.

aggregate outfile=* /break=cnt /total=max (cumfreql).

compute cut25=total/(100/25).

compute cut50=total/(100/50).

compute cut $75=$ total/ $(100 / 75)$.

match files file $=^{\prime} \mathrm{C}: \backslash$ temp $\backslash$ temp.sav'

/table $=*$

/by cnt.

exe. 


\section{* CREATE PERCENTile GROUPS.}

do repeat c=cumfreq1 cumfreq2 cumfreq3 cumfreq4 cumfreq5 /q=quart1 quart2 quart3 quart4 quart5.

if $(\mathrm{c}<\mathrm{cut} 25) \quad \mathrm{q}=1$.

if $(c>=$ cut25 \& $c<$ cut50) $q=2$.

if $(c>=$ cut50 \& $c<$ cut 75) $q=3$.

if $(c>=$ cut 75) $q=4$.

formats q $(f 1.0)$.

end repeat.

save outfile='C: \temp \quarters.sav' .

$\star \star \star$ COMPUTE STATISTIC $\star \star \star *$.

! do $! j=1$ ! to 5 .

get file='C: \temp \quarters.sav' /keep !grp !wgt !concat(!rwgt, 1) to ! concat (!rwgt, ! nrep) ! concat (pv, 1, ! pv)

! concat (pv, 2, ! pv) ! concat (pv, 3, ! pv) ! concat (pv, 4, ! pv) ! concat (pv, 5, ! pv) quart1 quart2 quart3 quart4 quart5.

weight by !wgt.

rename var (!concat ('quart', !j)=quart).

erase file=! quote (! concat ('C: \temp \temp', !j,'.sav')).

aggregate outfile = !quote(!concat ('C: \temp \temp', !j,'..sav')) /break=!grp quart

$/$ ! concat ('stat', !j) =! stat (! concat ('pv', !j, !pv)) .

! doend.

match files file='C: \temp \templ.sav'

/file='C: \temp \temp2.sav'

/file='c: \temp \temp3.sav'

/file='C: \temp \temp4.sav'

/file='C: \temp\temp5.sav'

/by ! grp quart.

erase file='C: \temp \all.sav'.

save outfile $=^{\prime} \mathrm{C}: \backslash$ temp \all.sav'.

* REPLICATES.

! do ! i= 1 ! to !nrep.

! do $! j=1$ ! to 5 .

get file='C: \temp \quarters.sav' /keep !grp !wgt !concat(!rwgt, 1) to

! concat (!rwgt, ! nrep) ! concat (pv, 1, ! pv)

! concat (pv, 2, ! pv) ! concat (pv, 3, ! pv) ! concat (pv, 4, ! pv) ! concat (pv, 5, ! pv) quart1 quart2 quart3 quart4 quart5.

weight by ! concat (!rwgt, ! i) .

rename var (!concat ('quart', !j)=quart).

sort cases by !grp quart.

erase file=! quote(!concat ('C:\temp \temp', !j,'.sav')).

aggregate outfile = !quote(!concat ('C: \temp \temp', !j,'.sav')) /break=!grp quart

/ ! concat ( 'statR', !j) =! stat (! concat ( 'pv', !j, ! pv)) .

! doend. 


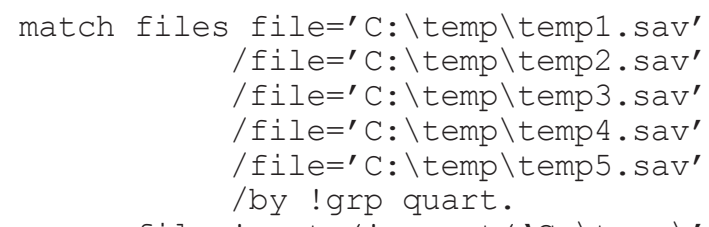

do repeat a=var1 to var5.

compute $\mathrm{a}=$ ! cons*a.

end repeat.

compute pv_var=mean (var1 to var5).

$\star * *$ CALCULATING MEASUREMENT VARIANCE (Bm) $* * *$.

compute stat=mean (stat1 to stat5).

do repeat a=stat1 to stat $5 / \mathrm{b}=$ pvar1 to pvar5.

compute $\mathrm{b}=(\mathrm{a}-\mathrm{stat}) * \star 2$.

end repeat.

compute pvmerr $=.25 *$ (sum(pvar1 to pvar5)).

$\star * *$ COMPUTE STANDARD ERROR $[\mathrm{V}=\mathrm{U}+(1+1 / \mathrm{M}) \mathrm{Bm}] * * *$.

compute $\mathrm{SE}=$ sqrt (pv_var+1.2*pvmerr) .

formats stat (f8.3)/ SE (f10.6).

list cases/var= !grp quart stat $\mathrm{SE}$.

!enddefine. 



\section{Appendices}

Appendix 1: PISA 2003 International Database

Appendix 2: Student Questionnaire

Appendix 3: Educational Career Questionnaire

Appendix 4: Information Communication Technology (ICT) Questionnaire

Appendix 5: School Questionnaire

Appendix 6: Student Questionnaire Data File Codebook

Appendix 7: School Questionnaire Data File Codebook

Appendix 8: Student Cognitive Test Data File Codebook

Appendix 9: Student and School Questionnaire Indices

Appendix 10: Scores Allocated to the Items 


\section{APPENDIX 1 - PISA 2003 INTERNATIONAL DATABASE}

\section{WHAT IS THE GENERAL STRUCTURE OF THE PISA 2003 INTERNATIONAL DATABASE?}

This document describes the international database of the OECD Programme for International Student Assessment (PISA) 2003. The database can be accessed through the PISA web page (www. pisa.oecd.org). The database comprises data collected in 2003 in 41 countries and processed in the second half of 2003 and in 2004. The first results were released in December 2004 (for the full set of results see OECD, 2004a).

The purpose of this document is to provide all of the necessary information to analyse the data in accordance with the methodologies used to collect and process the data. It does not provide detailed information regarding these methods.

The following sources can provide additional information about PISA:

- The PISA Web site (www.pisa.oecd.org) provides: i) descriptions about the programme, contact information, participating countries and results of PISA 2003 as well as PISA 2000; ii) the complete micro-level database, all questionnaires, publications and national reports of PISA 2003 and PISA 2000 , in a downloadable format; and iii) an opportunity for users to generate their own tables or request specific ones.

- Learning for Tomorrow's World - First Results from PISA 2003 (OECD, 2004a) includes the first results from PISA 2003. It presents evidence on student performance in reading, mathematical and scientific literacy and problem solving, reveals factors that influence the development of these skills at home and at school, and examines what the implications are for policy development.

- The PISA 2003 Assessment Framework - Mathematics, Reading, Science and Problem Solving Knowledge and Skills (OECD, 2003) describes the framework and instruments underlying the PISA 2003 assessment. It introduces the PISA approach to assessing mathematical, reading and scientific literary and problem solving with its three dimensions of processes, content and context. Further it presents tasks from the PISA 2003 assessment together with how these tasks were scored and how they relate to the conceptual framework underlying PISA.

- The PISA 2003 Technical Report (OECD, forthcoming) presents the methodology and procedures used in PISA.

The PISA database provides detailed information on all instruments used in PISA 2003 for:

- 30 OECD member countries: Australia, Austria, Belgium, Canada, the Czech Republic, Denmark, Finland, France, Germany, Greece, Hungary, Iceland, Ireland, Italy, Japan, Korea, Luxembourg, Mexico, the Netherlands, New Zealand, Norway, Poland, Portugal, the Slovak Republic, Spain, Sweden, Switzerland, Turkey, the United Kingdom and the United States.

- 11 OECD partner countries: Brazil, Hong Kong-China, Indonesia, Latvia, Liechtenstein, MacaoChina, the Russian Federation, Serbia, Thailand, Tunisia and Uruguay. 


\section{Test design}

In PISA 2003, a rotated test design was used to assess student performance in mathematical, reading and scientific literacy and problem solving (for the complete conceptual frameworks see OECD, 2003b). This type of test design ensures a wide coverage of content while at the same time keeping the testing burden on individual students low. Thirteen test booklets were distributed at random to students. These booklets included questions assessing reading literacy, mathematical literacy, scientific literacy and problem solving, but not all booklets assessed the same domains. Students were randomly assigned a testing booklet within each of the sampled schools.

- Booklets 1 and 2 contained reading and mathematics questions;

- Booklets 3 and 4 contained mathematics and problem solving questions;

- Booklets 5 and 6 contained mathematics and science questions;

- Booklets 7 and 8 contained reading, mathematics and science questions;

- Booklet 9 contained reading, mathematics, science and problem solving questions;

- Booklets 10 and 11 contained reading, mathematics and problem solving questions; and

- Booklets 12 and 13 contained mathematics, science and problem solving questions.

In addition to the thirteen two-hour booklets, a special one-hour booklet, referred to as the UH Booklet (or the Une Heure booklet) was prepared for use in schools catering exclusively to students with special needs. The UH booklet was shorter and contained items deemed most suitable for students with special educational needs. The UH booklet contained seven mathematics items, six reading items, eight science items and five problem solving items.

\section{Questionnaires}

\section{Student questionnaires}

A student questionnaire (see Appendix 2) was designed to collect information about the student's family, home environment, reading habits, school and everyday activities. This information was later analysed both independently and in relation to performance.

Additionally, the programme included two additional optional questionnaires for students. The first one was an educational career questionnaire (see Appendix 3) asking the students' past educational career, present educational settings and expected occupation. National centres were allowed to select any of the items included in this questionnaire for inclusion without having to administer all of the questions. The second one was an information communication technology (ICT) questionnaire (see Appendix 4), including questions regarding the students' use of, familiarity with and attitudes towards ICT. ICT was defined as the use of any equipment or software for processing or transmitting digital information that performs diverse general functions whose options can be specified or programmed by its user.

\section{School questionnaire}

The principals or head administrators of the participating schools responded to a school questionnaire (see Appendix 5) covering issues such as the demographics of the school, school staffing, the school environment, human and material educational resources in the school, selection and transfer policies, and educational and decision-making practices in the school. 


\section{Structure of the testing session}

The student testing session consisted of:

- Two 60-minute sessions assessing reading, mathematical and scientific literacy and problem solving;

- 35 minutes for the student questionnaire;

- Two minutes for the international option of educational career questionnaire; and

- Five minutes for the international option of ICT familiarity questionnaire.

The school principal or head administrator answered a 20 minutes school questionnaire.

\section{WHAT IS AVAILABLE FROM THE PISA 2003 INTERNATIONAL DATABASE?}

\section{What is available for downloading?}

The downloadable files are classified into six categories. Some of them are quite small, while others (e.g. the micro-level data files) are quite large, taking a long time to download. The six categories of file are:

\section{Questionnaires}

The following questionnaires are available: student questionnaire, educational career questionnaire, ICT familiarity questionnaire and school questionnaire. Appendices 2 to 5 of this document show these questionnaires, with the variable name of each item in the left-hand margin. For example:

ST03Q01 Q 3 Are you $<$ female $>$ or $<$ male $>$ ? $\quad<$ Female $>\quad<$ Male $>$

\section{Codebooks}

The codebooks are useful in relating the actual items from the instruments (assessment tests or questionnaires) to the data available in the data files as they identify the variable name with all possible values which are valid for that variable. In addition to the name of the variable, they also show its label, all possible responses (code and label), type of variable (e.g. string or numeric) and the columns where the values are shown in the actual data file. Three codebooks are available: the codebook for student questionnaire (see Appendix 6), the codebook for cognitive test item (see Appendix 8) and the codebook for school questionnaire (see Appendix 7). For example, in the case of the previous item (ST03Q01), the codebook shows:

\begin{tabular}{clll}
\hline ST03Q01 & Sex - Q3 & Female & $29-29$ \\
1 & Male & \\
2 & N/A & \\
7 & M/R & \\
8 & Mis & \\
9 & & \\
\hline
\end{tabular}


These files will read the raw text file, and convert it into a SAS ${ }^{\circledR}$ data file assigning label and values (valid and missing). The three SAS ${ }^{\circledR}$ control files will read and convert: the school questionnaire, the student questionnaire and the cognitive test item data files. These files have extension *.SAS.

\section{SPSS ${ }^{\circledR}$ Control files}

Similarly to the $S A S^{\circledR}$ control files, these files will read the raw text file, and convert it into a SPSS ${ }^{\circledR}$ data file assigning labels and values (valid and missing). The three SPSS ${ }^{\circledR}$ control files will read and convert: the school questionnaire, the student questionnaire and the cognitive test item data files. The files have extension *.SPS.

\section{Data files in text format}

The item by item database is available in text format, which once read by the SAS $^{\circledR}$ or SPSS ${ }^{\circledR}$ control files will be correctly formatted and labelled. As it is, it includes one row for each student with his or her responses to all items. These files have extension *.TXT and are in ASCII form.

\section{Compendia}

Compendia show the full item by country results for the three student questionnaires, the school questionnaire and the students' performance. The following three files are available: the test item compendium, the student questionnaire compendium and the school questionnaire compendium. There are two types of data for each item: percentages by categories and performances by categories. Standard errors are also reported for the percentages and for the means.

\section{WHICH FILES ARE INCLUDED IN THE PISA 2003 INTERNATIONAL DATABASE?}

The PISA international database consists of three data files. The files are in text (or ASCII) format and are accompanied by the corresponding SAS ${ }^{\circledR}$ and SPSS $^{\circledR}$ control (syntax) files, which can be used to read the text into a SAS ${ }^{\circledR}$ or SPSS ${ }^{\circledR}$ database. Besides the data collected through the international questionnaire, some countries collected data through national options, which are not included in the international database. These files are quite large as they include one record for each student or school.

\section{How are the files named?}

The data files in the international database are named according to the following convention:

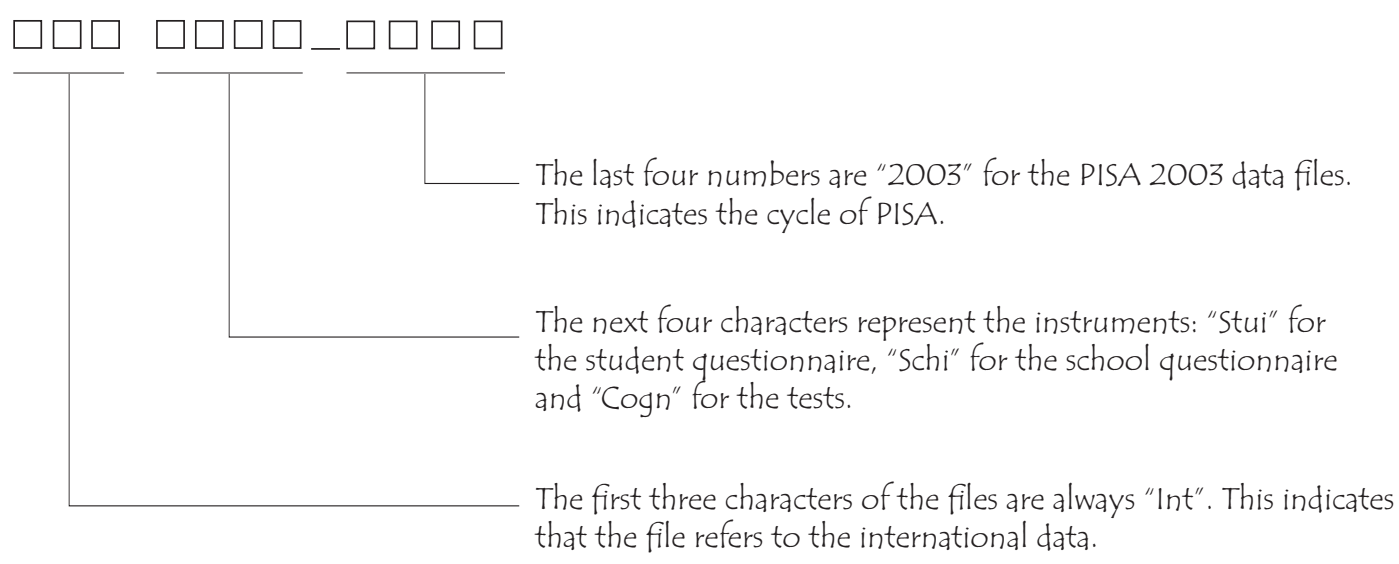




\section{Student questionnaire data file (filename: int_stui_2003.txt)}

For each student who participated in the assessment, the following information is available:

- Identification variables for the country, adjudicated sub-national region, stratum, school and student;

- The student responses on the three questionnaires, i.e. the student questionnaire and the two international options: educational career questionnaire and ICT questionnaire;

- The student indices (see Appendix 9) derived from the original questions in the questionnaires;

- The students' performance scores in mathematics, reading, science and problem solving; and

- The student weights and the 80 reading Fay's replicates for the computation of the sampling variance estimates.

\section{School questionnaire data file (filename: int_schi_2003.txt)}

For each school that participated in the assessment, the following information is available:

- The identification variables for the country, adjudicated sub-national region, stratum and school;

- The school responses on the school questionnaire;

- The school indices (see Appendix 9) derived from the original questions in the school questionnaire; and

- The school weight.

Cognitive test item data file (filename: int_cogn_2003.txt)

For each student who participated in the assessment, the following information is available:

- Identification variables for the country, adjudicated sub-national region, booklet ID, school and student; and

- The students' responses for each item included in the test, expressed in a one-digit format. ${ }^{1}$

\section{Which records are included in the international database?}

Records included in the database

Student level

- All PISA students who attended one of the two test sessions; and

- PISA students who only attended the questionnaire session are included if they provided a response to the father's occupation questions or the mother's occupation questions on the student questionnaire (Questions 7 to 10).

\section{School level}

- All participating schools - that is, any school where at least 25 per cent of the sampled eligible students were assessed - have a record in the school level international database, regardless of whether the school returned the school questionnaire.

\section{Records excluded from the database}

\section{Student level}

- Additional data collected by some countries for a national or international option such as a grade sample; 
- Sampled students who were reported as not eligible, students who were no longer at school, students who were excluded for physical, mental or linguistic reasons, and students who were absent on the testing day;

- Students who refused to participate in the assessment sessions; and

- Students from schools where less than 25 percent of the sampled and eligible students participated.

School level

- Schools where fewer than 25 per cent of the sampled eligible students participated in the testing sessions.

\section{How are missing data represented?}

The coding of the data distinguishes between four different types of missing data:

- Item level non-response: 9 for a one-digit variable, 99 for a two-digit variable, 999 for a three-digit variable, and so on. Missing codes are shown in the codebooks. This missing code is used if the student or school principal was expected to answer a question, but no response was actually provided;

- Multiple or invalid responses: 8 for a one-digit variable, 98 for a two-digit variable, 998 for a three-digit variable, and so on. This code is used for Multiple choice items in both test booklets and questionnaires where an invalid response was provided. This code is not used for open-ended questions;

- Not applicable: 7 for a one-digit variable, 97 for a two-digit variables, 997 for a three-digit variable, and so on for the student questionnaire data file and for the school data file. Code ' $n$ ' is used for a one-digit variable in the test booklet data file. This code is used when it was not possible for the student to answer the question. For instance, this code is used if a question was misprinted or if a question was deleted from the questionnaire by a national centre. The not-applicable codes and code ' $n$ ' are also used in the test booklet file for questions that were not included in the test booklet that the student received; and

- Not reached items: all consecutive missing values starting from the end of each test session were replaced by the non-reached code, 'r', except for the first value of the missing series, which is coded as missing.

\section{How are students and schools identified?}

The student identification from the student files consists of three variables, which together form a unique identifier for each student:

- The country identification variable labelled COUNTRY. The country codes used in PISA are the ISO 3166 country codes;

- The school identification variable labelled SCHOOLID. These are sequential numbers, which were randomly assigned for confidentiality reasons; and

- The student identification variable labelled STIDSTD. These are sequential numbers, which were randomly assigned for confidentiality reasons.

The variable labelled SUBNATIO has been included to differentiate adjudicated sub-national entities within countries. This variable is used for four countries as follows: 
- Italy: the value ' 1 ' is assigned to the region Veneto-Nord-Est, '2' to the region Trento-NordEst, '3' to the region Toscana-Centro, '4' to the region Piemonte-Nord-Ovest, '5' to the region Lombardia-Nord Ovest, ' 6 ' to the region Bolzano and the value ' 7 ' to all other (non-adjudicated) Italian regions;

- Spain: the value ' 1 ' to the non-adjudicated regions in Spain, '2' to Castilia and Leon, '3' to Catalonia and ' 4 ' is assigned to Basque Country; and

- United Kingdom: the value ' 1 ' is assigned to England, Northern Ireland and Wales, and the value ' 2 ' is assigned to Scotland.

The variable labelled STRATUM contains information on the explicit strata used for sampling. Some of them were combined into larger units for policy or confidentiality reasons.

The school identification consists of two variables, which together form a unique identifier for each school:

- The country identification variable labelled COUNTRY. The country codes used in PISA are the ISO 3166 country codes; and

- The school identification variable labelled SCHOOLID.

\section{THE STUDENT QUESTIONNAIRE FILE}

\section{The responses to the student questionnaires}

The student files contain the original variables collected through the student context questionnaires, i.e. the compulsory student questionnaire and the two international options: the education career questionnaire and the ICT questionnaire.

The names that are used to represent these variables in the international database are directly related to the international version of the context questionnaires. Each variable name consists of seven characters.

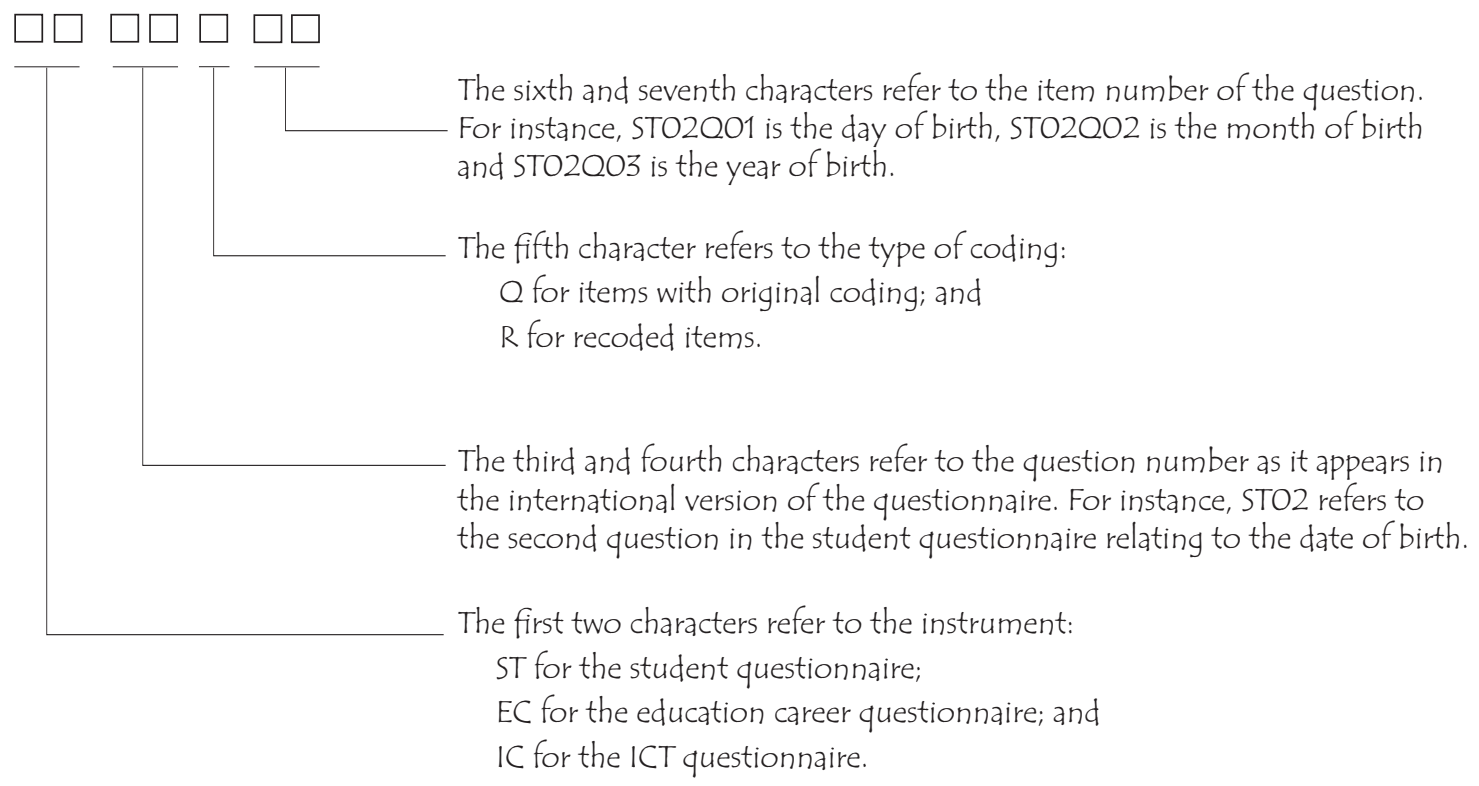


The weights and replicates

The weights

The variableW_FSTUWT is the final student weight. The sum of the weights constitutes an estimate of the size of the target population. If an analysis performed on the international level weighted by W_FSTUWT, large countries would have a stronger contribution to the results than small countries. Two country adjustment factors are included in the file:

- CNTFAC1 can be used for the computation of equal country weights. The weight W_FSTUWT*CNTFAC1 will give an equal weight of 1000 cases to each country so that smaller and larger countries contribute equally to the analysis. In order to obtain weights with equally weighted OECD countries, one needs to add the variable OECD indicating country membership as an additional multiplier (W_FSTUWT*CNTFAC1*OECD); and

- CNTFAC2 allows the computation of normalised or standardised weights. The weight W_FSTUWT*CNTFAC2 will give countries weights according to their sample sizes so that the sum of weights in each country is equal to the number of students in the database.

When analyses are carried out across countries, the country adjustment factors should also be applied to the Fay's replicates. The detail explanation of calculating weights is in Chapter 2.

\section{Fay's replicates}

Eighty Fay's replicates (W_FSTR1 to W_FSTR80) are included in the data files because they are needed to compute unbiased standard error estimates associated with any population parameter estimates. The standard error provides an estimate of the degree to which a statistic such as a mean score may be expected to vary about the true population mean. A 95 per cent confidence interval for a mean may be constructed in such a way that, if the sampling procedure were repeated a large number of times, and the sample statistic re-computed each time, the confidence interval would be expected to contain the population estimate 95 per cent of the time. Fay's replicates take into account the complex two-stage stratified sample design. If this is not done, one underestimates the standard error, thereby running the risk of obtaining statistical significance when in fact there is none. More detail description and application of Fay's replicates are found in Chapter 3.

\section{The student performance scores}

\section{Performance scores}

For each domain, i.e. mathematics, reading, science and problem solving, and four mathematics scales (change and relationships, space and shape, quantity and uncertainty), a set of five plausible values transformed to the international PISA metric are provided:

- PV1MATH to PV5MATH for mathematics ability;

- PV1MATH1 to PV5MATH1 for mathematics/ space and shape ability;

- PV1MATH2 to PV5MATH2 for mathematics/ change and relationships ability;

- PV1MATH3 to PV5MATH3 for mathematics/ uncertainty ability;

- PV1MATH4 to PV5MATH4 for mathematics/ quantity ability;

- PV1READ to PV5READ for reading ability; and

- PV1SCIE to PV5SCIE for science ability. 
The plausible values represent a set of random values for each selected student at random from an estimated ability distribution of students with similar item response patterns and backgrounds. They are intended to provide good estimates of parameters of student populations (such as country mean scores), rather than estimates of individual student performance.

Mathematics and problem solving plausible values were transformed to PISA scale using the data for the OECD countries participating in PISA 2003. This linear transformation used weighted data, with an additional adjustment factor so that each country contributes equally in the computation of the standardisation parameters.

The weighted average of five means and five standard deviations of plausible values for each scale is 500 and 100, respectively for the OECD countries but the means and variances of the individual plausible values are not exactly 500 and 100, respectively. The same transformation as for mathematics was applied to the four mathematics sub-scales.

PISA 2003 reading and science plausible values were mapped to PISA 2000 scale and the PISA 2000 transformation, that gives OECD mean 500 and standard deviation of 100 to the reading and science scales in PISA 2000.

For a full description of plausible values can be found in Chapter 5 and the application of plausible values for analysis is in Chapter 7.

\section{The student questionnaire indices}

Several of PISA's measures reflect indices that summarise students' responses. Two types of indices are provided in the student questionnaire file. Simple indices are constructed through the arithmetical transformation or recoding of one or more items. Scale indices are constructed through the scaling of items. For description of PISA student indices, see Appendix 9. The details on the methods and the reliabilities of the indices see the PISA 2003 Technical Report (OECD, forthcoming).

\section{Item deletions}

In the student questionnaire, Question 2 concerning students' dates of birth and Question 18(a) to (e) concerning possessions at home were deleted from the student data file. Question 11(a) to (e) were recoded into ST11R01 and Question 13(a) to (e) were recoded into ST13R01. Question 35(a) was used in computation of minutes of mathematics per week (MMINS).

In the educational career questionnaire, Question 8 was used to create the PISA 2003 index of expected occupational status at the age of 30 (BSMJ).

\section{THE SCHOOL FILE}

\section{The responses to the school questionnaire}

The school files contain the original variables collected through the school context questionnaire.

The names which are used to represent these variables in the international database are directly related to the international version of the school questionnaire. Each variable name consists of seven characters. 


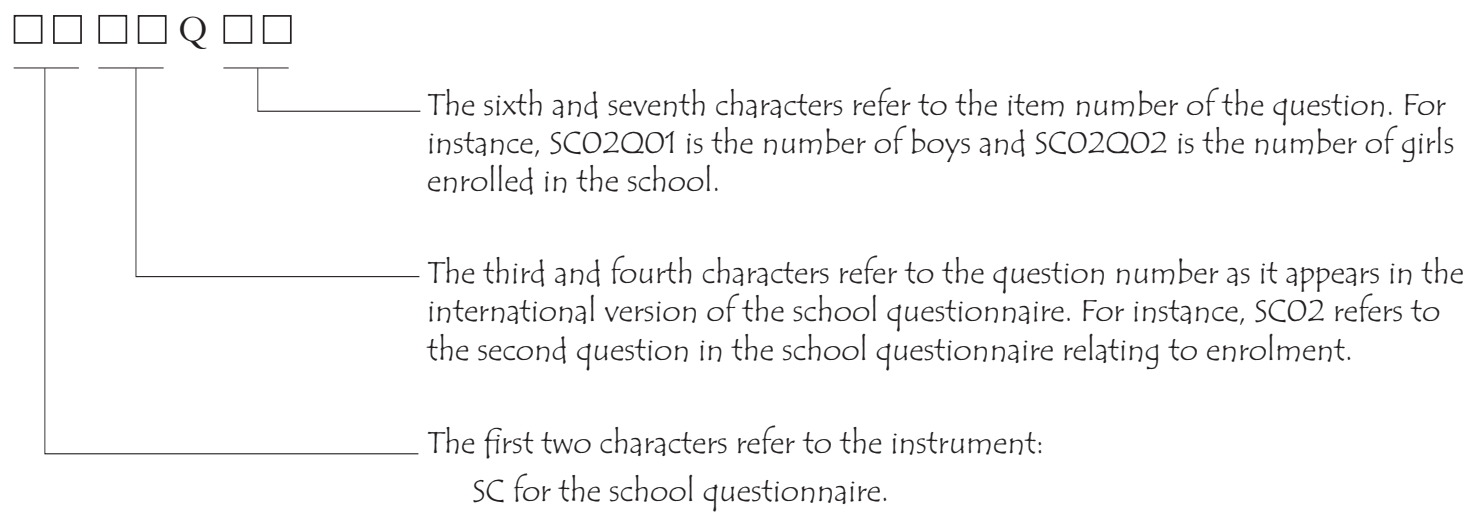

\section{The school weight}

The school base weight, SCWEIGHT, which has been adjusted for school non-response, is provided at the end of the school file. PISA uses an age-based sample instead of a grade-based sample. Additionally, the PISA sample of schools in some countries included primary schools, lower secondary schools, upper secondary schools, or even special education schools. For these two reasons, it is difficult to conceptually define the school population, except this it is the population of schools with at least one 15-year-old student. While in some countries, the population of schools with 15-year-olds is similar to the population of secondary schools, in other countries these two populations of schools are very different.

A recommendation is to analyse the school data at the student level. From a practical point of view, it means that the school data should be imported into the student data file. From a theoretical point of view, while it is possible to estimate the percentages of schools following a specific school characteristic, it is not meaningful. Instead, the recommendation is to estimate the percentages of students following the same school characteristic. For instance, the percentages of private schools versus public schools will not be estimated, but the percentages of students attending private school versus the percentage of students attending public schools will.

As school data will be imported in the student data file, the final weight and the 80 Fay's replicates will be used in a similar what to how they are used for the student data.

\section{The school questionnaire indices}

Several of PISA's measures reflect indices that summarise school principals' responses. Two types of indices are provided in the school questionnaire file. Simple indices are constructed through the arithmetical transformation or recoding of one or more items. Scale indices are constructed through the scaling of items. For a description of PISA indices, see Appendix 9. The details on the methods and the reliabilities of the indices see the PISA 2003 Technical Report (OECD, forthcoming).

\section{THE FILE WITH THE STUDENT TEST DATA}

The file with the test data contains individual students' responses to all items used for the international item calibration and in the generation of the plausible values. All item responses included in this file have a one-digit format, which contains the score for the student on that item. 
The PISA items are organised into units. Each unit consists of a piece of text or related texts, followed by one or more questions. Each unit is identified by a short label and by a long label. The units' short labels consist of four characters. The first character is R, M, S or X respectively for reading, mathematics, science or problem solving. The three next characters indicate the unit name. For example, M124 is a mathematics unit called Walking. The full item label (usually seven-digit) represents each particular question within a unit. Thus items within a unit have the same initial four characters: all items in the unit Walking begin with 'M124', plus a question number: for example, the third question in the Walking unit is M124Q03.

Users may notice that the question numbers in some cases are not sequential, and in other cases, that question numbers are missing. The initial item numbering was done before the field trial, with some changes occurring after it (the field trial took place a year before the main assessment). For example, during the development of the main study instruments, some items were re-ordered within a unit, while others were deleted from the item pool.

In this file, the items are sorted by domain and alphabetically by short label within domain. This means that the mathematics items appear at the beginning of the file, followed by the reading items, the science items and then the problem solving items. Within domains, units with smaller numeric labels appear before those with larger label, and within each unit, the first question will precede the second, and so on.

For items omitted by students, embedded missing and non-reached missing items were differentiated. All consecutive missing values clustered at the end of each booklet were replaced by a non-reached code ' $r$ ', except for the first value of the missing series. Embedded and non-reached missing items were treated differently in the scaling. Non-reached items for students who were reported to have left the session earlier than expected were considered not applicable in all analyses.

\section{Recoding of the assessment items}

Some of the items needed to be recoded prior to the national and international scaling processes:

- Double-digit coded items (mathematics, science and problem solving only) were truncated by retaining only the first digit, which corresponds to the score initially assigned to the item. An exception is item M462Q01 where code 13 was recoded into 0;

- Other items were recoded and/or combined. These items have been re-labelled. The character ' $\mathrm{T}$ ' was added to the end of the previous short label for such items;

- Numerical variables were recoded into scores, i.e. incorrect answer (0), correct answer (1), missing answer (9) or not applicable (7);

- Some questions consisted of several true/false or yes/no items. One question was also composed of several multiple-choice items (M833Q01). These items were combined into new variables. The new codes correspond to the number of correct answers on the subset of items; and

- Finally, four items, which comprised a subset of items (R219Q01, M192Q01, M520Q01 and M520Q03 ), were combined to form new variables. The combined codes correspond to the number of correct answers to each of the sub-items included in these four items. 


\section{Item deletions}

Assessment data were initially scaled by country, and item parameter estimates were analysed across countries. During the item adjudication process, some items were flagged for particular countries and a consultation process took place to perform additional checks on these items. The consultations resulted in the deletion of a few items at the national level and two items at the international level. At the international level the two deleted items were S327Q02 and M434Q01T. The nationally deleted items are listed in Table A1.1. These deleted items at the national level, as well as two deleted items at the international level were recoded as not applicable and were not included in either the international scaling or the generation of plausible values.

Table A1.1 - Items deleted at the national level

\begin{tabular}{|c|c|c|c|}
\hline Item & Country & Item & Country \\
\hline M144Q03 & Iceland (booklet 4 only) & R219Q01E & Tunisia \\
\hline M155Q01 & Korea & R219Q01T & Tunisia \\
\hline M179Q01T & Italy (Italian version only) & R227Q01 & $\begin{array}{l}\text { Spain (Catalonian and } \\
\text { Castilian versions), }\end{array}$ \\
\hline M273Q01 & Denmark (booklet 7 only) & S131Q02T & Russia \\
\hline M402Q02 & Hungary & S252Q02 & $\begin{array}{l}\text { Spain (Castilian, Galician, } \\
\text { and Valencian versions) }\end{array}$ \\
\hline M442Q02 & Uruguay & S268Q02T & Norway \\
\hline M603Q02 & Canada & S326Q01 & Portugal \\
\hline M704Q01T & Switzerland (Italian version only) & $\mathrm{X} 414 \mathrm{Q} 01$ & Russia \\
\hline M800Q01 & Uruguay & $\mathrm{X} 603 \mathrm{Q} 02 \mathrm{~T}$ & Italy (Italian version only) \\
\hline R055Q03 & $\begin{array}{l}\text { Austria, Luxembourg (German } \\
\text { version only), Germany, } \\
\text { Switzerland (German version } \\
\text { only), Belgium (German version } \\
\text { only), Italy (German version only), } \\
\text { Liechtenstein }\end{array}$ & X603Q03 & Italy (Italian version only) \\
\hline R102Q04a & Korea & R111Q6B & Tunisia \\
\hline
\end{tabular}

\section{International scores assigned to the items}

The final scores allocated to the different categories are presented in Appendix 10. The codes are grouped according o the scores they were assigned for the final international calibration.

\section{ADDITIONAL TECHNICAL INFORMATION AND GLOSSARY}

\section{Codebook}

A codebook is a document that identifies the variables and all possible values associated with them. In addition to the name of the variable, it also shows the variable label, all possible responses (i.e. in the case of multiple-choice items it shows the values for all alternatives and the full label of each alternative), type of variable (e.g. string or numeric) and the columns where the values are shown in the actual data file. 


\section{Compendia}

Compendia include a set of tables showing statistics for every item included in the questionnaires, and the relationship with performance. The tables show the percentage of students per category of response and the performance for the group of students in each category of response.

\section{Double-digit coding}

Students' responses could give valuable information about their ideas and thinking, besides being correct or incorrect. The marking guides for mathematics and science included a system of two-digit coding for marking so that the frequency of various types of correct and incorrect responses could be recoded. The first digit is the actual score. The second digit is used to categorise the different kinds of responses on the basis of the strategies used by the student to answer the item. There are two main advantages of using double-digit codes. Firstly, more information can be collected about students' misconceptions, common errors, and different approaches to solving problems. Secondly, double-digit coding allows a more structured way of presenting the codes, clearly indicating the hierarchical levels of groups of codes. The assessment data files including the second digit were available to national centres.

\section{ISO 3166}

For International Standardization Organization (ISO) country codes, see http://www.iso.org.

\section{SAS ${ }^{\circledR}$}

$\mathrm{SAS}^{\circledR}$ is a statistical package. For further information, see http://www.sas.com.

\section{SPSS $^{\circledR}$}

SPSS $^{\circledR}$ is a statistical package. For further information, see http://www.spss.com.

\section{WesVar ${ }^{\circledR}$}

WesVar ${ }^{\circledR}$ is a statistical package that computes estimates and their variance estimates from survey data using replication methods. The information generated can then be used to estimate sampling errors for different types of survey statistics. It can be used in conjunction with a wide range of complex sample designs, including multistage, stratified, and unequal probability samples. For further information, see http://www.westat.com/wesvar.

1. The responses from open-ended items could give valuable information about students' ideas and thinking, which could be fed back into curriculum planning. For this reason, the marking guides for these items in mathematics and science were designed to include a two-digit marking so that the frequency of various types of correct and incorrect response could be recorded. The first digit was the actual score. The second digit was used to categorise the different kings of response on the basis of the strategies used by the student to answer the item. The international database includes only the first digit. 


\section{APPENDIX 2 - STUDENT QUESTIONNAIRE}

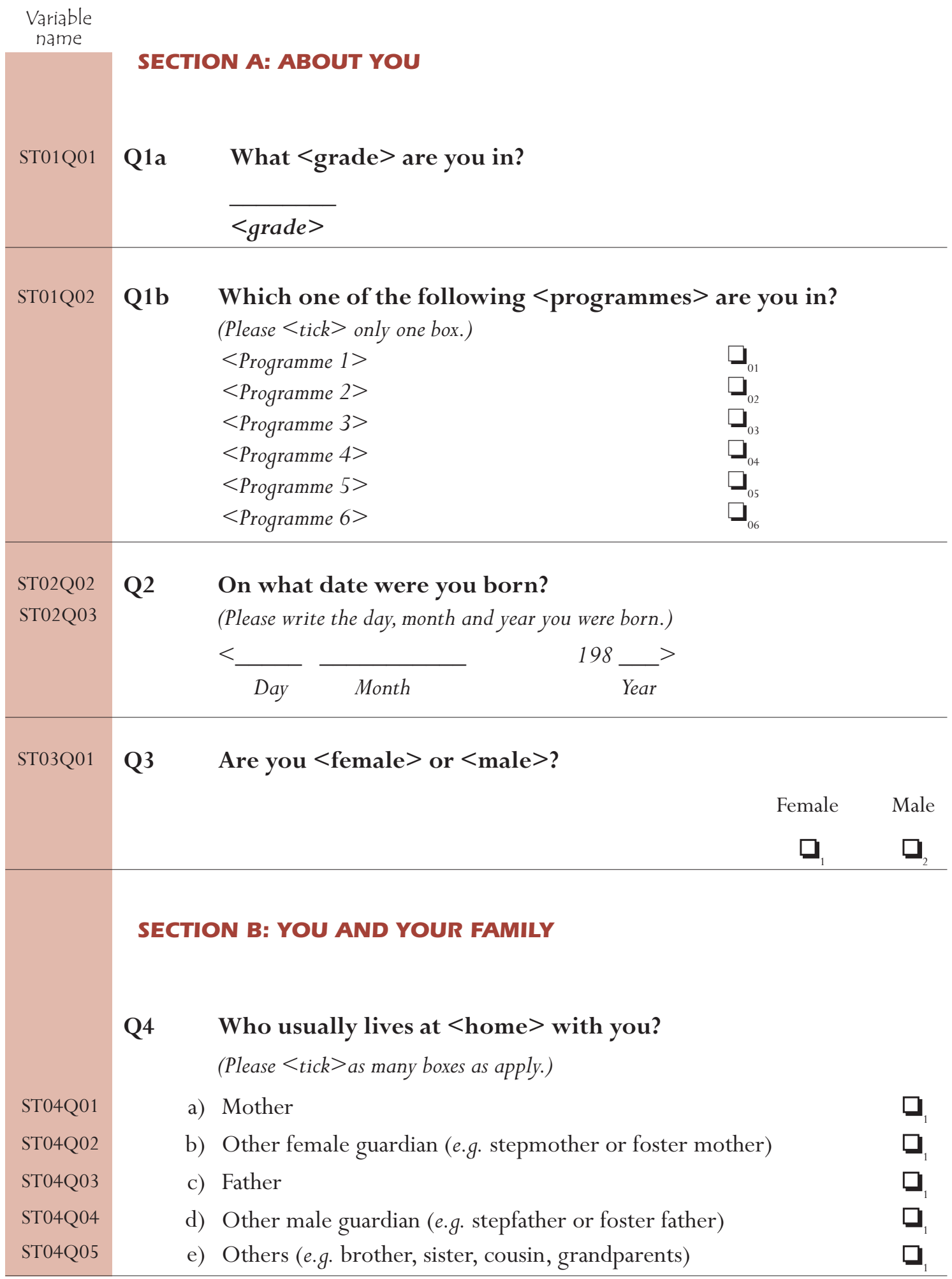




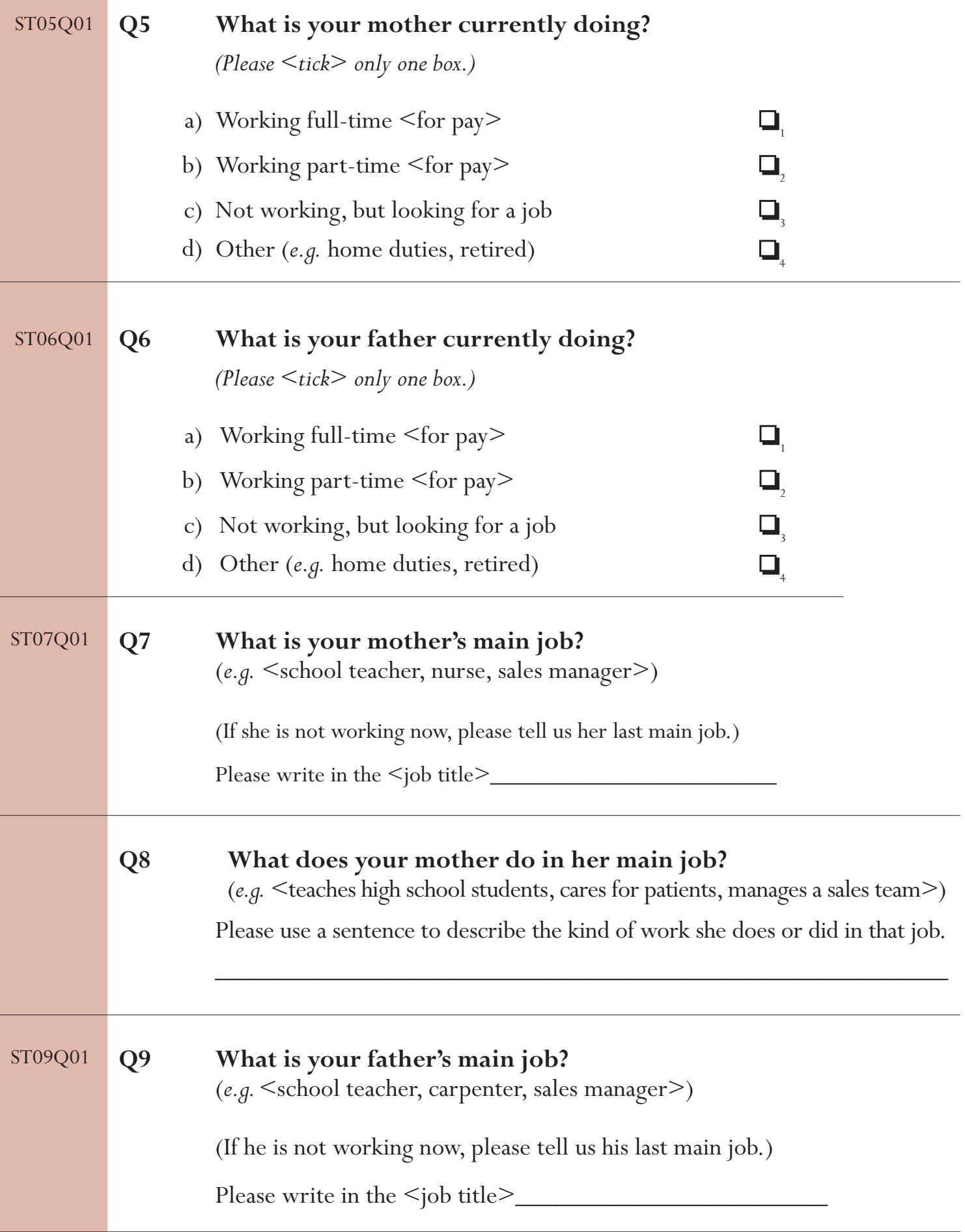

Q10 What does your father do in his main job?

(e.g. <teaches high school students, builds houses, manages a sales team>)

Please use a sentence to describe the kind of work he does or did in that job. 


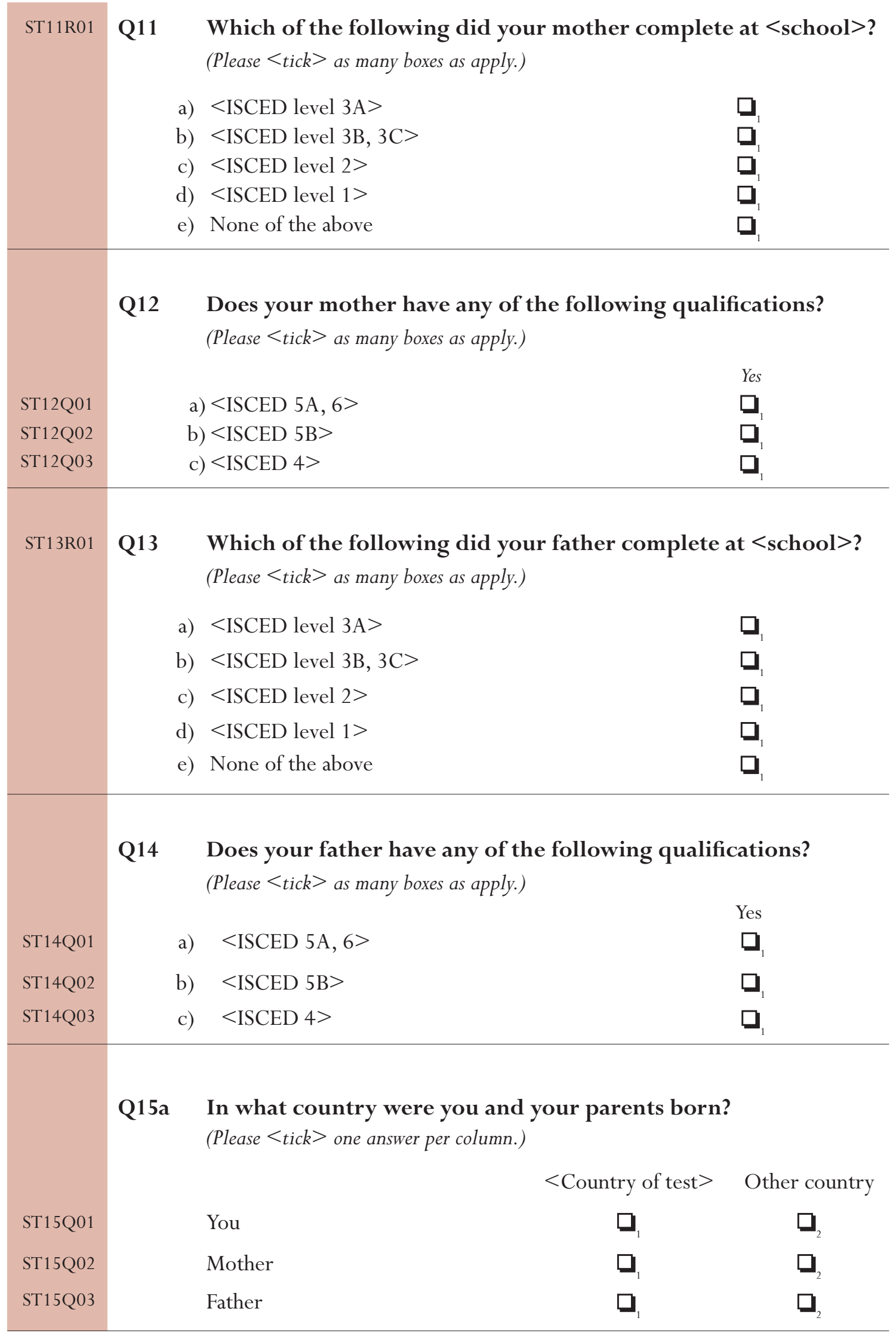




\begin{tabular}{|c|c|c|c|}
\hline \multirow[t]{2}{*}{ ST15Q04 } & \multirow[t]{2}{*}{ Q15b } & \multicolumn{2}{|c|}{$\begin{array}{l}\text { If you were NOT born in }<\text { country of test }>\text {, how old were you } \\
\text { when you arrived in }<\text { country of test }>\text { ? }\end{array}$} \\
\hline & & & Years \\
\hline \multirow[t]{8}{*}{ ST16Q01 } & \multirow[t]{8}{*}{ Q16 } & \multicolumn{2}{|l|}{$\begin{array}{l}\text { What language do you speak at home most of the time? } \\
\text { (Please }<\text { tick }>\text { only one box.) }\end{array}$} \\
\hline & & $<$ Test language $>$ & $\square_{01}$ \\
\hline & & $<$ Other official national languages $>$ & $\square_{02}$ \\
\hline & & $<$ Other national dialects or languages $>$ & $\square_{03}$ \\
\hline & & $<$ Other language $1>$ & $\square_{04}$ \\
\hline & & $<$ Other language $2>$ & $\square_{05}$ \\
\hline & & $<$ Other language $3>$ & $\square_{06}$ \\
\hline & & Other languages & $\square$ \\
\hline
\end{tabular}

Q17 Which of the following do you have in your home?

(Please $<$ tick $>$ as many boxes as apply.)

ST17Q01

ST17Q02

ST17Q03

ST17Q04

ST17Q05

ST17Q06

ST17Q07

ST17Q08

ST17Q09

ST17Q10

ST17Q11

ST17Q12

ST17Q13 a) A desk to study at

b) A room of your own

c) A quiet place to study

d) A computer you can use for school work

e) Educational software

f) A link to the Internet

g) Your own calculator

h) Classic literature (e.g. $<$ Shakespeare $>$ )

i) Books of poetry

j) Works of art (e.g. paintings)

k) Books to help with your school work

l) A dictionary

m) A dishwasher

n) <Country-specific item 1>

o) < Country-specific item 2>

p) < Country-specific item 3> 
Q18 How many of these do you have at your home?

(Please $<$ tick $>$ only one box in each row.)

None One Two Three or more
a) $<$ Cellular $>$ phone
b) Television
c) Computer
d) Motor car
e) Bathroom

$\begin{array}{llll}\square_{1} & \square_{2} & \square_{3} & \square \\ \square_{1} & \square_{2}^{2} & \square_{3}^{3} & \square \\ \square_{1} & \square_{2} & \square_{3} & \square \\ \square_{1} & \square_{2} & \square_{3} & \square \\ \square_{1} & \square_{2} & \square_{3} & \square\end{array}$

ST19Q01 Q19 How many books are there in your home?

There are usually about $<40$ books per metre $>$ of shelving. Do not include magazines, newspapers or your schoolbooks.

(Please $<$ tick $>$ only one box.)

$0-10$ books

11-25 books

26-100 books

101-200 books

201-500 books

More than 500 books

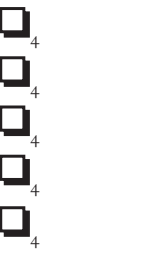

$101-200$ books
$201-500$ books
More than 500 books

SECTION C: YOUR EDUCATION

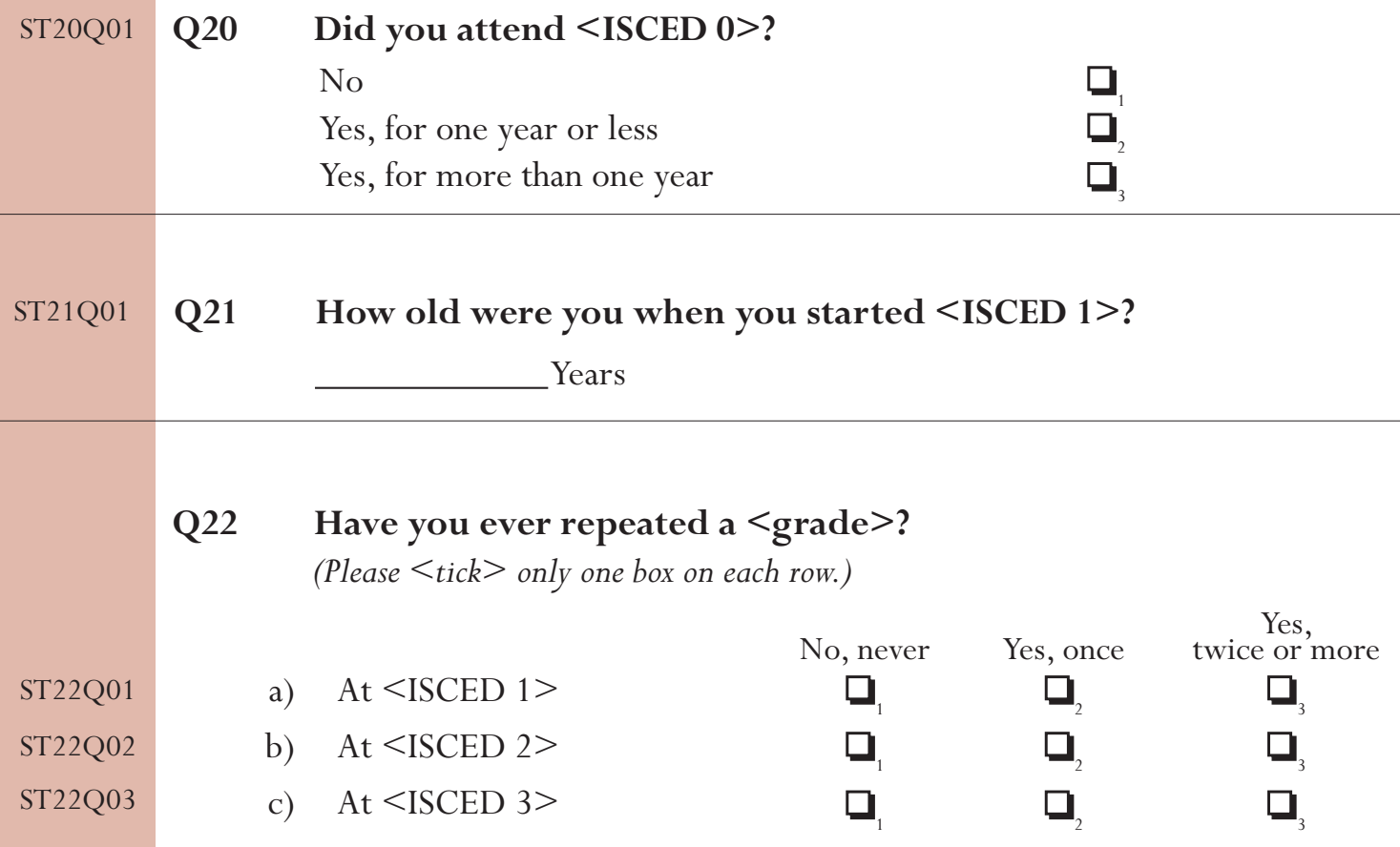


Q23 Which of the following do you expect to complete?

(Please $<$ tick $>$ as many as apply.)

ST23Q01

ST23Q02

ST23Q03

ST23Q04

ST23Q05

ST23Q06

a) $<$ ISCED level 2>

b) $<$ ISCED level 3B or $\mathrm{C}>$

c) $<$ ISCED level 3A>

d) $<$ ISCED level 4>

e) $<$ ISCED level 5B $>$

f) $<$ ISCED level 5A or 6>

Q24 Thinking about what you have learned in school:

To what extent do you agree with the following statements?

(Please $<$ tick $>$ only one box on each row.)

a) School has done little to prepare me for adult life when I leave school.

ST24Q02

ST24Q03

ST24Q04

b) School has been a waste of time.

c) School has helped give me confidence to make decisions.

d) School has taught me things which Strongly
agree Agree Disagree $\begin{gathered}\text { Strongly } \\ \text { disagree }\end{gathered}$ could be useful in a job.

\begin{tabular}{|c|c|c|c|}
\hline \multicolumn{4}{|c|}{ SECTION D: YOUR SCHOOL } \\
\hline & Q25 & $\begin{array}{l}\text { Which of the following are reasons why you attend this scl } \\
\text { (Please }<\text { tick }>\text { as many as apply.) }\end{array}$ & ol? \\
\hline ST25Q01 & a) & This is the local school for students who live in this area. & $\square_{1}$ \\
\hline ST25Q02 & b) & This school is known to be a better school than others in the area. & $\square_{1}$ \\
\hline ST25Q03 & c) & This school offers specific study programmes. & $\square_{1}$ \\
\hline ST25Q04 & d) & This school has a particular religious philosophy. & $\square$ \\
\hline ST25Q05 & e) & Previously, family members attended this school. & $\square_{1}$ \\
\hline ST25Q06 & f) & Other reasons. & $\square$ \\
\hline
\end{tabular}


Q26 Thinking about the teachers at your school:

To what extent do you agree with the following statements?

(Please $<$ tick $>$ only one box in each row.)

ST26Q01

ST26Q02

ST26Q03

ST26Q04

ST26Q05

a) Students get along well with most teachers.

Strongly
agree Agree Disagree $\begin{aligned} & \text { Strongly } \\ & \text { disagree }\end{aligned}$

b) Most teachers are interested in students' well-being.
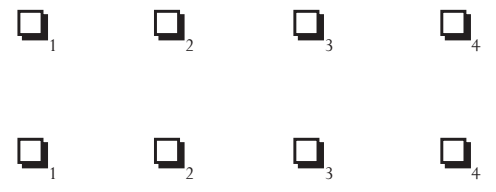

c) Most of my teachers really listen to what I have to say.

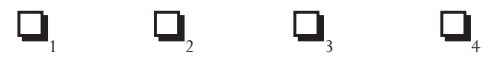

d) If I need extra help, I will receive it from my teachers.

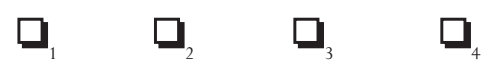

e) Most of my teachers treat me fairly.

Q27 My school is a place where:

(Please $<$ tick $>$ only one box in each row.)

Strongly
agree Agree Disagree $\begin{aligned} & \text { Strongly } \\ & \text { disagree }\end{aligned}$

ST27Q01

ST27Q02

ST27Q03

ST27Q04

ST27Q05

ST27Q06

a) I feel like an outsider (or left out of things).

$\square_{1} \quad \square_{2} \quad \square_{3} \quad \square_{4}$

b) I make friends easily.

c) I feel like I belong.

d) I feel awkward and out of place.

e) Other students seem to like me.

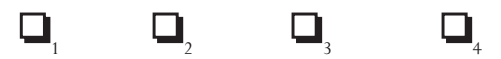

$\begin{array}{llll}\square_{1} & \square_{2} & \square_{3} & \square_{4}\end{array}$

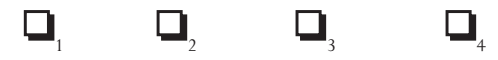

f) I feel lonely.

$\square_{1} \square_{2} \quad \square_{3} \quad \square_{4}$

ST28Q01 Q28 In the last two full weeks you were in school, how many times did you arrive late for school?

(Please $<$ tick $>$ only one box)

None

One or two times

Three or four times

Five or more times 
Q29 The following question asks about the time you spend studying and doing different kinds of homework outside of your regular classes. This should include all of your studying and homework.

On average, how many hours do you spend each week on the following?

When answering include time at the weekend too.

ST29Q01

ST29Q02

ST29Q03

ST29Q04

ST29Q05

ST29Q06

\section{ST2906}

ST30Q01

ST30Q02

ST30Q03

ST30Q04

ST30Q05

ST30Q06

ST30Q07

ST30Q08 a) Homework or other study set by your teachers

b) $<$ Remedial classes $>$ at school

c) $<$ Enrichment classes $>$ at school

d) Work with a $<$ tutor $>$

e) Attending < out-of-school> classes

f) Other study hours per week hours per week hours per week hours per week hours per week hours per week

\section{SECTION E: LEARNING MATHEMATICS}

Q30 Thinking about your views on mathematics:

\section{To what extent do you agree with the following statements?}

(Please $<$ tick $>$ only one box in each row.) Strongly
agree Agree Disagree $\begin{aligned} & \text { Strongly } \\ & \text { disagree }\end{aligned}$

a) I enjoy reading about mathematics.

b) Making an effort in mathematics is worth it because it will help me in the work that I want to do later on.

c) I look forward to my mathematics lessons.

d) I do mathematics because I enjoy it.

e) Learning mathematics is worthwhile for me because it will improve my career $<$ prospects, chances $>$.

f) I am interested in the things I learn in mathematics.

g) Mathematics is an important subject for me because I need it for what I want to study later on.

h) I will learn many things in mathematics that will help me get a job. 


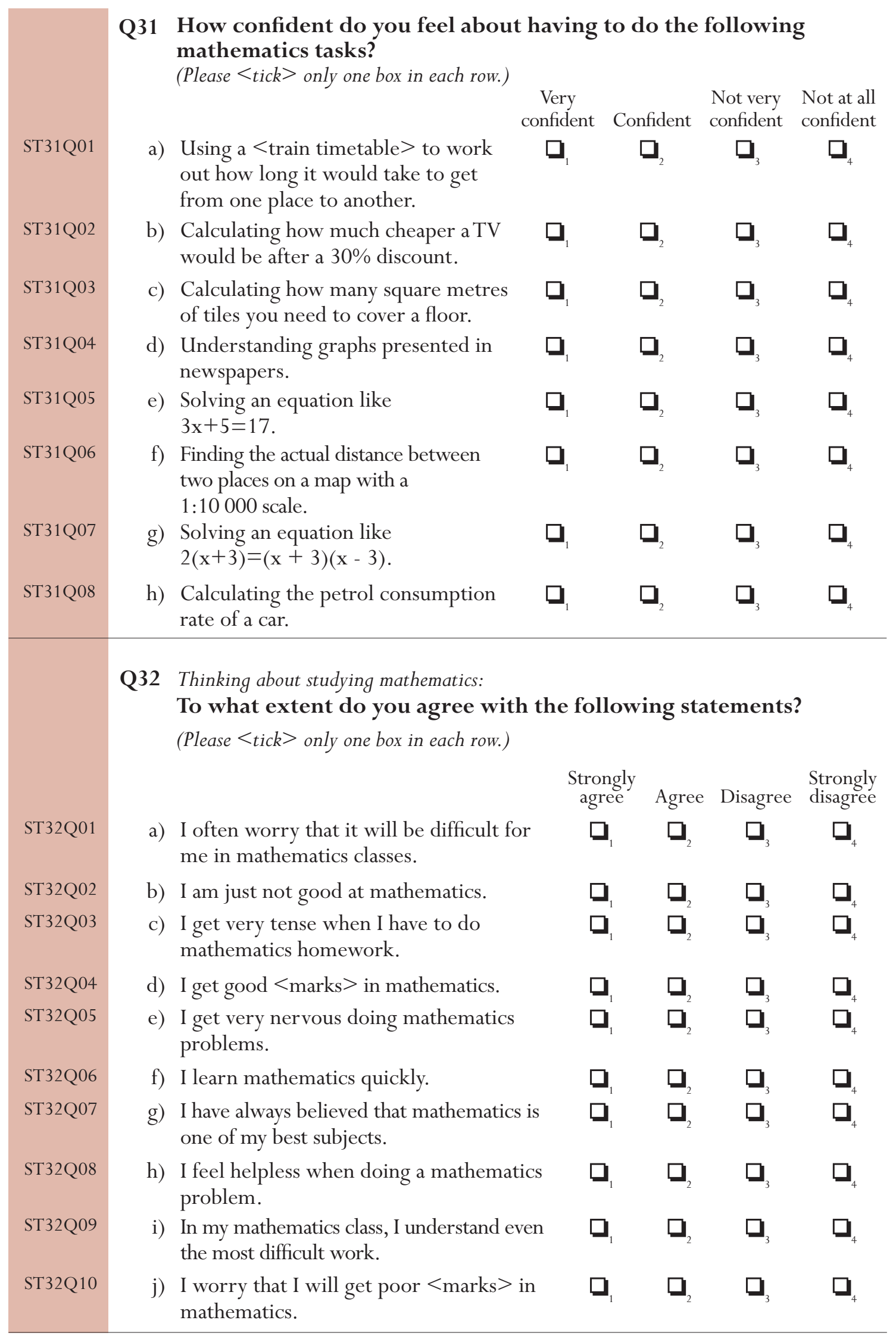

Using a $<$ train timetable $>$ to wor out how long it would take to get from one place to another.

Calculating how many square metres Un nou need to cover a floor.

sented in

Solving an equation like

two places on a map with a

1:10 000 scale.

Solving an equat

Calculating the petrol consumption rate of a car.

Q32 Thinking about studying mathematics:

To what extent do you agree with the following statements?

(Please $<$ tick $>$ only one box in each row.)

\section{Strongly Strongly}

ST32Q02

b) I am just not good at mathematics.

c) I get very tense when I have to do mathematics homework.

I get good $<$ marks $>$ in mathematics

I get very nervous doing mathematics problem.

In my mathematics class, I understand even mathematics. 
Q33 The following question asks about the time you spend studying and doing mathematics homework outside of your regular mathematics classes.

On average, how much time do you spend each week on the following?

When answering include time at the weekend too.

ST33Q01

ST33Q02

ST33Q03

ST33Q04

ST33Q05

ST33Q06

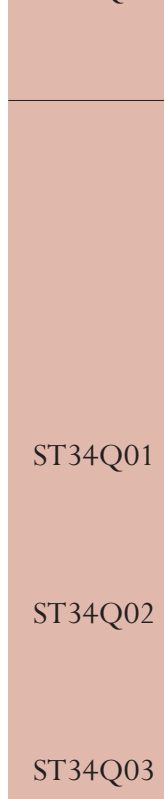

ST34Q04

ST34Q05

ST34Q06

ST34Q07 a) Homework or other study set by your mathematics teacher

b) $<$ Remedial classes $>$ in mathematics at school

c) $<$ Enrichment classes $>$ in mathematics at school

d) Work with a $<$ mathematics tutor $>$

e) Attending < out-of-school> mathematics classes

f) Other mathematics activities (e.g. < mathematics competitions, mathematics club $>$ ) hours per week hours per week hours per week hours per week hours per week hours per week

Q34 There are different ways of studying mathematics.

\section{To what extent do you agree with the following statements?}

(Please $<$ tick $>$ only one box in each row.) Strongly
agree Agree Disagree $\begin{gathered}\text { Strongly } \\ \text { disagree }\end{gathered}$

a) When I study for a mathematics test, I try to work out what are the most

$\square$ $\square \quad \square \quad \square$ important parts to learn.

b) When I am solving mathematics problems, I often think of new ways to get the answer.

c) When I study mathematics, I make myself check to see if I remember the work I have already done.

d) When I study mathematics, I try to figure out which concepts I still have not understood properly.

e) I think how the mathematics I have learnt can be used in everyday life.

f) I go over some problems in mathematics so often that I feel as if I could solve them in my sleep.

g) When I study for mathematics, I learn as much as I can off by heart. 


\begin{tabular}{|c|c|c|c|c|c|c|}
\hline & & & $\begin{array}{l}\text { Strongly } \\
\text { agree }\end{array}$ & Agree & Disagree & $\begin{array}{l}\text { Strongly } \\
\text { disagree }\end{array}$ \\
\hline ST34Q08 & h) & $\begin{array}{l}\text { I try to understand new concepts in } \\
\text { mathematics by relating them to things } \\
\text { I already know. }\end{array}$ & $\square_{1}$ & $\square_{2}$ & $\square_{3}$ & $\square_{4}$ \\
\hline ST34Q09 & i) & $\begin{array}{l}\text { In order to remember the method for } \\
\text { solving a mathematics problem, I go } \\
\text { through examples again and again. }\end{array}$ & $\square$ & $\square_{2}$ & $\square_{3}$ & $\square_{4}$ \\
\hline ST34Q10 & j) & $\begin{array}{l}\text { When I cannot understand something in } \\
\text { mathematics, I always search for more } \\
\text { information to clarify the problem. }\end{array}$ & $\square$ & $\square_{2}$ & $\square_{3}$ & $\square_{4}$ \\
\hline ST34Q11 & k) & $\begin{array}{l}\text { When I am solving a mathematics } \\
\text { problem, I often think about how the } \\
\text { solution might be applied to other } \\
\text { interesting questions. }\end{array}$ & $\square$ & $\square_{2}$ & $\square_{3}$ & $\square_{4}$ \\
\hline ST34Q12 & l) & $\begin{array}{l}\text { When I study mathematics, I start by } \\
\text { working out exactly what I need to } \\
\text { learn. }\end{array}$ & $\square$ & $\square_{2}$ & $\square_{3}$ & $\square_{4}$ \\
\hline ST34Q13 & $\mathrm{m})$ & $\begin{array}{l}\text { To learn mathematics, I try to } \\
\text { remember every step in a procedure. }\end{array}$ & $\square$ & $\square_{2}$ & $\square_{3}$ & $\square_{4}$ \\
\hline \multirow[t]{4}{*}{ ST34Q14 } & n) & $\begin{array}{l}\text { When learning mathematics, I try to } \\
\text { relate the work to things I have learnt } \\
\text { in other subjects. }\end{array}$ & $\square$ & $\square_{2}$ & $\square_{3}$ & $\square_{4}$ \\
\hline & \multicolumn{6}{|c|}{ SECTION F: YOUR <MATHEMATICS> CLASSES } \\
\hline & \multirow[t]{2}{*}{ Q35a } & \multicolumn{5}{|c|}{ How many minutes, on average, are there in a $<$ class period $>$ ? } \\
\hline & & Minutes in a $<$ class period $>$ : & & & minutes & \\
\hline \multirow[t]{2}{*}{ ST35Q02 } & \multirow[t]{2}{*}{ Q35b } & \multicolumn{5}{|c|}{$\begin{array}{l}\text { In the last full week you were in school, how many }<\text { class } \\
\text { periods }>\text { did you spend in }<\text { mathematics }>\text { ? }\end{array}$} \\
\hline & & Number of mathematics $<$ class periods $>$ : & & & \multicolumn{2}{|c|}{$<$ class periods $>$} \\
\hline \multirow[t]{2}{*}{ ST35Q03 } & \multirow[t]{2}{*}{ Q35c } & \multicolumn{5}{|c|}{$\begin{array}{l}\text { In the last full week you were in school, how many } \\
<\text { class periods }>\text { did you have }<\text { in total }>\text { ? }\end{array}$} \\
\hline & & $\begin{array}{l}\text { Number of } \mathbf{A L L}<\text { class periods }> \\
\text { (including your }<\text { mathematics }>\text { classes): }\end{array}$ & & & \multicolumn{2}{|c|}{$<$ class periods $>$} \\
\hline ST36Q01 & Q36 & \multicolumn{5}{|c|}{$\begin{array}{l}\text { On average, about how many students attend your } \\
<\text { mathematics }>\text { class? }\end{array}$} \\
\hline
\end{tabular}


Q37 Thinking about your $<$ mathematics $>$ classes: To what extent do you agree with the following statements?

(Please $<$ tick $>$ only one box in each row.)

ST37Q01

ST37Q02

ST37Q03

ST37Q04

ST37Q05

ST37Q06

ST37Q07

ST37Q08

ST37Q09

ST37Q10

a) I would like to be the best in my class in mathematics.

b) In mathematics I enjoy working with other students in groups.

c) I try very hard in mathematics because I want to do better in the exams than the others.

d) When we work on a project in mathematics, I think that it is a good idea to combine the ideas of all the students in a group.

e) I make a real effort in mathematics because I want to be one of the best.

f) I do my best work in mathematics when I work with other students.

g) In mathematics I always try to do better than the other students in my class.

h) In mathematics, I enjoy helping others to work well in a group.

i) In mathematics I learn most when I work with other students in my class.

j) I do my best work in mathematics when I try to do better than others.

Strongly agree

Agree

Disagree

Strongly

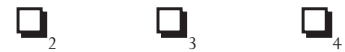

$\square$

$\square$

$\square$<smiles>[O]</smiles>

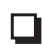

$\square$<smiles>[O]</smiles>

$\square$<smiles>[Hg]</smiles><smiles>[O]</smiles><smiles>[Hg]</smiles><smiles>[O]</smiles>

$\square$

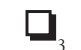<smiles>[18OH]</smiles><smiles>[Hg]</smiles><smiles>[Hg]</smiles><smiles>[Hg]</smiles>
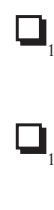

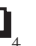

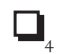

$\square$

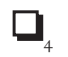

$\square_{4}$

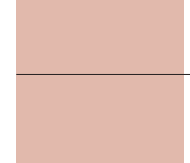

Q38 How often do these things happen in your < mathematics $>$ lessons?

(Please $<$ tick $>$ only one box in each row.)

$$
\begin{array}{cccc}
\text { Every } & \text { Most } & \text { Some } & \begin{array}{c}
\text { Never or } \\
\text { hardly } \\
\text { lesson }
\end{array}
\end{array}
$$

ST38Q01

ST38Q02

ST38Q03 a) The teacher shows an interest in every student's learning.

b) Students don't listen to what the teacher says.

c) The teacher gives extra help when students need it. $\square$
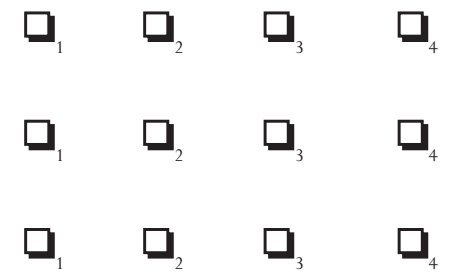


\begin{tabular}{|c|c|c|c|c|c|c|}
\hline & & & $\begin{array}{l}\text { Every } \\
\text { lesson }\end{array}$ & $\begin{array}{l}\text { Most } \\
\text { lessons }\end{array}$ & $\begin{array}{l}\text { Some } \\
\text { lessons }\end{array}$ & $\begin{array}{c}\text { Never or } \\
\text { hardly } \\
\text { ever }\end{array}$ \\
\hline ST38Q04 & d) & $\begin{array}{l}\text { Students work from books and other } \\
\text { printed material. }\end{array}$ & $\square$ & $\square_{2}$ & $\square_{3}$ & $\square_{4}$ \\
\hline ST38Q05 & e) & $\begin{array}{l}\text { The teacher helps students with their } \\
\text { learning. }\end{array}$ & $\square_{1}$ & $\square_{2}$ & $\square_{3}$ & $\square_{4}$ \\
\hline ST38Q06 & f) & There is noise and disorder. & $\square$ & $\square$ & $\square$ & $\square$ \\
\hline ST38Q07 & g) & $\begin{array}{l}\text { The teacher continues teaching until the } \\
\text { students understand. }\end{array}$ & $\square$ & $\square_{2}$ & $\square_{3}$ & $\square$ \\
\hline ST38Q08 & h) & $\begin{array}{l}\text { The teacher has to wait a long time for } \\
\text { students to < quieten down }>\text {. }\end{array}$ & $\square_{1}$ & $\square_{2}$ & $\square_{3}$ & $\square_{4}$ \\
\hline ST38Q09 & i) & Students cannot work well. & $\square$ & $\square$ & $\square$ & $\square$ \\
\hline ST38Q10 & & $\begin{array}{l}\text { The teacher gives students an opportunity } \\
\text { to express opinions. }\end{array}$ & $\square_{1}$ & $\square_{2}$ & $\square_{3}$ & $\square_{4}$ \\
\hline ST38Q11 & k) & $\begin{array}{l}\text { Students don't start working for a long } \\
\text { time after the lesson begins. }\end{array}$ & $\square_{1}$ & $\square_{2}$ & $\square_{3}$ & $\square$ \\
\hline
\end{tabular}





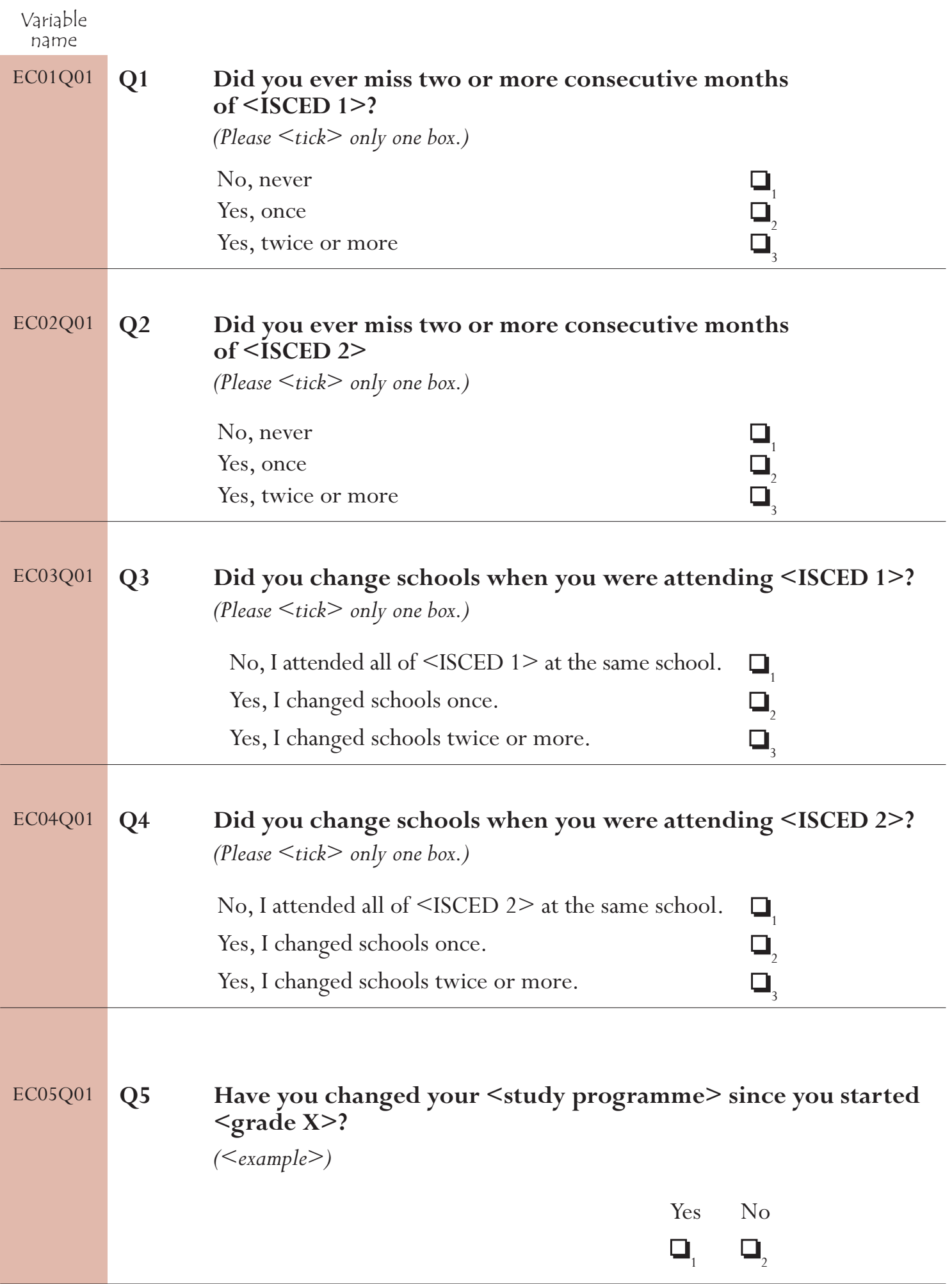




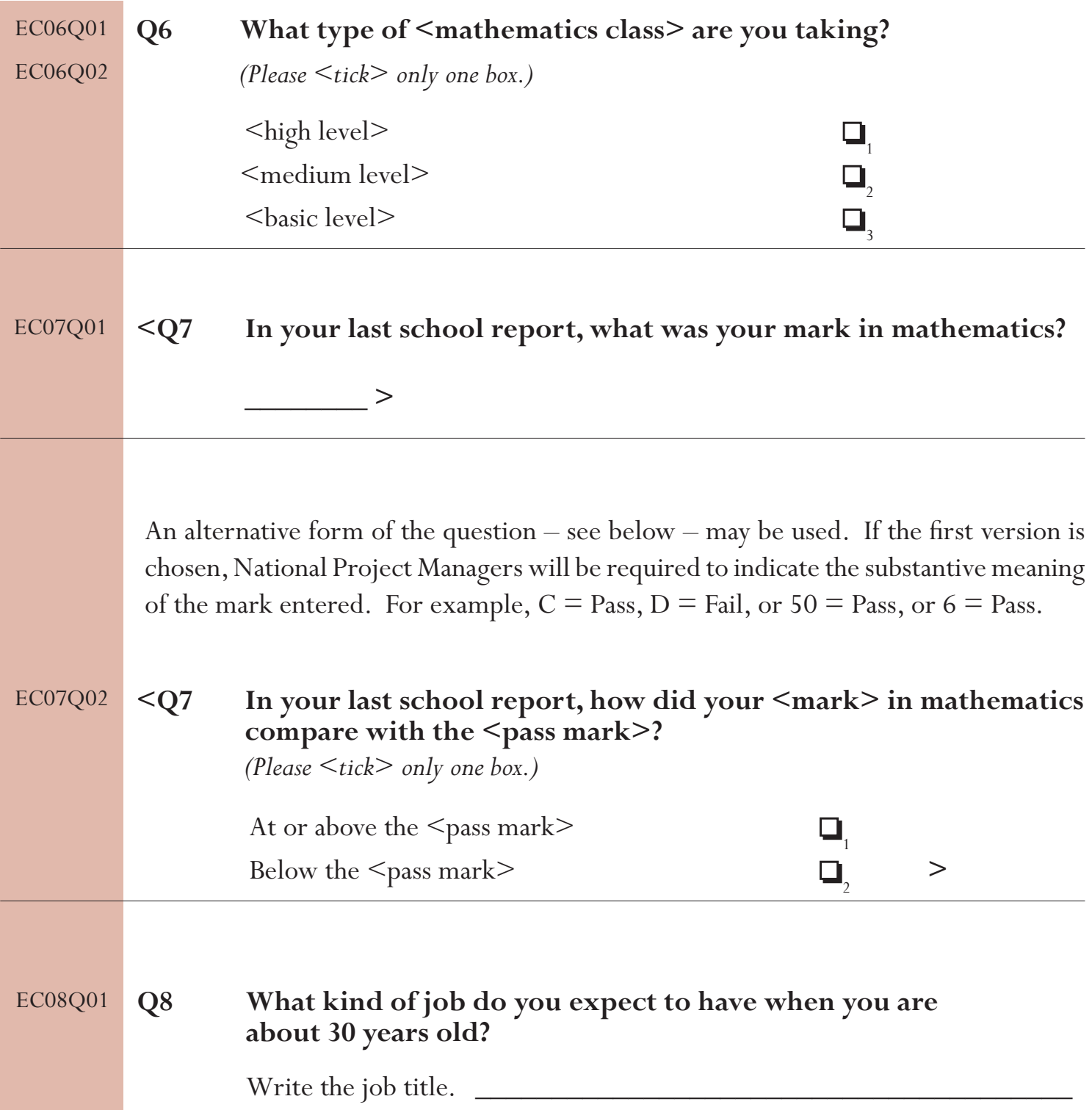




\section{APPENDIX 4 - INFORMATION COMMUNICATION TECHNOLOGY (ICT) QUESTIONNAIRE}

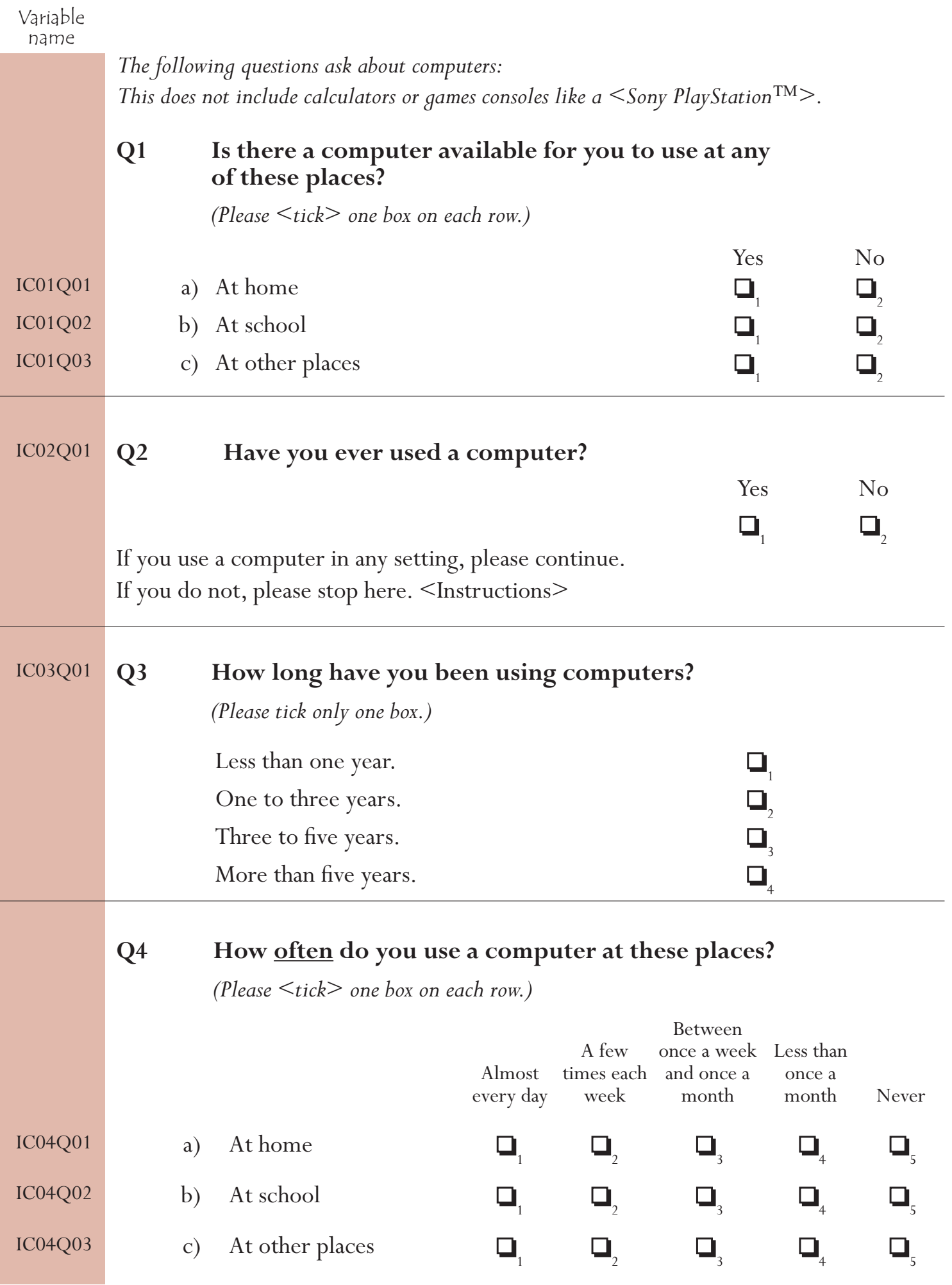


How often do you use:

(Please $<$ tick $>$ one box on each row.)

IC05Q02

IC05Q03

IC05Q04

IC05Q05

IC05Q06

IC05Q07

IC05Q08

IC05Q09

IC05Q10

IC05Q11

IC05Q12

a) The Internet to look up information about people, things, or ideas?

b) Games on a computer?

c) Word processing (e.g. $<$ Microsoft ${ }^{\circledR}$ Word $^{\circledR}$ or WordPerfect $\left.^{\circledR}>\right)$ ?

d) The Internet to collaborate with a group or team?

e) Spreadsheets (e.g. $<\mathrm{IBM}^{\circledR}$ Lotus $1-2-3^{\circledR}$ or Microsoft $^{\circledR}$ Excel $\left.^{\circledR}>\right)$ ?

f) The Internet to download software (including games)?

g) Drawing, painting or graphics programs on a computer?

h) Educational software such as mathematics programs?

i) The computer to help you learn school material?

j) The Internet to download music?

k) The computer for programming?

l) A computer for electronic communication (e.g. e-mail or "chat rooms")?

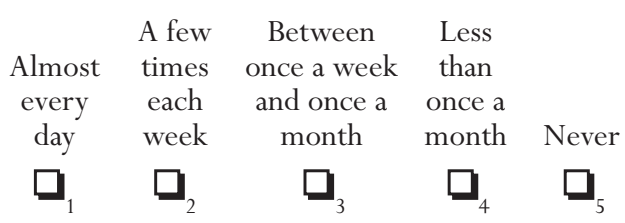

$\square$

$\square_{1} \square_{2}$

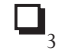

$\square_{4} \square_{5}$

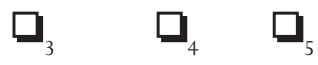

$\square \quad \square$

$\square_{1} \square_{2}$

$\square$

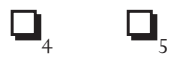

$\square$
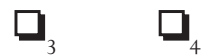

$\square$

$\square$
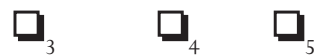

口 $\square$

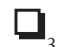

$\square_{4} \quad \square_{5}$
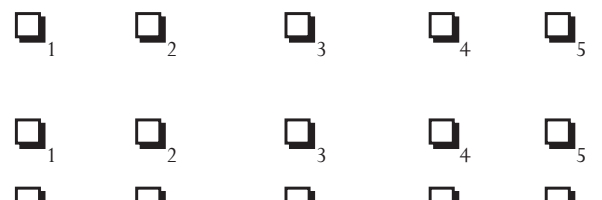

$\square_{1}$

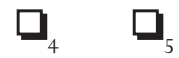

Q6 How well can you do each of these tasks on a computer?

(Please $<$ tick $>$ one box on each row.)

I can do I know what

I can do this this with this means I don't know very well by help from but I cannot what this myself. someone. do it. means.

IC06Q01

IC06Q02

IC06Q03

IC06Q04 a) Start a computer game.

b) Use software to find and get rid of computer viruses.

c) Open a file.

d) Create/edit a document.

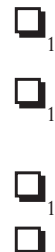

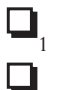

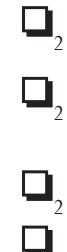

$\square_{2}$ $\square$
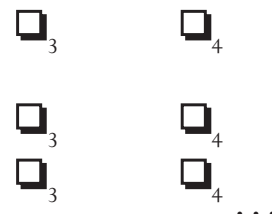


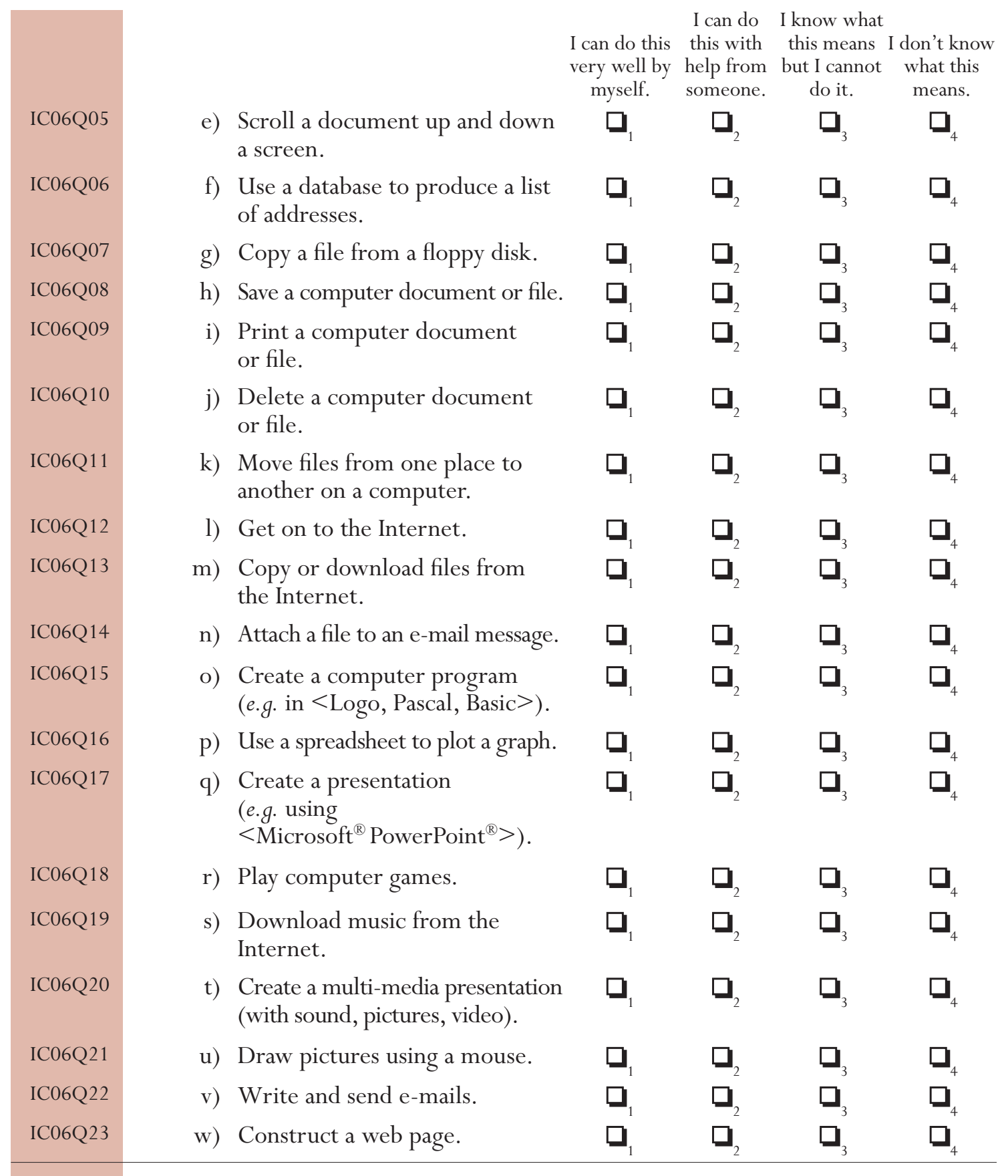

Use a database to produce a list or file.

Delete a computer document

Move files from one place to

Copy or down

Attach a file to an e-mail message.

o) Create a computer program (e.g. in <Logo, Pascal, Basic>).

p) Use a spreadsheet to plot a graph.

q) Create a presentation (e.g. using $<$ Microsoft $^{\circledR}$ PowerPoint $^{\circledR}>$ ).

r) Play computer games.

s) Download music from the Internet.

can do this this with this means I don't know ery well by help from but I cannot what this myself. someone. do it. means.

Create a multi-media presentation

Q7 Thinking about your experience with computers: To what extent do you agree with the following statements?

(Please $<$ tick $>$ one box on each row.)

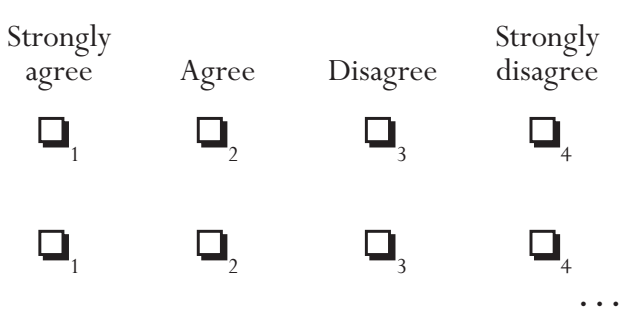




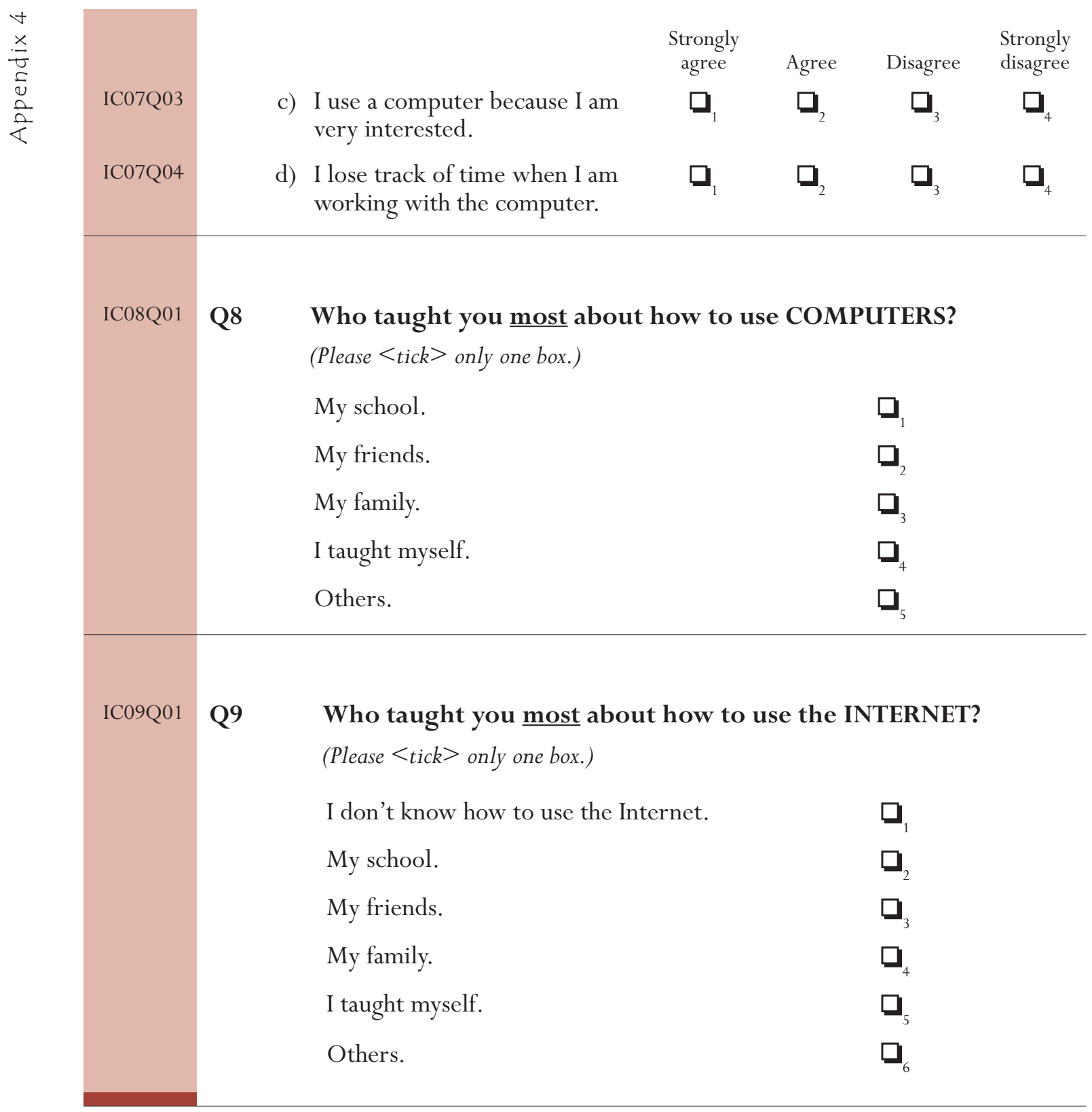




\section{APPENDIX 5 - SCHOOL QUESTIONNAIRE}

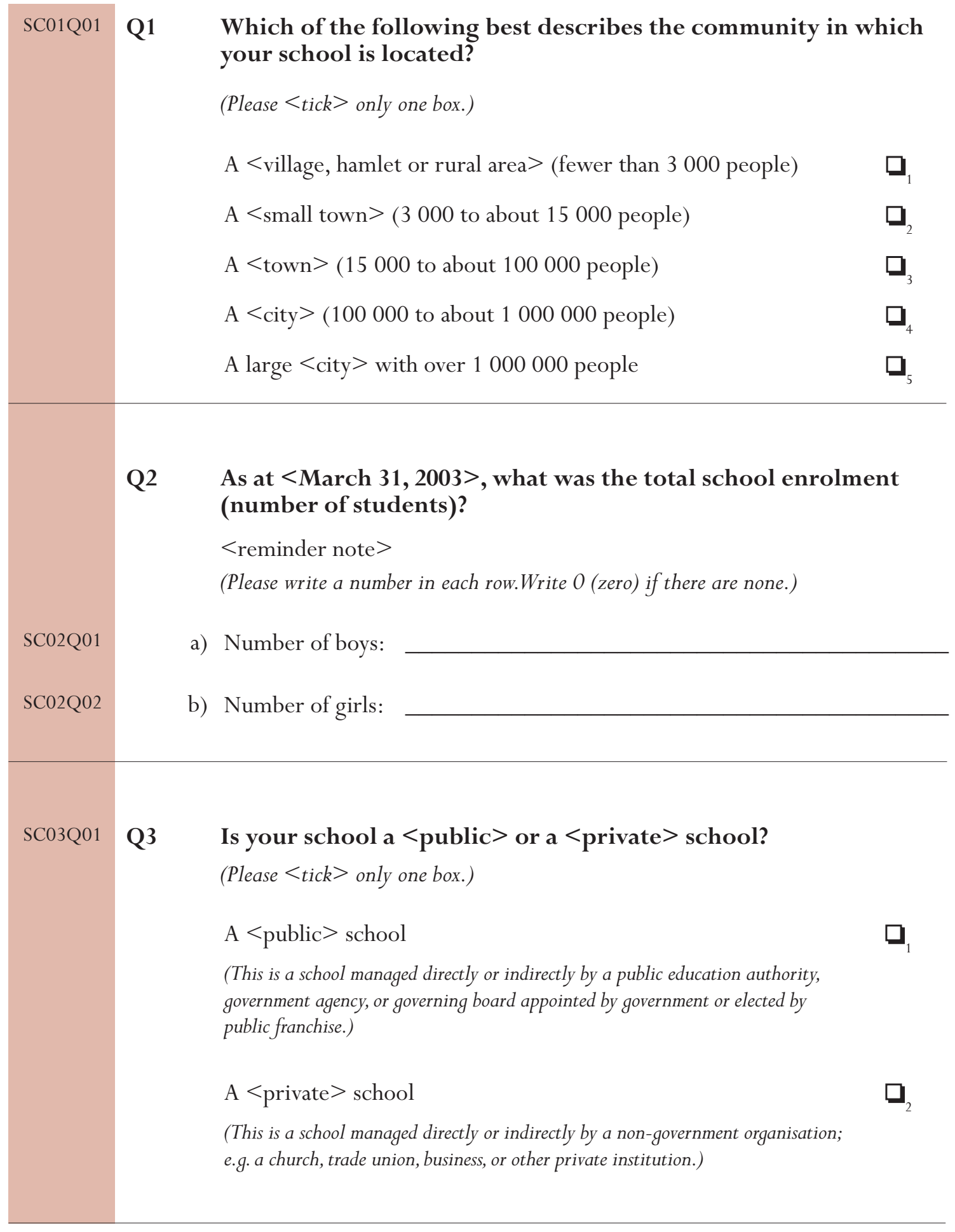


Q4 About what percentage of your total funding for a typical school year comes from the following sources?

$<$ reminder note $>$

(Please write a number in each row.Write 0 (zero) if no funding comes from that source.)
SC04Q01
a) Government (includes departments, local, regional, state and national)
SC04Q02 b) Student fees or school charges paid by parents
SC04Q03
c) Benefactors, donations, bequests, sponsorships, parent fund raising
SC04Q04 d) Other

Total $\quad 100 \%$

Q5 Are the following < grade levels $>$ found in your school?

(Please $<$ tick $>$ one box on each row.)

\begin{tabular}{|c|c|c|c|}
\hline & & & Yes \\
\hline SC05Q01 & a) & $<$ Grade $1>$ & $\square_{1}$ \\
\hline SC05Q02 & b) & $<$ Grade $2>$ & $\square$ \\
\hline SC05Q03 & c) & $<$ Grade $3>$ & $\square_{1}$ \\
\hline SC05Q04 & d) & $<$ Grade 4> & $\square$ \\
\hline SC05Q05 & e) & $<$ Grade $5>$ & $\square$ \\
\hline SC05Q06 & f) & $<$ Grade 6> & $\square$ \\
\hline SC05Q07 & g) & $<$ Grade $7>$ & $\square_{1}$ \\
\hline SC05Q08 & h) & $<$ Grade $8>$ & $\square_{1}$ \\
\hline SC05Q09 & i) & $<$ Grade 9> & $\square_{1}$ \\
\hline SC05Q10 & j) & $<$ Grade $10>$ & $\square_{1}$ \\
\hline SC05Q11 & k) & $<$ Grade $11>$ & $\square_{1}$ \\
\hline SC05Q12 & l) & $<$ Grade $12>$ & $\square_{1}$ \\
\hline SC05Q13 & $\mathrm{m})$ & $<$ Grade $13>$ & $\square_{1}$ \\
\hline SC05Q14 & n) & $<$ Ungraded school $>$ & $\square$ \\
\hline
\end{tabular}


Q6 About what percentage of students in your school repeated a $<$ grade $>$, at these $<$ ISCED levels $>$, last < academic $>$ year?

(Please write a number in each row.Write 0 (zero) if nobody repeated $a<$ grade $>$. $<$ Tick $>$ the not applicable box if the $<$ ISCED level $>$ does not appear in your school.) repeating a $<$ grade $>$ at $<$ ISCEDC $2>$ in this school last year was:

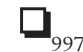

The approximate percentage of students repeating a $<$ grade $>$ at $<$ ISCEDC $3>$ in this school last year was:

The following is a list of programmes that may be in your school and that are available to 15 -year-old students.

- $<$ Programme 1 $>$

- $<$ Programme 2>

- $<$ Programme 3>

- $<$ Programme 4>

\section{Q7 For each of these programmes in your school:}

$<$ reminder note $>$

(Please write a number in each row for each programme in your school.)

a) How many <instructional $>$ weeks are in the school year?

b) How many hours in total are there in the school week? (include lunch breaks, < study hall time $>$, and after school activities)

c) How many hours for $<$ instruction $>$ are there in the school week? (exclude lunch breaks and after school activities)

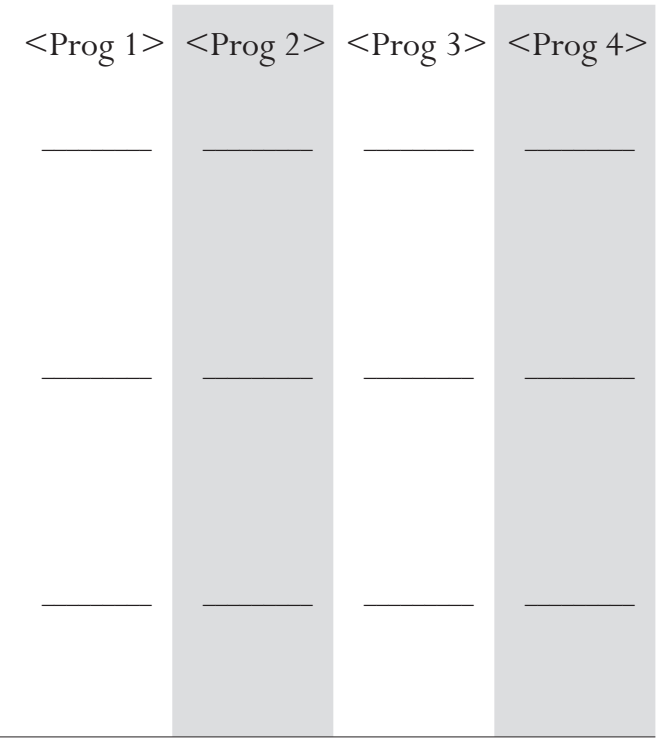


Q8 Is your school's capacity to provide instruction hindered by a shortage or inadequacy of any of the following?

(Please $<$ tick $>$ one box in each row.)

SC08Q01

SC08Q02

SC08Q03

SC08Q04

SC08Q05

SC08Q06

SC08Q07

SC08Q08

SC08Q09

SC08Q10

SC08Q11

SC08Q12

SC08Q13

SC08Q14

SC08Q15

SC08Q16

SC08Q17

SC08Q18

SC08Q19

SC08Q20 a) Availability of qualified mathematics teachers

b) Availability of qualified science teachers

c) Availability of qualified < test language> teachers

d) Availability of qualified < other national language $>$ teachers

e) Availability of qualified foreign language teachers

f) Availability of experienced teachers

g) Availability of < emergency/replacement $>$ teachers

h) Availability of support personnel

i) Instructional materials (e.g. textbooks)

j) Budget for supplies (e.g. paper, pencils)

k) School buildings and grounds

l) Heating/cooling and lighting systems

m) Instructional space (e.g. classrooms)

n) Special equipment for disabled students

o) Computers for instruction

p) Computer software for instruction

q) Calculators for instruction

r) Library materials

s) Audio-visual resources

t) Science laboratory equipment and materials
Not Very To some

at all little extent A lot

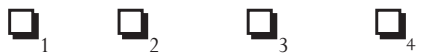

$\square$

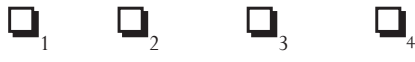

$\square_{1} \square_{2} \quad \square_{3} \quad \square_{4}$

$\square_{1} \quad \square_{2} \quad \square_{3} \quad \square_{4}$

$\square_{1} \square_{2} \square_{3}$

$\square_{1} \quad \square_{2} \quad \square_{3} \quad \square_{4}$

$\square \quad \square, \quad \square, \quad \square$

$\square_{1} \quad \square_{2} \quad \square_{3} \quad \square_{4}$

$\square_{1} \quad \square_{2} \quad \square_{3} \quad \square_{4}$

$\square_{1} \quad \square_{2} \quad \square_{3} \quad \square_{4}$

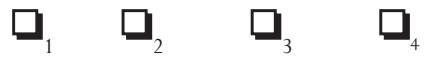

$\square_{1} \quad \square_{2} \quad \square_{3} \quad \square_{4}$
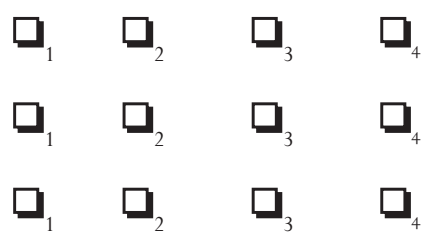

$\square_{1} \square_{2} \square_{3} \square_{4}$

$\square_{1} \quad \square_{2} \quad \square_{3} \square_{4}$

$\begin{array}{llll}\square_{1} & \square_{2} & \square_{3} & \square_{4}\end{array}$

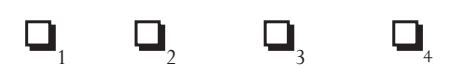


Q9 In your school, about how many computers are:

$<$ reminder note $>$

(Please write a number in each row.Write 0 (zero) if there are none.)

a) In the school altogether?

b) Available to 15-year-old students?

c) Available only to teachers?

d) Available only to administrative staff?

e) Connected to the Internet/World Wide Web?

f) Connected to a local area network (LAN)?

Q10 How much consideration is given to the following factors when students are admitted to your school?

(Please $<$ tick $>$ one box in each row.)

a) Residence in a particular area

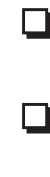

SC10Q03

b) Student's academic record (including placement tests)

c) Recommendation of feeder schools

d) Parents' endorsement of the instructional or religious philosophy of the school

e) Student need or desire for a special programme

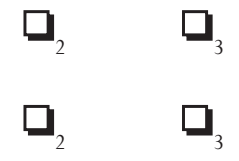

$\square$<smiles>[Hg]</smiles><smiles>[GeH3]</smiles><smiles>[Hg]</smiles>

SC10Q06

f) Attendance of other family members at the school (past or present)<smiles>[O]</smiles><smiles>[Li]</smiles><smiles>[Hg]</smiles><smiles>[CH]</smiles><smiles>[CH]1[CH-]CC1</smiles><smiles>[O]</smiles><smiles>[Hg]</smiles><smiles>[C]1[C+]=CC=C1</smiles> 
Q11 Think about the students in your school. How much do you agree with the following statements?

(Please $<$ tick $>$ one box in each row.)

Strongly
agree

SC11Q02

SC11Q03

SC11Q04

SC11Q05

SC11Q06

SC11Q07 as much as possible. a) Students enjoy being in school.

g) Students do their best to learn

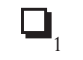

$\square_{2}$<smiles>[120Po]</smiles>

b) Students work with enthusiasm.

c) Students take pride in this school.

d) Students value academic achievement.

e) Students are cooperative and respectful.

f) Students value the education they can receive in this school.

$\square$<smiles>[Ca][Hg]</smiles>

$\square$

$\square$

$\square \square$

$\square_{2}$

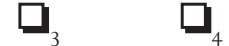

$\square$

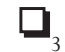

$\square_{4}$

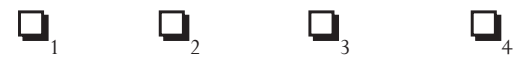

Strongly

disagree

\begin{tabular}{|c|c|c|c|c|c|c|c|c|}
\hline & \multirow[t]{3}{*}{ Q12 } & & \multicolumn{6}{|c|}{$\begin{array}{l}\text { Generally, in your school, how often are }<15 \text {-year-old }>\text { students } \\
\text { assessed using: }\end{array}$} \\
\hline & & & (Please $<$ tick $>$ only one box in eac & row.) & & & & \\
\hline & & & & Never & $\begin{array}{l}1 \text { to } \\
2 \text { times } \\
\text { a year }\end{array}$ & $\begin{array}{l}3 \text { to } \\
5 \text { times } \\
\text { a year }\end{array}$ & Monthly & $\begin{array}{c}\text { More than } \\
\text { once a } \\
\text { month }\end{array}$ \\
\hline SC12Q01 & & a) & Standardised tests? & $\square_{1}$ & $\square_{2}$ & $\square_{3}$ & $\square_{4}$ & $\square_{5}$ \\
\hline SC12Q02 & & b) & Teacher-developed tests? & $\square_{1}$ & $\square_{2}$ & $\square_{3}$ & $\square_{4}$ & $\square_{5}$ \\
\hline SC12Q03 & & c) & Teachers' judgmental ratings? & $\square$ & $\square$ & $\square$ & $\square$ & $\square$ \\
\hline SC12Q04 & & d) & Student $<$ portfolios $>$ ? & $\square$ & $\square_{2}$ & $\square_{3}$ & $\square_{4}$ & $\square_{5}$ \\
\hline SC12Q05 & & e) & $\begin{array}{l}\text { Student assignments/ } \\
\text { projects/homework? }\end{array}$ & $\square_{1}$ & $\square_{2}$ & $\square_{3}$ & $\square_{4}$ & $\square_{5}$ \\
\hline
\end{tabular}


Q13 In your school, are assessments of $<15$-year-old students $>$ used for any of the following purposes?

(Please $<$ tick> only one box in each row.)

SC13Q01

SC13Q02

SC13Q03

SC13Q04

SC13Q05

SC13Q06

SC13Q07

SC13Q08

a) To inform parents about their child's progress.

b) To make decisions about students' retention or promotion.

c) To group students for instructional purposes.

d) To compare the school to $<$ district or national $>$ performance.

e) To monitor the school's progress from year to year.

f) To make judgements about teachers' effectiveness.

g) To identify aspects of instruction or the curriculum that could be improved.

h) To compare the school with other schools.

Q14 About how many 15-year-old students in your school have a $<$ first language $>$ that is not $<$ the test language $>$ ?

(Please $<$ tick $>$ only one box.)

SC14Q01

SC14Q02

SC14Q03

SC14Q04

a) $40 \%$ or more

b) $20 \%$ or more but less than $40 \%$

c) $10 \%$ or more but less than $20 \%$

d) Less than $10 \%$

Q15 Schools with students whose $<$ first language $>$ is not $<$ the test language $>$ sometimes offer specific language options to these students.

Does your school offer any of the following options to 15 -yearold students whose $<$ first language $>$ is not $<$ the test language $>$ ?

(Please $<$ tick $>$ one box in each row.)

$\begin{array}{cccc}\begin{array}{c}\text { No, not } \\ \text { for any } \\ \text { languages }\end{array} & \begin{array}{c}\text { Yes } \\ \text { for one } \\ \text { language }\end{array} & \begin{array}{c}\text { Yes for 2 } \\ \text { or more } \\ \text { languages }\end{array} & \begin{array}{c}\text { Not } \\ \text { applicable }\end{array}\end{array}$

SC15Q01

a) Instruction in their

language is offered as a

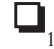

$\square$ separate subject.

b) Instruction in other parts of the curriculum is offered in their language. 
Q16 Schools sometimes organise instruction differently for students with different abilities and interests in mathematics.

Which of the following options describe what your school does for 15-year-old students in mathematics classes?

(Please $<$ tick $>$ one box in each row.)

$\begin{array}{ccc}\begin{array}{c}\text { For } \\ \text { all classes }\end{array} & \begin{array}{c}\text { For some } \\ \text { classes }\end{array} & \text { Not for } \\ \text { any classes }\end{array}$

SC16Q01

SC16Q02

SC16Q03

SC16Q04

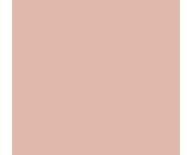

a) Mathematics classes study similar content, but at different levels of difficulty.

b) Different classes study different content or sets of mathematics topics that have different levels of difficulty.

c) Students are grouped by ability within their mathematics classes.
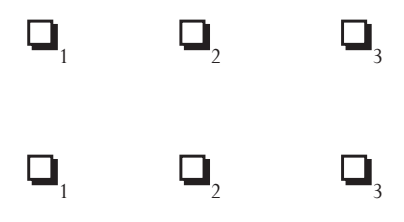

In mathematics classes, teachers use a pedagogy suitable for $<$ students with heterogeneous abilities $>$ (i.e. students are not grouped by ability).

Q17 In your school, do any of the following activities to promote engagement with mathematics occur?

(Please $<$ tick $>$ one box in each row)

$\begin{array}{llcl}\text { SC17Q01 } & \text { a) }<\text { Enrichment mathematics }> & \square_{1} & \square_{2} \\ \text { SC17Q02 } & \text { b) }<\text { Remedial mathematics }> & \square_{1} & \square_{2} \\ \text { SC17Q03 } & \text { c) }<\text { Mathematics competitions }> & \square_{1} & \square_{2} \\ \text { SC17Q04 } & \text { d) }<\text { Mathematics clubs }> & \square_{1} & \square_{2} \\ \text { SC17Q05 } & \text { e) } \quad \text { <Computer clubs }>\text { (specifically related to } & \square_{1} & \square_{2}\end{array}$


Q18 How many of the following are on the staff of your school?

Include both full-time and part-time teachers. A full-time teacher is employed at least $90 \%$ of the time as a teacher for the full school year. All other teachers should be considered part-time.

$<$ reminder note $>$

(Please write a number in each space provided.Write 0 (zero) if there is none.)

Full time PartTime
a) Teachers in TOTAL
b) Teachers fully certified by < the appropriate authority>
c) Teachers with an $<$ ISCED5A $>$ qualification in $<$ pedagogy $>$

\section{Q19 How many of the following are on the <MATHEMATICS staff $>$ of your school?}

Include both full-time and part-time teachers. A full-time teacher is employed at least $90 \%$ of the time as a teacher for the full school year. All other teachers should be considered part-time.

\section{Please count only those teachers who have taught or will teach} mathematics during the current school year.

$<$ reminder note $>$

(Please write a number in each space provided.Write $O$ (zero) if there are none.)

a) Teachers of mathematics in TOTAL

b) Teachers of mathematics with an $<$ ISCED5A $>$ qualification $<$ with a major $>$ in mathematics

c) Teachers of mathematics with an $<$ ISCED5A $>$ qualification $<$ but not a major $>$ in mathematics

d) Teachers of mathematics with an $<$ ISCED5A $>$ qualification in <pedagogy $>$

e) Teachers of mathematics with an $<$ ISCED5B $>$ but not an $<$ ISCED 5A $>$ qualification 
Q20 During the last year, have any of the following been used to monitor the practice of mathematics teachers at your school?

(Please $<$ tick $>$ one box in each row.)

SC20Q01

SC20Q02

SC20Q03

SC20Q04

a) Tests or assessments of student achievement

b) Teacher peer review (of lesson plans, assessment instruments, lessons)

c) Principal or senior staff observations of lessons

d) Observation of classes by inspectors or other persons external to the school

Yes $\quad$ No
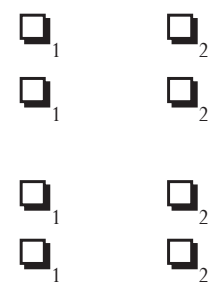

Q21 How much do you agree with these statements about innovation in your school?

(Please $<$ tick $>$ one box in each row.)

$\begin{array}{ccc}\text { Strongly } & & \text { Strongly } \\ \text { agree } & \text { Agree Disagree } & \text { disagree }\end{array}$
SC21Q01
SC21Q02
SC21Q03
a) Mathematics teachers are interested in trying new methods and teaching practices.
b) There is a preference among mathematics teachers to stay with well-known methods and practices.
c) There are frequent disagreements between "innovative" and "traditional" mathematics teachers.
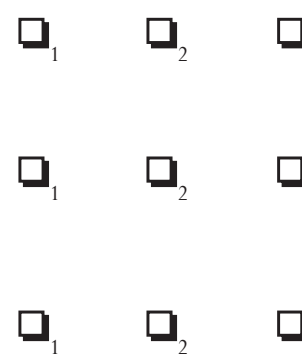

Q22 How much do you agree with these statements about teachers' expectations in your school?

(Please $<$ tick> one box in each row.)

$\begin{array}{ccc}\text { Strongly } & \text { Strongly } \\ \text { agree } & \text { Agree Disagree } & \text { disagree }\end{array}$

SC22Q01

a) There is consensus among mathematics teachers that academic achievement must be kept as high as possible.

SC22Q02

SC22Q03

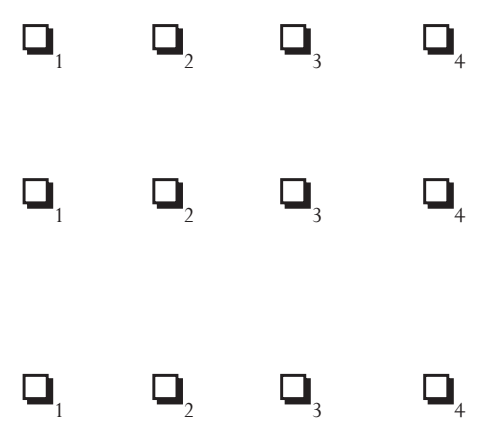

b) There is consensus among mathematics teachers that it is best to adapt academic standards to the students' level and needs.

c) There are frequent disagreements between mathematics teachers who consider each other to be "too demanding" or "too lax". 


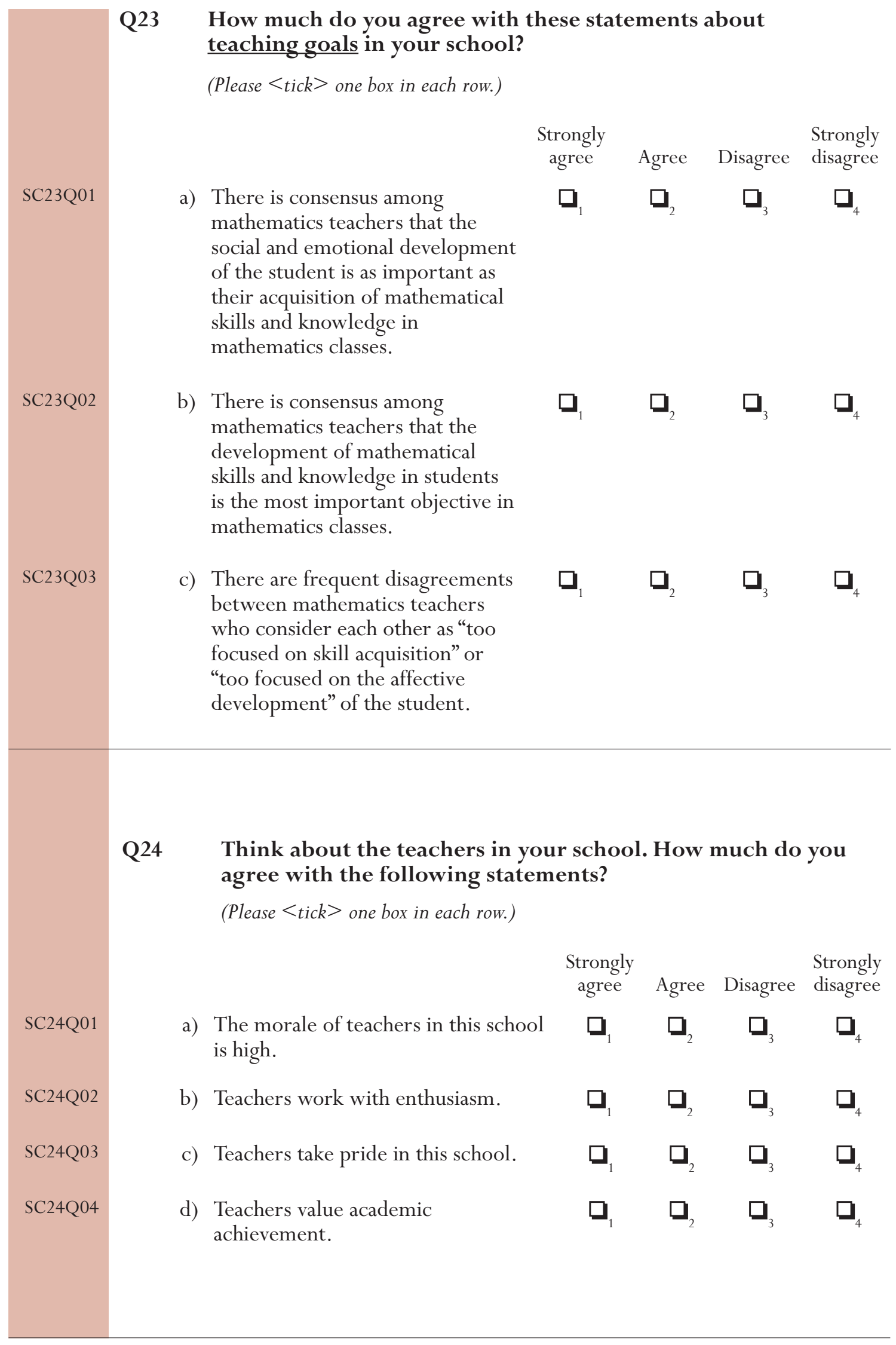

There is consensus among mathematics teachers that the social and emotional development the student is as important as ge in mathematics classes.

There is consensus among mathematics teachers that the development of mathematical skills and knowledge in students is the most important objective in mathematics classes.

There are frequent disagreement between mathematics teachers who consider each other as "too focused on skill acquisition" or "too focused on the affective development" of the student. agree Agree 
Q25 In your school, to what extent is the learning of students hindered by: (Please $<$ tick $>$ one box in each row.)

$\begin{array}{ccc}\text { Not } & \text { Very } & \text { To some } \\ \text { at all little } & \text { extent }\end{array}$ A lot

SC25Q01

SC25Q02

$\mathrm{SC} 25 \mathrm{Q} 03$

SC25Q04

SC25Q05

SC25Q06

SC25Q07

SC25Q08

SC25Q09

SC25Q10

SC25Q11

SC25Q12

SC25Q13

a) Teachers' low expectations of students?

b) Student absenteeism?

c) Poor student-teacher relations?

d) Disruption of classes by students?

e) Teachers not meeting individual students' needs?

f) Teacher absenteeism?

g) Students skipping classes?

h) Students lacking respect for teachers?

i) Staff resisting change?

j) Student use of alcohol or illegal drugs?

k) Teachers being too strict with students?

l) Students intimidating or bullying other students?

$\begin{array}{llll}\square_{1} & \square_{2} & \square_{3} & \square_{4} \\ \square_{1} & \square_{2} & \square_{3} & \square_{4} \\ \square_{1} & \square_{2} & \square_{3} & \square_{4} \\ \square_{1} & \square_{2} & \square_{3} & \square_{4} \\ \square_{1} & \square_{2} & \square_{3} & \square_{4}\end{array}$

m) Students not being encouraged to achieve their full potential?

\section{Q26 In your school, who has the main responsibility for:}

(Please $<$ tick $>$ as many boxes as appropriate in each row.)

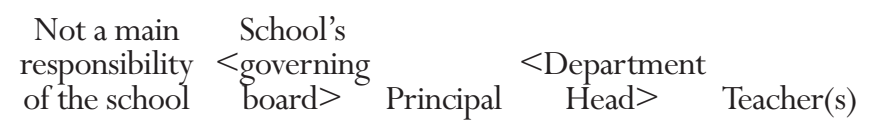

SC26Q01

SC26Q02

SC26Q03

SC26Q04

SC26Q05

SC26Q06 a) Selecting teachers for hire?

b) Firing teachers?

c) Establishing teachers' starting salaries?

d) Determining teachers' salary increases?

e) Formulating the school budget?

f) Deciding on budget allocations within the school?

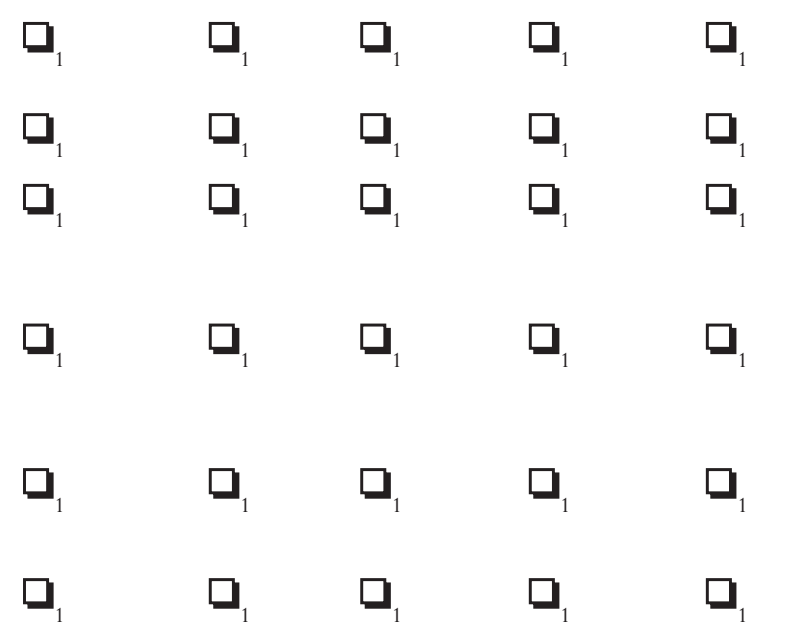




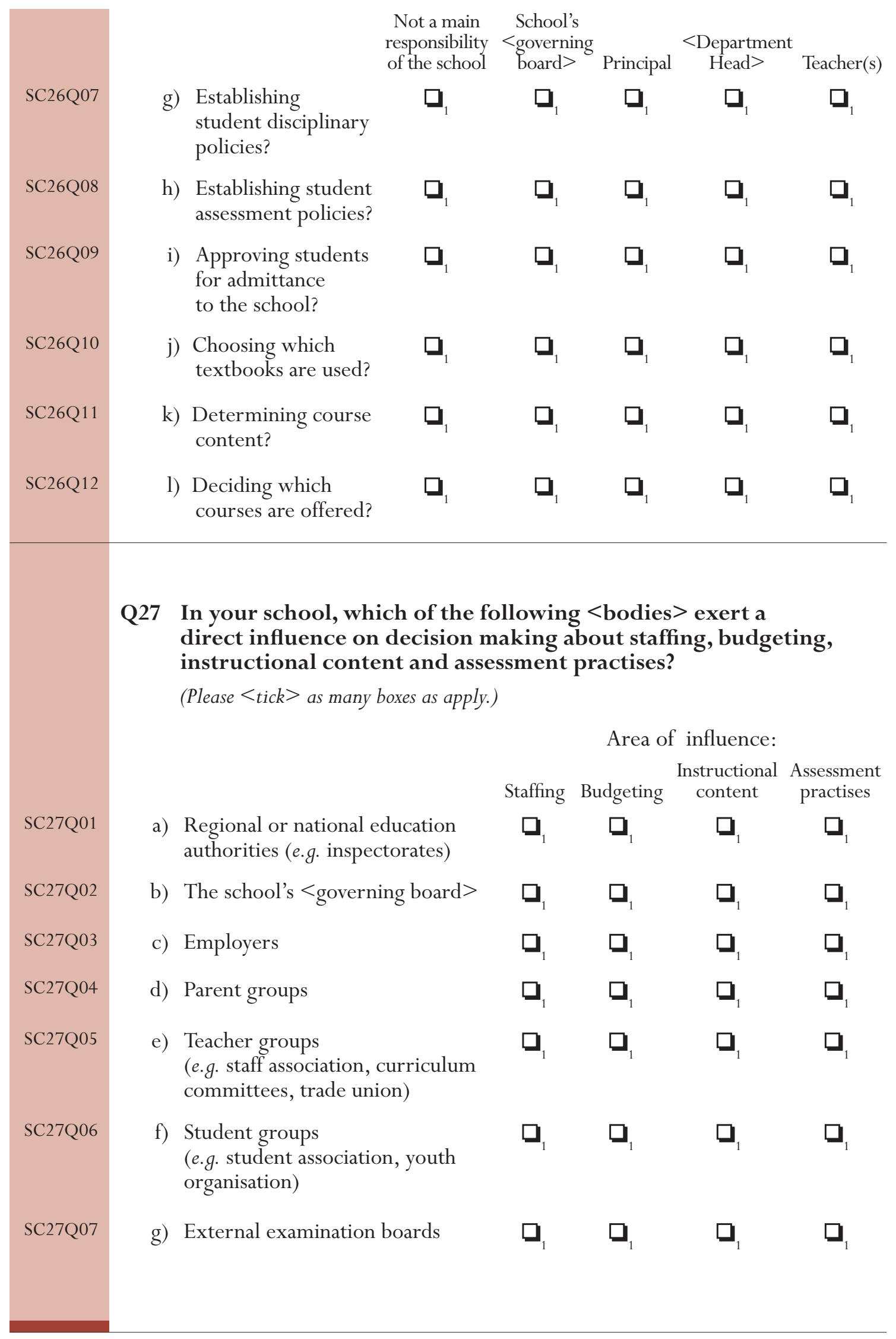
tudent disciplinary

Establishing student

Approving students for admittance to the school?

Choosing which textbooks are used?

Determining course content?

Deciding which courses are offered?

Not a main School's responsibility < governing 



\section{APPENDIX 6 - STUIDENT QUESTIONNAIRE DATA FILE CODEBOOK}

$\begin{array}{lll}\text { COUNTRY (1) } & \text { Country ID } \\ \text { Format: } & \text { A3 } & \text { Columns: 1-3 } \\ 008 & \text { Albania } \\ 032 & \text { Argentina } \\ 036 & \text { Australia } \\ 040 & \text { Austria } \\ 056 & \text { Belgium } \\ 076 & \text { Brazil } \\ 100 & \text { Bulgaria } \\ 124 & \text { Canada } \\ 152 & \text { Chile } \\ 203 & \text { Czech Republic } \\ 208 & \text { Denmark } \\ 246 & \text { Finland } \\ 250 & \text { France } \\ 276 & \text { Germany } \\ 300 & \text { Greece } \\ 344 & \text { Hong Kong-China } \\ 348 & \text { Hungary } \\ 352 & \text { Iceland } \\ 360 & \text { Indonesia } \\ 372 & \text { Ireland } \\ 376 & \text { Israel } \\ 380 & \text { Italy } \\ 392 & \text { Japan } \\ 410 & \text { Korea } \\ 428 & \text { Latvia } \\ 438 & \text { Liechtenstein } \\ 442 & \text { Luxembourg } \\ 446 & \text { Macao-China } \\ 484 & \text { Mexico } \\ 528 & \text { Netherlands } \\ 554 & \text { New Zealand } \\ 578 & \text { Norway } \\ 604 & \text { Peru } \\ 616 & \text { Poland } \\ & \end{array}$

$\begin{array}{ll}620 & \text { Portugal } \\ 643 & \text { Russian Federation } \\ 703 & \text { Slovakia } \\ 724 & \text { Spain } \\ 752 & \text { Sweden } \\ 756 & \text { Switzerland } \\ 764 & \text { Thailand } \\ 788 & \text { Tunisia } \\ 792 & \text { Turkey } \\ 807 & \text { Macedonia } \\ 826 & \text { United Kingdom } \\ 840 & \text { United States } \\ 858 & \text { Uruguay } \\ 891 & \text { Serbia }\end{array}$

\section{CNT (2) Country Alphanumeric ISO Code}

Format: A3 Columns: 4-6

SUBNATIO (3) Adjudicated sub-region

$\begin{array}{lll}\text { Format: } & \text { A4 } & \text { Columns: 7-10 } \\ 0360 & \text { Australia } \\ 0400 & \text { Austria } \\ 0560 & \text { Belgium } \\ 0760 & \text { Brazil } \\ 1240 & \text { Canada } \\ 2030 & \text { Czech Republic } \\ 2080 & \text { Denmark } \\ 2460 & \text { Finland } \\ 2500 & \text { France } \\ 2760 & \text { Germany } \\ 3000 & \text { Greece } \\ 3440 & \text { Hong Kong-China } \\ 3480 & \text { Hungary } \\ 3520 & \text { Iceland } \\ 3600 & \text { Indonesia } \\ 3720 & \text { Ireland } \\ 3801 & \text { Italy: Veneto-Nord Est }\end{array}$


Italy: Trento-Nord-Est

3803

Italy: Toscana-Centro

3804 Italy: Piemonte-Nord-Ovest

3805 Italy: Lombardia-Nord Ovest

3806 Italy: Bolzano

3807 Italy: Other regions

3920 Japan

$4100 \quad$ Korea

4280 Latvia

4380 Liechtenstein

4420 Luxembourg

4460 Macao SAR

4840 Mexico

5280 Netherlands

5540 New Zealand

5780 Norway

6160 Poland

6200 Portugal

6430 Russian Federation

7030 Slovak Republic

7241 Spain: Other regions

7242 Spain: Castilia y Leon

7243 Spain: Catalonia

7244 Spain: Basque Country

7520 Sweden

$7560 \quad$ Switzerland

7640 Thailand

7880 Tunisia

7920 Turkey

8261 United Kingdom: England, Wales \& Northern Ireland

8262 United Kingdom: Scotland

$8400 \quad$ United States

8580 Uruguay

8910 Serbia

\section{SCHOOLID (4) School ID}

Format: A5 Columns: 11-15

STIDSTD (5) Student ID

Format: A5 Columns: 16-20

$\begin{array}{lll}\text { ST01Q01 (6) } & \text { Grade Q1a } \\ \text { Format: } & \text { F2 } & \text { Columns: 22-23 } \\ & 97 & \text { N/A } \\ & 98 & \text { Invalid } \\ & 99 & \text { Missing }\end{array}$

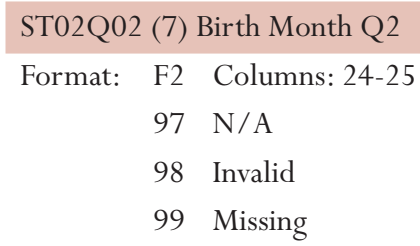

1 Tick

2 No tick

$7 \mathrm{~N} / \mathrm{A}$

8 Invalid

9 Missing 


\section{ST17Q07 (38) Possessions: calculator Q17g}

$\begin{array}{rll}\text { Format: } & \text { F1 } & \text { Column } \\ & 1 & \text { Tick } \\ 2 & \text { No tick } \\ 7 & \text { N/A } \\ 8 & \text { Invalid } \\ 9 & \text { Missing }\end{array}$

ST17Q08 (39) Possessions: literature Q17h

$\begin{array}{rll}\text { Format: } & \text { F1 } & \text { Columns: } \\ 1 & \text { Tick } \\ 2 & \text { No tick } \\ 7 & \text { N/A } \\ 8 & \text { Invalid } \\ 9 & \text { Missing }\end{array}$

ST17Q09 (40) Possessions: poetry Q17i

Format: F1 Columns: 76-76

1 Tick

2 No tick

7 N/A

8 Invalid

9 Missing

ST17Q10 (41) Possessions: art Q17j

Format: F1 Columns: 77-77

1 Tick

2 No tick

7 N/A

8 Invalid

9 Missing

ST17Q11 (42) Possessions: textbooks Q17k

Format: F1 Columns: 78-78

1 Tick

2 No tick

$7 \mathrm{~N} / \mathrm{A}$

8 Invalid

9 Missing

ST17Q12 (43) Possessions: dictionary Q17l

Format: F1 Columns: 79-79

1 Tick

2 No tick

7 N/A

8 Invalid

9 Missing

ST17Q13 (44) Possessions: dishwasher Q17m

Format: F1 Columns: 80-80

1 Tick

2 No tick

7 N/A

8 Invalid

9 Missing
ST17Q14 (45) Possessions: <Cntry item 1> Q17n

Format: A5 Columns: 81-85

04001 Austria: DVD

04002 Austria: No DVD

05611 Belgium (Fl.): DVD

05612 Belgium (Fl.): No DVD

05621 Belgium (Fr.): DVD

05622 Belgium (Fr.): No DVD

20301 Czech Republic: Own mobile phone

20302 Czech Republic: No own mobile phone

27601 Germany: Own garden

27602 Germany: No own garden

30001 Greece: Video camera

30002 Greece: No Video camera

34401 Hong Kong: Encyclopaedia

34402 Hong Kong: No encyclopaedia

34801 Hungary: Video casette recorder

34802 Hungary: No video casette recorder

37201 Ireland: Games console

37202 Ireland: No games console

38001 Italy: Ancient furniture

38002 Italy: No ancient furniture

41001 Korea: DVD

41002 Korea: No DVD

44201 Luxembourg: Satellite dish

44202 Luxembourg: No satellite dish

44601 Macao: Encyclopaedia

44602 Macao: No encyclopaedia

55401 New Zealand: Clothes dryer

55402 New Zealand: No clothes dryer

57801 Norway: Swimming pool

57802 Norway: No swimming pool

61601 Poland: Satellite or cable TV (>29 channels)

61602 Poland: No satellite or cable TV (>29 channels)

72401 Spain: Video

72402 Spain: No video

75601 Switzerland: Musical instrument

75602 Switzerland: No musical instrument

78801 Tunisia: Telephone

78802 Tunisia: No telephone 


$\begin{array}{ll}79201 & \text { Turkey: Central heating system } \\ 79202 & \text { Turkey: No central heating system } \\ 82621 & \text { Scotland: Musical instruments } \\ 82622 & \text { Scotland: No musical instruments } \\ 85801 & \text { Uruguay: Freezer } \\ 85802 & \text { Uruguay: No freezer } \\ 99997 & \text { N/A } \\ 99998 & \text { Invalid } \\ 99999 & \text { Missing }\end{array}$

ST17Q15 (46) Possessions: <Cntry item 2> Q17o

\begin{tabular}{|c|c|c|}
\hline Format: & A5 & Columns: 86-90 \\
\hline & 04001 & Austria: MP3 player \\
\hline & 04002 & Austria: No MP3 player \\
\hline & 05611 & Belgium (Fl.): Swimming pool \\
\hline & 05612 & Belgium (Fl.): No swimming pool \\
\hline & 20301 & $\begin{array}{l}\text { Czech Republic: Your own discman } \\
\text { or mp3 player }\end{array}$ \\
\hline & 20302 & $\begin{array}{l}\text { Czech Republic: No own discman } \\
\text { or mp3 player }\end{array}$ \\
\hline & 30001 & Greece: HiFi equipment \\
\hline & 30002 & Greece: No HiFi equipment \\
\hline & 34401 & $\begin{array}{l}\text { Hong Kong: Musical instrument } \\
\text { (e.g., piano, violin) }\end{array}$ \\
\hline & 34402 & $\begin{array}{l}\text { Hong Kong: No musical } \\
\text { instrument (e.g., piano, violin) }\end{array}$ \\
\hline & 34801 & Hungary: CD player \\
\hline & 34802 & Hungary: No CD player \\
\hline & 37201 & Ireland: VCR or DVD \\
\hline & 37202 & Ireland: No VCR or DVD \\
\hline & 38001 & Italy: DVD player \\
\hline & 38002 & Italy: No DVD player \\
\hline & 44201 & Luxembourg: Own television set \\
\hline & 44202 & Luxembourg: No own television set \\
\hline & 44601 & $\begin{array}{l}\text { Macao: Musical instrument } \\
\text { (e.g., piano, violin) }\end{array}$ \\
\hline & 44602 & $\begin{array}{l}\text { Macao: No musical instrument } \\
\text { (e.g., piano, violin) }\end{array}$ \\
\hline & 55401 & New Zealand: DVD \\
\hline & 55402 & New Zealand: No DVD \\
\hline & 57801 & Norway: Housekeeper \\
\hline & 57802 & Norway: No housekeeper \\
\hline & 61601 & Poland: VCR or DVD \\
\hline & 61602 & Poland: No VCR or DVD \\
\hline & 72401 & Spain: DVD \\
\hline
\end{tabular}

72402 Spain: No DVD

78801 Tunisia: Electricity

78802 Tunisia: No electricity

79201 Turkey: Washing machine

79202 Turkey: No washing machine

82621 Scotland: Cable/satellite TV

82622 Scotland: No Cable/satellite TV

85801 Uruguay: DVD

85802 Uruguay: No DVD

99997 N/A

99998 Invalid

99999 Missing

\section{ST17Q16 (47) Possessions: <Cntry item 3> Q17p}

Format: A5 Columns:

04001 Austria: Digital camera

04002 Austria: No digital camera

05611 Belgium (Fl.): CDs with classical music

05612 Belgium (Fl.): No CDs with classical music

30001 Greece: Air conditioning

30002 Greece: No air conditioning

34801 Hungary: DVD

34802 Hungary: No DVD

38001 Italy: Musical instrument (except flute)

38002 Italy: No musical instrument (except flute)

44201 Luxembourg: Own mobile phone

44202 Luxembourg: No own mobile phone

72401 Spain: Video console (Playstation, X-Box, Nintendo, etc.)

72402 Spain: No video console (Playstation, X-Box, Nintendo, etc.)

78801 Tunisia: Running water

78802 Tunisia: No running water

79201 Turkey: Vacuum cleaner

79202 Turkey: No vacuum cleaner

82621 Scotland: Kitchen range (eg AGA, Rayburn)

82622 Scotland: No kitchen range (eg AGA, Rayburn)

85801 Uruguay: Water heater

85802 Uruguay: No water heater

$99997 \mathrm{~N} / \mathrm{A}$

99998 Invalid

99999 Missing 


$$
\begin{array}{lll}
\text { ST19Q01 (48) How many books at home Q19 } \\
\text { Format: } & \text { F1 } & \text { Columns: } 96-96 \\
1 & 0-10 \text { books } \\
2 & 11-25 \text { books } \\
3 & 26-100 \text { books } \\
4 & 101-200 \text { books } \\
5 & 201-500 \text { books } \\
6 & \text { More than } 500 \text { books } \\
7 & \text { N/A } \\
8 & \text { Invalid } \\
9 & \text { Missing }
\end{array}
$$

$\begin{array}{lll}\text { ST20Q01 (49) } & \text { Attend }<\text { ISCED 0 }>\text { Q20 } \\ \text { Format: } & \text { F1 } & \text { Columns: 97-97 } \\ 1 & \text { No } \\ 2 & \text { Yes, one year or less } \\ 3 & \text { Yes, more than one year } \\ 7 & \text { N/A } \\ 8 & \text { Invalid } \\ 9 & \text { Missing }\end{array}$

$\begin{array}{lll}\text { ST21Q01 } & (50)<\text { ISCED 1> Years Q21 } \\ \text { Format: } & \text { F5.1 } & \text { Columns: 98-102 } \\ & 997.0 & \text { N/A } \\ & 998.0 & \text { Invalid } \\ & 999.0 & \text { Missing }\end{array}$

ST22Q01 (51) Repeat < ISCED 1> Q22a

Format: F1 Columns: 103-103

1 No, never

2 Yes, once

3 Yes, twice or more

7 N/A

8 Invalid

9 Missing

ST22Q02 (52) Repeat < ISCED 2> Q22b

Format: F1 Columns: 104-104

1 No, never

2 Yes, once

3 Yes, twice or more

7 N/A

8 Invalid

9 Missing

$\begin{array}{lll}\text { ST22Q03 } & \text { (53) } & \text { Repeat }<\text { ISCED 3 }>\text { Q22c } \\ \text { Format: } & \text { F1 } & \text { Columns: 105-105 } \\ 1 & \text { No, never } \\ 2 & \text { Yes, once } \\ 3 & \text { Yes, twice or more } \\ 7 & \text { N/A } \\ 8 & \text { Invalid } \\ 9 & \text { Missing }\end{array}$

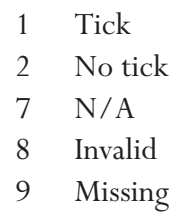

ST24Q01 (60) School: done little Q24a

Format: F1 Columns: 112-112

Strongly agree

Agree

Disagree

Strongly disagree

N/A

Invalid

9 Missing 
ST24Q02 (61) School: waste of time Q24b

$$
\begin{array}{rll}
\text { Format: } & \text { F1 } & \text { Columns: 113-113 } \\
1 & \text { Strongly agree } \\
2 & \text { Agree } \\
3 & \text { Disagree } \\
4 & \text { Strongly disagree } \\
7 & \text { N/A } \\
8 & \text { Invalid } \\
9 & \text { Missing }
\end{array}
$$

ST24Q03 (62) School: give confidence Q24c

$$
\begin{array}{rll}
\text { Format: } & \text { F1 } & \text { Columns: 114-114 } \\
1 & \text { Strongly agree } \\
2 & \text { Agree } \\
3 & \text { Disagree } \\
4 & \text { Strongly disagree } \\
7 & \text { N/A } \\
8 & \text { Invalid } \\
9 & \text { Missing }
\end{array}
$$

ST24Q04 (63) School: useful Q24d

$$
\begin{array}{rll}
\text { Format: } & \text { F1 } & \text { Columns: 115-115 } \\
1 & \text { Strongly agree } \\
2 & \text { Agree } \\
3 & \text { Disagree } \\
4 & \text { Strongly disagree } \\
7 & \text { N/A } \\
8 & \text { Invalid } \\
9 & \text { Missing }
\end{array}
$$

$\begin{array}{lll}\text { ST25Q01 (64) } & \text { Attend: lo } \\ \text { Format: } & \text { F1 } & \text { Columns: } \\ & 1 & \text { Tick } \\ 2 & \text { No tick } \\ 7 & \text { N/A } \\ 8 & \text { Invalid } \\ 9 & \text { Missing }\end{array}$

$\begin{array}{lll}\text { ST25Q02 (65) } & \text { Attend: } \\ \text { Format: } & \text { F1 } & \text { Column } \\ & 1 & \text { Tick } \\ 2 & \text { No tick } \\ 7 & \text { N/A } \\ 8 & \text { Invalid } \\ 9 & \text { Missing }\end{array}$

ST25Q03 (66) Attend: specific program Q25c

Format: F1 Columns: 118-118

1 Tick

2 No tick

7 N/A

8 Invalid

9 Missing

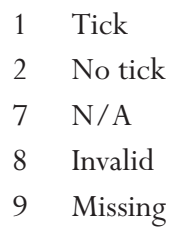




$$
\begin{array}{lll}
\text { ST26Q04 (73) } & \text { Give extra help Q26d } \\
\text { Format: } & \text { F1 } & \text { Columns: 125-125 } \\
& 1 & \text { Strongly agree } \\
2 & \text { Agree } \\
3 & \text { Disagree } \\
4 & \text { Strongly disagree } \\
7 & \text { N/A } \\
8 & \text { Invalid } \\
9 & \text { Missing }
\end{array}
$$

ST26Q05 (74) Treat me fairly Q26e

Format: F1 Columns: 126-126

1 Strongly agree

2 Agree

3 Disagree

4 Strongly disagree

7 N/A

8 Invalid

9 Missing

$$
\begin{array}{lll}
\text { ST27Q01 (75) } & \text { Feel an outsider Q27a } \\
\text { Format: } & \text { F1 } & \text { Columns: 127-127 } \\
& 1 & \text { Strongly agree } \\
2 & \text { Agree } \\
3 & \text { Disagree } \\
4 & \text { Strongly disagree } \\
7 & \text { N/A } \\
8 & \text { Invalid } \\
9 & \text { Missing }
\end{array}
$$

ST27Q02 (76) Make friends Q27b

$$
\begin{array}{rll}
\text { Format: } & \text { F1 } & \text { Columns: 128-128 } \\
1 & \text { Strongly agree } \\
2 & \text { Agree } \\
3 & \text { Disagree } \\
4 & \text { Strongly disagree } \\
7 & \text { N/A } \\
8 & \text { Invalid } \\
9 & \text { Missing }
\end{array}
$$

$\begin{array}{lll}\text { ST27Q03 (77) } & \text { Feel I belong Q27c } \\ \text { Format: } & \text { F1 } & \text { Columns: 129-129 } \\ & 1 & \text { Strongly agree } \\ 2 & \text { Agree } \\ 3 & \text { Disagree } \\ 4 & \text { Strongly disagree } \\ 7 & \text { N/A } \\ 8 & \text { Invalid } \\ 9 & \text { Missing }\end{array}$

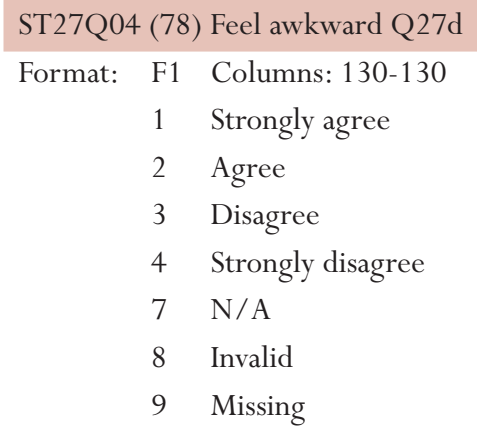


ST29Q03 (84) Hours all: <Enrichment> Q29C

Format: F3 Columns: 150-157

$997 \mathrm{~N} / \mathrm{A}$

998 Invalid

999 Missing

$\begin{array}{ll}\text { ST29Q04 (85) Hours all: tutor Q29d } \\ \text { Format: } & \text { F3 Columns: 158-165 } \\ & 997 \mathrm{~N} / \mathrm{A} \\ & 998 \text { Invalid } \\ & 999 \text { Missing }\end{array}$

ST29Q05 (86) Hours all: < out-of-school> Q29e

Format: F3 Columns: 166-173

$997 \mathrm{~N} / \mathrm{A}$

998 Invalid

999 Missing

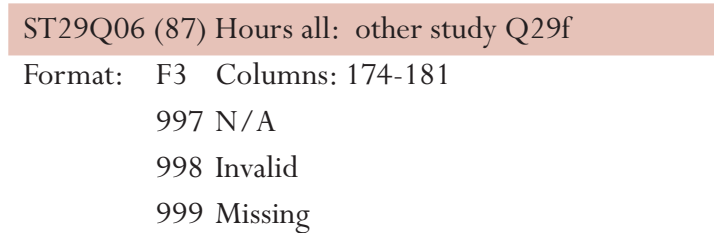

\section{ST30Q01 (88) Attitude: enjoy reading Q30a}

Format: F1 Columns: 182-182

1 Strongly agree

2 Agree

3 Disagree

4 Strongly disagree

7 N/A

8 Invalid

9 Missing

\section{ST30Q02 (89) Attitude: effort Q30b}

Format: F1 Columns: 183-183

1 Strongly agree

2 Agree

3 Disagree

4 Strongly disagree

7 N/A

8 Invalid

9 Missing

\section{ST30Q03 (90) Attitude: look forward Q30c}

Format: F1 Columns: 184-184

1 Strongly agree

2 Agree

3 Disagree

4 Strongly disagree

7 N/A

8 Invalid

9 Missing
ST30Q04 (91) Attitude: enjoy Maths Q30d

Format: F1 Columns: 185-185

1 Strongly agree

2 Agree

3 Disagree

4 Strongly disagree

7 N/A

8 Invalid

9 Missing

ST30Q05 (92) Attitude: career Q30e

Format: F1 Columns: 186-186

1 Strongly agree

2 Agree

3 Disagree

4 Strongly disagree

7 N/A

8 Invalid

9 Missing

ST30Q06 (93) Attitude: interested Q30f

Format: F1 Columns: 187-187

1 Strongly agree

2 Agree

3 Disagree

4 Strongly disagree

7 N/A

8 Invalid

9 Missing

\section{ST30Q07 (94) Attitude: further study Q30g}

Format: F1 Columns: 188-188

1 Strongly agree

2 Agree

3 Disagree

4 Strongly disagree

7 N/A

8 Invalid

9 Missing

ST30Q08 (95) Attitude: job Q30h

Format: F1 Columns: 189-189

1 Strongly agree

2 Agree

3 Disagree

4 Strongly disagree

7 N/A

8 Invalid

$9 \quad$ Missing 


$\begin{array}{lll}\text { ST31Q01 } & \text { (96) } & \text { Confident: timetable } \\ \text { Format: } & \text { F1 } & \text { Columns: 190-190 } \\ & 1 & \text { Very confident } \\ 2 & \text { Confident } \\ 3 & \text { Not very confident } \\ 4 & \text { Not at all confident } \\ 7 & \text { N/A } \\ 8 & \text { Invalid } \\ 9 & \text { Missing }\end{array}$

\section{ST31Q02 (97) Confident: discount Q31b}

Format: F1 Columns: 191-191

1 Very confident

2 Confident

3 Not very confident

4 Not at all confident

7 N/A

8 Invalid

9 Missing

$\begin{array}{lll}\text { ST31Q03 (98) } & \text { Confident: area Q31 } \\ \text { Format: } & \text { F1 } & \text { Columns: 192-192 } \\ & 1 & \text { Very confident } \\ 2 & \text { Confident } \\ 3 & \text { Not very confident } \\ 4 & \text { Not at all confident } \\ 7 & \text { N/A } \\ 8 & \text { Invalid } \\ 9 & \text { Missing }\end{array}$

\section{ST31Q04 (99) Confident: graphs Q31d}

Format: F1 Columns: 193-193

1 Very confident

2 Confident

3 Not very confident

4 Not at all confident

7 N/A

8 Invalid

9 Missing

$\begin{array}{lll}\text { ST31Q05 (100) Confident: linear } \\ \text { Format: } & \text { F1 } & \text { Columns: 194-194 } \\ & 1 & \text { Very confident } \\ 2 & \text { Confident } \\ 3 & \text { Not very confident } \\ 4 & \text { Not at all confident } \\ 7 & \text { N/A } \\ 8 & \text { Invalid } \\ 9 & \text { Missing }\end{array}$

$\begin{array}{lll}\text { ST31Q06 (101) Confident: distanc } \\ \text { Format: } & \text { F1 } & \text { Columns: 195-195 } \\ 1 & \text { Very confident } \\ 2 & \text { Confident } \\ 3 & \text { Not very confident } \\ 4 & \text { Not at all confident } \\ 7 & \text { N/A } \\ 8 & \text { Invalid } \\ 9 & \text { Missing }\end{array}$

ST31Q07 (102) Confident: quadratics Q31g

Format: F1 Columns: 196-196

1 Very confident

2 Confident

3 Not very confident

$4 \quad$ Not at all confident

7 N/A

8 Invalid

9 Missing

ST31Q08 (103) Confident: rate Q31h

Format: F1 Columns: 197-197

1 Very confident

2 Confident

3 Not very confident

4 Not at all confident

7 N/A

8 Invalid

9 Missing

ST32Q01 (104) Feel study: worry Q32a

Format: F1 Columns: 198-198

1 Strongly agree

2 Agree

3 Disagree

4 Strongly disagree

7 N/A

8 Invalid

9 Missing

ST32Q02 (105) Feel study: not good Q32b

Format: F1 Columns: 199-199

1 Strongly agree

2 Agree

3 Disagree

4 Strongly disagree

7 N/A

8 Invalid

9 Missing 


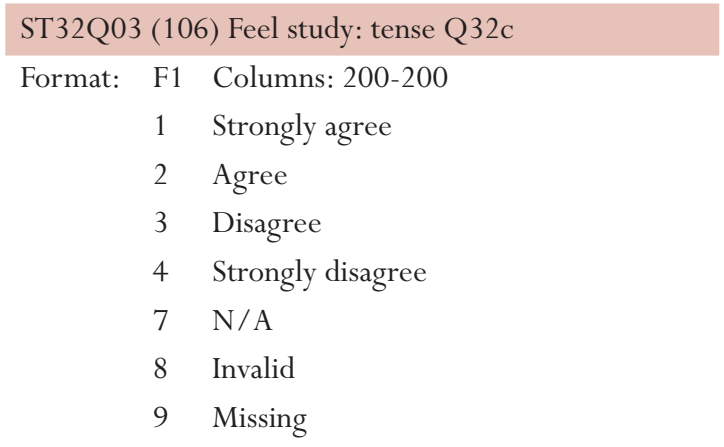

\section{ST32Q04 (107) Feel study: good < marks> Q32d}

Format: F1 Columns: 201-201

1 Strongly agree

2 Agree

3 Disagree

4 Strongly disagree

7 N/A

8 Invalid

9 Missing

\section{ST32Q05 (108) Feel study: nervous Q32e}

Format: F1 Columns: 202-202

1 Strongly agree

2 Agree

3 Disagree

4 Strongly disagree

7 N/A

8 Invalid

9 Missing

ST32Q06 (109) Feel study: quickly Q32f

Format: F1 Columns: 203-203

1 Strongly agree

2 Agree

3 Disagree

4 Strongly disagree

7 N/A

8 Invalid

9 Missing

ST32Q07 (110) Feel study: best subject Q32g

$\begin{array}{rll}\text { Format: } & \text { F1 } & \text { Columns: 204-204 } \\ 1 & \text { Strongly agree } \\ 2 & \text { Agree } \\ 3 & \text { Disagree } \\ 4 & \text { Strongly disagree } \\ 7 & \text { N/A } \\ 8 & \text { Invalid } \\ 9 & \text { Missing }\end{array}$

ST32Q08 (111) Feel study: helpless Q32h

Format: F1 Columns: 205-205

1 Strongly agree

2 Agree

3 Disagree

4 Strongly disagree

7 N/A

8 Invalid

9 Missing

ST32Q09 (112) Feel study: underst. diffc. Q32i

Format: F1 Columns: 206-206

1 Strongly agree

2 Agree

3 Disagree

4 Strongly disagree

7 N/A

8 Invalid

9 Missing

ST32Q10 (113) Feel study: poor < marks > Q32j

Format: F1 Columns: 207-207

1 Strongly agree

2 Agree

3 Disagree

4 Strongly disagree

7 N/A

8 Invalid

9 Missing

ST33Q01 (114) Hours maths: homework Q33a

Format: F3 Columns: 208-215

997 N/A

998 Invalid

999 Missing

ST33Q02 (115) Hours maths: < remedial> Q33b

Format: F3 Columns: 216-223

997 N/A

998 Invalid

999 Missing

ST33Q03 (116) Hours maths: <enrichment> Q33c

Format: F3 Columns: 224-231

997 N/A

998 Invalid

999 Missing

ST33Q04 (117) Hours maths: tutor Q33d

Format: F3 Columns: 232-239

$997 \mathrm{~N} / \mathrm{A}$

998 Invalid

999 Missing 


\section{ST33Q05 (118) Hours maths: <out-of-school> Q33e}

Format: F3 Columns: 240-247

997 N/A

998 Invalid

999 Missing

$\begin{array}{ll}\text { ST33Q06 (119) Hours maths: other Q33f } \\ \text { Format: } & \text { F3 Columns: 248-255 } \\ & 997 \text { N/A } \\ & 998 \text { Invalid } \\ & 999 \text { Missing }\end{array}$

\section{ST34Q01 (120) Learn: important parts Q34a}

Format: F1 Columns: 256-256

1 Strongly agree

2 Agree

3 Disagree

4 Strongly disagree

$7 \quad \mathrm{~N} / \mathrm{A}$

8 Invalid

9 Missing

\section{ST34Q02 (121) Learn: new ways Q34b}

Format: F1 Columns: 257-257

1 Strongly agree

2 Agree

3 Disagree

4 Strongly disagree

7 N/A

8 Invalid

9 Missing

ST34Q03 (122) Learn: check myself Q34c

Format: F1 Columns: 258-258

1 Strongly agree

2 Agree

3 Disagree

4 Strongly disagree

7 N/A

8 Invalid

9 Missing

ST34Q04 (123) Learn: concepts Q34d

Format: F1 Columns: 259-259

1 Strongly agree

2 Agree

3 Disagree

4 Strongly disagree

7 N/A

8 Invalid

$9 \quad$ Missing
ST34Q05 (124) Learn: everyday life Q34e

Format: F1 Columns: 260-260

1 Strongly agree

2 Agree

3 Disagree

4 Strongly disagree

7 N/A

8 Invalid

9 Missing

ST34Q06 (125) Learn: solve when sleep Q34f

Format: F1 Columns: 261-261

1 Strongly agree

2 Agree

3 Disagree

4 Strongly disagree

7 N/A

8 Invalid

9 Missing

ST34Q07 (126) Learn: by heart Q34g

Format: F1 Columns: 262-262

1 Strongly agree

2 Agree

3 Disagree

4 Strongly disagree

7 N/A

8 Invalid

9 Missing

ST34Q08 (127) Learn: by relating Q34h

Format: F1 Columns: 263-263

1 Strongly agree

2 Agree

3 Disagree

4 Strongly disagree

7 N/A

8 Invalid

9 Missing

ST34Q09 (128) Learn: examples Q34i

Format: F1 Columns: 264-264

1 Strongly agree

2 Agree

3 Disagree

4 Strongly disagree

7 N/A

8 Invalid

$9 \quad$ Missing 


$\begin{array}{lll}\text { ST34Q10 (129) Learn: clarify Q34j } \\ \text { Format: } & \text { F1 } & \text { Columns: 265-265 } \\ 1 & \text { Strongly agree } \\ 2 & \text { Agree } \\ 3 & \text { Disagree } \\ 4 & \text { Strongly disagree } \\ 7 & \text { N/A } \\ 8 & \text { Invalid } \\ 9 & \text { Missing }\end{array}$

ST34Q11 (130) Learn: applied Q34k

Format: F1 Columns: 266-266

1 Strongly agree

2 Agree

3 Disagree

4 Strongly disagree

7 N/A

8 Invalid

9 Missing

$$
\begin{array}{lll}
\text { ST34Q12 (131) Learn: exactly Q34l } \\
\text { Format: } & \text { F1 } & \text { Columns: 267-267 } \\
& 1 & \text { Strongly agree } \\
2 & \text { Agree } \\
3 & \text { Disagree } \\
4 & \text { Strongly disagree } \\
7 & \text { N/A } \\
8 & \text { Invalid } \\
9 & \text { Missing }
\end{array}
$$

ST34Q13 (132) Learn: procedure Q34m

Format: F1 Columns: 268-268

1 Strongly agree

2 Agree

3 Disagree

4 Strongly disagree

7 N/A

8 Invalid

9 Missing

\section{ST34Q14 (133) Learn: relate Q34n}

Format: F1 Columns: 269-269

1 Strongly agree

2 Agree

3 Disagree

4 Strongly disagree

7 N/A

8 Invalid

9 Missing
ST35Q02 (134) Maths < class periods> Q35b

Format: F3 Columns: 270-277

$997 \mathrm{~N} / \mathrm{A}$

998 Invalid

999 Missing

ST35Q03 (135) All < class periods> Q35c

Format: F3 Columns: 278-285

$997 \mathrm{~N} / \mathrm{A}$

998 Invalid

999 Missing

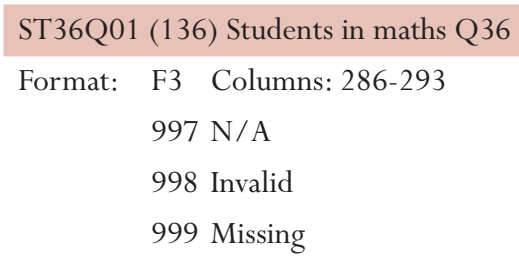

\section{ST37Q03 (139) Attitudes: exams Q37c}

Format: F1 Columns: 296-296

1 Strongly agree

2 Agree

3 Disagree

4 Strongly disagree

7 N/A

8 Invalid

$9 \quad$ Missing 


$\begin{array}{lll}\text { ST37Q04 (140) Attitudes: project } \\ \text { Format: } & \text { F1 } & \text { Columns: 297-297 } \\ & 1 & \text { Strongly agree } \\ 2 & \text { Agree } \\ 3 & \text { Disagree } \\ 4 & \text { Strongly disagree } \\ 7 & \text { N/A } \\ 8 & \text { Invalid } \\ 9 & \text { Missing }\end{array}$

ST37Q05 (141) Attitudes: effort Q37e

Format: F1 Columns: 298-298

1 Strongly agree

2 Agree

3 Disagree

4 Strongly disagree

7 N/A

8 Invalid

9 Missing

$\begin{array}{lll}\text { ST37Q06 (142) Attitudes: work w } \\ \text { Format: } & \text { F1 } & \text { Columns: 299-299 } \\ & 1 & \text { Strongly agree } \\ 2 & \text { Agree } \\ 3 & \text { Disagree } \\ 4 & \text { Strongly disagree } \\ 7 & \text { N/A } \\ 8 & \text { Invalid } \\ 9 & \text { Missing }\end{array}$

ST37Q07 (143) Attitudes: do better Q37g

Format: F1 Columns: 300-300

1 Strongly agree

2 Agree

3 Disagree

4 Strongly disagree

7 N/A

8 Invalid

9 Missing

$\begin{array}{lll}\text { ST37Q08 (144) Attitudes: helping } \\ \text { Format: } & \text { F1 } & \text { Columns: 301-301 } \\ & 1 & \text { Strongly agree } \\ 2 & \text { Agree } \\ 3 & \text { Disagree } \\ 4 & \text { Strongly disagree } \\ 7 & \text { N/A } \\ 8 & \text { Invalid } \\ 9 & \text { Missing }\end{array}$

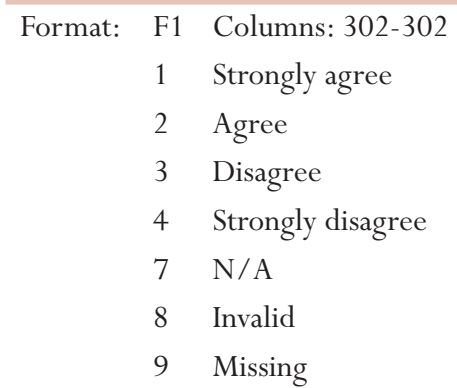




\section{ST38Q04 (150) Lesson: book work Q38d}

Format: F1 Columns: 307-307

1 Every lesson

2 Most lessons

3 Some lessons

4 Never or hardly ever

7 N/A

8 Invalid

9 Missing

ST38Q05 (151) Lesson: help learning Q38e

Format: F1 Columns: 308-308

1 Every lesson

2 Most lessons

3 Some lessons

4 Never or hardly ever

7 N/A

8 Invalid

9 Missing

\section{ST38Q06 (152) Lesson: noise Q38f}

Format: F1 Columns: 309-309

1 Every lesson

2 Most lessons

3 Some lessons

4 Never or hardly ever

7 N/A

8 Invalid

9 Missing

ST38Q07 (153) Lesson: understand Q38g

Format: F1 Columns: 310-310

1 Every lesson

2 Most lessons

3 Some lessons

4 Never or hardly ever

7 N/A

8 Invalid

9 Missing

ST38Q08 (154) Lesson: <quieten down> Q38h

$\begin{array}{lll}\text { Format: } & \text { F1 } & \text { Columns: 311-311 } \\ 1 & \text { Every lesson } \\ 2 & \text { Most lessons } \\ 3 & \text { Some lessons } \\ 4 & \text { Never or hardly ever } \\ 7 & \text { N/A } \\ 8 & \text { Invalid } \\ 9 & \text { Missing }\end{array}$

ST38Q09 (155) Lesson: can't work well Q38i

Format: F1 Columns: 312-312

1 Every lesson

2 Most lessons

3 Some lessons

4 Never or hardly ever

$7 \mathrm{~N} / \mathrm{A}$

8 Invalid

9 Missing

\section{ST38Q10 (156) Lesson: opinions Q38j}

Format: F1 Columns: 313-313

1 Every lesson

2 Most lessons

3 Some lessons

4 Never or hardly ever

7 N/A

8 Invalid

9 Missing

\section{ST38Q11 (157) Lesson: late start Q38k}

Format: F1 Columns: 314-314

1 Every lesson

2 Most lessons

3 Some lessons

4 Never or hardly ever

7 N/A

8 Invalid

9 Missing

EC01Q01 (158) Missing two months < ISCED 1> EC1

Format: F1 Columns: 315-315

1 No, never

2 Yes, once

3 Yes, twice or more

7 N/A

8 Invalid

$9 \quad$ Missing

EC02Q01 (159) Missing two months < ISCED 2> EC2

Format: F1 Columns: 316-316

1 No, never

2 Yes, once

3 Yes, twice or more

7 N/A

8 Invalid

$9 \quad$ Missing 
EC03Q01 (160) Change while in <ISCED 1> EC3

$\begin{array}{rll}\text { Format: } & \text { F1 } & \text { Columns: 317-317 } \\ & 1 & \text { No, all }<\text { ISCED } 1>\text { same school } \\ 2 & \text { Yes, changed once } \\ 3 & \text { Yes, changed twice or more } \\ 7 & \text { N/A } \\ 8 & \text { Invalid } \\ 9 & \text { Missing }\end{array}$

EC04Q01 (161) Change while in <ISCED 2> EC4

Format: F1 Columns: 318-318

1 No, all $<$ ISCED $1>$ same school

2 Yes, changed once

3 Yes, changed twice or more

7 N/A

8 Invalid

9 Missing

\section{EC05Q01 (162) Changed < study programme > since}

$$
<\text { Grade } \mathrm{X}>\text { EC5 }
$$

Format: F1 Columns: 319-319

1 Yes

2 No

7 N/A

8 Invalid

$9 \quad$ Missing

$\begin{array}{lll}\text { EC06Q01 } & (163) \text { Type }<\text { Mathemati } \\ \text { Format: } & \text { F1 } & \text { Columns: 320-320 } \\ & 1 & <\text { high level }> \\ 2 & <\text { medium level }> \\ 3 & <\text { basic level }> \\ 7 & \text { N/A } \\ 8 & \text { Invalid } \\ 9 & \text { Missing }\end{array}$

EC06Q02 (164) Type <Mathematics class $>$ National codes EC6

\section{Format: A4 Columns: 321-324}

0361 Australia: High level maths course

0362 Australia: Medium level maths course

0363 Australia: Low level maths course

2761 Germany: High level maths course

2762 Germany: Medium level maths course

2763 Germany: Low level maths course

3001 Greece: Advanced level maths course

3002 Greece: Medium level maths course

3003 Greece: Basic level maths course
3481 Hungary: Special or high level maths course

3482 Hungary: Normal maths course

3521 Iceland: Fast track maths course

3522 Iceland: Medium track maths course

3523 Iceland: Slow track maths course

4101 Korea: High level maths course

4102 Korea: Medium level maths course

4103 Korea: Basic level maths course

7031 Slovak Rep.: Extended maths teaching

7032 Slovak Rep.: Normal maths teaching

7033 Slovak Rep.: Reduced maths teaching

8261 Scotland: Nat. Qualifications higher

8262 Scotland: Intermediate/Access, standard grade

9997 N/A

9998 Invalid

9999 Missing

\section{EC07Q01 (165) Mark in <Mathematics> EC7}

Format: F8.3 Columns: 325-332

$\begin{array}{ll}997.000 & \text { N/A } \\ 998.000 & \text { Invalid } \\ 999.000 & \text { Missing }\end{array}$

\section{EC07Q02 (166) Pass mark in maths EC7}

$\begin{array}{cll}\text { Format: } & \text { F1 } & \text { Columns: 333-333 } \\ 1 & \text { At or above < pass mark> } \\ 2 & \text { Below < pass mark }> \\ 7 & \text { N/A } \\ 8 & \text { Invalid } \\ 9 & \text { Missing }\end{array}$

EC07Q03 (167) Mark in maths in percentages EC7

Format: F8.3 Columns: 334-341

$\begin{array}{ll}997.000 & \text { N/A } \\ 998.000 & \text { Invalid } \\ 999.000 & \text { Missing }\end{array}$

\section{EC08Q01 (168) Job at 30 EC8}

Format: A4 Columns: 342-345

$9997 \mathrm{~N} / \mathrm{A}$

9998 Invalid

9999 Missing 


$\begin{array}{cll}\text { IC01Q01 (169) Available at home IC1a } \\ \text { Format: } & \text { F1 } & \text { Columns: 346-346 } \\ 1 & \text { Yes } \\ 2 & \text { No } \\ 7 & \text { N/A } \\ 8 & \text { Invalid } \\ 9 & \text { Missing } \\ \text { IC01Q02 (170) Available at school IC1b } \\ \text { Format: } & \text { F1 } & \text { Columns: 347-347 } \\ 1 & \text { Yes } \\ 2 & \text { No } \\ 7 & \text { N/A } \\ 8 & \text { Invalid } \\ 9 & \text { Missing }\end{array}$

IC01Q03 (171) Available at other places IC1C

Format: F1 Columns: 348-348

1 Yes

2 No

$7 \quad \mathrm{~N} / \mathrm{A}$

$8 \quad$ Invalid

9 Missing

IC02Q01 (172) Used computer IC2

Format: F1 Columns: 349-349

1 Yes

2 No

$7 \quad \mathrm{~N} / \mathrm{A}$

8 Invalid

9 Missing

IC03Q01 (173) How long using computers IC3

Format: F1 Columns: 350-350

1 Less than 1 year

21 to 3 years

33 to 5 years

4 More than 5 years

$7 \quad \mathrm{~N} / \mathrm{A}$

8 Invalid

9 Missing

\section{IC04Q01 (174) Use often at home IC4a}

Format: F1 Columns: 351-351

1 Almost every day

2 A few times each week

3 Between 1 pwk \& 1 pmn

$4 \quad$ Less than 1 pmn

5 Never

$7 \quad \mathrm{~N} / \mathrm{A}$

8 Invalid

$9 \quad$ Missing
IC04Q02 (175) Use often at school IC4b

Format: F1 Columns: 352-352

Almost every day

A few times each week

Between 1 pwk \& 1 pmn

Less than 1 pmn

Never

N/A

Invalid

Missing

IC04Q03 (176) Use often at other places IC4c

Format: F1 Columns: 353-353

Almost every day

A few times each week

Between 1 pwk \& 1 pmn

Less than 1 pmn

Never

$\mathrm{N} / \mathrm{A}$

Invalid

Missing

IC05Q01 (177) How often: information IC5a

Format: F1 Columns: 354-354

Almost every day

A few times each week

Between 1 pwk \& 1 pmn

Less than 1 pmn

Never

N/A

Invalid

Missing

IC05Q02 (178) How often games IC5b

Format: F1 Columns: 355-355

Almost every day

A few times each week

Between 1 pwk \& 1 pmn

Less than 1 pmn

Never

N/A

Invalid

Missing

IC05Q03 (179) How often: Word IC5c

Format: F1 Columns: 356-356

Almost every day

A few times each week

Between 1 pwk \& 1 pmn

Less than 1 pmn

Never

N/A

Invalid

Missing 


$\begin{array}{lll}\text { IC05Q04 (180) } & \text { How often: group IC5d } \\ \text { Format: } & \text { F1 } & \text { Columns: 357-357 } \\ 1 & \text { Almost every day } \\ 2 & \text { A few times each week } \\ 3 & \text { Between 1 pwk \& 1 pmn } \\ 4 & \text { Less than 1 pmn } \\ 5 & \text { Never } \\ 7 & \text { N/A } \\ 8 & \text { Invalid } \\ 9 & \text { Missing }\end{array}$

\section{IC05Q05 (181) How often: spreadsheets IC5e}

$\begin{array}{lll}\text { Format: } & \text { F1 } & \text { Columns: 358-358 } \\ 1 & \text { Almost every day } \\ 2 & \text { A few times each week } \\ 3 & \text { Between 1 pwk \& 1 pmn } \\ 4 & \text { Less than 1 pmn } \\ 5 & \text { Never } \\ 7 & \text { N/A } \\ 8 & \text { Invalid } \\ 9 & \text { Missing }\end{array}$

IC05Q06 (182) How often: Internet software? IC5f

Format: F1 Columns: 359-359

1 Almost every day

2 A few times each week

3 Between 1 pwk \& 1 pmn

4 Less than 1 pmn

5 Never

$7 \quad \mathrm{~N} / \mathrm{A}$

8 Invalid

9 Missing

$\begin{array}{lll}\text { IC05Q07 (183) } & \text { How often: graphics IC5g } \\ \text { Format: } & \text { F1 } & \text { Columns: 360-360 } \\ & 1 & \text { Almost every day } \\ 2 & \text { A few times each week } \\ 3 & \text { Between 1 pwk \& 1 pmn } \\ 4 & \text { Less than 1 pmn } \\ 5 & \text { Never } \\ 7 & \text { N/A } \\ 8 & \text { Invalid } \\ 9 & \text { Missing }\end{array}$

IC05Q08 (184) How often: educ software IC5h

$\begin{array}{lll}\text { Format: } & \text { F1 } & \text { Columns: 361-361 } \\ 1 & \text { Almost every day } \\ 2 & \text { A few times each week } \\ 3 & \text { Between 1 pwk \& 1 pmn } \\ 4 & \text { Less than 1 pmn } \\ 5 & \text { Never } \\ 7 & \text { N/A } \\ 8 & \text { Invalid } \\ 9 & \text { Missing }\end{array}$

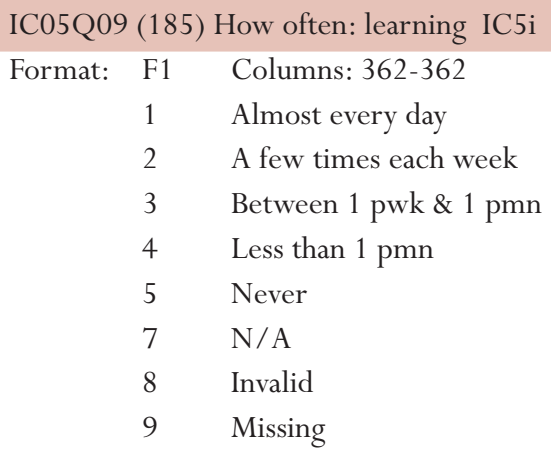

IC05Q11 (187) How often: programming IC5k

Format: F1 Columns: 364-364

1 Almost every day

2 A few times each week

3 Between 1 pwk \& 1 pmn

$4 \quad$ Less than 1 pmn

5 Never

$7 \quad \mathrm{~N} / \mathrm{A}$

$8 \quad$ Invalid

$9 \quad$ Missing

IC05Q12 (188) How often: chatrooms IC5I

Format: F1 Columns: 365-365

1 Almost every day

2 A few times each week

3 Between 1 pwk \& 1 pmn

$4 \quad$ Less than 1 pmn

5 Never

$7 \quad \mathrm{~N} / \mathrm{A}$

8 Invalid

$9 \quad$ Missing

IC06Q01 (189) How well: start game IC6a

Format: F1 Columns: 366-366

1 Can do well

2 Can do with help

3 Cannot do

4 Don $t$ know

$7 \quad \mathrm{~N} / \mathrm{A}$

8 Invalid

$9 \quad$ Missing 
IC06Q02 (190) How well: antiviruses IC6b

$\begin{array}{cll}\text { Format: } & \text { F1 } & \text { Columns: 367-367 } \\ 1 & \text { Can do well } \\ 2 & \text { Can do with help } \\ 3 & \text { Cannot do } \\ 4 & \text { Don t know } \\ 7 & \text { N/A } \\ 8 & \text { Invalid } \\ 9 & \text { Missing }\end{array}$

$\begin{array}{lll}\text { IC06Q03 (191) } & \text { How well: open file } \\ \text { Format: } & \text { F1 } & \text { Columns: 368-368 } \\ 1 & \text { Can do well } \\ 2 & \text { Can do with help } \\ 3 & \text { Cannot do } \\ 4 & \text { Don t know } \\ 7 & \text { N/A } \\ 8 & \text { Invalid } \\ 9 & \text { Missing }\end{array}$

\section{IC06Q04 (192) How well: edit IC6d}

Format: F1 Columns: 369-369

1 Can do well

2 Can do with help

3 Cannot do

4 Don $\mathrm{t}$ know

$7 \quad \mathrm{~N} / \mathrm{A}$

8 Invalid

9 Missing

IC06Q05 (193) How well: scroll IC6e

Format: F1 Columns: 370-370

1 Can do well

2 Can do with help

3 Cannot do

4 Don $\mathrm{t}$ know

$7 \quad \mathrm{~N} / \mathrm{A}$

8 Invalid

9 Missing

IC06Q06 (194) How well: addresses IC6f

Format: F1 Columns: 371-371

1 Can do well

2 Can do with help

3 Cannot do

4 Don $t$ know

$7 \quad \mathrm{~N} / \mathrm{A}$

8 Invalid

$9 \quad$ Missing
IC06Q07 (195) How well: copy IC6g

Format: F1 Columns: 372-372

Can do well

Can do with help

Cannot do

Don t know

N/A

Invalid

$9 \quad$ Missing

IC06Q08 (196) How well: save IC6h

Format: F1 Columns: 373-373

Can do well

Can do with help

Cannot do

Don $\mathrm{t}$ know

$7 \quad \mathrm{~N} / \mathrm{A}$

8 Invalid

$9 \quad$ Missing

IC06Q09 (197) How well: print IC6i

Format: F1 Columns: 374-374

1 Can do well

2 Can do with help

3 Cannot do

4 Don $t$ know

$7 \quad \mathrm{~N} / \mathrm{A}$

8 Invalid

$9 \quad$ Missing

IC06Q10 (198) How well: delete IC6j

Format: F1 Columns: 375-375

Can do well

Can do with help

Cannot do

Don $t$ know

N/A

8 Invalid

$9 \quad$ Missing

IC06Q11 (199) How well: move IC6k

Format: F1 Columns: 376-376

1 Can do well

2 Can do with help

3 Cannot do

4 Don $t$ know

$7 \quad \mathrm{~N} / \mathrm{A}$

$8 \quad$ Invalid

$9 \quad$ Missing 


$\begin{array}{lll}\text { IC06Q12 (200) } & \text { How well: Internet I } \\ \text { Format: } & \text { F1 } & \text { Columns: 377-377 } \\ 1 & \text { Can do well } \\ 2 & \text { Can do with help } \\ 3 & \text { Cannot do } \\ 4 & \text { Don t know } \\ 7 & \text { N/A } \\ 8 & \text { Invalid } \\ 9 & \text { Missing }\end{array}$

$\begin{array}{lll}\text { IC06Q13 (201) } & \text { How well: download } \\ \text { Format: } & \text { F1 } & \text { Columns: 378-378 } \\ 1 & \text { Can do well } \\ 2 & \text { Can do with help } \\ 3 & \text { Cannot do } \\ 4 & \text { Don t know } \\ 7 & \text { N/A } \\ 8 & \text { Invalid } \\ 9 & \text { Missing }\end{array}$

\section{IC06Q14 (202) How well: attach IC6n}

Format: F1 Columns: 379-379

1 Can do well

2 Can do with help

3 Cannot do

4 Don $\mathrm{t}$ know

$7 \quad \mathrm{~N} / \mathrm{A}$

8 Invalid

9 Missing

\section{IC06Q15 (203) How well: program IC6o}

$\begin{array}{cll}\text { Format: } & \text { F1 } & \text { Columns: 380-380 } \\ 1 & \text { Can do well } \\ 2 & \text { Can do with help } \\ 3 & \text { Cannot do } \\ 4 & \text { Don t know } \\ 7 & \text { N/A } \\ 8 & \text { Invalid } \\ 9 & \text { Missing }\end{array}$

\section{IC06Q16 (204) How well: spreadsheet plot IC6p}

Format: F1 Columns: 381-381

1 Can do well

2 Can do with help

3 Cannot do

4 Don t know

$7 \quad \mathrm{~N} / \mathrm{A}$

8 Invalid

$9 \quad$ Missing
IC06Q17 (205) How well: PowerPoint IC6q

$\begin{array}{lll}\text { Format: } & \text { F1 } & \text { Columns: 382-382 } \\ 1 & \text { Can do well } \\ 2 & \text { Can do with help } \\ 3 & \text { Cannot do } \\ 4 & \text { Don t know } \\ 7 & \text { N/A } \\ 8 & \text { Invalid } \\ 9 & \text { Missing }\end{array}$

IC06Q18 (206) How well: games IC6r

Format: F1 Columns: 383-383

1 Can do well

2 Can do with help

3 Cannot do

4 Don $t$ know

$7 \quad \mathrm{~N} / \mathrm{A}$

8 Invalid

$9 \quad$ Missing

IC06Q19 (207) How well: download music IC6s

Format: F1 Columns: 384-384

1 Can do well

2 Can do with help

3 Cannot do

4 Don $\mathrm{t}$ know

$7 \quad \mathrm{~N} / \mathrm{A}$

8 Invalid

$9 \quad$ Missing

IC06Q20 (208) How well: multimedia IC6t

Format: F1 Columns: 385-385

1 Can do well

2 Can do with help

3 Cannot do

4 Don $t$ know

$7 \quad \mathrm{~N} / \mathrm{A}$

8 Invalid

9 Missing

IC06Q21 (209) How well: draw IC6u

Format: F1 Columns: 386-386

1 Can do well

2 Can do with help

3 Cannot do

4 Don $t$ know

$7 \quad \mathrm{~N} / \mathrm{A}$

8 Invalid

$9 \quad$ Missing 


$\begin{array}{lll}\text { IC06Q22 } & \text { (210) } & \text { How well: e-mails IC6v } \\ \text { Format: } & \text { F1 } & \text { Columns: 387-387 } \\ 1 & \text { Can do well } \\ 2 & \text { Can do with help } \\ 3 & \text { Cannot do } \\ 4 & \text { Don t know } \\ 7 & \text { N/A } \\ 8 & \text { Invalid } \\ 9 & \text { Missing } \\ \text { IC06Q23 (211) How well: web page IC6w } \\ \text { Format: } & \text { F1 Columns: } 388-388 \\ 1 & \text { Can do well } \\ 2 & \text { Can do with help } \\ 3 & \text { Cannot do } \\ 4 & \text { Don t know } \\ 7 & \text { N/A } \\ 8 & \text { Invalid } \\ 9 & \text { Missing } \\ \end{array}$

IC07Q01 (212) Feel: important IC7a

$\begin{array}{rll}\text { Format: } & \text { F1 } & \text { Columns: 389-389 } \\ 1 & \text { Strongly agree } \\ 2 & \text { Agree } \\ 3 & \text { Disagree } \\ 4 & \text { Strongly disagree } \\ 7 & \text { N/A } \\ 8 & \text { Invalid } \\ 9 & \text { Missing }\end{array}$

IC07Q02 (213) Feel: fun IC7b

$\begin{array}{cll}\text { Format: } & \text { F1 } & \text { Columns: 390-390 } \\ 1 & \text { Strongly agree } \\ 2 & \text { Agree } \\ 3 & \text { Disagree } \\ 4 & \text { Strongly disagree } \\ 7 & \text { N/A } \\ 8 & \text { Invalid } \\ 9 & \text { Missing }\end{array}$

\section{IC07Q03 (214) Feel: interested IC7c}

$\begin{array}{cll}\text { Format: } & \text { F1 } & \text { Columns: 391-391 } \\ 1 & \text { Strongly agree } \\ 2 & \text { Agree } \\ 3 & \text { Disagree } \\ 4 & \text { Strongly disagree } \\ 7 & \text { N/A } \\ 8 & \text { Invalid } \\ 9 & \text { Missing }\end{array}$

IC07Q04 (215) Feel: forget time IC7d

$\begin{array}{cll}\text { Format: } & \text { F1 } & \text { Columns: 392-392 } \\ 1 & \text { Strongly agree } \\ 2 & \text { Agree } \\ 3 & \text { Disagree } \\ 4 & \text { Strongly disagree } \\ 7 & \text { N/A } \\ 8 & \text { Invalid } \\ 9 & \text { Missing }\end{array}$

IC08Q01 (216) Learn: computer IC8

Format: F1 Columns: 393-393

1 My school

2 My friends

3 My family

$4 \quad$ Taught myself

5 Others

$7 \quad \mathrm{~N} / \mathrm{A}$

8 Invalid

$9 \quad$ Missing

$\begin{array}{cll}\text { IC09Q01 } & \text { (217) } & \text { Learn: Internet IC9 } \\ \text { Format: } & \text { F1 } & \text { Columns: 394-394 } \\ & 1 & \text { Don t know how to use } \\ 2 & \text { My school } \\ 3 & \text { My friends } \\ 4 & \text { My family } \\ 5 & \text { Taught myself } \\ 6 & \text { Others } \\ 7 & \text { N/A } \\ 8 & \text { Invalid } \\ 9 & \text { Missing }\end{array}$

\section{SC07Q01 (218) Instructional weeks in year}

Format: F3 Columns: 395-397

997 N/A

998 Invalid

999 Missing

$\begin{array}{lll}\text { CLCUSE3A (219) } & \text { How much effort was invested in the test } \\ \text { Format: } & \text { F5 } & \text { Columns: 398-402 } \\ & 997 & \text { N/A } \\ 998 & \text { Invalid } \\ 999 & \text { Missing }\end{array}$

CLCUSE3B (220) How much effort would has been invested if marks were counted by schools

$\begin{array}{lll}\text { Format: } & \text { F5 } & \text { Columns: 403-407 } \\ & 997 & \text { N/A } \\ & 998 & \text { Invalid } \\ & 999 & \text { Missing }\end{array}$




\begin{tabular}{|c|c|c|}
\hline \multicolumn{3}{|c|}{ AGE (221) age of student } \\
\hline \multirow[t]{4}{*}{ Format: } & $\mathrm{F} 5.2$ & Columns: 408-412 \\
\hline & 97.00 & $\mathrm{~N} / \mathrm{A}$ \\
\hline & 98.00 & Invalid \\
\hline & 99.00 & Missing \\
\hline \multicolumn{3}{|c|}{ GRADE (222) Grade compared to modal grade in country } \\
\hline \multirow[t]{2}{*}{ Format: } & $\mathrm{F} 2$ & Columns: 413-414 \\
\hline & 9 & Missing \\
\hline \multicolumn{3}{|c|}{ ISCEDL (223) ISCED Level } \\
\hline \multirow[t]{7}{*}{ Format: } & F1 & Columns: 415-415 \\
\hline & 1 & ISCED level 1 \\
\hline & 2 & ISCED level 2 \\
\hline & 3 & ISCED level 3 \\
\hline & 7 & $\mathrm{~N} / \mathrm{A}$ \\
\hline & 8 & Invalid \\
\hline & 9 & Missing \\
\hline
\end{tabular}

\section{ISCEDD (224) ISCED designation}

Format: F1 Columns: 416-416

$\begin{array}{ll}1 & \text { A } \\ 2 & \text { B } \\ 3 & \text { C } \\ 4 & \text { M } \\ 7 & \text { N/A } \\ 8 & \text { Invalid } \\ 9 & \text { Missing }\end{array}$

\section{ISCEDO (225) ISCED orientation}

Format: F1 Columns: 417-417

1 General

2 Pre-vocational

3 Vocational

$7 \quad \mathrm{~N} / \mathrm{A}$

8 Invalid

$9 \quad$ Missing

\section{PROGN (226) Unique national programme code}

$\begin{array}{lll}\text { Format: } & \text { A6 } & \text { Columns: 418-423 } \\ \text { Value } & \text { Label } \\ 036001 & \text { AUS: <Year 10 in a general } \\ & \text { academic program } \\ 036002 & \text { AUS: <Year 10 in a general } \\ & \text { program (vocational) } \\ 036003 & \text { AUS:Year 11 or 12 in a general } \\ & \text { academic program } \\ 036004 & \begin{array}{l}\text { AUS:Year 11 or 12 in a VET } \\ \text { (vocational) course }\end{array}\end{array}$

040002 AUT: Hauptschule (Lower Secondary school)

040003 AUT: Polytechnische Schule (Vocational)

040004 AUT: Sonderschule (Special school (lower sec.))

040005 AUT: Sonderschul-Oberstufe (Special school (upper sec.))

040006 AUT:AHS-Unterstufe (Gymnasium Lower Secondary )

040007 AUT:AHS-Oberstufe (Gymnasium Upper Secondary)

040010 AUT: Berufsschule (Apprenticeship)

040011 AUT: BMS (Medium vocational school)

040012 AUT: Haushaltungs- und Hauswirtschaftsschulen (Medium voc.)

040014 AUT: BHS (Higher vocational school)

040015 AUT: Anst. Der Kindergarten-/ Sozialpadagogik (Voc. college)

056111 BEL: 1st year A of 1st stage of General Education (Fl.)

056112 BEL: 1st year B of 1st stage of General Education (Fl.)

056113 BEL: 2nd year of 1st stage, prep. voc. sec. education (Fl.)

056114 BEL: 2nd year of 1st stage, prep. reg. sec. education (Fl.)

056115 BEL: 2nd \& 3rd stage regular sec. education (Fl.)

056116 BEL: 2nd \& 3rd stage technical sec. education (Fl.)

056117 BEL: 2nd \& 3rd stage artistic sec. education (Fl.)

056118 BEL: 2nd \& 3rd stage vocational sec. ed. (Fl.)

056119 BEL: Part-time vocational sec. ed. for labour market (Fl.)

056120 BEL: Special sec. education (Fl.)

056197 BEL: Missing (Fl.)

056231 BEL: (1st grade of )General Education (Fr.) 


\begin{tabular}{|c|c|}
\hline 056232 & BEL: Special needs (Fr.) \\
\hline 056233 & BEL: Vocational Education (Fr.) \\
\hline 056234 & $\begin{array}{l}\text { BEL: Complementary year or } \\
\text { programme for } 1 \text { st degree (Fr.) }\end{array}$ \\
\hline 056235 & BEL: General Education (Fr.) \\
\hline \multirow[t]{2}{*}{056236} & BEL: Technical or Artistical \\
\hline & Education (transition) (Fr.) \\
\hline \multirow[t]{2}{*}{056237} & BEL:Technical or Artistical \\
\hline & Education (qualif.) (Fr.) \\
\hline 056238 & BEL: Vocational Education (Fr.) \\
\hline \multirow[t]{2}{*}{056239} & BEL: Vocational training for \\
\hline & labour market (Fr.) \\
\hline \multirow[t]{2}{*}{056242} & BEL: Special sec. education \\
\hline & (form 3 or $4-$ voc.) (Fr.) \\
\hline \multirow[t]{2}{*}{056244} & BEL: Special sec. education form \\
\hline & 3 (Germ.) \\
\hline \multirow[t]{2}{*}{056245} & BEL: Part-time Vocational \\
\hline & Education (Germ.) \\
\hline \multirow[t]{2}{*}{056246} & BEL: Vocational Education \\
\hline & (Germ.) \\
\hline 056297 & BEL: Missing (Fr.\&Germ.) \\
\hline 076001 & BRA: Lower sec. education \\
\hline 076002 & BRA: Upper sec. education \\
\hline 076097 & BRA: Missing \\
\hline 124102 & CAN: Grades $7-9$ \\
\hline 124103 & CAN: Grades $10-12$ \\
\hline 124197 & CAN: Missing \\
\hline 203001 & CZE: Basic school \\
\hline \multirow[t]{2}{*}{203002} & CZE: 6, 8-year gymnasium \& 8- \\
\hline & year conservatory (lower sec.) \\
\hline \multirow[t]{2}{*}{203003} & CZE: 6, 8-year gymnasium \\
\hline & (upper sec.) \\
\hline 203004 & CZE: 4- year gymnasium \\
\hline \multirow[t]{2}{*}{203005} & CZE: Voc/tech sec. school with \\
\hline & maturate \\
\hline 203006 & CZE: Conservatory (upper sec.) \\
\hline \multirow[t]{2}{*}{203007} & CZE:Voc/tech sec. school \\
\hline & without maturate \\
\hline 203008 & CZE: Special schools \\
\hline \multirow[t]{3}{*}{203009} & CZE: Practical schools, \\
\hline & vocational education \\
\hline & predominantly \\
\hline 208001 & DNK: Lower sec. \\
\hline 208002 & DNK: Continuation school \\
\hline
\end{tabular}

208004 DNK: Upper sec.

208097 DNK: Missing

246001 FIN: Comprehensive sec. school

250001 FRA: 5ème, 4ème, 3ème (lower sec.)

250002 FRA: SEGPA, CPA (special education)

250003 FRA: 2nde ou 1ère (générale ou techn.) (upper sec. general)

250004 FRA: Enseignement professionnel (upper sec. vocational)

250005 FRA: apprentissage (upper sec. vocational)

276001 DEU: Lower sec. access to upper sec. (compr., special educ.)

276002 DEU: Lower sec. no access to upper sec. (Hauptschule)

276003 DEU: Lower sec. no access to upper sec. (Realschule)

276004 DEU: Lower sec. access to upper sec. (Gymnasium)

276005 DEU: Lower sec. access to upper sec. (comprehensive)

276006 DEU: Lower sec. no access to upper sec. (Koop. Gesamtschule)

276009 DEU: Lower sec. no access to upper sec.

276010 DEU: Lower sec. no access to upper sec.

276011 DEU: Lower sec. no access to upper sec.

276012 DEU: Lower sec. no access to upper sec.

276013 DEU: Lower sec. with access to upper sec. (comprehensive)

276014 DEU: pre-vocational training year

276015 DEU: Vocational school (Berufsschule)

276016 DEU: Vocational school (Berufsfachschule)

276017 DEU: Upper sec. (Gymnasium)

276018 DEU: Upper sec. (comprehensive)

276097 DEU: Missing 


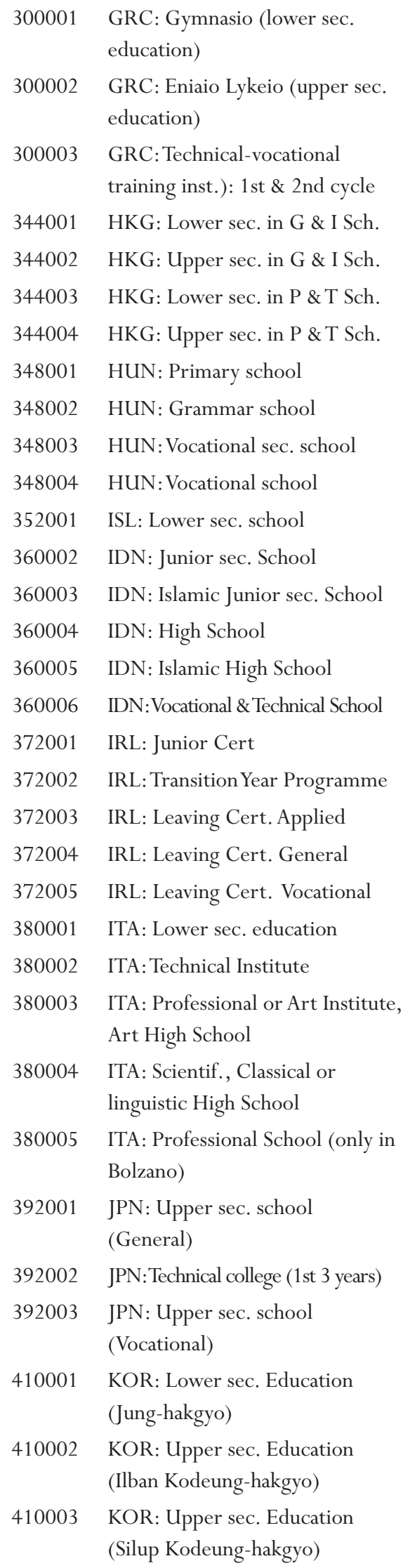

428001 LVA: Basic education

428004 LVA: General sec. education

428097 LVA: Missing

438001 LIE: sec. education, 1st stage

438002 LIE: Preparatory course for vocational education

438003 LIE: School prep. for the university entrance certificate

442001 LUX:Year 7 or 8 or 9

442002 LUX:Year 7 or 8 or 9

442003 LUX:Year 10 or 11, with mostly VET (vocational) subjects

442004 LUX:Year 10-12, in a program leading to an apprenticeship

442005 LUX:Year 10-12 in a program leading to higher education

442006 LUX:Year 10-12 in a program leading to university

446001 MAC: Lower sec. in G \& I Sch.

446002 MAC: Upper sec. in G \& I Sch.

446003 MAC: Lower sec. in P \& T Sch.

446004 MAC: Upper sec. in P \& T Sch.

484001 MEX: General Lower sec.

484002 MEX: Technical Lower sec.

484003 MEX: Lower sec. for workers

484004 MEX: General Lower sec. by Television

484005 MEX: Job Training

484006 MEX: General Baccalaureate or Upper sec.(Years prog.)

484007 MEX: General Baccalaureate or Upper sec.(semester prog.)

484008 MEX: General Baccalaureate or Upper sec.(two years prog.)

484009 MEX: Technical Baccalaureate or Upper sec. (semester prog.)

484010 MEX: Professional Technician (semester prog.)

528001 NLD: PRO

528002 NLD: VMBO

528003 NLD:VMBO BB

528004 NLD:VMBO KB

528005 NLD:VMBO GL

528006 NLD:VMBOTL 


\begin{tabular}{|c|c|}
\hline 28007 & NLD: HAVO $2 / 3$ \\
\hline 28008 & NLD: HAVO 4/5 \\
\hline 28009 & NLD: VWO $2 / 3$ \\
\hline 28010 & NLD: VWO 4/5 \\
\hline 28097 & NLD: Missing \\
\hline 54001 & NZL:Years 7 to 10 \\
\hline 54002 & NZL:Years 11 to 13 \\
\hline 4097 & NZL: Missing \\
\hline 8001 & NOR: Lower sec. \\
\hline 8002 & NOR: Upper sec. \\
\hline 16001 & POL: Gymnasium \\
\hline 16002 & $\begin{array}{l}\text { POL: Lyceum - General } \\
\text { education }\end{array}$ \\
\hline 620001 & PRT: Lower sec. \\
\hline 620003 & PRT: Upper sec. \\
\hline 620004 & $\begin{array}{l}\text { PRT: Vocational sec. } \\
\text { (technological) }\end{array}$ \\
\hline 620005 & $\begin{array}{l}\text { PRT: Vocational sec. } \\
\text { (professional) }\end{array}$ \\
\hline 643001 & $\begin{array}{l}\text { RUS: Basic general education } \\
\text { (Lower sec.) }\end{array}$ \\
\hline 643002 & $\begin{array}{l}\text { RUS: Sec. general education } \\
\text { (Upper sec.) }\end{array}$ \\
\hline 643003 & $\begin{array}{l}\text { RUS: Initial professional } \\
\text { education (prof. schools, etc.) }\end{array}$ \\
\hline 643004 & $\begin{array}{l}\text { RUS: Sec. professional education } \\
\text { (technikum, college, etc.) }\end{array}$ \\
\hline 643099 & RUS: Missing \\
\hline 703002 & SVK: Basic school (lower sec.) \\
\hline 703004 & $\begin{array}{l}\text { SVK: Vocational Basic school } \\
\text { (lower sec.) }\end{array}$ \\
\hline 703005 & SVK: sec. school (lower sec.) \\
\hline 703006 & SVK: sec. school (upper sec.) \\
\hline 703007 & SVK: High School (Gymnasium \\
\hline 703008 & SVK: sec. College \\
\hline 703009 & SVK: Technical College \\
\hline 703010 & SVK: Vocational College \\
\hline 724101 & ESP: Compulsory sec. Education \\
\hline 724102 & ESP: Baccalaureat \\
\hline 724201 & ESP: Compulsory sec. Education \\
\hline 724301 & ESP: Compulsory sec. Education \\
\hline 724401 & ESP: Compulsory sec. Education \\
\hline 2001 & SWE: Compulsory basic school \\
\hline
\end{tabular}

752002 SWE: Upper sec. school, general orientation

752003 SWE: Upper sec. school, vocational orientation

752004 SWE: Upper sec. school, the individual programme

752097 SWE: Missing

756001 CHE: sec. education, 1st stage

756002 CHE: Preparatory course for vocational education

756003 CHE: School prep. for the university entrance certificate

756004 CHE: Vocational baccalaureat, dual system 3-4 years

756005 CHE: Vocational education, dual system 3-4 years

756006 CHE: Intermediate diploma school 3-4 years

756007 CHE: Basic vocational education, dual system 1-2 years

756008 CHE: Intermediate Diploma School

756099 CHE: Missing

764001 THA: Lower sec. level

764002 THA: Upper sec. level

764003 THA: Vocational stream

788001 TUN: Enseignement de base (lower sec.)

788002 TUN: 2ndaire (upper sec.)

792001 TUR: Primary education (lower sec.)

792002 TUR: General high school (upper sec.)

792003 TUR: Anatolian high school (upper sec.)

792004 TUR: High school with foreign language (upper sec.)

792005 TUR: Science high schools (upper sec.)

792006 TUR: Vocational high schools

792007 TUR: Anatolian vocational high schools

792008 TUR: Technical high schools

792009 TUR: Anatolian technical high schools

826101 GBR: Studies toward Entry Level Certificates 
826102 GBR: Studies toward academic GCSEs eg history, Fr.

826103 GBR: Studies toward applied or vocational GCSEs

826104 GBR: Studies at GNVQ

Foundation or Intermed.

Level (6-unit)

826105 GBR: Studies toward NVQ Level 1 or 2

826106 GBR: Studies toward for AS or A Levels

826109 GBR: < Year 10

(England\&Wales) or $<$ Year 11

(North. Ireland)

826201 SCO: All students in S4

826202 SCO: S5 and studies at Higher level, A-level, or equivalent

826203 SCO: S5 and studies at Intermed., Access level or equivalent

840001

840002

840097

858001

858002

858003

858004

858005

858006

858007

858008

858009

858010

891001

891002

891003

891004

891005

891006

891007

891008

891009

891010

891097
USA: Grades $7-9$

USA: Grades $10-12$

USA: Missing

URY: Lower sec. (Plan 86)

URY: Lower sec. (Plan 96)

URY: Lower sec.

(Plan 96 technological)

URY: Vocational lower sec. (basic courses)

URY: Vocational lower sec. (basic professional)

URY: Rural lower sec.

URY: General upper sec.

URY: Technical upper sec.

URY: Vocational upper sec.

URY: Military School

YUG: Gymnasium

YUG: Technical

YUG: Technical Vocational

YUG: Medical

YUG: Medical Vocational

YUG: Economic

YUG: Agricultural

YUG: Agricultaral Vocational

YUG: Artistic

YUG: Missing
FAMSTRUC (227) Family Structure

$\begin{array}{rll}\text { Format: } & \text { F1 } & \text { Columns: 424-424 } \\ & 1 & \text { Single parent family } \\ 2 & \text { Nuclear family } \\ 3 & \text { Mixed family } \\ 4 & \text { Other } \\ 9 & \text { Missing }\end{array}$

BMMJ (228) ISCO code: mother

Format: F2 Columns: 425-426

$97 \mathrm{~N} / \mathrm{A}$

98 Invalid

99 Missing

BFMJ (229) ISCO code: father

Format: F2 Columns: 427-428

$97 \mathrm{~N} / \mathrm{A}$

98 Invalid

99 Missing

BSMJ (230) ISCO code: student

Format: F2 Columns: 429-430

$97 \mathrm{~N} / \mathrm{A}$

98 Invalid

99 Missing

HISEI (231) Highest parental occupational status

Format: F2 Columns: 431-432

99 Missing

MSECATEG (232) Mother: white collar/blue collar

classification

Format: F1 Columns: 433-433

1 White collar high skilled

2 White collar low skilled

3 Blue collar high skilled

4 Blue collar low skilled

9 Missing

FSECATEG (233) Father: white collar/blue collar classification

Format: F1 Columns: 434-434

1 White collar high skilled

2 White collar low skilled

3 Blue collar high skilled

4 Blue collar low skilled

9 Missing

HSECATEG (234) Highest parent: white collar/blue collar classification

Format: F1 Columns: 435-435

1 White collar high skilled

2 White collar low skilled

3 Blue collar high skilled

4 Blue collar low skilled

9 Missing 
SSECATEG (235) Self: white collar/blue collar classification

$$
\begin{array}{rll}
\text { Format: } & \text { F1 } & \text { Columns: 436-436 } \\
& 1 & \text { White collar high skilled } \\
2 & \text { White collar low skilled } \\
3 & \text { Blue collar high skilled } \\
4 & \text { Blue collar low skilled } \\
9 & \text { Missing }
\end{array}
$$

$\begin{array}{lll}\text { MISCED (236) } & \text { Educational level of } \\ \text { Format: } & \text { F1 } & \text { Columns: 437-437 } \\ & 0 & \text { None } \\ 1 & \text { ISCED 1 } \\ 2 & \text { ISCED 2 } \\ 3 & \text { ISCED 3B, C } \\ 4 & \text { ISCED 3A, ISCED } 4 \\ 5 & \text { ISCED 5B } \\ 6 & \text { ISCED 5A, 6 } \\ 9 & \text { Missing }\end{array}$

FISCED (237) Educational level of father (ISCED)

Format: F1 Columns: 438-438

$\begin{array}{ll}0 & \text { None } \\ 1 & \text { ISCED 1 } \\ 2 & \text { ISCED 2 } \\ 3 & \text { ISCED 3B, C } \\ 4 & \text { ISCED 3A, ISCED 4 } \\ 5 & \text { ISCED 5B } \\ 6 & \text { ISCED 5A, 6 } \\ 9 & \text { Missing }\end{array}$

HISCED (238) Highest educational level of parents

Format: F1 Columns: 439-439

0 None

1 ISCED 1

2 ISCED 2

3 ISCED 3B, C

4 ISCED 3A, ISCED 4

5 ISCED $5 \mathrm{~B}$

6 ISCED 5A, 6

9 Missing

PARED (239) Highest parental educt
schooling
$\begin{array}{lll}\text { Format: } & \text { F2 } & \text { Columns: 440-441 } \\ & 99 & \text { Missing }\end{array}$

ISO_S (240) ISO code country of birth: student

Format: A8 Columns:

$\begin{array}{ll}03608261 & \text { AUS: England } \\ 03608262 & \text { AUS: Scotland } \\ 03609996 & \text { AUS: Other } \\ 04000391 & \text { AUT: Former Yugoslavia } \\ 04009996 & \text { AUT: Other } \\ 05610021 & \text { BFL: An African country } \\ & \text { (not Maghreb) } \\ 05610151 & \text { BFL: A Maghreb country } \\ 05611501 & \text { BFL: Another country of the EU } \\ 05619996 & \text { BFL: Other } \\ 05620021 & \text { BFR: An African country } \\ 05620151 & \text { BFR: A Maghreb country } \\ 05621501 & \text { BFR: Another country of the EU } \\ 05629996 & \text { BFR: Other } \\ 07609996 & \text { BRA: Other } \\ 12419996 & \text { CAE: Other } \\ 12429996 & \text { CAF: Other } \\ 12439996 & \text { CAN: Other } \\ 20309996 & \text { CZE: Other } \\ 20800391 & \text { DNK: Former Yugoslavia } \\ 20809996 & \text { DNK: Other } \\ 24609996 & \text { FIN: Other } \\ 25009996 & \text { FRA: Other } \\ 27601501 & \text { DEU: Russia, Kazakhstan or }\end{array}$
another Republic of the Former Soviet Union

27608911 DEU: Montenegro

27608912 DEU: Serbia

27609996 DEU: Other

30001501 GRC: Republics of the Former Soviet Union

30009996 GRC: Other

34409996 HKG: Other

34809996 HUN: Other

35209996 ISL: Other

36009996 IDN: Other

37200701 IRL: Bosnia

37203761 IRL: Palestine

37208261 IRL: Northern Ireland

37208262 IRL: Great Britain

37209996 IRL: Other

38001501 ITA: An European country that is not member of the EU

38009996 ITA: Other

39209996 JPN: Other

41009996 KOR: Other

42809996 LVA: Other

44209996 LUX: Other

44609996 MAC: Other 


\begin{tabular}{|c|c|}
\hline 48409996 & MEX: Other \\
\hline 52801501 & NLD: Other European country \\
\hline 52809996 & NLD: Other \\
\hline 55409996 & NZL: Other \\
\hline 57809996 & NOR: Other \\
\hline 61609996 & POL: Other \\
\hline 62009996 & PRT: Other \\
\hline 64301501 & RUS: Republics of the Former \\
\hline & Soviet Union \\
\hline 64309996 & RUS: Other \\
\hline 70301501 & SVK: Other European country \\
\hline & of Europe \\
\hline 70309996 & SVK: Other \\
\hline 72419996 & ESC: Other \\
\hline 72429996 & ECL: Other \\
\hline 72439996 & ECT: Other \\
\hline 72449996 & EBS: Other \\
\hline 75209996 & SWE: Other \\
\hline 75600391 & CHE: FormerYugoslavia \\
\hline 75600392 & CHE: Albania or Kosovo \\
\hline 75601551 & CHE: Germany or Austria \\
\hline 75601552 & CHE: France or Belgium \\
\hline 75607560 & CHE: Switzerland \\
\hline 75609996 & CHE: Other \\
\hline 76409996 & THA: Other \\
\hline 78809996 & TUN: Other \\
\hline 79209996 & TUR: Other \\
\hline 82619996 & GRB: Other \\
\hline 82620301 & SCO: China (incl Hong Kong) \\
\hline 82621421 & SCO: Middle East \\
\hline 82621501 & SCO: Other European country \\
\hline 82628261 & SCO: England, Wales, N Ireland \\
\hline 82628262 & SCO: Scotland \\
\hline 82629996 & SCO: Other \\
\hline 84009996 & USA: Other \\
\hline 85809996 & URY: Other \\
\hline 89101491 & YUG: FormerYugoslavia \\
\hline 89109996 & YUG: Other \\
\hline 99990020 & Africa \\
\hline 99990080 & Albania \\
\hline 99990290 & Caribbean \\
\hline 99990320 & Argentina \\
\hline 99990360 & Australia \\
\hline 99990400 & Austria \\
\hline 99990500 & Bangladesh \\
\hline 99990560 & Belgium \\
\hline 99990600 & Bermuda \\
\hline 99990700 & Bosnia-Herzegovina \\
\hline 99990760 & Brazil \\
\hline 99991000 & Bulgaria \\
\hline 99991040 & Myanmar (Burma) \\
\hline
\end{tabular}

\begin{tabular}{|c|c|}
\hline 99991120 & Belarus \\
\hline 99991240 & Canada \\
\hline 99991440 & Sri Lanka \\
\hline 99991490 & FormerYugoslavia \\
\hline 99991510 & An East-European country \\
\hline 99991560 & China \\
\hline 99991840 & Cook Islands \\
\hline 99991910 & Croatia \\
\hline 99992030 & Czech Republic \\
\hline 99992080 & Denmark \\
\hline 99992330 & Estonia \\
\hline 99992420 & Fiji \\
\hline 99992460 & Finland \\
\hline 99992500 & France \\
\hline 99992680 & Georgia \\
\hline 99992760 & Germany \\
\hline 99993000 & Greece \\
\hline 99993440 & Hong Kong \\
\hline 99993480 & Hungary \\
\hline 99993520 & Iceland \\
\hline 99993560 & India \\
\hline 99993600 & Indonesia \\
\hline 99993640 & Iran \\
\hline 99993680 & Iraq \\
\hline 99993720 & Ireland \\
\hline 99993800 & Italy \\
\hline 99993880 & Jamaica \\
\hline 99993920 & Japan \\
\hline 99994000 & Jordan \\
\hline 99994100 & Korea \\
\hline 99994220 & Lebanon \\
\hline 99994280 & Latvia \\
\hline 99994340 & Libya \\
\hline 99994400 & Lithuania \\
\hline 99994420 & Luxembourg \\
\hline 99994460 & Macau \\
\hline 99994580 & Malaysia \\
\hline 99994840 & Mexico \\
\hline 99995160 & Namibia \\
\hline 99995280 & Netherlands \\
\hline 99995540 & New Zealand \\
\hline 99995660 & Nigeria \\
\hline 99995780 & Norway \\
\hline 99995860 & Pakistan \\
\hline 99996080 & Philippines \\
\hline 99996160 & Poland \\
\hline 99996200 & Portugal \\
\hline 99996420 & Romania \\
\hline 99996430 & Russia \\
\hline 99996820 & Saudi Arabia \\
\hline 99997030 & Slovakia \\
\hline
\end{tabular}




$\begin{array}{ll}99997040 & \text { Vietnam } \\ 99997050 & \text { Slovenia } \\ 99997100 & \text { South Africa } \\ 99997160 & \text { Zimbabwe } \\ 99997240 & \text { Spain } \\ 99997520 & \text { Sweden } \\ 99997560 & \text { Switzerland } \\ 99997640 & \text { Thailand } \\ 99997760 & \text { Tonga } \\ 99997800 & \text { Tunisia } \\ 99997920 & \text { Turkey } \\ 99998040 & \text { Ukraine } \\ 99998070 & \text { Macedonia } \\ 99998180 & \text { Egypt } \\ 99998260 & \text { United Kingdom } \\ 99998340 & \text { Tanzania } \\ 99998400 & \text { United States } \\ 99998580 & \text { Uruguay } \\ 99998820 & \text { Samoa } \\ 99998910 & \text { Yugoslavia } \\ 99998940 & \text { Zambia }\end{array}$

ISO_M (241) ISO code country of birth: mother Format: A8 Columns:

See ISO_S

ISO_F (242) ISO code country of birth: father

Format: A8 Columns:

See ISO_S

\section{IMMIG (243) Country of birth}

$\begin{array}{lll}\text { Format: } & \text { F1 } & \text { Columns: 466-466 } \\ & 1 & \text { Native students } \\ 2 & \text { First-generation students } \\ 3 & \text { Non-native students } \\ 9 & \text { Missing }\end{array}$

LANG (244) Foreign language spoken at home

$\begin{array}{rll}\text { Format: } & \text { F1 } & \text { Columns: 467-467 } \\ & 0 & \text { Test language or other national language } \\ 1 & \text { Foreign language } \\ 7 & \text { N/A } \\ 8 & \text { Invalid } \\ 9 & \text { Missing }\end{array}$

\section{LANGN (245) Language at home, national}

$\begin{array}{ll}\text { Format: } & \text { A6 Columns: 468-473 } \\ & \text { Value Label } \\ & 036001 \text { AUS: English } \\ 036002 & \text { AUS: Indigenous Australian } \\ & \text { languages } \\ & 036003 \text { AUS: Italian }\end{array}$

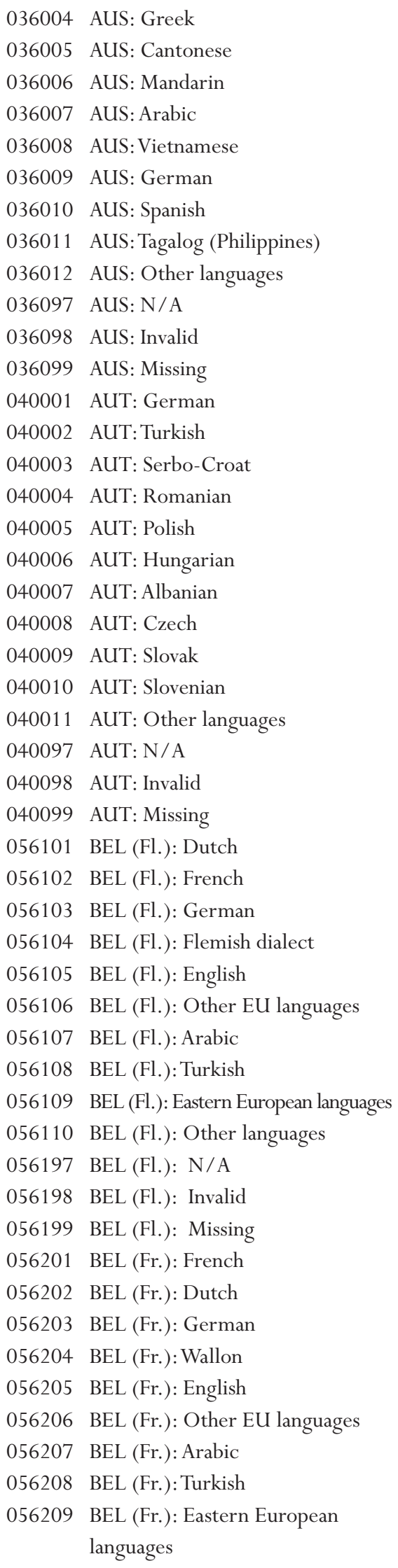




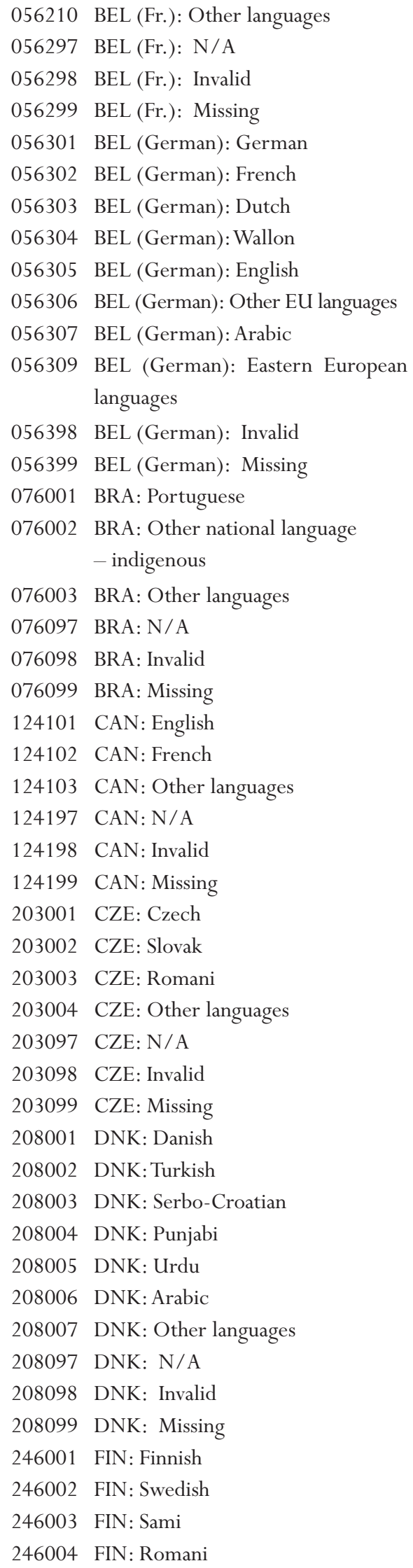

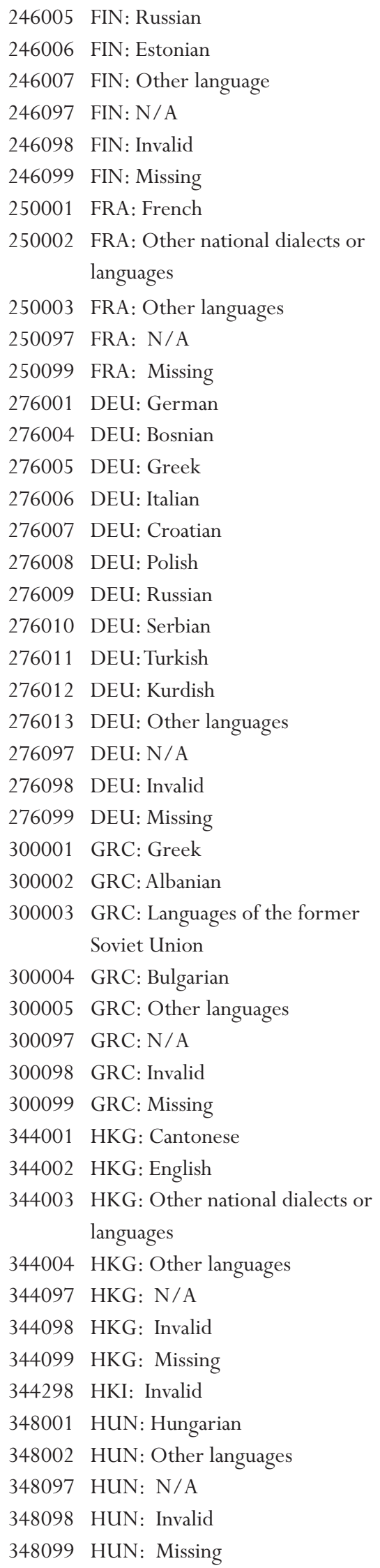




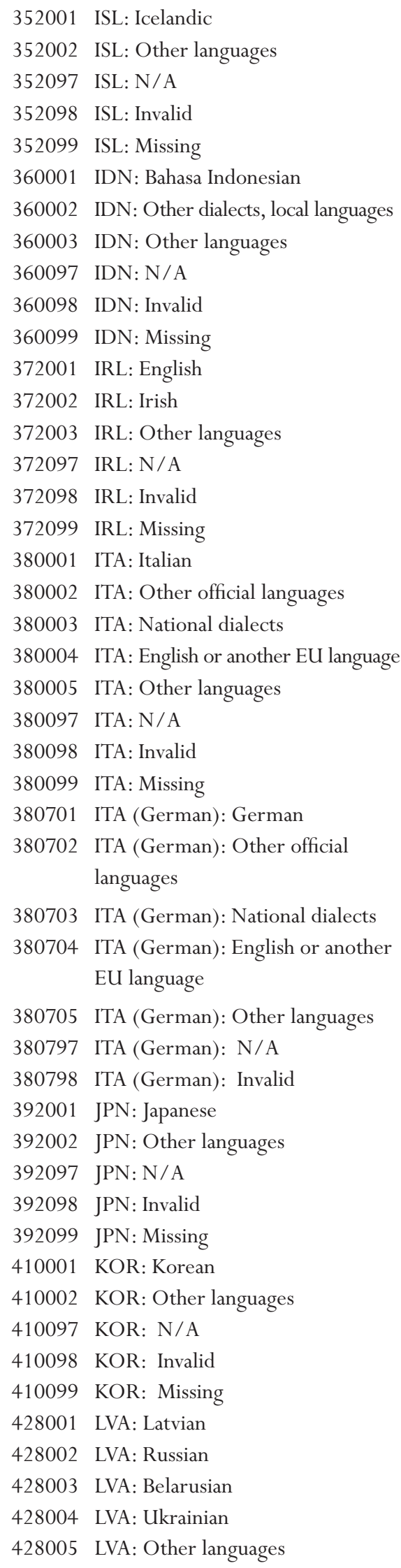

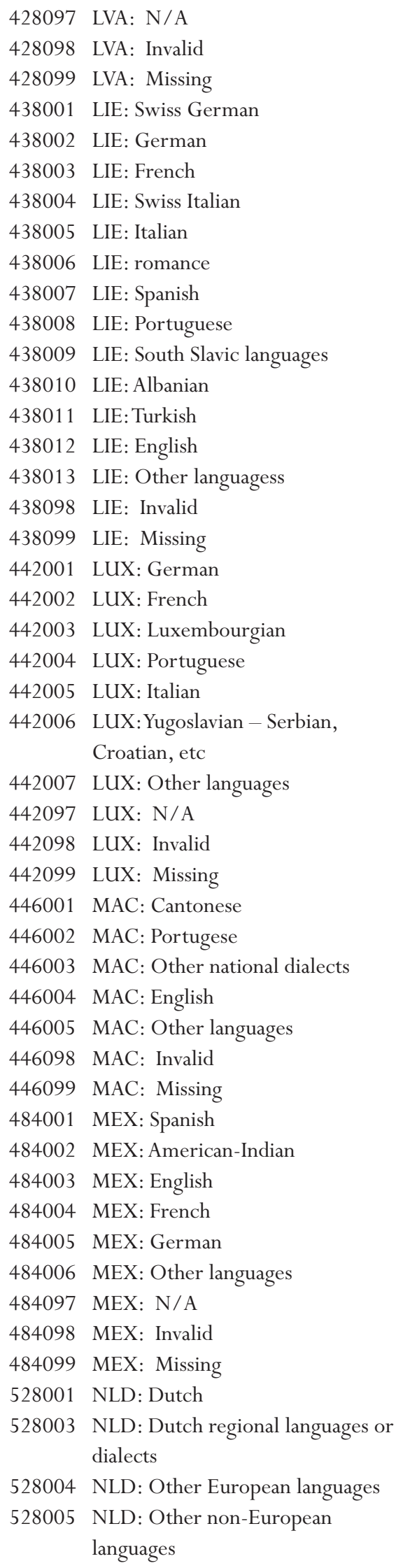




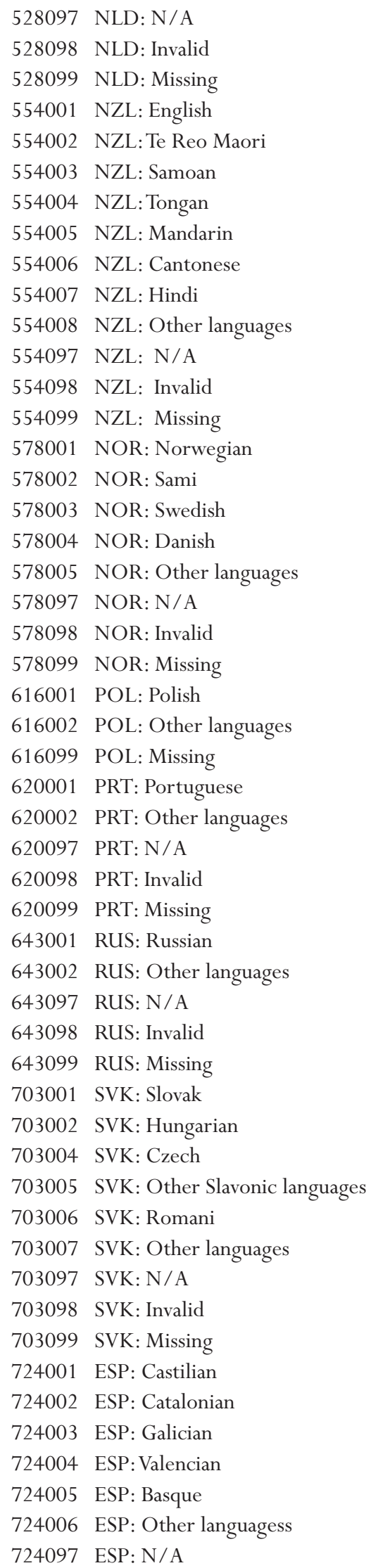

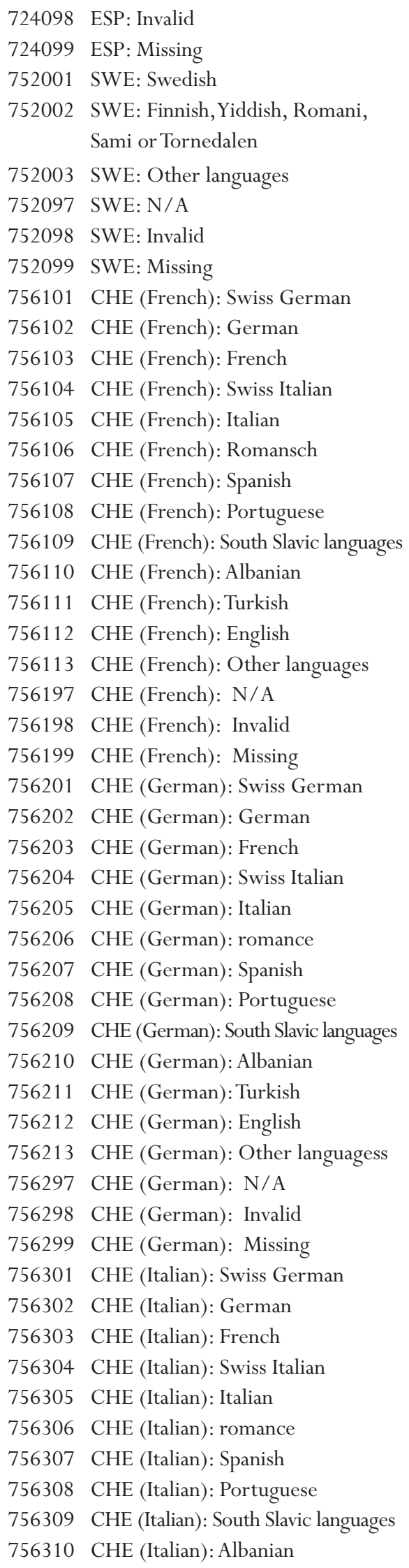


756311 CHE (Italian): Turkish

756312 CHE (Italian): English

756313 CHE (Italian): Other languages

756397 CHE (Italian): N/A

756398 CHE (Italian): Invalid

756399 CHE (Italian): Missing

764001 THA: Thai central

764002 THA: Other Thai dialects

764003 THA: Other languages

764099 THA: Missing

788001 TUN: Arabic

788002 TUN: Arabic, Tunisian dialect

788003 TUN: French

788004 TUN: Other languages

788097 TUN: N/A

788098 TUN: Invalid

788099 TUN: Missing

792001 TUR:Turkish

792002 TUR: Other national dialects or languages

792003 TUR: English

792004 TUR: French

792005 TUR: German

792006 TUR: Other languages

792097 TUR: N/A

792098 TUR: Invalid

792099 TUR: Missing

826101 GBR (Eng., Wales, NI): English

826102 GBR (Eng., Wales, NI): Irish

826103 GBR (Eng., Wales, NI): Ulster Scots

826104 GBR (Eng., Wales, NI): Welsh

826105 GBR (Eng., Wales, NI): Other languages

826197 GBR (Eng., Wales, NI): N/A

826198 GBR (Eng., Wales, NI): Invalid

826199 GBR (Eng., Wales, NI): Missing

826201 GBR (Scotland): English or Scots

826202 GBR (Scotland): Gaelic

826204 GBR (Scotland): Arabic

826205 GBR (Scotland): Bengali

826206 GBR (Scotland): Cantonese or Mandarin

826207 GBR (Scotland): Gujarati

826208 GBR (Scotland): Hindi

826209 GBR (Scotland): Malay

826210 GBR (Scotland): Punjabi

826211 GBR (Scotland): Urdu

826212 GBR (Scotland): Other European languages

826213 GBR (Scotland): Other nonEuropean languages
826297 GBR (Scotland): N/A

826298 GBR (Scotland): Invalid

826299 GBR (Scotland): Missing

840001 USA: English

840002 USA: Spanish

840003 USA: Other languages

840097 USA: N/A

840098 USA: Invalid

840099 USA: Missing

858001 URY: Spanish

858002 URY: Portuguese

858003 URY: English

858004 URY: Other languages

858097 URY: N/A

858098 URY: Invalid

858099 URY: Missing

891001 YUG: Serbian

891002 YUG: Hungarian

891003 YUG: Albanian

891004 YUG: Romanian

891005 YUG: Slovak

891006 YUG: Other languages

891097 YUG: N/A

891098 YUG: Invalid

891099 YUG: Missing
MMINS (247) Minutes of maths per week

Format: F5 Columns: 475-482

997 N/A

998 Invalid

999 Missing

TMINS (248) Total minutes of instructional time $\mathrm{p} / \mathrm{w}$

Format: F5 Columns: 483-490

997 N/A

998 Invalid

999 Missing

PCMATH (249) Ratio of maths and total

instructional time

Format: F8.3 Columns: 491-496

$997.000 \mathrm{~N} / \mathrm{A}$

998.000 Invalid

999.000 Missing 


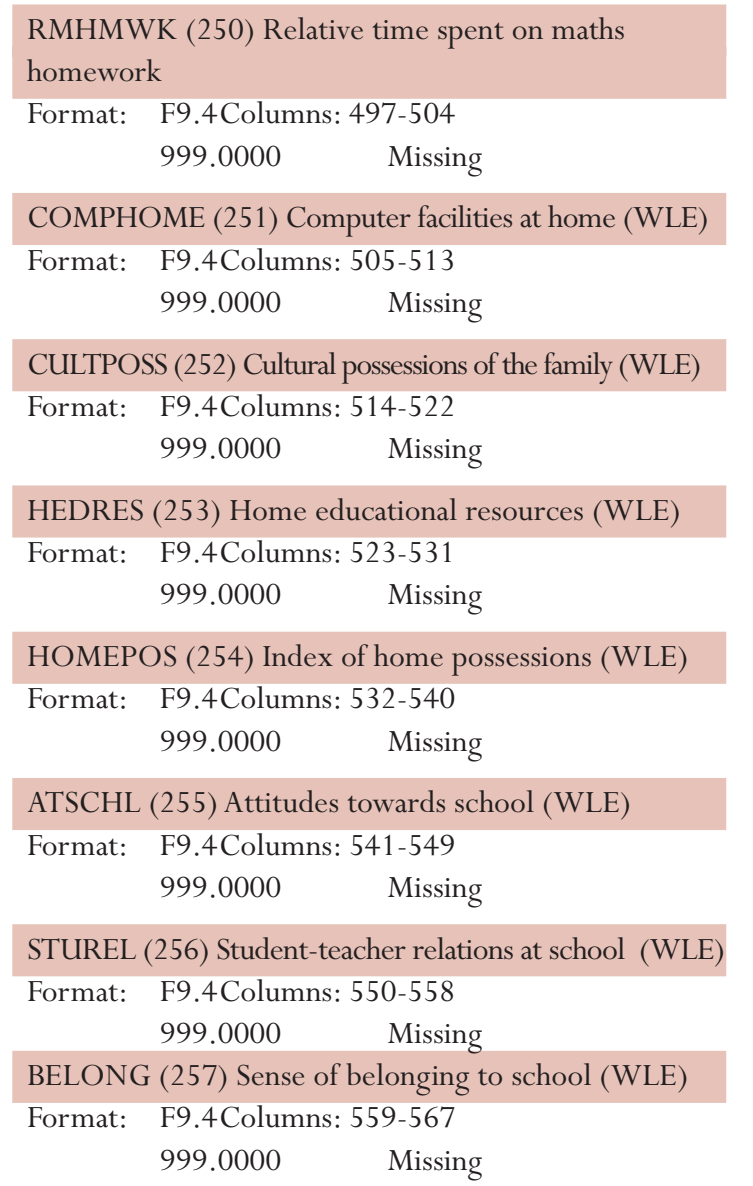

INTMAT (258) Interest in mathematics (WLE)

Format: F9.4Columns: 568-576 $999.0000 \quad$ Missing

INSTMOT (259) Instrumental motivation in mathematics (WLE)

Format: F9.4Columns: 577-585 999.0000 Missing

MATHEFF (260) Mathematics self-efficacy (WLE)

Format: F9.4Columns: 586-594 999.0000 Missing

ANXMAT (261) Mathematics anxiety (WLE)

Format: F9.4Columns: 595-603 999.0000 Missing

SCMAT (262) Mathematics self-concept (WLE)

Format: F9.4Columns: 604-612 999.0000 Missing

\section{CSTRAT (263) Control strategies (WLE)}

Format: F9.4Columns: 613-621 999.0000 Missing

ELAB (264) Elaboration strategies (WLE)

Format: F9.4Columns: 622-630 $999.0000 \quad$ Missing
MEMOR (265) Memorisation strategies (WLE)

Format: F9.4Columns: 631-639

$$
999.0000 \quad \text { Missing }
$$

COMPLRN (266) Competitive learning (WLE)

Format: F9.4Columns: 640-648

$$
999.0000 \quad \text { Missing }
$$

COOPLRN (267) Co-operative learning (WLE)

Format: F9.4Columns: 649-657

$$
999.0000 \quad \text { Missing }
$$

TEACHSUP (268) Teacher support in maths lessons (WLE)

Format: F9.4Columns: 658-666

$$
999.0000 \quad \text { Missing }
$$

DISCLIM (269) Disciplinary climate in maths lessons (WLE)

Format: F9.4Columns: 667-675

$$
999.0000 \quad \text { Missing }
$$

INTUSE (270) ICT: Internet/entertainment use (WLE)

Format: F9.4Columns: 676-684

$$
999.0000 \quad \text { Missing }
$$

PRGUSE (271) ICT: Programs/software use (WLE)

Format: F9.4Columns: 685-693

$$
999.0000 \quad \text { Missing }
$$

ROUTCONF (272) ICT: Confidence in routine tasks (WLE)

Format: F9.4Columns: 694-702

$$
999.0000 \quad \text { Missing }
$$

INTCONF (273) ICT: Confidence in internet tasks (WLE)

Format: F9.4Columns: 703-711

$$
\text { 999.0000 Missing }
$$

HIGHCONF (274) ICT: Confidence in high-level tasks (WLE)

Format: F9.4Columns: 712-720

$$
999.0000 \quad \text { Missing }
$$

ATTCOMP (275) ICT: Attitudes towards computers (WLE)

Format: F9.4Columns: 721-729

$$
999.0000 \quad \text { Missing }
$$

ESCS (276) Index of Socio-Economic and Cultural Status

Format: F10.5 Columns: 730-739

$$
999.00000 \quad \text { Missing }
$$

PV1MATH (277) Plausible value in math

Format: F9.4Columns: 740-748

$$
9997.0000 \quad \mathrm{~N} / \mathrm{A}
$$

PV2MATH (278) Plausible value in math

Format: F9.4Columns: 749-757

$9997.0000 \quad \mathrm{~N} / \mathrm{A}$ 
PV3MATH (279) Plausible value in math

Format: F9.4Columns: 758-766

$9997.0000 \quad \mathrm{~N} / \mathrm{A}$

PV4MATH (280) Plausible value in math

Format: F9.4Columns: 767-775

$9997.0000 \quad \mathrm{~N} / \mathrm{A}$

PV5MATH (281) Plausible value in math

Format: F9.4Columns: 776-784

$9997.0000 \quad \mathrm{~N} / \mathrm{A}$

PV1MATH1 (282) Plausible value in math - Space and Shape

Format: F9.4Columns: 785-793

$9997.0000 \quad \mathrm{~N} / \mathrm{A}$

PV2MATH1 (283) Plausible value in math - Space and Shape

Format: F9.4Columns: 794-802

$9997.0000 \quad \mathrm{~N} / \mathrm{A}$

PV3MATH1 (284) Plausible value in math - Space and Shape

Format: F9.4Columns: 803-811

$9997.0000 \quad \mathrm{~N} / \mathrm{A}$

PV4MATH1 (285) Plausible value in math - Space and Shape

Format: F9.4Columns: 812-820

$9997.0000 \quad \mathrm{~N} / \mathrm{A}$

PV5MATH1 (286) Plausible value in math - Space and Shape

Format: F9.4Columns: 821-829

$9997.0000 \quad \mathrm{~N} / \mathrm{A}$

PV1MATH2 (287) Plausible value in math - Change and

Relationships

Format: F9.4Columns: 830-838

$9997.0000 \quad \mathrm{~N} / \mathrm{A}$

PV2MATH2 (288) Plausible value in math - Change and

Relationships

Format: F9.4Columns: 839-847

$9997.0000 \quad \mathrm{~N} / \mathrm{A}$

PV3MATH2 (289) Plausible value in math - Change and

Relationships

Format: F9.4Columns: 848-856

$9997.0000 \quad \mathrm{~N} / \mathrm{A}$

PV4MATH2 (290) Plausible value in math - Change and

Relationships

Format: F9.4Columns: 857-865

$9997.0000 \quad \mathrm{~N} / \mathrm{A}$

PV5MATH2 (291) Plausible value in math - Change and

Relationships

Format: F9.4Columns: 866-874

$9997.0000 \quad \mathrm{~N} / \mathrm{A}$
PV1MATH3 (292) Plausible value in math - Uncertainty

Format: F9.4Columns: 875-883

$9997.0000 \quad \mathrm{~N} / \mathrm{A}$

PV2MATH3 (293) Plausible value in math - Uncertainty

Format: F9.4Columns: 884-892

$9997.0000 \quad \mathrm{~N} / \mathrm{A}$

PV3MATH3 (294) Plausible value in math - Uncertainty

Format: F9.4Columns: 893-901

$9997.0000 \quad \mathrm{~N} / \mathrm{A}$

PV4MATH3 (295) Plausible value in math - Uncertainty

Format: F9.4Columns: 902-910

$9997.0000 \quad \mathrm{~N} / \mathrm{A}$

PV5MATH3 (296) Plausible value in math - Uncertainty

Format: F9.4Columns: 911-919

$9997.0000 \quad \mathrm{~N} / \mathrm{A}$

PV1MATH4 (297) Plausible value in math - Quantity

Format: F9.4Columns: 920-928

$9997.0000 \quad \mathrm{~N} / \mathrm{A}$

PV2MATH4 (298) Plausible value in math - Quantity

Format: F9.4Columns: 929-937

$9997.0000 \quad \mathrm{~N} / \mathrm{A}$

PV3MATH4 (299) Plausible value in math - Quantity

Format: F9.4Columns: 938-946

$9997.0000 \quad \mathrm{~N} / \mathrm{A}$

PV4MATH4 (300) Plausible value in math - Quantity

Format: F9.4Columns: 947-955 9997.0000

$\mathrm{N} / \mathrm{A}$

PV5MATH4 (301) Plausible value in math - Quantity

Format: F9.4Columns: 956-964

$9997.0000 \quad \mathrm{~N} / \mathrm{A}$

PV1READ (302) Plausible value in reading

Format: F9.4Columns: 965-973

$9997.0000 \quad \mathrm{~N} / \mathrm{A}$

PV2READ (303) Plausible value in reading

Format: F9.4Columns: 974-982

$9997.0000 \quad \mathrm{~N} / \mathrm{A}$

PV3READ (304) Plausible value in reading

Format: F9.4Columns: 983-991

$9997.0000 \quad \mathrm{~N} / \mathrm{A}$

PV4READ (305) Plausible value in reading

Format: F9.4Columns: 992-1000

$9997.0000 \quad \mathrm{~N} / \mathrm{A}$

PV5READ (306) Plausible value in reading

Format: F9.4Columns: 1001-1009

$9997.0000 \quad \mathrm{~N} / \mathrm{A}$ 
PV1SCIE (307) Plausible value in science

Format: F9.4Columns: 1010-1018

$9997.0000 \quad \mathrm{~N} / \mathrm{A}$

PV2SCIE (308) Plausible value in science

Format: F9.4Columns: 1019-1027

$9997.0000 \quad \mathrm{~N} / \mathrm{A}$

PV3SCIE (309) Plausible value in science

Format: F9.4Columns: 1028-1036 $9997.0000 \quad \mathrm{~N} / \mathrm{A}$

PV4SCIE (310) Plausible value in science

Format: F9.4Columns: 1037-1045

$9997.0000 \quad \mathrm{~N} / \mathrm{A}$

PV5SCIE (311) Plausible value in science

Format: F9.4Columns: 1046-1054 $9997.0000 \quad \mathrm{~N} / \mathrm{A}$

PV1PROB (312) Plausible value in problem solving

Format: F9.4Columns: 1055-1063 $9997.0000 \quad \mathrm{~N} / \mathrm{A}$

PV2PROB (313) Plausible value in problem solving

Format: F9.4Columns: 1064-1072

$9997.0000 \quad \mathrm{~N} / \mathrm{A}$

PV3PROB (314) Plausible value in problem solving

Format: F9.4Columns: 1073-1081 $9997.0000 \quad \mathrm{~N} / \mathrm{A}$

PV4PROB (315) Plausible value in problem solving

Format: F9.4Columns: 1082-1090 $9997.0000 \quad \mathrm{~N} / \mathrm{A}$

PV5PROB (316) Plausible value in problem solving

Format: F9.4Columns: 1091-1099

$9997.0000 \quad \mathrm{~N} / \mathrm{A}$

W_FSTUWT (317) Student final weight

Format: F9.4Columns: 1100-1108 $9997.0000 \quad \mathrm{~N} / \mathrm{A}$

CNTFAC1 (318) Country weight factor for equal weights (1000)

Format: F8.6Columns: 1109-1116

CNTFAC2 (319) Country weight factor for normalised weights (sample size)

Format: F8.6Columns: 1117-1124

OECD (320) OECD country indicator

Format: F1 Columns: 1125-1125

0 Partner country

1 OECD country
UH (321) One-hour booklet indicator

Format: F1 Columns: 1126-1126

0 Two-hour booklet

1 One-hour booklet

W_FSTR1 (322) BRR replicate

Format: F9.4Columns: 1127-1135

$9997.0000 \quad \mathrm{~N} / \mathrm{A}$

W_FSTR2 (323) BRR replicate

Format: F9.4Columns: 1136-1144 $9997.0000 \quad \mathrm{~N} / \mathrm{A}$

W_FSTR3 (324) BRR replicate

Format: F9.4Columns: 1145-1153 $9997.0000 \quad \mathrm{~N} / \mathrm{A}$

W_FSTR4 (325) BRR replicate

Format: F9.4Columns: 1154-1162 $9997.0000 \quad \mathrm{~N} / \mathrm{A}$

W_FSTR5 (326) BRR replicate

Format: F9.4Columns: 1163-1171 $9997.0000 \quad \mathrm{~N} / \mathrm{A}$

W_FSTR6 (327) BRR replicate

Format: F9.4Columns: 1172-1180 $9997.0000 \quad \mathrm{~N} / \mathrm{A}$

W_FSTR7 (328) BRR replicate

Format: F9.4Columns: 1181-1189 $9997.0000 \quad \mathrm{~N} / \mathrm{A}$

W_FSTR8 (329) BRR replicate

Format: F9.4Columns: 1190-1198 $9997.0000 \quad \mathrm{~N} / \mathrm{A}$

W_FSTR9 (330) BRR replicate

Format: F9.4Columns: 1199-1207 9997.0000

$\mathrm{N} / \mathrm{A}$

W_FSTR10 (331) BRR replicate

Format: F9.4Columns: 1208-1216 $9997.0000 \quad \mathrm{~N} / \mathrm{A}$

W_FSTR11 (332) BRR replicate

Format: F9.4Columns: 1217-1225 $9997.0000 \quad \mathrm{~N} / \mathrm{A}$

W_FSTR12 (333) BRR replicate

Format: F9.4Columns: 1226-1234 $9997.0000 \quad \mathrm{~N} / \mathrm{A}$

W_FSTR13 (334) BRR replicate

Format: F9.4Columns: 1235-1243 $9997.0000 \quad \mathrm{~N} / \mathrm{A}$ 
W_FSTR14 (335) BRR replicate

Format: F9.4Columns: 1244-1252

$9997.0000 \quad \mathrm{~N} / \mathrm{A}$

W_FSTR15 (336) BRR replicate

Format: F9.4Columns: 1253-1261

$9997.0000 \quad \mathrm{~N} / \mathrm{A}$

W_FSTR16 (337) BRR replicate

Format: F9.4Columns: 1262-1270

$9997.0000 \quad \mathrm{~N} / \mathrm{A}$

W_FSTR17 (338) BRR replicate

Format: F9.4Columns: 1271-1279

$9997.0000 \quad \mathrm{~N} / \mathrm{A}$

W_FSTR18 (339) BRR replicate

Format: F9.4Columns: 1280-1288 $9997.0000 \quad \mathrm{~N} / \mathrm{A}$

W_FSTR19 (340) BRR replicate

Format: F9.4Columns: 1289-1297

$9997.0000 \quad \mathrm{~N} / \mathrm{A}$

W_FSTR20 (341) BRR replicate

Format: F9.4Columns: 1298-1306 $9997.0000 \quad \mathrm{~N} / \mathrm{A}$

W_FSTR21 (342) BRR replicate

Format: F9.4Columns: 1307-1315 $9997.0000 \quad \mathrm{~N} / \mathrm{A}$

W_FSTR22 (343) BRR replicate

Format: F9.4Columns: 1316-1324 $9997.0000 \quad \mathrm{~N} / \mathrm{A}$

W_FSTR23 (344) BRR replicate

Format: F9.4Columns: 1325-1333

$9997.0000 \quad \mathrm{~N} / \mathrm{A}$

W_FSTR24 (345) BRR replicate

Format: F9.4Columns: 1334-1342 $9997.0000 \quad \mathrm{~N} / \mathrm{A}$

W_FSTR25 (346) BRR replicate

Format: F9.4Columns: 1343-1351 $9997.0000 \quad \mathrm{~N} / \mathrm{A}$

W_FSTR26 (347) BRR replicate

Format: F9.4Columns: 1352-1360 $9997.0000 \quad \mathrm{~N} / \mathrm{A}$

W_FSTR27 (348) BRR replicate

Format: F9.4Columns: 1361-1369

$9997.0000 \quad \mathrm{~N} / \mathrm{A}$

W_FSTR28 (349) BRR replicate

Format: F9.4Columns: 1370-1378

$9997.0000 \quad \mathrm{~N} / \mathrm{A}$
W_FSTR29 (350) BRR replicate

Format: F9.4Columns: 1379-1387 $9997.0000 \quad \mathrm{~N} / \mathrm{A}$

W_FSTR30 (351) BRR replicate

Format: F9.4Columns: 1388-1396 $9997.0000 \quad \mathrm{~N} / \mathrm{A}$

W_FSTR31 (352) BRR replicate

Format: F9.4Columns: 1397-1405 $9997.0000 \quad \mathrm{~N} / \mathrm{A}$

W_FSTR32 (353) BRR replicate

Format: F9.4Columns: 1406-1414 $9997.0000 \quad \mathrm{~N} / \mathrm{A}$

W_FSTR33 (354) BRR replicate

Format: F9.4Columns: 1415-1423 $9997.0000 \quad \mathrm{~N} / \mathrm{A}$

W_FSTR34 (355) BRR replicate

Format: F9.4Columns: 1424-1432 $9997.0000 \quad \mathrm{~N} / \mathrm{A}$

W_FSTR35 (356) BRR replicate

Format: F9.4Columns: 1433-1441 $9997.0000 \quad \mathrm{~N} / \mathrm{A}$

W_FSTR36 (357) BRR replicate

Format: F9.4Columns: 1442-1450 $9997.0000 \quad \mathrm{~N} / \mathrm{A}$

W_FSTR37 (358) BRR replicate

Format: F9.4Columns: 1451-1459 $9997.0000 \quad \mathrm{~N} / \mathrm{A}$

W_FSTR38 (359) BRR replicate

Format: F9.4Columns: 1460-1468 $9997.0000 \quad \mathrm{~N} / \mathrm{A}$

W_FSTR39 (360) BRR replicate

Format: F9.4Columns: 1469-1477 $9997.0000 \quad \mathrm{~N} / \mathrm{A}$

W_FSTR40 (361) BRR replicate

Format: F9.4Columns: 1478-1486 $9997.0000 \quad \mathrm{~N} / \mathrm{A}$

W_FSTR41 (362) BRR replicate

Format: F9.4Columns: 1487-1495 $9997.0000 \quad \mathrm{~N} / \mathrm{A}$

W_FSTR42 (363) BRR replicate

Format: F9.4Columns: 1496-1504 $9997.0000 \quad \mathrm{~N} / \mathrm{A}$

W_FSTR43 (364) BRR replicate

Format: F9.4Columns: 1505-1513 $9997.0000 \quad \mathrm{~N} / \mathrm{A}$ 
W_FSTR44 (365) BRR replicate

Format: F9.4Columns: 1514-1522

$9997.0000 \quad \mathrm{~N} / \mathrm{A}$

W_FSTR45 (366) BRR replicate

Format: F9.4Columns: 1523-1531

$9997.0000 \quad \mathrm{~N} / \mathrm{A}$

W_FSTR46 (367) BRR replicate

Format: F9.4Columns: 1532-1540

$9997.0000 \quad \mathrm{~N} / \mathrm{A}$

W_FSTR47 (368) BRR replicate

Format: F9.4Columns: 1541-1549 $9997.0000 \quad \mathrm{~N} / \mathrm{A}$

W_FSTR48 (369) BRR replicate

Format: F9.4Columns: 1550-1558

$9997.0000 \quad \mathrm{~N} / \mathrm{A}$

W_FSTR49 (370) BRR replicate

Format: F9.4Columns: 1559-1567 $9997.0000 \quad \mathrm{~N} / \mathrm{A}$

W_FSTR50 (371) BRR replicate

Format: F9.4Columns: 1568-1576 $9997.0000 \quad \mathrm{~N} / \mathrm{A}$

W_FSTR51 (372) BRR replicate

Format: F9.4Columns: 1577-1585

$9997.0000 \quad \mathrm{~N} / \mathrm{A}$

W_FSTR52 (373) BRR replicate

Format: F9.4Columns: 1586-1594 $9997.0000 \quad \mathrm{~N} / \mathrm{A}$

W_FSTR53 (374) BRR replicate

Format: F9.4Columns: 1595-1603 $9997.0000 \quad \mathrm{~N} / \mathrm{A}$

W_FSTR54 (375) BRR replicate

Format: F9.4Columns: 1604-1612

$9997.0000 \quad \mathrm{~N} / \mathrm{A}$

W_FSTR55 (376) BRR replicate

Format: F9.4Columns: 1613-1621 $9997.0000 \quad \mathrm{~N} / \mathrm{A}$

W_FSTR56 (377) BRR replicate

Format: F9.4Columns: 1622-1630 $9997.0000 \quad \mathrm{~N} / \mathrm{A}$

W_FSTR57 (378) BRR replicate

Format: F9.4Columns: 1631-1639

$9997.0000 \quad \mathrm{~N} / \mathrm{A}$

W_FSTR58 (379) BRR replicate

Format: F9.4Columns: 1640-1648 $9997.0000 \quad \mathrm{~N} / \mathrm{A}$
W_FSTR59 (380) BRR replicate

Format: F9.4Columns: 1649-1657 $9997.0000 \quad \mathrm{~N} / \mathrm{A}$

W_FSTR60 (381) BRR replicate

Format: F9.4Columns: 1658-1666 $9997.0000 \quad \mathrm{~N} / \mathrm{A}$

W_FSTR61 (382) BRR replicate

Format: F9.4Columns: 1667-1675 $9997.0000 \quad \mathrm{~N} / \mathrm{A}$

W_FSTR62 (383) BRR replicate

Format: F9.4Columns: 1676-1684 $9997.0000 \quad \mathrm{~N} / \mathrm{A}$

W_FSTR63 (384) BRR replicate

Format: F9.4Columns: 1685-1693 $9997.0000 \quad \mathrm{~N} / \mathrm{A}$

W_FSTR64 (385) BRR replicate

Format: F9.4Columns: 1694-1702 $9997.0000 \quad \mathrm{~N} / \mathrm{A}$

W_FSTR65 (386) BRR replicate

Format: F9.4Columns: 1703-1711 $9997.0000 \quad \mathrm{~N} / \mathrm{A}$

W_FSTR66 (387) BRR replicate

Format: F9.4Columns: 1712-1720 $9997.0000 \quad \mathrm{~N} / \mathrm{A}$

W_FSTR67 (388) BRR replicate

Format: F9.4Columns: 1721-1729 $9997.0000 \quad \mathrm{~N} / \mathrm{A}$

W_FSTR68 (389) BRR replicate

Format: F9.4Columns: 1730-1738 $9997.0000 \quad \mathrm{~N} / \mathrm{A}$

W_FSTR69 (390) BRR replicate

Format: F9.4Columns: 1739-1747 $9997.0000 \quad \mathrm{~N} / \mathrm{A}$

W_FSTR70 (391) BRR replicate

Format: F9.4Columns: 1748-1756 $9997.0000 \quad \mathrm{~N} / \mathrm{A}$

W_FSTR71 (392) BRR replicate

Format: F9.4Columns: 1757-1765 $9997.0000 \quad \mathrm{~N} / \mathrm{A}$

W_FSTR72 (393) BRR replicate

Format: F9.4Columns: 1766-1774 $9997.0000 \quad \mathrm{~N} / \mathrm{A}$

W_FSTR73 (394) BRR replicate

Format: F9.4Columns: 1775-1783

$9997.0000 \quad \mathrm{~N} / \mathrm{A}$ 


\begin{tabular}{|c|c|c|c|c|}
\hline \multicolumn{3}{|c|}{ W_FSTR74 (395) BRR replicate } & \multirow[t]{2}{*}{04012} & \multirow{2}{*}{$\begin{array}{l}\text { Austria: BMS (gewerblich- } \\
\text { technisch-kunstgewerbl.) }\end{array}$} \\
\hline \multicolumn{3}{|c|}{ Format: F9.4Columns: 1784-1792 } & & \\
\hline \multicolumn{3}{|c|}{9997.0000} & 04013 & Austria: BMS (kaufmännisch \\
\hline \multicolumn{3}{|c|}{ W_FSTR75 (396) BRR replicate } & & /Handelschulen) \\
\hline \multicolumn{3}{|c|}{ Format: F9.4Columns: 1793-1801 } & 04014 & Austria: BMS (wirtschafts- \\
\hline & \multirow{2}{*}{\multicolumn{2}{|c|}{$9997.0000 \quad$ N/A }} & & /sozialberufl.) \\
\hline & & & 04015 & Austria: BMS (land-/forstwirtschaftl.) \\
\hline \multicolumn{3}{|c|}{ W_FSTR76 (397) BRR replicate } & 04016 & Austria: BHS (techn.-gewerblich) \\
\hline \multicolumn{3}{|c|}{ Format: F9.4Columns: 1802-1810 } & 04017 & Austria: BHS (kaufmännisch) \\
\hline \multirow{2}{*}{\multicolumn{3}{|c|}{9997.0000}} & 04018 & Austria: BHS (wirtschafts-/sozialberufl) \\
\hline & & & 04019 & Austria: BHS (land-/ forstwirtschaftl.) \\
\hline \multicolumn{3}{|c|}{ W_FSTR77 (398) BRR replicate } & 04020 & Austria: Anstalt der Kindergarten- \\
\hline \multicolumn{3}{|c|}{ Format: F9.4Columns: 1811-1819 } & & /Sozialpädagogik \\
\hline & \multicolumn{2}{|c|}{$9997.0000 \quad \mathrm{~N} / \mathrm{A}$} & 04021 & Austria: Moderately small schools \\
\hline \multicolumn{3}{|c|}{ W_FSTR78 (399) BRR replicate } & 04022 & Austria: Very small schools \\
\hline \multicolumn{3}{|c|}{ Format: F9.4Columns: 1820-1828 } & 05601 & Belgium (Flemish): Only general \\
\hline \multirow{2}{*}{\multicolumn{3}{|c|}{$9997.0000 \quad \mathrm{~N} / \mathrm{A}$}} & \multirow{3}{*}{05602} & education, private \\
\hline & & & & Belgium (Flemish): Only general \\
\hline \multicolumn{3}{|c|}{ W_FSTR79 (400) BRR replicate } & & education, public \\
\hline \multicolumn{3}{|c|}{ Format: F9.4Columns: 1829-1837 } & \multirow{2}{*}{05603} & Belgium (Flemish): Gen.-techn.- \\
\hline & 9997.0 & $\mathrm{~N} / \mathrm{A}$ & & vocat.-arts, private \\
\hline \multicolumn{3}{|c|}{ W_FSTR80 (401) BRR replicate } & 05604 & Belgium (Flemish): Gen.-techn.- \\
\hline \multicolumn{3}{|c|}{ Format: F9.4Columns: 1838-1846 } & \multirow[b]{2}{*}{05605} & vocat.-arts, public \\
\hline & 9997.0 & $00 \quad \mathrm{~N} / \mathrm{A}$ & & $\begin{array}{l}\text { Belgium (Flemish): Techn., arts, not } \\
\text { general, private }\end{array}$ \\
\hline $\begin{array}{l}\text { WVARST } \\
(1-80)\end{array}$ & RR $(402$ & Randomised final variance stratum & 05606 & $\begin{array}{l}\text { Belgium (Flemish): Techn.-vocat.- } \\
\text { arts, not gen., public }\end{array}$ \\
\hline Format: & $\mathrm{F} 2 \mathrm{Co}$ & umns: $1847-1848$ & 05607 & Belgium (Flemish): Special \\
\hline UNIT $(4$ & 3) Final & variance unit $(1,2,3)$ & & education - private \\
\hline Format: & $\mathrm{F} 1 \quad \mathrm{Co}$ & umns: 1849-1849 & 05608 & $\begin{array}{l}\text { Belgium (Flemish): Special } \\
\text { education - public }\end{array}$ \\
\hline STRATU & M (404) & tratum & 05609 & Belgium (Flemish): Part time \\
\hline Format: & A5 $\mathrm{Co}$ & umns: $\quad 1851-1855$ & & vocational \\
\hline & 03601 & Australia: ACT & 05610 & Belgium (Flemish): Moderately \\
\hline & 03602 & Australia: NSW & & small schools \\
\hline & 03603 & Australia: VIC & 05611 & Belgium (Flemish): Very small \\
\hline & 03604 & Australia: QLD & & schools \\
\hline & 03605 & Australia: SA & 05612 & Belgium (French): Organised by \\
\hline & 03606 & Australia: WA & & community \\
\hline & 03607 & Australia:TAS & 05613 & Belgium (French): Organised by \\
\hline & 03608 & Australia: NT & & community, spec. ed. \\
\hline & 04002 & Austria: Hauptschule & 05614 & Belgium (French): Official schools \\
\hline & 04003 & Austria: Polytechn. Schule & & subsidised by comm. \\
\hline & 04005 & Austria: Gymnasium & 05615 & Belgium (French): Subsidised \\
\hline & 04006 & Austria: Realgymnasium & & special education \\
\hline & 04007 & Austria: Oberstufenrealgymnasium & 05616 & Belgium (French): Subsidised \\
\hline & 04009 & Austria: Berufsschule (techn.-gewerbl.) & & confessional \\
\hline & 04010 & $\begin{array}{l}\text { Austria: Berufsschule (kaufmänn. } \\
\text { /Handel/Verkehr) }\end{array}$ & 05617 & $\begin{array}{l}\text { Belgium (French): Subsidised } \\
\text { confessional - spec. ed. }\end{array}$ \\
\hline
\end{tabular}


05618 Belgium (French): Subsidised nonconfessional

05619 Belgium (French): Subsidised nonconfessional - spec. ed.

05620 Belgium (German)

07601 Brazil: Central (private)

07602 Brazil: Central (public)

07603 Brazil: North (private)

07604 Brazil: North (public)

07605 Brazil: Northeast (private)

07606 Brazil: Northeast (public)

07607 Brazil: South (private)

07608 Brazil: South (public)

07609 Brazil: Southeast (private)

07610 Brazil: Southeast (public)

07611 Brazil: Small schools

07612 Brazil: Very small schools

12400 Canada

20301 Czech Republic: Stratum 1

20302 Czech Republic: Stratum 2

20303 Czech Republic: Stratum 3

20304 Czech Republic: Stratum 4

20305 Czech Republic: Stratum 5

20306 Czech Republic: Stratum 6

20307 Czech Republic: Stratum 7

20308 Czech Republic: Stratum 8

20309 Czech Republic: Stratum 9

20310 Czech Republic: Stratum 10

20311 Czech Republic: Stratum 11

20312 Czech Republic: Stratum 12

20313 Czech Republic: Stratum 13

20314 Czech Republic: Stratum 14

20315 Czech Republic: Stratum 15

20316 Czech Republic: Stratum 16

20317 Czech Republic: Stratum 17

20318 Czech Republic: Stratum 18

20319 Czech Republic: Stratum 19

20320 Czech Republic: Stratum 20

20321 Czech Republic: Stratum 21

20322 Czech Republic: Stratum 22

20323 Czech Republic: Stratum 23

20324 Czech Republic: Stratum 24

20325 Czech Republic: Stratum 25

20326 Czech Republic: Stratum 26

20327 Czech Republic: Stratum 27

20328 Czech Republic: Stratum 28

20329 Czech Republic: Stratum 29

20330 Czech Republic: Stratum 30

20331 Czech Republic: Stratum 31
20332

20333

20334

20801

20802

20803

24601

24602

24603

24604

24605

24606

24607

24608

24609

24610

24611

24612

25001

25002

25003

25004

25005

25006

27601

27602

27603

27604

27605

27606

27607

27608

27609

27610

27611

27612

27613

27614

27615

27616

27617

27618

30001

30002

30003

30004

30005

30006
Czech Republic: Stratum 32

Czech Republic: Stratum 33

Czech Republic: Stratum 34

Denmark: Large schools

Denmark: Moderately small schools

Denmark: Very Small schools

Finland: Stratum 1

Finland: Stratum 2

Finland: Stratum 3

Finland: Stratum 4

Finland: Stratum 5

Finland: Stratum 6

Finland: Stratum 7

Finland: Stratum 8

Finland: Stratum 9

Finland: Stratum 10

Finland: Stratum 11

Finland: Stratum 12

France: Lycées généraux et

technologiques

France: Collèges

France: Lycées professionnels

France: Lycées agricoles

France: Moderately small schools

France: Very small schools

Germany: Stratum 1

Germany: Stratum 2

Germany: Stratum 3

Germany: Stratum 4

Germany: Stratum 5

Germany: Stratum 6

Germany: Stratum 7

Germany: Stratum 8

Germany: Stratum 9

Germany: Stratum 10

Germany: Stratum 11

Germany: Stratum 12

Germany: Stratum 13

Germany: Stratum 14

Germany: Stratum 15

Germany: Stratum 16

Germany: Stratum 17

Germany: Stratum 18

Greece: Stratum 1

Greece: Stratum 2

Greece: Stratum 3

Greece: Stratum 4

Greece: Stratum 5

Greece: Stratum 6 
30009 Greece: Stratum 9

30010 Greece: Stratum 10

30011 Greece: Stratum 11

30012 Greece: Stratum 12

34401 Hong Kong-China: Stratum 1

34402 Hong Kong-China: Stratum 2

34403 Hong Kong-China: Stratum 3

34801 Hungary: Stratum 1

34802 Hungary: Stratum 2

34803 Hungary: Stratum 3

34804 Hungary: Stratum 4

34805 Hungary: Stratum 5

35201 Iceland: Stratum 1

35202 Iceland: Stratum 2

35203 Iceland: Stratum 3

35204 Iceland: Stratum 4

35205 Iceland: Stratum 5

35206 Iceland: Stratum 6

35207 Iceland: Stratum 7

35208 Iceland: Stratum 8

35209 Iceland: Stratum 9

36001 Indonesia: Stratum 1

36002 Indonesia: Stratum 2

36003 Indonesia: Stratum 3

36004 Indonesia: Stratum 4

36005 Indonesia: Stratum 5

36006 Indonesia: Stratum 6

36007 Indonesia: Stratum 7

36008 Indonesia: Stratum 8

36009 Indonesia: Stratum 9

36010 Indonesia: Stratum 10

36011 Indonesia: Stratum 11

36012 Indonesia: Stratum 12

36013 Indonesia: Stratum 13

36014 Indonesia: Stratum 14

36015 Indonesia: Stratum 15

36016 Indonesia: Stratum 16

36017 Indonesia: Stratum 17

36018 Indonesia: Stratum 18

36019 Indonesia: Stratum 19

36020 Indonesia: Stratum 20

36021 Indonesia: Stratum 21

36022 Indonesia: Stratum 22

36023 Indonesia: Stratum 23

36024 Indonesia: Stratum 24

36025 Indonesia: Stratum 25

36026 Indonesia: Stratum 26
36027

36028

37201

Indonesia: Stratum 27

Indonesia: Stratum 28

Ireland: Stratum 1

37202 Ireland: Stratum 2

37203 Ireland: Stratum 3

38001 Italy, Piemonte-Nord-Ovest: Stratum 1

38002 Italy, Piemonte-Nord-Ovest: Stratum 2

38003 Italy, Piemonte-Nord-Ovest: Stratum 3

38004 Italy, Piemonte-Nord-Ovest: Stratum 4

38005 Italy, Lombardia-Nord Ovest: Stratum 5

38006 Italy, Lombardia-Nord Ovest: Stratum 6

38007 Italy, Lombardia-Nord Ovest: Stratum 7

38008 Italy, Lombardia-Nord Ovest: Stratum 8

38009 Italy, Other regions: Stratum 9

38010 Italy, Other regions: Stratum 10

38011 Italy, Other regions: Stratum 11

38012 Italy, Other regions: Stratum 12

38013 Italy, Other regions: Stratum 13

38014 Italy, Veneto-Nord Est: Stratum 14

38015 Italy, Veneto-Nord Est: Stratum 15

38016 Italy, Veneto-Nord Est: Stratum 16

38017 Italy, Veneto-Nord Est: Stratum 17

38018 Italy, Veneto-Nord Est: Stratum 18

38019 Italy, Trento-Nord-Est: Stratum 19

38020 Italy, Trento-Nord-Est: Stratum 20

38021 Italy, Bolzano: Stratum 21

38022 Italy, Bolzano: Stratum 22

38023 Italy, Other regions: Stratum 23

38024 Italy, Other regions: Stratum 24

38025 Italy, Other regions: Stratum 25

38026 Italy, Other regions: Stratum 26

38028 Italy, Toscana-Centro: Stratum 28

38029 Italy, Toscana-Centro: Stratum 29

38030 Italy, Toscana-Centro: Stratum 30

38031 Italy, Toscana-Centro: Stratum 31

38032 Italy, Other regions: Stratum 32

38033 Italy, Other regions: Stratum 33

38034 Italy, Other regions: Stratum 34

38035 Italy, Other regions: Stratum 35

38036 Italy, Other regions: Stratum 36

38037 Italy, Other regions: Stratum 37

38038 Italy, Other regions: Stratum 38

38039 Italy, Other regions: Stratum 39

38040 Italy, Other regions: Stratum 40

38041 Italy, Other regions: Stratum 41

38042 Italy, Other regions: Stratum 42

38043 Italy, Other regions: Stratum 43

38044 Italy, Other regions: Stratum 44

39201 Japan: Public \& Academic Course 
39202 Japan: Public \& Practical Course

39203 Japan: Private \& Academic Course

39204 Japan: Private \& Practical Course

41001 Korea: Metropolitan General

41002 Korea: Metropolitan Vocational

41003 Korea: Urban General

41004 Korea: Urban Vocational

41005 Korea: Urban General \& Vocational

41006 Korea: Rural General

41007 Korea: Rural Vocational

41008 Korea: Rural General \& Vocational

41009 Korea: Moderately small schools

42801 Latvia: Large schools

42802 Latvia: Moderately small schools

42803 Latvia: Very small schools

43840 Liechtenstein

44201 Luxembourg: Stratum 1

44202 Luxembourg: Stratum 2

44203 Luxembourg: Stratum 3

44601 Macao-China: Stratum 1

44602 Macao-China: Stratum 2

44603 Macao-China: Stratum 3

48401 Mexico: Aguascalientes

48402 Mexico: Baja California

48403 Mexico: Baja California Sur

48404 Mexico: Campeche Coahuila

48405 Mexico: Coahuila

48406 Mexico: Colima

48407 Mexico: Chiapas

48408 Mexico: Chihuahua

48409 Mexico: Distrito Federal

48410 Mexico: Durango

48411 Mexico: Guanajuato

48412 Mexico: Guerrero

48413 Mexico: Hidalgo

48414 Mexico: Jalisco

48415 Mexico: Mexico

48417 Mexico: Morelos

48418 Mexico: Nayarit

48419 Mexico: Nuevo Leon

48420 Mexico: Oaxaca

48421 Mexico: Puebla

48422 Mexico: Queretaro

48423 Mexico: Quintana Roo

48424 Mexico: San Luis Potosi

48425 Mexico: Sinaloa

48426 Mexico: Sonora

48427 Mexico: Tabasco

48428 Mexico: Tamaulipas
48429

48430

48431

48432

48433

48434

52801

52802

55401

57801

57802

57803

57804

61601

61602

62001

62002

62003

62004

62005

62006

62007

62008

62009

64303

64304

64307

64308

64314

64316

64318

64321

64322

64323

64324

64325

64326

64327

64331

64332

64334

64335

64336

64337

64338

64342

64343

64347

64348
Mexico: Tlaxcala

Mexico: Veracruz

Mexico:Yucatan

Mexico: Zacatecas

Mexico: Small schools

Mexico: Very small schools

Netherlands: Lower secondary schools

Netherlands: Upper secondary schools

New Zealand

Norway: Stratum 1

Norway: Stratum 2

Norway: Stratum 3

Norway: Stratum 4

Poland: Gimnazja

Poland: Licea (vss)

Portugal: Açores

Portugal: DREA

Portugal: DREALG

Portugal: DREC

Portugal: DREL

Portugal: DREN

Portugal: Madeira

Portugal: Small schools

Portugal: Very small schools

Russian Federation: Stratum 3

Russian Federation: Stratum 4

Russian Federation: Stratum 7

Russian Federation: Stratum 8

Russian Federation: Stratum 14

Russian Federation: Stratum 16

Russian Federation: Stratum 18

Russian Federation: Stratum 21

Russian Federation: Stratum 22

Russian Federation: Stratum 23

Russian Federation: Stratum 24

Russian Federation: Stratum 25

Russian Federation: Stratum 26

Russian Federation: Stratum 27

Russian Federation: Stratum 31

Russian Federation: Stratum 32

Russian Federation: Stratum 34

Russian Federation: Stratum 35

Russian Federation: Stratum 36

Russian Federation: Stratum 37

Russian Federation: Stratum 38

Russian Federation: Stratum 42

Russian Federation: Stratum 43

Russian Federation: Stratum 47

Russian Federation: Stratum 48 
64350 Russian Federation: Stratum 50

64351 Russian Federation: Stratum 51

64352 Russian Federation: Stratum 52

64353 Russian Federation: Stratum 53

64354 Russian Federation: Stratum 54

64355 Russian Federation: Stratum 55

64356 Russian Federation: Stratum 56

64358 Russian Federation: Stratum 58

64359 Russian Federation: Stratum 59

64361 Russian Federation: Stratum 61

64363 Russian Federation: Stratum 63

64364 Russian Federation: Stratum 64

64366 Russian Federation: Stratum 66

64370 Russian Federation: Stratum 70

64371 Russian Federation: Stratum 71

64374 Russian Federation: Stratum 74

64376 Russian Federation: Stratum 76

64377 Russian Federation: Stratum 77

64378 Russian Federation: Stratum 78

64386 Russian Federation: Stratum 86

70301 Slovak Republic: Stratum 1

70302 Slovak Republic: Stratum 2

70303 Slovak Republic: Stratum 3

70304 Slovak Republic: Stratum 4

70305 Slovak Republic: Stratum 5

70306 Slovak Republic: Stratum 6

70307 Slovak Republic: Stratum 7

70308 Slovak Republic: Stratum 8

70309 Slovak Republic: Stratum 9

70310 Slovak Republic: Stratum 10

70311 Slovak Republic: Stratum 11

70312 Slovak Republic: Stratum 12

70313 Slovak Republic: Stratum 13

70314 Slovak Republic: Stratum 14

70315 Slovak Republic: Stratum 15

70316 Slovak Republic: Stratum 16

70317 Slovak Republic: Stratum 17

70318 Slovak Republic: Stratum 18

70319 Slovak Republic: Stratum 19

70320 Slovak Republic: Stratum 20

72401 Spain: Stratum 1

72402 Spain: Stratum 2

72403 Spain: Stratum 3

72404 Spain, Castilia y Leon: Stratum 4

72405 Spain, Castilia y Leon: Stratum 5

72406 Spain, Castilia y Leon: Stratum 6

72407 Spain, Castilia y Leon: Stratum 7

72408 Spain, Catalonia: Stratum 8

72409 Spain, Catalonia: Stratum 9
72410

72411

72412

72413

72414

72415

72416

72417

72418

72419

72420

72421

72422

72423

72424

72425

72426

72427

72428

72429

72430

72431

72432

72433

72434

72435

72436

72437

72438

72439

72440

72441

72442

72443

72444

72445

75201

75202

75203

75204

75205

75206

75207

75208

75209

75601

75602

75603

75604
Spain, Catalonia: Stratum 10

Spain, Basque Country: Stratum 11

Spain, Basque Country: Stratum 12

Spain, Basque Country: Stratum 13

Spain, Basque Country: Stratum 14

Spain, Basque Country: Stratum 15

Spain, Basque Country: Stratum 16

Spain, Basque Country: Stratum 17

Spain, Basque Country: Stratum 18

Spain: Stratum 19

Spain: Stratum 20

Spain: Stratum 21

Spain: Stratum 22

Spain: Stratum 23

Spain: Stratum 24

Spain: Stratum 25

Spain: Stratum 26

Spain: Stratum 27

Spain: Stratum 28

Spain: Stratum 29

Spain: Stratum 30

Spain: Stratum 31

Spain: Stratum 32

Spain: Stratum 33

Spain: Stratum 34

Spain: Stratum 35

Spain: Stratum 36

Spain: Stratum 37

Spain: Stratum 38

Spain: Stratum 39

Spain: Stratum 40

Spain: Stratum 41

Spain: Stratum 42

Spain: Stratum 43

Spain: Stratum 44

Spain: Stratum 45

Sweden: Stratum 1

Sweden: Stratum 2

Sweden: Stratum 3

Sweden: Stratum 4

Sweden: Stratum 5

Sweden: Stratum 6

Sweden: Stratum 7

Sweden: Stratum 8

Sweden: Stratum 9

Switzerland (German): Stratum 1

Switzerland (German): Stratum 2

Switzerland (German): Stratum 3

Switzerland (German): Stratum 4 
75605 Switzerland (German): Stratum 5

75606 Switzerland (German): Stratum 6

75607 Switzerland (German): Stratum 7

75608 Switzerland (German): Stratum 8

75609 Switzerland (German): Stratum 9

75610 Switzerland (German): Stratum 10

75611 Switzerland (German): Stratum 11

75612 Switzerland (German): Stratum 12

75613 Switzerland (German): Stratum 13

75614 Switzerland (German): Stratum 14

75615 Switzerland (French): Stratum 15

75616 Switzerland (French): Stratum 16

75617 Switzerland (French): Stratum 17

75618 Switzerland (French): Stratum 18

75619 Switzerland (French): Stratum 19

75620 Switzerland (French): Stratum 20

75621 Switzerland (French): Stratum 21

75623 Switzerland (French): Stratum 23

75624 Switzerland (French): Stratum 24

75625 Switzerland (French): Stratum 25

75626 Switzerland (French): Stratum 26

75628 Switzerland (French): Stratum 28

75629 Switzerland (Italian): Stratum 29

75631 Switzerland (Italian): Stratum 31

75632 Switzerland (Italian): Stratum 32

75634 Switzerland (Italian): Stratum 34

76401 Thailand: Stratum 1

76402 Thailand: Stratum 2

76403 Thailand: Stratum 3

76404 Thailand: Stratum 4

76405 Thailand: Stratum 5

76406 Thailand: Stratum 6

76407 Thailand: Stratum 7

76408 Thailand: Stratum 8

76409 Thailand: Stratum 9

76410 Thailand: Stratum 10

76411 Thailand: Stratum 11

76412 Thailand: Stratum 12

76413 Thailand: Stratum 13

76414 Thailand: Stratum 14

76415 Thailand: Stratum 15

78801 Tunisia: East

78802 Tunisia: West

79201 Turkey: Stratum 1

79202 Turkey: Stratum 2

79203 Turkey: Stratum 3

79204 Turkey: Stratum 4

79205 Turkey: Stratum 5

79206 Turkey: Stratum 6
Turkey: Stratum 7

Turkey: Stratum 8

Turkey: Stratum 9

Turkey: Stratum 10

Turkey: Stratum 11

Turkey: Stratum 12

Turkey: Stratum 13

Turkey: Stratum 14

Turkey: Stratum 15

Turkey: Stratum 16

Turkey: Stratum 17

Turkey: Stratum 18

Turkey: Stratum 19

Turkey: Stratum 20

Turkey: Stratum 21

Turkey: Stratum 22

Turkey: Stratum 23

Turkey: Stratum 24

Turkey: Stratum 25

Turkey: Stratum 26

Turkey: Stratum 27

Turkey: Stratum 28

Turkey: Stratum 29

Turkey: Stratum 30

Turkey: Stratum 31

Turkey: Stratum 32

Turkey: Stratum 33

Turkey: Stratum 34

Turkey: Stratum 35

Turkey: Stratum 36

Turkey: Stratum 37

Turkey: Stratum 38

Turkey: Stratum 39

Turkey: Stratum 40

Turkey: Stratum 41

Turkey: Stratum 42

Turkey: Stratum 43

Scotland: Stratum 1

Scotland: Stratum 2

Scotland: Stratum 3

Scotland: Stratum 4

Scotland: Stratum 5

England

N.Ireland

N.Ireland: Very large schools

Wales

United States

Uruguay: Liceos Publicos (Mdeo/AM)

Uruguay: Liceos Publicos

(cap. dptos interior) 
85803 Uruguay: Liceos Publicos (rural)

85804 Uruguay: Escuelas tecnicas

(Mdeo/AM)

85805 Uruguay: Escuelas tecnicas (interior)

85806 Uruguay: Colegios privados

85808 Uruguay: Small schools

85809 Uruguay: Very small schools

89101 Serbia: Stratum 1

89102 Serbia: Stratum 2

89103 Serbia: Stratum 3

89104 Serbia: Stratum 4

89105 Serbia: Stratum 5

89106 Serbia: Stratum 6

89107 Serbia: Stratum 7

89108 Serbia: Stratum 8 


\section{COUNTRY (1) Country ID}

Format: A3 Columns: 1-3

See Appendix 6: Student questionnaire data file codebook

\section{CNT (2) Nation code}

Format: A3 Columns: 4-6

SUBNATIO (3) Adjudicated sub-region

Format: A4 Columns: 7-10

See Appendix 6: Student questionnaire data file codebook

\section{SCHOOLID (4) School ID}

Format: A5 Columns: 11-15

$$
\begin{array}{lll}
\text { SC01Q01 } & \text { (5) } & \text { School location Q1 } \\
\text { Format: } & \text { F1 } & \text { Columns: } 17-17 \\
& 1 & \text { Village (less } 3000) \\
2 & \text { Small town }(3000 \text { to } 15000) \\
3 & \text { Town }(15000 \text { to } 100000) \\
4 & \text { City }(100000 \text { to } 1000000) \\
5 & \text { Large city (more } 1000000) \\
7 & \text { N/A } \\
8 & \text { Invalid } \\
9 & \text { Missing }
\end{array}
$$

\section{SC02Q01 (6) Number of boys Q2a}

Format: F8 Columns: 18-25

997 N/A

998 Invalid

999 Missing

$$
\begin{aligned}
& 997 \text { N/A } \\
& 998 \text { Invalid } \\
& 999 \text { Missing }
\end{aligned}
$$$$
\text { SC02Q02 (7) Number of girls Q2b }
$$$$
\text { Format: F8 Columns: 26-33 }
$$

\section{SC03Q01 (8) Public or private Q3}

Format: F1 Columns: 34-34

1 Public

2 Private

7 N/A

8 Invalid

9 Missing

\section{N/A \\ 998 Invalid \\ 999 Missing \\ $997 \mathrm{~N} / \mathrm{A}$ \\ 998 Invalid \\ 999 Missing}

SC04Q01 (9) Funding Government Q4a

Format: F8.3Columns: 35-42

SC04Q02 (10) Funding Student fees Q4b

Format: F8.3Columns: 43-50

$\begin{array}{ll}\text { SC04Q03 (11) Funding Benefactors Q4c } \\ \text { Format: } & \text { F8.3 Columns: 51-58 } \\ & 997 \text { N/A } \\ & 998 \text { Invalid } \\ & 999 \text { Missing }\end{array}$

\section{SC04Q04 (12) Funding Other Q4d}

Format: F8.3Columns: 59-66 $997 \mathrm{~N} / \mathrm{A}$

998 Invalid

999 Missing

$\begin{array}{lll}\text { SC05Q01 } & \text { (13) } & \text { Grade } 1 \\ \text { Format: } & \text { F1 } & \text { Column } \\ & 1 & \text { Yes } \\ 2 & \text { No } \\ 7 & \text { N/A } \\ 8 & \text { Invalid } \\ 9 & \text { Missing }\end{array}$

$\begin{array}{lll}\text { SC05Q02 } & \text { (14) } & \text { Grade } 2 \\ \text { Format: } & \text { F1 } & \text { Column } \\ & 1 & \text { Yes } \\ 2 & \text { No } \\ 7 & \text { N/A } \\ 8 & \text { Invalid } \\ 9 & \text { Missing }\end{array}$

$\begin{array}{lll}\text { SC05Q03 (15) } & \text { Grade } 3 \text { Q5c } \\ \text { Format: } & \text { F1 } & \text { Columns: 69-69 } \\ & 1 & \text { Yes } \\ 2 & \text { No } \\ 7 & \text { N/A } \\ 8 & \text { Invalid } \\ 9 & \text { Missing }\end{array}$




$\begin{array}{lll}\text { SC05Q04 (16) } & \text { Grade } 4 \text { Q5d } \\ \text { Format: } & \text { F1 } & \text { Columns: 70-70 } \\ & 1 & \text { Yes } \\ 2 & \text { No } \\ 7 & \text { N/A } \\ 8 & \text { Invalid } \\ 9 & \text { Missing }\end{array}$

$\begin{array}{lll}\text { SC05Q05 (17) } & \text { Grade 5 Q5e } \\ \text { Format: } & \text { F1 } & \text { Columns: 71-71 } \\ & 1 & \text { Yes } \\ 2 & \text { No } \\ 7 & \text { N/A } \\ 8 & \text { Invalid } \\ 9 & \text { Missing }\end{array}$

\section{SC05Q06 (18) Grade 6 Q5f}

Format: F1 Columns: 72-72

1 Yes

2 No

7 N/A

8 Invalid

9 Missing

\section{SC05Q07 (19) Grade 7 Q5g}

$\begin{array}{rll}\text { Format: } & \text { F1 } & \text { Column } \\ 1 & \text { Yes } \\ 2 & \text { No } \\ 7 & \text { N/A } \\ 8 & \text { Invalid } \\ 9 & \text { Missing }\end{array}$

\section{SC05Q08 (20) Grade 8 Q5h}

Format: F1 Columns: 74-74

1 Yes

2 No

7 N/A

8 Invalid

9 Missing

\section{SC05Q09 (21) Grade 9 Q5i}

Format: F1 Columns: 75-75

1 Yes

2 No

$7 \mathrm{~N} / \mathrm{A}$

8 Invalid

9 Missing

\section{SC05Q10 (22) Grade 10 Q5j}

Format: F1 Columns: 76-76

1 Yes

$2 \mathrm{No}$

7 N/A

8 Invalid

9 Missing

\section{SC05Q11 (23) Grade 11 Q5k}

Format: F1 Columns: 77-77

1 Yes

2 No

7 N/A

8 Invalid

9 Missing

\section{SC05Q12 (24) Grade 12 Q5I}

Format: F1 Columns: 78-78

1 Yes

2 No

7 N/A

8 Invalid

9 Missing

\section{SC05Q13 (25) Grade 13 Q5m}

Format: F1 Columns: 79-79

1 Yes

2 No

7 N/A

8 Invalid

9 Missing

SC05Q14 (26) Ungraded school Q5n

Format: F1 Columns: 80-80

1 Yes

$2 \mathrm{No}$

7 N/A

8 Invalid

$9 \quad$ Missing

SC06Q01 (27) Repeat < grade> at < ISCED2> Q6a

Format: F8.3Columns: 81-88

$997 \mathrm{~N} / \mathrm{A}$

998 Invalid

999 Missing

SC06Q02 (28) Repeat < grade> at < ISCED3> Q6b

Format: F8.3Columns: 89-96

997 N/A

998 Invalid

999 Missing

SC08Q01 (29) Shortage: maths teacher Q8a

Format: F1 Columns: 97-97

1 Not at all

2 Very little

3 To some extent

4 A lot

7 N/A

8 Invalid

$9 \quad$ Missing 


$\begin{array}{lll}\text { SC08Q02 (30) } & \text { Shortage: science } \\ \text { Format: } & \text { F1 } & \text { Columns: } 98-98 \\ & 1 & \text { Not at all } \\ & 2 & \text { Very little } \\ 3 & \text { To some extent } \\ 4 & \text { A lot } \\ 7 & \text { N / A } \\ 8 & \text { Invalid } \\ 9 & \text { Missing }\end{array}$

SC08Q03 (31) Shortage: test lang. teacher Q8c

Format: F1 Columns: 99-99

1 Not at all

2 Very little

3 To some extent

4 A lot

7 N/A

8 Invalid

9 Missing

SC08Q04 (32) Shortage: other national lang. teacher Q8d

Format: F1 Columns: 100-100

1 Not at all

2 Very little

3 To some extent

4 A lot

7 N/A

8 Invalid

9 Missing

SC08Q05 (33) Shortage: foreign lang. teacher Q8e

Format: F1 Columns: 101-101

1 Not at all

2 Very little

3 To some extent

4 A lot

$7 \mathrm{~N} / \mathrm{A}$

8 Invalid

9 Missing

\section{SC08Q06 (34) Shortage: experienced teacher Q8f}

Format: F1 Columns: 102-102

1 Not at all

2 Very little

3 To some extent

4 A lot

7 N/A

8 Invalid

9 Missing

\section{SC08Q07 (35) Shortage: emergency teacher Q8g}

Format: F1 Columns: 103-103

1 Not at all

2 Very little

3 To some extent

4 A lot

7 N/A

8 Invalid

9 Missing
SC08Q08 (36) Shortage: support personnel Q8h

Format: F1 Columns: 104-104

Not at all

Very little

To some extent

A lot

$\mathrm{N} / \mathrm{A}$

Invalid

9 Missing

SC08Q09 (37) Shortage: textbooks Q8i

Format: F1 Columns: 105-105

Not at all

Very little

To some extent

A lot

N/A

8 Invalid

9 Missing

SC08Q10 (38) Shortage: supplies Q8j

Format: F1 Columns: 106-106

1 Not at all

2 Very little

3 To some extent

4 A lot

7 N/A

8 Invalid

9 Missing

SC08Q11 (39) Shortage: buildings Q8k

Format: F1 Columns: 107-107

1 Not at all

2 Very little

3 To some extent

4 A lot

7 N/A

8 Invalid

9 Missing

SC08Q12 (40) Shortage: heating Q81

Format: F1 Columns: 108-108

1 Not at all

2 Very little

3 To some extent

4 A lot

7 N/A

8 Invalid

9 Missing

SC08Q13 (41) Shortage: classrooms Q8m

Format: F1 Columns: 109-109

1 Not at all

2 Very little

3 To some extent

4 A lot

$7 \mathrm{~N} / \mathrm{A}$

8 Invalid

9 Missing 
SC08Q14 (42) Shortage: special equipment Q8n

Format: F1 Columns: 110-110

1 Not at all

2 Very little

3 To some extent

4 A lot

7 N/A

8 Invalid

9 Missing

\section{SC08Q15 (43) Shortage: computers Q8o}

Format: F1 Columns: 111-111

1 Not at all

2 Very little

3 To some extent

4 A lot

7 N/A

8 Invalid

9 Missing

SC08Q16 (44) Shortage: computer software Q8p

Format: F1 Columns: 112-112

1 Not at all

2 Very little

3 To some extent

4 A lot

7 N/A

8 Invalid

$9 \quad$ Missing

SC08Q17 (45) Shortage: calculators Q8q

Format: F1 Columns: 113-113

1 Not at all

2 Very little

3 To some extent

4 A lot

7 N/A

8 Invalid

9 Missing

SC08Q18 (46) Shortage: library material Q8r

Format: F1 Columns: 114-114

1 Not at all

2 Very little

3 To some extent

4 A lot

7 N/A

8 Invalid

9 Missing

SC08Q19 (47) Shortage: audio-vidio Q8s

Format: F1 Columns: 115-115

1 Not at all

2 Very little

3 To some extent

4 A lot

7 N/A

8 Invalid

$9 \quad$ Missing
SC08Q20 (48) Shortage: lab equipment Q8t

Format: F1 Columns: 116-116

1 Not at all

2 Very little

3 To some extent

4 A lot

7 N/A

8 Invalid

9 Missing

SC09Q01 (49) Computers: altogether Q9a

Format: F8 Columns: 117-124

$9997 \quad$ N/A

9998 Invalid

9999 Missing

SC09Q02 (50) Computers: students Q9b

Format: F8 Columns: 125-132

$9997 \quad \mathrm{~N} / \mathrm{A}$

9998 Invalid

9999 Missing

SC09Q03 (51) Computers: teachers Q9C

Format: F8 Columns: 133-140

$\begin{array}{ll}9997 & \mathrm{~N} / \mathrm{A} \\ 9998 & \text { Invalid } \\ 9999 & \text { Missing }\end{array}$

9999 Missing

SC09Q04 (52) Computers: admin Q9d

Format: F8 Columns: 141-148

$9997 \quad \mathrm{~N} / \mathrm{A}$

9998 Invalid

9999 Missing

SC09Q05 (53) Computers: with Web Q9e

Format: F8 Columns: 149-156

$9997 \quad \mathrm{~N} / \mathrm{A}$

9998 Invalid

9999 Missing

SC09Q06 (54) Computers: with LAN Q9f

Format: F8 Columns: 157-164

$\begin{array}{ll}9997 & \text { N/A } \\ 9998 & \text { Invalid } \\ 9999 & \text { Missing }\end{array}$

SC10Q01 (55) Admittance: residence Q10a

Format: F1 Columns: 165-165

1 Prerequisite

2 High priority

3 Considered

4 Not considered

$7 \mathrm{~N} / \mathrm{A}$

8 Invalid

$9 \quad$ Missing 


$\begin{array}{lll}\text { SC10Q02 (56) Admittance: student record Q10b } \\ \text { Format: } & \text { F1 } & \text { Columns: 166-166 } \\ & 1 & \text { Prerequisite } \\ 2 & \text { High priority } \\ 3 & \text { Considered } \\ 4 & \text { Not considered } \\ 7 & \text { N/A } \\ 8 & \text { Invalid } \\ 9 & \text { Missing } \\ \text { SC10Q03 } & (57) \text { Admittance: recommendation Q10c } \\ \text { Format: } & \text { F1 } & \text { Columns: 167-167 } \\ 1 & \text { Prerequisite } \\ 2 & \text { High priority } \\ 3 & \text { Considered } \\ 4 & \text { Not considered } \\ 7 & \text { N/A } \\ 8 & \text { Invalid } \\ 9 & \text { Missing }\end{array}$

SC10Q04 (58) Admittance: parents' endorsement Q10d

Format: F1 Columns: 168-168

1 Prerequisite

2 High priority

3 Considered

4 Not considered

7 N/A

8 Invalid

9 Missing

SC10Q05 (59) Admittance: special programme Q10e

Format: F1 Columns: 169-169

1 Prerequisite

2 High priority

3 Considered

4 Not considered

7 N/A

8 Invalid

9 Missing

SC10Q06 (60) Admittance: family preference Q10f

Format: F1 Columns: 170-170

1 Prerequisite

2 High priority

3 Considered

4 Not considered

7 N/A

8 Invalid

9 Missing

\section{SC10Q07 (61) <Country Specific> Q10g}

Format: F1 Columns: 171-171

1 Prerequisite

2 High priority

3 Considered

4 Not considered

7 N/A

8 Invalid

$9 \quad$ Missing
SC11Q01 (62) Students: enjoy Q11a

Format: F1 Columns: 172-172

Strongly agree

Agree

Disagree

Strongly disagree

N/A

Invalid

9 Missing

SC11Q02 (63) Students: enthusiasm Q11b

Format: F1 Columns: 173-173

1 Strongly agree

2 Agree

3 Disagree

4 Strongly disagree

7 N/A

8 Invalid

9 Missing

SC11Q03 (64) Students: take pride Q11C

Format: F1 Columns: 174-174

Strongly agree

Agree

Disagree

Strongly disagree

7 N/A

8 Invalid

9 Missing

SC11Q04 (65) Students: value academic Q11d

Format: F1 Columns: 175-175

Strongly agree

Agree

Disagree

Strongly disagree

$\mathrm{N} / \mathrm{A}$

8 Invalid

9 Missing

SC11Q05 (66) Students: respectful Q11e

Format: F1 Columns: 176-176

Strongly agree

Agree

Disagree

Strongly disagree

$\mathrm{N} / \mathrm{A}$

Invalid

9 Missing

SC11Q06 (67) Students: value education Q11f

Format: F1 Columns: 177-177

Strongly agree

Agree

Disagree

Strongly disagree

$\mathrm{N} / \mathrm{A}$

8 Invalid

9 Missing 


$\begin{array}{lll}\text { SC11Q07 (68) } & \text { Students: learn Q11g } \\ \text { Format: } & \text { F1 } & \text { Columns: 178-178 } \\ & 1 & \text { Strongly agree } \\ 2 & \text { Agree } \\ 3 & \text { Disagree } \\ 4 & \text { Strongly disagree } \\ 7 & \text { N/A } \\ 8 & \text { Invalid } \\ 9 & \text { Missing }\end{array}$

SC12Q01 (69) Standardised test Q12a

Format: F1 Columns: 179-179

1 Never

21 to 2 times a year

33 to 5 times a year

4 Monthly

5 More once a month

7 N/A

8 Invalid

9 Missing

\section{SC12Q02 (70) Teacher's test Q12b}

Format: F1 Columns: 180-180

1 Never

21 to 2 times a year

33 to 5 times a year

4 Monthly

5 More once a month

7 N/A

8 Invalid

9 Missing

\section{SC12Q03 (71) Teacher's ratings Q12c}

Format: F1 Columns: 181-181

1 Never

21 to 2 times a year

33 to 5 times a year

4 Monthly

5 More once a month

7 N/A

8 Invalid

9 Missing

\section{SC12Q04 (72) Students'portfolios Q12d}

Format: F1 Columns: 182-182

1 Never

21 to 2 times a year

33 to 5 times a year

4 Monthly

5 More once a month

7 N/A

8 Invalid

9 Missing
SC12Q05 (73) Student assignments Q12e

Format: F1 Columns: 183-183

Never

1 to 2 times a year

3 to 5 times a year

Monthly

More once a month

$\mathrm{N} / \mathrm{A}$

Invalid

Missing

SC13Q01 (74) Assessment: inform parents Q13a

Format: F1 Columns: 184-184

1 Yes

$2 \mathrm{No}$

7 N/A

8 Invalid

9 Missing

SC13Q02 (75) Assessment: retention Q13b

Format: F1 Columns: 185-185

1 Yes

2 No

7 N/A

8 Invalid

9 Missing

SC13Q03 (76) Assessment: group students Q13C

Format: F1 Columns: 186-186

1 Yes

2 No

7 N/A

8 Invalid

9 Missing

SC13Q04 (77) Assessment: compare to national Q13d

Format: F1 Columns: 187-187

$$
\begin{array}{ll}
1 & \text { Yes } \\
2 & \text { No } \\
7 & \text { N/A } \\
8 & \text { Invalid } \\
9 & \text { Missing }
\end{array}
$$

SC13Q05 (78) Assessment: school's progress Q13e

Format: F1 Columns: 188-188

1 Yes

2 No

7 N/A

8 Invalid

9 Missing

SC13Q06 (79) Assessment: teachers' effectiveness Q13f

Format: F1 Columns: 189-189

Yes

No

$\mathrm{N} / \mathrm{A}$

8 Invalid

9 Missing 


\begin{tabular}{|c|c|c|}
\hline \multicolumn{3}{|c|}{ SC13Q07 (80) Assessment: improve curriculum Q13g } \\
\hline \multirow[t]{6}{*}{ Format: } & F1 & Columns: $190-190$ \\
\hline & 1 & Yes \\
\hline & 2 & No \\
\hline & 7 & $\mathrm{~N} / \mathrm{A}$ \\
\hline & 8 & Invalid \\
\hline & 9 & Missing \\
\hline \multicolumn{3}{|c|}{ SC13Q08 (81) Assessment: compare to other schools Q13h } \\
\hline \multirow[t]{6}{*}{ Format: } & $\mathrm{F} 1$ & Columns: 191-191 \\
\hline & 1 & Yes \\
\hline & 2 & No \\
\hline & 7 & $\mathrm{~N} / \mathrm{A}$ \\
\hline & 8 & Invalid \\
\hline & 9 & Missing \\
\hline
\end{tabular}

SC14Q01 (82) Language percent Q14

Format: F1 Columns: 192-192

$140 \%$ or more

2 more $20 \%$ less $40 \%$

3 more $10 \%$ less $20 \%$

4 Less than $10 \%$

7 N/A

8 Invalid

9 Missing

SC15Q01 (83) Separate subject Q15a

Format: F1 Columns: 193-193

1 No

2 Yes for one

3 Yes for 2 or more

4 Not Applicable

7 N/A

8 Invalid

9 Missing

\section{SC15Q02 (84) Other parts Q15b}

Format: F1 Columns: 194-194

1 No

2 Yes for one

3 Yes for 2 or more

4 Not Applicable

7 N/A

8 Invalid

9 Missing

SC16Q01 (85) Streaming by levels Q16a

Format: F1 Columns: 195-195

1 For all classes

2 For some classes

3 Not for any classes

7 N/A

8 Invalid

9 Missing
SC16Q02 (86) Streaming by content Q16b

Format: F1 Columns: 196-196

For all classes

For some classes

Not for any classes

$\mathrm{N} / \mathrm{A}$

Invalid

9 Missing

SC16Q03 (87) Grouped by ability Q16c

Format: F1 Columns: 197-197

1 For all classes

2 For some classes

3 Not for any classes

7 N/A

8 Invalid

9 Missing

SC16Q04 (88) Not grouped by ability Q16d

Format: F1 Columns: 198-198

1 For all classes

2 For some classes

3 Not for any classes

7 N/A

8 Invalid

9 Missing

SC17Q01 (89) Enrichment mathematics Q17a

Format: F1 Columns: 199-199

1 Yes

2 No

7 N/A

8 Invalid

9 Missing

SC17Q02 (90) Remedial mathematics Q17b

Format: F1 Columns: 200-200

1 Yes

2 No

7 N/A

8 Invalid

9 Missing

SC17Q03 (91) Mathematics competitions Q17c

Format: F1 Columns: 201-201

1 Yes

2 No

7 N/A

8 Invalid

9 Missing

SC17Q04 (92) Mathematics clubs Q17d

Format: F1 Columns: 202-202

1 Yes

2 No

7 N/A

8 Invalid

9 Missing 


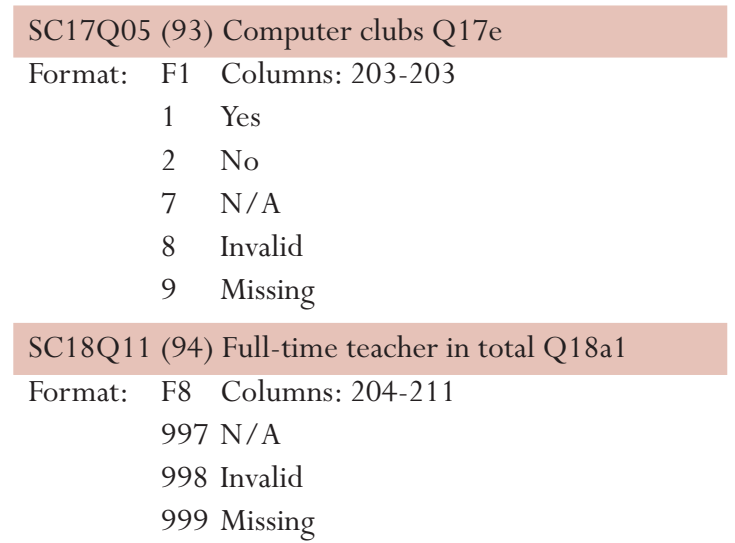

SC18Q12 (95) Full-time teacher fully certified Q18b1

Format: F8 Columns: 212-219

$997 \mathrm{~N} / \mathrm{A}$

998 Invalid

999 Missing

SC18Q13 (96) Full-time teacher ISCED5A in pedagogy
$\begin{array}{ll}\text { Q18c1 } \\ \text { Format: } & \text { F8 Columns: 220-227 } \\ & 997 \text { N/A } \\ & 998 \text { Invalid } \\ & 999 \text { Missing }\end{array}$

SC18Q21 (97) Part-time teacher in total Q18a2

Format: F8 Columns: 228-235

$997 \mathrm{~N} / \mathrm{A}$

998 Invalid

999 Missing

SC18Q22 (98) Part-time teacher fully certified Q18b2

Format: F8 Columns: 236-243

$997 \mathrm{~N} / \mathrm{A}$

998 Invalid

999 Missing

SC18Q23 (99) Part-time teacher ISCED5A in pedagogy
Q18c2
$\begin{array}{ll}\text { Format: } & \text { F8 Columns: 244-251 } \\ & 997 \mathrm{~N} / \mathrm{A} \\ & 998 \text { Invalid } \\ & 999 \text { Missing }\end{array}$

SC19Q11 (100) Full-time maths teacher Q19a1

Format: F8 Columns: 252-259

$997 \mathrm{~N} / \mathrm{A}$

998 Invalid

999 Missing

SC19Q12 (101) Full-time teacher ISCED5A maths Q19b1

Format: F8 Columns: 260-267

997 N/A

998 Invalid

999 Missing
SC19Q13 (102) Full-time maths teacher ISCED5A

no major Q19c1

Format: F8 Columns: 268-275

$997 \mathrm{~N} / \mathrm{A}$

998 Invalid

999 Missing

SC19Q14 (103) Full-time maths teacher ISCED5A

in pedagogy Q19d1

Format: F8 Columns: 276-283

997 N/A

998 Invalid

999 Missing

SC19Q15 (104) Full-time maths teacher ISCED5B

Q19e1

Format: F8 Columns: 284-291

997 N/A

998 Invalid

999 Missing

SC19Q21 (105) Part-time maths teacher Q19a2

Format: F8 Columns: 292-299

997 N/A

998 Invalid

999 Missing

SC19Q22 (106) Part-time teacher ISCED5A maths

Q19b2

Format: F8 Columns: 300-307

997 N/A

998 Invalid

999 Missing

SC19Q23 (107) Part-time maths teacher ISCED5A no major Q19c2

Format: F8 Columns: 308-315

997 N/A

998 Invalid

999 Missing

SC19Q24 (108) Part-time maths teacher ISCED5A

in pedagogy Q19d2

Format: F8 Columns: 316-323

997 N/A

998 Invalid

999 Missing

SC19Q25 (109) Part-time maths teacher ISCED5B

Q19e2

Format: F8 Columns: 324-331

997 N/A

998 Invalid

999 Missing 


\begin{tabular}{|c|c|c|}
\hline \multicolumn{3}{|c|}{ SC20Q01 (110) By students' achievement Q20a } \\
\hline \multirow[t]{6}{*}{ Format: } & F1 & Columns: $332-332$ \\
\hline & 1 & Yes \\
\hline & 2 & No \\
\hline & 7 & $\mathrm{~N} / \mathrm{A}$ \\
\hline & 8 & Invalid \\
\hline & 9 & Missing \\
\hline \multicolumn{3}{|c|}{ SC20Q02 (111) By teacher review Q20b } \\
\hline \multirow[t]{6}{*}{ Format: } & $\mathrm{F} 1$ & Columns: 333-333 \\
\hline & 1 & Yes \\
\hline & 2 & No \\
\hline & 7 & $\mathrm{~N} / \mathrm{A}$ \\
\hline & 8 & Invalid \\
\hline & 9 & Missing \\
\hline
\end{tabular}

SC20Q03 (112) By principal Q20c

Format: F1 Columns: 334-334

1 Yes

$2 \mathrm{No}$

7 N/A

8 Invalid

9 Missing

\begin{tabular}{|c|c|c|}
\hline \multicolumn{3}{|c|}{ SC20Q04 (113) By inspe } \\
\hline Format: & F1 & Columns \\
\hline & 1 & Yes \\
\hline & 2 & No \\
\hline & 7 & $\mathrm{~N} / \mathrm{A}$ \\
\hline & 8 & Invalid \\
\hline & 9 & Missing \\
\hline
\end{tabular}

SC21Q01 (114) Innovative teachers Q21a

Format: F1 Columns: 336-336

1 Strongly agree

2 Agree

3 Disagree

4 Strongly disagree

7 N/A

8 Invalid

9 Missing

SC21Q02 (115) Traditional teachers Q21b

Format: F1 Columns: 337-337

1 Strongly agree

2 Agree

3 Disagree

4 Strongly disagree

7 N/A

8 Invalid

$9 \quad$ Missing
SC21Q03 (116) Innovation disagreements Q21C

Format: F1 Columns: 338-338

Strongly agree

Agree

Disagree

Strongly disagree

7 N/A

8 Invalid

9 Missing

SC22Q01 (117) Require high achievement Q22a

Format: F1 Columns: 339-339

1 Strongly agree

2 Agree

3 Disagree

4 Strongly disagree

7 N/A

8 Invalid

9 Missing

\section{SC22Q02 (118) Adapt standards Q22b}

Format: F1 Columns: 340-340

Strongly agree

Agree

Disagree

Strongly disagree

7 N/A

8 Invalid

9 Missing

\section{SC22Q03 (119) Frequent disagreements Q22C}

Format: F1 Columns: 341-341

Strongly agree

Agree

Disagree

Strongly disagree

7 N/A

8 Invalid

9 Missing

SC23Q01 (120) Goals social development Q23a

Format: F1 Columns: 342-342

1 Strongly agree

2 Agree

3 Disagree

4 Strongly disagree

7 N/A

8 Invalid

9 Missing

SC23Q02 (121) Goals maths skills Q23b

Format: F1 Columns: 343-343

Strongly agree

Agree

Disagree

4 Strongly disagree

7 N/A

8 Invalid

9 Missing 


$\begin{array}{lll}\text { SC23Q03 (122) Goals disagreements Q23c } \\ \text { Format: } & \text { F1 } & \text { Columns: 344-344 } \\ & 1 & \text { Strongly agree } \\ 2 & \text { Agree } \\ 3 & \text { Disagree } \\ 4 & \text { Strongly disagree } \\ 7 & \text { N/A } \\ 8 & \text { Invalid } \\ 9 & \text { Missing }\end{array}$

SC24Q01 (123) High morale Q24a

Format: F1 Columns: 345-345

1 Strongly agree

2 Agree

3 Disagree

4 Strongly disagree

7 N/A

8 Invalid

9 Missing

SC24Q02 (124) Enthusiasm Q24b

Format: F1 Columns: 346-346

1 Strongly agree

2 Agree

3 Disagree

4 Strongly disagree

7 N/A

8 Invalid

9 Missing

SC24Q03 (125) Pride in school Q24c

Format: F1 Columns: 347-347

1 Strongly agree

2 Agree

3 Disagree

4 Strongly disagree

7 N/A

8 Invalid

9 Missing

SC24Q04 (126) Academic Achievement Q24d

Format: F1 Columns: 348-348

1 Strongly agree

2 Agree

3 Disagree

4 Strongly disagree

7 N/A

8 Invalid

9 Missing

SC25Q01 (127) Low expectations Q25a

Format: F1 Columns: 349-349

1 Not at all

2 Very Little

3 To some extent

4 A lot

7 N/A

8 Invalid

9 Missing
SC25Q02 (128) Student absenteeism Q25b

Format: F1 Columns: 350-350

Not at all

Very Little

To some extent

A lot

$\mathrm{N} / \mathrm{A}$

Invalid

9 Missing

SC25Q03 (129) Stud-teacher relations Q25C

Format: F1 Columns: 351-351

Not at all

Very Little

To some extent

A lot

N/A

Invalid

9 Missing

SC25Q04 (130) Disruption of classes Q25d

Format: F1 Columns: 352-352

Not at all

Very Little

To some extent

A lot

N/A

Invalid

9 Missing

SC25Q05 (131) Not meeting needs Q25e

Format: F1 Columns: 353-353

1 Not at all

2 Very Little

3 To some extent

4 A lot

7 N/A

8 Invalid

9 Missing

SC25Q06 (132) Teacher absenteeism Q25f

Format: F1 Columns: 354-354

1 Not at all

2 Very Little

3 To some extent

4 A lot

7 N/A

8 Invalid

9 Missing

SC25Q07 (133) Skipping classes Q25g

Format: F1 Columns: 355-355

1 Not at all

2 Very Little

3 To some extent

4 A lot

7 N/A

8 Invalid

9 Missing 


$\begin{array}{lll}\text { SC25Q08 (134) Lack of respect Q25h } \\ \text { Format: } & \text { F1 } & \text { Columns: 356-356 } \\ 1 & \text { Not at all } \\ 2 & \text { Very Little } \\ 3 & \text { To some extent } \\ 4 & \text { A lot } \\ 7 & \text { N/A } \\ 8 & \text { Invalid } \\ 9 & \text { Missing }\end{array}$

SC25Q09 (135) Resisting change Q25i

Format: F1 Columns: 357-357

1 Not at all

2 Very Little

3 To some extent

4 A lot

7 N/A

8 Invalid

9 Missing

SC25Q10 (136) Use of alcohol Q25j

Format: F1 Columns: 358-358

1 Not at all

2 Very Little

3 To some extent

4 A lot

7 N/A

8 Invalid

9 Missing

\section{SC25Q11 (137) Teacher strictness Q25k}

Format: F1 Columns: 359-359

1 Not at all

2 Very Little

3 To some extent

4 A lot

7 N/A

8 Invalid

9 Missing

\section{SC25Q12 (138) Bullying Q25l}

Format: F1 Columns: 360-360

1 Not at all

2 Very Little

3 To some extent

4 A lot

$7 \mathrm{~N} / \mathrm{A}$

8 Invalid

9 Missing

\section{SC25Q13 (139) Lack encouragement Q25m}

Format: F1 Columns: 361-361

1 Not at all

2 Very Little

3 To some extent

4 A lot

7 N/A

8 Invalid

9 Missing
SC26Q01 (140) Hiring teachers Q26a

Format: A5 Columns: 362-366

Value Label

77777 N/A

88888 Invalid

99999 Missing

SC26Q02 (141) Firing teachers Q26b

Format: A5 Columns: 367-371

$\begin{array}{ll}\text { Value } & \text { Label } \\ 77777 & \text { N/A } \\ 88888 & \text { Invalid } \\ 99999 & \text { Missing }\end{array}$

SC26Q03 (142) Teacher salaries Q26c

Format: A5 Columns: 372-376

Value Label

77777 N/A

88888 Invalid

99999 Missing

SC26Q04 (143) Salary increase Q26d

Format: A5 Columns: 377-381

Value Label

77777 N/A

88888 Invalid

99999 Missing

SC26Q05 (144) Budget formulation Q26e

Format: A5 Columns: 382-386

Value Label

77777 N/A

88888 Invalid

99999 Missing

SC26Q06 (145) Budget allocation Q26f

Format: A5 Columns: 387-391

Value Label

77777 N/A

88888 Invalid

99999 Missing

SC26Q07 (146) Disciplinary policies Q26g

Format: A5 Columns: 392-396

Value Label

77777 N/A

88888 Invalid

99999 Missing

SC26Q08 (147) Assessment: policies Q26h

Format: A5 Columns: 397-401

Value Label

77777 N/A

88888 Invalid

99999 Missing 
SC26Q09 (148) Student admittance Q26i

Format: A5 Columns: 402-406

Value Label

77777 N/A

88888 Invalid

99999 Missing

SC26Q10 (149) Textbooks Q26j

Format: A5 Columns: 407-411

Value Label

77777 N/A

88888 Invalid

99999 Missing

SC26Q11 (150) Course content Q26k

Format: A5 Columns: 412-416

Value Label

77777 N/A

88888 Invalid

99999 Missing

SC26Q12 (151) Course offer Q26l

Format: A5 Columns: 417-421

Value Label

77777 N/A

88888 Invalid

99999 Missing

SC27Q01 (152) Decision making: national Q27a

Format: A4 Columns: 422-425

$\begin{array}{ll}7777 & \text { N/A } \\ 8888 & \text { Invalid } \\ 9999 & \text { Missing }\end{array}$

9999 Missing

SC27Q02 (153) Decision making: local Q27b

Format: A4 Columns: 426-429

$7777 \quad \mathrm{~N} / \mathrm{A}$

8888 Invalid

9999 Missing

SC27Q03 (154) Decision making: employers Q27C

Format: A4 Columns: 430-433

7777 N/A

8888 Invalid

9999 Missing

SC27Q04 (155) Decision making: parents Q27d

Format: A4 Columns: 434-437

$7777 \quad \mathrm{~N} / \mathrm{A}$

$8888 \quad$ Invalid

9999 Missing

SC27Q05 (156) Decision making: teachers Q27e

Format: A4 Columns: 438-441

$7777 \quad \mathrm{~N} / \mathrm{A}$

8888 Invalid

9999 Missing
SC27Q06 (157) Decision making: students Q27f

Format: A4 Columns: 442-445

$7777 \quad \mathrm{~N} / \mathrm{A}$

$8888 \quad$ Invalid

9999 Missing

SC27Q07 (158) Decision making: external Q27g

Format: A4 Columns: 446-449

$7777 \quad \mathrm{~N} / \mathrm{A}$

8888 Invalid

9999 Missing

SCHLSIZE (159) School size

Format: F8 Columns: 450-457

99997 N/A

99998 Invalid

99999 Missing

PCGIRLS (160) Proportion of girls

Format: F8.3Columns: 458-465

$997 \mathrm{~N} / \mathrm{A}$

998 Invalid

999 Missing

SCHLTYPE (161) School ownership

Format: F1 Columns: 466-466

1 Private independent

2 Private government-dependent

3 Public

$7 \quad \mathrm{~N} / \mathrm{A}$

9 Missing

RATCOMP (162) Computer ratio to school size

Format: F8.3Columns: 467-474

$97 \mathrm{~N} / \mathrm{A}$

98 Invalid

99 Missing

COMPWEB (163) Proportion of computers connected to WEB

Format: F8.3Columns: 475-482

$7 \mathrm{~N} / \mathrm{A}$

9 Missing

COMPLAN (164) Proportion of computers connected to LAN

Format: F8.3Columns: 483-490

$7 \mathrm{~N} / \mathrm{A}$

9 Missing

STRATIO (165) Student/teacher ratio

Format: F8.3Columns: 491-498

997 N/A

998 Invalid

999 Missing 
PROPCERT (166) Proportion of certified teachers

Format: F8.3Columns: 499-506

$997 \mathrm{~N} / \mathrm{A}$

998 Invalid

999 Missing

PROPQPED (167) Proportion of teachers with ISCED

$5 \mathrm{~A}$ in pedagogy

Format: F8.3Columns: 507-514

$997 \mathrm{~N} / \mathrm{A}$

998 Invalid

999 Missing

SMRATIO (168) Maths student/teacher ratio

Format: F8.3Columns: 515-522

$997 \mathrm{~N} / \mathrm{A}$

998 Invalid

999 Missing

PROPMATH (169) Proportion of maths teachers

Format: F8.3Columns: 523-530

$997 \mathrm{~N} / \mathrm{A}$

998 Invalid

999 Missing

PROPMA5A (170) Proportion of math teachers with a ISCED 5A level in maths

Format: F8.3Columns: 531-538

997 N/A

998 Invalid

999 Missing

ASSESS (171) Estimated number of assessments per year

Format: F1 Columns: 539-539

$1<20$

$2 \quad 20-39$

$3>40$

$7 \quad \mathrm{~N} / \mathrm{A}$

9 Missing

SELECT (172) School selectivity

Format: F1 Columns: 540-540

0 Not considered

1 At least one considered

2 At least one high priority

3 At least one pre-requiste

7 N/A

9 Missing

ABGROUP (173) Streaming within schools

Format: F1 Columns: 541-541

1 Not for any classes

2 For some classes

3 For all classes

7 N/A

9 Missing
EXCOURSE (174) School offering extension courses

(number of types)

Format: F1 Columns: 542-542

9 Missing

MACTIV (175) School offering maths activities (number of types)

Format: F1 Columns: 543-543

9 Missing

\section{AUTRES (176) Resource autonomy}

Format: F1 Columns: 544-544

9 Missing

\section{AUTCURR (177) Curricular autonomy}

Format: F1 Columns: 545-545

9 Missing

MSTREL (178) Index of poor student-teacher relations (school average)

Format: F5.2Columns: 546-550

$$
\begin{array}{ll}
7 & \text { N/A } \\
9 & \text { Missing }
\end{array}
$$

TCSHORT (179) Shortage of teachers (WLE)

Format: F8.3Columns: 551-558

997 Missing

SCMATBUI (180) Quality of material resources (WLE)

Format: F8.3Columns: 559-566

997 Missing

SCMATEDU (181) Quality of educational resources (WLE)

Format: F8.3Columns: 567-574

997 Missing

STMORALE (182) Student morale (WLE)

Format: F8.3Columns: 575-582

997 Missing

TCMORALE (183) Teacher morale (WLE)

Format: F8.3 Columns: 583-590

997 Missing

STUDBEHA (184) Student behaviours (WLE)

Format: F8.3Columns: 591-598

997 Missing

TEACBEHA (185) Teacher behaviours (WLE)

Format: F8.3Columns: 599-606

997 Missing

TCHCONS (186) Mathematics teacher consensus (WLE)

Format: F8.3Columns: 607-614 997 Missing

SCHAUTON (187) School autonomy (WLE)

Format: F8.3Columns: 615-622

997 Missing 
$\sqrt{\wedge} \quad$ TCHPARTI (188) Teacher participation (WLE)

$\begin{array}{lll}\stackrel{x}{U} & \text { Format: } & \text { F8.3Columns: 623-630 } \\ & & 997 \text { Missing }\end{array}$

SCWEIGHT (189) School weight

Format: F8.5 Columns: 631-638

997 Missing

STRATUM (190) Stratum

Format: A5 Columns: 640-644

See Appendix 6: Student questionnaire data file codebook 


\section{APPENDIX 8 - STUDENT COGNITIVE TEST DATA FILE CODEBOOK}

\section{COGNITIVE DATA}

Country (1) COUNTRY THREE-DIGIT ISO CODE

Format A3 Columns 1-3

See Appendix 6: Student questionnaire data file codebook

CNT (2) COUNTRY ALPHANUMERIC ISO CODE

Format A3 Columns 4-6

\section{Subnatio (3) SUB-NATION CODE}

FormatA4 Columns 7-10

See Appendix 6: Student questionnaire data file codebook

\section{Schoolid (4) SCHOOL ID}

Format A5 Columns 11-15

\section{Stidstd (5) STUDENT ID}

Format A5 Columns 16-20

BOOKID (6) BOOKLET ID

Format A2 Columns 22-23

\begin{tabular}{clll} 
M033Q01 (7) A VIEW WITH A ROOM Q1 & \multicolumn{2}{l}{ Multiple Choice } \\
Format A1 & Columns 24-24 & Booklet 1 & Q1 \\
1 & No Credit & Booklet 5 & Q43 \\
2 & No Credit & Booklet 11 & Q25 \\
3 & No Credit & Booklet 13 & Q10 \\
4 & Full Credit & & \\
8 & M/R & & \\
9 & Missing & & \\
n & N/A & & \\
r & Not reached & &
\end{tabular}

M034Q01T (8) BRICKS Q1
$\begin{array}{rlll}\text { Format A1 } & \text { Columns 25-25 } & \text { Coded Response } \\ 0 & \text { No Credit } & \text { Booklet 1 } & \text { Q22 } \\ 1 & \text { Full Credit } & \text { Booklet 2 } & \text { Q10 } \\ 8 & \text { Invalid } & \text { Booklet 6 } & \text { Q52 } \\ 9 & \text { Missing } & \text { Booklet 12 } & \text { Q29 } \\ \text { n } & \text { N/A } & & \\ \text { r } & \text { Not reached } & & \end{array}$

\begin{tabular}{clll} 
M124Q01 (9) WALKING Q1 & \multicolumn{2}{l}{ Coded Response } \\
FormatA1 & Columns 26-26 & Booklet 2 & Q16 \\
0 & No Credit & Booklet 3 & Q4 \\
1 & No Credit & Booklet 7 & Q37 \\
2 & Full Credit & Booklet 13 & Q25 \\
9 & Missing & & \\
n & N/A & & \\
r & Not reached & &
\end{tabular}




$\begin{array}{clll}\text { M124Q03T (10) WALKING Q3 } & \text { Coded Response } \\ \text { FormatA1 } & \text { Columns 27-27 } & \text { Booklet 2 } & \text { Q17 } \\ 0 & \text { No Credit } & \text { Booklet 3 } & \text { Q5 } \\ 1 & \text { Partial Credit } & \text { Booklet 7 } & \text { Q38 } \\ 2 & \text { Partial Credit } & \text { Booklet 13 } & \text { Q26 } \\ 3 & \text { Full Credit } & & \\ 9 & \text { Missing } & & \\ \text { n } & \text { N/A } & & \\ \text { r } & \text { Not reached } & & \end{array}$

\begin{tabular}{|c|c|c|}
\hline \multirow{2}{*}{$\begin{array}{l}\text { M144Q01T (11) CUBE PAINTING Q1 } \\
\text { FormatA1 Columns 28-28 }\end{array}$} & \multicolumn{2}{|c|}{ Coded Response } \\
\hline & & \\
\hline $0 \quad$ No Credit & Booklet 1 & Q33 \\
\hline 1 Full Credit & Booklet 3 & Q21 \\
\hline $9 \quad$ Missing & Booklet 4 & Q9 \\
\hline n $\quad \mathrm{N} / \mathrm{A}$ & Booklet 8 & Q59 \\
\hline r Not reached & & \\
\hline
\end{tabular}

\begin{tabular}{|c|c|c|c|}
\hline \multirow{2}{*}{\multicolumn{2}{|c|}{$\begin{array}{l}\text { M144Q02T (12) CUBE PAINTING Q2 } \\
\text { FormatA1 Columns 29-29 }\end{array}$}} & \multicolumn{2}{|c|}{ Coded Response } \\
\hline & & \multirow{2}{*}{\multicolumn{2}{|c|}{ Booklet $1 \quad$ Q34 }} \\
\hline 0 & No Credit & & \\
\hline 1 & Full Credit & \multirow{4}{*}{$\begin{array}{l}\text { Booklet } 3 \\
\text { Booklet } 4 \\
\text { Booklet } 8\end{array}$} & Q22 \\
\hline 8 & Invalid & & Q10 \\
\hline 9 & Missing & & \multirow[t]{2}{*}{ Q60 } \\
\hline $\mathrm{n}$ & $\mathrm{N} / \mathrm{A}$ & & \\
\hline $\mathrm{r}$ & Not reached & & \\
\hline
\end{tabular}

\begin{tabular}{|c|c|c|c|}
\hline \multirow{2}{*}{\multicolumn{2}{|c|}{$\begin{array}{l}\text { M144Q03 (13) CUBE PAINTING Q3 } \\
\text { FormatA1 Columns 30-30 }\end{array}$}} & \multicolumn{2}{|c|}{ Multiple Choice } \\
\hline & & & \\
\hline 1 & Full Credit & Booklet 1 & Q35 \\
\hline 2 & No Credit & Booklet 3 & Q23 \\
\hline 3 & No Credit & Booklet 4 & Q11 \\
\hline 4 & No Credit & Booklet 8 & Q61 \\
\hline 8 & $\mathrm{M} / \mathrm{R}$ & & \\
\hline 9 & Missing & & \\
\hline $\mathrm{n}$ & $\mathrm{N} / \mathrm{A}$ & & \\
\hline $\mathrm{r}$ & Not reached & & \\
\hline
\end{tabular}

\begin{tabular}{|c|c|c|c|}
\hline M144Q0 & 4T (14) CUBE PAINTING Q4 & Coded Res & \\
\hline FormatA1 & Columns 31-31 & & \\
\hline 0 & No Credit & Booklet 1 & Q36 \\
\hline 1 & Full Credit & Booklet 3 & Q24 \\
\hline 8 & Invalid & Booklet 4 & Q12 \\
\hline 9 & Missing & Booklet 8 & Q62 \\
\hline $\mathrm{n}$ & $\mathrm{N} / \mathrm{A}$ & & \\
\hline $\mathrm{r}$ & Not reached & & \\
\hline
\end{tabular}

\begin{tabular}{|c|c|c|c|}
\hline M145Q0 & $1 \mathrm{~T}$ (15) CUBES Q1 & Complex M & iple Choice \\
\hline FormatA1 & Columns 32-32 & & \\
\hline 0 & No Credit & Booklet 1 & Q13 \\
\hline 1 & No Credit & Booklet 2 & Q1 \\
\hline 2 & No Credit & Booklet 6 & Q43 \\
\hline 3 & No Credit & Booklet 12 & Q20 \\
\hline 4 & No Credit & & \\
\hline 5 & No Credit & & \\
\hline 6 & Full Credit & & \\
\hline 8 & Invalid & & \\
\hline 9 & Missing & & \\
\hline $\mathrm{n}$ & $\mathrm{N} / \mathrm{A}$ & & \\
\hline $\mathrm{r}$ & Not reached & & \\
\hline
\end{tabular}




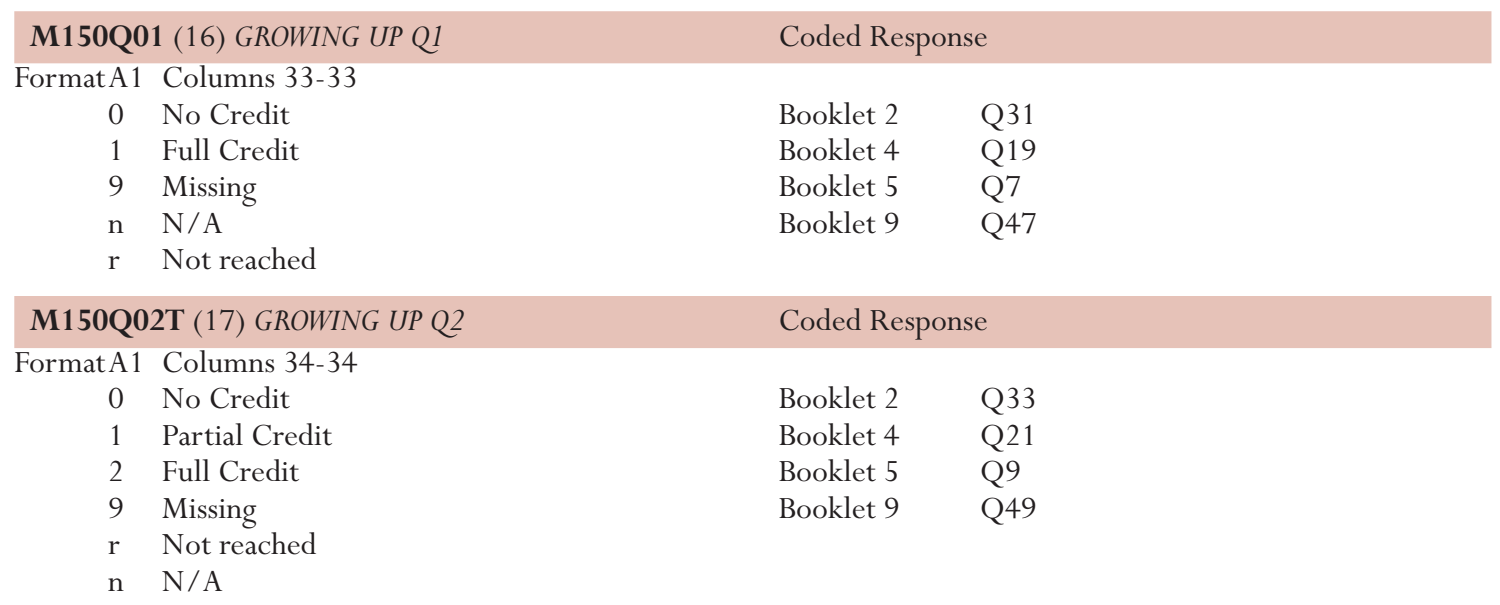

$\begin{array}{cllc}\text { M150Q03T (18) GROWING UP Q3 } & \text { Coded Response } \\ \text { FormatA1 } & \text { Columns 35-35 } & \text { Booklet 2 } & \text { Q32 } \\ 0 & \text { No Credit } & \text { Booklet 4 } & \text { Q20 } \\ 1 & \text { Full Credit } & \text { Booklet 5 } & \text { Q8 } \\ 9 & \text { Missing } & \text { Booklet 9 } & \text { Q48 } \\ \text { n } & \text { N/A } & & \\ \text { r } & \text { Not reached } & \end{array}$

\begin{tabular}{|c|c|c|}
\hline \multirow{2}{*}{$\begin{array}{l}\text { M155Q01 (19) POPULATION PYRAMIDS Q1 } \\
\text { FormatA1 Columns 36-36 }\end{array}$} & \multicolumn{2}{|c|}{ Coded Response } \\
\hline & & \\
\hline $0 \quad$ No Credit & Booklet 3 & Q29 \\
\hline 1 Full Credit & Booklet 5 & Q18 \\
\hline $9 \quad$ Missing & Booklet 6 & Q5 \\
\hline n $\quad \mathrm{N} / \mathrm{A}$ & Booklet 10 & Q42 \\
\hline r Not reached & & \\
\hline
\end{tabular}

\begin{tabular}{|c|c|c|}
\hline \multirow{2}{*}{$\begin{array}{l}\text { M155Q02T (20) POPULATION PYRAMIDS Q2 } \\
\text { FormatA1 Columns 37-37 }\end{array}$} & \multicolumn{2}{|c|}{ Coded Response } \\
\hline & & \\
\hline $0 \quad$ No Credit & Booklet 3 & Q28 \\
\hline 1 Partial Credit & Booklet 5 & Q17 \\
\hline 2 Full Credit & Booklet 6 & Q4 \\
\hline $9 \quad$ Missing & Booklet 10 & Q41 \\
\hline $\mathrm{n} \quad \mathrm{N} / \mathrm{A}$ & & \\
\hline r Not reached & & \\
\hline
\end{tabular}

\begin{tabular}{|c|c|c|}
\hline M155Q03T (21) POPULATION PYRAMIDS Q3 & \multicolumn{2}{|c|}{ Coded Response } \\
\hline \multicolumn{3}{|l|}{ FormatA1 Columns 38-38 } \\
\hline $0 \quad$ No Credit & Booklet 3 & Q30 \\
\hline 1 Partial Credit & Booklet 5 & Q19 \\
\hline 2 Full Credit & Booklet 6 & Q6 \\
\hline $9 \quad$ Missing & Booklet 10 & Q43 \\
\hline $\mathrm{n} \quad \mathrm{N} / \mathrm{A}$ & & \\
\hline r Not reached & & \\
\hline
\end{tabular}

\begin{tabular}{|c|c|c|c|}
\hline \multicolumn{2}{|c|}{ M155Q04T (22) POPULATION PYRAMIDS Q4 } & \multicolumn{2}{|c|}{ Complex Multiple Cho } \\
\hline FormatA1 & Columns 39-39 & & \\
\hline 0 & No Credit & Booklet 3 & Q31 \\
\hline 1 & No Credit & Booklet 5 & Q20 \\
\hline 2 & No Credit & Booklet 6 & Q7 \\
\hline 3 & No Credit & Booklet 10 & Q44 \\
\hline 4 & Full Credit & & \\
\hline 8 & Invalid & & \\
\hline 9 & Missing & & \\
\hline $\mathrm{n}$ & $\mathrm{N} / \mathrm{A}$ & & \\
\hline $\mathrm{r}$ & Not reached & & \\
\hline
\end{tabular}




\begin{tabular}{|c|c|c|}
\hline \multirow{2}{*}{$\begin{array}{l}\text { M179Q01T (23) ROBBERIES Q1 } \\
\text { FormatA1 Columns 40-40 }\end{array}$} & \multicolumn{2}{|c|}{ Coded Response } \\
\hline & & \\
\hline $0 \quad$ No Credit & Booklet 1 & $\mathrm{Q}^{9}$ \\
\hline Partial Credit & Booklet 5 & Q5 \\
\hline Full Credit & Booklet 11 & Q \\
\hline Missing & Booklet 13 & Q1 \\
\hline n $\quad \mathrm{N} / \mathrm{A}$ & & \\
\hline r Not reached & & \\
\hline
\end{tabular}

\begin{tabular}{clll} 
M192Q01T (24) CONTAINERS Q1 & \multicolumn{2}{c}{ Complex Multiple Ch } \\
Format A1 & Columns 41-41 & & \\
0 & No Credit & Booklet 1 & Q20 \\
1 & No Credit & Booklet 2 & Q8 \\
2 & Full Credit & Booklet 6 & Q50 \\
3 & Full Credit & Booklet 12 & Q27 \\
8 & Invalid & & \\
9 & Missing & & \\
$\mathrm{n}$ & N/A & & \\
$\mathrm{r}$ & Not reached & &
\end{tabular}

M266Q01T (25) CARPENTER Q1 Complex Multiple Choice

$\begin{array}{clll}\text { FormatA1 } & \text { Columns 42-42 } & & \\ 0 & \text { No Credit } & \text { Booklet 4 } & \text { Q31 } \\ 1 & \text { No Credit } & \text { Booklet 6 } & \text { Q18 } \\ 2 & \text { No Credit } & \text { Booklet 7 } & \text { Q6 } \\ 3 & \text { No Credit } & \text { Booklet 11 } & \text { Q43 } \\ 4 & \text { Full Credit } & & \\ 8 & \text { Invalid } & & \\ 9 & \text { Missing } & & \\ \mathrm{n} & \text { N/A } & & \\ \mathrm{r} & \text { Not reached } & & \end{array}$

\begin{tabular}{clll} 
M273Q01T (26) PIPELINES Q1 & \multicolumn{2}{l}{ Coded Response } \\
FormatA1 & Columns 43-43 & Booklet 4 & Q37 \\
0 & No Credit & Booklet 6 & Q24 \\
1 & Full Credit & Booklet 7 & Q12 \\
8 & Invalid & Booklet 11 & Q49 \\
9 & Missing & & \\
$\mathrm{n}$ & N/A & & \\
$\mathrm{r}$ & Not reached & &
\end{tabular}

\begin{tabular}{clll} 
M302Q01T (27) CAR DRIVE Q1 & \multicolumn{2}{l}{ Coded Response } \\
FormatA1 & Columns 44-44 & Booklet 4 & Q26 \\
0 & No Credit & Booklet 6 & Q13 \\
1 & Full Credit & Booklet 7 & Q1 \\
9 & Missing & Booklet 11 & Q38 \\
n & N/A & & \\
r & Not reached
\end{tabular}

$\begin{array}{clll}\text { M302Q02 (28) CAR DRIVE Q2 } & \text { Coded Response } \\ \text { FormatA1 } & \text { Columns 45-45 } & & \\ 0 & \text { No Credit } & \text { Booklet 4 } & \text { Q27 } \\ 1 & \text { Full Credit } & \text { Booklet 6 } & \text { Q14 } \\ 9 & \text { Missing } & \text { Booklet 7 } & \text { Q2 } \\ \text { n } & \text { N/A } & \text { Booklet 11 } & \text { Q39 } \\ \text { r } & \text { Not reached } & & \end{array}$




\begin{tabular}{clll} 
M302Q03 (29) CAR DRIVE Q3 & \multicolumn{2}{l}{ Coded Response } \\
FormatA1 & Columns 46-46 & Booklet 4 & Q28 \\
0 & No Credit & Booklet 6 & Q15 \\
1 & Full Credit & Booklet 7 & Q3 \\
9 & Missing & Booklet 11 & Q40 \\
n & N/A & & \\
r & Not reached & &
\end{tabular}

$\begin{array}{clll}\text { M305Q01 (30) MAP Q1 } & \text { Multiple Choice } \\ \text { FormatA1 } & \text { Columns 47-47 } & \text { Booklet 2 } & \text { Q13 } \\ 1 & \text { No Credit } & \text { Booklet 3 } & \text { Q1 } \\ 2 & \text { No Credit } & \text { Booklet 7 } & \text { Q34 } \\ 3 & \text { Full Credit } & \text { Booklet 13 } & \text { Q22 } \\ 4 & \text { No Credit } & & \\ 8 & \text { M/R } & & \\ 9 & \text { Missing } & & \\ \mathrm{n} & \text { N/A } & & \\ \mathrm{r} & \text { Not reached } & & \end{array}$

\begin{tabular}{clll} 
M402Q01 (31) INTERNET RELAY CHAT Q1 & \multicolumn{2}{l}{ Coded Response } \\
FormatA1 & Columns 48-48 & Booklet 1 & Q7 \\
0 & No Credit & Booklet 5 & Q49 \\
1 & Full Credit & Booklet 11 & Q31 \\
9 & Missing & Booklet 13 & Q16 \\
n & N/A & & \\
r & Not reached & &
\end{tabular}

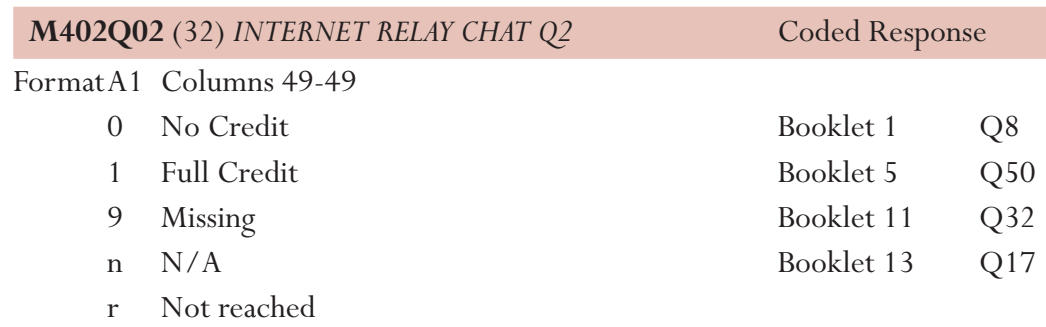

\begin{tabular}{clll} 
M406Q01 (33) RUNNING TRACKS Q1 & \multicolumn{2}{l}{ Coded Response } \\
FormatA1 & Columns 50-50 & Booklet 2 & Q28 \\
0 & No Credit & Booklet 4 & Q16 \\
1 & Full Credit & Booklet 5 & Q4 \\
9 & Missing & Booklet 9 & Q44 \\
n & N/A & & \\
r & Not reached & &
\end{tabular}

\begin{tabular}{clll} 
M406Q02 (34) RUNNING TRACKS Q2 & \multicolumn{2}{l}{ Coded Response } \\
$\begin{array}{clll}\text { FormatA1 } & \text { Columns 51-51 } & & \\
0 & \text { No Credit } & \text { Booklet 2 } & \text { Q29 } \\
1 & \text { Full Credit } & \text { Booklet 4 } & \text { Q17 } \\
9 & \text { Missing } & \text { Booklet 5 } & \text { Q5 } \\
\text { n } & \text { N/A } & \text { Booklet 9 } & \text { Q45 } \\
\text { r } & \text { Not reached } & & \end{array}$
\end{tabular}




\begin{tabular}{clll} 
M406Q03 (35) RUNNING TRACKS Q3 & \multicolumn{2}{l}{ Coded Response } \\
FormatA1 & Columns 52-52 & Booklet 2 & Q30 \\
0 & No Credit & Booklet 4 & Q18 \\
1 & Full Credit & Booklet 5 & Q6 \\
9 & Missing & Booklet 9 & Q46 \\
n & N/A & & \\
r & Not reached & &
\end{tabular}

M408Q01T (36) LOTTERIES Q1
$\begin{array}{clll}\text { FormatA1 } & \text { Columns 53-53 } & \text { Complex Multiple Ch } \\ 0 & \text { No Credit } & \text { Booklet 1 } & \text { Q14 } \\ 1 & \text { No Credit } & \text { Booklet 2 } & \text { Q2 } \\ 2 & \text { No Credit } & \text { Booklet 6 } & \text { Q44 } \\ 3 & \text { No Credit } & \text { Booklet 12 } & \text { Q21 } \\ 4 & \text { Full Credit } & & \\ 8 & \text { Invalid } & & \\ 9 & \text { Missing } & & \\ \mathrm{n} & \text { N/A } & & \\ \mathrm{r} & \text { Not reached } & & \end{array}$

\begin{tabular}{clll} 
M411Q01 (37) DIVING Q1 & \multicolumn{2}{l}{ Coded Response } \\
FormatA1 & Columns 54-54 & Booklet 2 & Q36 \\
0 & No Credit & Booklet 4 & Q24 \\
1 & Full Credit & Booklet 5 & Q12 \\
9 & Missing & Booklet 9 & Q52 \\
n & N/A & & \\
r & Not reached & &
\end{tabular}

\begin{tabular}{clll} 
M411Q02 (38) DIVING Q2 & \multicolumn{2}{c}{ Multiple Choice } \\
FormatA1 & Columns 55-55 & Booklet 2 & Q37 \\
1 & No Credit & Booklet 4 & Q25 \\
2 & No Credit & Booklet 5 & Q13 \\
3 & No Credit & Booklet 9 & Q53 \\
4 & Full Credit & & \\
8 & M/R & & \\
9 & Missing & & \\
n & N/A & & \\
r & Not reached & &
\end{tabular}

\begin{tabular}{clll} 
M413Q01 (39) EXCHANGE RATE Q1 & \multicolumn{2}{l}{ Coded Response } \\
FormatA1 & Columns 56-56 & Booklet 2 & Q25 \\
0 & No Credit & Booklet 4 & Q13 \\
1 & Full Credit & Booklet 5 & Q1 \\
9 & Missing & Booklet 9 & Q41 \\
n & N/A & Booklet UH & Q16 \\
r & Not reached & &
\end{tabular}

M413Q02 (40) EXCHANGE RATE Q2 Coded Response

FormatA1 Columns 57-57

0 No Credit

1 Full Credit

9 Missing

n N/A

r Not reached

Booklet $2 \quad$ Q26

Booklet $4 \quad$ Q14

Booklet $5 \quad$ Q2

Booklet $9 \quad$ Q42

Booklet UH Q17 


M413Q03T (41) EXCHANGE RATE Q3
\begin{tabular}{clll} 
FormatA1 & Columns 58-58 & \multicolumn{2}{l}{ Coded Response } \\
0 & No Credit & Booklet 2 & Q27 \\
1 & Full Credit & Booklet 4 & Q15 \\
9 & Missing & Booklet 5 & Q3 \\
n & N/A & Booklet 9 & Q43 \\
r & Not reached & Booklet UH & Q18
\end{tabular}

\begin{tabular}{|c|c|c|c|}
\hline \multicolumn{2}{|c|}{ M420Q01T (42) TRANSPORT Q1 } & \multicolumn{2}{|c|}{ Complex Multiple Ch } \\
\hline \multicolumn{4}{|c|}{ FormatA1 Columns 59-59 } \\
\hline 0 & No Credit & Booklet 3 & Q34 \\
\hline 1 & No Credit & Booklet 5 & Q23 \\
\hline 2 & No Credit & Booklet 6 & Q10 \\
\hline 3 & No Credit & Booklet 10 & Q47 \\
\hline 4 & Full Credit & & \\
\hline 8 & $\mathrm{M} / \mathrm{R}$ & & \\
\hline 9 & Missing & & \\
\hline $\mathrm{n}$ & $\mathrm{N} / \mathrm{A}$ & & \\
\hline $\mathrm{r}$ & Not reached & & \\
\hline
\end{tabular}

\begin{tabular}{clll} 
M421Q01 (43) HEIGHT Q1 & \multicolumn{2}{l}{ Coded Response } \\
$\begin{array}{clll}\text { Format A1 } & \text { Columns 60-60 } & \text { Booklet 1 } & \text { Q26 } \\
\text { 0 } & \text { No Credit } & \text { Booklet 3 } & \text { Q14 } \\
1 & \text { Full Credit } & \text { Booklet 4 } & \text { Q2 } \\
9 & \text { Missing } & \text { Booklet 8 } & \text { Q52 } \\
\text { n } & \text { N/A } & & \\
\text { r } & \text { Not reached } & & \end{array}$
\end{tabular}

M421Q02T (44) HEIGHT Q2 Complex Multiple Choice

FormatA1 Columns 61-61

$\begin{array}{llll}0 & \text { No Credit } & \text { Booklet 1 } & \text { Q27 } \\ 1 & \text { No Credit } & \text { Booklet 3 } & \text { Q15 } \\ 2 & \text { No Credit } & \text { Booklet 4 } & \text { Q3 } \\ 3 & \text { No Credit } & \text { Booklet 8 } & \text { Q53 }\end{array}$

4 Full Credit

$8 \mathrm{M} / \mathrm{R}$

9 Missing

n N/A

r Not reached

\begin{tabular}{clll} 
M421Q03 (45) HEIGHT Q3 & \multicolumn{2}{c}{ Multiple Choice } \\
FormatA1 & Columns 62-62 & Booklet 1 & Q28 \\
1 & No Credit & Booklet 3 & Q16 \\
2 & No Credit & Booklet 4 & Q4 \\
3 & No Credit & Booklet 8 & Q54 \\
4 & Full Credit & & \\
5 & No Credit & & \\
8 & M/R & & \\
9 & Missing & & \\
n & N/A & & \\
r & Not reached & &
\end{tabular}




\begin{tabular}{clll} 
M423Q01 (46) TOSSING COINS Q1 & \multicolumn{2}{l}{ Multiple Choice } \\
Format A1 & Columns 63-63 & Booklet 1 & Q23 \\
1 & Full Credit & Booklet 2 & Q11 \\
2 & No Credit & Booklet 6 & Q53 \\
3 & No Credit & Booklet 12 & Q30 \\
4 & No Credit & & \\
8 & M/R & & \\
9 & Missing & & \\
n & N/A & & \\
r & Not reached & &
\end{tabular}

\begin{tabular}{|c|c|c|}
\hline M438Q01 (47) EXPORTS Q1 & \multicolumn{2}{|c|}{ Coded Response } \\
\hline FormatA1 Columns 64-64 & & \\
\hline $0 \quad$ No Credit & Booklet 2 & Q21 \\
\hline 1 Full Credit & Booklet 3 & Q9 \\
\hline $9 \quad$ Missing & Booklet 7 & Q42 \\
\hline $\mathrm{n} \quad \mathrm{N} / \mathrm{A}$ & Booklet 13 & Q30 \\
\hline r Not reached & & \\
\hline
\end{tabular}

\begin{tabular}{clll} 
M438Q02 (48) EXPORTS Q2 & \multicolumn{2}{l}{ Multiple Choice } \\
FormatA1 & Columns 65-65 & & \\
1 & No Credit & Booklet 2 & Q22 \\
2 & No Credit & Booklet 3 & Q10 \\
3 & No Credit & Booklet 7 & Q43 \\
4 & No Credit & & \\
5 & Full Credit & & \\
8 & M/R & & \\
9 & Missing & & \\
$\mathrm{n}$ & N/A & & \\
$\mathrm{r}$ & Not reached & &
\end{tabular}

M442Q02 (49) BRAILLE Q2
\begin{tabular}{clll} 
FormatA1 & Columns 66-66 & \multicolumn{2}{l}{ Coded Response } \\
0 & No Credit & Booklet 3 & Q32 \\
1 & Full Credit & Booklet 5 & Q21 \\
9 & Missing & Booklet 6 & Q8 \\
n & N/A & Booklet 10 & Q45 \\
r & Not reached & &
\end{tabular}

M446Q01 (50) THERMOMETER CRICKET Q1
\begin{tabular}{clll} 
FormatA1 & Columns 67-67 & \multicolumn{2}{l}{ Coded Response } \\
0 & No Credit & Booklet 1 & Q18 \\
1 & Full Credit & Booklet 2 & Q6 \\
9 & Missing & Booklet 6 & Q48 \\
n & N/A & Booklet 12 & Q25 \\
r & Not reached & &
\end{tabular}

\section{M446Q02 (51) THERMOMETER CRICKET Q2 Coded Response}

FormatA1 Columns 68-68

0 No Credit

1 Full Credit

Booklet $1 \quad$ Q19

9 Missing

Booklet $2 \quad$ Q7

n $\quad \mathrm{N} / \mathrm{A}$

Booklet $6 \quad$ Q49

r Not reached

Booklet 12 Q26 


$\begin{array}{clll}\text { M447Q01 (52) TILE ARRANGEMENT Q1 } & \text { Multiple Choice } \\ \text { FormatA1 } & \text { Columns 69-69 } & \text { Booklet 3 } & \text { Q36 } \\ 1 & \text { No Credit } & \text { Booklet 5 } & \text { Q2 } \\ 2 & \text { No Credit } & \text { Booklet 6 } & \text { Q12 } \\ 3 & \text { No Credit } & \text { Booklet 10 } & \text { Q49 } \\ 4 & \text { Full Credit } & & \\ \text { r } & \text { Not reached } & & \\ 8 & \text { M/R } & \\ 9 & \text { Missing } & \\ \mathrm{n} & \text { N/A } & \\ \mathrm{r} & \text { Not reached } & \end{array}$

\begin{tabular}{clll} 
M462Q01T (53) THIRD SIDE Q1 & \multicolumn{2}{l}{ Coded Response } \\
FormatA1 Columns 70-70 & Booklet 2 & Q20 \\
0 & No Credit & Booklet 3 & Q8 \\
1 & Partial Credit & Booklet 7 & Q41 \\
2 & Full Credit & Booklet 13 & Q29 \\
9 & Missing & Booklet UH & Q13 \\
n & N/A & & \\
r & Not reached & &
\end{tabular}

\begin{tabular}{clll} 
M464Q01T (54) THE FENCE Q1 & \multicolumn{2}{l}{ Coded Response } \\
FormatA1 & Columns 71-71 & Booklet 1 & Q10 \\
0 & No Credit & Booklet 5 & Q52 \\
1 & Full Credit & Booklet 11 & Q34 \\
8 & Invalid & Booklet 13 & Q19 \\
9 & Missing & & \\
$\mathrm{n}$ & N/A & & \\
$\mathrm{r}$ & Not reached & &
\end{tabular}

\begin{tabular}{|c|c|c|c|}
\hline \multicolumn{2}{|c|}{ M467Q01 (55) COLOURED CANDIES Q1 } & \multicolumn{2}{|c|}{ Multiple Choice } \\
\hline \multicolumn{4}{|c|}{ FormatA1 Columns 72-72 } \\
\hline 1 & No Credit & Booklet 1 & Q2 \\
\hline 2 & Full Credit & Booklet 5 & Q44 \\
\hline 3 & No Credit & Booklet 11 & Q26 \\
\hline 4 & No Credit & Booklet 13 & Q11 \\
\hline 8 & $\mathrm{M} / \mathrm{R}$ & & \\
\hline 9 & Missing & & \\
\hline $\mathrm{n}$ & $\mathrm{N} / \mathrm{A}$ & & \\
\hline $\mathrm{r}$ & Not reached & & \\
\hline
\end{tabular}

M468Q01T (56) SCIENCE TESTS Q1_Coded Response

$\begin{array}{clll}\text { Format A1 } & \text { Columns 73-73 } & & \\ 0 & \text { No Credit } & \text { Booklet 3 } & \text { Q35 } \\ 1 & \text { Full Credit } & \text { Booklet 5 } & \text { Q24 } \\ 8 & \text { Invalid } & \text { Booklet 6 } & \text { Q11 } \\ 9 & \text { Missing } & \text { Booklet 10 } & \text { Q48 } \\ \mathrm{n} & \text { N/A } & & \\ \mathrm{r} & \text { Not reached } & & \end{array}$

$\begin{array}{clll}\text { M474Q01 (57) RUNNING TIME Q1 } & \text { Coded Response } \\ \text { FormatA1 } & \text { Columns 74-74 } & & \\ 0 & \text { No Credit } & \text { Booklet 2 } & \text { Q15 } \\ 1 & \text { Full Credit } & \text { Booklet 3 } & \text { Q3 } \\ 9 & \text { Missing } & \text { Booklet 7 } & \text { Q36 } \\ \text { n } & \text { N/A } & \text { Booklet 13 } & \text { Q24 } \\ \text { r } & \text { Not reached } & & \end{array}$




\begin{tabular}{|c|c|c|c|}
\hline M484Q0 & $1 \mathrm{~T}$ (58) BOOKSHELVES Q1 & Coded Res & \\
\hline Format A 1 & Columns 75-75 & & \\
\hline 0 & No Credit & Booklet 3 & Q27 \\
\hline 1 & Full Credit & Booklet 5 & Q16 \\
\hline 8 & Invalid & Booklet 6 & Q3 \\
\hline 9 & Missing & Booklet 10 & Q40 \\
\hline $\mathrm{n}$ & $\mathrm{N} / \mathrm{A}$ & & \\
\hline $\mathrm{r}$ & Not reached & & \\
\hline
\end{tabular}

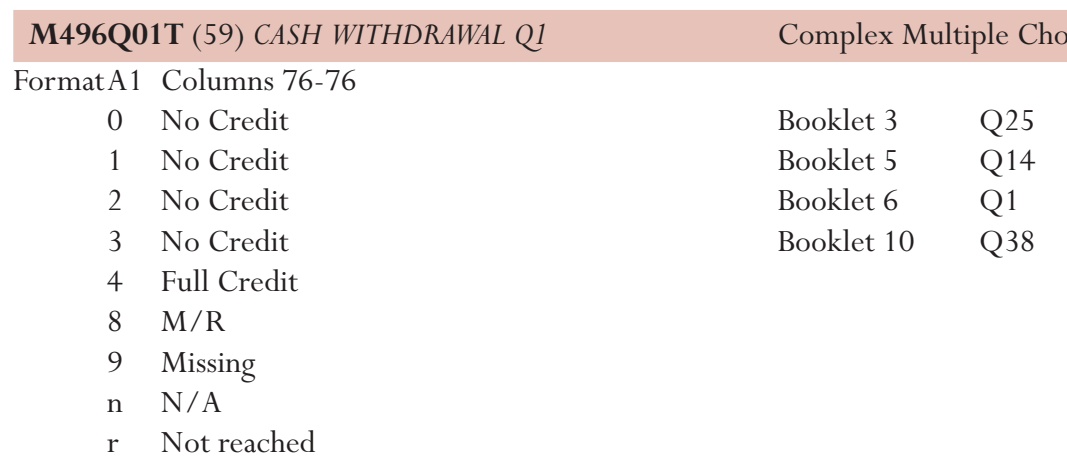

\begin{tabular}{clll} 
M496Q02 (60) CASH WITHDRAWAL Q2 & \multicolumn{2}{l}{ Coded Response } \\
FormatA1 & Columns 77-77 & Booklet 3 & Q26 \\
0 & No Credit & Booklet 5 & Q15 \\
1 & Full Credit & Booklet 6 & Q2 \\
9 & Missing & Booklet 10 & Q39 \\
n & N/A & & \\
r & Not reached & &
\end{tabular}

\begin{tabular}{clll} 
M505Q01 (61) LITTER Q1 & \multicolumn{2}{l}{ Coded Response } \\
FormatA1 & Columns 78-78 & Booklet 2 & Q19 \\
0 & No Credit & Booklet 3 & Q7 \\
1 & Full Credit & Booklet 7 & Q40 \\
9 & Missing & Booklet 13 & Q28 \\
n & N/A & & \\
r & Not reached & &
\end{tabular}

\begin{tabular}{clll} 
M509Q01 (62) EARTHQUAKE Q1 & \multicolumn{2}{l}{ Multiple Choice } \\
FormatA1 & Columns 79-79 & & \\
1 & No Credit & Booklet 3 & Q33 \\
2 & No Credit & Booklet 5 & Q22 \\
3 & Full Credit & Booklet 6 & Q9 \\
4 & No Credit & Booklet 10 & Q46 \\
8 & M/R & & \\
9 & Missing & & \\
$\mathrm{n}$ & N/A & & \\
$\mathrm{r}$ & Not reached & &
\end{tabular}

\begin{tabular}{clll} 
M510Q01T (63) CHOICES Q1 & \multicolumn{2}{c}{ Coded Response } \\
FormatA1 & Columns 80-80 & Booklet 2 & Q14 \\
0 & No Credit & Booklet 3 & Q2 \\
1 & Full Credit & Booklet 7 & Q35 \\
8 & Invalid & Booklet 13 & Q23 \\
9 & Missing & & \\
n & N/A & & \\
r & Not reached & &
\end{tabular}




\begin{tabular}{clll} 
M513Q01 (64) TEST SCORES Q1 & \multicolumn{2}{l}{ Coded Response } \\
FormatA1 & Columns 81-81 & Booklet 4 & Q32 \\
0 & No Credit & Booklet 6 & Q19 \\
1 & Full Credit & Booklet 7 & Q7 \\
9 & Missing & Booklet 11 & Q44 \\
n & N/A & & \\
r & Not reached & &
\end{tabular}

M520Q01T (65) SKATEBOARD Q1_ Coded Response

FormatA1 Columns 82-82
0 No Credit
1 Partial Credit
2 Full Credit
8 Invalid
9 Missing
n $\mathrm{N} / \mathrm{A}$
r Not reached

Booklet $1 \quad$ Q15

Booklet 2 Q3

Booklet $6 \quad$ Q45

Booklet 12

Q22

M520Q02 (66) SKATEBOARD Q2
$\begin{array}{rlll}\text { Format A1 } & \text { Columns 83-83 } & \text { Multiple Choice } \\ 1 & \text { No Credit } & \text { Booklet 1 } & \text { Q16 } \\ 2 & \text { No Credit } & \text { Booklet 2 } & \text { Q4 } \\ 3 & \text { No Credit } & \text { Booklet 6 } & \text { Q46 } \\ 4 & \text { Full Credit } & \text { Booklet 12 } & \text { Q23 } \\ 8 & \text { M/R } & & \\ 9 & \text { Missing } & & \\ \text { n } & \text { N/A } & & \\ \text { r } & \text { Not reached } & & \end{array}$

M520Q03T (67) SKATEBOARD Q3 Coded Response

FormatA1 Columns 84-84
0 No Credit
Booklet $1 \quad$ Q17
1 No Credit
Booklet 2 Q5
2 No Credit
Booklet $6 \quad$ Q47
3 No Credit
Booklet $12 \quad$ Q24
4 Full Credit
$8 \mathrm{M} / \mathrm{R}$
9 Missing
n N/A
r Not reached

\section{M547Q01T (68) STAIRCASE Q1_ Coded Response}

FormatA1 Columns 85-85

$\begin{array}{llll}0 & \text { No Credit } & \text { Booklet 2 } & \text { Q23 } \\ 1 & \text { Full Credit } & \text { Booklet 3 } & \text { Q11 } \\ 8 & \text { Invalid } & \text { Booklet 7 } & \text { Q44 } \\ 9 & \text { Missing } & \text { Booklet 13 } & \text { Q32 } \\ \text { n } & \text { N/A } & & \\ \text { r } & \text { Not reached } & & \end{array}$




\begin{tabular}{clll} 
M555Q02T (69) NUMBER CUBES Q2 & \multicolumn{2}{c}{ Complex Multiple Cho } \\
FormatA1 & Columns 86-86 & & \\
0 & No Credit & Booklet 1 & Q24 \\
1 & No Credit & Booklet 2 & Q12 \\
2 & No Credit & Booklet 6 & Q54 \\
3 & No Credit & Booklet 12 & Q31 \\
4 & Full Credit & & \\
8 & M/R & & \\
9 & Missing & & \\
n & N/A & & \\
r & Not reached & &
\end{tabular}

\begin{tabular}{clll} 
M559Q01 (70) TELEPHONE RATES Q1 & \multicolumn{2}{c}{ Multiple Choice } \\
FormatA1 & Columns 87-87 & Booklet 1 & Q32 \\
1 & No Credit & Booklet 3 & Q20 \\
2 & No Credit & Booklet 4 & Q8 \\
3 & No Credit & Booklet 8 & Q58 \\
4 & Full Credit & & \\
8 & M/R & & \\
9 & Missing & & \\
$\mathrm{n}$ & N/A & & \\
$\mathrm{r}$ & Not reached & &
\end{tabular}

\begin{tabular}{clll} 
M564Q01 (71) CHAIR LIFT Q1 & \multicolumn{2}{l}{ Multiple Choice } \\
FormatA1 & Columns 88-88 & Booklet 1 & Q11 \\
1 & No Credit & Booklet 5 & Q53 \\
2 & Full Credit & Booklet 11 & Q35 \\
3 & No Credit & Booklet 13 & Q20 \\
4 & No Credit & Booklet UH & Q14 \\
8 & M/R & & \\
9 & Missing & & \\
n & N/A & & \\
r & Not reached & &
\end{tabular}

\begin{tabular}{clll} 
M564Q02 (72) CHAIR LIFT Q2 & \multicolumn{2}{l}{ Multiple Choice } \\
Format A1 & Columns 89-89 & Booklet 1 & Q12 \\
1 & No Credit & Booklet 5 & Q54 \\
2 & No Credit & Booklet 11 & Q36 \\
3 & Full Credit & Booklet 13 & Q21 \\
4 & No Credit & Booklet UH & Q15 \\
5 & No Credit & & \\
8 & M/R & & \\
9 & Missing & & \\
n & N/A & & \\
r & Not reached & &
\end{tabular}

\begin{tabular}{clll} 
M571Q01 (73) STOP THE CAR Q1 & \multicolumn{2}{l}{ Multiple Choice } \\
FormatA1 & Columns 90-90 & Booklet 1 & Q31 \\
1 & No Credit & Booklet 3 & Q19 \\
2 & No Credit & Booklet 4 & Q7 \\
3 & No Credit & Booklet 8 & Q57 \\
4 & Full Credit & & \\
8 & M/R & & \\
9 & Missing & & \\
$\mathrm{n}$ & N/A & & \\
$\mathrm{r}$ & Not reached & &
\end{tabular}




\begin{tabular}{clll} 
M598Q01 (74) MAKING A BOOKLET Q1 & \multicolumn{2}{l}{ Coded Response } \\
$\begin{array}{clll}\text { FormatA1 } & \text { Columns 91-91 } & & \\
0 & \text { No Credit } & \text { Booklet 2 } & \text { Q34 } \\
1 & \text { Full Credit } & \text { Booklet 4 } & \text { Q22 } \\
9 & \text { Missing } & \text { Booklet 5 } & \text { Q10 } \\
\text { n } & \text { N/A } & \text { Booklet 9 } & \text { Q50 } \\
\text { r } & \text { Not reached } & & \end{array}$
\end{tabular}

\begin{tabular}{|c|c|c|c|}
\hline \multicolumn{2}{|c|}{ M603Q01T (75) NUMBER CHECK Q1 } & \multicolumn{2}{|c|}{ Complex Multiple Cl } \\
\hline Format A1 & Columns 92-92 & & \\
\hline 0 & No Credit & Booklet 4 & Q29 \\
\hline 1 & No Credit & Booklet 6 & Q16 \\
\hline 2 & No Credit & Booklet 7 & Q4 \\
\hline 3 & Full Credit & Booklet 11 & Q41 \\
\hline 8 & $\mathrm{M} / \mathrm{R}$ & & \\
\hline 9 & Missing & & \\
\hline $\mathrm{n}$ & $\mathrm{N} / \mathrm{A}$ & & \\
\hline $\mathrm{r}$ & Not reached & & \\
\hline
\end{tabular}

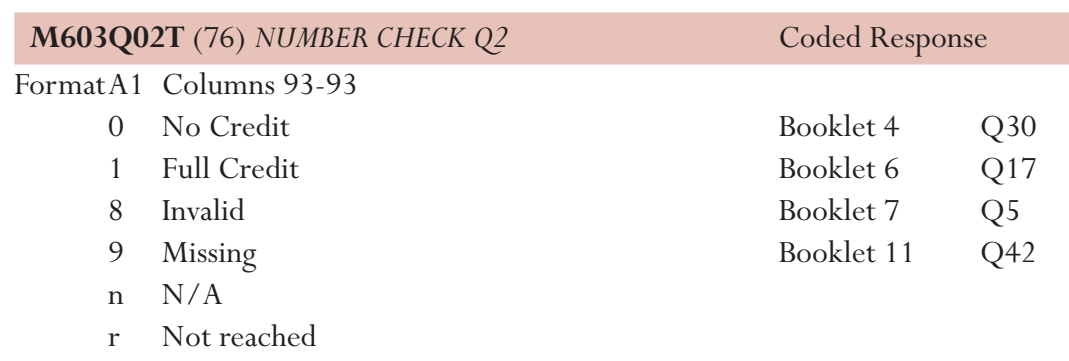

\section{M702Q01 (77) SUPPORT FOR PRESIDENT Q1_ Coded Response}

FormatA1 Columns 94-94

$\begin{array}{llll}0 & \text { No Credit } & \text { Booklet 1 } & \text { Q21 } \\ 1 & \text { No Credit } & \text { Booklet 2 } & \text { Q9 } \\ 2 & \text { Full Credit } & \text { Booklet 6 } & \text { Q51 } \\ 9 & \text { Missing } & \text { Booklet 12 } & \text { Q28 } \\ \text { n } & \text { N/A } & & \\ \text { r } & \text { Not reached } & & \end{array}$

M704Q01T (78) THE BEST CAR Q1 Coded Response

$\begin{array}{clll}\text { Format A1 } & \text { Columns 95-95 } & & \\ 0 & \text { No Credit } & \text { Booklet 1 } & \text { Q29 } \\ 1 & \text { Full Credit } & \text { Booklet 3 } & \text { Q17 } \\ 8 & \text { Invalid } & \text { Booklet 4 } & \text { Q5 } \\ 9 & \text { Missing } & \text { Booklet 8 } & \text { Q55 } \\ \text { n } & \text { N/A } & & \\ \mathrm{r} & \text { Not reached } & & \end{array}$

M704Q02T (79) THE BEST CAR Q2 Coded Response

FormatA1 Columns 96-96

0 No Credit

1 Full Credit

8 Invalid

9 Missing

n N/A

r Not reached

$\begin{array}{ll}\text { Booklet 1 } & \text { Q30 } \\ \text { Booklet 3 } & \text { Q18 } \\ \text { Booklet 4 } & \text { Q6 } \\ \text { Booklet 8 } & \text { Q56 }\end{array}$

Booklet $1 \quad$ Q30

Booklet $4 \quad$ Q6

Booklet 8 - 


\begin{tabular}{clll} 
M710Q01 (80) FORECAST OF RAIN Q1 & \multicolumn{2}{l}{ Multiple Choice } \\
FormatA1 & Columns 97-97 & Booklet 2 & Q35 \\
1 & No Credit & Booklet 4 & Q23 \\
2 & No Credit & Booklet 5 & Q11 \\
3 & No Credit & Booklet 9 & Q51 \\
4 & Full Credit & & \\
5 & No Credit & & \\
8 & M/R & & \\
9 & Missing & & \\
$\mathrm{n}$ & N/A & & \\
$\mathrm{r}$ & Not reached & &
\end{tabular}

$\begin{array}{clll}\text { M800Q01 } & \text { (81) COMPUTER GAME Q1 } & \text { Multiple Choice } \\ \text { FormatA1 } & \text { Columns 98-98 } & \text { Booklet 1 } & \text { Q25 } \\ 1 & \text { No Credit } & \text { Booklet 3 } & \text { Q13 } \\ 2 & \text { No Credit } & \text { Booklet 4 } & \text { Q1 } \\ 3 & \text { Full Credit } & \text { Booklet 8 } & \text { Q51 } \\ 4 & \text { No Credit } & \text { Booklet UH } & \text { Q12 } \\ 8 & \text { M/R } & & \\ 9 & \text { Missing } & & \\ \text { n } & \text { N/A } & & \\ \text { r } & \text { Not reached } & & \end{array}$

\begin{tabular}{|c|c|c|}
\hline M803Q01T (82) LABELS Q1 & \multicolumn{2}{|c|}{ Coded Response } \\
\hline FormatA1 Columns 99-99 & & \\
\hline $0 \quad$ No Credit & Booklet 4 & Q36 \\
\hline 1 Full Credit & Booklet 6 & Q23 \\
\hline $8 \quad$ Invalid & Booklet 7 & Q11 \\
\hline $9 \quad$ Missing & Booklet 11 & $\mathrm{Q} 48$ \\
\hline $\mathrm{n} \quad \mathrm{N} / \mathrm{A}$ & & \\
\hline r Not reached & & \\
\hline
\end{tabular}

M806Q01T (83) STEP PATTERN Q1_ Coded Response

FormatA1 Columns 100-100

$\begin{array}{llll}0 & \text { No Credit } & \text { Booklet 2 } & \text { Q24 } \\ 1 & \text { Full Credit } & \text { Booklet 3 } & \text { Q12 } \\ 8 & \text { Invalid } & \text { Booklet 7 } & \text { Q45 } \\ 9 & \text { Missing } & \text { Booklet 13 } & \text { Q33 }\end{array}$

n $\mathrm{N} / \mathrm{A}$

r Not reached

\begin{tabular}{clll} 
M810Q01T (84) BICYCLES Q1 & \multicolumn{2}{l}{ Coded Response } \\
Format A1 & Columns 101-101 & Booklet 1 & Q3 \\
0 & No Credit & Booklet 5 & Q45 \\
1 & Full Credit & Booklet 11 & Q27 \\
8 & Invalid & Booklet 13 & Q12 \\
9 & Missing & & \\
$\mathrm{n}$ & N/A & & \\
$\mathrm{r}$ & Not reached & &
\end{tabular}

\begin{tabular}{clll} 
M810Q02T (85) BICYCLES Q2 & \multicolumn{2}{l}{ Coded Response } \\
FormatA1 & Columns 102-102 & Booklet 1 & Q4 \\
0 & No Credit & Booklet 5 & Q46 \\
1 & Full Credit & Booklet 11 & Q28 \\
8 & Invalid & Booklet 13 & Q13 \\
9 & Missing & & \\
$\mathrm{n}$ & N/A & & \\
$\mathrm{r}$ & Not reached & &
\end{tabular}




\begin{tabular}{clll} 
M810Q03T (86) BICYCLES Q3 & \multicolumn{2}{l}{ Coded Response } \\
FormatA1 & Columns 103-103 & Booklet 1 & Q5 \\
0 & No Credit & Booklet 5 & Q47 \\
1 & Partial Credit & Booklet 11 & Q29 \\
2 & Full Credit & Booklet 13 & Q14 \\
9 & Missing & & \\
r & Not reached & & \\
$\mathrm{n}$ & N/A & &
\end{tabular}

\begin{tabular}{clll} 
M828Q01 (87) CARBON DIOXIDE Q1 & \multicolumn{2}{l}{ Coded Response } \\
FormatA1 & Columns 104-104 & & \\
0 & No Credit & Booklet 4 & Q33 \\
1 & Full Credit & Booklet 6 & Q20 \\
9 & Missing & Booklet 7 & Q8 \\
n & N/A & Booklet 11 & Q45 \\
r & Not reached & &
\end{tabular}

\begin{tabular}{clll} 
M828Q02 (88) CARBON DIOXIDE Q2 & \multicolumn{2}{l}{ Coded Response } \\
FormatA1 & Columns 105-105 & & \\
0 & No Credit & Booklet 4 & Q34 \\
1 & Full Credit & Booklet 6 & Q21 \\
9 & Missing & Booklet 7 & Q9 \\
n & N/A & Booklet 11 & Q46 \\
r & Not reached & &
\end{tabular}

\begin{tabular}{clll} 
M828Q03 (89) CARBON DIOXIDE Q3 & \multicolumn{2}{l}{ Coded Response } \\
FormatA1 & Columns 106-106 & Booklet 4 & Q35 \\
0 & No Credit & Booklet 6 & Q22 \\
1 & Full Credit & Booklet 7 & Q10 \\
9 & Missing & Booklet 11 & Q47 \\
n & N/A & & \\
r & Not reached & &
\end{tabular}

\begin{tabular}{clll} 
M833Q01T (90) SEEINGTHE TOWER Q1 & \multicolumn{2}{c}{ Complex Multiple Cho } \\
FormatA1 & Columns 107-107 & Booklet 1 & Q6 \\
0 & No Credit & Booklet 5 & Q48 \\
1 & No Credit & Booklet 11 & Q30 \\
2 & No Credit & Booklet 13 & Q15 \\
3 & No Credit & & \\
4 & No Credit & & \\
5 & Full Credit & & \\
8 & M/R & & \\
9 & Missing & & \\
$\mathrm{n}$ & N/A & &
\end{tabular}

\begin{tabular}{|c|c|c|c|}
\hline \multicolumn{2}{|c|}{ R055Q01 (91) DRUGGED SPIDERS Q1 } & \multicolumn{2}{|c|}{ Multiple Choice } \\
\hline \multicolumn{4}{|c|}{ FormatA1 Columns 108-108 } \\
\hline 1 & No Credit & Booklet 2 & Q46 \\
\hline 2 & No Credit & Booklet 8 & Q44 \\
\hline 3 & No Credit & Booklet 10 & Q22 \\
\hline 4 & Full Credit & Booklet 11 & Q9 \\
\hline 8 & $\mathrm{M} / \mathrm{R}$ & Booklet UH & Q8 \\
\hline 9 & Missing & & \\
\hline $\mathrm{n}$ & $\mathrm{N} / \mathrm{A}$ & & \\
\hline $\mathrm{r}$ & Not reached & & \\
\hline
\end{tabular}




$\begin{array}{clll}\text { R055Q02 } & \text { (92) DRUGGED SPIDERS Q2 } & \text { Coded Response } \\ \text { FormatA1 } & \text { Columns 109-109 } & & \\ 0 & \text { No Credit } & \text { Booklet 2 } & \text { Q47 } \\ 1 & \text { Full Credit } & \text { Booklet 8 } & \text { Q45 } \\ 9 & \text { Missing } & \text { Booklet 10 } & \text { Q23 } \\ \text { n } & \text { N/A } & \text { Booklet 11 } & \text { Q10 } \\ \text { r } & \text { Not reached } & \text { Booklet UH } & \text { Q9 }\end{array}$

$\begin{array}{clll}\text { R055Q03 } & \text { (93) DRUGGED SPIDERS Q3 } & \text { Coded Response } \\ \text { Format A1 } & \text { Columns 110-110 } & & \\ 0 & \text { No Credit } & \text { Booklet 2 } & \text { Q48 } \\ 1 & \text { No Credit } & \text { Booklet 8 } & \text { Q46 } \\ 2 & \text { Full Credit } & \text { Booklet 10 } & \text { Q24 } \\ 9 & \text { Missing } & \text { Booklet 11 } & \text { Q11 } \\ \text { n } & \text { N/A } & \text { Booklet UH } & \text { Q10 } \\ \text { r } & \text { Not reached } & & \end{array}$

R055Q05 (94) DRUGGED SPIDERS Q5 Coded Response

FormatA1 Columns 111-111

$\begin{array}{llll}0 & \text { No Credit } & \text { Booklet 2 } & \text { Q49 } \\ 1 & \text { Full Credit } & \text { Booklet 8 } & \text { Q47 } \\ 9 & \text { Missing } & \text { Booklet 10 } & \text { Q25 } \\ \text { n } & \text { N/A } & \text { Booklet 11 } & \text { Q12 } \\ \text { r } & \text { Not reached } & \text { Booklet UH } & \text { Q11 }\end{array}$

\begin{tabular}{clll} 
R067Q01 & (95) AESOP Q1 & \multicolumn{2}{l}{ Multiple Choice } \\
FormatA1 & Columns 112-112 & Booklet 1 & Q39 \\
1 & No Credit & Booklet 7 & Q32 \\
2 & No Credit & Booklet 9 & Q21 \\
3 & Full Credit & Booklet 10 & Q3 \\
4 & No Credit & & \\
8 & M/R & & \\
9 & Missing & & \\
$\mathrm{n}$ & N/A & & \\
$\mathrm{r}$ & Not reached & &
\end{tabular}

\begin{tabular}{clll} 
R067Q04 & (96) AESOP Q4 & \multicolumn{2}{l}{ Coded Response } \\
FormatA1 & Columns 113-113 & Booklet 1 & Q40 \\
0 & No Credit & Booklet 7 & Q33 \\
1 & Partial Credit & Booklet 9 & Q22 \\
2 & Full Credit & Booklet 10 & Q4 \\
9 & Missing & & \\
n & N/A & & \\
r & Not reached & &
\end{tabular}

\begin{tabular}{clll} 
R067Q05 & (97) AESOP Q5 & \multicolumn{2}{l}{ Coded Response } \\
FormatA1 & Columns 114-114 & Booklet 1 & Q41 \\
0 & No Credit & Booklet 7 & Q34 \\
1 & Partial Credit & Booklet 9 & Q23 \\
2 & Full Credit & Booklet 10 & Q5 \\
9 & Missing & & \\
n & N/A & & \\
r & Not reached & &
\end{tabular}




\begin{tabular}{clll} 
R102Q04A (98) SHIRTS Q4A & \multicolumn{2}{l}{ Coded Response } \\
$\begin{array}{clll}\text { FormatA1 } & \text { Columns 115-115 } & & \\
0 & \text { No Credit } & \text { Booklet 1 } & \text { Q42 } \\
1 & \text { Full Credit } & \text { Booklet 7 } & \text { Q35 } \\
9 & \text { Missing } & \text { Booklet 9 } & \text { Q24 } \\
\text { n } & \text { N/A } & \text { Booklet 10 } & \text { Q6 } \\
\text { r } & \text { Not reached } & & \end{array}$
\end{tabular}

\begin{tabular}{|c|c|c|c|}
\hline \multicolumn{2}{|c|}{ R102Q05 (99) SHIRTS Q5 } & \multicolumn{2}{|c|}{ Coded Response } \\
\hline FormatA1 & Columns1 16-116 & & \\
\hline 0 & No Credit & Booklet 1 & Q43 \\
\hline 1 & Full Credit & Booklet 7 & Q36 \\
\hline 9 & Missing & Booklet 9 & Q25 \\
\hline $\mathrm{n}$ & $\mathrm{N} / \mathrm{A}$ & Booklet 10 & Q7 \\
\hline $\mathrm{r}$ & Not reached & & \\
\hline
\end{tabular}

$\begin{array}{clll}\text { R102Q07 } & \text { (100) SHIRTS Q7 } & \text { Multiple Choice } \\ \text { FormatA1 } & \text { Columns 117-117 } & & \\ 1 & \text { No Credit } & \text { Booklet 1 } & \text { Q44 } \\ 2 & \text { No Credit } & \text { Booklet 7 } & \text { Q37 } \\ 3 & \text { Full Credit } & \text { Booklet 9 } & \text { Q26 } \\ 4 & \text { No Credit } & \text { Booklet 10 } & \text { Q8 } \\ 8 & \text { M/R } & & \\ 9 & \text { Missing } & & \\ \mathrm{n} & \text { N/A } & & \\ \mathrm{r} & \text { Not reached } & & \end{array}$

\begin{tabular}{clll} 
R104Q01 (101) TELEPHONE Q1 & \multicolumn{2}{l}{ Coded Response } \\
FormatA1 & Columns 118-118 & Booklet 2 & Q50 \\
0 & No Credit & Booklet 8 & Q48 \\
1 & Full Credit & Booklet 10 & Q26 \\
9 & Missing & Booklet 11 & Q13 \\
$\mathrm{n}$ & N/A & & \\
$\mathrm{r}$ & Not reached & &
\end{tabular}

\begin{tabular}{clll} 
R104Q02 (102) TELEPHONE Q2 & \multicolumn{2}{l}{ Coded Response } \\
FormatA1 & Columns 119-119 & Booklet 2 & Q51 \\
0 & No Credit & Booklet 8 & Q49 \\
1 & Full Credit & Booklet 10 & Q27 \\
9 & Missing & Booklet 11 & Q14 \\
n & N/A & & \\
r & Not reached & &
\end{tabular}

R104Q05 (103) TELEPHONE Q5 Coded Response

FormatA1 Columns 120-120

$\begin{array}{llll}0 & \text { No Credit } & \text { Booklet 2 } & \text { Q52 } \\ 1 & \text { Partial Credit } & \text { Booklet 8 } & \text { Q50 } \\ 2 & \text { Full Credit } & \text { Booklet 10 } & \text { Q28 } \\ 9 & \text { Missing } & \text { Booklet 11 } & \text { Q15 }\end{array}$

n $\mathrm{N} / \mathrm{A}$

r Not reached 


R111Q01
$\begin{array}{clll}\text { FormatA1 } 104) \text { EXCHANGE Q1 } & \text { Columns 121-121 } & \text { Multiple Choice } \\ 1 & \text { No Credit } & \text { Booklet 2 } & \text { Q42 } \\ 2 & \text { No Credit } & \text { Booklet 8 } & \text { Q40 } \\ 3 & \text { No Credit } & \text { Booklet 10 } & \text { Q18 } \\ 4 & \text { Full Credit } & \text { Booklet 11 } & \text { Q5 } \\ 8 & \text { M/R } & & \\ 9 & \text { Missing } & & \\ \mathrm{n} & \text { N/A } & & \\ \mathrm{r} & \text { Not reached } & & \end{array}$

\begin{tabular}{clll} 
R111Q02B (105) EXCHANGE Q2B & \multicolumn{2}{l}{ Coded Response } \\
$\begin{array}{clll}\text { FormatA1 } & \text { Columns 122-122 } & & \\
0 & \text { No Credit } & \text { Booklet 2 } & \text { Q43 } \\
1 & \text { Partial Credit } & \text { Booklet 8 } & \text { Q41 } \\
2 & \text { Full Credit } & \text { Booklet 10 } & \text { Q19 } \\
9 & \text { Missing } & \text { Booklet 11 } & \text { Q6 } \\
\text { n } & \text { N/A } & & \\
\text { r } & \text { Not reached } & & \end{array}$
\end{tabular}

\begin{tabular}{|c|c|c|c|}
\hline R111Q06 & B (106) EXCHANGE Q6B & Coded Resp & \\
\hline FormatA1 & Columns 123-123 & & \\
\hline 0 & No Credit & Booklet 2 & Q45 \\
\hline 1 & Partial Credit & Booklet 8 & Q43 \\
\hline 2 & Full Credit & Booklet 10 & Q21 \\
\hline 9 & Missing & Booklet 11 & Q8 \\
\hline $\mathrm{n}$ & $\mathrm{N} / \mathrm{A}$ & & \\
\hline $\mathrm{r}$ & Not reached & & \\
\hline
\end{tabular}

R219Q01T (107) EMPLOYMENT Q1 Coded Response

$\begin{array}{clll}\text { FormatA1 } & \text { Columns 124-124 } & & \\ 0 & \text { No Credit } & \text { Booklet 1 } & \text { Q37 } \\ 1 & \text { No Credit } & \text { Booklet 7 } & \text { Q30 } \\ 2 & \text { No Credit } & \text { Booklet 9 } & \text { Q19 } \\ 3 & \text { No Credit } & \text { Booklet 10 } & \text { Q1 } \\ 4 & \text { Full Credit } & \text { Booklet UH } & \text { Q6 } \\ 8 & \text { M/R } & & \\ 9 & \text { Missing } & & \\ \mathrm{n} & \text { N/A } & & \\ \mathrm{r} & \text { Not reached } & & \end{array}$

R219Q01E (108) EMPLOYMENT Q1E_Coded Response

$\begin{array}{clll}\text { FormatA1 } & \text { Columns 125-125 } & & \\ 0 & \text { No Credit } & \text { Booklet 1 } & \text { Q37 } \\ 1 & \text { Full Credit } & \text { Booklet 7 } & \text { Q30 } \\ 9 & \text { Missing } & \text { Booklet 9 } & \text { Q19 } \\ \text { n } & \text { N/A } & \text { Booklet 10 } & \text { Q1 } \\ \text { r } & \text { Not reached } & \text { Booklet UH } & \text { Q6 }\end{array}$

\begin{tabular}{clll} 
R219Q02 (109) EMPLOYMENT Q2 & \multicolumn{2}{l}{ Coded Response } \\
FormatA1 & Columns 126-126 & & \\
0 & No Credit & Booklet 1 & Q38 \\
1 & Full Credit & Booklet 7 & Q31 \\
9 & Missing & Booklet 9 & Q20 \\
n & N/A & Booklet 10 & Q2 \\
r & Not reached & Booklet UH & Q7
\end{tabular}




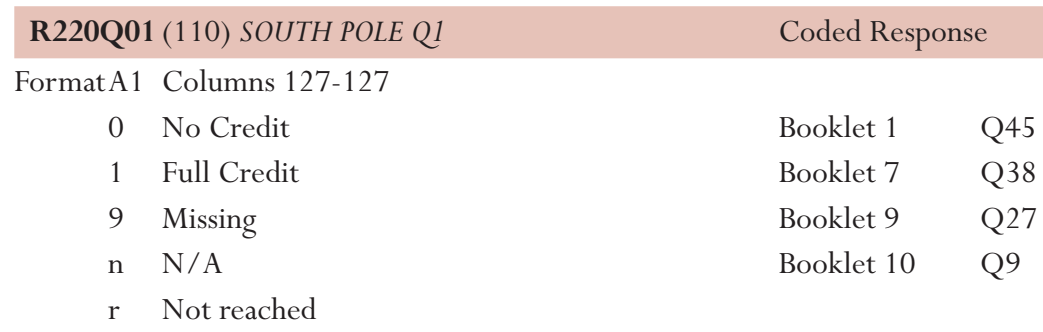

\begin{tabular}{|c|c|c|c|}
\hline \multicolumn{2}{|c|}{ R220Q02B (111) SOUTH POLE Q2B } & \multicolumn{2}{|c|}{ Multiple Choice } \\
\hline Format A 1 & Columns 128-128 & & \\
\hline 1 & Full Credit & Booklet 1 & Q46 \\
\hline 2 & No Credit & Booklet 7 & Q39 \\
\hline 3 & No Credit & Booklet 9 & Q28 \\
\hline 4 & No Credit & Booklet 10 & Q10 \\
\hline 8 & $\mathrm{M} / \mathrm{R}$ & & \\
\hline 9 & Missing & & \\
\hline $\mathrm{n}$ & $\mathrm{N} / \mathrm{A}$ & & \\
\hline $\mathrm{r}$ & Not reached & & \\
\hline
\end{tabular}

R220Q04 (112) SOUTH POLE Q4
$\begin{array}{rlll}\text { FormatA1 } & \text { Columns 129-129 } & \text { Multiple Choice } \\ 1 & \text { No Credit } & \text { Booklet 1 } & \text { Q47 } \\ 2 & \text { No Credit } & \text { Booklet 7 } & \text { Q40 } \\ 3 & \text { No Credit } & \text { Booklet 9 } & \text { Q29 } \\ 4 & \text { Full Credit } & \text { Booklet 10 } & \text { Q11 } \\ 8 & \text { M/R } & & \\ 9 & \text { Missing } & & \\ \text { n } & \text { N/A } & & \\ \text { r } & \text { Not reached } & & \end{array}$

\begin{tabular}{clll} 
R220Q05 (113) SOUTH POLE Q5 & \multicolumn{2}{l}{ Multiple Choice } \\
$\begin{array}{clll}\text { Format A1 } & \text { Columns 130-130 } & & \\
1 & \text { No Credit } & \text { Booklet 1 } & \text { Q48 } \\
2 & \text { No Credit } & \text { Booklet 7 } & \text { Q41 } \\
3 & \text { Full Credit } & \text { Booklet 9 } & \text { Q30 } \\
4 & \text { No Credit } & \text { Booklet 10 } & \text { Q12 } \\
8 & \text { M/R } & & \\
9 & \text { Missing } & & \\
\mathrm{n} & \text { N/A } & & \end{array}$
\end{tabular}

R220Q06 (114) SOUTH POLE Q6
\begin{tabular}{rlll} 
FormatA1 & Column 131-131 & \multicolumn{2}{l}{ Multiple Choice } \\
1 & No Credit & Booklet 1 & Q49 \\
2 & No Credit & Booklet 7 & Q42 \\
3 & Full Credit & Booklet 9 & Q31 \\
4 & No Credit & Booklet 10 & Q13 \\
8 & M/R & & \\
9 & Missing & & \\
$\mathrm{n}$ & N/A & & \\
$\mathrm{r}$ & Not reached & &
\end{tabular}




$\begin{array}{clll}\text { R227Q01 } & \text { (115) OPTICIAN Q1 } & \text { Multiple Choice } \\ \text { FormatA1 } & \text { Columns 132-132 } & \text { Booklet 2 } & \text { Q38 } \\ 1 & \text { No Credit } & \text { Booklet 8 } & \text { Q36 } \\ 2 & \text { Full Credit } & \text { Booklet 10 } & \text { Q14 } \\ 3 & \text { No Credit } & \text { Booklet 11 } & \text { Q1 } \\ 4 & \text { No Credit } & & \\ 8 & \text { M/R } & & \\ 9 & \text { Missing } & & \\ \text { n } & \text { N/A } & & \\ \text { r } & \text { Not reached } & & \end{array}$

R227Q02T (116) OPTICIAN Q2 Complex Multiple Choice

FormatA1 Columns 133-133

0 No Credit

1 No Credit

2 No Credit

3 No Credit

4 No Credit

5 Partial Credit

6 Partial Credit

7 Full Credit

$8 \mathrm{M} / \mathrm{R}$

9 Missing

n N/A

r Not reached

\begin{tabular}{clll} 
R227Q03 & (117) OPTICIAN Q3 & \multicolumn{2}{l}{ Coded Response } \\
FormatA1 & Columns 134-134 & Booklet 2 & Q40 \\
0 & No Credit & Booklet 8 & Q38 \\
1 & Full Credit & Booklet 10 & Q16 \\
9 & Missing & Booklet 11 & Q3 \\
n & N/A & & \\
r & Not reached & &
\end{tabular}

\begin{tabular}{clll} 
R227Q06 (118) OPTICIAN Q6 & \multicolumn{2}{l}{ Coded Response } \\
FormatA1 & Columns 135-135 & Booklet 2 & Q41 \\
0 & No Credit & Booklet 8 & Q39 \\
1 & Full Credit & Booklet 10 & Q17 \\
9 & Missing & Booklet 11 & Q4 \\
n & N/A & & \\
r & Not reached & &
\end{tabular}

\begin{tabular}{clll} 
S114Q03T (119) GREENHOUSE Q3 & \multicolumn{2}{l}{ Coded Response } \\
FormatA1 & Columns 136-136 & Booklet 5 & Q38 \\
0 & No Credit & Booklet 7 & Q25 \\
1 & Full Credit & Booklet 8 & Q13 \\
9 & Missing & Booklet 12 & Q44 \\
n & N/A & & \\
r & Not reached & &
\end{tabular}

\begin{tabular}{clll} 
S114Q04T (120) GREENHOUSE Q4 & \multicolumn{2}{l}{ Coded Response } \\
FormatA1 & Columns 137-137 & Booklet 5 & Q39 \\
0 & No Credit & Booklet 7 & Q26 \\
1 & Partial Credit & Booklet 8 & Q14 \\
2 & Full Credit & Booklet 12 & Q45 \\
9 & Missing & & \\
n & N/A & & \\
r & Not reached & &
\end{tabular}




\section{S114Q05T (121) GREENHOUSE Q5}

FormatA1 Columns 138-138
0 No Credit
1 Full Credit
9 Missing
n N/A
r Not reached

\section{Coded Response}

$\begin{array}{ll}\text { Booklet 5 } & \text { Q40 } \\ \text { Booklet 7 } & \text { Q27 } \\ \text { Booklet 8 } & \text { Q15 } \\ \text { Booklet 12 } & \text { Q46 }\end{array}$

Multiple Choice

$\begin{array}{clll}\text { S128Q01 } & \text { (122) CLONING Q1 } & \text { Multiple Choice } \\ \text { FormatA1 } & \text { Columns 139-139 } & \text { Booklet 6 } & \text { Q27 } \\ 1 & \text { Full Credit } & \text { Booklet 8 } & \text { Q20 } \\ 2 & \text { No Credit } & \text { Booklet 9 } & \text { Q3 } \\ 3 & \text { No Credit } & \text { Booklet 13 } & \text { Q36 } \\ 4 & \text { No Credit } & & \\ 8 & \text { M/R } & & \\ 9 & \text { Missing } & & \\ \mathrm{n} & \text { N/A } & & \\ \mathrm{r} & \text { Not reached } & & \end{array}$

S128Q02
\begin{tabular}{clll} 
Format 1 123) CLONING Q2 & Columns 140-140 & \multicolumn{2}{c}{ Multiple Choice } \\
1 & Full Credit & Booklet 6 & Q28 \\
2 & No Credit & Booklet 8 & Q21 \\
3 & No Credit & Booklet 9 & Q4 \\
4 & No Credit & Booklet 13 & Q37 \\
8 & M/R & & \\
9 & Missing & & \\
$\mathrm{n}$ & N/A & & \\
$\mathrm{r}$ & Not reached & & \\
\end{tabular}

S128Q03T (124) CLONING Q3 Complex Multiple Choice

FormatA1 Columns 141-141
0 No Credit
1 No Credit
2 Full Credit
$8 \mathrm{M} / \mathrm{R}$
9 Missing
n N/A
r Not reached

Booklet $6 \quad$ Q29

Booklet $8 \quad$ Q22

Booklet 9 Q5

Booklet $13 \quad$ Q38

\begin{tabular}{|c|c|c|c|}
\hline S129Q01 & (125) DAYLIGHT Q1 & Multiple Ch & \\
\hline FormatA1 & Columns 142-142 & & \\
\hline 1 & Full Credit & Booklet 6 & Q25 \\
\hline 2 & No Credit & Booklet 8 & Q18 \\
\hline 3 & No Credit & Booklet 9 & Q1 \\
\hline 4 & No Credit & Booklet 13 & Q34 \\
\hline 8 & $\mathrm{M} / \mathrm{R}$ & & \\
\hline 9 & Missing & & \\
\hline $\mathrm{n}$ & $\mathrm{N} / \mathrm{A}$ & & \\
\hline $\mathrm{r}$ & Not reached & & \\
\hline
\end{tabular}




\begin{tabular}{|c|c|c|c|}
\hline \multicolumn{2}{|c|}{ S129Q02T (126) DAYLIGHT Q2 } & \multicolumn{2}{|c|}{ Coded Response } \\
\hline \multicolumn{4}{|c|}{ FormatA1 Columns 143-143 } \\
\hline 0 & No Credit & Booklet 6 & Q26 \\
\hline 1 & Partial Credit & Booklet 8 & Q19 \\
\hline 2 & Full Credit & Booklet 9 & Q2 \\
\hline 9 & Missing & Booklet 13 & Q35 \\
\hline $\mathrm{n}$ & N/A & & \\
\hline $\mathrm{r}$ & Not reached & & \\
\hline
\end{tabular}

\begin{tabular}{|c|c|c|c|}
\hline S131Q02 & T (127) GOOD VIBRATIONS Q2 & Coded Resp & \\
\hline Format A1 & Columns 144-144 & & \\
\hline 0 & No Credit & Booklet 6 & Q30 \\
\hline 1 & Full Credit & Booklet 8 & Q23 \\
\hline 9 & Missing & Booklet 9 & Q6 \\
\hline $\mathrm{n}$ & $\mathrm{N} / \mathrm{A}$ & Booklet 13 & Q39 \\
\hline $\mathrm{r}$ & Not reached & & \\
\hline
\end{tabular}

\begin{tabular}{|c|c|c|c|}
\hline \multirow{2}{*}{\multicolumn{2}{|c|}{$\begin{array}{l}\text { S131Q04T (128) GOOD VIBRATIONS Q4 } \\
\text { FormatA1 Columns 145-145 }\end{array}$}} & \multicolumn{2}{|c|}{ Coded Response } \\
\hline & & & \\
\hline 0 & No Credit & Booklet 6 & Q31 \\
\hline 1 & Full Credit & Booklet 8 & Q24 \\
\hline 9 & Missing & Booklet 9 & Q7 \\
\hline $\mathrm{n}$ & N/A & Booklet 13 & Q40 \\
\hline $\mathrm{r}$ & Not reached & & \\
\hline
\end{tabular}

$\begin{array}{rlll}\text { S133Q01 } & \text { (129) RESEARCH Q1 } & \text { Multiple Choice } \\ \text { FormatA1 } & \text { Columns 146-146 } & & \\ 1 & \text { No Credit } & \text { Booklet 5 } & \text { Q35 } \\ 2 & \text { No Credit } & \text { Booklet 7 } & \text { Q22 } \\ 3 & \text { Full Credit } & \text { Booklet 8 } & \text { Q10 } \\ 4 & \text { No Credit } & \text { Booklet 12 } & \text { Q41 } \\ 8 & \text { M/R } & \text { Booklet UH } & \text { Q22 } \\ 9 & \text { Missing } & & \\ \text { n } & \text { N/A } & & \\ \text { r } & \text { Not reached } & & \end{array}$

$\begin{array}{rlll}\text { S133Q03 } & \text { (130) RESEARCH Q3 } & \text { Multiple Choice } \\ \text { FormatA1 } & \text { Columns 147-147 } & & \\ 1 & \text { Full Credit } & \text { Booklet 5 } & \text { Q36 } \\ 2 & \text { No Credit } & \text { Booklet 7 } & \text { Q23 } \\ 3 & \text { No Credit } & \text { Booklet 8 } & \text { Q11 } \\ 4 & \text { No Credit } & \text { Booklet 12 } & \text { Q42 } \\ 8 & \text { M/R } & \text { Booklet UH } & \text { Q23 } \\ 9 & \text { Missing } & & \\ \text { n } & \text { N/A } & & \\ \text { r } & \text { Not reached } & & \end{array}$

S133Q04T (131) RESEARCH Q4 Complex Multiple Choice

FormatA1 Columns148-148

0 No Credit

1 No Credit

2 No Credit

3 Full Credit

$8 \mathrm{M} / \mathrm{R}$

9 Missing

n N/A

r Not reached 


\begin{tabular}{|c|c|c|}
\hline S213Q01T (132) CLOTHES Q1 & Complex M & ple $\mathrm{Cl}$ \\
\hline FormatA1 Columns 149-149 & & \\
\hline $0 \quad$ No Credit & Booklet 5 & Q41 \\
\hline No Credit & Booklet 7 & Q28 \\
\hline No Credit & Booklet 8 & Q16 \\
\hline No Credit & Booklet 12 & Q47 \\
\hline 4 Full Credit & & \\
\hline $8 \mathrm{M} / \mathrm{R}$ & & \\
\hline 9 Missing & & \\
\hline $\mathrm{n} \quad \mathrm{N} / \mathrm{A}$ & & \\
\hline r Not reached & & \\
\hline
\end{tabular}

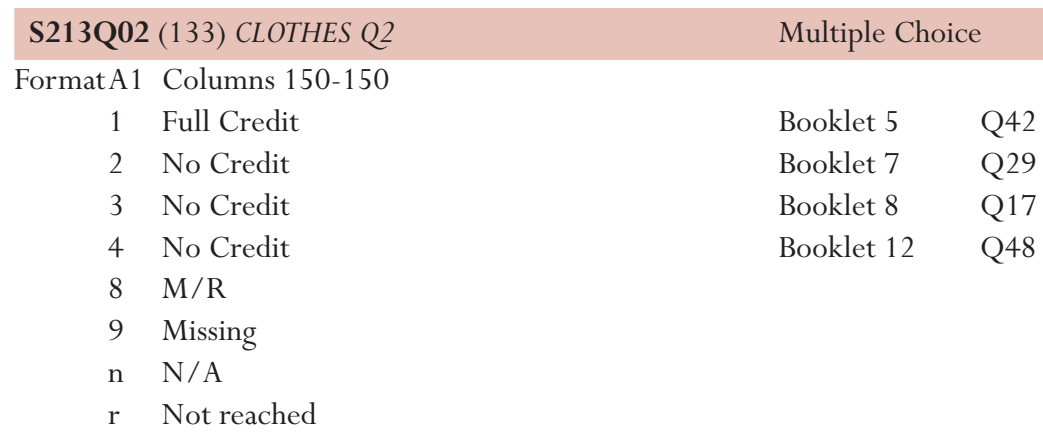

\begin{tabular}{clll} 
S252Q01 & (134) SOUTH RAINEA Q1 & \multicolumn{2}{l}{ Multiple Choice } \\
FormatA1 & Columns 151-151 & Booklet 5 & Q26 \\
1 & No Credit & Booklet 7 & Q13 \\
2 & No Credit & Booklet 8 & Q1 \\
3 & Full Credit & Booklet 12 & Q32 \\
4 & No Credit & Booklet UH & Q19 \\
8 & M/R & & \\
9 & Missing & & \\
$\mathrm{n}$ & N/A & & \\
$\mathrm{r}$ & Not reached & &
\end{tabular}

S252Q02 (135) SOUTH RAINEA Q2 Multiple Choice

FormatA1 Columns 152-152

1 Full Credit

Booklet $5 \quad$ Q27

2 No Credit

Booklet $7 \quad$ Q14

3 No Credit

Booklet $8 \quad$ Q2

4 No Credit

Booklet 12 Q33

$8 \mathrm{M} / \mathrm{R}$

Booklet UH Q20

9 Missing

n N/A

r Not reached

S252Q03T (136) SOUTH RAINEA Q3 Complex Multiple Choice

FormatA1 Columns 153-153

0 No Credit

1 No Credit

2 Full Credit

$8 \mathrm{M} / \mathrm{R}$

9 Missing

n N/A

r Not reached

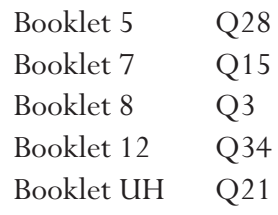




$\begin{array}{clll}\text { S256Q01 } & \text { (137) SPOONS Q1 } & \text { Multiple Choice } \\ \text { FormatA1 } & \text { Columns 154-154 } & & \\ 1 & \text { Full Credit } & \text { Booklet 6 } & \text { Q32 } \\ 2 & \text { No Credit } & \text { Booklet 8 } & \text { Q25 } \\ 3 & \text { No Credit } & \text { Booklet 9 } & \text { Q8 } \\ 4 & \text { No Credit } & \text { Booklet 13 } & \text { Q41 } \\ 8 & \text { M/R } & \text { Booklet UH } & \text { Q26 } \\ 9 & \text { Missing } & & \\ \text { n } & \text { N/A } & & \\ \text { r } & \text { Not reached } & & \end{array}$

$\begin{array}{clll}\text { S268Q01 } & \text { (138) ALGAE Q1 } & \text { Multiple Choice } \\ \text { FormatA1 } & \text { Columns 155-155 } & & \\ 1 & \text { No Credit } & \text { Booklet 6 } & \text { Q40 } \\ 2 & \text { No Credit } & \text { Booklet 8 } & \text { Q33 } \\ 3 & \text { Full Credit } & \text { Booklet 9 } & \text { Q16 } \\ 4 & \text { No Credit } & \text { Booklet 13 } & \text { Q49 } \\ 8 & \text { M/R } & & \\ 9 & \text { Missing } & \\ \text { n } & \text { N/A } & & \\ \text { r } & \text { Not reached } & & \end{array}$

$\begin{array}{clll}\text { S268Q02T (139) ALGAE Q2 } & \text { Coded Response } \\ \text { FormatA1 } & \text { Columns 156-156 } & & \\ 0 & \text { No Credit } & \text { Booklet 6 } & \text { Q41 } \\ 1 & \text { Full Credit } & \text { Booklet 8 } & \text { Q34 } \\ 9 & \text { Missing } & \text { Booklet 9 } & \text { Q17 } \\ \text { n } & \text { N/A } & \text { Booklet 13 } & \text { Q50 } \\ \text { r } & \text { Not reached } & & \end{array}$

$\begin{array}{clll}\text { S268Q06 } & \text { (140) ALGAE Q6 } & \text { Multiple Choice } \\ \text { FormatA1 } & \text { Columns 157-157 } & & \\ 1 & \text { No Credit } & \text { Booklet 6 } & \text { Q42 } \\ 2 & \text { Full Credit } & \text { Booklet 8 } & \text { Q35 } \\ 3 & \text { No Credit } & \text { Booklet 9 } & \text { Q18 } \\ 4 & \text { No Credit } & \text { Booklet 13 } & \text { Q51 } \\ 8 & \text { M/R } & & \\ 9 & \text { Missing } & \\ \text { n } & \text { N/A } & \\ \text { r } & \text { Not reached } & & \end{array}$

S269Q01 (141) EARTH'S TEMPERATURE Q1 Coded Response

$\begin{array}{clll}\text { Format A1 } & \text { Columns158-158 } & & \\ 0 & \text { No Credit } & \text { Booklet 6 } & \text { Q37 } \\ 1 & \text { Full Credit } & \text { Booklet 8 } & \text { Q30 } \\ 9 & \text { Missing } & \text { Booklet 9 } & \text { Q13 } \\ \text { n } & \text { N/A } & \text { Booklet 13 } & \text { Q46 } \\ \text { r } & \text { Not reached } & \text { Booklet UH } & \text { Q25 }\end{array}$

\begin{tabular}{|c|c|c|c|}
\hline \multirow{2}{*}{\multicolumn{2}{|c|}{$\begin{array}{l}\text { S269Q03T (142) EARTH'S TEMPERATURE Q3 } \\
\text { FormatA1 Columns 159-159 }\end{array}$}} & \multicolumn{2}{|c|}{ Coded Response } \\
\hline & & & \\
\hline 0 & No Credit & Booklet 6 & Q38 \\
\hline 1 & Full Credit & Booklet 8 & Q31 \\
\hline 9 & Missing & Booklet 9 & Q14 \\
\hline $\mathrm{n}$ & $\mathrm{N} / \mathrm{A}$ & Booklet 13 & Q47 \\
\hline $\mathrm{r}$ & Not reached & & \\
\hline
\end{tabular}




S269Q04T (143) EARTH'S TEMPERATURE Q4
$\begin{array}{clll}\text { FormatA1 } & \text { Columns 160-160 } & \text { Complex Multiple Choi } \\ 0 & \text { No Credit } & \text { Booklet 6 } & \text { Q39 } \\ 1 & \text { No Credit } & \text { Booklet 8 } & \text { Q32 } \\ 2 & \text { No Credit } & \text { Booklet 13 } & \text { Q15 } \\ 3 & \text { No Credit } & & \\ 4 & \text { Full Credit } & & \\ 8 & \text { M/R } & & \\ 9 & \text { Missing } & & \\ \text { n } & \text { N/A } & & \\ \text { r } & \text { Not reached } & & \end{array}$

\begin{tabular}{clll} 
S304Q01 & (144) WATER Q1 & \multicolumn{2}{l}{ Coded Response } \\
FormatA1 & Columns 161-161 & & \\
0 & No Credit & Booklet 6 & Q33 \\
1 & Full Credit & Booklet 8 & Q26 \\
9 & Missing & Booklet 9 & Q9 \\
n & N/A & Booklet 13 & Q42 \\
r & Not reached & &
\end{tabular}

\begin{tabular}{|c|c|c|}
\hline S304Q02 (145) WATER Q2 & \multicolumn{2}{|c|}{ Multiple Choice } \\
\hline FormatA1 Columns 162-162 & & \\
\hline 1 No Credit & Booklet 6 & Q34 \\
\hline 2 No Credit & Booklet 8 & Q27 \\
\hline 3 Full Credit & Booklet 9 & Q10 \\
\hline $4 \quad$ No Credit & Booklet 13 & Q43 \\
\hline $8 \mathrm{M} / \mathrm{R}$ & & \\
\hline 9 Missing & & \\
\hline $\mathrm{n} \quad \mathrm{N} / \mathrm{A}$ & & \\
\hline r Not reached & & \\
\hline
\end{tabular}

\begin{tabular}{clll} 
S304Q03a (146) WATER Q3A & \multicolumn{2}{l}{ Coded Response } \\
$\begin{array}{clll}\text { FormatA1 } & \text { Columns 163-163 } & & \\
0 & \text { No Credit } & \text { Booklet 6 } & \text { Q35 } \\
1 & \text { Full Credit } & \text { Booklet 8 } & \text { Q28 } \\
9 & \text { Missing } & \text { Booklet 9 } & \text { Q11 } \\
\text { n } & \text { N/A } & \text { Booklet 13 } & \text { Q44 } \\
\text { r } & \text { Not reached } & & \end{array}$
\end{tabular}

S304Q03b (147) WATER Q3B_ Coded Response

$\begin{array}{clll}\text { FormatA1 } & \text { Columns 164-164 } & & \\ 0 & \text { No Credit } & \text { Booklet 6 } & \text { Q36 } \\ 1 & \text { Full Credit } & \text { Booklet 8 } & \text { Q29 } \\ 9 & \text { Missing } & \text { Booklet 9 } & \text { Q12 } \\ \text { n } & \text { N/A } & \text { Booklet 13 } & \text { Q45 } \\ \text { r } & \text { Not reached } & & \end{array}$

S326Q01 (148) MILK Q1 Coded Response

FormatA1 Columns 165-165

0 No Credit

1 Full Credit

9 Missing

n $\mathrm{N} / \mathrm{A}$

Booklet $5 \quad$ Q31

Booklet $7 \quad$ Q18

r Not reached

Booklet $8 \quad$ Q6

Booklet $12 \quad$ Q37 


\begin{tabular}{clll} 
S326Q02 & (149) MILK Q2 & \multicolumn{2}{c}{ Coded Response } \\
FormatA1 & Columns 166-166 & Booklet 5 & Q32 \\
0 & No Credit & Booklet 7 & Q19 \\
1 & Full Credit & Booklet 8 & Q7 \\
9 & Missing & Booklet 12 & Q38 \\
n & N/A & & \\
r & Not reached & &
\end{tabular}

\begin{tabular}{|clll} 
S326Q03 & $($ 150) MILK Q3 & Multiple Choice \\
FormatA1 & Columns 167-167 & & \\
1 & No Credit & Booklet 5 & Q33 \\
2 & Full Credit & Booklet 7 & Q20 \\
3 & No Credit & Booklet 8 & Q8 \\
4 & No Credit & Booklet 12 & Q39 \\
8 & M/R & & \\
9 & Missing & & \\
n & N/A & & \\
r & Not reached & &
\end{tabular}

\begin{tabular}{clll} 
S326Q04T (151) MILK Q4 & \multicolumn{2}{c}{ Complex Multiple Cho } \\
FormatA1 & Columns 168-168 & Booklet 5 & Q34 \\
0 & No Credit & Booklet 7 & Q21 \\
1 & No Credit & Booklet 8 & Q9 \\
2 & No Credit & Booklet 12 & Q40 \\
3 & Full Credit & & \\
8 & M/R & & \\
9 & Missing & & \\
$\mathrm{n}$ & N/A & & \\
$\mathrm{r}$ & Not reached & &
\end{tabular}

S327Q01T (152) TIDAL ENERGY Q1
$\begin{array}{clll}\text { FormatA1 } & \text { Columns 169-169 } & \text { Complex Multiple Choice } \\ 0 & \text { No Credit } & \text { Booklet 5 } & \text { Q29 } \\ 1 & \text { No Credit } & \text { Booklet 7 } & \text { Q16 } \\ 2 & \text { Full Credit } & \text { Booklet 8 } & \text { Q4 } \\ 8 & \text { M/R } & \text { Booklet 12 } & \text { Q35 } \\ 9 & \text { Missing } & & \\ \text { n } & \text { N/A } & & \\ \text { r } & \text { Not reached } & & \end{array}$

\begin{tabular}{clll} 
X402Q01T (153) LIBRARY SYSTEM Q1 & \multicolumn{2}{l}{ Coded Response } \\
FormatA1 & Columns 170-170 & Booklet 4 & Q40 \\
0 & No Credit & Booklet 10 & Q31 \\
1 & Full Credit & Booklet 12 & Q13 \\
8 & Invalid & Booklet 13 & Q3 \\
9 & Missing & & \\
$\mathrm{n}$ & N/A & & \\
$\mathrm{r}$ & Not reached & &
\end{tabular}

X402Q02T (154) LIBRARY SYSTEM Q2
\begin{tabular}{clll} 
FormatA1 & Columns 171-171 & \multicolumn{2}{l}{ Coded Response } \\
0 & No Credit & Booklet 4 & Q41 \\
1 & Partial Credit & Booklet 10 & Q32 \\
2 & Partial Credit & Booklet 12 & Q14 \\
3 & Full Credit & Booklet 13 & Q4 \\
9 & N/A & & \\
n & Missing & & \\
r & Not reached & &
\end{tabular}




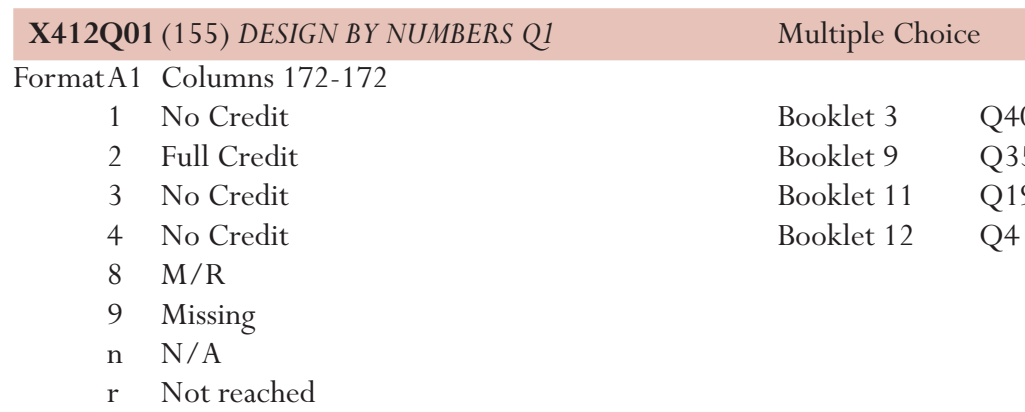

\begin{tabular}{|clll} 
X412Q02 & (156) DESIGN BY NUMBERS Q2 & Multiple Choice \\
FormatA1 & Columns 173-173 & Booklet 3 & Q41 \\
1 & No Credit & Booklet 9 & Q36 \\
2 & No Credit & Booklet 11 & Q20 \\
3 & No Credit & Booklet 12 & Q5 \\
4 & Full Credit & & \\
8 & M/R & & \\
9 & Missing & & \\
$\mathrm{n}$ & N/A & & \\
$\mathrm{r}$ & Not reached & &
\end{tabular}

\begin{tabular}{clll} 
X412Q03 & (157) DESIGN BY NUMBERS Q3 & \multicolumn{2}{l}{ Coded Response } \\
FormatA1 & Columns 174-174 & Booklet 3 & Q42 \\
0 & No Credit & Booklet 9 & Q37 \\
1 & Partial Credit & Booklet 11 & Q21 \\
2 & Full Credit & Booklet 12 & Q6 \\
9 & Missing & & \\
n & N/A & & \\
r & Not reached & &
\end{tabular}

\begin{tabular}{clll} 
X414Q01 & (158) COURSE DESIGN Q1 & \multicolumn{2}{l}{ Coded Response } \\
FormatA1 & Columns 175-175 & Booklet 4 & Q39 \\
0 & No Credit & Booklet 10 & Q30 \\
1 & Partial Credit & Booklet 12 & Q12 \\
2 & Full Credit & Booklet 13 & Q2 \\
9 & Missing & & \\
n & N/A & & \\
r & Not reached & &
\end{tabular}

X415Q01T (159) TRANSIT SYSTEM Q1
$\begin{array}{clll}\text { FormatA1 } & \text { Columns 176-176 } & \text { Coded Response } \\ 0 & \text { No Credit } & \text { Booklet 4 } & \text { Q38 } \\ 1 & \text { Partial Credit } & \text { Booklet 10 } & \text { Q29 } \\ 2 & \text { Full Credit } & \text { Booklet 12 } & \text { Q11 } \\ 9 & \text { Missing } & \text { Booklet 13 } & \text { Q1 } \\ \text { n } & \text { N/A } & \text { Booklet UH } & \text { Q5 } \\ \text { r } & \text { Not reached } & & \end{array}$

\begin{tabular}{|c|c|c|c|}
\hline X417Q01 & (160) CHILDREN'S CAMP Q1 & Coded Resp & \\
\hline Format A1 & Columns 177-177 & & \\
\hline 0 & No Credit & Booklet 3 & Q39 \\
\hline 1 & Partial Credit & Booklet 9 & Q34 \\
\hline 2 & Full Credit & & \\
\hline 9 & Missing & Booklet 11 & Q18 \\
\hline $\mathrm{n}$ & $\mathrm{N} / \mathrm{A}$ & Booklet 12 & Q3 \\
\hline $\mathrm{r}$ & Not reached & & \\
\hline
\end{tabular}




\begin{tabular}{|c|c|c|c|}
\hline \multirow{2}{*}{\multicolumn{2}{|c|}{$\begin{array}{l}\text { X423Q01T (161) FREEZER Q1 } \\
\text { FormatA1 Columns 178-178 }\end{array}$}} & \multicolumn{2}{|c|}{ Complex Multiple Choice } \\
\hline & & & \\
\hline 0 & No Credit & Booklet 3 & Q43 \\
\hline 1 & No Credit & Booklet 9 & Q38 \\
\hline 2 & No Credit & Booklet 11 & Q22 \\
\hline 3 & No Credit & Booklet 12 & Q7 \\
\hline 4 & No Credit & & \\
\hline 5 & Full Credit & & \\
\hline 6 & Full Credit & & \\
\hline 8 & $\mathrm{M} / \mathrm{R}$ & & \\
\hline 9 & Missing & & \\
\hline $\mathrm{n}$ & $\mathrm{N} / \mathrm{A}$ & & \\
\hline $\mathrm{r}$ & Not reached & & \\
\hline
\end{tabular}

X423Q02T (162) FREEZER Q2 Complex Multiple Choice

$\begin{array}{clll}\text { Format A1 } & \text { Columns 179-179 } & & \\ 0 & \text { No Credit } & \text { Booklet 3 } & \text { Q44 } \\ 1 & \text { No Credit } & \text { Booklet 9 } & \text { Q39 } \\ 2 & \text { No Credit } & \text { Booklet 11 } & \text { Q23 } \\ 3 & \text { Full Credit } & \text { Booklet 12 } & \text { Q8 } \\ 8 & \text { M/R } & & \\ 9 & \text { Missing } & & \\ \text { n } & \text { N/A } & & \\ \text { r } & \text { Not reached } & & \end{array}$

$\begin{array}{clll}\text { X430Q01 } & \text { (163) ENERGY NEEDS Q1 } & \text { Coded Response } \\ \text { Format A1 } & \text { Columns 180-180 } & & \\ 0 & \text { No Credit } & \text { Booklet 3 } & \text { Q37 } \\ 1 & \text { Full Credit } & \text { Booklet 9 } & \text { Q32 } \\ 9 & \text { Missing } & \text { Booklet 11 } & \text { Q16 } \\ \mathrm{n} & \text { N/A } & \text { Booklet 12 } & \text { Q1 } \\ \text { r } & \text { Not reached } & \text { Booklet UH } & \text { Q3 }\end{array}$

$\begin{array}{clll}\text { X430Q02 (164) ENERGY NEEDS Q2 } & \text { Coded Response } \\ \text { Format A1 } & \text { Columns 181-181 } & & \\ 0 & \text { No Credit } & \text { Booklet 3 } & \text { Q38 } \\ 1 & \text { Partial Credit } & \text { Booklet 9 } & \text { Q33 } \\ 2 & \text { Full Credit } & \text { Booklet 11 } & \text { Q17 } \\ 9 & \text { Missing } & \text { Booklet 12 } & \text { Q2 } \\ \text { n } & \text { N/A } & \text { Booklet UH } & \text { Q4 } \\ \text { r } & \text { Not reached } & & \end{array}$

\begin{tabular}{|c|c|c|c|}
\hline X601Q0 & IT (165) CINEMA OUTING Q1 & Complex Mu & iple Choi \\
\hline Format A1 & Columns $182-182$ & & \\
\hline 0 & No Credit & Booklet 3 & Q45 \\
\hline 1 & No Credit & Booklet 9 & Q40 \\
\hline 2 & No Credit & Booklet 11 & Q24 \\
\hline 3 & No Credit & Booklet 12 & Q9 \\
\hline 4 & No Credit & Booklet UH & Q1 \\
\hline 5 & Partial Credit & & \\
\hline 6 & Full Credit & & \\
\hline 8 & $\mathrm{M} / \mathrm{R}$ & & \\
\hline 9 & Missing & & \\
\hline $\mathrm{n}$ & $\mathrm{N} / \mathrm{A}$ & & \\
\hline $\mathrm{r}$ & Not reached & & \\
\hline
\end{tabular}




\begin{tabular}{|c|c|c|c|}
\hline \multicolumn{2}{|c|}{ X601Q02 (166) CINEMA OUTING Q2 } & \multicolumn{2}{|c|}{ Multiple Choice } \\
\hline FormatA1 & Columns 183-183 & & \\
\hline 1 & No Credit & Booklet 3 & Q46 \\
\hline 2 & No Credit & Booklet 9 & Q41 \\
\hline 3 & Full Credit & Booklet 11 & Q25 \\
\hline 4 & No Credit & Booklet 12 & Q10 \\
\hline 5 & No Credit & Booklet UH & Q2 \\
\hline 8 & $\mathrm{M} / \mathrm{R}$ & & \\
\hline 9 & Missing & & \\
\hline $\mathrm{n}$ & $\mathrm{N} / \mathrm{A}$ & & \\
\hline $\mathrm{r}$ & Not reached & & \\
\hline
\end{tabular}

X602Q01 (167) HOLIDAY Q1 Coded Response

FormatA1 Columns 184-184

0 No Credit

Booklet $4 \quad$ Q42

1 Full Credit

Booklet $10 \quad$ Q33

9 Missing

Booklet 12 Q15

n N/A

Booklet $13 \quad$ Q5

r Not reached

\begin{tabular}{clll} 
X602Q02 & $(168)$ HOLIDAY Q2 & \multicolumn{2}{l}{ Coded Response } \\
FormatA1 & Columns 185-185 & Booklet 4 & Q43 \\
0 & No Credit & & \\
1 & Partial Credit & Booklet 10 & Q34 \\
2 & Full Credit & Booklet 12 & Q16 \\
9 & Missing & Booklet 13 & Q6 \\
n & N/A & & \\
r & Not reached & &
\end{tabular}

X603Q01 (169) IRRIGATION Q1 Coded Response

FormatA1 Columns 186-186

0 No Credit

1 Full Credit

Booklet $4 \quad$ Q44

9 Missing

Booklet $10 \quad$ Q35

n N/A

Booklet $12 \quad \mathrm{Q} 17$

$r \quad$ Not reached

Booklet $13 \quad$ Q7

\begin{tabular}{|c|c|c|c|}
\hline X603Q02 & T (170) IRRIGATION Q2 & Complex M & ple Ch \\
\hline FormatA1 & Columns 187-187 & & \\
\hline 0 & No Credit & Booklet 4 & Q45 \\
\hline 1 & No Credit & Booklet 10 & Q36 \\
\hline 2 & No Credit & Booklet 12 & Q18 \\
\hline 3 & Full Credit & Booklet 13 & Q8 \\
\hline 8 & $\mathrm{M} / \mathrm{R}$ & & \\
\hline 9 & Missing & & \\
\hline $\mathrm{n}$ & $\mathrm{N} / \mathrm{A}$ & & \\
\hline $\mathrm{r}$ & Not reached & & \\
\hline
\end{tabular}

\begin{tabular}{clll} 
X603Q03 & (171) IRRIGATION Q3 & \multicolumn{2}{l}{ Coded Response } \\
FormatA1 & Columns 188-188 & Booklet 4 & Q46 \\
0 & No Credit & Booklet 12 & Q37 \\
1 & Full Credit & Booklet 10 & Q19 \\
9 & Missing & Booklet 12 & Q9 \\
n & N/A & & \\
r & Not reached & &
\end{tabular}


MSCALE (172) MATH SCALABLE

FormatA1 Columns 189-189

RSCALE (173) READING SCALABLE

FormatA1 Columns 190-190

SSCALE (174) SCIENCE SCALABLE

FormatA1 Columns 191-191

PSCALE (175) PROBLEM SOLVING SCALABLE

FormatA1 Columns 192-192

\section{CLCUSE1 (176) CALCULATOR USE}

FormatA1 Columns 193-193

1 No calculator

2 A simple calculator

3 A scientific calculator

4 A programmable calculator

5 A graphics calculator

$8 \mathrm{M} / \mathrm{R}$

9 Missing

n $\mathrm{N} / \mathrm{A}$

\section{CLCUSE3a (177) EFFORT-REAL:A}

FormatF3 Columns 194-196

CLCUSE3b (178) EFFORT-REAL: B

FormatF3 Columns 197-199 


\section{APPENDIX $9 \cdot$ STUDENT AND SCHOOL QUESTIONNAIRE INDICES}

Several of PISA's measures reflect indices that summarise responses from students or school principals to a series of related questions. The questions were selected from larger constructs on the basis of theoretical considerations and previous research. Structural equation modelling was used to confirm the theoretically expected behaviour of the indices and to validate their comparability across countries. For this purpose, a model was estimated separately for each country and, collectively, for all OECD countries.

This section explains the indices derived from the student and school context questionnaires that are used in this report. For a description of other PISA indices and details on the methods see the PISA 2003 Technical Report (OECD, forthcoming).

Two types of indices are distinguished:

- Simple indices constructed through the arithmetical transformation or recoding of one or more items: here, item responses are used to calculate meaningful variables; for example, the recoding of ISCO-88 codes into the international socio-economic index of occupational status (ISEI) or the calculation of student/teacher ratio based on information from the school questionnaire.

- Scale indices constructed through the scaling of items. All of these indices are derived via IRT scaling of either dichotomous (Yes/No) or Likert-type items. Unless otherwise indicated, where an index involves multiple questions and student responses, the index was scaled using a weighted maximum likelihood estimate, using a one-parameter item response model (referred to as a WARM estimator; see Warm, 1985) with three stages:

- The question parameters were estimated from equal-sized sub-samples of students from each OECD country.

- The estimates were computed for all students and all schools by anchoring the question parameters obtained in the preceding step.

- The indices were then standardised so that the mean of the index value for the OECD student population was zero and the standard deviation was one (countries being given equal weight in the standardisation process).

It is important to note that negative values in an index do not necessarily imply that students responded negatively to the underlying questions. A negative value merely indicates that a group of students (or all students, collectively, in a single country) or principals responded less positively than all students or principals did on average across OECD countries. Likewise, a positive value on an index indicates that a group of students or principals responded more favourably, or more positively, than students or principals did, on average, in OECD countries.

Terms enclosed in brackets $<>$ in the following descriptions were replaced in the national versions of the student and school questionnaires by the appropriate national equivalent. For example, the term < qualification at ISCED level 5A> was translated in the United States into "Bachelor's Degree, 
post-graduate certificate program, Master's degree program or first professional degree program”. Similarly the term < classes in the language of assessment> in Luxembourg was translated into "German classes" or "French classes" depending on whether students received the German or French version of the assessment instruments.

For the reliabilities of the indices, see the PISA 2003 Technical Report (OECD, forthcoming).

\section{STUDENT-LEVEL SIMPLE INDICES}

\section{Student background}

Age (AGE)

Similar to PISA 2000, the PISA 2003 index of age (AGE) is calculated as the difference between year and month of the testing and the year and month of a student's birth (ST02Q02 and ST02Q03).

\section{Study programme (ISCEDL, ISCEDD, ISCEDO and PROGN)}

The PISA 2003 indices of study programme are derived from students' responses to the item ST01Q02 asking study programmes available to 15 -year-old students in each country. All study programmes are classified by ISCED (OECD 1999). All national programmes are included in a separate index of unique study programme code (PROGN) where the first three digits are the ISO code for a country, the fourth digit the sub-national category and the last two digits the nationally specific programme code.

The following indices are derived from the data on study programmes:

- The PISA 2003 index of programme level (ISCEDL) indicates whether students are on the lower or upper secondary level (ISCED 3 or ISCED 2).

- The PISA 2003 index of programme designation (ISCEDD) indicates the designation of the study programme: (1) = 'A' (general programmes designed to give access to the next programme level); $(2)=$ 'B' (programmes designed to give access to vocational studies at the next programme level); (3) = 'C' (programmes designed to give direct access to the labour market); (4) = "M" (modular programmes that combine any or all of these characteristics).

- The PISA 2003 index of programme orientation (ISCEDO) indicates whether the programme's curricular content is general (1), pre-vocational (2) or vocational (3).

\section{Family structure (FAMSTRUC)}

The PISA 2003 index of family structure (FAMSTRUC) is simplified the PISA 2000 index of family structure. Students' responses to the items ST04Q01-ST04Q05 are recoded into the index of family structure with four categories: (1) a single parent family (students reporting to live with only one of the following: mother, female guardian, father, male guardian), (2) a nuclear family (students living with a father and a mother), (3) a mixed family (a father and a guardian, a mother and a guardian, or two guardians) and (4) other responses, except the non-responses which are maintained as missing or not applicable.

\section{Highest occupational status of parents (BMMJ, BFMJ, HISEI, MSECATEG, FSECATEG and HSECATEG)}

The occupational data for both the student's mother and student's father were obtained by asking openended questions ST07Q01 (from Q7 and Q8) in the student questionnaire for mothers' occupational status and ST09Q01(from Q9 and Q10) in the student questionnaire for fathers' occupational status. 
The responses were coded in accordance with the four-digit International Standard Classification of Occupation (ISCO 1988) (ILO, 1990) and then mapped to the international socio-economic index of occupational status (ISEI) (Ganzeboom et al.,1992). Three indices are obtained from these scores.

The PISA 2003 index of mother's occupational status (BMMJ) and the PISA 2003 index of father's occupational status (BFMJ) are derived from recoding ISCO codes into the ISEI. These indices are similar to the PISA 2000 indices of mother's occupation and father's occupation. The PISA 2003 index of the highest occupational level of parents (HISEI) corresponds to the higher ISEI score of either parent or to the only available parent's ISEI score. Higher values on these indices indicate higher level of occupational status.

These indices are also recoded into four occupational categories: (1) white collar high skilled occupation; (2) white collar low skilled occupation; (3) blue collar high skilled occupation; and (4) blue collar low skilled occupation, except the non-responses which are maintained as missing or not applicable. Indices with these categories are provided for mother (MSECATEG), father (FSECATEG) and either one of the parents having higher occupational status (HSECATEG).

\section{Educational level of parents (MISCED, FISCED, HISCED and PARED)}

The PISA 2003 indices of parents' educational level are derived from students' responses to the items ST11RQ01 and ST12Q01-ST12Q03 for mothers' educational level and ST13RQ01 and ST14Q01-ST14Q03 for fathers' educational level. The students' responses to these items are coded in accordance with the International Standard Classification of Education (ISCED 1997) (OECD 1999 ) in order to obtain internationally comparable categories of educational attainment. The format of these items in PISA 2003 is different from the format used in PISA 2000.

Table A9.1 — Levels of parental education converted into years of schooling

\begin{tabular}{|c|c|c|c|c|c|c|c|}
\hline & $\begin{array}{l}\overline{0} \\
0 \\
0 \\
0 \\
0 \\
0 \\
0 \\
0 \infty \\
0 \\
0 \\
0 \\
0 \\
0\end{array}$ & 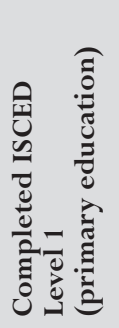 & 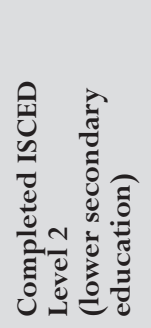 & 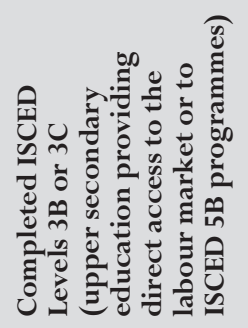 & 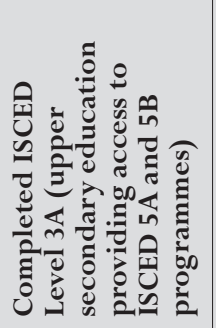 & 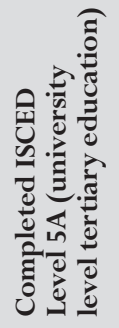 & 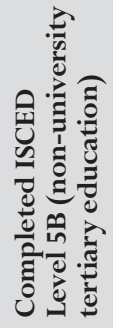 \\
\hline \multicolumn{8}{|l|}{ OECD countries } \\
\hline Australia & 0.0 & 6.5 & 10.0 & 11.5 & 12.0 & 15.0 & 14.0 \\
\hline Austria & 0.0 & 4.0 & 8.0 & 11.0 & 13.0 & 17.0 & 15.0 \\
\hline Belgium & 0.0 & 6.0 & 8.0 & 12.0 & 12.0 & 16.0 & 15.0 \\
\hline Canada & 0.0 & 6.0 & 9.0 & 12.0 & 12.0 & 17.0 & 15.0 \\
\hline Czech Republic & 0.0 & 5.0 & 9.0 & 12.0 & 13.0 & 17.0 & 16.0 \\
\hline Denmark & 0.0 & 6.0 & 9.5 & 12.5 & 12.5 & 16.5 & 15.5 \\
\hline Finland & 0.0 & 6.0 & 9.0 & 12.0 & 12.0 & 15.5 & 14.5 \\
\hline France & 0.0 & 5.0 & 9.0 & 11.0 & 12.0 & 15.0 & 14.0 \\
\hline Germany & 0.0 & 4.0 & 10.0 & 12.0 & 12.5 & 17.0 & 15.0 \\
\hline Greece & 0.0 & 6.0 & 9.0 & 11.5 & 12.0 & 17.0 & 15.5 \\
\hline Hungary & 0.0 & 4.0 & 8.0 & 10.5 & 12.0 & 16.5 & 13.5 \\
\hline Iceland & 0.0 & 7.0 & 10.0 & 13.0 & 14.0 & 17.0 & 16.5 \\
\hline Ireland & 0.0 & 6.0 & 9.0 & a & 12.0 & 16.0 & 14.0 \\
\hline Italy & 0.0 & 5.0 & 8.0 & 11.0 & 13.0 & 17.0 & 16.0 \\
\hline
\end{tabular}

a: The category does not apply in the country concerned. Data are therefore missing. 

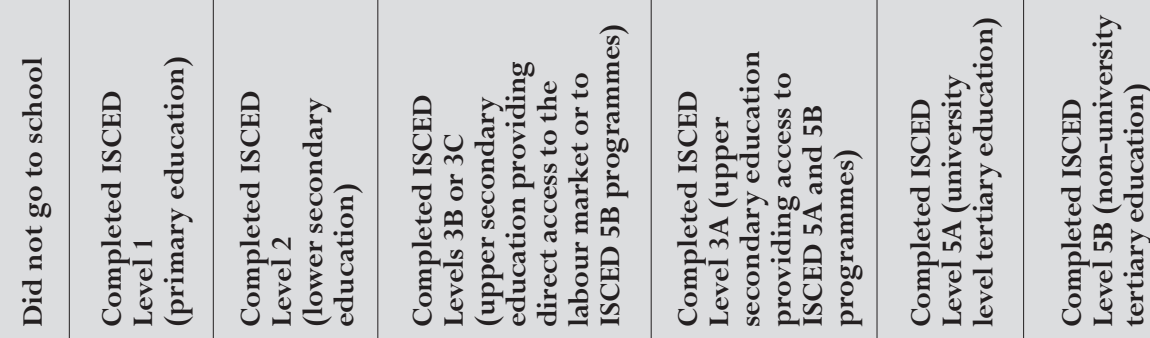

\begin{tabular}{|c|c|c|c|c|c|c|c|}
\hline Japan & 0.0 & 6.0 & 9.0 & 12.0 & 12.0 & 16.0 & 14.0 \\
\hline Korea & 0.0 & 6.0 & 9.0 & 12.0 & 12.0 & 16.0 & 15.0 \\
\hline Luxembourg & 0.0 & 6.0 & 9.0 & 12.0 & 13.0 & 17.0 & 17.0 \\
\hline Mexico & 0.0 & 6.0 & 9.0 & 12.0 & 12.0 & 16.0 & 14.0 \\
\hline Netherlands & 0.0 & 6.0 & 10.0 & a & 12.0 & 15.0 & $\mathrm{a}$ \\
\hline New Zealand & 0.0 & 6.0 & 10.0 & 12.0 & 13.0 & 16.0 & 16.0 \\
\hline Norway & 0.0 & 7.0 & 10.0 & 13.0 & 13.0 & 17.0 & 15.0 \\
\hline Poland & 0.0 & $\mathrm{a}$ & 8.0 & 11.0 & 12.0 & 16.0 & 15.0 \\
\hline Portugal & 0.0 & 6.0 & 9.0 & 12.0 & 12.0 & 17.0 & 15.0 \\
\hline Slovak Republic & 0.0 & 4.0 & 9.0 & 12.0 & 12.5 & 17.0 & 15.0 \\
\hline Spain & 0.0 & 6.0 & 10.0 & 12.0 & 12.0 & 15.0 & 14.0 \\
\hline Sweden & 0.0 & 6.0 & 9.0 & 12.0 & 12.0 & 15.5 & 14.0 \\
\hline Switzerland & 0.0 & 6.0 & 9.0 & 12.0 & 12.5 & 15.0 & 14.0 \\
\hline Turkey & 0.0 & 5.0 & 8.0 & 11.0 & 11.0 & 16.0 & 14.0 \\
\hline United States & 0.0 & 6.0 & 9.0 & $\mathrm{a}$ & 12.0 & 16.0 & 15.0 \\
\hline United Kingdom & 0.0 & 6.0 & 9.0 & 11.0 & 12.0 & 16.0 & 15.0 \\
\hline \multicolumn{8}{|l|}{ Partner countries } \\
\hline Brazil & 0.0 & 4.0 & 8.0 & 11.0 & 11.0 & 16.0 & 14.5 \\
\hline Hong Kong-China & 0.0 & 6.0 & 9.0 & 11.0 & 13.0 & 16.0 & 14.0 \\
\hline Indonesia & 0.0 & 6.0 & 9.0 & 12.0 & 12.0 & 16.0 & 15.0 \\
\hline Latvia & 0.0 & 4.0 & 9.0 & 12.0 & 12.0 & 16.0 & 16.0 \\
\hline Liechtenstein & 0.0 & 5.0 & 9.0 & 11.0 & 12.0 & 15.0 & 14.0 \\
\hline Macao-China & 0.0 & 6.0 & 9.0 & 11.0 & 13.0 & 16.0 & 14.0 \\
\hline Russian Federation & 0.0 & 4.0 & 9.0 & 11.5 & 12.0 & 15.0 & $\mathrm{a}$ \\
\hline Serbia & 0.0 & 4.0 & 8.0 & 11.0 & 12.0 & 16.0 & 14.0 \\
\hline Thailand & 0.0 & 6.0 & 9.0 & 12.0 & 12.0 & 16.0 & 14.0 \\
\hline Tunisia & 0.0 & 6.0 & 9.0 & 12.0 & 13.0 & 17.0 & 16.0 \\
\hline Uruguay & 0.0 & 6.0 & 9.0 & 11.0 & 12.0 & 16.0 & 15.0 \\
\hline
\end{tabular}

a: The category does not apply in the country concerned. Data are therefore missing.

Indices are constructed by taking always the highest level for each father or mother and have the following categories: (0) None; (1) ISCED 1 (primary education); (2) ISCED 2 (lower secondary); (3) ISCED Level 3B or 3C (vocational/pre-vocational upper secondary); (4) ISCED 3A (upper secondary) and/or ISCED 4 (non-tertiary post-secondary); (5) ISCED 5B (vocational tertiary); and (6) ISCED 5A, 6 (theoretically oriented tertiary and post-graduate). Indices with these categories are provided for mother (MISCED) and father (FISCED) of the student. The index of the highest educational level of parents (HISCED) corresponds to the higher ISCED level of either parent.

The highest level of educational attainment of parents is also converted into an index of years of schooling (PARED) using the conversion coefficients shown in Table A9.1. 
As in PISA 2000, students reported the country of birth for themselves as well as their mothers and fathers (ST15Q01-ST15Q03). This time, national centres were encouraged to collect more detailed information on countries of birth, for example by including a list of countries where higher frequencies were expected. A variable with ISO codes (where applicable) is added to the international database. Indices with these ISO codes are provided for students (ISO_S) and mothers (ISO_M) and fathers (ISO_F) of the students.

The PISA 2003 index of immigrant background (IMMIG) has the following categories: (1) "native" students (those students born in the country of assessment or who had at least one parent born in the country) $)^{1}$; (2) "first generation" students (those born in the country of assessment but whose parent(s) were born in another country; and (3) "non-native" students (those students born outside the country of assessment and whose parents were also born in another country). Students with missing responses for either the student or for both parents, or for all three questions are given missing values.

\section{Language background (LANG and LANGN)}

The PISA 2003 index of foreign language spoken at home (LANG) is derived from students' responses to the item ST16Q01 asking if the language spoken at home most of the time was the language of assessment, another official national language, another national dialect or language, or another language. In order to derive this index, responses are grouped into two categories: (1) language spoken at home most of the time is different from the language of assessment, from other official national languages and from other national dialects or languages; and (0) the language spoken at home most of the time is the language of assessment, is another official national language, or other national dialect or language.

Some countries collected more detailed information on language use at home, which is included in the database as the PISA 2003 index of language at home (national) (LANGN) with international language codes.

\section{Learning and instruction}

\section{Relative grade (GRADE)}

The PISA 2003 index of students' relative grades (GRADE) is derived both from the Student Questionnaire (ST01Q01) and from the Student Tracking Forms.

In order to adjust for between-country variation, the index of relative grade indicates whether students are at the modal grade in a country (value of 0 ), or whether they are below or above the modal grade ( $+\mathrm{x}$ grades, $-\mathrm{x}$ grades) .

\section{Expected educational level (SISCED)}

In PISA 2003, for the first time, students were asked about their educational aspirations. Students' responses to the items ST23Q01-ST23Q06 measuring expected educational levels are classified according to ISCED (OECD 1999).

The PISA 2003 index of expected educational level has the following categories: (1) None; (2) ISCED 2 (lower secondary); (3) ISCED Level 3B or 3C (vocational/prevocational upper secondary); (4) ISCED 3A (upper secondary) or ISCED 4 (non-tertiary post-secondary); (5) ISCED 5B (vocational tertiary); and (6) ISCED 5A, 6 (theoretically oriented tertiary and post-graduate). 
As part of the optional questionnaire on educational career, students in 24 countries were asked to write down their expected occupation and a description of this job (EC08Q01). The students' responses are coded to four-digit ISCO codes (ILO, 1990) and then mapped to the international socio-economic index of occupational status (ISEI) (Ganzeboom et al., 1992). The PISA 2003 index of expected occupational status (BSMJ) is derived from recoding ISCO codes into ISEI scores. Higher values on this index indicate higher level of expected occupational status.

This index is also recoded into an index with four categories of expected occupational status (SSECATEG): (1) white collar high skilled occupation; (2) white collar low skilled occupation; (3) blue collar high skilled occupation; and (4) blue collar low skilled occupation, except the nonresponses which are maintained as missing or not applicable.

\section{Relative time spent on mathematics homework (RMHMWK)}

The PISA 2003 index of relative time spent on mathematics homework (RMHMWK) is derived from students' responses to the items ST29Q01 and ST33Q01 measuring time spent for mathematics and overall homework in hours. A value on this index indicates a ratio of time spent on mathematics homework to overall time spent on homework.

\section{Minutes of mathematics instruction (MMINS)}

The PISA 2003 index of minutes of mathematics instruction (MMINS) is calculated by multiplying the average length of a class period by the number of class periods receiving mathematics instruction. This index is derived from students' responses to the items ST35Q01 and ST35Q02 measuring average length of a class period and their instructional time in mathematics in class periods. In some countries the amount of instructional time in mathematics varies across the year. This index indicates current instruction minutes in mathematics received by each student.

\section{Minutes of overall school instruction (TMINS)}

The PISA 2003 index of minutes of overall school instruction (TMINS) is calculated by multiplying the average length of a class period by the number of class periods receiving instruction in all subjects (including mathematics). This index is derived from students' responses to the item ST35Q03 measuring the average length of a class period and the item below measuring the number of class periods per week.

\section{Relative instructional time on mathematics (PCMATH)}

The PISA 2003 index of relative instructional time on mathematics (PCMATH) is calculated by dividing the instructional time in minutes on mathematics by the overall instructional time in minutes.

\section{STUDENT-LEVEL SCALE INDICES}

\section{Student background}

\section{Computer facilities at home (COMPHOME)}

The PISA 2003 index of computer facilities at home (COMPHOME) is derived from students' responses to the three items listed below. These variables are binary and the scale construction is done through IRT scaling. Positive values on this index indicate higher levels of computer facilities at home. 


\begin{tabular}{ll}
\hline Q17 & Which of the following do you have in your home? \\
ST17Q04 & Yes \\
ST17Q05 & e) Educational software \\
ST17Q06 & f) A link to the Internet \\
\hline
\end{tabular}

Home educational resources (HEDRES)

The PISA 2003 index of home educational resources (HEDRES) is derived from students' responses to the five items listed below. A slightly modified set of items for the PISA 2000 index of home educational resources are used for this PISA 2003 index. These variables are binary and the scale construction is done through IRT scaling. Positive values on this index indicate higher levels of home educational resources.

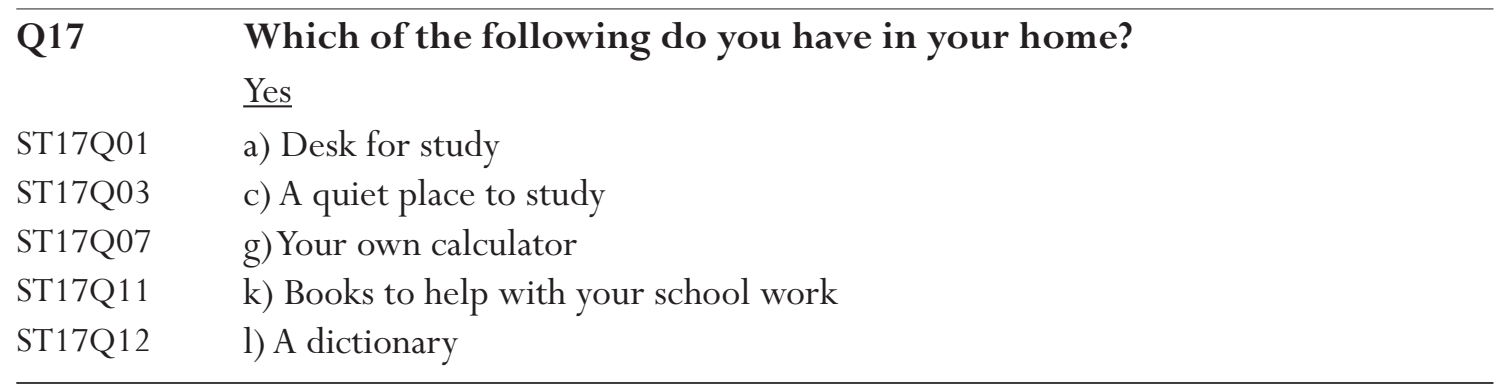

\section{Home possessions (HOMEPOS)}

The PISA 2003 index of home possessions (HOMEPOS) is derived from students' responses to the 14 items listed below. These variables are binary and the scale construction is done through IRT scaling. Positive values on this index indicate higher levels of home possessions.

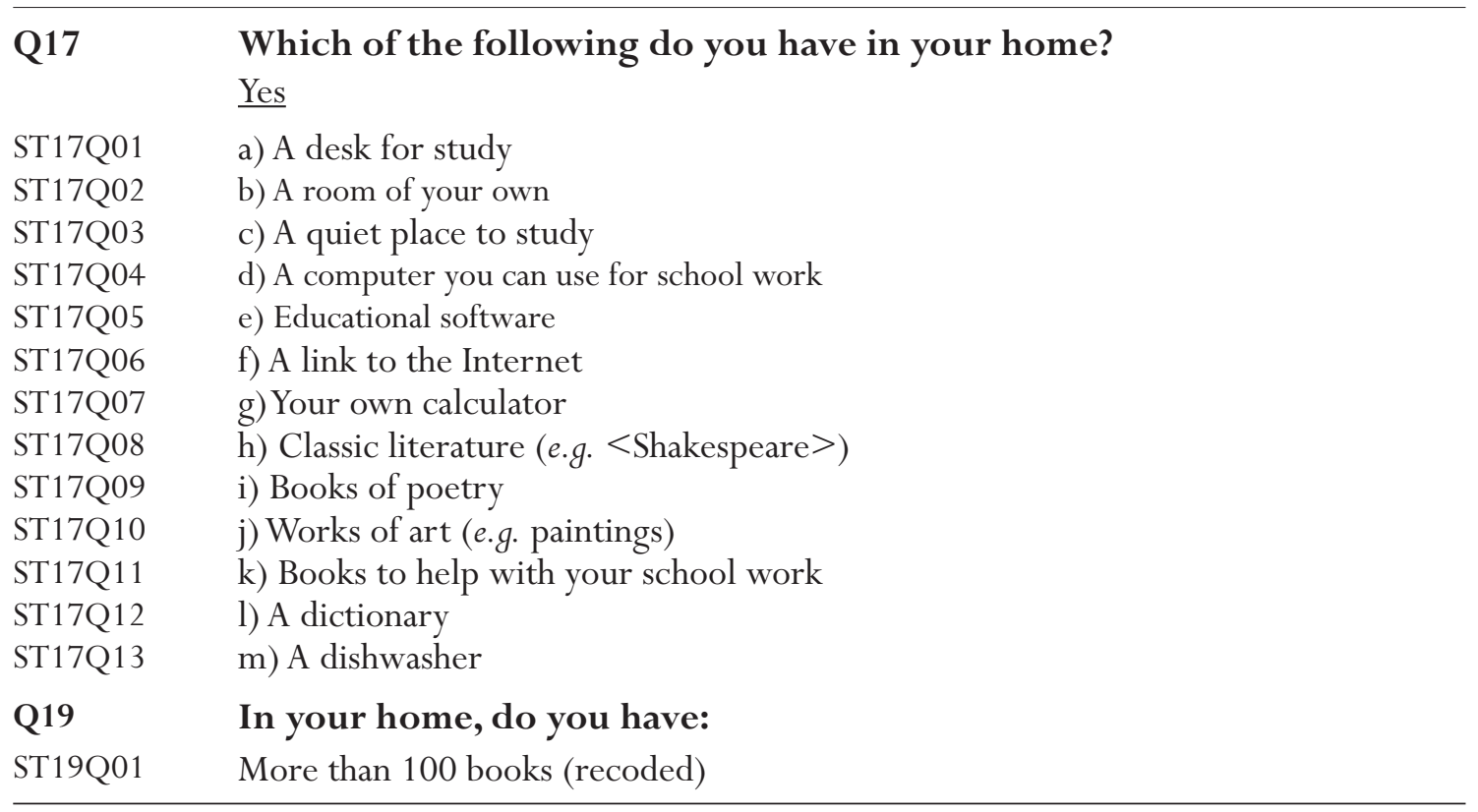




\section{Cultural possessions (CULTPOSS)}

The PISA 2003 index of cultural possession (CULTPOSS), which retains items used for the PISA 2000 index of cultural possessions, is derived from students' responses to the three items listed below. These variables are binary and the scale construction is done through IRT scaling. Positive values on this index indicate higher levels of cultural possessions.

\section{Q17 Which of the following do you have in your home?}

$\underline{\text { Yes }}$

ST17Q08 h) Classic literature (e.g. $<$ Shakespeare $>$ )

ST17Q09 i) Books of poetry

ST17Q10 j) Works of art (e.g. paintings)

\section{Economic, social and cultural status (ESCS)}

The PISA 2003 index of economic, social and cultural status (ESCS) is derived from three variables related to family background: the index of highest level of parental education in number of years of education according to the ISCED classification (PARED), the index of highest parental occupation status (HISEI) and the index of home possessions (HOMEPOS). Missing values for these three variables are imputed and then transformed to an international metric with OECD averages of 0 and OECD standard deviations of 1 . These OECD-standardised variables were used for a principal component analysis in order to obtain ESCS scores applying an OECD population weight giving each OECD country a weight of 1000. The PISA index of economic, social and cultural status (ESCS) is computed for PISA 2003 and also re-computed for the PISA 2000 data, but items and the wording of items are slightly different between PISA 2000 and PISA 2003. Further details concerning ESCS are found in PISA 2003 Technical Report (OECD, forthcoming).

\section{School climate}

\section{Attitudes towards school (ATSCHL)}

The PISA 2003 index of students' attitudes towards school (ATSCHL) is derived from students' responses to four items listed below. A four-point scale with the response categories recoded as "strongly agree" (=0); "agree" (=1); "disagree" $(=2)$; and "strongly disagree" $(=3)$ is used. As items ST24Q03 and ST24Q04 are inverted for IRT scaling, positive values on this index indicate students' positive attitudes toward school.

\section{Q24 Thinking about what you have learned in school:}

To what extent do you agree with the following statements?

\begin{tabular}{c|c|c|c}
\hline Strongly agree & Agree & Disagree & Strongly disagree \\
\hline
\end{tabular}

ST24Q01 a) School has done little to prepare me for adult life when I leave school.

ST24Q02 b) School has been a waste of time.

ST24Q03 c) School helped give me confidence to make decisions. $(+)$

ST24Q04 d) School has taught me things which could be useful in a job. $(+)$

(+) Item inverted for IRT scaling. 
The PISA 2003 index of student-teacher relations (STUREL) is derived from students' responses to the five items presented below. A four-point scale with the response categories recoded as "strongly agree" $(=0)$; "agree" $(=1)$; "disagree" $(=2)$; and "strongly disagree" $(=3)$ is used. All items are inverted for IRT scaling and positive values on this index indicate students' perception of good student-teacher relations at a school.

\section{Q26 Thinking about the teachers at your school:}

To what extent do you agree with the following statements?

\begin{tabular}{ll|c|c}
\hline \multicolumn{1}{c|}{ Strongly agree } & Agree & Disagree & Strongly disagree \\
\hline ST26Q01 & a) Students get along well with most teachers. $(+)$ \\
ST26Q02 & b) Most teachers are interested in students' well-being. $(+)$ & \\
ST26Q03 & c) Most of my teachers really listen to what I have to say. $(+)$ & \\
ST26Q04 & d) If I need extra help, I will receive it from my teachers. $(+)$ & \\
ST26Q05 & e) Most of my teachers treat me fairly. $(+)$ & \\
\hline
\end{tabular}

$(+)$ Item inverted for IRT scaling.

\section{Sense of belonging (BELONG)}

The PISA 2003 index of sense of belonging at school (BELONG) is derived from students' responses to the six items presented below. A four-point scale with the response categories recoded as "strongly agree" (=0); "agree" (=1); "disagree" $(=2)$; and "strongly disagree" $(=3)$ is used. As Items ST27Q02 and ST27Q03 are inverted for IRT scaling, positive values on this index indicate students' positive feelings about school.

\section{Q27 My school is a place where:}

\begin{tabular}{ll|l|c}
\hline \multicolumn{1}{c|}{ Strongly agree } & \multicolumn{1}{c|}{ Agree } & Disagree & Strongly disagree \\
\hline ST27Q01 & a) I feel like an outsider (or left out of things). & \\
ST27Q02 & b) I make friends easily. $(+)$ & \\
ST27Q03 & c) I feel like I belong. $(+)$ \\
ST27Q04 & d) I feel awkward and out of place. \\
ST27Q05 & e) Other students seem to like me. \\
ST27Q06 & f) I feel lonely.
\end{tabular}

$(+)$ Item inverted for IRT scaling.

\section{Self-related cognitions in mathematics}

Interest in and enjoyment of mathematics (INTMAT)

The PISA 2003 index of interest in and enjoyment of mathematics (INTMAT) is derived from students' responses to the four items listed below. A four-point scale with the response categories recoded as "strongly agree" $(=0)$; "agree" $(=1)$; "disagree" $(=2)$; and "strongly disagree" $(=3)$ is used. All items are inverted for IRT scaling and positive values on this index indicate higher levels of interest and enjoyment in mathematics. The PISA 2000 index of interest in mathematics was derived from a different set of items. 
Q30 Thinking about your views on mathematics:

To what extent do you agree with the following statements?

\begin{tabular}{|c|c|c|c|}
\hline \multicolumn{2}{|c|}{ Strongly agree } & Agree & Disagree \\
\hline ST30Q01 & \multicolumn{3}{|c|}{ a) I enjoy reading about mathematics. $(+)$} \\
\hline ST30Q03 & \multicolumn{3}{|c|}{ c) I look forward to my mathematics lessons. $(+)$} \\
\hline ST30Q04 & \multicolumn{3}{|c|}{ d) I do mathematics because I enjoy it. $(+)$} \\
\hline ST30Q06 & \multicolumn{3}{|c|}{ f) I am interested in the things I learn in mathematics. $(+)$} \\
\hline
\end{tabular}

(+) Item inverted for IRT scaling.

\section{Instrumental motivation in mathematics (INSTMOT)}

The PISA 2003 index of instrumental motivation in mathematics (INSTMOT) is derived from students' responses to the four items listed below. A four-point scale with the response categories recoded as "strongly agree" (=0), "agree" $(=1)$, "disagree" $(=2)$ and "strongly disagree" $(=3)$ is used. All items are inverted for IRT scaling and positive values on this index indicate higher levels of instrumental motivation to learn mathematics.

Q30 Thinking about your views on mathematics:

To what extent do you agree with the following statements?

\begin{tabular}{l|c|c|c} 
Strongly agree & Agree & Disagree & Strongly disagree
\end{tabular}

ST30Q02 b) Making an effort in mathematics is worth it because it will help me in the work that I want to do later on. $(+)$

ST30Q05 e) Learning mathematics is worthwhile for me because it will improve my career $<$ prospects, chances $>$. $(+)$

ST30Q07 g) Mathematics is an important subject for me because I need it for what I want to study later on. $(+)$

ST30Q08 h) I will learn many things in mathematics that will help me get a job. $(+)$

(+) Item inverted for IRT scaling.

\section{Mathematics self-efficacy (MATHEFF)}

The PISA 2003 index of mathematics self-efficacy (MATHEFF) is derived from students' responses to the eight items measuring the students' confidence with mathematical tasks as listed below. A four-point scale with the response categories recoded as "very confident" $(=0)$, "confident" $(=1)$, "not very confident" (=2) and "not at all confident" (=3) is used. All items are inverted for IRT scaling and positive values on this index indicate higher levels of self-efficacy in mathematics.

\begin{tabular}{|c|c|c|c|c|}
\hline Q31 & How co & you feel & having to do th & lowing calculations? \\
\hline & confident & Confident & Not very confident & Not at all confident \\
\hline ST31Q01 & a) Using & W & 8 & dville to Zedtown $(+)$ \\
\hline ST31Q02 & b) Calcul & ach cheap & would be after a & cent discount $(+)$ \\
\hline ST31Q03 & c) Calcul & hany square & s of tiles you need & ver a floor. $(+)$ \\
\hline ST31Q04 & d) Under & aphs preser & newspapers. $(+)$ & \\
\hline ST31Q05 & e) Solving & $n$ like $3 x+$ & 7. $(+)$ & \\
\hline ST31Q06 & f) Finding & distance be & two places on a $n$ & a $1: 10,000$ scale. $(+)$ \\
\hline ST31Q07 & g) Solving & like $2(x$ & $+3)(x-3)$ & \\
\hline ST31Q08 & h) Calcul & trol consul & rate of a car. $(+)$ & \\
\hline
\end{tabular}

(+) Item inverted for IRT scaling. 
The PISA 2003 index of mathematics anxiety, which is concerned with feelings of helplessness and emotional stress when dealing with mathematics, is derived from students' responses to the five items presented below. A four-point scale with the response categories recoded as "strongly agree" $(=0)$, "agree" (=1), "disagree" $(=2)$ and "strongly disagree" $(=3)$ is used. All items are inverted for IRT scaling and positive values on this index indicate higher levels of mathematics anxiety.

Q32 Thinking about studying mathematics: To what extent do you agree with the following statements?

\begin{tabular}{ll|c}
\hline \multicolumn{1}{c|}{ Strongly agree } & Agree & Disagree \\
\hline ST32Q01 & a) I often worry that it will be difficult for me in mathematics classes. $(+)$ \\
ST32Q03 & c) I get very tense when I have to do mathematics homework. $(+)$ \\
ST32Q05 & e) I get very nervous doing mathematics problems. $(+)$ \\
ST32Q08 & h) I feel helpless when doing a mathematics problem. $(+)$ \\
ST32Q10 & j) I worry that I will get poor $<$ marks $>$ in mathematics. $(+)$
\end{tabular}

(+) Item inverted for IRT scaling.

\section{Mathematics self-concept (SCMAT)}

The PISA 2003 index of mathematics self-concept is derived from students' responses to the five items. A four-point scale with the response categories recoded as "strongly agree" $(=0)$, "agree" $(=1)$, “disagree" (=2) and "strongly disagree" (=3) is used. Items ST32Q04, ST32Q06 and ST32Q07 are inverted for IRT scaling and positive values on this index indicate a positive self-concept in mathematics. The PISA 2000 index of self-concept in mathematics was derived from a different set of items.

\section{Q32 Thinking about studying mathematics:}

To what extent do you agree with the following statements?

\begin{tabular}{|c|c|c|c|c|}
\hline \multicolumn{2}{|c|}{ Strongly agree (1) } & Agree (2) & Disagree (3) & Strongly disagree (4) \\
\hline T32Q02 & \multicolumn{4}{|c|}{ b) I am just not good at mathematics. } \\
\hline ST32Q04 & \multicolumn{4}{|c|}{ d) I get good $<$ marks $>$ in mathematics. $(+)$} \\
\hline ST32Q06 & \multicolumn{4}{|c|}{ f) I learn mathematics quickly. $(+)$} \\
\hline ST32Q07 & \multicolumn{4}{|c|}{ g) I have always believed that mathematics is one of my best subjects. $(+)$} \\
\hline ST32Q09 & \multicolumn{4}{|c|}{ i) In my mathematics class, I understand even the most difficult work. $(+)$} \\
\hline
\end{tabular}

$(+)$ Item inverted for IRT scaling.

\section{Learning strategies and preferences in mathematics}

Memorisation/rehearsal learning strategies (MEMOR)

The PISA 2003 index of memorisation/rehearsal learning strategies is derived from students' responses to the four items measuring preference for memorisation/rehearsal as a learning strategy for mathematics as listed below. A four-point scale with the response categories recoded as "strongly agree" $(=0)$, "agree" $(=1)$, "disagree" $(=2)$ and "strongly disagree" $(=3)$ is used. All items are inverted for IRT scaling and positive values on this index indicate preferences for this learning strategy. The PISA 2000 index of memorisation strategies was derived from a different set of items asking not only asking about mathematics, but about learning strategies in general. 
Q34 There are different ways of studying mathematics.

To what extent do you agree with the following statements?

\begin{tabular}{|c|c|c|c|c|}
\hline \multicolumn{2}{|c|}{ Strongly agree } & Agree & Disagree & Strongly disagree \\
\hline ST34Q06 & \multicolumn{4}{|c|}{$\begin{array}{l}\text { f) I go over some problems in mathematics so often that I feel as if I could solve } \\
\text { them in my sleep. }(+)\end{array}$} \\
\hline ST34Q07 & \multicolumn{4}{|c|}{$\begin{array}{l}\text { g) When I study for mathematics, I try to learn the answers to problems off by } \\
\text { heart. }(+)\end{array}$} \\
\hline ST34Q09 & \multicolumn{4}{|c|}{$\begin{array}{l}\text { i) In order to remember the method for solving a mathematics problem, I go } \\
\text { through examples again and again. }(+)\end{array}$} \\
\hline ST34Q13 & \multicolumn{4}{|c|}{ m) To learn mathematics, I try to remember every step in a procedure. $(+)$} \\
\hline
\end{tabular}

$(+)$ Item inverted for IRT scaling.

\section{Elaboration learning strategies (ELAB)}

The PISA 2003 index of elaboration learning strategies is derived from students' responses to the five items measuring preference for elaboration as a learning strategy as presented below. A fourpoint scale with the response categories recoded as "strongly agree" $(=0)$, "agree" $(=1)$, "disagree" $(=2)$ and "strongly disagree" (=3) is used. All items are inverted for IRT scaling and positive values on this index indicate preferences for this learning strategy. The PISA 2000 index of elaboration strategies was derived from a different set of items asking about learning strategies in general.

\section{Q34 There are different ways of studying mathematics.}

To what extent do you agree with the following statements?

\begin{tabular}{ll|c|c}
\hline \multicolumn{1}{c}{ Strongly agree } & Agree & Disagree & Strongly disagree \\
\hline ST34Q02 & $\begin{array}{l}\text { b) When I am solving mathematics problems, I often think of new ways to get the } \\
\text { answer. }(+)\end{array}$ \\
ST34Q05 & e) I think how the mathematics I have learnt can be used in everyday life. $(+)$ \\
ST34Q08 & $\begin{array}{l}\text { h) I try to understand new concepts in mathematics by relating them to things I } \\
\text { already know. }(+)\end{array}$ \\
ST34Q11 & $\begin{array}{l}\text { k) When I am solving a mathematics problem, I often think about how the solution } \\
\text { might be applied to other interesting questions. }(+)\end{array}$ \\
ST34Q14 & n) When learning mathematics, I try to relate the work to things I have learnt in \\
other subjects. $(+)$
\end{tabular}

$(+)$ Item inverted for IRT scaling.

\section{Control learning strategies (CSTRAT)}

The PISA 2003 index of control learning strategies (CSTRAT) is derived from students' responses to the five items measuring preference for control as a learning strategy as listed below. A four-point scale with the response categories recoded as "strongly agree" $(=0)$, "agree" $(=1)$, "disagree" $(=2)$ and "strongly disagree" (=3) is used. All of them are inverted for IRT scaling and positive values on this index indicate preferences for this learning strategy. The PISA 2000 index of control strategies was derived from a different set of items asking about learning strategies in general. 
To what extent do you agree with the following statements?

\begin{tabular}{|c|c|c|c|c|}
\hline \multicolumn{2}{|c|}{ Strongly agree } & Agree & Disagree & Strongly disagree \\
\hline ST34Q01 & \multicolumn{4}{|c|}{$\begin{array}{l}\text { a) When I study for a mathematics test, I try to work out what are the most } \\
\text { important parts to learn. }(+)\end{array}$} \\
\hline ST34Q03 & \multicolumn{4}{|c|}{$\begin{array}{l}\text { c) When I study mathematics, I make myself check to see if I remember the work } \\
\text { I have already done. }(+)\end{array}$} \\
\hline ST34Q04 & \multicolumn{4}{|c|}{$\begin{array}{l}\text { d) When I study mathematics, I try to figure out which concepts I still have not } \\
\text { understood properly. }(+)\end{array}$} \\
\hline ST34Q10 & \multicolumn{4}{|c|}{$\begin{array}{l}\text { j) When I cannot understand something in mathematics, I always search for more } \\
\text { information to clarify the problem. }(+)\end{array}$} \\
\hline ST34Q12 & \multicolumn{4}{|c|}{ l) When I study mathematics, I start by working out exactly what I need to learn. $(+)$} \\
\hline
\end{tabular}

$(+)$ Item inverted for IRT scaling.

\section{Preference for competitive learning situations (COMPLRN)}

The PISA index of preference for competitive learning situation (COMPLRN) is derived from students' responses to the five items measuring preferences for competitive learning situations as listed below. A four-point scale with the response categories recoded as "strongly agree" $(=0)$, "agree" (=1), "disagree" (=2) and "strongly disagree" (=3) is used. All items are inverted for IRT scaling and positive values on this index indicate preferences for competitive learning situations. The PISA 2000 index of competitive learning was derived from a different set of items asking about learning situations in general.

\section{Q37 Thinking about your <mathematics> classes:}

To what extent do you agree with the following statements?

\begin{tabular}{|c|c|c|c|c|}
\hline \multicolumn{2}{|c|}{ Strongly agree } & Agree & Disagree & Strongly disagree \\
\hline ST37Q01 & \multicolumn{4}{|c|}{ a) I would like to be the best in my class in mathematics. $(+)$} \\
\hline ST37Q03 & \multicolumn{4}{|c|}{$\begin{array}{l}\text { c) I try very hard in mathematics because I want to do better in the exams than the } \\
\text { others. }(+)\end{array}$} \\
\hline ST37Q05 & \multicolumn{4}{|c|}{ e) I make a real effort in mathematics because I want to be one of the best. $(+)$} \\
\hline ST37Q07 & \multicolumn{4}{|c|}{ g) In mathematics I always try to do better than the other students in my class. $(+)$} \\
\hline ST37Q10 & \multicolumn{4}{|c|}{ j) I do my best work in mathematics when I try to do better than others. $(+)$} \\
\hline
\end{tabular}

$(+)$ Item inverted for IRT scaling.

\section{Preference for co-operative learning situations (COOPLRN)}

The PISA index of preference for co-operative learning situation (COOPLRN) is derived from students' responses to the five items measuring preferences for co-operative learning situations as listed below. A four-point scale with the response categories recoded as "strongly agree" $(=0)$, "agree" $(=1)$, "disagree" $(=2)$ and "strongly disagree" $(=3)$ is used. All of them are inverted for IRT scaling and positive values on this index indicate preferences for co-operative learning situations. The PISA 2000 index of co-operative learning was derived from a different set of items asking about learning situations in general. 
Q37 Thinking about your < mathematics>classes:

To what extent do you agree with the following statements?

\begin{tabular}{|c|c|c|c|c|}
\hline \multicolumn{2}{|c|}{ Strongly agree } & Agree & Disagree & Strongly disagree \\
\hline T37Q02 & \multicolumn{4}{|c|}{ b) In mathematics I enjoy working with other students in groups. $(+)$} \\
\hline ST37Q04 & \multicolumn{4}{|c|}{$\begin{array}{l}\text { d) When we work on a project in mathematics, I think that it is a good idea to } \\
\text { combine the ideas of all the students in a group. }(+)\end{array}$} \\
\hline ST37Q06 & \multicolumn{4}{|c|}{ f) I do my best work in mathematics when I work with other students. $(+)$} \\
\hline ST37Q08 & \multicolumn{4}{|c|}{ h) In mathematics, I enjoy helping others to work well in a group. $(+)$} \\
\hline ST37Q09 & \multicolumn{4}{|c|}{ i) In mathematics I learn most when I work with other students in my class. $(+)$} \\
\hline
\end{tabular}

$(+)$ Item inverted for IRT scaling.

\section{Classroom climate}

\section{Teacher support (TEACHSUP)}

The PISA 2003 index of teacher support in mathematics lessons is derived from students' responses to the five items listed below. These are the slightly modified items used for the PISA 2000 index of teacher support in language lessons. A four-point scale with the response categories recoded as "every lesson" (=0), "most lessons" (=1), "some lessons" $(=2)$ and "never or hardly ever" (=3) is used. All items are inverted and positive values on this index indicate students' perceptions of higher levels of teacher support.

\section{Q38 How often do these things happen in your < mathematics $>$ lessons?}

\begin{tabular}{ll|c|c}
\hline \multicolumn{1}{c|}{ Every lesson } & \multicolumn{1}{c|}{ Most lessons } & Some lessons & Never or hardly ever \\
\hline ST38Q01 & a) The teacher shows an interest in every student's learning. $(+)$ \\
ST38Q03 & c) The teacher gives extra help when students need it. $(+)$ \\
ST38Q05 & e) The teacher helps students with their learning. $(+)$ \\
ST38Q07 & g) The teacher continues teaching until the students understand. $(+)$ \\
ST38Q10 & j) The teacher gives students an opportunity to express opinions. $(+)$
\end{tabular}

(+) Item inverted for IRT scaling.

\section{Disciplinary climate (DISCLIM)}

The PISA 2003 index of disciplinary climate in mathematics lessons is derived from students' responses to the five items listed below. These are the slightly modified items used for the PISA 2000 index of disciplinary climate in language lessons. A four-point scale with the response categories recoded as "every lesson" (=0), "most lessons" (=1), "some lessons" $(=2)$ and "never or hardly ever" $(=3)$ is used. The items are not inverted for IRT scaling and positive values on this index indicate perceptions of a positive disciplinary climate.

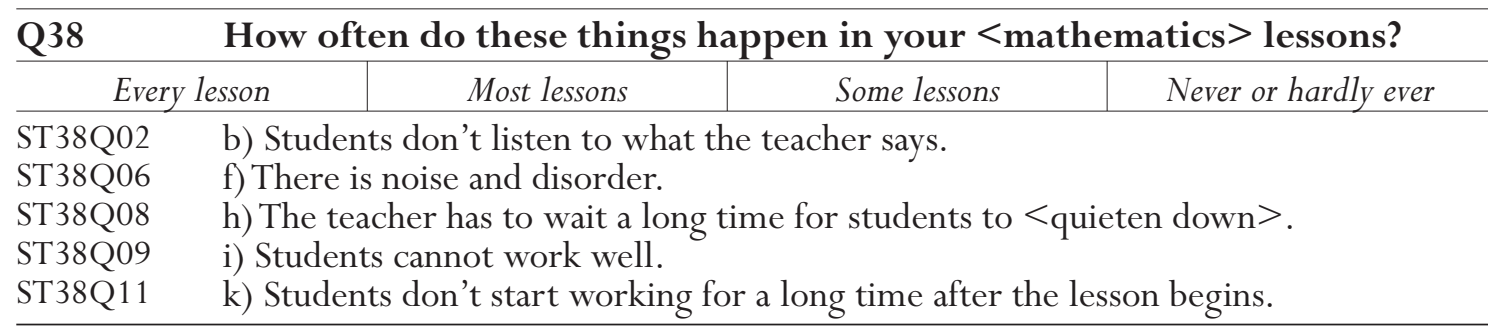




\section{ICT Internet/entertainment use (INTUSE)}

The PISA 2003 index of ICT internet/entertainment use (INTUSE) is derived from students' responses to the six items measuring the frequency of different types ICT use as listed below. A fivepoint scale with the response categories recoded as "almost every day" $(=0)$, "a few times each week" $(=1)$, "between once a week and once a month" $(=2)$, "less than once a month" $(=3)$ and "never" $(=4)$ is used. All items are inverted for IRT scaling and positive values on this index indicate high frequencies of ICT internet/entertainment use.

Q5 How often do you use:

\begin{tabular}{l|c|c|c|c} 
Almost every day & $\begin{array}{c}\text { A few times each } \\
\text { week }\end{array}$ & $\begin{array}{c}\text { Between once a week } \\
\text { and once a month }\end{array}$ & $\begin{array}{c}\text { Less than once a } \\
\text { month }\end{array}$ & Never \\
\hline
\end{tabular}

IC05Q01 a) The Internet to look up information about people, things, or ideas? $(+)$

IC05Q02 b) Games on a computer? (+)

IC05Q04 d) The Internet to collaborate with a group or team? (+)

IC05Q06 f) The Internet to download software? (+)

IC05Q10 j) The Internet to download music? (+)

IC05Q12 l) A computer for electronic communication (e.g. e-mail or "chat rooms")? (+)

$(+)$ Item inverted for IRT scaling.

\section{ICT program/software use (PRGUSE)}

The PISA 2003 index of ICT program/software use (PRGUSE) is derived from students' responses to the six items listed below. A five-point scale with the response categories recoded as "almost every day" $(=0)$, "a few times each week" $(=1)$, "between once a week and once a month" $(=2)$, "less than once a month" (=3) and "never" (=4) is used. All items are inverted for IRT scaling and positive values on this index indicate high frequencies of ICT program/software use.

\begin{tabular}{|c|c|c|c|c|c|}
\hline \multicolumn{6}{|c|}{ Q5 How often do you use: } \\
\hline \multicolumn{2}{|c|}{ Almost every day } & A few times each week & $\begin{array}{l}\text { Between once a week } \\
\text { and once a month }\end{array}$ & $\begin{array}{l}\text { Less than once } \\
\text { a month }\end{array}$ & Never \\
\hline IC05Q03 & \multicolumn{5}{|c|}{ c) Word processing (e.g. Microsoft ${ }^{\circledR} \operatorname{Word}^{\circledR}$ or WordPerfect $\left.{ }^{\circledR}\right)$ ? $(+)$} \\
\hline IC05Q05 & \multicolumn{5}{|c|}{ e) Spreadsheets (e.g. IBM ${ }^{\circledR}$ Lotus 1-2-3 ${ }^{\circledR}$ or Microsoft $^{\circledR}$ Excel $\left.^{\circledR}\right)$ ? $(+)$} \\
\hline IC05Q07 & \multicolumn{5}{|c|}{ g) Drawing, painting or graphics programs on a computer? $(+)$} \\
\hline IC05Q08 & \multicolumn{5}{|c|}{ h) Educational software such as mathematics programs? $(+)$} \\
\hline IC05Q09 & \multicolumn{5}{|c|}{ i) The computer to help you learn school material? $(+)$} \\
\hline IC05Q11 & \multicolumn{5}{|c|}{ k) The computer for programming? $(+)$} \\
\hline
\end{tabular}

(+) Item inverted for IRT scaling. 


\section{Confidence in ICT routine tasks (ROUTCONF)}

The PISA index of confidence in ICT routine tasks (ROUTCONF) is derived from students' responses to the 11 items on self-confidence with ICT tasks. A four-point scale with the response categories recoded as "I can do this very well by myself" $(=0)$, "I can do this with help from someone" $(=1)$, "I know what this means but I cannot do it" $(=2)$ and "I don't know what this means" (=3) is used. All items are inverted for IRT scaling and positive values on this index indicate high self-confidence in ICT routine tasks.

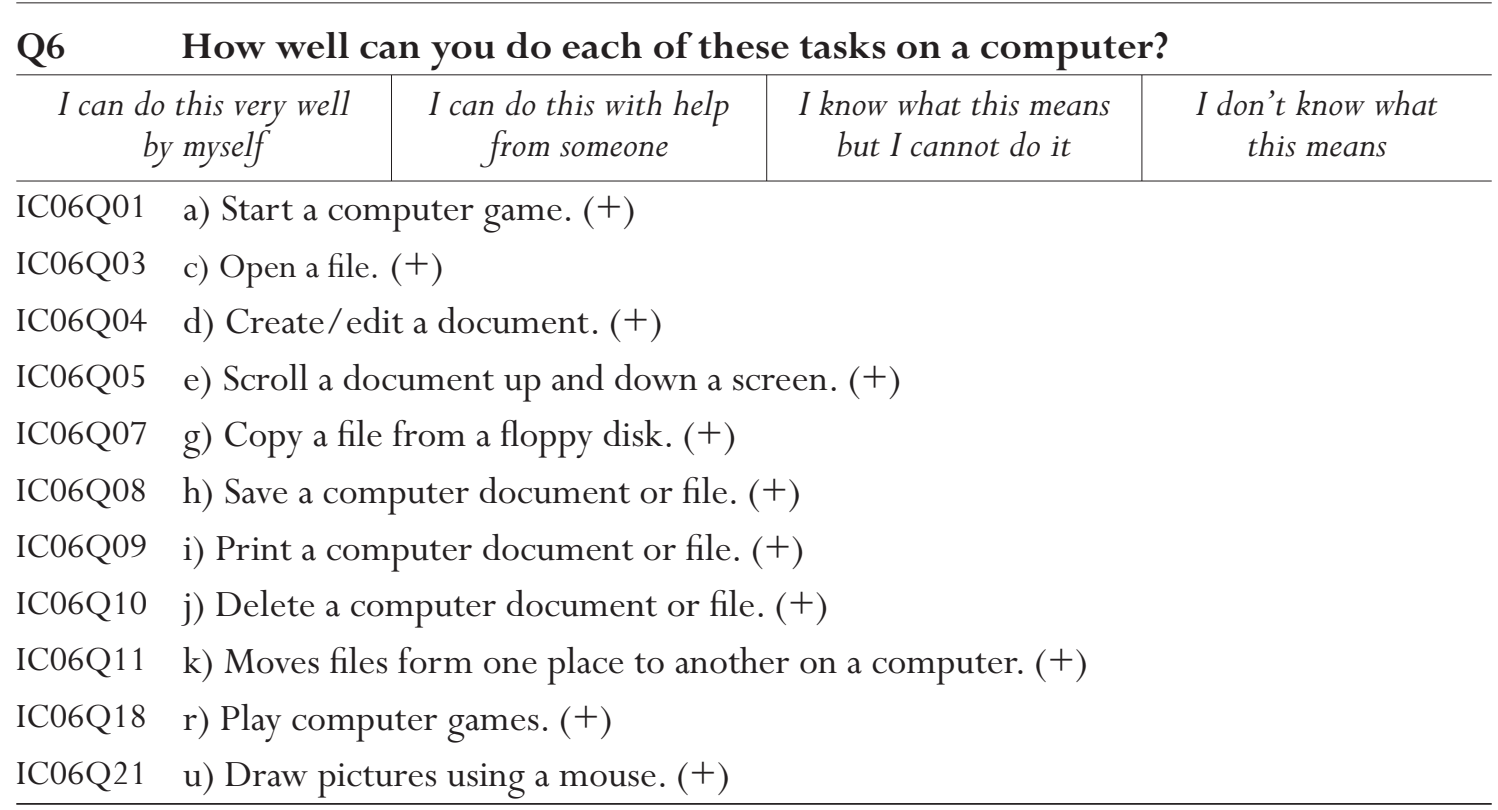

$(+)$ Item inverted for IRT scaling.

\section{Confidence in ICT Internet tasks (INTCONF)}

The PISA 2003 index of confidence in ICT internet tasks is derived from students' responses to the five items listed below. A four-point scale with the response categories recoded as "I can do this very well by myself" $(=0)$, "I can do this with help from someone" $(=1)$, "I know what this means but I cannot do it" (=2), and "I don't know what this means" $(=3)$ is used. All items are inverted for IRT scaling and positive values on this index indicate high self-confidence in ICT internet tasks.

\begin{tabular}{l|c|c|c}
\hline Q6 How well can you do each of these tasks on a computer? \\
\hline $\begin{array}{c}\text { I can do this very well } \\
\text { by myself }\end{array}$ & $\begin{array}{c}\text { I can do this with help } \\
\text { from someone }\end{array}$ & $\begin{array}{c}\text { I know what this means } \\
\text { but I cannot do it }\end{array}$ & $\begin{array}{c}\text { I don't know what } \\
\text { this means }\end{array}$ \\
\hline
\end{tabular}

IC06Q12 l) Get on to the Internet. (+)

IC06Q13 m) Copy or download files from the Internet. (+)

IC06Q14 n) Attach a file to an e-mail message. $(+)$

IC06Q19 s) Download music from the Internet. $(+)$

IC06Q22 v) Write and send e-mails. (+)

(+) Item inverted for IRT scaling. 
The PISA 2003 index of confidence in ICT high level tasks (HIGHCONF) is derived from students' responses to the seven questions listed below. A four-point scale with the response categories recoded as "I can do this very well by myself" $(=0)$, "I can do this with help from someone" $(=1)$, "I know what this means but I cannot do it" (=2), and “I don't know what this means" (=3) is used. All items are inverted for IRT scaling and positive values on this index indicated high self-confidence in ICT high level tasks.

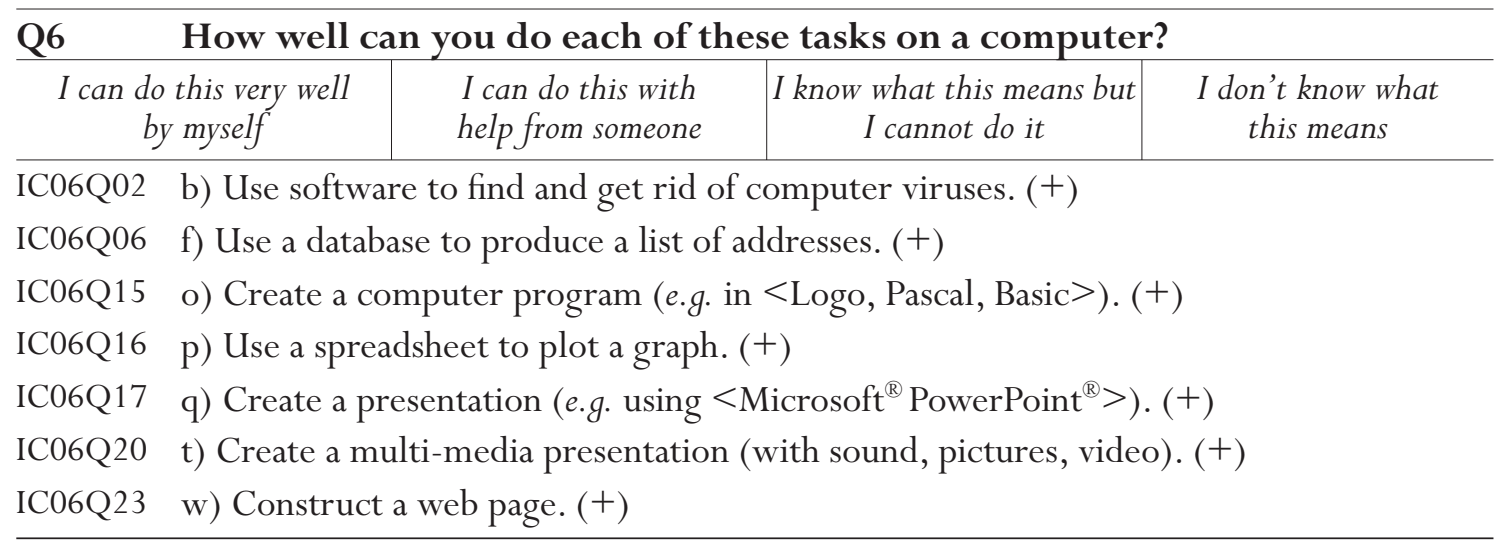

(+) Item inverted for IRT scaling.

\section{Attitudes toward computers (ATTCOMP)}

The PISA 2003 index of attitudes toward computers is derived from students' responses to the four items listed below. A four-point scale with the response categories recoded as "strongly agree" $(=0)$, "agree" $(=1)$, "disagree" $(=2)$, and "strongly agree" $(=3)$ is used. All items are inverted for IRT scaling and positive values on the index indicate positive attitudes toward computers. Due to the modifications in the item format and wording, this PISA 2003 index is not entirely comparable to the PISA 2000 index of interest in computers which was using a dichotomous form (Yes/No).

\section{Q7 Thinking about your experience with computers:}

To what extent do you agree with the following statements?

\begin{tabular}{l|c|c}
\hline \multicolumn{1}{c|}{ Strongly agree } & Agree & Disagree \\
\hline IC07Q01 & a) It is very important to me to work with a computer. $(+)$ \\
IC07Q02 & b) To play or work with a computer is really fun. $(+)$ \\
IC07Q03 & c) I use a computer because I am very interested. $(+)$ \\
IC07Q04 & d) I lose track of time when I am working with the computer. $(+)$ \\
\hline
\end{tabular}

(+) Item inverted for IRT scaling.

\section{SCHOOL-LEVEL SIMPLE INDICES}

\section{School characteristics}

\section{School size (SCHLSIZE)}

Similar to PISA 2000, the PISA 2003 index of school size (SCHLSIZE) is derived from summing school principals' responses to the number of girls and boys at a school (SC02Q01 and SC02Q02). Values on this index indicate total enrolment at school. 
Proportion of girls enrolled at school (PCGIRLS)

Similar to PISA 2000, the PISA 2003 index of the proportion of girls enrolled at school (PCGIRLS) is derived from school principals' responses regarding the number of girls divided by the total of girls and boys at a school (SC02Q01 and SC02Q02).

\section{School type (SCHLTYPE)}

Similar to PISA 2000, the PISA 2003 index of school type (SCHLTYPE) has three categories: (1) public schools controlled and managed by a public education authority or agency, (2) governmentdependent private schools controlled by non-government organisation or with a governing board not selected by a government agency which receive more than 50 per cent of their core funding from government agencies and (3) independent private schools controlled by a non-government organisation or with a governing board not selected by a government agency, which receive less than 50 per cent of their core funding from government agencies. This index is derived from school principals' responses to the items (SC03Q01 and SC04Q01 to SC04Q04) classifying schools into either public or private and identifying source of funding.

\section{Indicators of school resources}

\section{Availability of computers (RATCOMP, COMPWEB and COMPLAN)}

Similar to PISA 2000, the PISA 2003 index of availability of computers (RATCOMP) is derived from school principals' responses to the items measuring the availability of computers. It is calculated by dividing the number of computers at school (SC09Q01) by the number of students at school (SC02Q01 plus SC02Q02).

In addition, the following PISA 2003 indices on computer availability were developed as in PISA 2000:

- The PISA 2003 index of proportion of computers connected to a Local Area Network(COMPWEB) is derived from school principals' responses to the number of computers connected to the Web (SC09Q05) divided by the total number of computers (SC09Q01).

- The PISA 2003 index of proportion of computers connected to LAN (COMPLAN) is derived from school principals' responses to the number of computers connected to a local network (SC09Q06) divided by the total number of computers (SC09Q01).

\section{Quantity of teaching staff at school (STRATIO, PROPCERT and PROPOPED)}

As in PISA 2000, school principals are asked to report the number of full-time and part-time teachers at school in PISA 2003. The PISA 2003 indices of quantity of teaching staff at school are derived from questions asking about teachers in general (SC18Q11-SC18Q13 for full time staff and SC18Q21-SC18Q23 for part-time staff).

The PISA 2003 index of student/teacher ratio (STRATIO) is derived from school principals' reports of the school size (sum of SC02Q01 and SC02Q02) divided by the total number of teachers. The number of part-time teachers (SC18Q21) contributes 0.5 and the number of full-time teachers (SC18Q11) 1.0 to the total number of teachers. Values on this index indicate the number of students per teacher. 
The PISA 2003 index of proportion of fully certified teachers (PROPCERT) is derived from school principals' reports of the number of fully certified teachers (SC18Q12 plus 0.5* SC18Q22) divided by the total number of teachers (SC18Q11 plus 0.5* SC18Q21).

The PISA 2003 index of proportion of teachers who have an ISCED 5A qualification in pedagogy (PROPQPED) is derived from school principals' reports of the number of this kind of teachers (SC18Q13 plus $0.5 *$ SC18Q23) divided by the total number of teachers (SC18Q11 plus $0.5 *$ SC18Q21).

\section{Quantity of teaching staff for mathematics at school (SMRATIO, PROPMATH and PROPMA5A)}

The PISA 2003 indices of quantity of teaching staff for mathematics at school are derived from questions asking school principals to report the number of full-time and part-time teachers in total and with certain characteristics (SC19Q11-SC19Q15 for full-time staff and SC19Q21-SC19Q25 for part-time staff).

The PISA 2003 index of student/mathematics teacher ratio (SMRATIO) is derived from the school principals' reports of the school size (sum of SC02Q01 and SC02Q02) divided by the total number of mathematics teachers. The number of part-time mathematics teachers (SC19Q21) contributes 0.5 and the number of full-time mathematics teachers (SC19Q11) 1.0 to the total number of teachers.

The PISA 2003 index of proportion of mathematics teachers (PROPMATH) is derived from school principals' reports of the number of mathematics teachers (SC19Q11 plus $0.5 *$ SC19Q21) divided by the total number of teachers (SC18Q11 plus $0.5 *$ SC18Q21).

The PISA 2003 index of proportion of mathematics teachers with an ISCED5A qualification and a major in mathematics (PROPMA5A) is derived from school principals' reports of the number of the mathematics teachers with this qualification (SC19Q12 plus $0.5 *$ SC19Q22) divided by the total number of mathematics teachers (SC19Q11 plus 0.5* SC19Q21).

\section{Admittance policies and instructional context}

\section{School selectivity (SELECT)}

The PISA 2003 index of school selectivity (SELECT) is derived from school principals' responses to the items SC10Q02 and SC10Q06. Based on school principals' responses to these two items, schools are categorised into four different categories: (1) schools where none of these factors is considered for student admittance, (2) schools considering at least one of these factors, (3) schools giving high priority to at least one of these factors, and (4) schools where at least one of these factors is a pre-requisite for student admittance. Item SC10Q01 was not included because "residence in a particular area" is not a factor for selecting individual students. These items are similar to those used in PISA 2000, but the wording is slightly different.

\section{Use of assessments (ASSESS)}

The PISA 2003 index of use of assessments (ASSESS) is derived from school principals' responses to the items SC12Q01-SC12Q05. All five items are recoded into numerical values, which approximately reflect frequency of assessments per year ("Never" $=0, " 1-2$ times a year" $=1.5, " 3-5$ times a year" $=4$, "Monthly" $=8$, and "More than once a month" $=12$ ). This index is calculated as the sum of these 
$\underset{\times}{a}$ recoded items and then divided into three categories: (1) less than 20 times a year, (2) 20-39 times a year, and (3) more than 40 times a year.

\section{Ability grouping (ABGROUP)}

The PISA index of ability grouping between classes (ABGROUP) is derived from items SC16Q01 and SC16Q02 measuring the extent to which their school organises instruction differently for student with different abilities. Based on school principals' response to these two items, schools are assigned three categories: (1) schools with no ability grouping between any classes, (2) schools with one of these forms of ability grouping between classes for some classes, and (3) schools with one of these forms of ability grouping for all classes.

\section{School offering mathematics activities (MACTIV)}

The PISA 2003 index of mathematics activity index (MACTIV) is derived from five items (SC17Q01SC17Q05) measuring what activities to promote engagement with mathematics occur at their school. The number of different activities occurring at school is counted.

\section{School offering extension courses (number of types) (EXCOURSE)}

The PISA 2003 index of school offering extension courses (EXCOURSE) is derived from two items (SC17Q01 and SC17Q02) which are also used for the index of school offering mathematics activities (MACTIV). This index is computed as the sum of extension course types offered at school: (0) none, (1) either remedial or enrichment, and (2) both.

\section{School management}

\section{Resource autonomy (AUTRES)}

The PISA 2003 index of resource autonomy (AUTRES) is derived from school principals' responses to the six items (SC26Q01-SC26Q06) measuring who has the main responsibility for different types of decisions regarding the management of the school. This index indicates the number of decisions related to school resources that are a school responsibility.

\section{Curricular autonomy (AUTCURR)}

The PISA 2003 index of curricular autonomy (AUTCURR) is derived from school principals' responses to the four items (SC26Q08, SC26Q10-SC26Q12) measuring who has the main responsibility for different types of decisions regarding the management of the school. This index indicates the number of decisions related to curriculum that are a school responsibility.

\section{School climate}

Poor student-teacher relations (school average) (MSTREL)

The PISA 2003 index of poor student-teacher relations at school is derived from students' responses to the five items (ST26Q01-ST26Q05) measuring students' perception of various aspects of studentteacher relationships. The four-point scale with the response categories "strongly agree", "agree", "disagree", and "strongly disagree" was recoded into binary variables with "strongly disagree" coded 1 and other valid responses coded 0 . These responses were summarised by taking the average item response per student and computing the mean for each school. 


\section{SCHOOL-LEVEL SCALE INDICES}

\section{School resources}

\section{Quality of schools' physical infrastructure (SCMATBUI)}

The PISA 2003 index of quality of schools' physical infrastructure (SCMATBUI) is derived from school principals' responses to the three items below measuring the school principal's perceptions of potential factors hindering instruction at school. Similar items were used in PISA 2000, but the question format and item wording have been modified for PISA 2003. A four-point scale with the response categories recoded as "not at all” (=0), "very little" $(=1)$, "to some extent" $(=2)$, and "a lot" $(=3)$ is used. All items are inverted for IRT scaling and positive values on this index indicate positive evaluations of this aspect.

Q8 Is your school's capacity to provide instruction hindered by a shortage or inadequacy of any of the following?

\begin{tabular}{|c|c|c|c|}
\hline Not at all & Very little & To some extent & A lot \\
\hline \multicolumn{4}{|c|}{ SC08Q11 k) School buildings and grounds (+) } \\
\hline SC08Q12 l) Heati & \multicolumn{3}{|c|}{ l) Heating/cooling and lighting systems $(+)$} \\
\hline SC08Q13 m) Inst & \multicolumn{3}{|c|}{ m) Instructional space (e.g. classrooms) $(+)$} \\
\hline
\end{tabular}

$(+)$ Item inverted for IRT scaling.

\section{Quality of schools' educational resources (SMATEDU)}

The PISA 2003 index of quality of schools' educational resources (SMATEDU) is derived from school principals' responses to the seven items below measuring the school principal's perceptions of potential factors hindering instruction at school. Similar items were used in PISA 2000, but question format and item wording have been modified for PISA 2003. A four-point scale with the response categories recoded as "not at all" $(=0)$, "very little" $(=1)$, "to some extent" $(=2)$, and "a lot" $(=3)$ is used. All items are inverted for IRT scaling and positive values on this index indicate positive evaluations of this aspect.

\section{Q8 Is your school's capacity to provide instruction hindered by a shortage or inadequacy of any of the following?}

\begin{tabular}{l|c|c|c}
\hline \multicolumn{1}{c|}{ Not at all } & Very little & To some extent & A lot \\
\hline SC08Q09 & i) Instructional materials $(e . g$. textbooks $)(+)$ \\
SC08Q15 & o) Computers for instruction $(+)$ \\
SC08Q16 & p) Computer software for instruction $(+)$ \\
SC08Q17 & q) Calculators for instruction $(+)$ \\
SC08Q18 & r) Library materials $(+)$ \\
SC08Q19 & s) Audio-visual resources $(+)$ \\
SC08Q20 & t) Science laboratory equipment and materials $(+)$
\end{tabular}

$(+)$ Item inverted for IRT scaling. 


\section{Teacher shortage (TCSHORT)}

The PISA 2003 index of teacher shortage (TCSHORT) is derived from school principals' responses to the following four items measuring the school principal's perceptions of potential factors hindering instruction at school. Similar items were used in PISA 2000 but question format and item wording have been modified for PISA 2003. Furthermore, for PISA 2003 these items were administered together with the items on the quality of physical environment and educational resources. A fourpoint scale with the response categories recoded as "not at all" (=0), "very little" $(=1)$, "to some extent" (=2), and "a lot" (=3) is used. The items are not inverted for IRT scaling and positive values on this index indicate school principal's reports of teacher shortage at a school.

\begin{tabular}{|c|c|c|c|c|}
\hline \multirow[t]{2}{*}{ Q8 } & \multicolumn{4}{|c|}{$\begin{array}{l}\text { Is your school's capacity to provide instruction hindered by a shortage } \\
\text { or inadequacy of any of the following? }\end{array}$} \\
\hline & ot at all & Very little & To some extent & A lot \\
\hline \multicolumn{5}{|c|}{ SC08Q01 a) Availability of qualified mathematics teachers } \\
\hline SC08Q02 & \multicolumn{4}{|c|}{ b) Availability of qualified science teachers } \\
\hline SC08Q03 & \multicolumn{4}{|c|}{ c) Availability of qualified $<$ test language $>$ teachers } \\
\hline SC08Q05 & \multicolumn{4}{|c|}{ e) Availability of qualified foreign language teachers } \\
\hline SC08Q06 & \multicolumn{4}{|c|}{ f) Availability of experienced teachers } \\
\hline
\end{tabular}

\section{School climate}

School principals' perceptions of teacher morale and commitment (TCMORALE)

The PISA 2003 index of school principals' perceptions of teacher morale and commitment (TCMORALE) is derived from school principals' responses to the following four items measuring the school principal's perceptions of teachers at school. Similar items were used in PISA 2000, but question format has been modified for PISA 2003. The categories "disagree" and "strongly disagree" were collapsed into one category for IRT scaling because of very few responses in the category of "strongly disagree". Response categories of four-point scale items are recoded as "strongly agree" $(=0)$, "agree" (=1), and "disagree/strongly disagree" $(=2)$. All items are inverted for IRT scaling and positive values on this index indicate principals' reports of higher levels of teacher morale and commitment.

\section{Q24 Think about the teachers in your school:}

How much do you agree with the following statements?

\begin{tabular}{|c|c|c|c|}
\hline Strongly agree & Agree & Disagree & Strongly disagree \\
\hline \multicolumn{4}{|c|}{ SC24Q01 a) The morale of teachers in this school is high. $(+)$} \\
\hline \multicolumn{4}{|c|}{ SC24Q02 b) Teachers work with enthusiasm. $(+)$} \\
\hline \multicolumn{4}{|c|}{ SC24Q03 c) Teachers take pride in this school. $(+)$} \\
\hline \multicolumn{4}{|l|}{ SC24Q04 d) Teache1 } \\
\hline
\end{tabular}

(+) Item inverted for IRT scaling.

\section{School principals' perceptions of student morale and commitment (STMORALE)}

The PISA 2003 index of school principals' perceptions of student morale and commitment (STMORALE) is derived from school principals' responses to the following seven items measuring the school principal's perceptions of students at school. The items are, in part, a parallel to those on 
teacher morale and commitment. The categories "disagree" and "strongly disagree" were collapsed into one category for IRT scaling because of very few responses in the category of "strongly disagree". Response categories of four-point scale items are recoded as "strongly agree" $(=1)$, "agree" $(=2)$, and "disagree/strongly disagree" (=3). All items are inverted for IRT scaling and positive values on this index indicate principals' reports of higher levels of teacher morale and commitment.

\section{Q11 Think about the students in your school:}

How much do you agree with the following statements?

\begin{tabular}{|c|c|c|c|}
\hline Strongly agree & Agree & Disagree & Strongly disagree \\
\hline \multicolumn{4}{|c|}{ SC11Q01 a) Students enjoy being in school. $(+)$} \\
\hline SC11Q02 b) Studer & \multicolumn{3}{|c|}{ b) Students work with enthusiasm. $(+)$} \\
\hline SC11Q03 c) Studen & \multicolumn{3}{|c|}{ c) Students take pride in this school. $(+)$} \\
\hline SC11Q04 d) Studer & \multicolumn{3}{|c|}{ d) Students value academic achievement. $(+)$} \\
\hline SC11Q05 e) Studen & \multicolumn{3}{|c|}{ e) Students are cooperative and respectful. $(+)$} \\
\hline SC11Q06 f) Studen & \multicolumn{3}{|c|}{ f) Students value the education they can receive in this school. $(+)$} \\
\hline SC11Q07 g) Studen & \multicolumn{3}{|c|}{ g) Students do their best to learn as much as possible. $(+)$} \\
\hline
\end{tabular}

$(+)$ Item inverted for IRT scaling.

\section{School principals' perceptions of teacher-related factors affecting school climate (TEACBEHA)}

The PISA 2003 index of school principals' perceptions of teacher-related factors affecting school climate (TEACBEHA) is derived from school principals' responses to the following seven items measuring the school principal's perceptions of potential factors hindering the learning of students at school. These items were used in PISA 2000, but the question format and the wording of some items have been modified for PISA 2003. A four-point scale with the response categories recoded as "strongly agree" $(=0)$, "agree" $(=1)$, "disagree" $(=2)$, and "strongly disagree" $(=3)$ is used. All items are inverted for IRT scaling and positive values on this index indicate higher level of school principals' perceptions of teacher-related factors hindering students' learning.

Q25 In your school, to what extent is the learning of students hindered by:

\begin{tabular}{|c|c|c|c|}
\hline Not at all & Very little & To some extent & A lot \\
\hline \multicolumn{4}{|c|}{ ST25Q01 a) Teachers' low expectations of students? $(+)$} \\
\hline ST25Q03 c) Poor & \multicolumn{3}{|c|}{ c) Poor student-teacher relations? $(+)$} \\
\hline ST25Q05 e) Teac & \multicolumn{3}{|c|}{ e) Teachers not meeting individual students' needs? $(+)$} \\
\hline ST25Q06 f) Teach & \multicolumn{3}{|c|}{ f) Teacher absenteeism? $(+)$} \\
\hline ST25Q09 i) Staff & \multicolumn{3}{|c|}{ i) Staff resisting change? $(+)$} \\
\hline ST25Q11 k) Teac & \multicolumn{3}{|c|}{ k) Teachers being too strict with students? $(+)$} \\
\hline ST25Q13 m) Stu & \multicolumn{3}{|c|}{ m) Students not being encouraged to achieve their full potential? $(+)$} \\
\hline
\end{tabular}

$(+)$ Item inverted for IRT scaling.

\section{School principals' perceptions of student-related factors affecting school climate (STUDBEHA)}

The PISA 2003 index of school principals' perceptions of student-related factors affecting school climate (STUDBEHA) is derived from school principals' responses to the following six items measuring the school principals' perceptions of potential factors hindering the learning of students at school. These items were used in PISA 2000, but the question format and the wording of some items have been modified for PISA 2003. A four-point scale with the response categories recoded 
as "strongly agree" (=0), "agree" (=1), "disagree" $(=2)$, and "strongly disagree" $(=3)$ is used. All items are inverted for IRT scaling and positive values on this index indicate higher level of school principals' perceptions of student-related factors hindering students' learning.

Q25 In your school, to what extent is the learning of students hindered by:

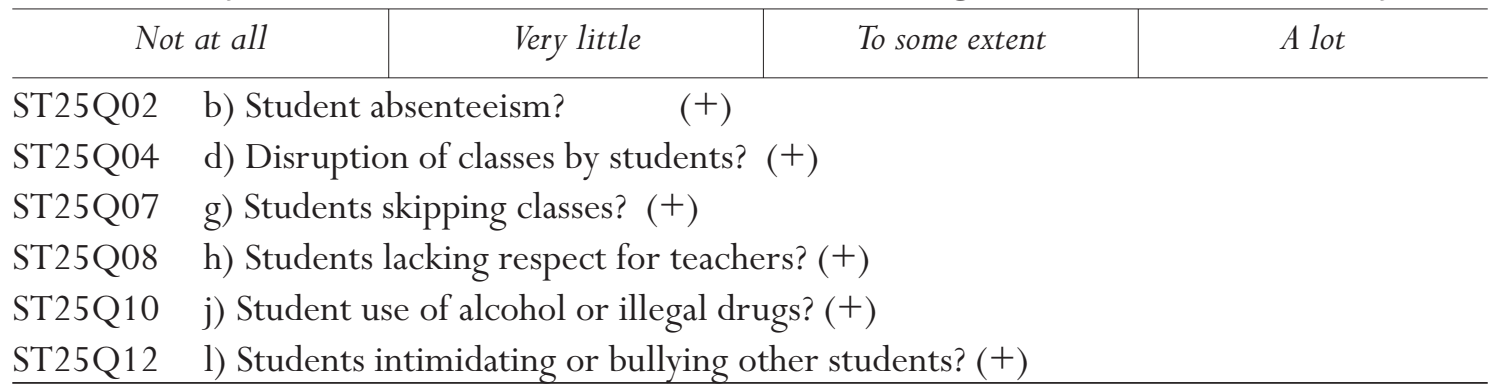

(+) Item inverted for IRT scaling.

\section{School principals' perceptions of teacher consensus on mathematics teaching (TCHCONS)}

The PISA 2003 index of school principals' perceptions of teacher consensus on mathematics teaching (TCHCONS) is derived from school principals' responses to the following three items asking about the school principals' views on having frequent disagreement among teachers regarding innovation, teacher expectations and teaching goals. A four-point scale with the response categories recoded as "strongly agree" (=0), "agree" $(=1)$, “disagree" $(=2)$, and "strongly disagree" $(=3)$ is used. All three items are not inverted for IRT scaling and positive values on this index indicate that higher levels of consensus among teachers are perceived by school principals.

\begin{tabular}{ll}
\hline \multicolumn{1}{c}{$\begin{array}{c}\text { Strongly agree } \\
\text { Q21 }\end{array}$} & $\begin{array}{l}\text { How much do you agree with these statements about innovation in your } \\
\text { school? }\end{array}$ \\
\cline { 2 - 2 } ST21Q03 & $\begin{array}{l}\text { c) There are frequent disagreements between "innovative" and "traditional" mathematics } \\
\text { teachers }\end{array}$ \\
\hline Q22 & $\begin{array}{l}\text { How much do you agree with these statements about teachers" } \\
\text { expectations in your school? }\end{array}$ \\
ST22Q03 & $\begin{array}{l}\text { c) There are frequent disagreements between mathematics teachers who consider each } \\
\text { other to be "too demanding" or "too lax". }\end{array}$ \\
\hline Q23 & $\begin{array}{l}\text { How much do you agree with these statements about teaching goals in } \\
\text { your school? }\end{array}$ \\
ST23Q03 & $\begin{array}{l}\text { c) There are frequent disagreements between mathematics teachers who consider } \\
\text { each other as "too focused on skill acquisition" or "too focused on the affective } \\
\text { development" of the student. }\end{array}$ \\
\hline
\end{tabular}

\section{School management}

\section{School autonomy (SCHAUTON)}

Similar to PISA 2000, the PISA 2003 index of school autonomy (SCHAUTON) is derived from school principals' responses to the 12 items (SC26Q01-SC26Q12) asking who has the main responsibility for different types of decisions regarding the management of the school. These items were used 
in PISA 2000, but the wording has been slightly modified for PISA 2003. As for PISA 2000, the category of "not a main responsibility of the school" (the first column) is recoded to 0 and those with ticks in other columns but not in the first were recoded to 1 . The recoded items are scaled using IRT and positive values on this index indicate school principals' perception of higher levels of school autonomy in decision making.

\section{Teacher participation (TCHPARTI)}

Similar to PISA 2000, the PISA 2003 index of teacher participation (TCHPARTI) is derived from school principals' responses to the 12 items (SC26Q01-SC26Q12) asking who has the main responsibility for different types of decisions regarding the management of the school. These items were used in PISA 2000, but the wording has been slightly modified for PISA 2003. As for PISA 2000, the category of "teacher" (the last column) indicating that teacher have a main responsibility is recoded to 1 and those with ticks in other columns but not in the last were recoded to 0 . The recoded items are scaled using IRT and positive values on this index indicate school principals' perception of higher levels of teacher participation in decision-making.

\section{Final student weight (W_FSTUWT)}

In the international data files, the variable W_FSTUWT is the final student weight. The sum of the weights constitutes an estimate of the size of the target population, i.e. the number of 15 -year-old students in grade 7 or above in that country attending school.

1. Students who were born abroad but had at least one parent born in the country of test are also classified as "native students". 



\section{APPENDIX 10 - SCORES ALLOCATED TO THE ITEMS}

\begin{tabular}{|c|c|c|c|c|c|}
\hline Item ID & Label & Type & Score 1 & Score 2 & Score 3 \\
\hline M033Q01 & P2000 A View with a Room & Multiple choice & 4 & & \\
\hline M034Q01T & P2000 Bricks & Closed-constructed response & 1 & & \\
\hline M124Q01 & P2000 Walking & Open-constructed response & 2 & & \\
\hline M124Q03T & P2000 Walking & Open-constructed response & 1 & 2 & 3 \\
\hline M144Q01T & P2000 Cube Painting & Closed-constructed response & 1 & & \\
\hline M144Q02T & P2000 Cube Painting & Closed-constructed response & 1 & & \\
\hline M144Q03 & P2000 Cube Painting & Multiple choice & 1 & & \\
\hline M144Q04T & P2000 Cube Painting & Closed-constructed response & 1 & & \\
\hline M145Q01T & P2000 Cubes & Closed-constructed response & 6 & & \\
\hline M150Q01 & P2000 Growing Up & Closed-constructed response & 1 & & \\
\hline M150Q02T & P2000 Growing Up & Closed-constructed response & 1 & 2 & \\
\hline M150Q03T & P2000 Growing Up & Open-constructed response & 1 & & \\
\hline M155Q01 & P2000 Population Pyramids & Open-constructed response & 1 & & \\
\hline M155Q02T & P2000 Population Pyramids & Open-constructed response & 1 & 2 & \\
\hline M155Q03T & P2000 Population Pyramids & Open-constructed response & 1 & 2 & \\
\hline M155Q04T & P2000 Population Pyramids & Complex multiple choice & 4 & & \\
\hline M179Q01T & P2000 Robberies & Open-constructed response & 1 & 2 & \\
\hline M192Q01T & P2000 Containers & Complex multiple choice & 2,3 & & \\
\hline M266Q01T & P2000 Carpenter & Complex multiple choice & 4 & & \\
\hline M273Q01T & P2000 Pipelines & Complex multiple choice & 1 & & \\
\hline M302Q01T & Car Drive & Closed-constructed response & 1 & & \\
\hline M302Q02 & Car Drive & Closed-constructed response & 1 & & \\
\hline M302Q03 & Car Drive & Open-constructed response & 1 & & \\
\hline M305Q01 & Map & Multiple choice & 3 & & \\
\hline M402Q01 & Internet Relay Chat & Short response & 1 & & \\
\hline M402Q02 & Internet Relay Chat & Short response & 1 & & \\
\hline M406Q01 & Running Tracks & Open-constructed response & 1 & & \\
\hline M406Q02 & Running Tracks & Open-constructed response & 1 & & \\
\hline M406Q03 & Running Tracks & Open-constructed response & 1 & & \\
\hline M408Q01T & Lotteries & Complex multiple choice & 4 & & \\
\hline M411Q01 & Diving & Short response & 1 & & \\
\hline M411Q02 & Diving & Multiple choice & 4 & & \\
\hline M413Q01 & Exchange Rate & Short response & 1 & & \\
\hline M413Q02 & Exchange Rate & Short response & 1 & & \\
\hline M413Q03T & Exchange Rate & Open-constructed response & 1 & & \\
\hline
\end{tabular}




\begin{tabular}{|c|c|c|c|c|c|}
\hline Item ID & Label & Type & Score 1 & Score 2 & Score 3 \\
\hline M420Q01T & Transport & Complex multiple choice & 4 & & \\
\hline M421Q01 & Height & Open-constructed response & 1 & & \\
\hline M421Q02T & Height & Complex multiple choice & 4 & & \\
\hline M421Q03 & Height & Multiple choice & 4 & & \\
\hline M423Q01 & Tossing Coins & Multiple choice & 1 & & \\
\hline M438Q01 & Exports & Closed-constructed response & 1 & & \\
\hline M438Q02 & Exports & Multiple choice & 5 & & \\
\hline M442Q02 & Braille & Closed-constructed response & 1 & & \\
\hline M446Q01 & Thermometer Cricket & Short response & 1 & & \\
\hline M446Q02 & Thermometer Cricket & Open-constructed response & 1 & & \\
\hline M447Q01 & Tile Arrangement & Multiple choice & 4 & & \\
\hline M462Q01T & Third Side & Open-constructed response & 1 & 2 & \\
\hline M464Q01T & The Fence & Short response & 1 & & \\
\hline M467Q01 & Coloured Candies & Multiple choice & 2 & & \\
\hline M468Q01T & Science Tests & Short response & 1 & & \\
\hline M474Q01 & Running Time & Closed-constructed response & 1 & & \\
\hline M484Q01T & Bookshelves & Short response & 1 & & \\
\hline M496Q01T & Cash Withdrawal & Complex multiple choice & 4 & & \\
\hline M496Q02 & Cash Withdrawal & Short response & 1 & & \\
\hline M505Q01 & Litter & Open-constructed response & 1 & & \\
\hline M509Q01 & Earthquake & Multiple choice & 3 & & \\
\hline M510Q01T & Choices & Short response & 1 & & \\
\hline M513Q01 & Test Scores & Open-constructed response & 1 & & \\
\hline M520Q01T & Skateboard & Short response & 1 & 2 & \\
\hline M520Q02 & Skateboard & Multiple choice & 4 & & \\
\hline M520Q03T & Skateboard & Short response & 4 & & \\
\hline M547Q01T & Staircase & Short response & 1 & & \\
\hline M555Q02T & Number Cubes & Complex multiple choice & 4 & & \\
\hline M559Q01 & Telephone Rates & Multiple choice & 4 & & \\
\hline M564Q01 & Chair Lift & Multiple choice & 2 & & \\
\hline M564Q02 & Chair Lift & Multiple choice & 3 & & \\
\hline M571Q01 & Stop the Car & Multiple choice & 4 & & \\
\hline M598Q01 & Making a Booklet & Closed-constructed response & 1 & & \\
\hline M603Q01T & Number Check & Complex multiple choice & 3 & & \\
\hline M603Q02T & Number Check & Short response & 1 & & \\
\hline M702Q01 & Support for President & Open-constructed response & 2 & & \\
\hline M704Q01T & The Best Car & Short response & 1 & & \\
\hline M704Q02T & The Best Car & Open-constructed response & 1 & & \\
\hline M710Q01 & Forecast of Rain & Multiple choice & 4 & & \\
\hline M800Q01 & Computer Game & Multiple choice & 3 & & \\
\hline M803Q01T & Labels & Short response & 1 & & \\
\hline
\end{tabular}




\begin{tabular}{|c|c|c|c|c|c|}
\hline Item ID & Label & Type & Score 1 & Score 2 & Score 3 \\
\hline M806Q01T & Step Pattern & Short response & 1 & & \\
\hline M810Q01T & Bicycles & Short response & 1 & & \\
\hline M810Q02T & Bicycles & Short response & 1 & & \\
\hline M810Q03T & Bicycles & Open-constructed response & 1 & 2 & \\
\hline M828Q01 & Carbon Dioxide & Open-constructed response & 1 & & \\
\hline M828Q02 & Carbon Dioxide & Short response & 1 & & \\
\hline M828Q03 & Carbon Dioxide & Short response & 1 & & \\
\hline M833Q01T & Seeing the Tower & Complex multiple choice & 5 & & \\
\hline R055Q01 & Drugged Spiders & Multiple choice & 4 & & \\
\hline R055Q02 & Drugged Spiders & Open-constructed response & 1 & & \\
\hline R055Q03 & Drugged Spiders & Open-constructed response & 2 & & \\
\hline R055Q05 & Drugged Spiders & Open-constructed response & 1 & & \\
\hline R067Q01 & Aesop & Multiple choice & 3 & & \\
\hline R067Q04 & Aesop & Open-constructed response & 1 & 2 & \\
\hline R067Q05 & Aesop & Open-constructed response & 1 & 2 & \\
\hline R102Q04A & Shirts & Open-constructed response & 1 & & \\
\hline R102Q05 & Shirts & Closed-constructed response & 1 & & \\
\hline R102Q07 & Shirts & Multiple choice & 3 & & \\
\hline R104Q01 & Telephone & Closed-constructed response & 1 & & \\
\hline R104Q02 & Telephone & Closed-constructed response & 1 & & \\
\hline R104Q05 & Telephone & Short response & 1 & 2 & \\
\hline R111Q01 & Exchange & Multiple choice & 4 & & \\
\hline R111Q02B & Exchange & Open-constructed response & 1 & 2 & \\
\hline R111Q06B & Exchange & Open-constructed response & 1 & 2 & \\
\hline R219Q01E & Employment & Short response & 1 & & \\
\hline R219Q01T & Employment & Closed-constructed response & 4 & & \\
\hline R219Q02 & Employment & Open-constructed response & 1 & & \\
\hline R220Q01 & South Pole & Short response & 1 & & \\
\hline R220Q02B & South Pole & Multiple choice & 1 & & \\
\hline R220Q04 & South Pole & Multiple choice & 4 & & \\
\hline R220Q05 & South Pole & Multiple choice & 3 & & \\
\hline R220Q06 & South Pole & Multiple choice & 3 & & \\
\hline R227Q01 & Optician & Multiple choice & 2 & & \\
\hline $\mathrm{R} 227 \mathrm{Q} 02 \mathrm{~T}$ & Optician & Complex multiple choice & 5,6 & 7 & \\
\hline R227Q03 & Optician & Open-constructed response & 1 & & \\
\hline R227Q06 & Optician & Short response & 1 & & \\
\hline S114Q03T & P2000 Greenhouse & Open-constructed response & 1 & & \\
\hline S114Q04T & P2000 Greenhouse & Open-constructed response & 1 & 2 & \\
\hline S114Q05T & P2000 Greenhouse & Open-constructed response & 1 & & \\
\hline S128Q01 & P2000 Cloning & Multiple choice & 1 & & \\
\hline S128Q02 & P2000 Cloning & Multiple choice & 1 & & \\
\hline
\end{tabular}




\begin{tabular}{|c|c|c|c|c|c|}
\hline Item ID & Label & Type & Score 1 & Score 2 & Score 3 \\
\hline S128Q03T & P2000 Cloning & Complex multiple choice & 2 & & \\
\hline S129Q01 & P2000 Daylight & Multiple choice & 1 & & \\
\hline S129Q02T & P2000 Daylight & Short response & 1 & 2 & \\
\hline S131Q02T & P2000 Good Vibrations & Open-constructed response & 1 & & \\
\hline S131Q04T & P2000 Good Vibrations & Open-constructed response & 1 & & \\
\hline S133Q01 & P2000 Research & Multiple choice & 3 & & \\
\hline S133Q03 & P2000 Research & Multiple choice & 1 & & \\
\hline S133Q04T & P2000 Research & Complex multiple choice & 3 & & \\
\hline S213Q01T & P2000 Clothes & Complex multiple choice & 4 & & \\
\hline S213Q02 & P2000 Clothes & Multiple choice & 1 & & \\
\hline S252Q01 & P2000 South Rainea & Multiple choice & 3 & & \\
\hline S252Q02 & P2000 South Rainea & Multiple choice & 1 & & \\
\hline $\mathrm{S} 252 \mathrm{Q} 03 \mathrm{~T}$ & P2000 South Rainea & Complex multiple choice & 2 & & \\
\hline S256Q01 & P2000 Spoons & Multiple choice & 1 & & \\
\hline S268Q01 & P2000 Algae & Multiple choice & 3 & & \\
\hline$S 268 Q 02 T$ & P2000 Algae & Open-constructed response & 1 & & \\
\hline S268Q06 & P2000 Algae & Multiple choice & 2 & & \\
\hline S269Q01 & P2000 Earth's Temperature & Open-constructed response & 1 & & \\
\hline S269Q03T & P2000 Earth’s Temperature & Open-constructed response & 1 & & \\
\hline$S 269 Q 04 T$ & P2000 Earth’s Temperature & Complex multiple choice & 4 & & \\
\hline S304Q01 & Water & Open-constructed response & 1 & & \\
\hline S304Q02 & Water & Multiple choice & 3 & & \\
\hline S304Q03a & Water & Open-constructed response & 1 & & \\
\hline S304Q03b & Water & Open-constructed response & 1 & & \\
\hline S326Q01 & Milk & Open-constructed response & 1 & & \\
\hline S326Q02 & Milk & Open-constructed response & 1 & & \\
\hline S326Q03 & Milk & Multiple choice & 2 & & \\
\hline S326Q04T & Milk & Complex multiple choice & 3 & & \\
\hline S327Q01T & Tidal Energy & Complex multiple choice & 2 & & \\
\hline $\mathrm{X} 402 \mathrm{Q} 01 \mathrm{~T}$ & Library System & Closed-constructed response & 1 & & \\
\hline $\mathrm{X} 402 \mathrm{Q} 02 \mathrm{~T}$ & Library System & Open-constructed response & 1 & 2 & 3 \\
\hline $\mathrm{X} 412 \mathrm{Q} 01$ & Design by Numbers & Multiple choice & 2 & & \\
\hline $\mathrm{X} 412 \mathrm{Q} 02$ & Design by Numbers & Multiple choice & 4 & & \\
\hline $\mathrm{X} 412 \mathrm{Q} 03$ & Design by Numbers & Open-constructed response & 1 & 2 & \\
\hline X414Q01 & Course Design & Open-constructed response & 1 & 2 & \\
\hline $\mathrm{X} 415 \mathrm{Q} 01 \mathrm{~T}$ & Transit System & Open-constructed response & 1 & 2 & \\
\hline X417Q01 & Children’s Camp & Open-constructed response & 1 & 2 & \\
\hline $\mathrm{X} 423 \mathrm{Q} 01 \mathrm{~T}$ & Freezer & Multiple choice & 5,6 & & \\
\hline $\mathrm{X} 423 \mathrm{Q} 02 \mathrm{~T}$ & Freezer & Multiple choice & 3 & & \\
\hline X430Q01 & Energy Needs & Closed-constructed response & 1 & & \\
\hline $\mathrm{X} 430 \mathrm{Q} 02$ & Energy Needs & Open-constructed response & 1 & 2 & \\
\hline
\end{tabular}




\begin{tabular}{|c|c|c|c|c|c|}
\hline Item ID & Label & Type & Score 1 & Score 2 & Score 3 \\
\hline X601Q01T & Cinema Outing & Multiple choice & 5 & 6 & \\
\hline X601Q02 & Cinema Outing & Multiple choice & 3 & & \\
\hline $\mathrm{X} 602 \mathrm{Q} 01$ & Holiday & Closed-constructed response & 1 & & \\
\hline $\mathrm{X} 602 \mathrm{Q} 02$ & Holiday & Open-constructed response & 1 & 2 & \\
\hline X603Q01 & Irrigation & Open-constructed response & 1 & & \\
\hline $\mathrm{X603Q} Q 2 \mathrm{~T}$ & Irrigation & Multiple choice & 3 & & \\
\hline $\mathrm{X} 603 \mathrm{Q} 03$ & Irrigation & Open-constructed response & 1 & & \\
\hline
\end{tabular}



Baumert, J., S. Gruehn, S. Heyn, O. Köller and K.U. Schnabel (1997), Bildungsverläufe und Psychosoziale Entwicklung im Jugendalter (BIJU): Dokumentation - Band 1, Max-Planck-Institut für Bildungsforschung, Berlin.

Baumert, J., S. Heyn and O. Köller (1994), Das Kieler Lernstrategien-Inventar (KSI), Institut für die Pädagogik der Naturwissenschaften an der Universität Kiel, Kiel.

Beaton A.E., I.V.S. Mullis, M.O. Martin, E.J. Gonzalez, D.L. Kelly and T.A. Smith (1997), Mathematics Achievement in the Middle SchoolYears: IEA's Third International Mathematics and Science Study (TIMSS), Boston College, Chestnut Hill.

Beaton, A.E. (1987), The NAEP 1983-1984 Technical Report, Educational Testing Service, Princeton.

Beaton, A.E. (1988), The NAEP 1985-86 Reading Anomaly: A Technical Report, Educational Testing Service, National Assessment of Educational Progress, Princeton.

Bloom, B.S. (1979), Caractéristiques individuelles et apprentissage scolaire, Éditions Labor, Bruxelles.

Bryk, A.S. and S.W. Raudenbush (1992), Hierarchical Linear Models in Social and Behavioral Research: Applications and Data Analysis Methods, Sage Publications, Beverly Hills.

Cornfield, J. (1951), "A Method for Estimating Comparative Rates from Clinical Data. Applications to Cancer of the Lung, Breast, and Cervix", Journal of the National Cancer Institute Vol. 11, Oxford University Press, Oxford, pp. 1269-1275.

Dunn, O.J. (1961), "Multiple Comparisons among Means", Journal of the American Statistical Association, Vol. 56, American Statistical Association, Alexandria, pp. 52-64.

Eignor, D., C. Taylor, I. Kirsch and J. Jamieson (1998), "Development of a Scale for Assessing the Level of Computer Familiarity of TOEFL Students", TOEFL Research Report No. 60, Educational Testing Service, Princeton.

Ganzeboom, H.B.G., P. de Graaf and D. J.Treiman, with J. de Leeuw, (1992), "A Standard International Socioeconomic Index of Occupational Status”, Social Science Research, Vol. 21(1), Academic Press, New York, pp. 1-56.

Goldstein, H. (1995), Multilevel Statistical Models, second edition, Edward Arnold, London.

Goldstein, H. (1997), "Methods in School Effectiveness Research", School Effectiveness and School Improvement, 8, Swets \& Zeitlinger, Lisse, The Netherlands, pp.369-395.

Gonzalez, E.J. and A.M. Kennedy (2003), PIRLS 2001 User Guide for the International Database, Boston College, Chestnut Hill.

Guilford, J.P. (1954), Psychometric Methods, MacGraw Hill, New York.

Husen, T. (1967), International Study of Achievement in Mathematics: a Comparison of Twelve Countries, Almqvist \& Wiksells, Uppsala.

Judkins, D.R. (1990), "Fay's Method of Variance Estimation”, Journal of Official Statistics, Vol. 6, No. 3, Statistics Sweden, Stockholm, pp. 223-239.

Kish, L. and M.R. Frankel (1974), "Inference from Complex Sample”, Journal of the Royal Statistical Society (B), 36, p. 1-37. Royal Statistical Society, London.

Kish, L. (1987), Statistical Design for Research, John Wiley \& Sons, New York.

Marsh, H.W., R.J. Shavelson and B.M. Byrne (1992), "A Multidimensional, Hierarchical Self-concept”, in R. P. Lipka andT. M. Brinthaupt (eds.), Studying the Self: Self-Perspectives across the Life-Span, State University of New York Press, Albany. 
Monseur, C. and R.J. Adams (2002) "Plausible Values: How to Deal with Their Limitations", paper presented at the International Objective Measurement Workshop, New Orleans, 6-7 April.

OECD (Organisation for Economic Co-operation and Development) (1998), Education at a Glance - OECD Indicators, OECD, Paris.

OECD (1999a), Measuring Student Knowledge and Skills - A New Framework for Assessment, OECD, Paris.

OECD (1999b), Classifying Educational Programmes - Manual for ISCED-97 Implementation in OECD Countries, OECD, Paris.

OECD (2001), Knowledge and Skills for Life - First Results from PISA 2000, OECD, Paris.

OECD (2002a), Programme for International Student Assessment - Manual for the PISA 2000 Database, OECD, Paris.

OECD (2002b), Sample Tasks from the PISA 2000 Assessment - Reading, Mathematical and Scientific Literacy, OECD, Paris.

OECD (2002c), Programme for International Student Assessment - PISA 2000 Technical Report, OECD, Paris.

OECD (2002d), Reading for Change: Performance and Engagement across Countries, OECD, Paris.

OECD (2003a), Literacy Skills for the World of Tomorrow - Further Results from PISA 2000, OECD, Paris.

OECD (2003b), The PISA 2003 Assessment Framework - Mathematics, Reading, Science and Problem Solving Knowledge and Skills, OECD, Paris.

OECD (2004a), Learning for Tomorrow's World - First Results from PISA 2003, OECD, Paris.

OECD (2004b), Problem Solving for Tomorrow'sWorld - First Measures of Cross-Curricular Competencies from PISA 2003, OECD, Paris.

OECD (forthcoming), PISA 2003 Technical Report, OECD, Paris.

Owens, L. and J. Barnes (1992), Learning Preferences Scales, Australian Council for Educational Research, Camberwell.

Rust, K. and S. Krawchuk (2002), Replicate Variance Estimation Methods for International Surveys of Student Achievement, International Conference on Improving Surveys, Copenhagen.

Rust, K.F. and J.N.K. Rao (1996), "Variance Estimation for Complex Surveys Using Replication Techniques", Statistical Methods in Medical Research, Vol. 5, Hodder Arnold, London, pp. 283-310.

Rust, K.F. (1996), TIMSS 1995 working paper.

Warm, T.A. (1989), "Weighted Likelihood Estimation of Ability in Item Response Theory", Psychometrika, Vol. 54(3), Psychometric Society, Williamsburg, Va., etc., pp. 427-450.

Westat (2000), WesVar complex samples 4.0. Westat. Rockville.

Wright, B.D. and M.H. Stone (1979), Best Test Design: Rasch Measurement, MESA Press, Chicago.

Wu, M. and R.J. Adams (2002), "Plausible Values - Why They Are Important", paper presented at the International Objective Measurement Workshop, New Orleans, 6-7 April.

Wu, M.L., R.J. Adams and M.R. Wilson (1997), Conquest: Multi-Aspect Test Software [computer program], Australian Council for Educational Research, Camberwell. 


\author{
OECD PUBLICATIONS, 2 rue André-Pascal, PARIS CEDEX 16 \\ PRINTED IN FRANCE \\ (982005031P) ISBN 92-64-01065-3 - No. 541312005
}

\begin{abstract}
Although the OECD has attempted to provide accurate information in this Manual, the OECD assumes no responsibility for the accuracy of the information or for any errors or omissions contained therein. The contents of this Manual may be changed at any time without notice. Mention of non-OECD products or services is for information purposes only and constitutes neither an endorsement nor a recommendation.
\end{abstract}

OECD, PISA, OECD/PISA and their respective logos are trademarks belonging to the OECD and should not be used without permission. All names and logos of other parties or products are used for identification purposes only and may be trademarks of their respective owners. In particular:

SAS and all other SAS Institute Inc. product or service names are registered trademarks or trademarks of SAS Institute Inc. in the United States and/or other countries.

SPSS is either a registered trademark or a trademark of SPSS Inc. in the United States and/or other countries.

Microsoft Excel, Microsoft Word and Microsoft PowerPoint are either a registered trademark or a trademark of Microsoft Corporation in the United States and/or other countries.

Lotus 1-2-3 is either a registered trademark or a trademark of IBM in the United States and/or other countries.

WordPerfect is a registered trademark or trademark of Corel Corporation and/or its subsidiaries in Canada, the United States and/or other countries. 


\section{PISA 2003 Data Analysis Manual SPSS $^{\circledR}$ USERS}

The OECD Programme for International Student Assessment's (PISA) 2003 survey collected data on students' performances in reading, mathematics, science and problem solving, as well as contextual information on students' background, home characteristics and school factors which could influence performance. The first results were released in December 2004 and presented in two volumes: Learning for Tomorrow's World First Results from PISA 2003 (OECD, 2004) and Problem Solving for Tomorrow's World - First Measures of Cross-Curricular Competencies from PISA 2003 (OECD, 2004).

This publication is an essential tool for researchers, as it provides all the information required to understand the PISA 2003 database and perform analyses in accordance with the complex methodologies used to collect and process the data. It includes detailed information on how to analyse the PISA 2003 data, enabling researchers to both reproduce the initial results and to undertake further analyses. The publication includes:

- Introductory chapters explaining the statistical theories and concepts required to analyse the PISA data, including full chapters on how to apply replicate weights and undertake analyses using plausible values;

- Worked examples providing full syntax in SPSS ${ }^{\circledR}$; and

- Comprehensive description of the OECD PISA 2003 international database.

The PISA 2003 database comprises micro-level data on student performance for 41 countries collected in 2003, together with students' responses to the PISA 2003 questionnaires and the test questions. It can be downloaded from www.pisa.oecd.org.

\section{THE OECD PROGRAMME FOR INTERNATIONAL STUDENT ASSESSMENT (PISA)}

PISA is a collaborative process among the 30 member countries of the OECD and nearly 30 partner countries. It brings together scientific expertise from the participating countries and is steered by their governments on the basis of shared, policy-driven interests. PISA is an unprecedented attempt to measure student achievement, as is evident from some of its features:

- The literacy approach: PISA aims to define each assessment area (mathematics, science, reading and problem solving) not mainly in terms of mastery of the school curriculum, but in terms of the knowledge and skills needed for full participation in society.

- A long-term commitment: It will enable countries to monitor regularly and predictably their progress in meeting key learning objectives.

- The age-group covered: By assessing 15-year-olds, i.e. young people near the end of their compulsory education, PISA provides a significant indication of the overall performance of school systems.

- The relevance to lifelong learning: PISA does not limit itself to assessing students' knowledge and skills but also asks them to report on their own motivation to learn, their beliefs about themselves and their learning strategies.

OECD's books, periodicals and statistical databases are now available via www.SourceOECD.org, our online library.

This book is available to subscribers to the following SourceOECD themes:

Education and Skills

Emerging Economies

Transition Economies

Ask your librarian for more details on how to access OECD books on line, or write to us at:

SourceOECD@oecd.org

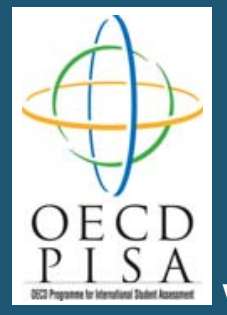

www.oecd.org

www.pisa.oecd.org

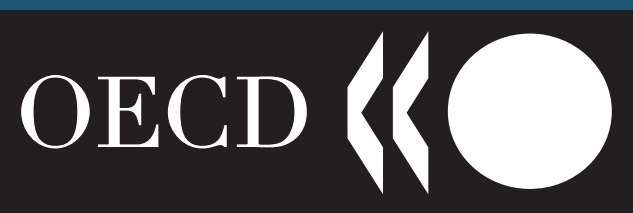

ISBN 92-64-01065-3

$982005031 \mathrm{P}$ 